\title{
Synthesis of Sterically Hindered Primary Amines by Concurrent Tandem Photoredox Catalysis
}

Michael C. Nicastri, ${ }^{1}$ Dan Lehnherr, ${ }^{*}, 2$ Yu-hong Lam, ${ }^{3}$ Daniel A. DiRocco, ${ }^{2}$ Tomislav Rovis ${ }^{*}, 1$

${ }^{1}$ Department of Chemistry, Columbia University, New York, New York, 10027, United States ${ }^{2}$ Process Research and Development, Merck \& Co., Inc., Rahway, New Jersey, 07065, United States ${ }^{3}$ Computational and Structural Chemistry, Merck \& Co., Inc., Rahway, New Jersey, 07065, United States

\section{dan.lehnherr@merck.com tr2504@columbia.edu}

\section{Table of contents}

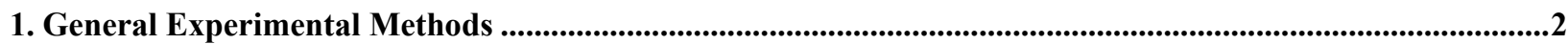

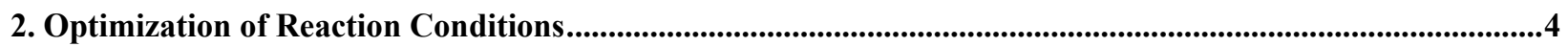

2.1 High-throughput Experimentation: Photocatalyst Screen (Selected Data) ……............................................

2.2 The Effect of Super-stoichiometric Equivalents of 4-cyanopyridine................................................................

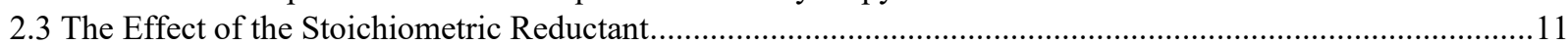

3. $O$-Benzoyl Oxime \& Iminium Hydrochloride Substrate Scope ........................................................................16

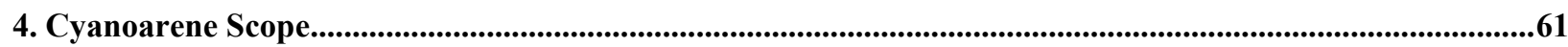

5. Synthesis of $O$-Benzoyl Oximes Starting Materials ....................................................................................................83

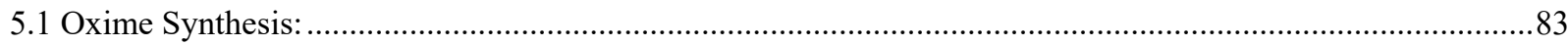

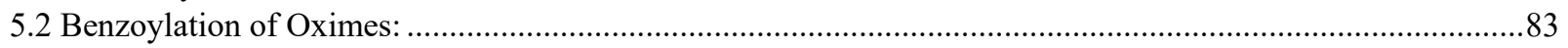

6. Synthesis of Iminium Hydrochlorides...................................................................................................................126

7. UV-Vis Absorption/Emission Spectra and Stern-Volmer Plot ..............................................................................128

8. DFT Computational Study.....................................................................................................................................129

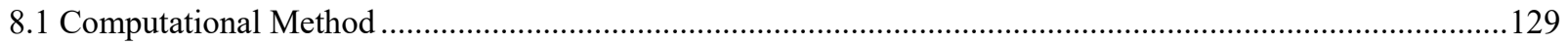

8.3 DFT Illustrations of Alpha-Amino Radical Without and With ortho-Methyl Substituent .............................132

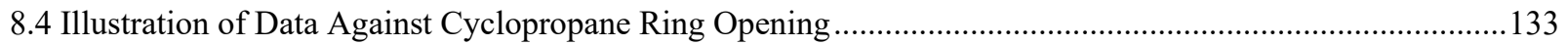

8.5 Cartesian Coordinates and Energies of DFT Optimized Structures..............................................................134

9. References ……..............................................................................................................................................................204 


\section{General Experimental Methods}

Heating of reaction mixtures was performed using a temperature-controlled hotplate equipped with stirring and an active thermocouple. Stirring of reaction mixtures was performed using magnetic stirring, unless noted otherwise. Preparative scale photochemical reactions run in $4 \mathrm{~mL}$ vials refer to ChemGlass vials part number CG4912-01 (1 Dram, $15 \times 45 \mathrm{~mm}, 13-425$ Thread) equipped with pressure relief septa caps (prevents pressure over 150 psig); glass made from 33 expansion borosilicate glass which conforms to ASTM E-438 Type 1-Class A, USP Type 1 and ISO 3585; vials were equipped with a magnetic stir bar (Biotage ${ }^{\circledR}$ Stirbars, part number 355543). Evaporation and concentration in vacuo were done using variable vacuum via a vacuum controlled (ca. $400-40 \mathrm{mmHg}$ ).

Materials. Reagents were purchased in reagent grade from commercial suppliers (Advanced ChemBlocks, Inc.; Alfa Aesar; Angene International Limited; AsaTech, Inc.; AURUM Pharmatech LLC; ChemBridge Corporation; CombiBlocks, Inc.; Enamine LLC; Key Organics Inc.; Life Chemicals, Inc.; Matrix Scientific; Princeton BioMolecular Research, Inc.; Sigma-Aldrich; TCI America) and used without further purification, unless otherwise described. Anhydrous solvents (acetonitrile, dimethylsulfoxide) were obtained from Sigma-Aldrich as part of their Sure/SealTM bottles product line. NMR solvents, specifically, $d_{6}$-DMSO (anhydrous, 99.9\% $d$-content, catalog 570672-50G) and $\mathrm{CD}_{3} \mathrm{CN}$ (anhydrous, $99.8 \% d$-content, catalog 151807-10X1ML), were purchased from Sigma-Aldrich Isotope Laboratories in sealed ampules and used as received. HPLC and UPLC eluent mixtures made use of HPLC grade $\mathrm{MeCN}$ and $\mathrm{H}_{2} \mathrm{O}$.

Instrumentation. Proton nuclear magnetic resonance $\left({ }^{1} \mathrm{H}\right.$ NMR) spectra, carbon nuclear magnetic resonance $\left({ }^{13} \mathrm{C}\right.$ NMR) spectra, proton coupled fluorine nuclear magnetic resonance $\left({ }^{19} \mathrm{~F} \mathrm{NMR}\right)$, and proton decoupled fluorine nuclear magnetic resonance $\left({ }^{19} \mathrm{~F}\left\{{ }^{1} \mathrm{H}\right\}\right.$ NMR) were recorded at $25{ }^{\circ} \mathrm{C}$ (unless stated otherwise) on a Bruker DRX-500 spectrometer. Chemical shifts for protons are reported in parts per million downfield from tetramethylsilane and are referenced to residual proton of the NMR solvent according to values reported in the literature. ${ }^{1}$ Chemical shifts for carbon are reported in parts per million downfield from tetramethylsilane and are referenced to the carbon resonances of the NMR solvent. For samples in $\mathrm{CDCl}_{3}$ the residual solvent signal was referenced to $7.26 \mathrm{ppm}$ for ${ }^{1} \mathrm{H}$ and 77.0 ppm for ${ }^{13} \mathrm{C}$, for samples in $\mathrm{CD}_{3} \mathrm{CN}$ the residual solvent signal was referenced to $1.94 \mathrm{ppm}$ for ${ }^{1} \mathrm{H}$ and $1.32 \mathrm{ppm}$ for ${ }^{13} \mathrm{C}$, and for samples in $d_{6}$-DMSO the residual solvent signal was referenced to $2.50 \mathrm{ppm}$ for ${ }^{1} \mathrm{H}$ and $39.52 \mathrm{ppm}$ for ${ }^{13} \mathrm{C}$. Data are represented as follows: chemical shift, integration, multiplicity $(\mathrm{br}=$ broad, $\mathrm{s}=$ singlet, $\mathrm{d}=$ doublet, $\mathrm{t}=$ triplet, $\mathrm{q}=$ quartet, quint $=$ quintet, $\mathrm{m}=$ multiplet, $\mathrm{dt}=$ doublet of triplets), coupling constants $(J)$ in $\mathrm{Hertz}(\mathrm{Hz})$. Quantitative ${ }^{1} \mathrm{H}$ NMR analysis ( ${ }^{1} \mathrm{H}$ qNMR) refers to standard ${ }^{1} \mathrm{H}$ NMR using the following parameters: $\mathrm{d} 1=60$ seconds, number of scans $=8$. Details of the electrochemistry equipment setup are described below. LRMS data was obtained using a Waters Acquity UPLC interfaced with a Waters Xevo G2 QTof ESI with the source temperature set to $120{ }^{\circ} \mathrm{C}$. UV-vis absorption and emission spectra were collected using a Horiba Duetta Simultaneous EEM Fluorescence and UV-Vis-NIR Spectrophotometer using a $1 \mathrm{~cm} \times 1 \mathrm{~cm}$ quartz cuvette.

Photochemistry Equipment. Photochemical reactions were conducted on four different scales: $0.01 \mathrm{mmol} ; 0.1 \mathrm{mmol}$; $0.3 \mathrm{mmol}$; and $3.6 \mathrm{mmol}$, in three separate photoreactor setups. High-throughput experimentation reactions $(0.01$ mmol scale) were conducted as described by DiRocco et. al. ${ }^{2}$ The reaction optimization and scope reactions, conducted, at $0.1 \mathrm{mmol}$ and $0.3 \mathrm{mmol}$ respectively, were irradiated for 2.5 hours in a temperature controlled $\left(40{ }^{\circ} \mathrm{C}\right)$ TAK 120 photoreactor with $455 \mathrm{~nm}$ light (5 W per vial), enabling up to 10 reactions to be run in parallel at a time. ${ }^{3} \mathrm{~A}$ large scale reaction $(3.6 \mathrm{mmol})$ was conducted in a PennOC $\mathrm{m} 1$ photoreactor which can accommodate a $40 \mathrm{~mL}$ glass vial. ${ }^{4}$

Abbreviations. $\mathrm{DMSO}=$ dimethylsulfoxide, $\mathrm{ESI}=$ electrospray ionization $\left(\mathrm{ESI}^{+}\right.$and $\mathrm{ESI}^{-}$denote positive or negative mode, respectively), EtOAc = ethyl acetate, $\mathrm{GC}=$ gas chromatography, $\mathrm{HPLC}=$ high pressure liquid chromatography, $\mathrm{UPLC}=$ ultrahigh pressure liquid chromatography, UPLC-MS $=\mathrm{HR}=$ high resolution, $\mathrm{LC}=$ liquid chromatography, $\mathrm{LED}=$ light emitting diode, $\mathrm{LR}=$ low-resolution, $\mathrm{MeCN}=$ acetonitrile, $\mathrm{MeOH}=$ methanol, $\mathrm{MS}=$ mass spectrometry, $\mathrm{NA}=$ not available, $\mathrm{ND}=$ not detected, $\mathrm{PTFE}=$ polytetrafluoroethylene, $\mathrm{qNMR}=$ quantitative $\mathrm{NMR}, \mathrm{QToF}=$ quadrupole time of flight, $\mathrm{RBF}=$ round bottom flask, $\mathrm{rpm}=$ rounds per minute, $\mathrm{RT}=$ room temperature $\left(\mathrm{ca} .25^{\circ} \mathrm{C}\right)$, $\mathrm{THF}=$ tetrahydrofuran, $\mathrm{v} / \mathrm{v}=$ volume per volume percent, $\mathrm{w} / \mathrm{v}=$ weight per volume percent, DIPA = diisopropylamine, DIPEA = diisopropylethylamine, $\mathrm{BzOH}=$ benzoic acid.

Symbols. $\delta=$ parts per million ( $\mathrm{ppm}), T=$ time, $t_{r}=$ retention time, $t_{r r}=$ relative retention time, $[\text { compound }]_{\mathrm{o}}=$ initial concentration of specific compound, [compound $]_{\mathrm{T}}=$ total initial concentration of specific compound assuming no speciation of the compound has occurred. 
UPLC MS Method for Assay Yield and LRMS Determination. Samples were dissolved in MeCN and $1 \mu \mathrm{L}$ injection volumes were used. The Waters UPLC was equipped with an ACQUITY UPLCTM BEH C18 VanGuard Precolumn (130 Å pore size, $1.7 \mu \mathrm{m}$ particle size, $2.1 \mathrm{~mm} \times 5 \mathrm{~mm}$ length; part number for 3/pkg: 186003975$)$ flowing into an ACQUITY UPLC ${ }^{\text {TM }}$ HSS T3 Column $(100 \AA$ pore size, $1.8 \mu \mathrm{m}$ particle size, $2.1 \mathrm{~mm}$ internal diameter $\times 50$ mm length; part number 186003538 ) operating at $55^{\circ} \mathrm{C}$ with a $0.8 \mathrm{~mL} / \mathrm{min}$ flow rate of a binary eluent mixture (eluent $\mathrm{A}$ and $\mathrm{B}$, prepared as described below). The 4 min method used the following eluent gradient: gradient from $95 \% \mathrm{~A}$, $5 \% \mathrm{~B}$ at $t=0 \mathrm{~min}$ to $80 \% \mathrm{~A}, 20 \% \mathrm{~B}$ at $t=1.50 \mathrm{~min}$, followed by a gradient to reach $0.1 \% \mathrm{~A}, 99.9 \% \mathrm{~B}$ at $t=3.90 \mathrm{~min}$, and a subsequent change to $95 \% \mathrm{~A}, 5 \% \mathrm{~B}$ at $t=3.91 \mathrm{~min}$ until $t=4.00 \mathrm{~min}$. Two different eluent systems were used: (system 1) eluent $\mathrm{A}=$ aqueous mobile phase $\left(3960 \mathrm{~mL} \mathrm{H}_{2} \mathrm{O}, 40 \mathrm{~mL} \mathrm{pH} 3.5\right.$ buffer, details below), eluent $\mathrm{B}=$ organic mobile phase $\left(3600 \mathrm{~mL} \mathrm{MeCN}, 360 \mathrm{~mL} \mathrm{H}_{2} \mathrm{O}, 40 \mathrm{~mL}\right.$ pH 3.5 buffer, details below. The $\mathrm{pH} 3.5$ buffer is prepared by dissolving ammonium formate $(12.6 \mathrm{~g}, 0.200 \mathrm{~mol})$ and formic acid $(7.9 \mathrm{~mL}, 9.6 \mathrm{~g}, 0.21 \mathrm{~mol})$ in $1000 \mathrm{~mL} \mathrm{H}_{2} \mathrm{O}$. For analysis of the reaction mixture containing product $5 \mathrm{~s}$ derived via the oxime photochemistry, a different mobile phase gradient in the context of a 6 min method. The 6 min method used the following eluent gradient: gradient from $95 \% \mathrm{~A}$, $5 \% \mathrm{~B}$ at $t=0 \mathrm{~min}$ to $80 \% \mathrm{~A}, 20 \% \mathrm{~B}$ at $t=2.25 \mathrm{~min}$, followed by a gradient to reach $0.1 \% \mathrm{~A}, 99.9 \% \mathrm{~B}$ at $t=5.85 \mathrm{~min}$, and a subsequent change to $95 \% \mathrm{~A}, 5 \% \mathrm{~B}$ at $t=5.87 \mathrm{~min}$ until $t=6.00 \mathrm{~min}$.

UPLC-MS Method for Yield Determination. Reactions were diluted in volumetric flasks with a known amount of biphenyl (Sigma Aldrich, B34656) as an internal standard ratio. Aliquots of this reaction-internal standard mixture were then diluted in vials for UPLC-MS analysis. Samples were then analyzed by the UPLC MS Method for Assay Yield and LRMS Determination. Analysis of the chromatograph for each reaction mixture yielded LRMS data for product identification and a corresponding peak which was analyzed for UV-Vis absorption. The raw area under the curve for the product peak at $210 \mathrm{~nm}$ was then compared to the raw area under the curve for the biphenyl internal standard. The ratio between these two values was then utilized to determine yield via calibration of the UPLC-MS from an authentic sample isolated from the reaction mixtures, or obtained from other chemical syntheses.

Reactions were diluted with $\mathrm{MeCN}$ in a $25 \mathrm{~mL}$ flask containing $5 \mathrm{~mL}$ of a $12.5 \mathrm{mM}$ biphenyl solution in $\mathrm{MeCN}$. The theoretical yield in mmol was then utilized to calculate the concentration of product in the $25 \mathrm{~mL}$ flask when the reaction was quantitative. Authentic sample was then utilized to develop a calibration curve between a concentration range which encompassed $0 \%$ to $>100 \%$ yield. The yield of the reaction was then determined by comparing the obtained product to internal standard ratio vs. the calibration curve.

Caution! Cyanide may be produced as a byproduct in these reactions. Cyanide is toxic and could lead to the release of HCN gas (extremely toxic). Reactions and their aqueous work-up should be conducted in well-ventilated enclosures (e.g. fumehood) and aqueous cyanide-containing waste should be kept basic and disposed of in accordance with local guidelines. 


\section{Optimization of Reaction Conditions}

\subsection{High-throughput Experimentation: Photocatalyst Screen (Selected Data)}

In a glove box under a nitrogen atmosphere, the components of the reaction were added to a $20 \mathrm{~mL}$ vial containing a stir bar. The oxime 3a $(0.66 \mathrm{mmol}, 2.2$ equiv.) was added followed by 4-cyanopyridine (4a) ( $0.3 \mathrm{mmol}$, $1 \mathrm{eq})$. The contents of the vial were then dissolved in DMSO (3 mL, 0.1 M) and stirred until homogeneous. After stirring, DIPA (157 $\mu \mathrm{L}, 0.9 \mathrm{mmol}, 3.6$ equiv.). The reaction was stirred for 10 minutes until the reaction solution was homogeneous. This was repeated three further times, where acetone, acetonitrile, and 1,2-dichloroethane were used as the reaction solvent. Pre-dosed photocatalysts (22 photocatalysts and 2 control reactions consisting of well with no catalyst and either the presence or absence of light) in $1 \mathrm{~mL}$ titer vials for a subtotal of 24 different conditions. These 24 conditions were screened with the stock solutions created in 4 different solvents (DMSO, MeCN, DMF, 1,2dichloroethane) in a single 96-well plate array. The reactions were irradiated with blue light (455 nm, $30 \mathrm{~mA}$ LED, $37^{\circ} \mathrm{C}$ ) for $20 \mathrm{~h}$. The reactions were quenched by opening the vials and exposing the contents to oxygen. Triethylamine $(100 \mu \mathrm{L})$ was added to each reaction to quench any potential hydrogencyanide that was evolved in the reaction. An aliquot from each reaction $(100 \mu \mathrm{L})$ was diluted into a solution of biphenyl in acetonitrile $(500 \mu \mathrm{L}, 2 \mathrm{mM}$ biphenyl). Each reaction was analyzed by UPLC-MS. The reaction yield was determined via calibration of the UV-Vis absorption signal for product.

Caution! Cyanide may be produced as a byproduct in these reactions. Cyanide is toxic and could lead to the release of HCN gas (extremely toxic). Reactions and their aqueous work-up should be conducted in well-ventilated enclosures (e.g. fumehood) and aqueous cyanide-containing waste should be kept basic and disposed of in accordance with local guidelines.

Results can be found on the following page. 
Table S1. Selected results of reaction discovery via high-throughput experimentation

\begin{tabular}{|c|c|c|c|}
\hline Photocatalyst & Solvent & Reductant & Yield \\
\hline 4-Cz-IPN & Acetone & DIPA & $30 \%$ \\
\hline 4-DPA-IPN & Acetone & DIPA & $0 \%$ \\
\hline Mes-Acri & Acetone & DIPA & $0 \%$ \\
\hline tBu-Acri-N-Ph & Acetone & DIPA & $0 \%$ \\
\hline tetraMeO-Acri-N-Ph & Acetone & DIPA & $0 \%$ \\
\hline tetraMeO-Acri-N-diMeOPh & Acetone & DIPA & $0 \%$ \\
\hline $\mathrm{Ru}(\mathrm{bpz})_{3}\left(\mathrm{PF}_{6}\right)_{2}$ & Acetone & DIPA & $0 \%$ \\
\hline $\mathrm{Ru}(\mathrm{bpy})_{3}\left(\mathrm{PF}_{6}\right)_{2}$ & Acetone & DIPA & $0 \%$ \\
\hline $\mathrm{Ru}(\mathrm{bpm})_{3}(\mathrm{Cl})_{2}$ & Acetone & DIPA & $0 \%$ \\
\hline $\mathrm{Ru}(\text { phen })_{3} \mathrm{Cl}_{2} \times \mathrm{H}_{2} \mathrm{O}$ & Acetone & DIPA & $0 \%$ \\
\hline $\mathrm{Ru}\left(\mathrm{p}-\mathrm{CF}_{3}-\mathrm{bpy}\right)_{3}\left(\mathrm{BF}_{4}\right)_{2}$ & Acetone & DIPA & $0 \%$ \\
\hline $\operatorname{Ir}(\mathrm{ppy})_{2}(\mathrm{dtbpy})\left(\mathrm{PF}_{6}\right)$ & Acetone & DIPA & $0 \%$ \\
\hline $\operatorname{Ir}\left[\mathrm{dF}\left(\mathrm{CF}_{3}\right) \mathrm{ppy}\right]_{2}(\mathrm{dtbpy}) \mathrm{PF}_{6}$ & Acetone & DIPA & $0 \%$ \\
\hline $\operatorname{Ir}(\mathrm{ppy})_{3}$ & Acetone & DIPA & $0 \%$ \\
\hline $\operatorname{Ir}(\mathrm{dF}-\mathrm{ppy})_{3}$ & Acetone & DIPA & $0 \%$ \\
\hline $\operatorname{Ir}[\mathrm{dF}(\mathrm{F}) \mathrm{ppy}]_{2}(\mathrm{dtbpy}) \mathrm{PF}_{6}$ & Acetone & DIPA & $0 \%$ \\
\hline $\mathrm{Ir}[\mathrm{dF}(\mathrm{H}) \mathrm{ppy}]_{2}(\mathrm{dtbpy}) \mathrm{PF}_{6}$ & Acetone & DIPA & $0 \%$ \\
\hline $\operatorname{Ir}[\mathrm{dF}(\mathrm{Me}) \mathrm{ppy}]_{2}(\mathrm{dtbpy}) \mathrm{PF}_{6}$ & Acetone & DIPA & $0 \%$ \\
\hline Rhodamine 6G & Acetone & DIPA & $0 \%$ \\
\hline $\mathrm{N}$-Et-Flavinium & Acetone & DIPA & $5 \%$ \\
\hline Ph-phenathiazine & Acetone & DIPA & $0 \%$ \\
\hline DCA & Acetone & DIPA & $0 \%$ \\
\hline No cat & Acetone & DIPA & $0 \%$ \\
\hline No cat/ no light & Acetone & DIPA & $0 \%$ \\
\hline 4-Cz-IPN & DMSO & DIPA & $2 \%$ \\
\hline 4-DPA-IPN & DMSO & DIPA & $4 \%$ \\
\hline Mes-Acri & DMSO & DIPA & $0 \%$ \\
\hline tBu-Acri-N-Ph & DMSO & DIPA & $0 \%$ \\
\hline tetraMeO-Acri-N-Ph & DMSO & DIPA & $0 \%$ \\
\hline tetraMeO-Acri-N-diMeOPh & DMSO & DIPA & $0 \%$ \\
\hline $\mathrm{Ru}(\mathrm{bpz})_{3}\left(\mathrm{PF}_{6}\right)_{2}$ & DMSO & DIPA & $0 \%$ \\
\hline $\mathrm{Ru}(\mathrm{bpy})_{3}\left(\mathrm{PF}_{6}\right)_{2}$ & DMSO & DIPA & $0 \%$ \\
\hline $\mathrm{Ru}(\mathrm{bpm})_{3}(\mathrm{Cl})_{2}$ & DMSO & DIPA & $0 \%$ \\
\hline $\mathrm{Ru}(\text { phen })_{3} \mathrm{Cl}_{2} \times \mathrm{H}_{2} \mathrm{O}$ & DMSO & DIPA & $0 \%$ \\
\hline $\mathrm{Ru}\left(\mathrm{p}-\mathrm{CF}_{3}-\mathrm{bpy}\right)_{3}\left(\mathrm{BF}_{4}\right)_{2}$ & DMSO & DIPA & $0 \%$ \\
\hline $\operatorname{Ir}(\mathrm{ppy})_{2}(\mathrm{dtbpy})\left(\mathrm{PF}_{6}\right)$ & DMSO & DIPA & $43 \%$ \\
\hline $\operatorname{Ir}\left[\mathrm{dF}\left(\mathrm{CF}_{3}\right) \mathrm{ppy}\right]_{2}(\mathrm{dtbpy}) \mathrm{PF}_{6}$ & DMSO & DIPA & $50 \%$ \\
\hline $\operatorname{Ir}(\mathrm{ppy})_{3}$ & DMSO & DIPA & $3 \%$ \\
\hline $\operatorname{Ir}(\mathrm{dF}-\mathrm{ppy})_{3}$ & DMSO & DIPA & $0 \%$ \\
\hline $\operatorname{Ir}\left[\mathrm{dF}(\mathrm{F}) \mathrm{ppy}_{2}(\mathrm{dtbpy}) \mathrm{PF}_{6}\right.$ & DMSO & DIPA & $7 \%$ \\
\hline $\operatorname{Ir}\left[\mathrm{dF}(\mathrm{H}) \mathrm{ppy}_{2}(\mathrm{dtbpy}) \mathrm{PF}_{6}\right.$ & DMSO & DIPA & $68 \%$ \\
\hline $\operatorname{Ir}[\mathrm{dF}(\mathrm{Me}) \mathrm{ppy}]_{2}(\mathrm{dtbpy}) \mathrm{PF}_{6}$ & DMSO & DIPA & $71 \%$ \\
\hline Rhodamine $6 \mathrm{G}$ & DMSO & DIPA & $0 \%$ \\
\hline N-Et-Flavinium & DMSO & DIPA & $0 \%$ \\
\hline Ph-phenathiazine & DMSO & DIPA & $0 \%$ \\
\hline DCA & DMSO & DIPA & $0 \%$ \\
\hline
\end{tabular}




\begin{tabular}{|c|c|c|c|}
\hline No cat & DMSO & DIPA & $0 \%$ \\
\hline No cat/ no light & DMSO & DIPA & $0 \%$ \\
\hline 4-Cz-IPN & $\mathrm{MeCN}$ & DIPA & $4 \%$ \\
\hline 4-DPA-IPN & $\mathrm{MeCN}$ & DIPA & $0 \%$ \\
\hline Mes-Acri & $\mathrm{MeCN}$ & DIPA & $0 \%$ \\
\hline tBu-Acri-N-Ph & $\mathrm{MeCN}$ & DIPA & $0 \%$ \\
\hline tetraMeO-Acri-N-Ph & $\mathrm{MeCN}$ & DIPA & $0 \%$ \\
\hline tetraMeO-Acri-N-diMeOPh & $\mathrm{MeCN}$ & DIPA & $0 \%$ \\
\hline $\mathrm{Ru}(\mathrm{bpz})_{3}\left(\mathrm{PF}_{6}\right)_{2}$ & $\mathrm{MeCN}$ & DIPA & $0 \%$ \\
\hline $\mathrm{Ru}(\mathrm{bpy})_{3}\left(\mathrm{PF}_{6}\right)_{2}$ & $\mathrm{MeCN}$ & DIPA & $0 \%$ \\
\hline $\mathrm{Ru}(\mathrm{bpm})_{3}(\mathrm{Cl})_{2}$ & $\mathrm{MeCN}$ & DIPA & $0 \%$ \\
\hline $\mathrm{Ru}(\text { phen })_{3} \mathrm{Cl}_{2} \times \mathrm{xH}_{2} \mathrm{O}$ & $\mathrm{MeCN}$ & DIPA & $0 \%$ \\
\hline $\mathrm{Ru}\left(\mathrm{p}-\mathrm{CF}_{3}-\mathrm{bpy}\right)_{3}\left(\mathrm{BF}_{4}\right)_{2}$ & $\mathrm{MeCN}$ & DIPA & $0 \%$ \\
\hline $\operatorname{Ir}(\mathrm{ppy})_{2}(\mathrm{dtbpy})\left(\mathrm{PF}_{6}\right)$ & $\mathrm{MeCN}$ & DIPA & $0 \%$ \\
\hline $\operatorname{Ir}[\mathrm{dF}(\mathrm{CF} 3) \mathrm{ppy}]_{2}(\mathrm{dtbpy}) \mathrm{PF}_{6}$ & $\mathrm{MeCN}$ & DIPA & $0 \%$ \\
\hline $\operatorname{Ir}(\mathrm{ppy})_{3}$ & $\mathrm{MeCN}$ & DIPA & $0 \%$ \\
\hline $\operatorname{Ir}(\mathrm{dF}-\mathrm{ppy})_{3}$ & $\mathrm{MeCN}$ & DIPA & $0 \%$ \\
\hline $\mathrm{Ir}[\mathrm{dF}(\mathrm{F}) \text { ppy }]_{2}(\mathrm{dtbpy}) \mathrm{PF}_{6}$ & $\mathrm{MeCN}$ & DIPA & $6 \%$ \\
\hline $\operatorname{Ir}\left[\mathrm{dF}(\mathrm{H}) \mathrm{ppy}_{2}(\mathrm{dtbpy}) \mathrm{PF}_{6}\right.$ & $\mathrm{MeCN}$ & DIPA & $7 \%$ \\
\hline $\operatorname{Ir}[\mathrm{dF}(\mathrm{Me}) \mathrm{ppy}]_{2}(\mathrm{dtbpy}) \mathrm{PF}_{6}$ & $\mathrm{MeCN}$ & DIPA & $0 \%$ \\
\hline Rhodamine $6 G$ & $\mathrm{MeCN}$ & DIPA & $0 \%$ \\
\hline N-Et-Flavinium & $\mathrm{MeCN}$ & DIPA & $0 \%$ \\
\hline Ph-phenathiazine & $\mathrm{MeCN}$ & DIPA & $0 \%$ \\
\hline DCA & $\mathrm{MeCN}$ & DIPA & $0 \%$ \\
\hline No cat & $\mathrm{MeCN}$ & DIPA & $0 \%$ \\
\hline No cat/ no light & $\mathrm{MeCN}$ & DIPA & $0 \%$ \\
\hline 4-Cz-IPN & 1,2-dichloroethane & DIPA & $4 \%$ \\
\hline 4-DPA-IPN & 1,2-dichloroethane & DIPA & $0 \%$ \\
\hline Mes-Acri & 1,2-dichloroethane & DIPA & $0 \%$ \\
\hline tBu-Acri-N-Ph & 1,2-dichloroethane & DIPA & $0 \%$ \\
\hline tetraMeO-Acri-N-Ph & 1,2-dichloroethane & DIPA & $0 \%$ \\
\hline tetraMeO-Acri-N-diMeOPh & 1,2-dichloroethane & DIPA & $0 \%$ \\
\hline $\mathrm{Ru}(\mathrm{bpz})_{3}\left(\mathrm{PF}_{6}\right)_{2}$ & 1,2-dichloroethane & DIPA & $0 \%$ \\
\hline $\mathrm{Ru}(\mathrm{bpy})_{3}\left(\mathrm{PF}_{6}\right)_{2}$ & 1,2-dichloroethane & DIPA & $0 \%$ \\
\hline $\mathrm{Ru}(\mathrm{bpm})_{3}(\mathrm{Cl})_{2}$ & 1,2-dichloroethane & DIPA & $0 \%$ \\
\hline $\mathrm{Ru}(\text { phen })_{3} \mathrm{Cl}_{2} \mathrm{xH}_{2} \mathrm{O}$ & 1,2-dichloroethane & DIPA & $0 \%$ \\
\hline $\mathrm{Ru}\left(\mathrm{p}-\mathrm{CF}_{3}-\mathrm{bpy}\right)_{3}\left(\mathrm{BF}_{4}\right)_{2}$ & 1,2-dichloroethane & DIPA & $0 \%$ \\
\hline $\operatorname{Ir}(\mathrm{ppy})_{2}(\mathrm{dtbpy})\left(\mathrm{PF}_{6}\right)$ & 1,2-dichloroethane & DIPA & $2 \%$ \\
\hline $\operatorname{Ir}[\mathrm{dF}(\mathrm{CF} 3) \mathrm{ppy}]_{2}(\mathrm{dtbpy}) \mathrm{PF}_{6}$ & 1,2-dichloroethane & DIPA & $0 \%$ \\
\hline $\operatorname{Ir}(\mathrm{ppy})_{3}$ & 1,2-dichloroethane & DIPA & $0 \%$ \\
\hline $\operatorname{Ir}(\mathrm{dF}-\mathrm{ppy})_{3}$ & 1,2-dichloroethane & DIPA & $0 \%$ \\
\hline $\operatorname{Ir}\left[\mathrm{dF}(\mathrm{F}) \mathrm{ppy}_{2} \mathrm{z}_{2}(\mathrm{dtbpy}) \mathrm{PF}_{6}\right.$ & 1,2-dichloroethane & DIPA & $0 \%$ \\
\hline $\operatorname{Ir}[\mathrm{dF}(\mathrm{H}) \mathrm{ppy}]_{2}(\mathrm{dtbpy}) \mathrm{PF}_{6}$ & 1,2-dichloroethane & DIPA & $19 \%$ \\
\hline $\operatorname{Ir}[\mathrm{dF}(\mathrm{Me}) \mathrm{ppy}]_{2}(\mathrm{dtbpy}) \mathrm{PF}_{6}$ & 1,2-dichloroethane & DIPA & $0 \%$ \\
\hline Rhodamine 6G & 1,2-dichloroethane & DIPA & $0 \%$ \\
\hline $\mathrm{N}$-Et-Flavinium & 1,2-dichloroethane & DIPA & $0 \%$ \\
\hline Ph-phenathiazine & 1,2-dichloroethane & DIPA & $0 \%$ \\
\hline DCA & 1,2-dichloroethane & DIPA & $0 \%$ \\
\hline
\end{tabular}




\begin{tabular}{|c|c|c|c|}
\hline No cat & 1,2-dichloroethane & DIPA & $0 \%$ \\
\hline No cat/ no light & 1,2-dichloroethane & DIPA & $0 \%$ \\
\hline 4-Cz-IPN & DMSO & DIPEA & $9 \%$ \\
\hline Eosin $Y$ & DMSO & DIPA & $0 \%$ \\
\hline Eosin $Y$ & 1,2-dichloroethane & DIPA & $0 \%$ \\
\hline $\operatorname{Eosin} Y$ & $\mathrm{MeCN}$ & DIPA & $0 \%$ \\
\hline Eosin $Y$ & Acetone & DIPA & $0 \%$ \\
\hline 4-DPA-IPN & DMSO & DIPEA & $16 \%$ \\
\hline Mes-Acri & DMSO & DIPEA & $2 \%$ \\
\hline tBu-Acri-N-Ph & DMSO & DIPEA & $0 \%$ \\
\hline tetraMeO-Acri-N-Ph & DMSO & DIPEA & $3 \%$ \\
\hline tetraMeO-Acri-N-diMeOPh & DMSO & DIPEA & $0 \%$ \\
\hline $\mathrm{Ru}(\mathrm{bpz}) 3(\mathrm{PF} 6) 2$ & DMSO & DIPEA & $0 \%$ \\
\hline $\mathrm{Ru}(\mathrm{bpy}) 3(\mathrm{PF} 6) 2$ & DMSO & DIPEA & $11 \%$ \\
\hline $\mathrm{Ru}(\mathrm{bpm}) 3(\mathrm{Cl}) 2$ & DMSO & DIPEA & $0 \%$ \\
\hline $\mathrm{Ru}($ phen $) 3 \mathrm{Cl} 2 \times \mathrm{H} 2 \mathrm{O}$ & DMSO & DIPEA & $2 \%$ \\
\hline Ru(p-CF3-bpy)3 (BF4)2 & DMSO & DIPEA & $0 \%$ \\
\hline $\operatorname{Ir}(\mathrm{ppy}) 2(\mathrm{dtbpy})(\mathrm{PF} 6)$ & DMSO & DIPEA & $3 \%$ \\
\hline $\operatorname{Ir}[\mathrm{dF}(\mathrm{CF} 3) \mathrm{ppy}] 2(\mathrm{dtbpy}) \mathrm{PF} 6$ & DMSO & DIPEA & $13 \%$ \\
\hline $\operatorname{Ir}($ ppy)3 & DMSO & DIPEA & $7 \%$ \\
\hline $\operatorname{Ir}(\mathrm{dF}-\mathrm{ppy}) 3$ & DMSO & DIPEA & $4 \%$ \\
\hline $\operatorname{Ir}[\mathrm{dF}(\mathrm{F}) \mathrm{ppy}] 2(\mathrm{dtbpy}) \mathrm{PF} 6$ & DMSO & DIPEA & $5 \%$ \\
\hline $\operatorname{Ir}[\mathrm{dF}(\mathrm{H}) \mathrm{ppy}] 2$ (dtbpy)PF6 & DMSO & DIPEA & $5 \%$ \\
\hline $\operatorname{Ir}[\mathrm{dF}(\mathrm{Me}) \mathrm{ppy}] 2(\mathrm{dtbpy}) \mathrm{PF} 6$ & DMSO & DIPEA & $7 \%$ \\
\hline Rhodamine 6G & DMSO & DIPEA & $0 \%$ \\
\hline $\mathrm{N}$-Et-Flavinium & DMSO & DIPEA & $0 \%$ \\
\hline Ph-phenathiazine & DMSO & DIPEA & $0 \%$ \\
\hline DCA & DMSO & DIPEA & $0 \%$ \\
\hline No cat & DMSO & DIPEA & $0 \%$ \\
\hline No cat/ no light & DMSO & DIPEA & $0 \%$ \\
\hline
\end{tabular}


2.2 The Effect of Super-Stoichiometric Equivalents of 4-Cyanopyridine

A stock solution of 4-cyanopyridine 4a was made in DMSO (344 mg in $2.2 \mathrm{~mL}$ DMSO, $0.5 \mathrm{M}$ ) in a $20 \mathrm{~mL}$ vial containing a stir bar. In separate vials, stock solutions of the photocatalyst $(36 \mathrm{mg}$ in $1200 \mu \mathrm{L}, 6 \mathrm{E}-3 \mathrm{mmol}$ per $200 \mu \mathrm{L}$ ), benzoic acid ( $220 \mathrm{mg}$ in $1200 \mu \mathrm{L}, 0.1 \mathrm{mmol}$ in $200 \mu \mathrm{L}$ ), and model oxime 3a (431 mg in $4.5 \mathrm{~mL}, 0.4 \mathrm{M}$ ) were made in DMSO. Each component of the reaction was added to a $4 \mathrm{~mL}$ vial: $200 \mu \mathrm{L}$ of photocatalyst solution, $200 \mu \mathrm{L}$ benzoic acid solution, $750 \mu \mathrm{L}$ of oxime solution, and $200 \mu \mathrm{L}$ per equivalent of 4-cyanopyridine solution. DMSO was added to each reaction to yield a final reaction volume of $3 \mathrm{~mL}$. The reaction was stirred in the glovebox for 10 minutes yielding a homogeneous solution. DIPA $(153 \mu \mathrm{L}, 1.1 \mathrm{mmol}, 3.6$ equiv) was added to the vial. The reaction was stirred for 10 minutes before being removed from the glovebox and having its cap sealed by parafilm. The reaction was placed into the photoreactor and irradiated with blue light $(455 \mathrm{~nm}, 5 \mathrm{~W}$ per reaction) for two hours. After the two hours, the reaction was quenched by opening the vial and exposing the contents to oxygen. Triethylamine was added to each reaction to quench any hydrogen cyanide that was evolved in the reaction. An aliquot from each reaction $(100 \mu \mathrm{L})$ was diluted into a solution of biphenyl in acetonitrile $(500 \mu \mathrm{L}, 2 \mathrm{mM}$ biphenyl). Each reaction was analyzed by UPLC-MS. The reaction yield was determined via calibration of the UV-Vis absorption signal for product.

Table S2. Testing the role of cyanoarene stoichiometry
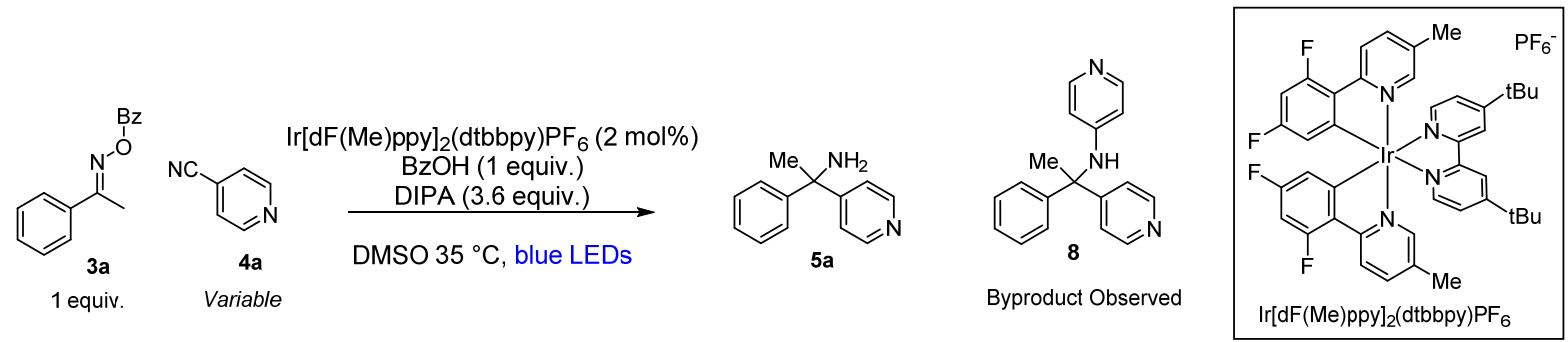

\begin{tabular}{|c|c|c|c|c|}
\hline $\begin{array}{c}\text { Equivalents of 4- } \\
\text { cyanopyridine }\end{array}$ & Yield & 4-cyanopyridine to IS & Byproduct to IS & Byproduct to Product \\
\hline 1 & $39 \%$ & 0.00 & 0.40 & 0.22 \\
\hline 1.5 & $64 \%$ & 0.00 & 0.67 & 0.23 \\
\hline 2 & $86 \%$ & 0.00 & 0.98 & 0.25 \\
\hline 2.5 & $89 \%$ & 0.49 & 1.05 & 0.26 \\
\hline 3 & $89 \%$ & 1.92 & 1.09 & 0.27 \\
\hline
\end{tabular}




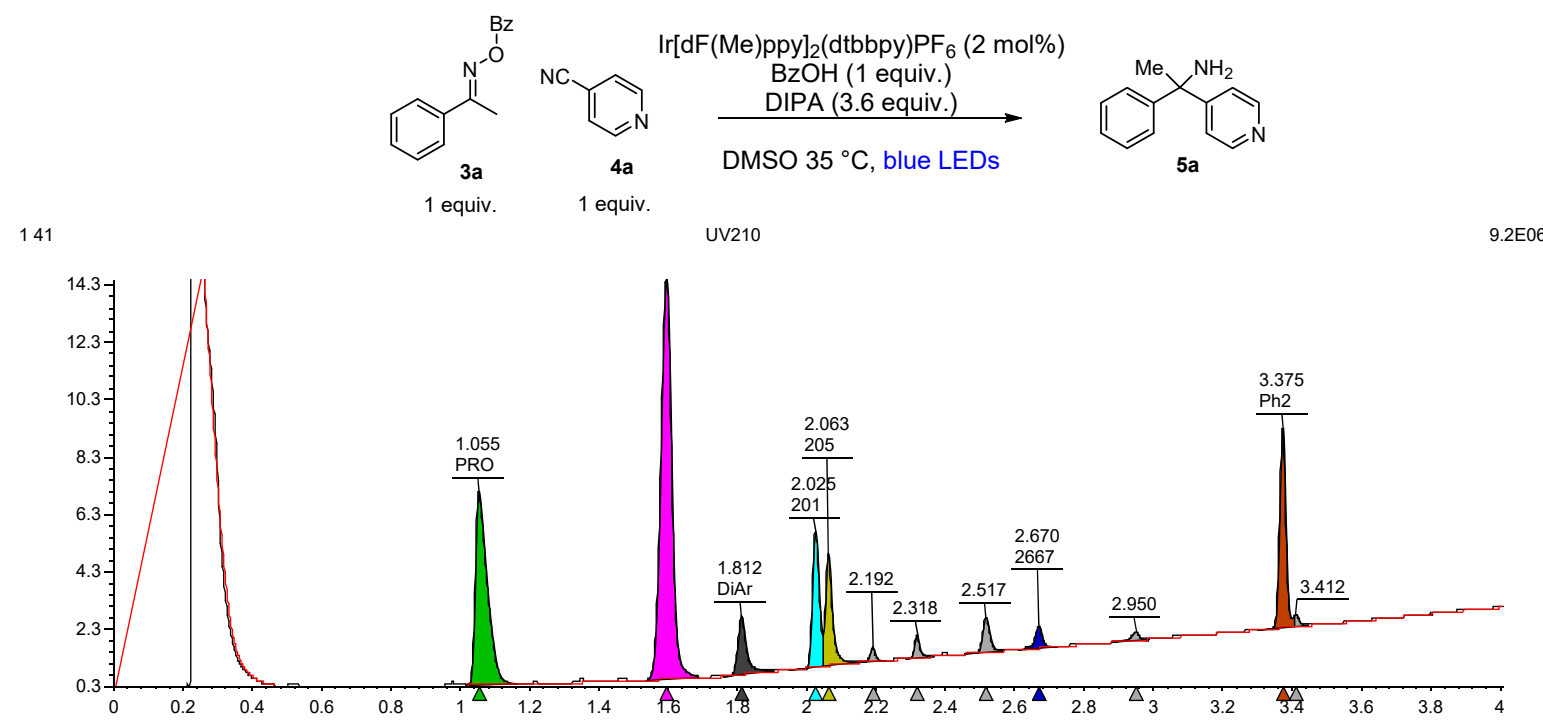

Product $(t=1.055)$. Theoretical m/z $[\mathrm{M}+\mathrm{H}]=199.1230$. Observed: $[\mathrm{M}+\mathrm{H}] 199.1,\left[\mathrm{M}-\mathrm{NH}_{2}\right] 182.1$ (fragment).

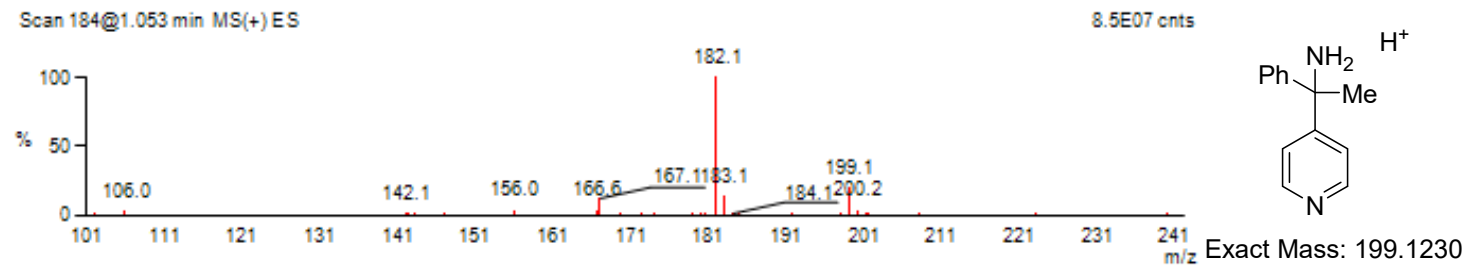

$\operatorname{DiAr}(t=1.81)$. Theoretical m/z $[\mathrm{M}+\mathrm{H}]=276.1495$. Observed: [M+H]276.2, [M- 4pyr] 199.1 (fragment) [M-NH $2_{2}^{-}$ 4pyr] 182.1 (fragment).

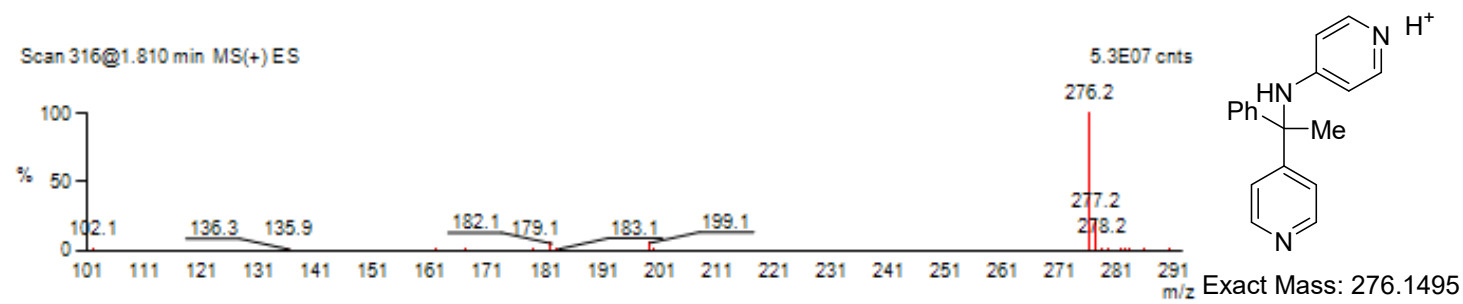

(201) \& (205) $(t=2.01 \& t=2.05)$ Theoretical $\mathrm{m} / \mathrm{z}[\mathrm{M}+\mathrm{H}]=224.1182$. Observed: $[\mathrm{M}+\mathrm{H}] 224.2,\left[\mathrm{M}-\mathrm{NH}_{2}\right] 202.2$ (fragment). Masses consistent for both observed byproducts.
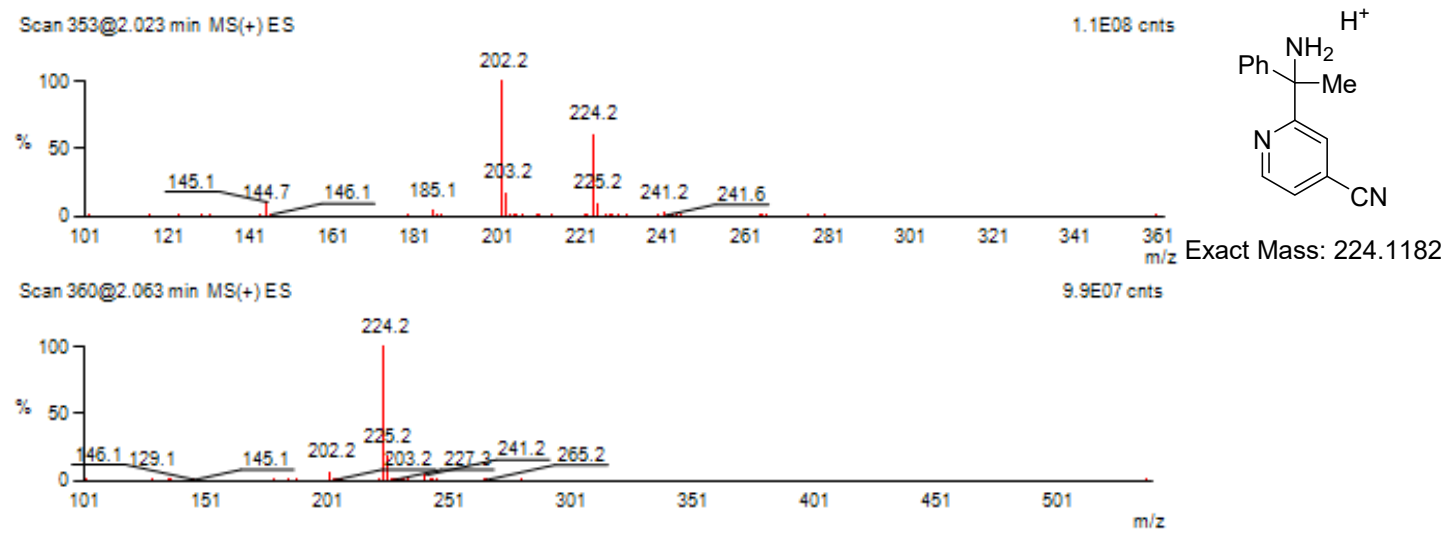

Figure S1. UPLC-MS analysis of a coupling reaction with 1:1 stoichiometry of 3a:4a 


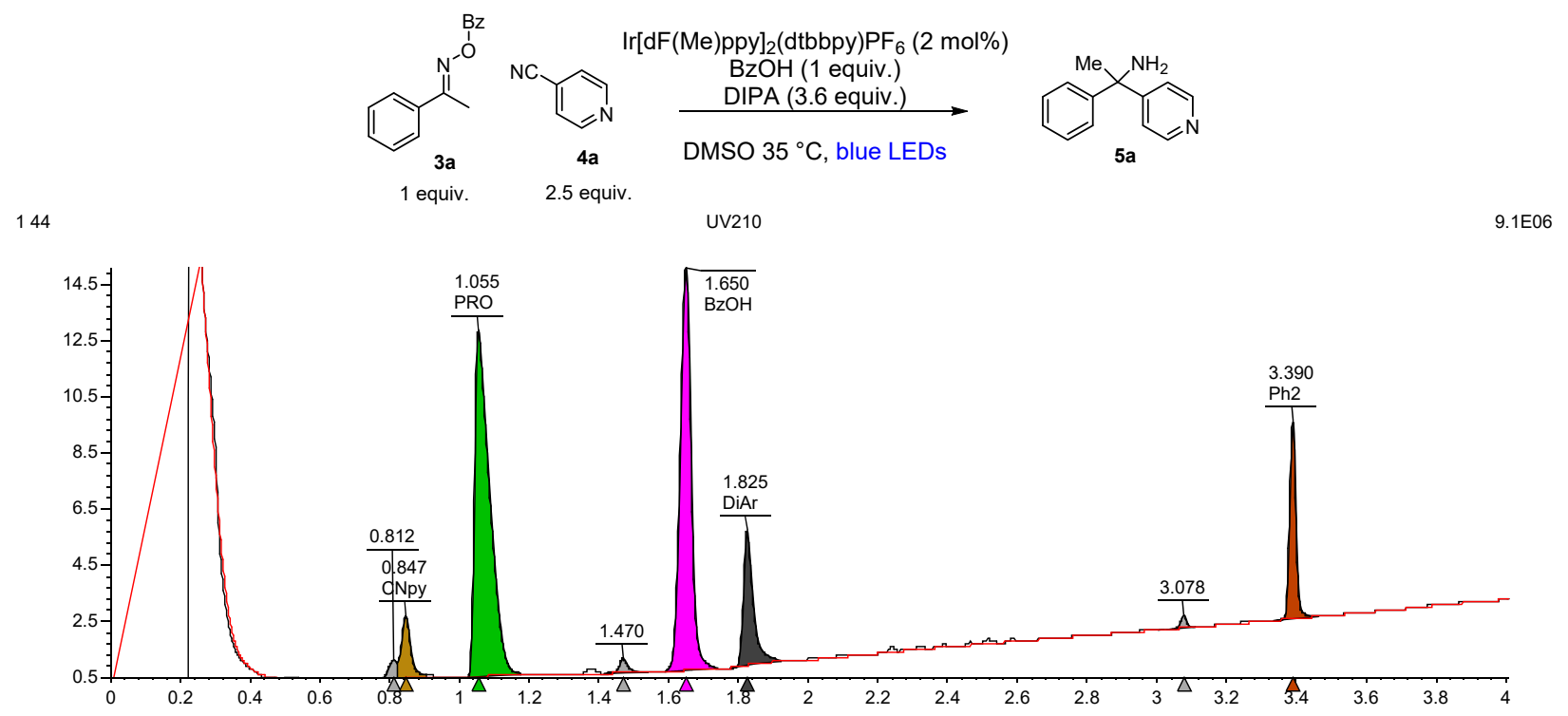

Product $(t=1.055)$. Theoretical m/z $[\mathrm{M}+\mathrm{H}]=199.1230$. Observed: $[\mathrm{M}+\mathrm{H}] 199.1$, $\left[\mathrm{M}-\mathrm{NH}_{2}\right] 182.1$ (fragment).

Scan $185 @ 1.059$ min MS(+) ES

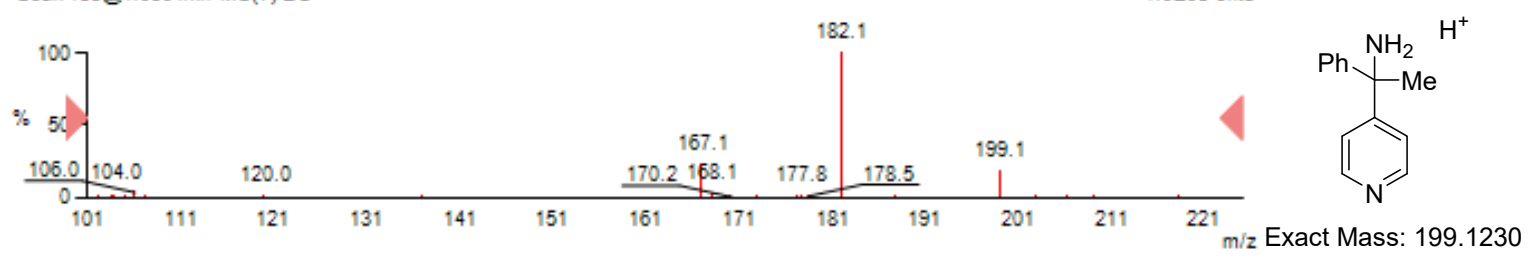

$\operatorname{DiAr}(t=1.81)$. Theoretical m/z $[\mathrm{M}+\mathrm{H}]=276.1495$. Observed: [M+H]276.2, [M- 4pyr] 199.1 (fragment) [M-NH${ }_{2}^{-}$ 4pyr] 182.1 (fragment).

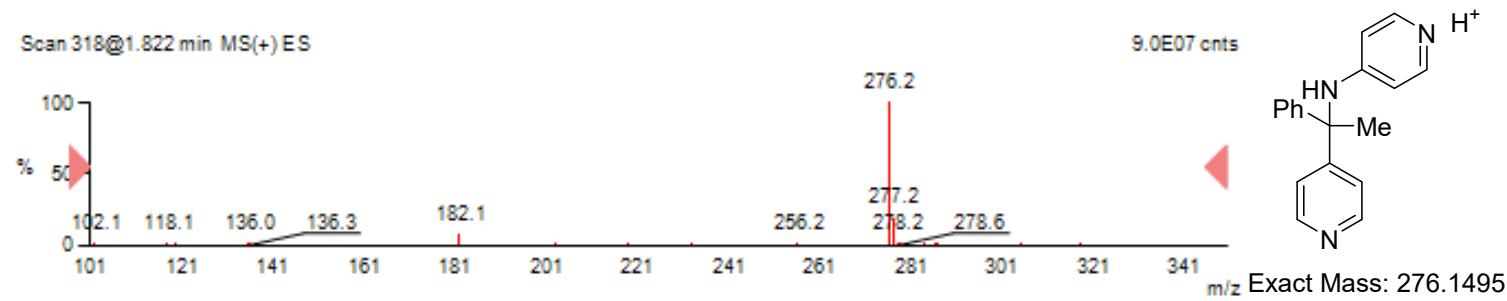

Figure S2. UPLC-MS analysis of a coupling reaction with 1:2.5 stoichiometry of 3a:4a 


\subsection{The Effect of the Stoichiometric Reductant}

In a glovebox under an atmosphere of nitrogen, solid reagents were added to a 4-mL vial: model oxime substrate (3a) (72 mg, $0.3 \mathrm{mmol}, 1.0$ equiv.); benzoic acid (37 mg, $0.1 \mathrm{mmol}, 1.0$ equiv.); photocatalyst $\operatorname{Ir}[\mathrm{dF}(\mathrm{Me}) \text { ppy }]_{2} \mathrm{dtbbpyPF}$ (6 mg, 2E-3 mmol, $2 \mathrm{~mol} \%$ ); 4-cyanopyridine (4a) (69 mg, $0.66 \mathrm{mmol}, 2.2$ equiv.). The reactants were then dissolved by the addition of DMSO $(3 \mathrm{~mL})$ and stirred for 10 minutes until the solution was homogeneous. The bases were then added to their respective reactions: DIPA $(153 \mu \mathrm{L}, 1.1 \mathrm{mmol})$, DIPEA $(191 \mu \mathrm{L}$, $1.1 \mathrm{mmol}$ ), Hantzsch ester (279 mg, $1.1 \mathrm{mmol}$ ); or tris-4-methoxyphenyl amine (369 mg, $1.1 \mathrm{mmol}$ ). The reaction was stirred for 10 minutes before being removed from the glovebox and having its cap sealed by parafilm. The reaction was placed into the photoreactor and irradiated with blue light ( $455 \mathrm{~nm}, 5 \mathrm{~W}$ per reaction). At 2.5 hours the reaction was considered complete. The reaction is quenched by opening the vial and exposing the vial contents to oxygen. Triethylamine was added to each reaction to quench any hydrogen cyanide that was evolved in the reaction. The reaction is then added to a $25 \mathrm{~mL}$ volumetric flask along with a solution of biphenyl in MeCN $(5 \mathrm{~mL}, 12.5 \mathrm{mM}$ biphenyl). The contents of the flask were then diluted until the volume reached $25 \mathrm{~mL}$. Aliquots $(20 \mu \mathrm{L}, 50 \mu \mathrm{L}$, and $100 \mu \mathrm{L}$ ) of the diluted reaction were then taken to measure by UPLC-MS. The reaction yield was determined by UPLC-MS calibration relative to a pure sample of model substrate $\mathbf{5}$.

Table S3. Testing the Effect of Alternative Stoichiometric Reductants
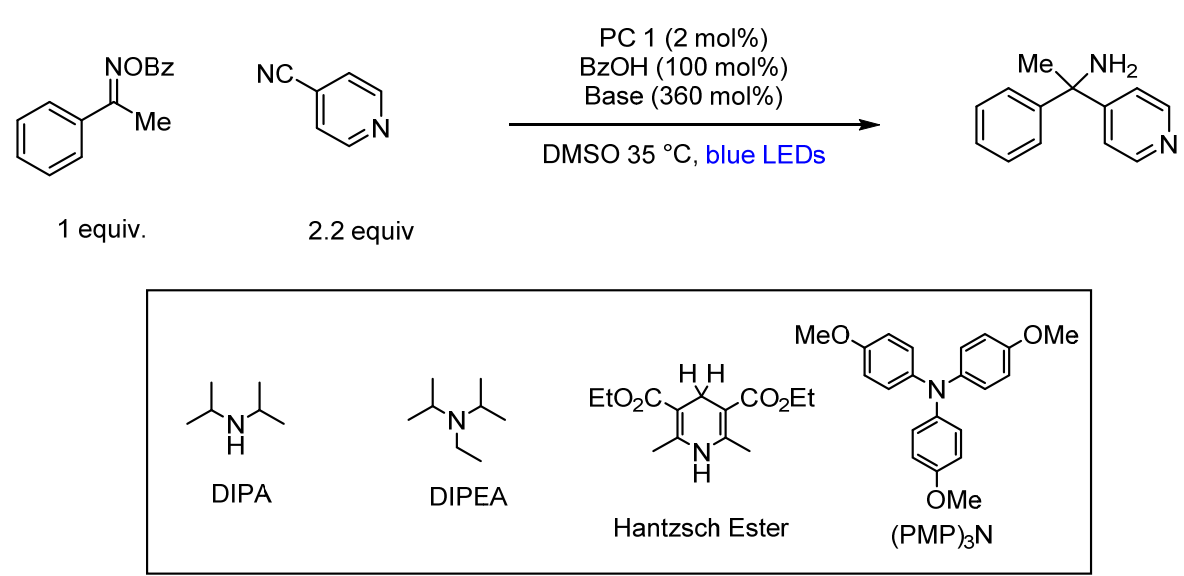

\begin{tabular}{|c|c|}
\hline Base / Reductant & Yield \\
\hline Diisopropylamine (DIPA) & $86 \%$ \\
\hline Diisopropylethylamine (DIPEA) & $27 \%$ \\
\hline Hantzsch Ester & $26 \%$ \\
\hline Tris-para-methoxyphenylamine (PMP) ${ }_{3} \mathrm{~N}$ & $0 \%$ \\
\hline
\end{tabular}




\subsection{Evidence for Iminyl Radicals}

Scheme S1. Evidence of the dimerization of Iminyl Radicals

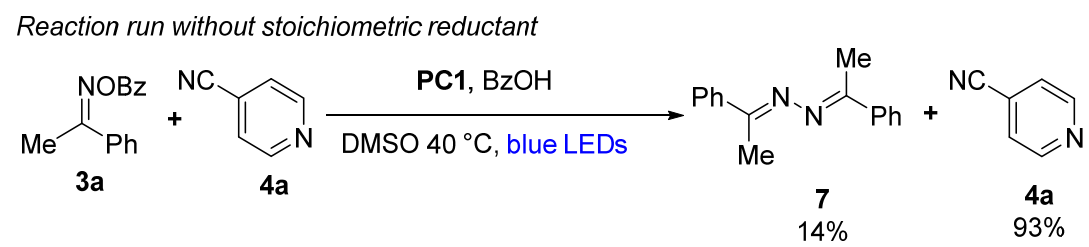

Reaction without DIPA

In a glovebox under an atmosphere of nitrogen, solid reagents were added to a 4-mL vial: model oxime substrate 3a (72 mg, $0.3 \mathrm{mmol}, 1.0$ equiv.); benzoic acid (37 mg, $0.1 \mathrm{mmol}, 1.0$ equiv.); photocatalyst $\operatorname{Ir}[\mathrm{dF}(\mathrm{Me}) \text { ppy }]_{2} \mathrm{dtbbpyPF}_{6}$ (6 mg, 2E-3 mmol, $\left.2 \mathrm{~mol} \%\right)$; 4-cyanopyridine $4 \mathrm{a}(69 \mathrm{mg}, 0.66 \mathrm{mmol}, 2.2$ equiv.). The reactants were then dissolved by the addition of DMSO $(3 \mathrm{~mL})$ and stirred for 10 minutes until the solution was homogeneous. The reaction was then irradiated for $2.5 \mathrm{~h}$ in the TAK 120 photoreactor $\left(455 \mathrm{~nm}, 5 \mathrm{~W}, 40^{\circ} \mathrm{C}\right)$. The reaction is quenched by opening the vial and exposing the vial contents to oxygen.

The reaction was then added to a $25 \mathrm{~mL}$ volumetric flask along with a solution of biphenyl in $\mathrm{MeCN}(5 \mathrm{~mL}$, $12.5 \mathrm{mM}$ biphenyl). The contents of the flask were then diluted until the volume reached $25 \mathrm{~mL}$. Aliquots $(20 \mu \mathrm{L}, 50$ $\mu \mathrm{L}$, and $100 \mu \mathrm{L}$ ) of the diluted reaction were then taken to measure by UPLC-MS. The remainder of the diluted reaction was then concentrated under reduced pressure to remove the $\mathrm{MeCN}$. The remaining crude oil is dissolved in methylene chloride $(50 \mathrm{~mL})$. Isopropyl amine $(2 \mathrm{~mL})$ was then added to the reaction to raise the $\mathrm{pH}$, deprotonate the desired product, and prevent the formation of hydrogen cyanide. The methylene chloride solution was washed with water $(3 \times 50 \mathrm{~mL})$ followed by a wash with brine $(50 \mathrm{~mL})$. The remaining organic fraction was then dried over anhydrous sodium sulfate, filtered, and concentrated under reduced pressure to afford a crude product.

The crude product was purified by FLASH chromatography using a Teledyne-Isco Combiflash, [12 $\mathrm{g}$ column, $\mathrm{CH}_{2} \mathrm{Cl}_{2}$ (Solvent A), $\mathrm{MeOH}$ (Solvent B)]. The column utilized a gradient elution: 5 min (100\% A), 5 min (100\% A to $97.5 \%$ A: $2.5 \%$ B), 10 min (97.5\% A: $2.5 \%$ B to $95 \%$ A: $5 \%$ B), $10 \min$ (95\% A: 5\% B), 10 (Hold 95\% A: $5 \%$ B), 5 min (95\% A: $5 \%$ B to $90 \%$ A: $10 \%$ B), 5 min (Hold 90\% A: 10\% B). The product fractions were collected and concentrated under reduced pressure to afford the pure product 7 (10 mg, 14\%). ${ }^{1} \mathrm{H}$ NMR $\left(500 \mathrm{MHz}, \mathrm{CD}_{3} \mathrm{CN}\right) \delta$ $7.93(\mathrm{dd}, J=6.8,3.0 \mathrm{~Hz}, 4 \mathrm{H}), 7.50-7.40(\mathrm{~m}, 6 \mathrm{H}), 2.28(\mathrm{~s}, 6 \mathrm{H}) .{ }^{13} \mathrm{C} \mathrm{NMR}\left(126 \mathrm{MHz}, \mathrm{CD}_{3} \mathrm{CN}\right) \delta 158.35,139.49$, 130.64, 129.37, 127.56, 15.18. MS (ESI) $\mathrm{m} / \mathrm{z}$ calcd. for $\mathrm{C}_{16} \mathrm{H}_{17} \mathrm{~N}_{2}\left([\mathrm{M}+\mathrm{H}]^{+}\right)$237.1, found 237.7. 


\section{Observation of Azine (Dimer of Iminyl Radicals) via UPLC-MS}

11
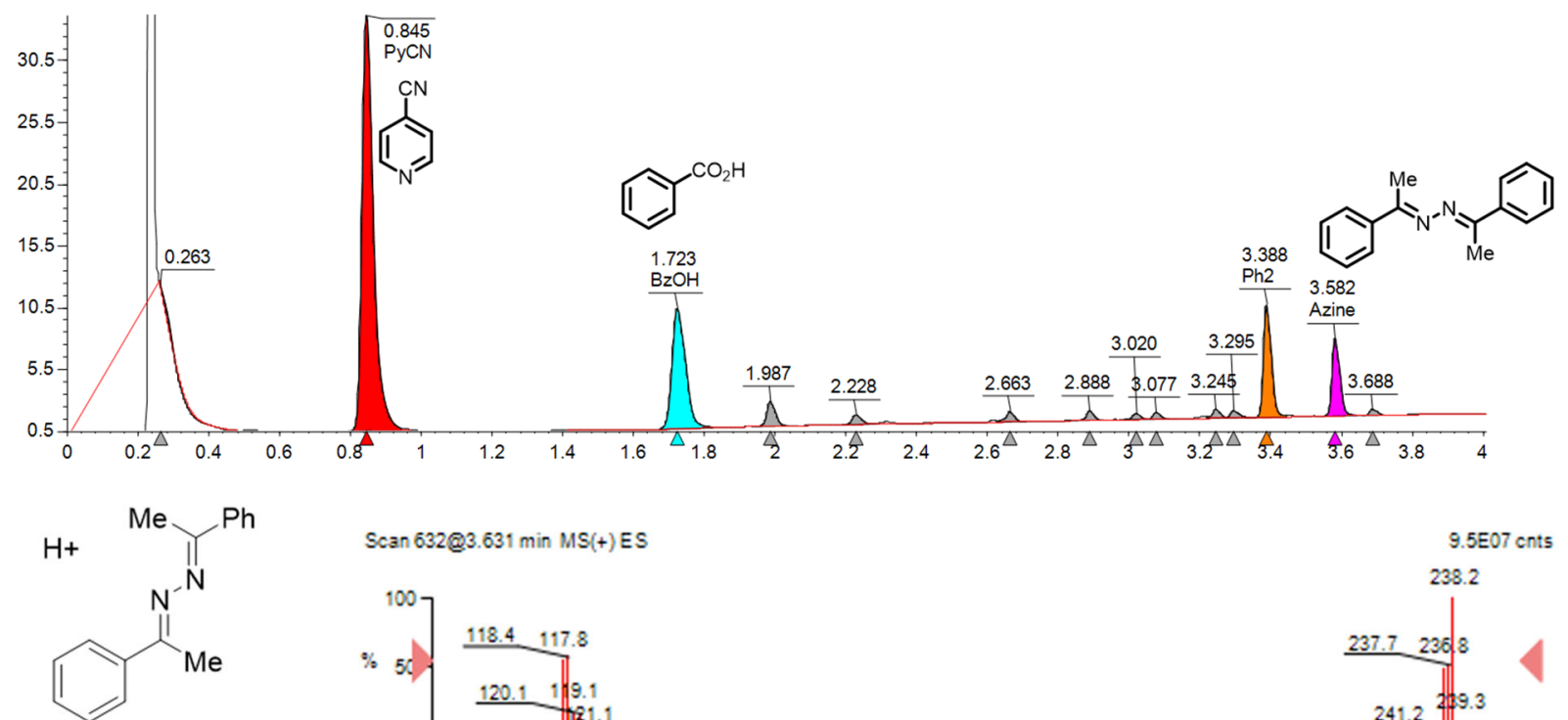

Exact Mass: 237.1386 Molecular Weight: 237.3255

Scan $632 @ 3.631 \mathrm{~min} M S(+) E S$

$9.5 E 07$ cnts

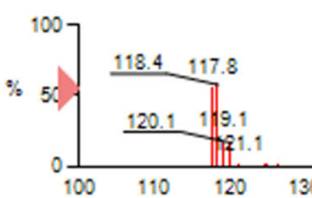

238.2
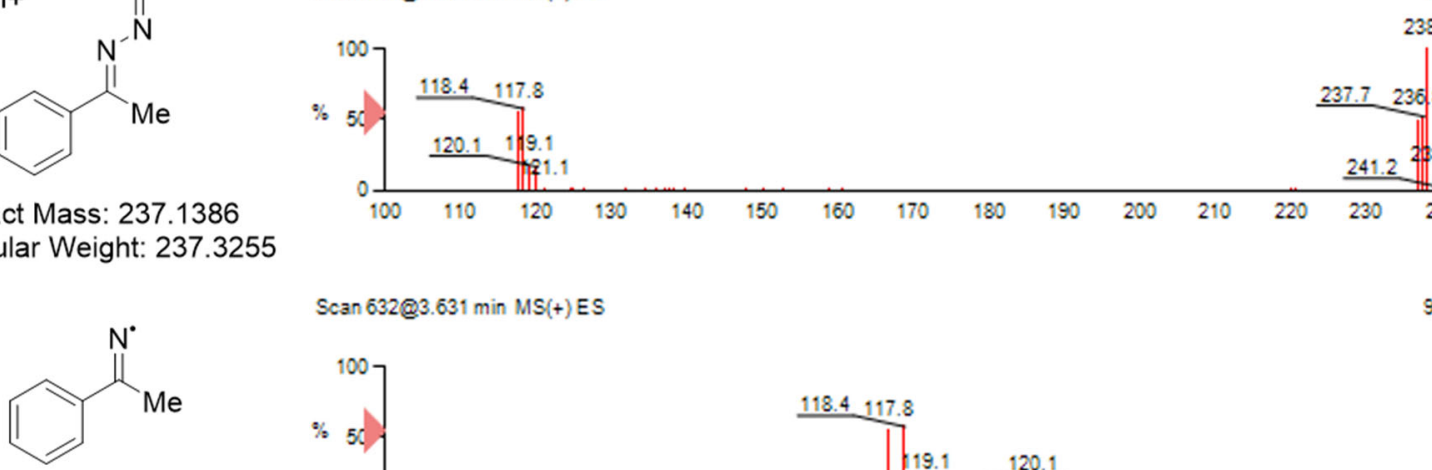

Exact Mass: 118.0657 Molecular Weight: 118.1590

Scan 632@3.631 min MS(+)ES

$9.5 E 07 \mathrm{cnts}$

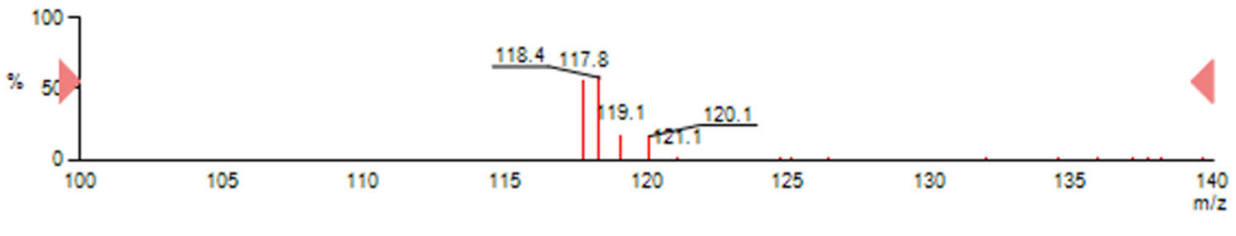

Figure S3. UPLC chromatograph with compound assignments for key UV signals. Low resolution mass spectrum $(\mathrm{ESI}+)$ for the retention time identified as the azine and identification of a fragmentation pattern for N-N bond cleavage of the dimeric structure. 


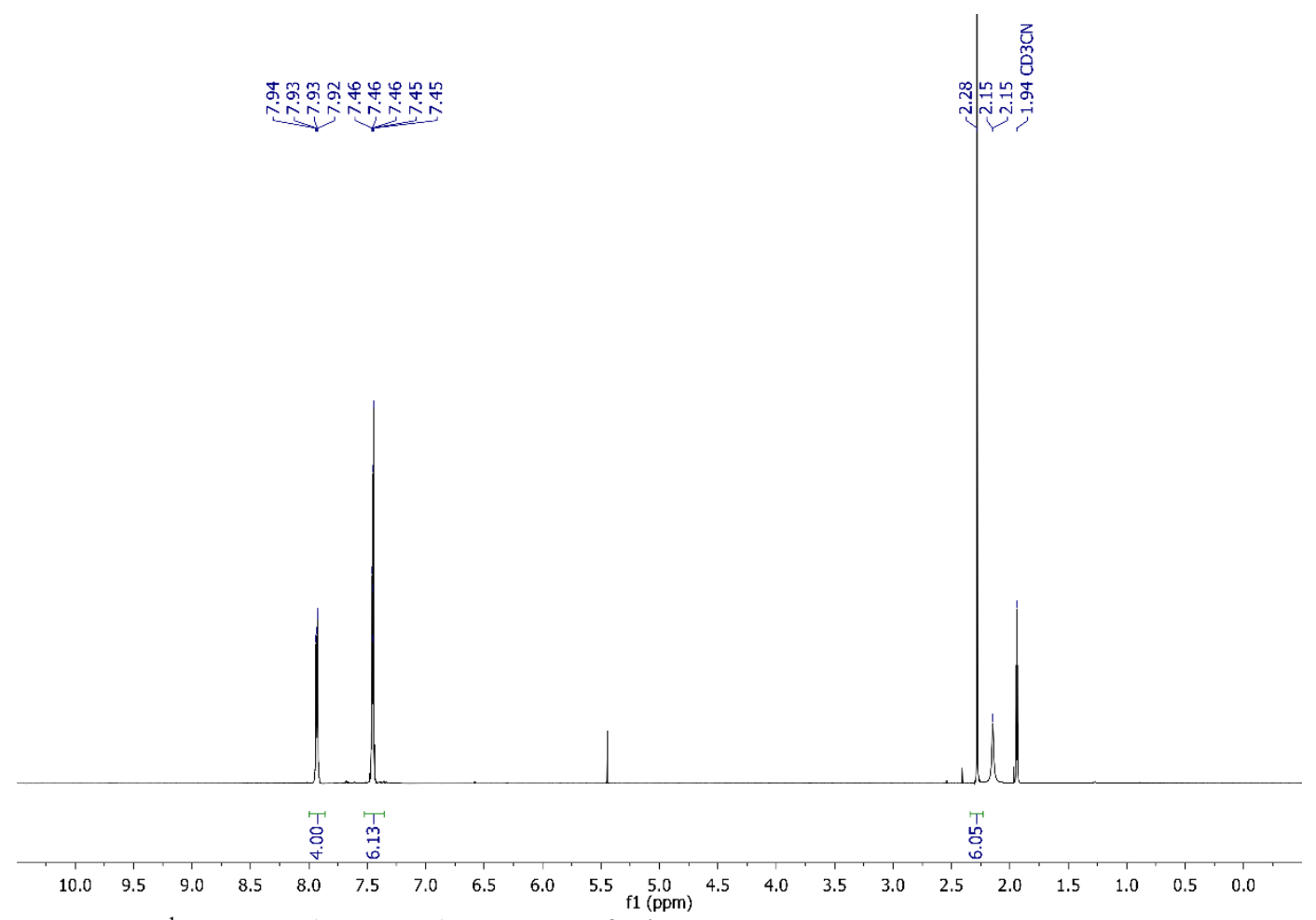

Figure S4. ${ }^{1} \mathrm{H}$ qNMR $(500 \mathrm{MHz})$ spectrum of 7 in $\mathrm{CD}_{3} \mathrm{CN}$.

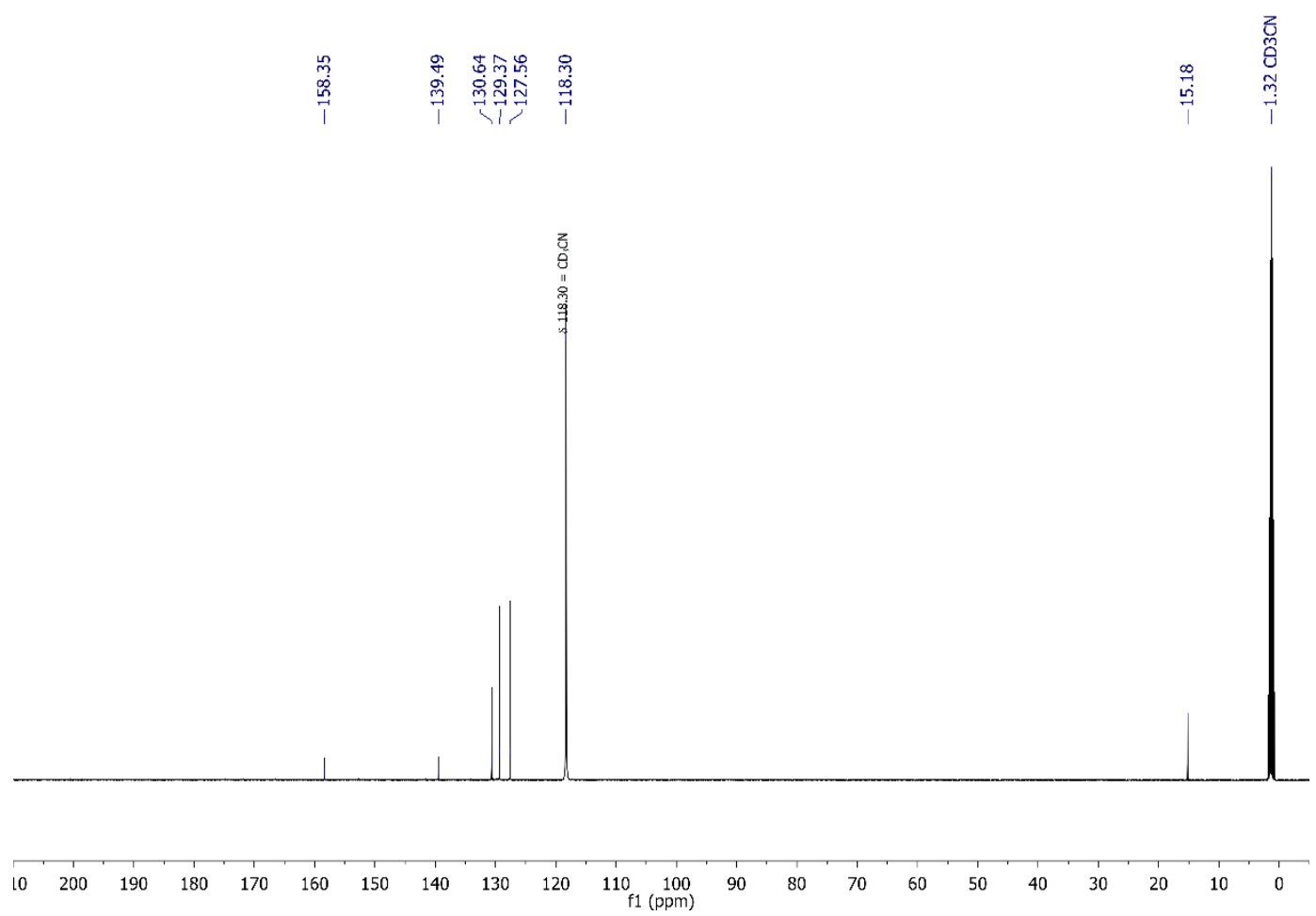

Figure S5. ${ }^{13} \mathrm{C}$ NMR $(126 \mathrm{MHz})$ spectrum of 7 in $\mathrm{CD}_{3} \mathrm{CN}$. 


\subsection{Comparison of Oxime Consumption Without DIPA Reductant}

In a glovebox under an atmosphere of nitrogen, solid reagents were added to a 4-mL vial: model oxime substrate 3a (72 mg, $0.3 \mathrm{mmol}, 1.0$ equiv.); benzoic acid (37 mg, $0.1 \mathrm{mmol}, 1.0$ equiv.); photocatalyst (2E-3 mmol, 2 mol\%) (Table 4); 4-cyanopyridine 4a (69 $\mathrm{mg}, 0.66 \mathrm{mmol}, 2.2$ equiv.). The reactants were then dissolved by the addition of DMSO $(3 \mathrm{~mL})$ and stirred for 10 minutes until the solution was homogeneous. The reaction was then irradiated for $2.5 \mathrm{~h}$ in the TAK 120 photoreactor $\left(455 \mathrm{~nm}, 5 \mathrm{~W}, 40{ }^{\circ} \mathrm{C}\right)$. The reaction was quenched by opening the vial and exposing the vial contents to oxygen.

The reaction was then added to a $25 \mathrm{~mL}$ volumetric flask along with a solution of biphenyl in MeCN $(5 \mathrm{~mL}$, $12.5 \mathrm{mM}$ biphenyl). The contents of the flask were then diluted until the volume reached $25 \mathrm{~mL}$. Aliquots $(20 \mu \mathrm{L}, 50$ $\mu \mathrm{L}$, and $100 \mu \mathrm{L}$ ) of the diluted reaction were then taken to measure by UPLC-MS. For each reaction, the ratio of absorbance of the starting oxime was compared to the constant biphenyl internal standard was calculated. The amount of oxime remaining in each reaction was determined by comparing the product to internal standard for each reaction to the product to internal standard ratio of a reaction without photocatalyst added.

Table S4. Comparison of Photocatalysts Triplet Energies in the Coupling Reaction

\begin{tabular}{|c|c|c|c|c|c|c|}
\hline Entry & Photocatalyst & $\begin{array}{l}\text { Amount of } \\
\text { Photocatalyst }\end{array}$ & $\begin{array}{c}\text { Triplet } \\
\text { Energy }\left(\mathrm{E}^{\mathrm{\top}}\right) \\
{\left[\mathrm{kcal} \mathrm{mol}^{-1}\right]}\end{array}$ & $\begin{array}{l}\text { Ered } \\
\text { (V) vs. } \\
\text { SCE }\end{array}$ & $\begin{array}{l}E^{*} \text { ox } \\
\text { (V) vs. } \\
\text { SCE }\end{array}$ & $\begin{array}{c}\text { Oxime } \\
\text { Remaining } \\
(\%)\end{array}$ \\
\hline 1 & PC1 & $6 \mathrm{mg}$ & 55.8 & -1.42 & 0.97 & 0 \\
\hline 2 & PC3 & $6 \mathrm{mg}$ & 61.8 & -1.37 & 1.21 & 51 \\
\hline 3 & PC4 & $4 \mathrm{mg}$ & 58.1 & -2.2 & 0.31 & 0 \\
\hline 4 & PC5 & $5 \mathrm{mg}$ & 46 & -1.33 & 0.77 & 98 \\
\hline 5 & PC6 & $4 \mathrm{mg}$ & 43.6 & -1.06 & 0.83 & 98 \\
\hline
\end{tabular}




\section{O-Benzoyl Oxime \& Iminium Hydrochloride Substrate Scope}

\subsection{General Reaction Procedure A:}

Reactions were conducted in a $4 \mathrm{~mL}$ vial (Chemglass), with a PTFE stir bar, and a vial cap containing a septum. All reaction contents were measured inside of a glovebox where the atmosphere contained $<0.1 \mathrm{ppm}$ oxygen. $O$-benzoyl oxime substrate (3a-3r) $(0.3 \mathrm{mmol}, 1.0$ equiv.); benzoic acid ( $37 \mathrm{mg}, 0.1 \mathrm{mmol}, 1.0$ equiv.); photocatalyst (PC1) $\operatorname{Ir}\left[\mathrm{dF}(\mathrm{Me})\right.$ ppy $_{2} \mathrm{dtbbpyPF}_{6}(6 \mathrm{mg}, 2 \mathrm{E}-3 \mathrm{mmol}, 2 \mathrm{~mol} \%$ ); and cyanoarene (4a-4ai) (0.66 mmol, 2.2 equiv.) were added to a $4 \mathrm{~mL}$ vial. The reactants were then dissolved by the addition of DMSO $(3 \mathrm{~mL})$ and stirred for 10 minutes until the solution was homogeneous. DIPA $(153 \mu \mathrm{L}, 1.1 \mathrm{mmol}, 3.6$ equiv. $)$ was then added to the reaction. The reaction was capped and stirred for ten minutes until the DIPA was miscible with the reaction. The reaction vessel was then removed from the glovebox and sealed with parafilm to further protect the reaction from oxygen. The reaction was then placed into the photoreactor and irradiated with blue light ( $455 \mathrm{~nm}, 5 \mathrm{~W}$ per reaction) for 2.5 hours.

Caution! Cyanide may be produced as a byproduct in these reactions. Cyanide is toxic and could lead to the release of HCN gas (extremely toxic). Reactions and their aqueous work-up should be conducted in well-ventilated enclosures (e.g. fumehood) and aqueous cyanide-containing waste should be kept basic and disposed of in accordance with local guidelines.

At 2.5 hours the reaction is considered complete, and the reaction was quenched by opening the vial and exposing the vial contents to oxygen. The reaction was then added to a $25 \mathrm{~mL}$ volumetric flask along with a solution of biphenyl in $\mathrm{MeCN}$ ( $5 \mathrm{~mL}, 12.5 \mathrm{mM}$ biphenyl). The contents of the flask were then diluted until the volume reached $25 \mathrm{~mL}$. Aliquots $(20 \mu \mathrm{L}, 50 \mu \mathrm{L}$, and $100 \mu \mathrm{L})$ of the diluted reaction were then taken to measure by UPLC-MS. The remainder of the diluted reaction was then concentrated under reduced pressure to remove the $\mathrm{MeCN}$. The remaining crude oil is dissolved in methylene chloride $(50 \mathrm{~mL})$. Isopropyl amine $(2 \mathrm{~mL})$ was then added to the reaction to raise the $\mathrm{pH}$, deprotonate the desired product, and prevent the formation of hydrogen cyanide. The methylene chloride solution was washed with water $(3 \times 50 \mathrm{~mL})$ followed by a wash with brine $(50 \mathrm{~mL})$. The remaining organic fraction was then dried over anhydrous sodium sulfate, filtered, and concentrated under reduced pressure to afford a crude product.

The crude product was purified by FLASH chromatography using a Teledyne-Isco Combiflash, [12 $\mathrm{g}$ column, $\mathrm{CH}_{2} \mathrm{Cl}_{2}$ (Solvent A), $\mathrm{MeOH}$ (Solvent B)]. The column utilized a gradient elution: 5 min (100\% A), 5 min (100\% A to $97.5 \%$ A: $2.5 \%$ B), $10 \min (97.5 \%$ A: $2.5 \%$ B to $95 \%$ A: $5 \%$ B), $10 \min (95 \%$ A: $5 \%$ B), 10 (Hold 95\% A: $5 \%$ B), 5 min (95\% A: $5 \%$ B to $90 \%$ A: $10 \%$ B), 5 min (Hold 90\% A: 10\% B). The product fractions were collected and concentrated under reduced pressure to afford the pure product. After characterization, the pure product is used to produce a calibration curve according to the general UPLC calibration procedure. 


\subsection{General Iminium Salt Reaction Procedure:}

Reactions were conducted in a $4 \mathrm{~mL}$ vial (Chemglass), with a PTFE stir bar, and a vial cap containing a septum. All reaction contents were measured inside of a glovebox where the atmosphere contained $<0.1 \mathrm{ppm}$ oxygen. Iminium $\mathrm{HCl}$ salt ( $0.66 \mathrm{mmol}, 2.2$ equiv.); photocatalyst $\operatorname{Ir}[\mathrm{dF}(\mathrm{Me}) \text { ppy }]_{2} \mathrm{dtbbpyPF}_{6}(6 \mathrm{mg}, 2 \mathrm{E}-3 \mathrm{mmol}, 2 \mathrm{~mol} \%$ ); and 4-cyanopyridine ( $31 \mathrm{mg}, 0.3 \mathrm{mmol}, 1.0$ equiv.) were added to a $4 \mathrm{~mL}$ vial. The reactants were then dissolved by the addition of DMSO $(3 \mathrm{~mL})$ and stirred for 10 minutes until the solution was homogeneous. DIPA (153 $\mu \mathrm{L}, 1.1 \mathrm{mmol}$, 3.6 equiv.) was then added to the reaction. The reaction was capped and stirred for ten minutes until the DIPA was miscible with the reaction. The reaction vessel was then removed from the glovebox and sealed with parafilm to further protect the reaction from oxygen. The reaction was then placed into the photoreactor and irradiated with blue light (455 nm, $5 \mathrm{~W}$ per reaction).

Caution! Cyanide may be produced as a byproduct in these reactions. Cyanide is toxic and could lead to the release of HCN gas (extremely toxic). Reactions and their aqueous work-up should be conducted in well-ventilated enclosures (e.g. fumehood) and aqueous cyanide-containing waste should be kept basic and disposed of in accordance with local guidelines.

At 2.5 hours the reaction as considered complete, and the reaction was quenched by opening the vial and exposing the vial contents to oxygen. The reaction was then added to a $25 \mathrm{~mL}$ volumetric flask along with a solution of biphenyl in MeCN (5 mL, $12.5 \mathrm{mM}$ biphenyl). Isopropyl amine $(2 \mathrm{~mL})$ was then added to the reaction to raise the $\mathrm{pH}$, deprotonate the desired product, and prevent the formation of hydrogen cyanide. The contents of the flask were then diluted until the volume reached $25 \mathrm{~mL}$. Aliquots $(20 \mu \mathrm{L}, 50 \mu \mathrm{L}$, and $100 \mu \mathrm{L})$ of the diluted reaction were then taken to measure by UPLC-MS. The yield of the reaction was determined via UPLC-MS calibration. 


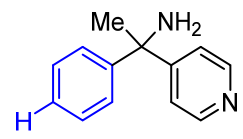

Compound 5a was synthesized following General Procedure A using 4-pyridinecarbonitrile (733 mg, $7.04 \mathrm{mmol}$ ), PC1 (72 mg, 7.2E-2 mmol), benzoic acid (444 mg, $3.2 \mathrm{mmol})$, DIPA (1.836 mL, $1.1 \mathrm{mmol})$ and $O$-benzoyl oxime (3a) $(765 \mathrm{mg}, 3.2 \mathrm{mmol})$ in DMSO (36 mL). The product was obtained as a viscous brown oil $(513 \mathrm{mg}$, $70 \%)$. The yield of product $5 \mathbf{a}$ was determined to be $80 \%$ by a calibrated UPLC-MS ${ }^{1} \mathrm{H}$ NMR $\left(500 \mathrm{MHz}, \mathrm{CD}_{3} \mathrm{CN}\right) \delta 8.45(\mathrm{~d}, J=$ $5.9 \mathrm{~Hz}, 2 \mathrm{H}), 7.40(\mathrm{~d}, J=7.7 \mathrm{~Hz}, 2 \mathrm{H}), 7.35(\mathrm{~d}, J=6.0 \mathrm{~Hz}, 2 \mathrm{H}), 7.31(\mathrm{t}, J=7.7 \mathrm{~Hz}, 2 \mathrm{H}), 7.22(\mathrm{t}, J=7.3 \mathrm{~Hz}, 1 \mathrm{H}), 2.07$ (s, 2H), 1.79 (s, 3H). ${ }^{13} \mathrm{C}$ NMR $\left(126 \mathrm{MHz}, \mathrm{CD}_{3} \mathrm{CN}\right) \delta 160.09,150.51,149.96,129.16,127.49,127.06,122.30,58.73$, 31.31. MS (ESI) $\mathrm{m} / \mathrm{z}$ calcd. for $\mathrm{C}_{13} \mathrm{H}_{15} \mathrm{~N}_{2}\left([\mathrm{M}+\mathrm{H}]^{+}\right)$199.1, found 199.2.

Compound $\mathbf{5 a}$ was also synthesized from the corresponding iminium $\mathrm{HCl}$ salt $\mathbf{6 a}$ and cyanoarene $\mathbf{4 a}$. The reaction was conducted by following the General Iminium Salt Reaction Procedure using iminium $\mathrm{HCl}$ salt 6a (156 mg. 0.66 mmol, 2.2 equiv.); photocatalyst $\operatorname{Ir}[\mathrm{dF}(\mathrm{Me}) \text { ppy }]_{2} \mathrm{dtbbpyPF}_{6}(6 \mathrm{mg}, 2 \mathrm{E}-3 \mathrm{mmol}, 2 \mathrm{~mol} \%)$; DIPA (153 $\mu \mathrm{L}, 1.1 \mathrm{mmol}$, 3.6 equiv.); and 4-cyanopyridine ( $31 \mathrm{mg}, 0.3 \mathrm{mmol}, 1.0$ equiv.). The yield of product $\mathbf{5 a}$ was determined to be $81 \%$ by a calibrated UPLC-MS. 


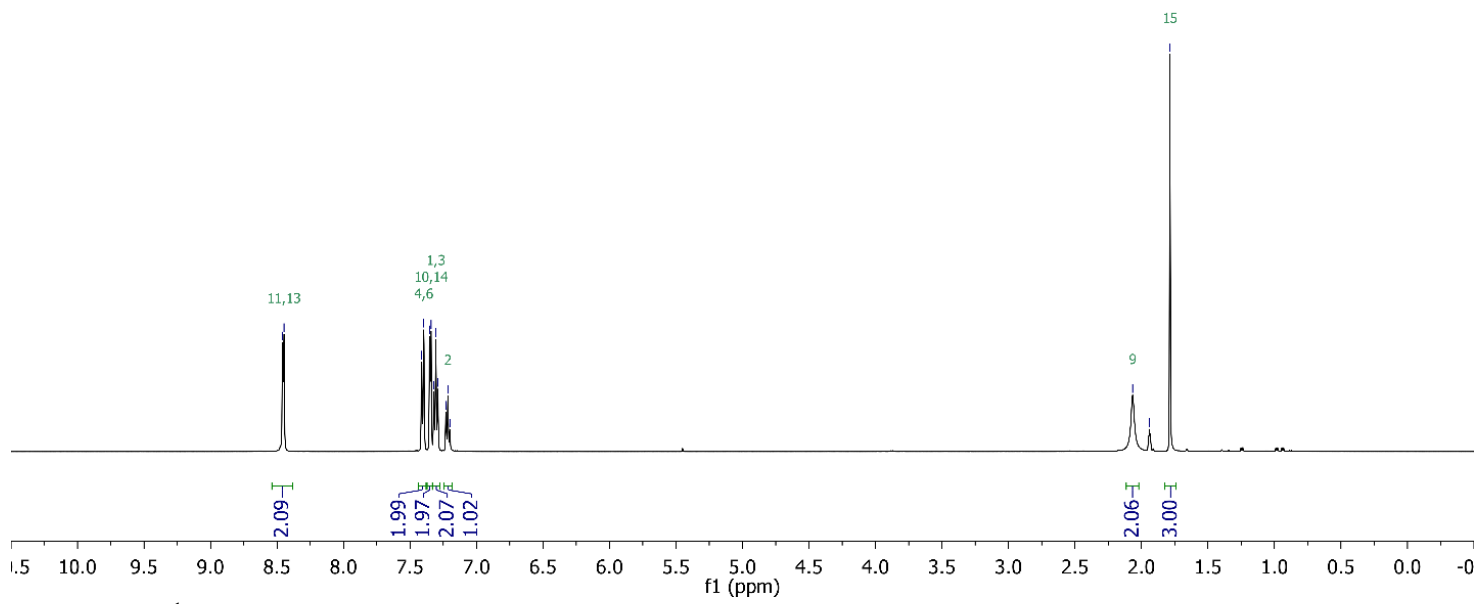

Figure S6. ${ }^{1} \mathrm{H}$ qNMR $(500 \mathrm{MHz})$ spectrum of $\mathbf{5 a}$ in $\mathrm{CD}_{3} \mathrm{CN}$.
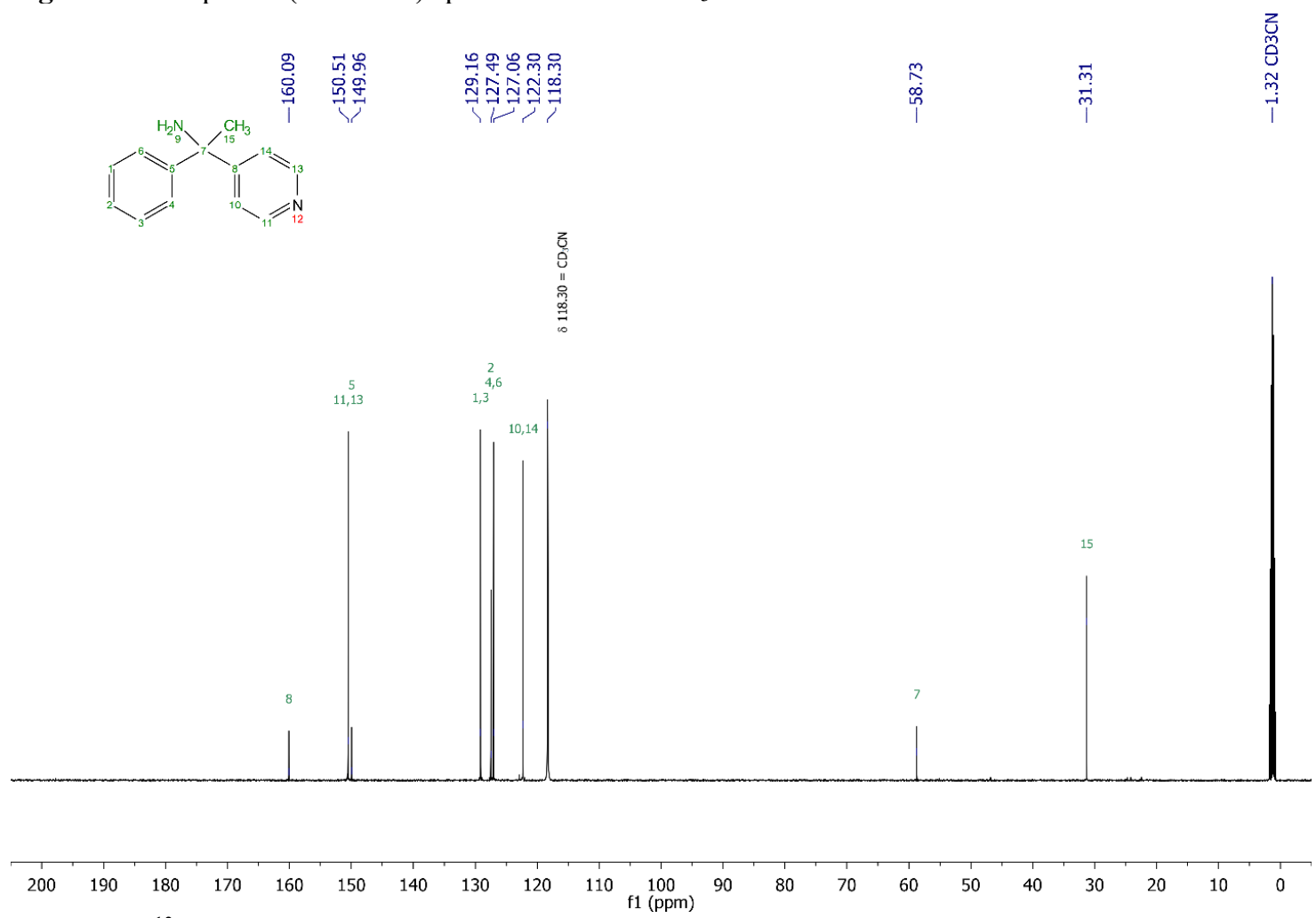

Figure S7. ${ }^{13} \mathrm{C}$ NMR (126 MHz) spectrum of 5a in $\mathrm{CD}_{3} \mathrm{CN}$. 


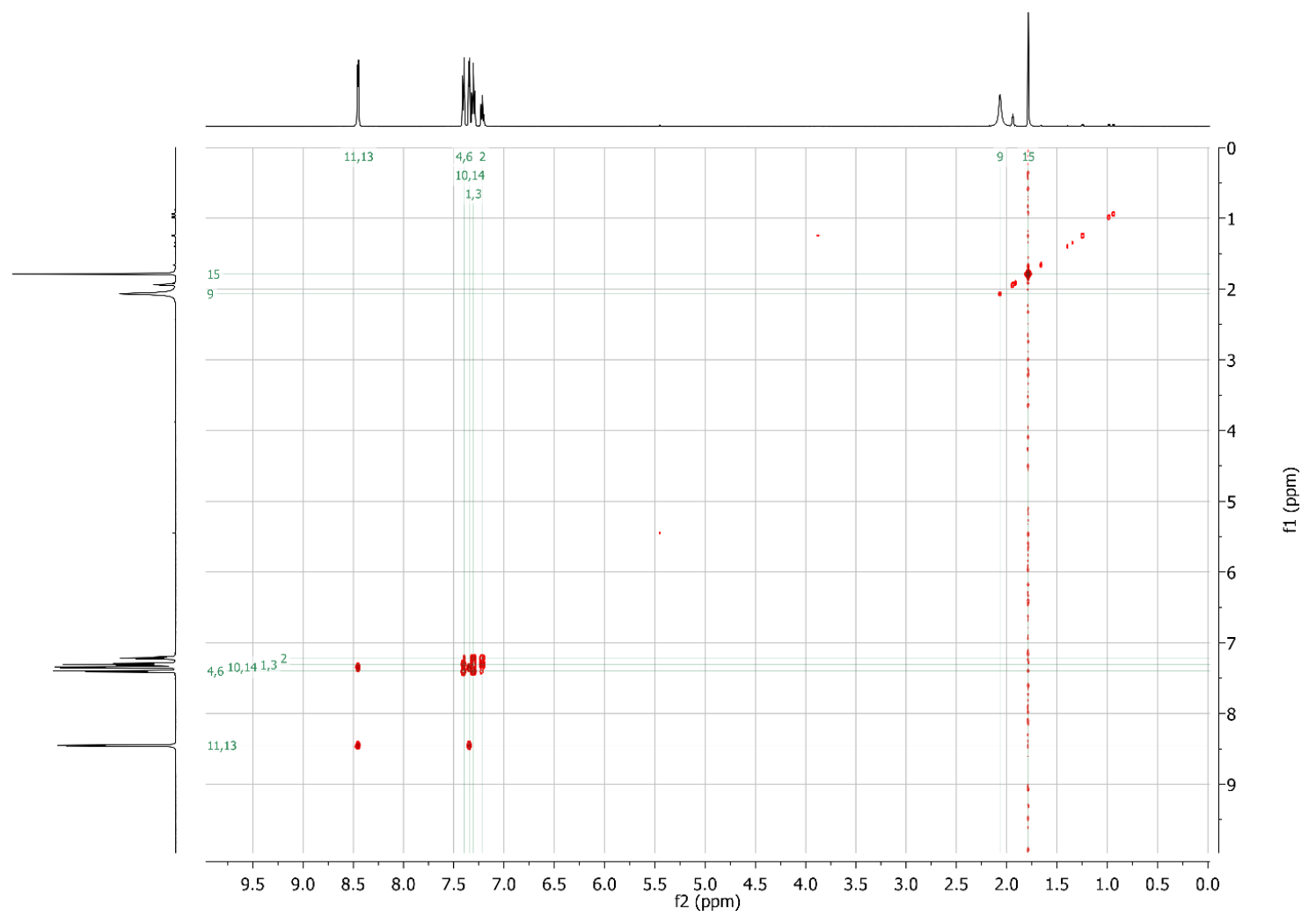

Figure S8. COSY NMR (126 MHz) spectrum of $\mathbf{5 a}$ in $\mathrm{CD}_{3} \mathrm{CN}$.

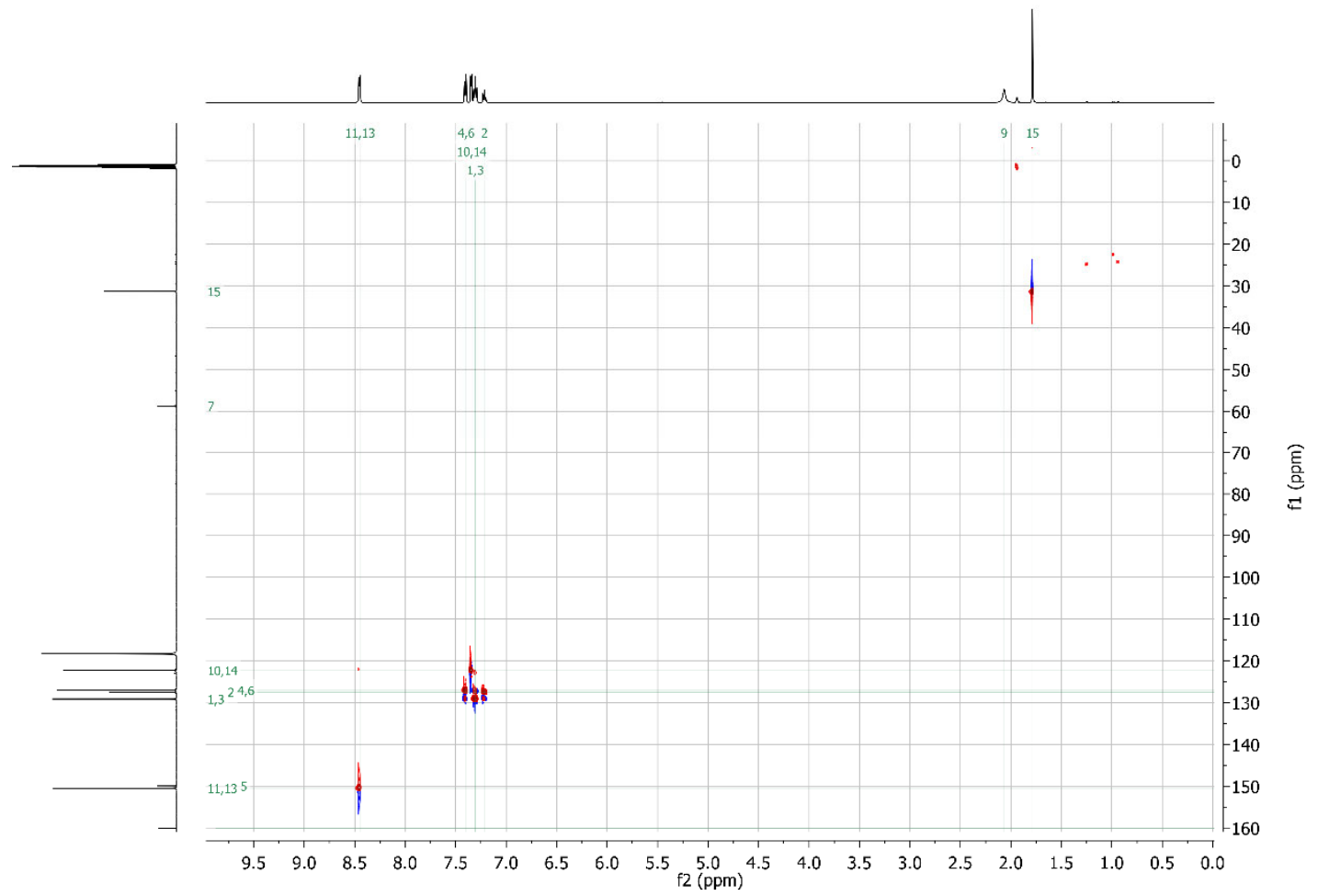

Figure S9. HSQC NMR (126 MHz) spectrum of $\mathbf{5 a}$ in $\mathrm{CD}_{3} \mathrm{CN}$. 


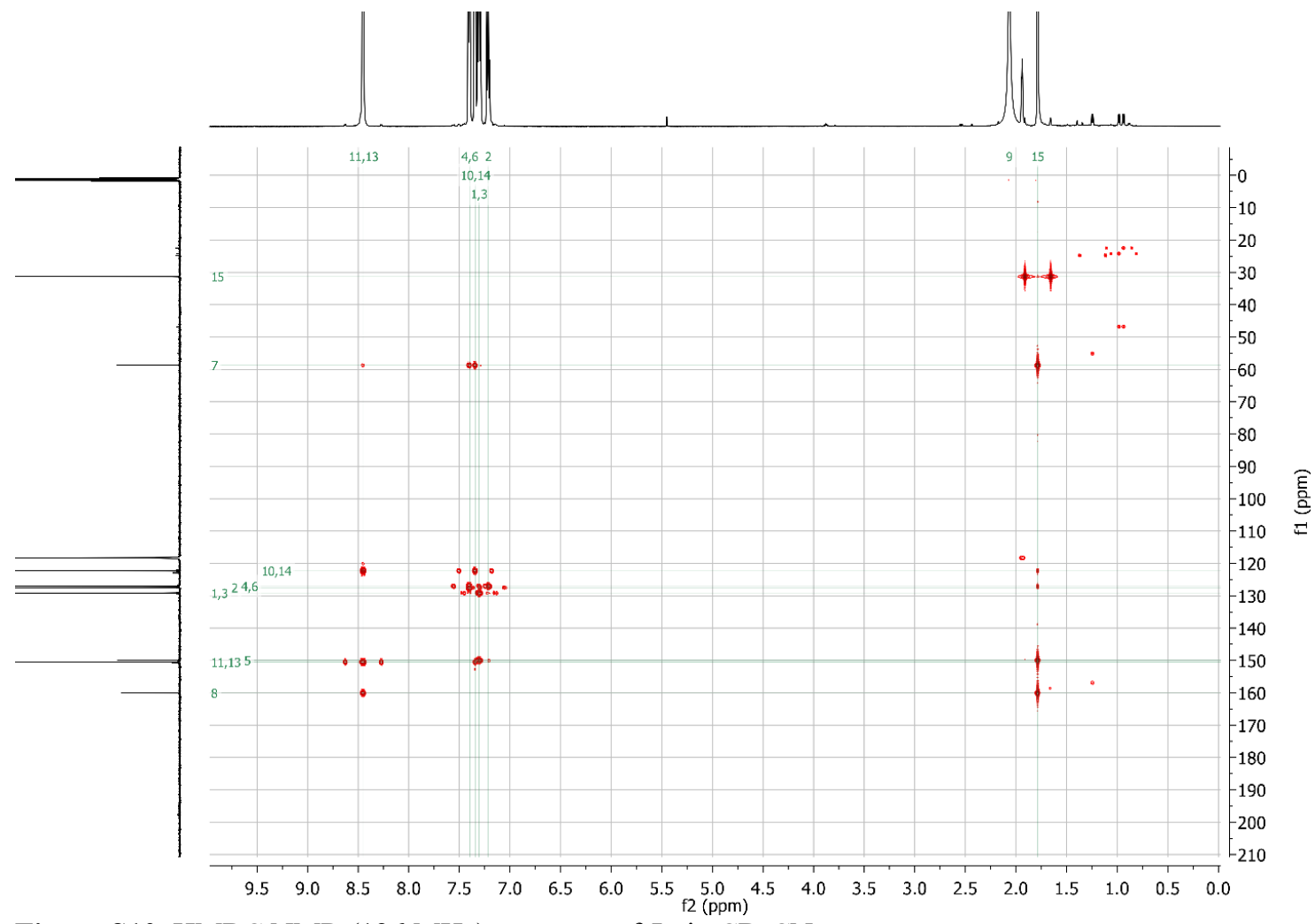

Figure S10. HMBC NMR (126 MHz) spectrum of $\mathbf{5 a}$ in $\mathrm{CD}_{3} \mathrm{CN}$. 
<smiles>CC(N)(c1ccncc1)c1ccc(C(F)(F)F)cc1</smiles>

Compound $\mathbf{5 b}$ was synthesized following General Procedure A using 4-pyridinecarbonitrile 4a (69 mg, $0.66 \mathrm{mmol})$, PC1 (6 mg, 6E-3 mmol), benzoic acid (37 mg, $0.3 \mathrm{mmol})$, DIPA (153 $\mu \mathrm{L}, 1.1 \mathrm{mmol})$ and $O$-benzoyl oxime (3b) $(88$ $\mathrm{mg}, 0.3 \mathrm{mmol})$ in DMSO $(3 \mathrm{~mL})$. The product was purified according to the general chromatography method for oximes. The product was obtained as a film on a vial $(50 \mathrm{mg}, 64 \%)$. The yield of product $\mathbf{5 b}$ was determined to be 91\% by a calibrated UPLC-MS ${ }^{1} \mathrm{H}$ NMR $\left(500 \mathrm{MHz}, \mathrm{CD}_{3} \mathrm{CN}\right) \delta 8.47(\mathrm{~d}, J=5.4 \mathrm{~Hz}, 2 \mathrm{H}), 7.67-7.55(\mathrm{~m}, 4 \mathrm{H}), 7.34(\mathrm{~d}$, $J=5.6 \mathrm{~Hz}, 2 \mathrm{H}), 2.14(\mathrm{~s}, 2 \mathrm{H}), 1.81(\mathrm{~s}, 3 \mathrm{H}) .{ }^{13} \mathrm{C}$ NMR $\left(126 \mathrm{MHz}, \mathrm{CD}_{3} \mathrm{CN}\right) \delta 159.14,154.45,150.71,129.03(\mathrm{q}, J=$ $32.2 \mathrm{~Hz}), 126.57$ (q, $J=271.6,270.8,254.6 \mathrm{~Hz}), 126.00$ (q, $J=3.7 \mathrm{~Hz}), 122.27,118.30,58.88,31.09 .{ }^{19} \mathrm{~F}$ NMR $(471$ $\left.\mathrm{MHz}, \mathrm{CD}_{3} \mathrm{CN}\right) \delta-62.89$. MS (ESI) $\mathrm{m} / \mathrm{z}$ calcd. for $\mathrm{C}_{14} \mathrm{H}_{13} \mathrm{~F}_{3} \mathrm{~N}_{2}\left([\mathrm{M}+\mathrm{H}]^{+}\right)$267.1, found 267.2.

Compound $\mathbf{5 b}$ was also synthesized from the corresponding iminium $\mathrm{HCl}$ salt $\mathbf{6} \mathbf{b}$ and cyanoarene $4 \mathbf{a}$. The reaction was conducted by following the General Iminium Salt Reaction Procedure using iminium $\mathrm{HCl}$ salt (6b) (211 mg, 0.66 mmol, 2.2 equiv.); photocatalyst $\operatorname{Ir}\left[\mathrm{dF}(\mathrm{Me}) \mathrm{ppy}_{2} \mathrm{dtbbpyPF}_{6}(6 \mathrm{mg}, 2 \mathrm{E}-3 \mathrm{mmol}, 2 \mathrm{~mol} \%\right.$ ); DIPA (153 $\mu \mathrm{L}, 1.1 \mathrm{mmol}$, 3.6 equiv.); and 4-cyanopyridine ( $31 \mathrm{mg}, 0.3 \mathrm{mmol}, 1.0$ equiv.). The yield of product $\mathbf{5 b}$ was determined to be $14 \%$ by a calibrated UPLC-MS. 


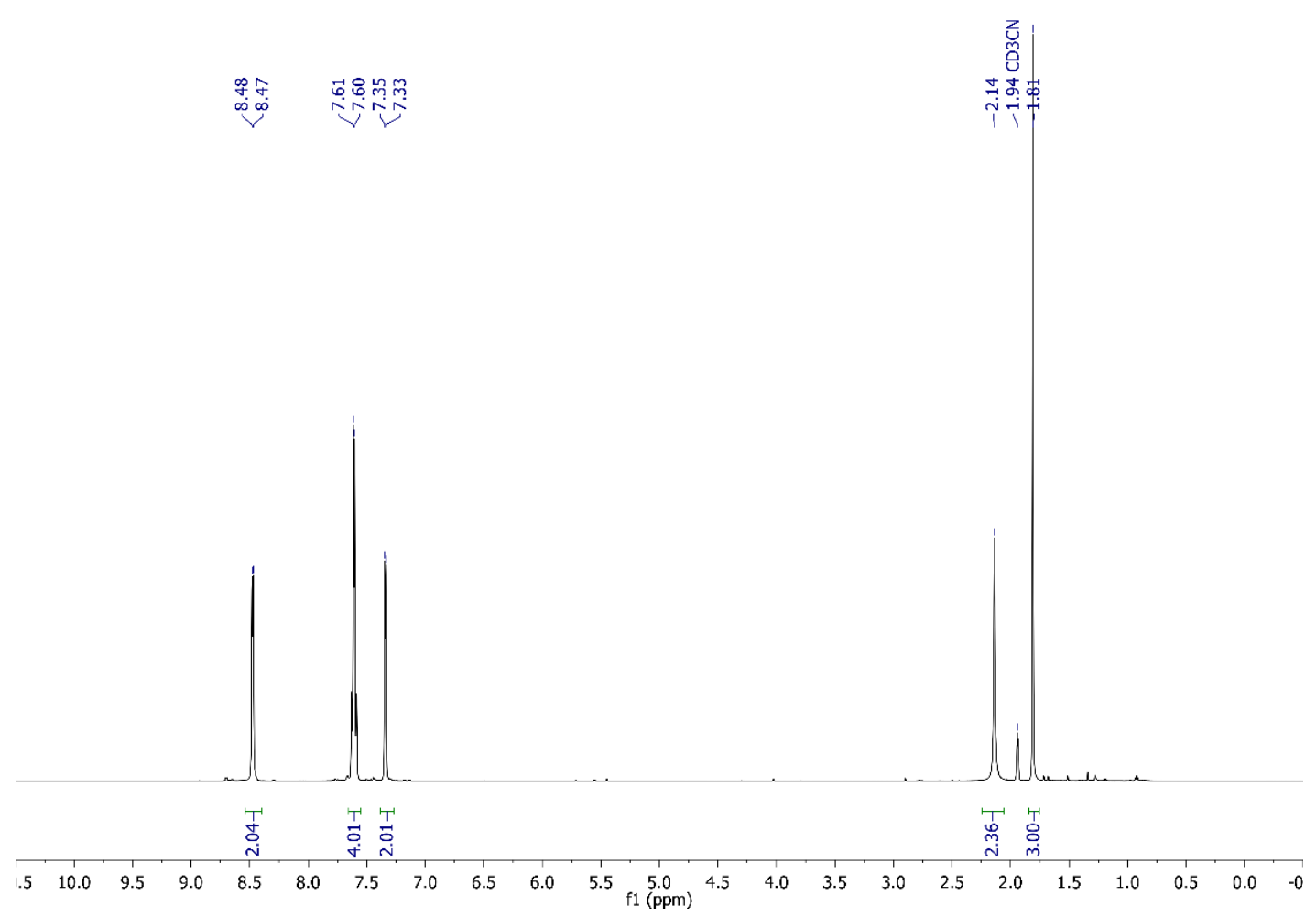

Figure S11. ${ }^{1} \mathrm{H}$ qNMR $(500 \mathrm{MHz})$ spectrum of $\mathbf{5 b}$ in $\mathrm{CD}_{3} \mathrm{CN}$.

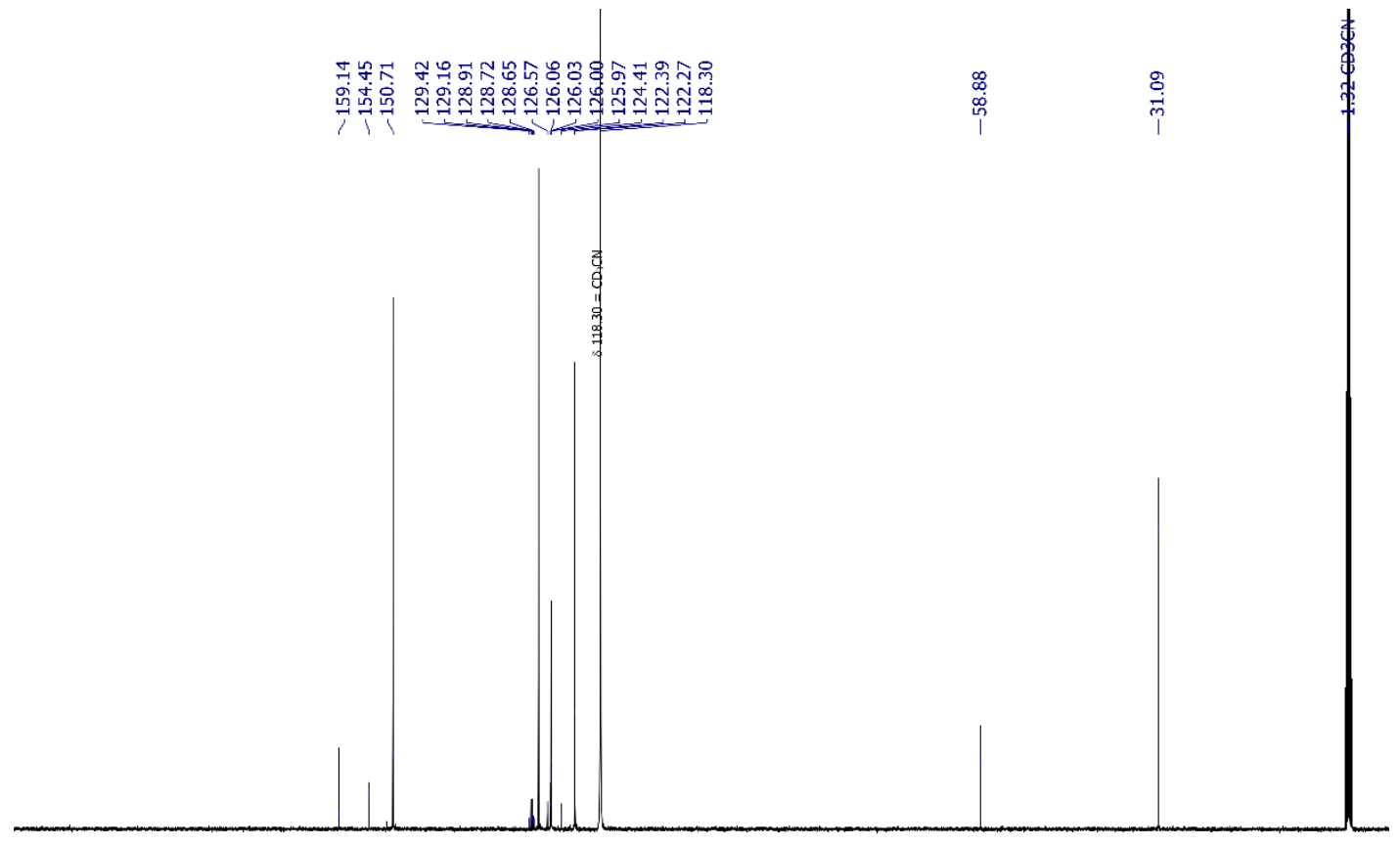

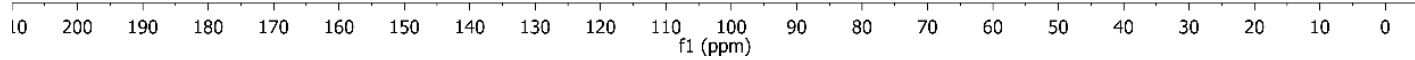

Figure S12. ${ }^{13} \mathrm{C}$ NMR $(126 \mathrm{MHz})$ spectrum of $\mathbf{5 b}$ in $\mathrm{CD}_{3} \mathrm{CN}$. 


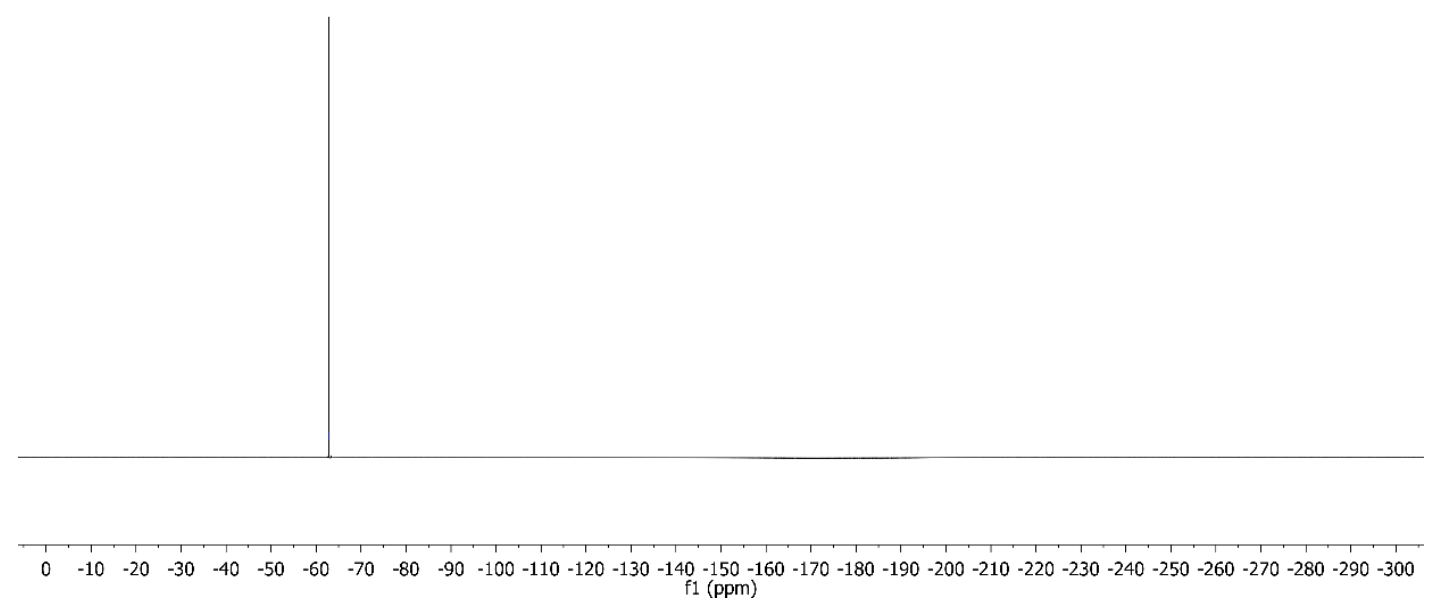

Figure S13. ${ }^{19} \mathrm{~F}$ NMR $(471 \mathrm{MHz})$ spectrum of compound $\mathbf{5 b}$ in $\mathrm{CD}_{3} \mathrm{CN}$. 
<smiles>CC(N)(c1ccncc1)c1ccc(Cl)cc1</smiles>

Compound 5c was synthesized following General Procedure A using 4-pyridinecarbonitrile 4a (69 $\mathrm{mg}, 0.66 \mathrm{mmol})$, PC1 (6 mg, 6E-3 mmol), benzoic acid (37 mg, $0.3 \mathrm{mmol})$, DIPA $(153 \mu \mathrm{L}, 1.1 \mathrm{mmol})$ and $O$-benzoyl oxime (3c) $(82$ $\mathrm{mg}, 0.3 \mathrm{mmol})$ in DMSO $(3 \mathrm{~mL})$. The product was purified according to the general chromatography method for oximes. The product was obtained as a film on a vial $(51 \mathrm{mg}, 73 \%)$. The yield of product $\mathbf{5 f}$ was determined to be $98 \%$ by a calibrated UPLC-MS ${ }^{1} \mathrm{H}$ NMR $\left(500 \mathrm{MHz}\right.$, Acetonitrile- $\left.d_{3}\right) \delta 8.46(\mathrm{~d}, J=6.2 \mathrm{~Hz}, 2 \mathrm{H}), 7.38(\mathrm{~d}, J=8.8 \mathrm{~Hz}$, 2H), $7.33(\mathrm{~d}, J=6.2 \mathrm{~Hz}, 2 \mathrm{H}), 7.31-7.28(\mathrm{~m}, 2 \mathrm{H}), 2.18(\mathrm{~s}, 3 \mathrm{H}), 1.76(\mathrm{~s}, 3 \mathrm{H}) .{ }^{13} \mathrm{C}$ NMR $\left(126 \mathrm{MHz}, \mathrm{CD}_{3} \mathrm{CN}\right) \delta 159.54$, $150.59,148.86,132.78,129.02,128.99,122.23,118.30,58.51,41.38,31.24$. MS (ESI) m/z calcd. for $\mathrm{C}_{13} \mathrm{H}_{14} \mathrm{ClN}_{2}([\mathrm{M}$ $+\mathrm{H}]^{+}$) 233.1, found 233.2.

Compound $\mathbf{5 c}$ was also synthesized from the corresponding iminium $\mathrm{HCl}$ salt $\mathbf{6 c}$ and cyanoarene $4 \mathbf{a}$. The reaction was conducted by following the General Iminium Salt Reaction Procedure using iminium $\mathrm{HCl}$ salt (6c) (125 mg, 0.66 mmol, 2.2 equiv.); photocatalyst $\operatorname{Ir}[\mathrm{dF}(\mathrm{Me}) \text { ppy }]_{2} \mathrm{dtbbpyPF}_{6}$ (6 mg, 2E-3 mmol, $2 \mathrm{~mol} \%$ ); DIPA (153 $\mu \mathrm{L}, 1.1 \mathrm{mmol}$, 3.6 equiv.); and 4-cyanopyridine ( $31 \mathrm{mg}, 0.3 \mathrm{mmol}, 1.0$ equiv.). The yield of product $\mathbf{5 c}$ was determined to be $66 \%$ by a calibrated UPLC-MS. 


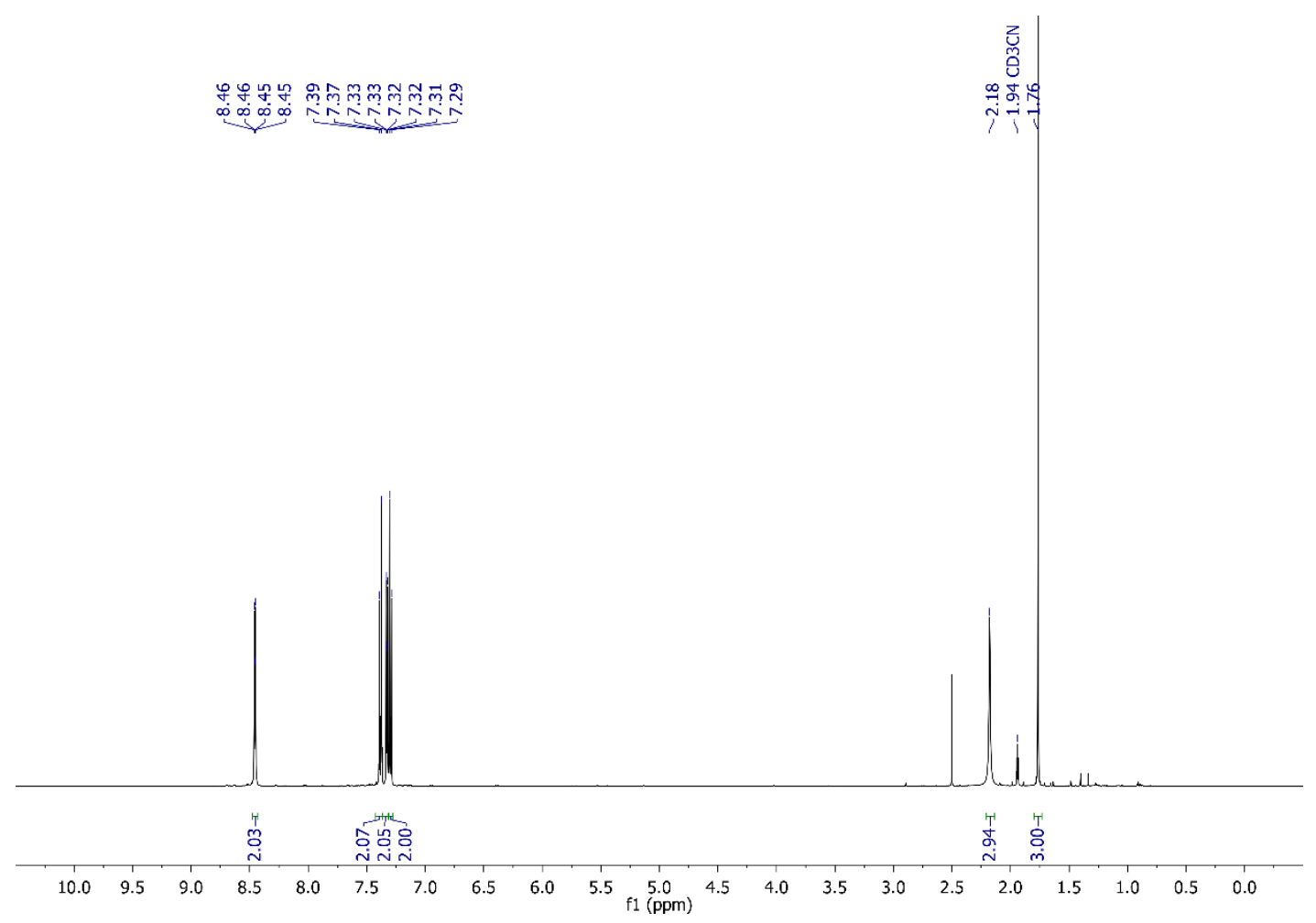

Figure S14. ${ }^{1} \mathrm{H}$ qNMR $(500 \mathrm{MHz})$ spectrum of $\mathbf{5 c}$ in $\mathrm{CD}_{3} \mathrm{CN}$.

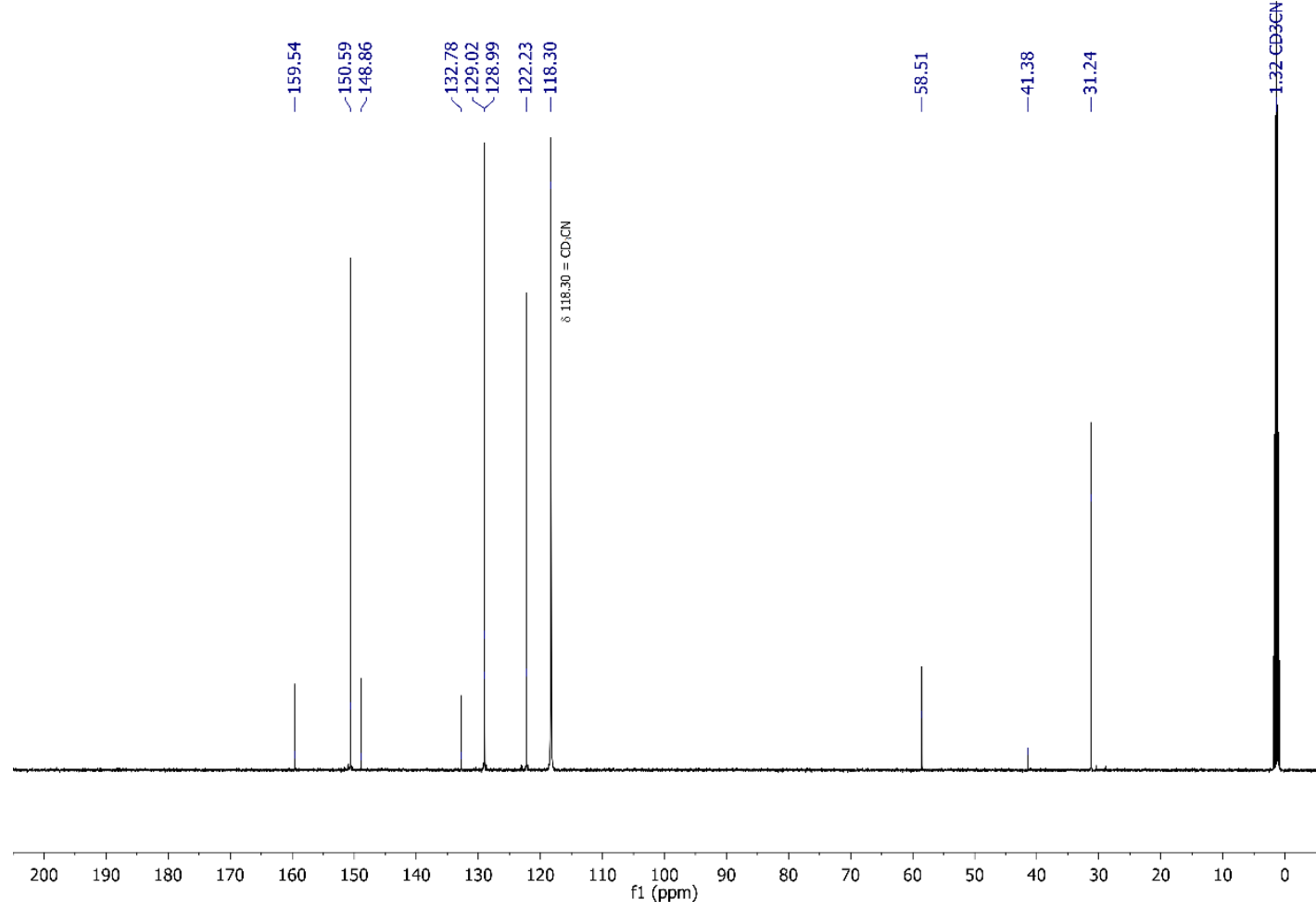

Figure S15. ${ }^{13} \mathrm{C}$ NMR $(126 \mathrm{MHz})$ spectrum of $\mathbf{5} \mathbf{c}$ in $\mathrm{CD}_{3} \mathrm{CN}$. 


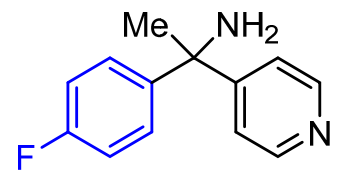

Synthesis of 5d was synthesized following General Procedure A using 4-pyridinecarbonitrile $4 \mathbf{a}(69 \mathrm{mg}, 0.66 \mathrm{mmol})$, PC1 (6 mg, 6E-3 mmol), benzoic acid (37 mg, $0.3 \mathrm{mmol})$, DIPA (153 $\mu \mathrm{L}, 1.1 \mathrm{mmol})$ and $O$-benzoyl oxime (3d) (77 $\mathrm{mg}, 0.3 \mathrm{mmol})$ in DMSO $(3 \mathrm{~mL})$. The product was purified according to the general chromatography method for oximes. The product was obtained as a film on a vial $(50 \mathrm{mg}, 77 \%)$. The yield of product $\mathbf{5} \mathbf{f}$ was determined to be $94 \%$ by a calibrated UPLC-MS. ${ }^{1} \mathrm{H}$ NMR $\left(500 \mathrm{MHz}, \mathrm{CD}_{3} \mathrm{CN}\right) \delta 8.46(\mathrm{dd}, J=4.6,1.5 \mathrm{~Hz}, 2 \mathrm{H}), 7.41(\mathrm{dd}, J=8.9,5.4$ $\mathrm{Hz}, 2 \mathrm{H}), 7.34$ (dd, $J=4.6,1.6 \mathrm{~Hz}, 2 \mathrm{H}), 7.03$ (t, $J=8.9 \mathrm{~Hz}, 2 \mathrm{H}), 2.09$ (s, 3H), $1.78(\mathrm{~s}, 3 \mathrm{H}) .{ }^{13} \mathrm{C} \mathrm{NMR}(126 \mathrm{MHz}$, $\left.\mathrm{CD}_{3} \mathrm{CN}\right) \delta 163.32,161.39,159.85,150.58,146.07,146.05,129.16,129.09,122.23,118.31,115.66,115.49,58.43$, 31.49. ${ }^{19} \mathrm{~F}$ NMR (471 MHz, $\left.\mathrm{CD}_{3} \mathrm{CN}\right) \delta$-118.40. MS (ESI) $\mathrm{m} / \mathrm{z}$ calcd. for $\mathrm{C}_{13} \mathrm{H}_{14} \mathrm{FN}_{2}\left([\mathrm{M}+\mathrm{H}]^{+}\right) 217.1$, found 217.2

Compound 5d was also synthesized from the corresponding iminium $\mathrm{HCl}$ salt $\mathbf{6 d}$ and cyanoarene $4 \mathbf{a}$. The reaction was conducted by following the General Iminium Salt Reaction Procedure using iminium $\mathrm{HCl} \mathrm{salt} \mathrm{(6d)(174} \mathrm{mg,} 0.66$ mmol, 2.2 equiv.); photocatalyst $\operatorname{Ir}[\mathrm{dF}(\mathrm{Me}) \text { ppy }]_{2} \mathrm{dtbbpyPF}_{6}(6 \mathrm{mg}, 2 \mathrm{E}-3 \mathrm{mmol}, 2 \mathrm{~mol} \%)$; DIPA (153 $\mu \mathrm{L}, 1.1 \mathrm{mmol}$, 3.6 equiv.); and 4-cyanopyridine ( $31 \mathrm{mg}, 0.3 \mathrm{mmol}, 1.0$ equiv.). The yield of product $\mathbf{5 d}$ was determined to be $87 \%$ by a calibrated UPLC-MS. 


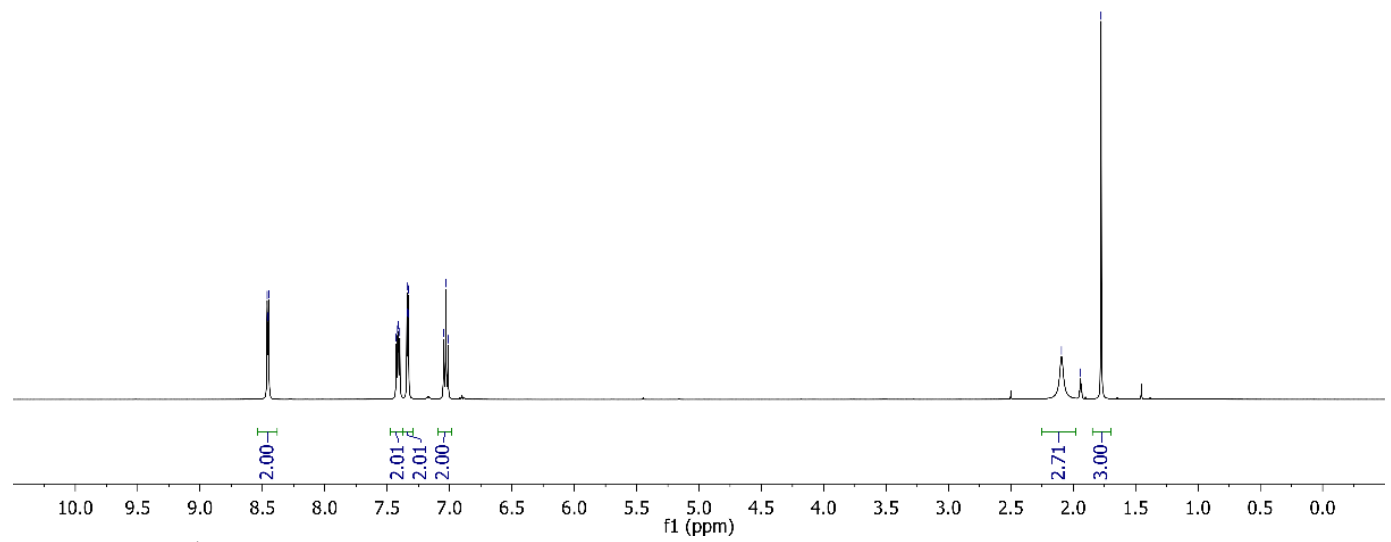

Figure S16. ${ }^{1} \mathrm{H}$ qNMR $(500 \mathrm{MHz})$ spectrum of $\mathbf{5 d}$ in $\mathrm{CD}_{3} \mathrm{CN}$.

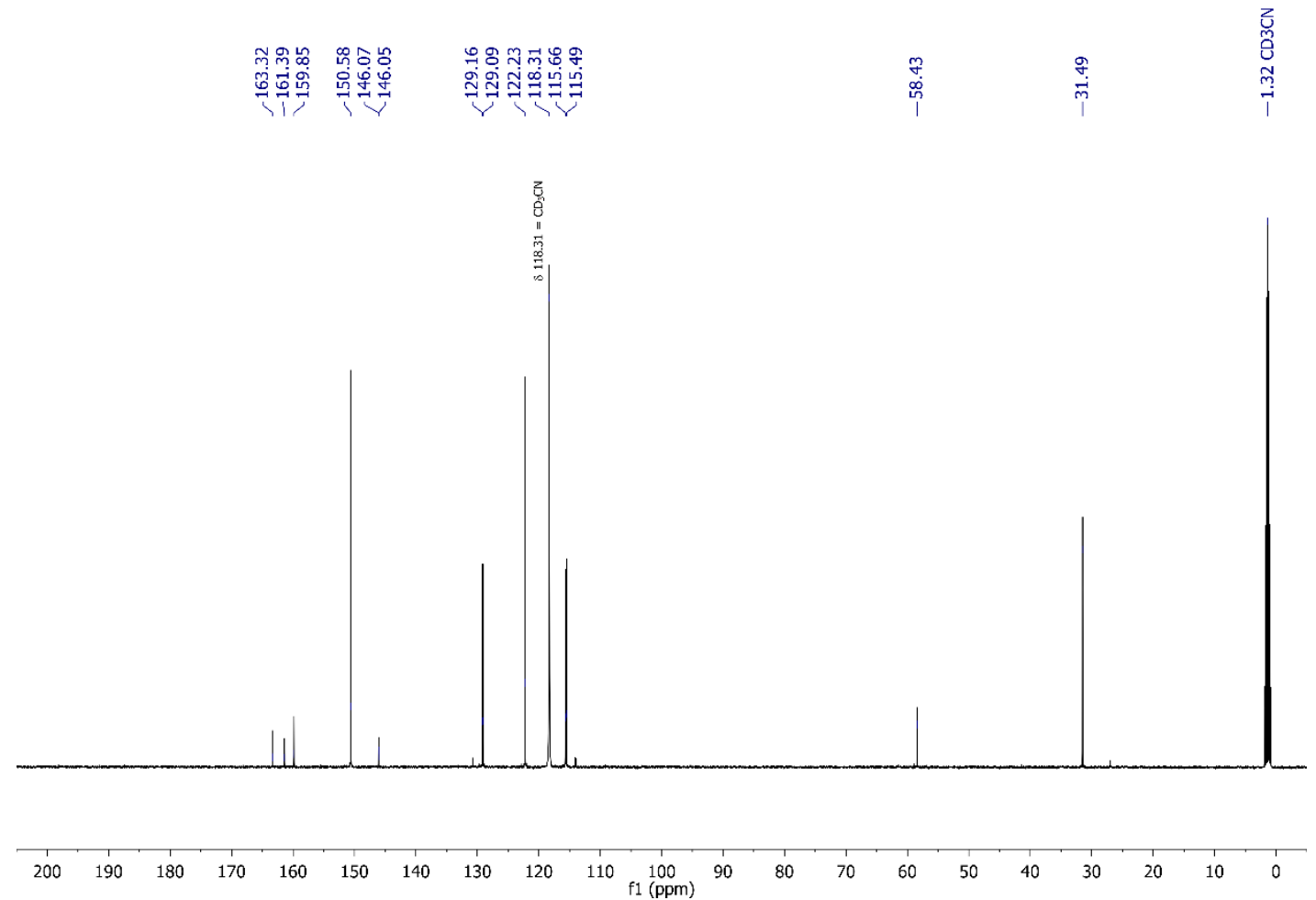

Figure S17. ${ }^{13} \mathrm{C}$ NMR $(126 \mathrm{MHz})$ spectrum of $\mathbf{5 d}$ in $\mathrm{CD}_{3} \mathrm{CN}$. 

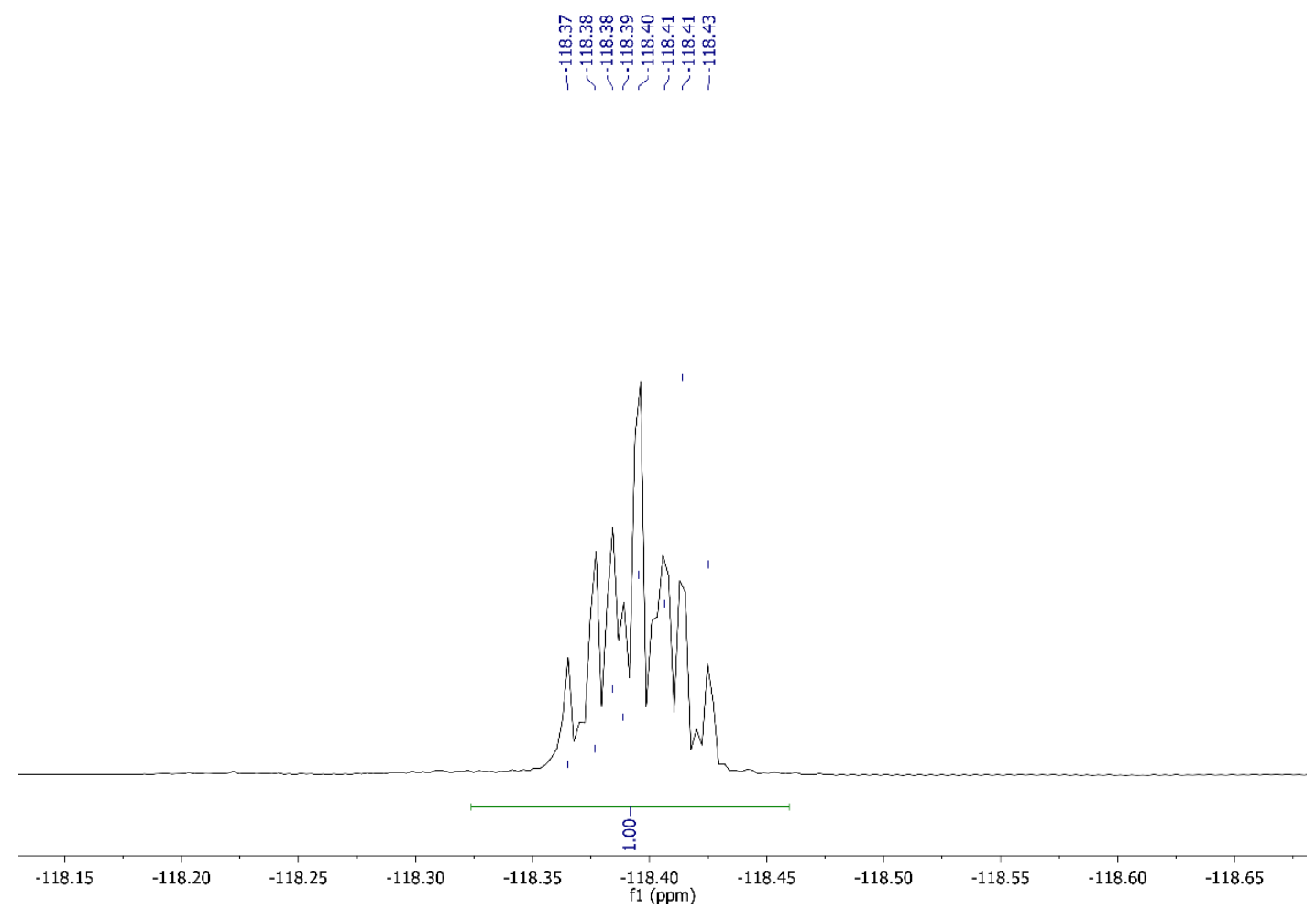

Figure S18. ${ }^{19} \mathrm{~F}$ NMR $(471 \mathrm{MHz})$ spectrum of compound $\mathbf{5 d}$ in $\mathrm{CD}_{3} \mathrm{CN}$. 
<smiles>Cc1ccc(C(C)(N)c2ccncc2)cc1</smiles>

Synthesis of 5e was synthesized following General Procedure A using 4-pyridinecarbonitrile (69 $\mathrm{mg}, 0.66 \mathrm{mmol})$, PC1 (6 mg, 6E-3 mmol), benzoic acid (37 mg, $0.3 \mathrm{mmol})$, DIPA $(153 \mu \mathrm{L}, 1.1 \mathrm{mmol})$ and $O$-benzoyl oxime (3e) $(76$ $\mathrm{mg}, 0.3 \mathrm{mmol})$ in DMSO $(3 \mathrm{~mL})$. The product was purified according to the general chromatography method for oximes. The product was obtained as a film on a vial $(50 \mathrm{mg}, 79 \%)$. The yield of product $\mathbf{5 e}$ was determined to be $98 \%$ by a calibrated UPLC-MS ${ }^{1} \mathrm{H}$ NMR $\left(500 \mathrm{MHz}, \mathrm{CD}_{3} \mathrm{CN}\right) \delta 8.44(\mathrm{~d}, J=6.2 \mathrm{~Hz}, 2 \mathrm{H}), 7.34(\mathrm{~d}, J=6.2 \mathrm{~Hz}, 2 \mathrm{H})$, $7.27(\mathrm{~d}, J=8.3 \mathrm{~Hz}, 2 \mathrm{H}), 7.12(\mathrm{~d}, J=8.0 \mathrm{~Hz}, 2 \mathrm{H}), 2.29(\mathrm{~s}, 3 \mathrm{H}), 1.76(\mathrm{~s}, 3 \mathrm{H}) .{ }^{13} \mathrm{C} \mathrm{NMR}\left(126 \mathrm{MHz}, \mathrm{CD}_{3} \mathrm{CN}\right) \delta 160.26$, $150.47,147.05,137.09,129.74,126.99,122.27,58.46,31.37,20.94$. MS (ESI) m/z calcd. for $\mathrm{C}_{14} \mathrm{H}_{17} \mathrm{~N}_{2}\left([\mathrm{M}+\mathrm{H}]^{+}\right)$ 213.1, found 213.2

Compound 5e was also synthesized from the corresponding iminium $\mathrm{HCl}$ salt $\mathbf{6 e}$ and cyanoarene 4a. The reaction was conducted by following the General Iminium Salt Reaction Procedure using iminium HCl salt (6e) $(112 \mathrm{mg}, 0.66$ mmol, 2.2 equiv.); photocatalyst $\operatorname{Ir}[\mathrm{dF}(\mathrm{Me}) \text { ppy }]_{2} \mathrm{dtbbpyPF}_{6}(6 \mathrm{mg}, 2 \mathrm{E}-3 \mathrm{mmol}, 2 \mathrm{~mol} \%)$; DIPA (153 $\mu \mathrm{L}, 1.1 \mathrm{mmol}$, 3.6 equiv.); and 4-cyanopyridine ( $31 \mathrm{mg}, 0.3 \mathrm{mmol}, 1.0$ equiv.). The yield of product $\mathbf{5 e}$ was determined to be $93 \%$ by a calibrated UPLC-MS. 


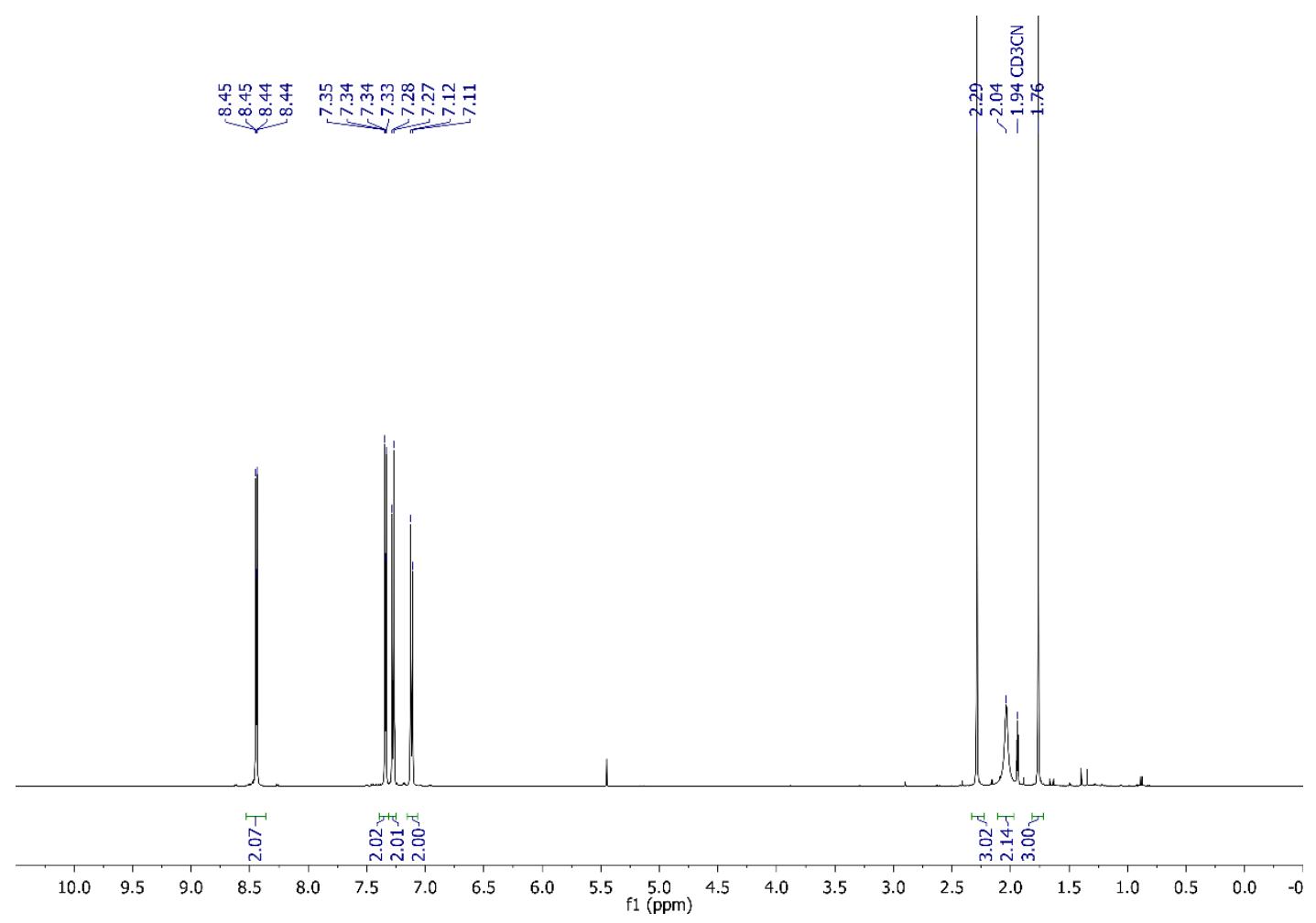

Figure S19. ${ }^{1} \mathrm{H}$ qNMR $(500 \mathrm{MHz})$ spectrum of $5 \mathbf{e}$ in $\mathrm{CD}_{3} \mathrm{CN}$.
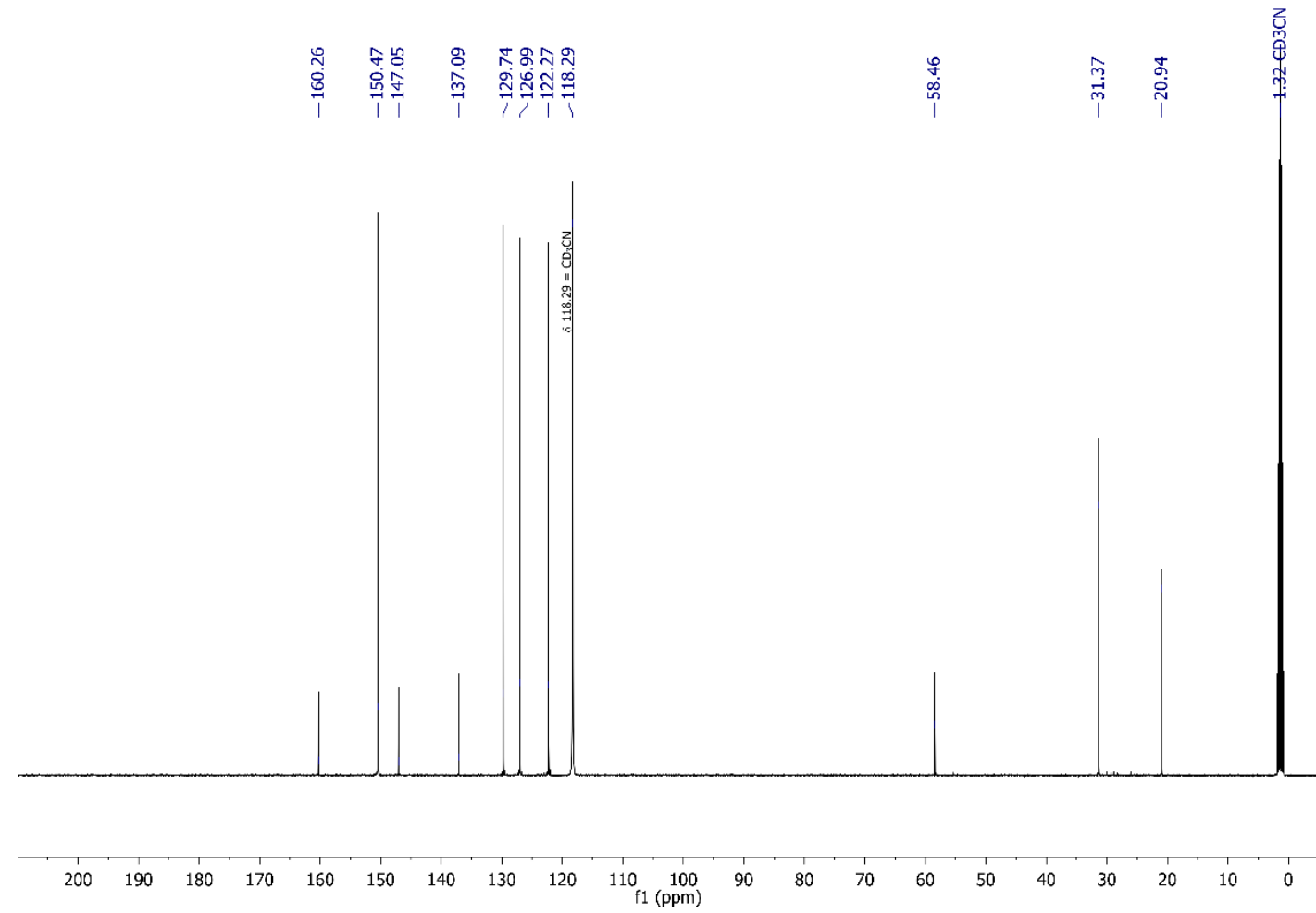

Figure S20. ${ }^{13} \mathrm{C}$ NMR $(126 \mathrm{MHz})$ spectrum of $\mathbf{5 e}$ in $\mathrm{CD}_{3} \mathrm{CN}$. 
<smiles>COc1ccc(C(C)(N)c2ccncc2)cc1</smiles>

Synthesis of $\mathbf{5 f}$ was synthesized following General Procedure A using 4-pyridinecarbonitrile 4a (69 $\mathrm{mg}, 0.66 \mathrm{mmol})$, PC1 (6 mg, 6E-3 mmol), benzoic acid (37 mg, $0.3 \mathrm{mmol})$, DIPA ( $153 \mu \mathrm{L}, 1.1 \mathrm{mmol})$ and $O$-benzoyl oxime (3f) $(81$ $\mathrm{mg}, 0.3 \mathrm{mmol})$ in DMSO $(3 \mathrm{~mL})$. The product was purified according to the general chromatography method for oximes. The product was obtained as a film on a vial $(44 \mathrm{mg}, 64 \%)$. The yield of product $\mathbf{5} \mathbf{f}$ was determined to be $79 \%$ by a calibrated UPLC-MS ${ }^{1} \mathrm{H}$ NMR $\left(500 \mathrm{MHz}, \mathrm{CD}_{3} \mathrm{CN}\right) \delta 8.44(\mathrm{~d}, \mathrm{~J}=6.0 \mathrm{~Hz}, 2 \mathrm{H}), 7.34(\mathrm{~d}, \mathrm{~J}=6.1 \mathrm{~Hz}, 2 \mathrm{H}), 7.31$ $(\mathrm{d}, \mathrm{J}=8.9 \mathrm{~Hz}, 2 \mathrm{H}), 6.85(\mathrm{~d}, \mathrm{~J}=8.8 \mathrm{~Hz}, 2 \mathrm{H}), 3.75(\mathrm{~s}, 3 \mathrm{H}), 2.06(\mathrm{~s}, 2 \mathrm{H}), 1.76(\mathrm{~s}, 3 \mathrm{H}) .{ }^{13} \mathrm{C} \mathrm{NMR}\left(126 \mathrm{MHz}, \mathrm{CD}_{3} \mathrm{CN}\right)$ $160.37,159.23,150.47,142.05,128.26,122.24,118.30,114.34,58.25,55.85,31.54$. MS (ESI) m/z calcd. for $\mathrm{C}_{14} \mathrm{H}_{16} \mathrm{~N}_{2} \mathrm{O}\left([\mathrm{M}+\mathrm{H}]^{+}\right)$229.1, found 229.2.

Compound $\mathbf{5 f}$ was also synthesized from the corresponding iminium $\mathrm{HCl}$ salt $\mathbf{6 f}$ and cyanoarene 4a. The reaction was conducted by following the General Iminium Salt Reaction Procedure using iminium HCl salt (6f) (186 mg, 0.66 mmol, 2.2 equiv.); photocatalyst $\operatorname{Ir}[\mathrm{dF}(\mathrm{Me}) \text { ppy }]_{2} \mathrm{dtbbpyPF}_{6}(6 \mathrm{mg}, 2 \mathrm{E}-3 \mathrm{mmol}, 2 \mathrm{~mol} \%$ ); DIPA (153 $\mu \mathrm{L}, 1.1 \mathrm{mmol}$, 3.6 equiv.); and 4-cyanopyridine $\mathbf{4 a}(31 \mathrm{mg}, 0.3 \mathrm{mmol}, 1.0$ equiv.). The yield of product $\mathbf{5 f}$ was determined to be $94 \%$ by a calibrated UPLC-MS. 


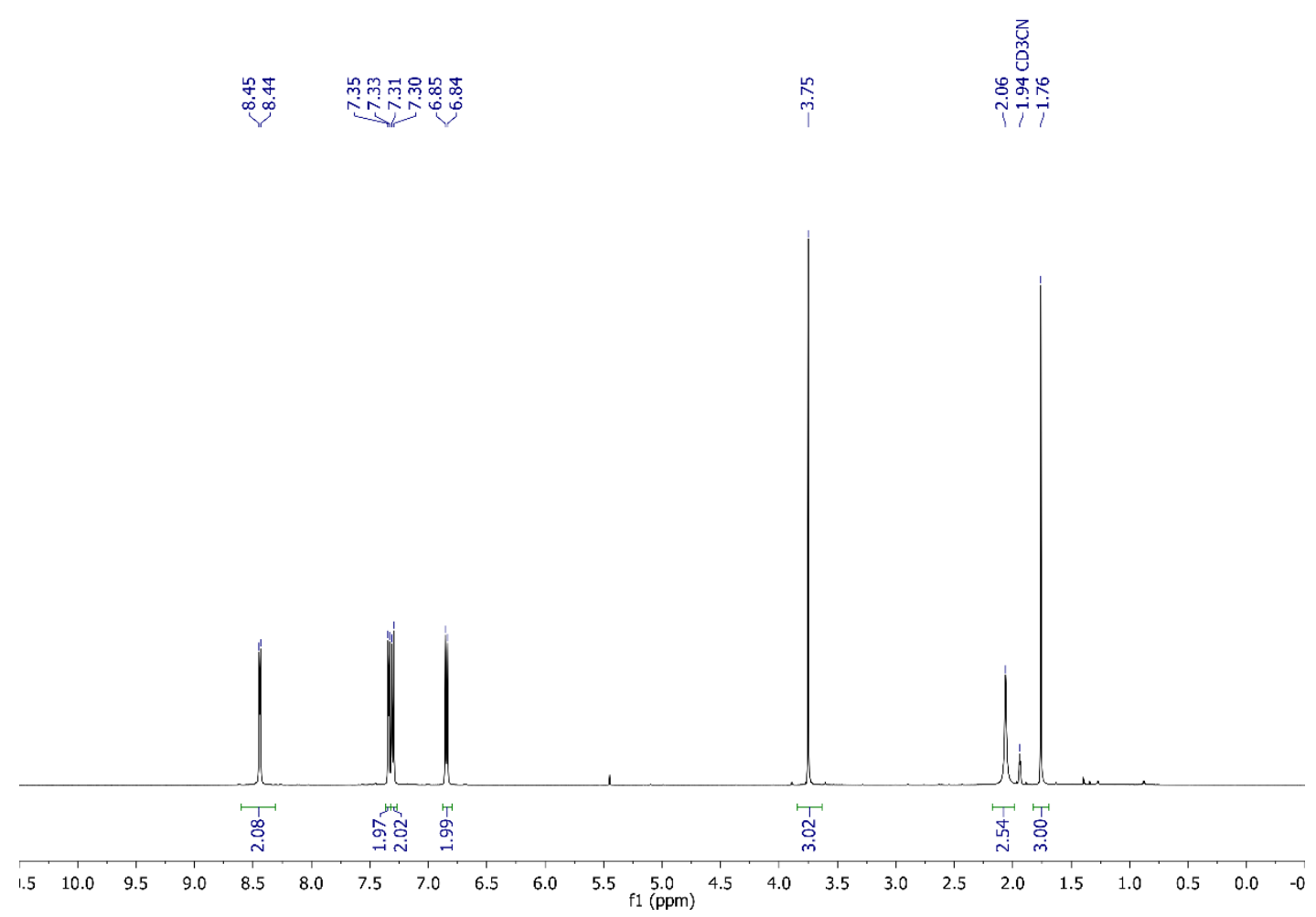

Figure S21. ${ }^{1} \mathrm{H}$ qNMR $(500 \mathrm{MHz})$ spectrum of $\mathbf{5} f$ in $\mathrm{CD}_{3} \mathrm{CN}$.
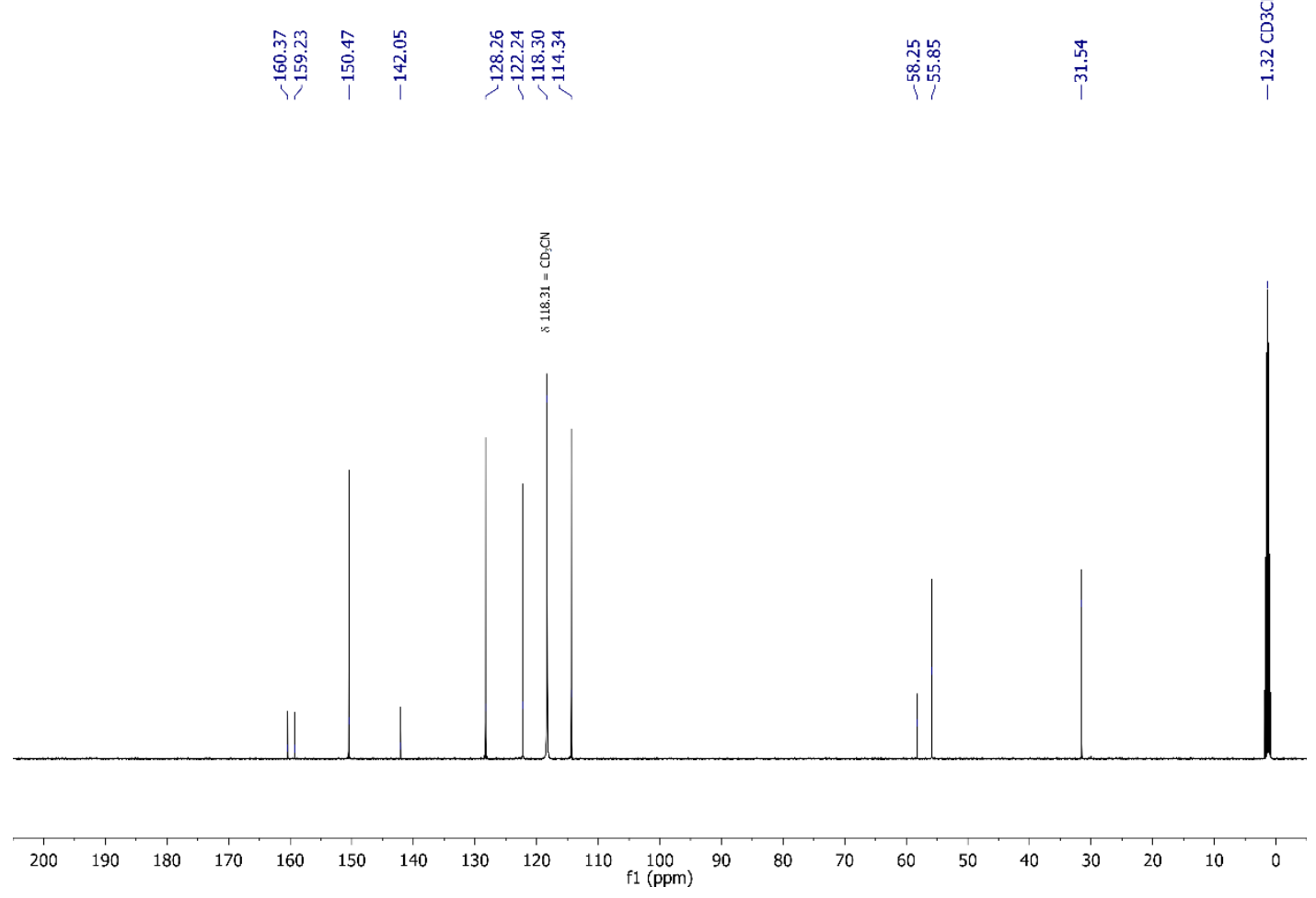

Figure S22. ${ }^{13} \mathrm{C}$ NMR $(126 \mathrm{MHz})$ spectrum of $\mathbf{5}$ in $\mathrm{CD}_{3} \mathrm{CN}$. 
<smiles>Cc1ccccc1C(C)(N)c1ccncc1</smiles>

Synthesis of $\mathbf{5 g}$ was synthesized following General Procedure A using 4-pyridinecarbonitrile 4a (69 mg, $0.66 \mathrm{mmol})$, PC1 $(6 \mathrm{mg}, 6 \mathrm{E}-3 \mathrm{mmol})$, benzoic acid $(37 \mathrm{mg}, 0.3 \mathrm{mmol})$, DIPA $(153 \mu \mathrm{L}, 1.1 \mathrm{mmol})$ and $O$-benzoyl oxime $(\mathbf{3 g})(76$ $\mathrm{mg}, 0.3 \mathrm{mmol})$ in DMSO $(3 \mathrm{~mL})$. The product was purified according to the general chromatography method for oximes. The product was obtained as a film on a vial $(21 \mathrm{mg}, 30 \%)$. The yield of product $\mathbf{5 g}$ was determined to be $32 \%$ by a calibrated UPLC-MS ${ }^{1} \mathrm{H}$ NMR $\left(500 \mathrm{MHz}, \mathrm{CD}_{3} \mathrm{CN}\right) \delta 8.43(\mathrm{~d}, J=5.5 \mathrm{~Hz}, 2 \mathrm{H}), 7.75(\mathrm{~d}, J=7.8 \mathrm{~Hz}, 1 \mathrm{H})$, $7.26(\mathrm{t}, J=7.6 \mathrm{~Hz}, 1 \mathrm{H}), 7.21(\mathrm{~d}, J=5.1 \mathrm{~Hz}, 3 \mathrm{H}), 7.09(\mathrm{~d}, J=7.4 \mathrm{~Hz}, 1 \mathrm{H}), 2.08(\mathrm{~s}, 3 \mathrm{H}), 1.86(\mathrm{~s}, 3 \mathrm{H}), 1.73(\mathrm{~s}, 3 \mathrm{H}) .{ }^{13} \mathrm{C}$ NMR $\left(126 \mathrm{MHz}, \mathrm{CD}_{3} \mathrm{CN}\right) \delta 160.67,150.57,146.52,137.35,133.32,128.25,126.94,126.59,121.74,118.30,59.31$, 32.93, 21.80. MS (ESI) M/Z CALCD. FOR $\mathrm{C}_{14} \mathrm{H}_{17} \mathrm{~N}_{2}\left([\mathrm{M}+\mathrm{H}]^{+}\right)$213.1, found 213.2

Compound $\mathbf{5 g}$ was also synthesized from the corresponding iminium $\mathrm{HCl}$ salt $\mathbf{6 g}$ and cyanoarene $\mathbf{4 a}$. The reaction was conducted by following the General Iminium Salt Reaction Procedure using iminium $\mathrm{HCl}$ salt (6g) (112 mg, 0.66 mmol, 2.2 equiv.); photocatalyst $\operatorname{Ir}\left[\mathrm{dF}(\mathrm{Me}) \mathrm{ppy}_{2} \mathrm{dtbbpyPF}_{6}(6 \mathrm{mg}, 2 \mathrm{E}-3 \mathrm{mmol}, 2 \mathrm{~mol} \%)\right.$; DIPA (153 $\mu \mathrm{L}, 1.1 \mathrm{mmol}$, 3.6 equiv.); and 4-cyanopyridine ( $31 \mathrm{mg}, 0.3 \mathrm{mmol}, 1.0$ equiv.). The yield of product $\mathbf{5 g}$ was determined to be $82 \%$ by a calibrated UPLC-MS. 


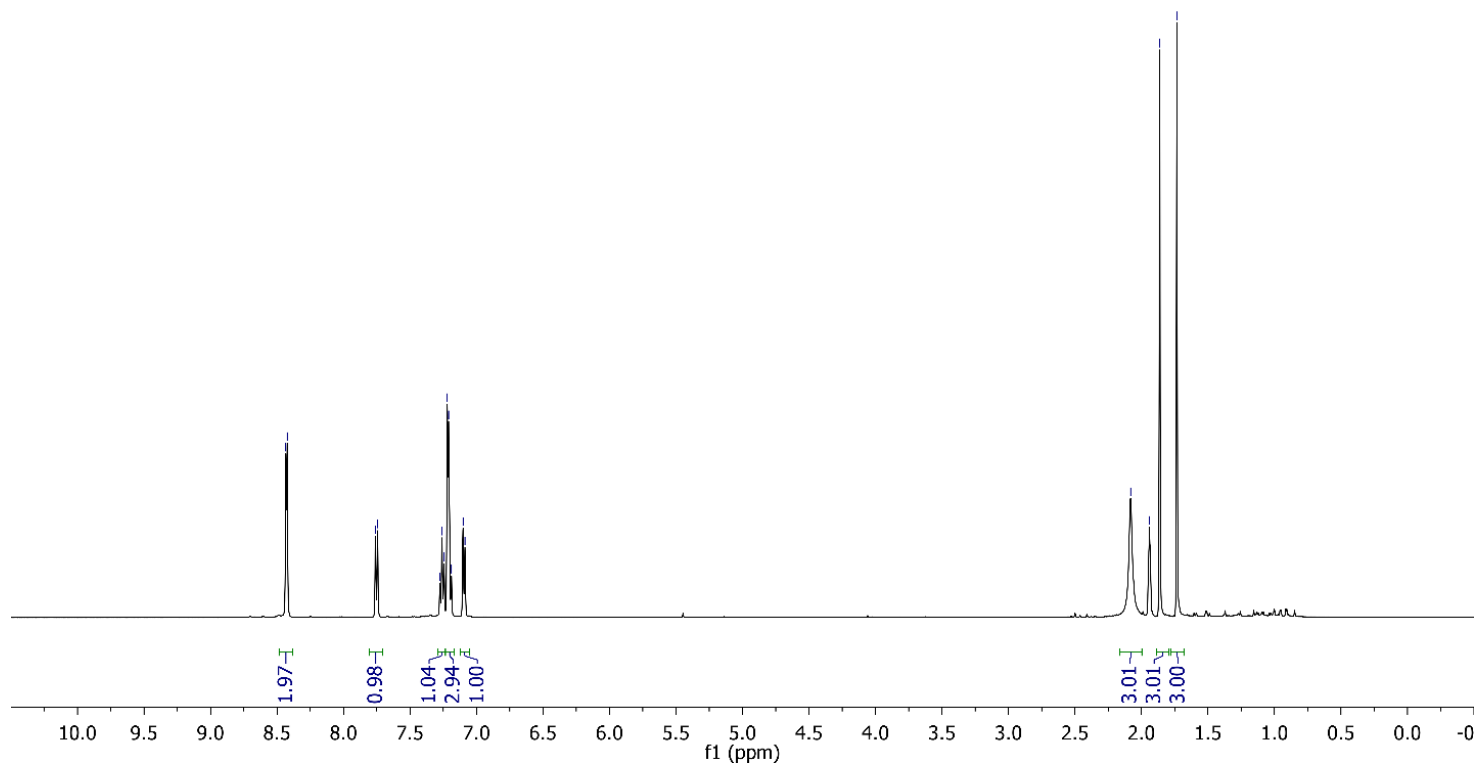

Figure S23. ${ }^{1} \mathrm{H}$ qNMR $(500 \mathrm{MHz})$ spectrum of $\mathbf{5 g}$ in $\mathrm{CD}_{3} \mathrm{CN}$.

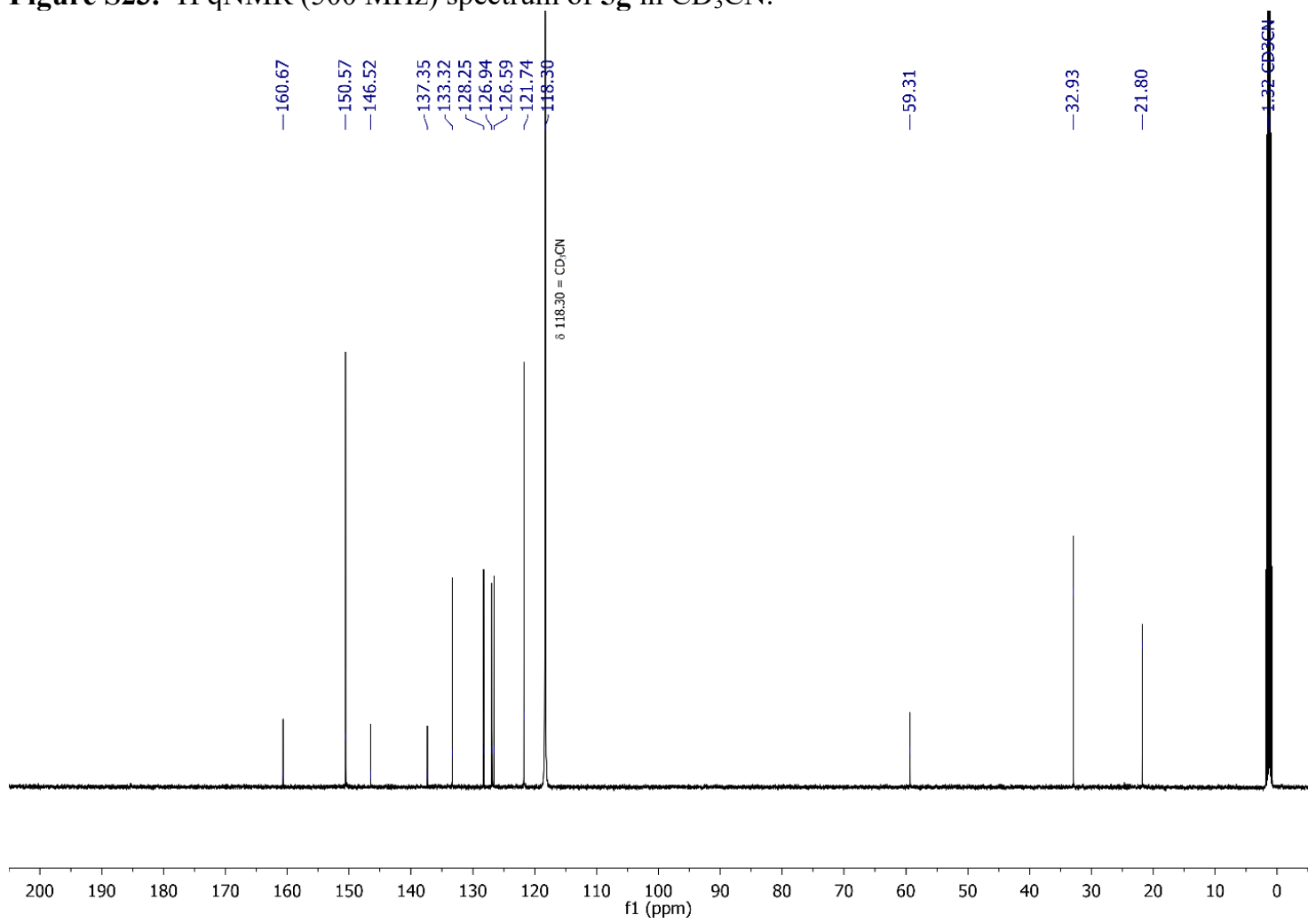

Figure S24. ${ }^{13} \mathrm{C}$ NMR $(126 \mathrm{MHz})$ spectrum of $\mathbf{5 g}$ in $\mathrm{CD}_{3} \mathrm{CN}$. 
<smiles>CC(N)(c1ccncc1)c1ccc2ccccc2c1</smiles>

Compound 5h was synthesized following General Procedure A using 4-pyridinecarbonitrile 4a (69 mg, $0.66 \mathrm{mmol})$, PC1 (6 mg, 6E-3 mmol), benzoic acid (37 mg, $0.3 \mathrm{mmol})$, DIPA (153 $\mu \mathrm{L}, 1.1 \mathrm{mmol})$ and $O$-benzoyl oxime (3h) $(87$ $\mathrm{mg}, 0.3 \mathrm{mmol})$ in DMSO $(3 \mathrm{~mL})$. The product was purified according to the general chromatography method for oximes. The product was obtained as a film on a vial (37 mg, 50\%). The yield of product $\mathbf{5 h}$ was determined to be $50 \%$ by a calibrated UPLC-MS ${ }^{1} \mathrm{H}$ NMR $\left(500 \mathrm{MHz}, \mathrm{CD}_{3} \mathrm{CN}\right) \delta 8.45(\mathrm{~d}, J=6.2 \mathrm{~Hz}, 2 \mathrm{H}), 8.02(\mathrm{~d}, J=1.5 \mathrm{~Hz}, 1 \mathrm{H}), 7.90-$ $7.85(\mathrm{~m}, 1 \mathrm{H}), 7.84-7.81(\mathrm{~m}, 1 \mathrm{H}), 7.76(\mathrm{~d}, J=8.7 \mathrm{~Hz}, 1 \mathrm{H}), 7.48(\mathrm{pd}, J=6.9,1.5 \mathrm{~Hz}, 2 \mathrm{H}), 7.39-7.35(\mathrm{~m}, 3 \mathrm{H}), 2.18$ (s, 3H), 1.87 (s, 3H). ${ }^{13} \mathrm{C} \mathrm{NMR}\left(126 \mathrm{MHz}, \mathrm{CD}_{3} \mathrm{CN}\right) \delta 159.81,150.56,147.31,134.11,133.09,129.03,128.75,128.33$, 127.22, 126.93, 126.48, 124.77, 122.41, 58.93, 31.19. MS (ESI) m/z calcd. for $\mathrm{C}_{17} \mathrm{H}_{17} \mathrm{~N}_{2}\left([\mathrm{M}+\mathrm{H}]^{+}\right) 249.1$, found 249.2 .

Compound $\mathbf{5 h}$ was also synthesized from the corresponding iminium $\mathrm{HCl}$ salt $\mathbf{6} \mathbf{h}$ and cyanoarene $\mathbf{4 a}$. The reaction was conducted by following the General Iminium Salt Reaction Procedure using iminium $\mathrm{HCl}$ salt (6h) (136 mg, 0.66 mmol, 2.2 equiv.); photocatalyst $\operatorname{Ir}[\mathrm{dF}(\mathrm{Me}) \text { ppy }]_{2} \mathrm{dtbbpyPF}_{6}$ (6 mg, $2 \mathrm{E}-3 \mathrm{mmol}, 2 \mathrm{~mol} \%$ ); DIPA (153 $\mu \mathrm{L}, 1.1 \mathrm{mmol}$, 3.6 equiv.); and 4-cyanopyridine ( $31 \mathrm{mg}, 0.3 \mathrm{mmol}, 1.0$ equiv.). The yield of product $\mathbf{5 h}$ was determined to be $98 \%$ by a calibrated UPLC-MS. 


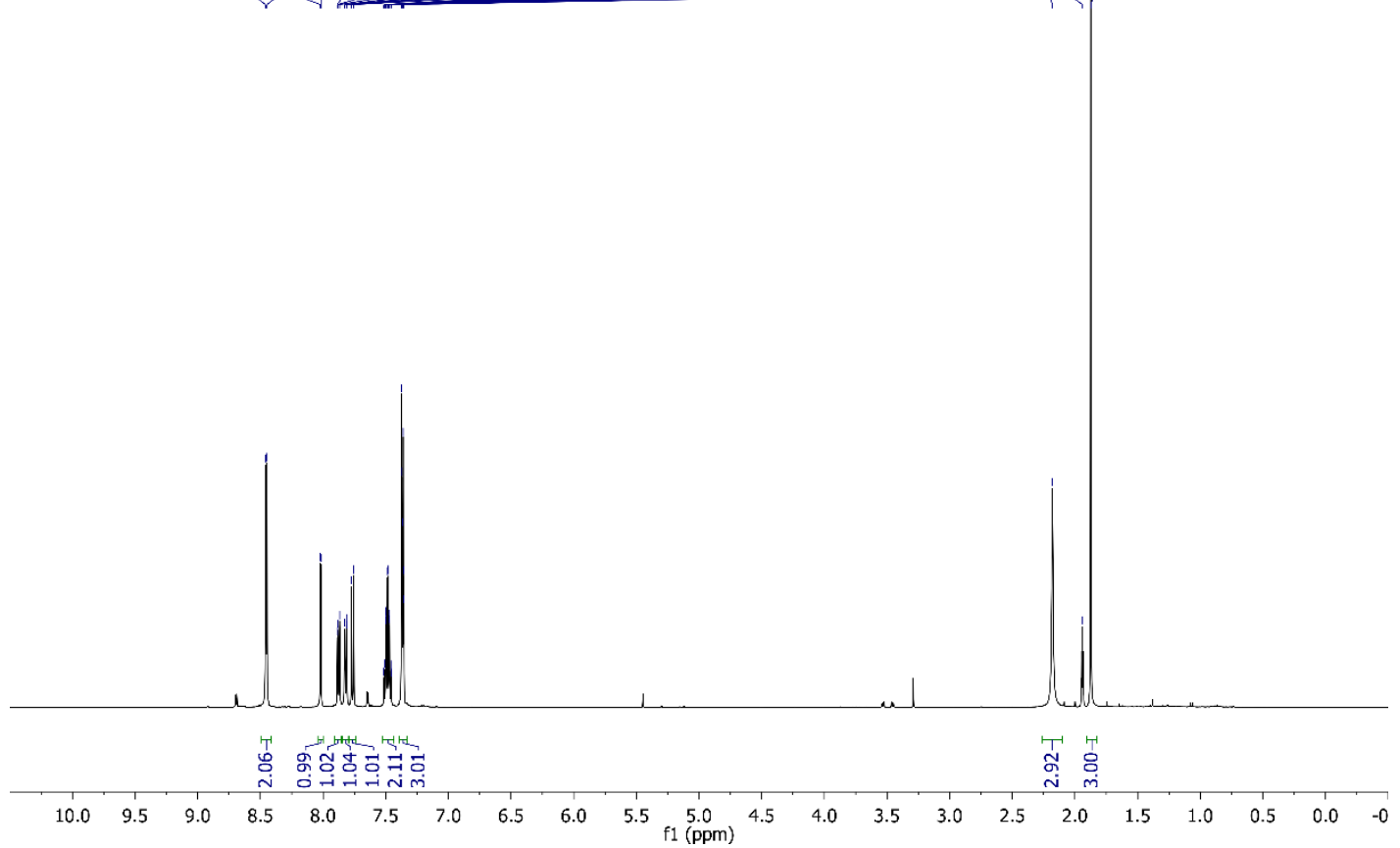

Figure S25. ${ }^{1} \mathrm{H}$ qNMR $(500 \mathrm{MHz})$ spectrum of $\mathbf{5 h}$ in $\mathrm{CD}_{3} \mathrm{CN}$.
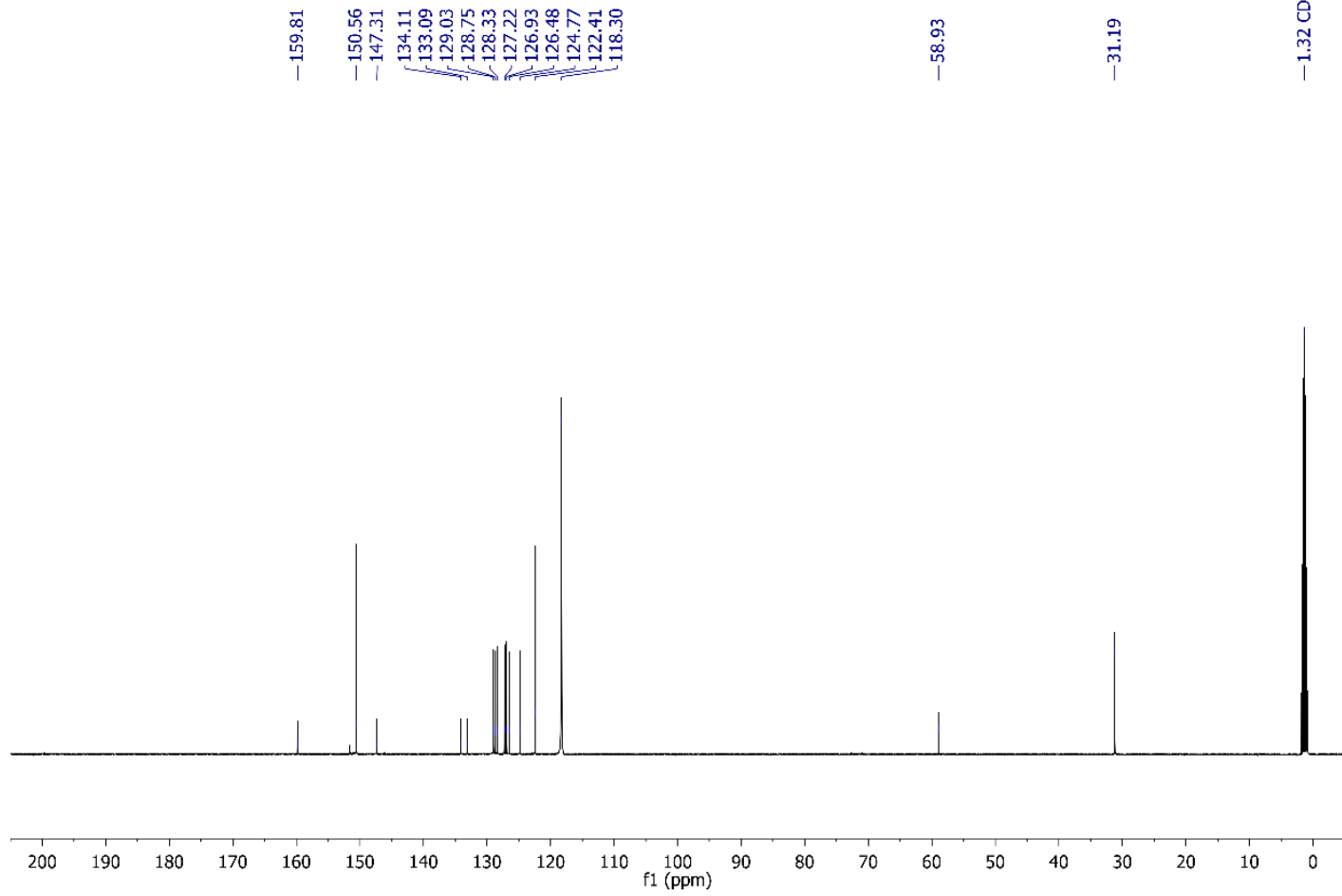

Figure S26. ${ }^{13} \mathrm{C}$ NMR $(126 \mathrm{MHz})$ spectrum of $\mathbf{5 h}$ in $\mathrm{CD}_{3} \mathrm{CN}$. 


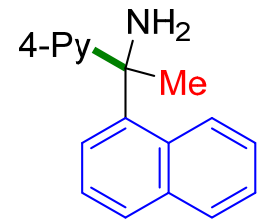

Compound $\mathbf{5 i}$ was also synthesized from the corresponding iminium $\mathrm{HCl}$ salt $\mathbf{6} \mathbf{i}$ and cyanoarene $\mathbf{4 a}$. The reaction was conducted by following the General Iminium Salt Reaction Procedure using iminium HCl salt (6i) (130 mg, 0.66 mmol, 2.2 equiv.); photocatalyst $\operatorname{Ir}[\mathrm{dF}(\mathrm{Me}) \text { ppy }]_{2} \mathrm{dtbbpyPF}_{6}(6 \mathrm{mg}, 2 \mathrm{E}-3 \mathrm{mmol}, 2 \mathrm{~mol} \%)$; DIPA (153 $\mu \mathrm{L}, 1.1 \mathrm{mmol}$, 3.6 equiv.); and 4-cyanopyridine (31 $\mathrm{mg}, 0.3 \mathrm{mmol}, 1.0$ equiv.). The yield of product $\mathbf{5 i}$ was determined to be $76 \%$ by a calibrated UPLC-MS. Product characterization matched as described. ${ }^{5}{ }^{1} \mathrm{H}$ NMR $\left(500 \mathrm{MHz}, \mathrm{CD}_{3} \mathrm{CN}\right) \delta 8.38(\mathrm{~d}, J=$ $5.9 \mathrm{~Hz}, 2 \mathrm{H}), 7.93(\mathrm{~d}, J=7.3 \mathrm{~Hz}, 1 \mathrm{H}), 7.86(\mathrm{~d}, J=8.2 \mathrm{~Hz}, 2 \mathrm{H}), 7.71(\mathrm{~d}, J=8.7 \mathrm{~Hz}, 1 \mathrm{H}), 7.55(\mathrm{t}, J=7.8 \mathrm{~Hz}, 1 \mathrm{H}), 7.35$ $(\mathrm{t}, J=7.4 \mathrm{~Hz}, 1 \mathrm{H}), 7.27(\mathrm{~d}, J=6.0 \mathrm{~Hz}, 2 \mathrm{H}), 7.18$ (t, $J=7.8 \mathrm{~Hz}, 1 \mathrm{H}), 2.19$ (br s, 2H), 1.87 (s, 3H). ${ }^{13} \mathrm{C} \mathrm{NMR}(126$ $\left.\mathrm{MHz}, \mathrm{CD}_{3} \mathrm{CN}\right) \delta 161.08,150.66,143.96,136.00,131.30,129.96,129.47,127.97,126.07,125.02,121.62,59.44$, 33.86. MS (ESI) $\mathrm{m} / \mathrm{z}$ calcd. for $\mathrm{C}_{17} \mathrm{H}_{17} \mathrm{~N}_{2}\left([\mathrm{M}+\mathrm{H}]^{+}\right) 249.1$, found 249.2 . 


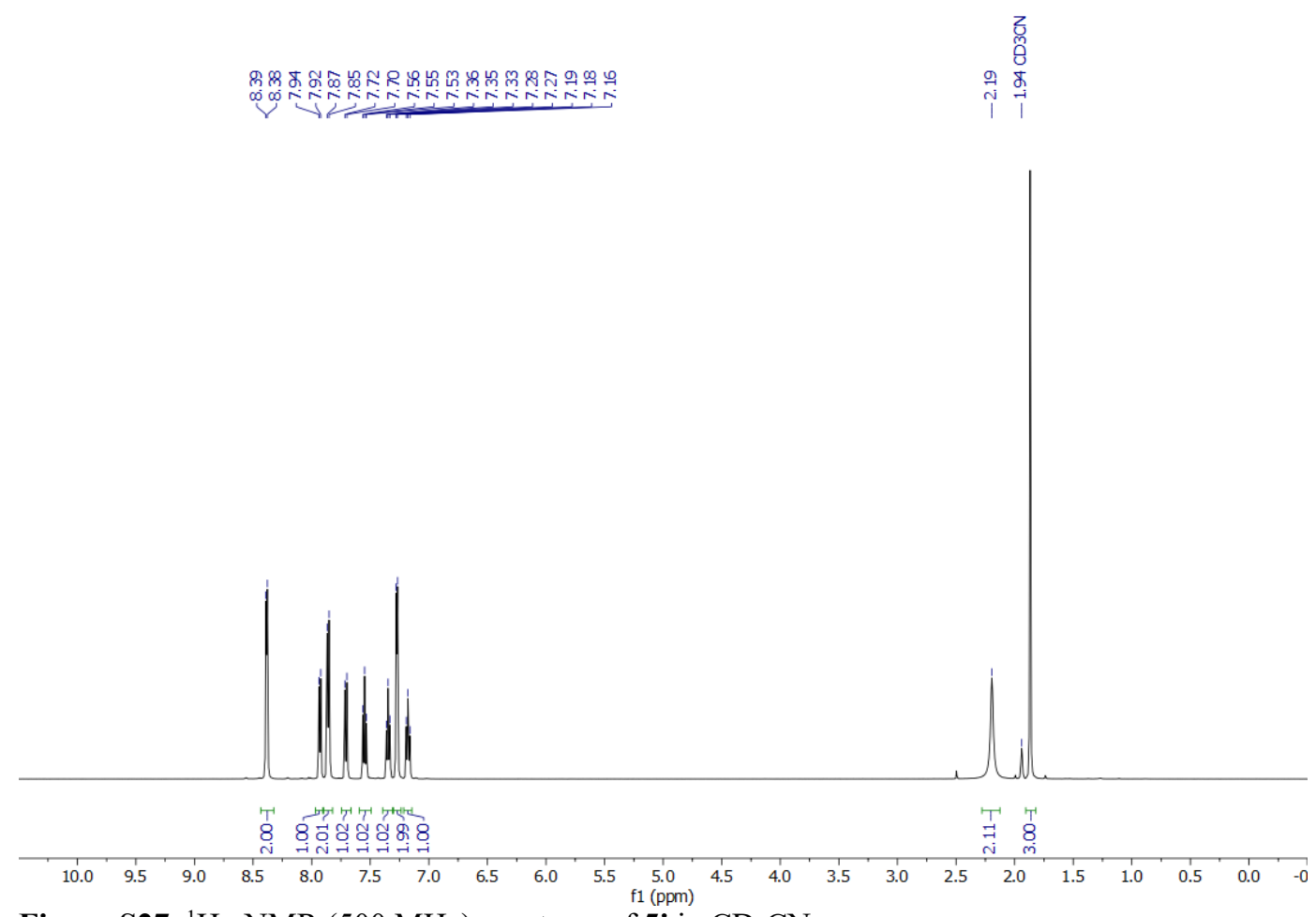

Figure S27. ${ }^{1} \mathrm{H}$ qNMR $(500 \mathrm{MHz})$ spectrum of $\mathbf{5 i}$ in $\mathrm{CD}_{3} \mathrm{CN}$.
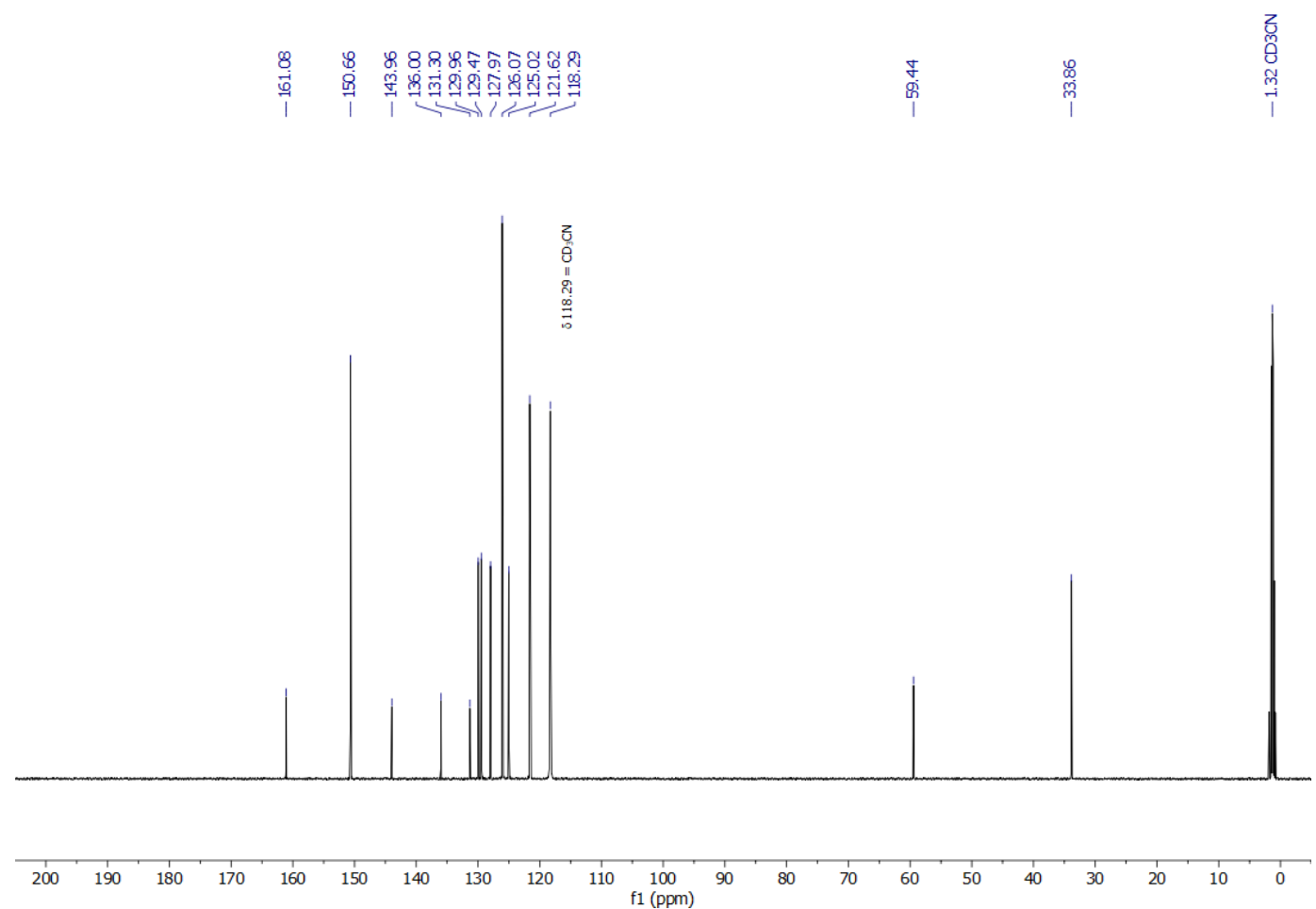

Figure S28. ${ }^{13} \mathrm{C}$ NMR $(126 \mathrm{MHz})$ spectrum of $\mathbf{5 i}$ in $\mathrm{CD}_{3} \mathrm{CN}$. 


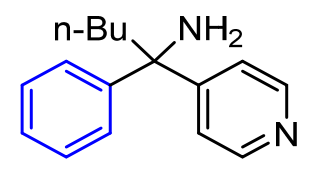

Compound 5l was synthesized following General Procedure A using 4-pyridinecarbonitrile 4a (69 mg, 0.66 mmol), PC1 (6 mg, 6E-3 mmol), benzoic acid (37 mg, $0.3 \mathrm{mmol})$, DIPA (153 $\mu \mathrm{L}, 1.1 \mathrm{mmol})$ and $O$-benzoyl oxime (3I) (84 $\mathrm{mg}, 0.3 \mathrm{mmol})$ in DMSO $(3 \mathrm{~mL})$. The product was purified according to the general chromatography method for oximes. The product was obtained as a film on a vial (51 mg, 71\%). The yield of product $\mathbf{5 l}$ was determined to be $94 \%$ by a calibrated UPLC-MS. ${ }^{1} \mathrm{H}$ NMR $\left(500 \mathrm{MHz}, \mathrm{CD}_{3} \mathrm{CN}\right) \delta 8.45-8.43(\mathrm{~m}, 2 \mathrm{H}), 7.43-7.40(\mathrm{~m}, 2 \mathrm{H}), 7.36-7.34(\mathrm{~m}, 2 \mathrm{H})$, $7.30(\mathrm{t}, J=7.8 \mathrm{~Hz}, 2 \mathrm{H}), 7.22-7.18(\mathrm{~m}, 1 \mathrm{H}), 2.25-2.16(\mathrm{~m}, 2 \mathrm{H}), 1.37-1.27(\mathrm{~m}, 2 \mathrm{H}), 1.23-1.08(\mathrm{~m}, 2 \mathrm{H}), 0.86(\mathrm{t}, J=$ $7.4 \mathrm{~Hz}, 3 \mathrm{H}) .{ }^{13} \mathrm{C}$ NMR $\left(126 \mathrm{MHz}, \mathrm{CD}_{3} \mathrm{CN}\right) \delta 159.27,150.41,149.04,129.11,127.39,127.38,122.67,61.37,42.23$, 26.92, 23.80, 14.35. MS (ESI) $\mathrm{m} / \mathrm{z}$ calcd. for $\mathrm{C}_{16} \mathrm{H}_{17} \mathrm{~N}_{2}\left([\mathrm{M}+\mathrm{H}]^{+}\right)$241.2, found 241.2.

Compound $\mathbf{5 l}$ was also synthesized from the corresponding iminium $\mathrm{HCl}$ salt $\mathbf{6} \mathbf{l}$ and cyanoarene $\mathbf{4 a}$. The reaction was conducted by following the General Iminium Salt Reaction Procedure using iminium $\mathrm{HCl} \mathrm{salt} \mathrm{(61)} \mathrm{(130} \mathrm{mg,} 0.66$ mmol, 2.2 equiv.); photocatalyst $\operatorname{Ir}\left[\mathrm{dF}(\mathrm{Me})\right.$ ppy ${ }_{2} \mathrm{dtbbpyPF}_{6}$ (6 mg, 2E-3 mmol, $2 \mathrm{~mol} \%$ ); DIPA (153 $\mu \mathrm{L}, 1.1 \mathrm{mmol}$, 3.6 equiv.); and 4-cyanopyridine ( $31 \mathrm{mg}, 0.3 \mathrm{mmol}, 1.0$ equiv.). The yield of product $\mathbf{5 l}$ was determined to be $76 \%$ by a calibrated UPLC-MS. 


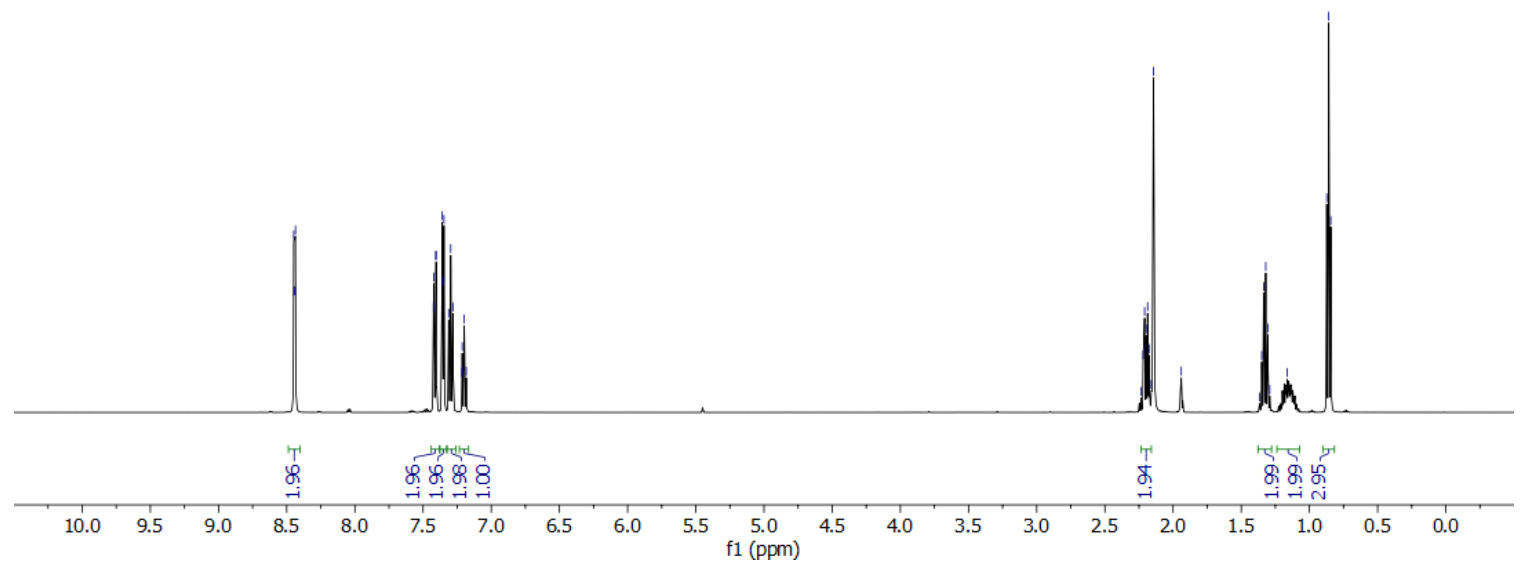

Figure S29. ${ }^{1} \mathrm{H}$ qNMR $(500 \mathrm{MHz})$ spectrum of $\mathbf{5 l}$ in $\mathrm{CD}_{3} \mathrm{CN}$.

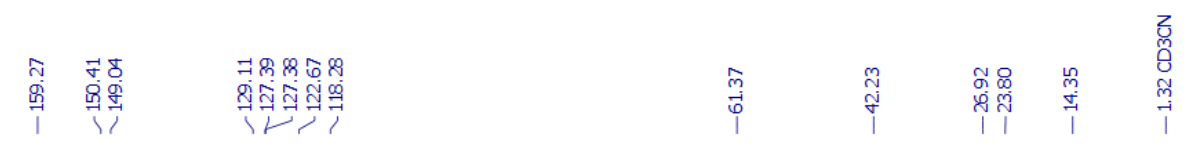

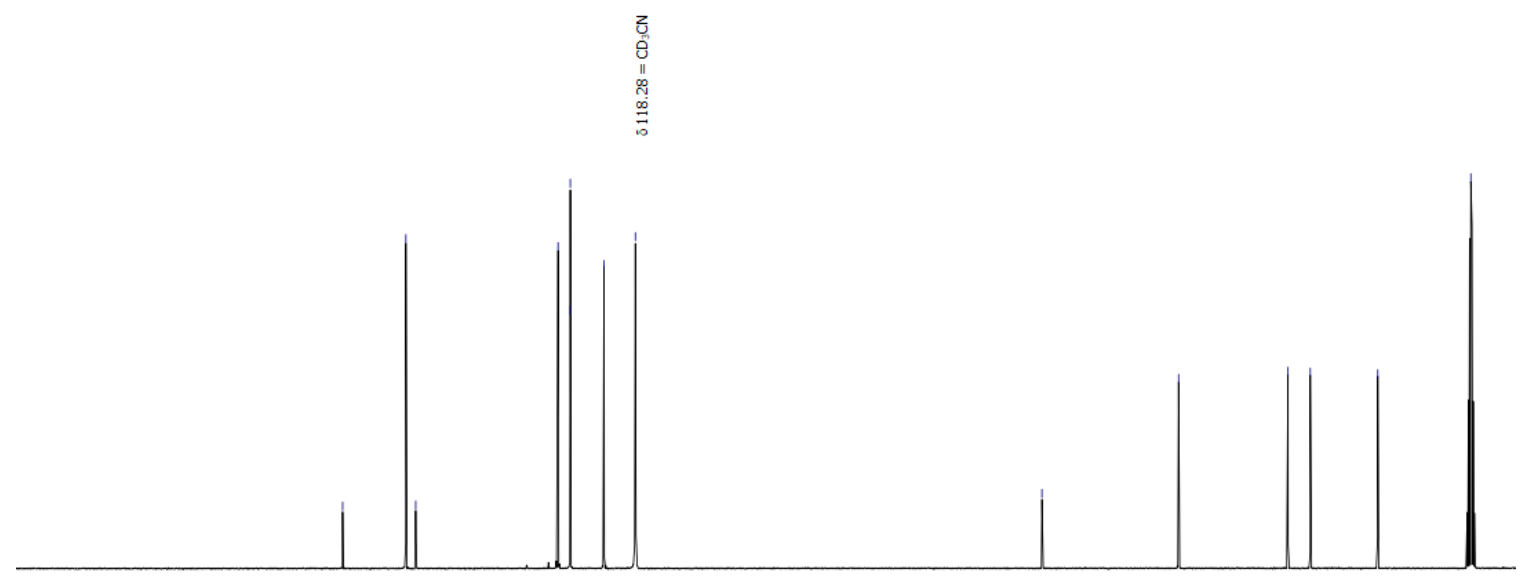

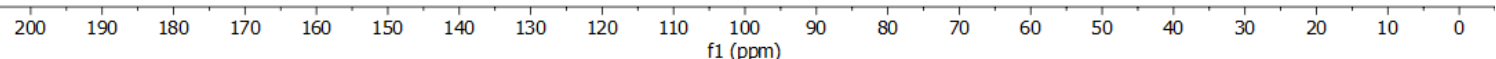

Figure S30. ${ }^{13} \mathrm{C}$ NMR (126 MHz) spectrum of $\mathbf{5 l}$ in $\mathrm{CD}_{3} \mathrm{CN}$. 
<smiles>[14CH3]C(N)(c1ccccc1)C(Br)(Br)Br</smiles>

Compound $\mathbf{5 m}$ was also synthesized from the corresponding iminium $\mathrm{HCl}$ salt $\mathbf{6 m}$ and cyanoarene $\mathbf{4 a}$. The reaction was conducted by following the General Iminium Salt Reaction Procedure using iminium $\mathrm{HCl}$ salt (6m)(130 mg, 0.66 mmol, 2.2 equiv.); photocatalyst $\operatorname{Ir}[\mathrm{dF}(\mathrm{Me}) \text { ppy }]_{2} \mathrm{dtbbpyPF}_{6}$ (6 mg, 2E-3 mmol, $\left.2 \mathrm{~mol} \%\right)$; DIPA (153 $\mu \mathrm{L}, 1.1 \mathrm{mmol}$, 3.6 equiv.); and 4-cyanopyridine ( $31 \mathrm{mg}, 0.3 \mathrm{mmol}, 1.0$ equiv.). $\mathrm{MS}$ (ESI) $\mathrm{m} / z$ calcd. for $\mathrm{C}_{16} \mathrm{H}_{21} \mathrm{~N}_{2}\left([\mathrm{M}+\mathrm{H}]^{+}\right) 241.2$, found 242.3 . The product $5 \mathrm{~m}$ was synthesized in $84 \%$ yield (retention time $\mathrm{t}=2.310, \mathrm{~m} / \mathrm{z}=242.3(\mathrm{M}+\mathrm{H}]^{+}$) as determined by a calibrated UPLC generated from an authentic sample using the UPLC-MS Method for Yield Determination. ${ }^{5} \mathrm{H}$ NMR $\left(500 \mathrm{MHz}, \mathrm{CD}_{3} \mathrm{CN}\right) \delta 8.41(\mathrm{dd}, J=1.6,4.6 \mathrm{~Hz}, 2 \mathrm{H}), 7.43-7.39(\mathrm{~m}, 2 \mathrm{H}), 7.39-7.36(\mathrm{dd}, J=$ $1.7,4.6 \mathrm{~Hz}, 2 \mathrm{H}), 7.28-7.23(\mathrm{~m}, 2 \mathrm{H}), 7.22-7.18(\mathrm{~m}, 1 \mathrm{H}), 2.09$ (br s, $2 \mathrm{H}), 1.15(\mathrm{~s}, 9 \mathrm{H}) .{ }^{13} \mathrm{C} \mathrm{NMR}\left(126 \mathrm{MHz}, \mathrm{CD}_{3} \mathrm{CN}\right)$ $\delta 158.48,149.63,147.96,130.22,128.04,127.16,125.42,67.85,38.68,28.17$. MS (ESI) m/z calcd. for $\mathrm{C}_{16} \mathrm{H}_{21} \mathrm{~N}_{2}([\mathrm{M}$ $+\mathrm{H}]^{+}$) 241.2, found 241.3. 


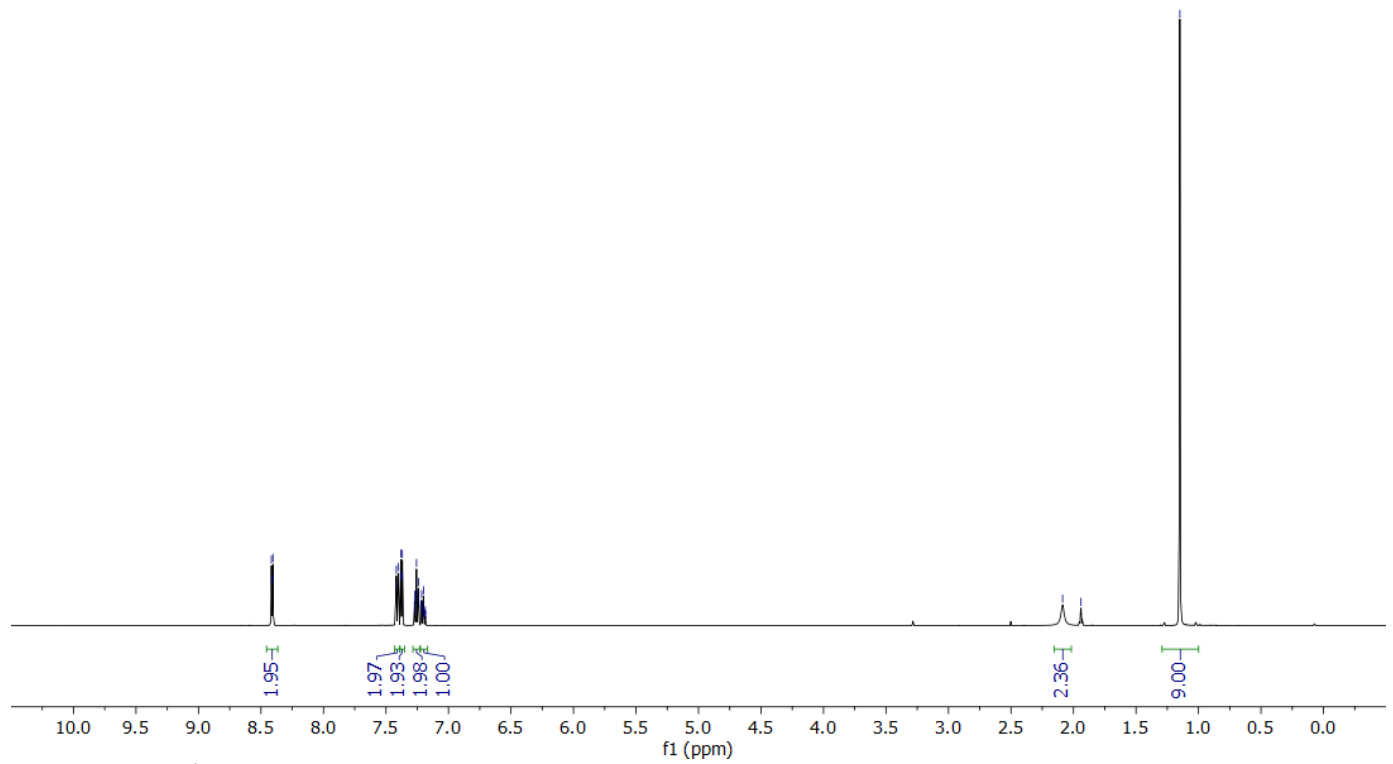

Figure S31. ${ }^{1} \mathrm{H}$ qNMR $(500 \mathrm{MHz})$ spectrum of $5 \mathbf{m}$ in $\mathrm{CD}_{3} \mathrm{CN}$.
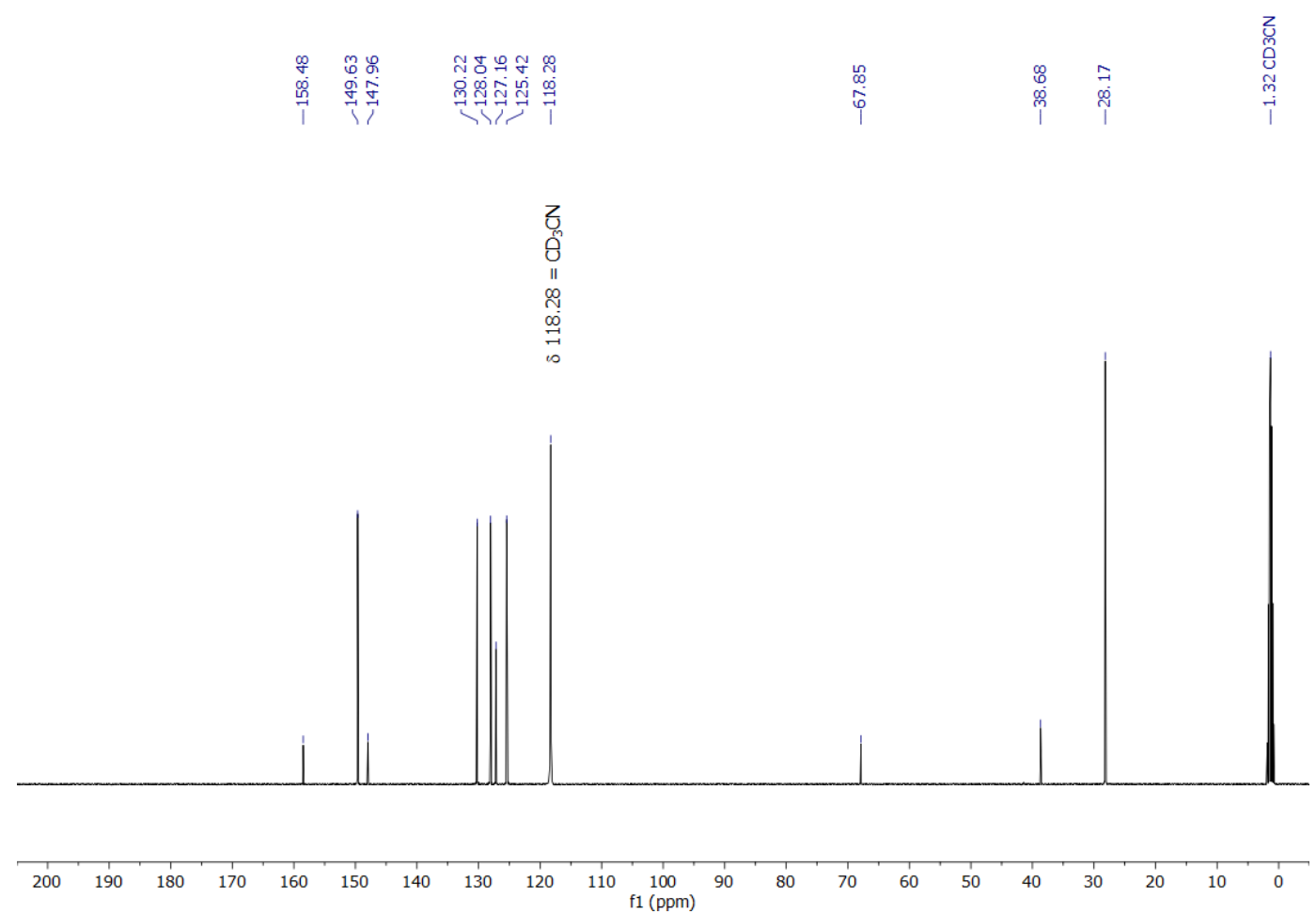

Figure S32. ${ }^{13} \mathrm{C}$ NMR (126 MHz) spectrum of $\mathbf{5 m}$ in $\mathrm{CD}_{3} \mathrm{CN}$. 


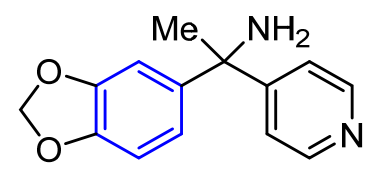

Compound 5j was synthesized following General Procedure A using 4-pyridinecarbonitrile 4a (69 mg, 0.66 mmol), PC1 (6 mg, 6E-3 mmol), benzoic acid (37 mg, $0.3 \mathrm{mmol})$, DIPA (153 $\mu \mathrm{L}, 1.1 \mathrm{mmol})$ and $O$-benzoyl oxime (3j) (73 $\mathrm{mg}, 0.3 \mathrm{mmol})$ in DMSO $(3 \mathrm{~mL})$. The product was purified according to the general chromatography method for oximes. The product was obtained as a film on a vial $(25 \mathrm{mg}, 34 \%)$. The yield of product $\mathbf{5} \mathbf{j}$ was determined to be $42 \%$ by a calibrated UPLC-MS $1 \mathrm{H}$ NMR: ${ }^{1} \mathrm{H}$ NMR $\left(500 \mathrm{MHz}, \mathrm{CD}_{3} \mathrm{CN} \delta 8.44(\mathrm{~d}, J=6.2 \mathrm{~Hz}, 2 \mathrm{H}), 7.34(\mathrm{~d}, J=6.2\right.$ $\mathrm{Hz}, 2 \mathrm{H}), 6.92-6.86(\mathrm{~m}, 2 \mathrm{H}), 6.75(\mathrm{~d}, J=8.1 \mathrm{~Hz}, 1 \mathrm{H}), 5.91(\mathrm{q}, J=1.0 \mathrm{~Hz}, 2 \mathrm{H}), 2.04(\mathrm{~s}, 2 \mathrm{H}), 1.74(\mathrm{~s}, 3 \mathrm{H}) .{ }^{13} \mathrm{C} \mathrm{NMR}$ $\left(126 \mathrm{MHz}, \mathrm{CD}_{3} \mathrm{CN}\right) \delta 160.09,150.51,148.54,147.06,144.20,122.18,120.15,108.42,108.00,102.27,58.60,31.55$. MS (ESI) $\mathrm{m} / \mathrm{z}$ calcd. for $\mathrm{C}_{14} \mathrm{H}_{15} \mathrm{~N}_{2} \mathrm{O}_{2}\left([\mathrm{M}+\mathrm{H}]^{+}\right)$243.1, found 243.2. 


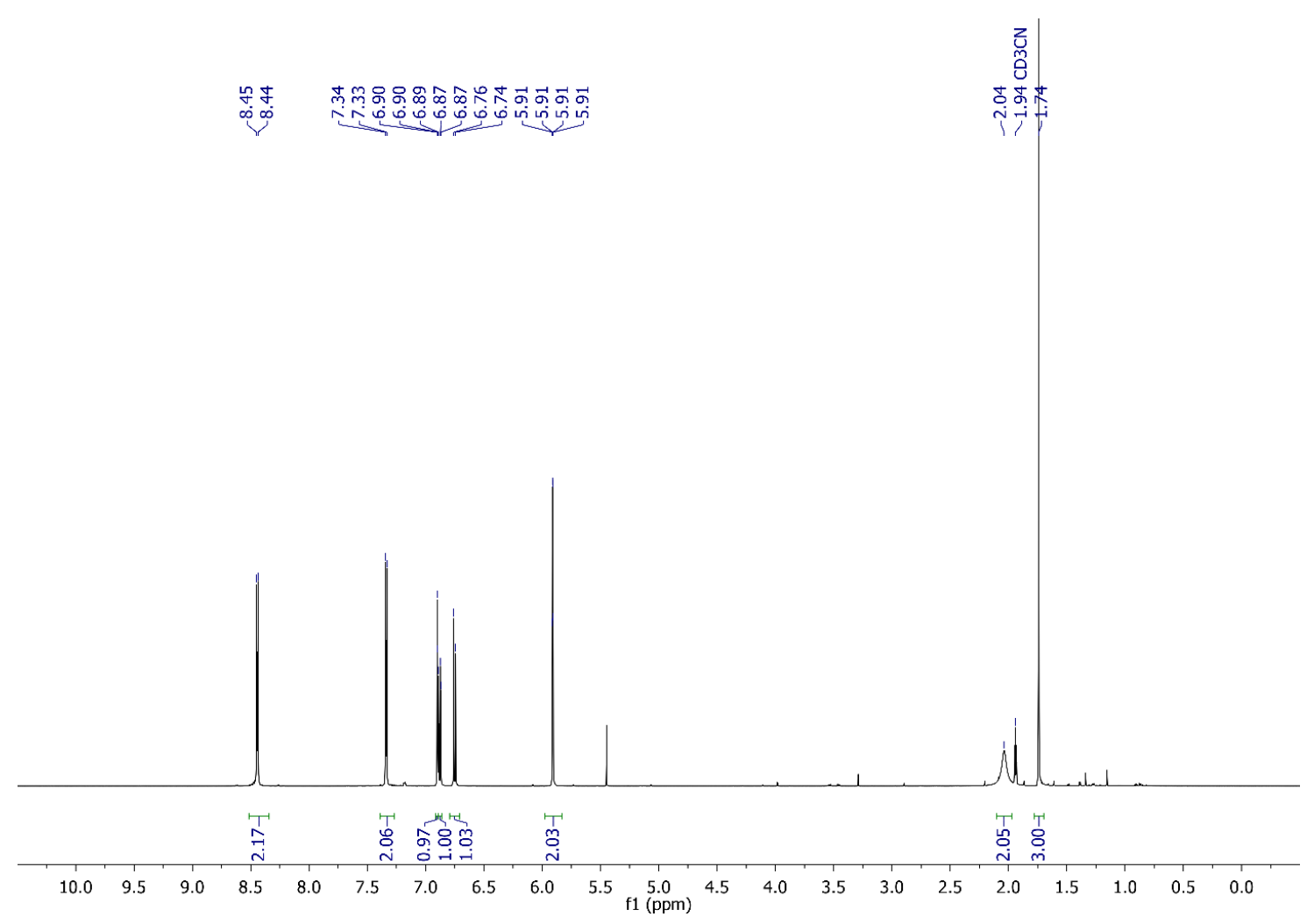

Figure S33. ${ }^{1} \mathrm{H}$ qNMR $(500 \mathrm{MHz})$ spectrum of $\mathbf{5 j}$ in $\mathrm{CD}_{3} \mathrm{CN}$.

\begin{tabular}{|c|c|c|c|c|}
\hline 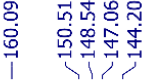 & 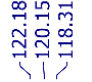 & 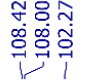 & $\begin{array}{c}8 \\
0 \\
\infty \\
\infty \\
1\end{array}$ & 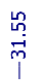 \\
\hline
\end{tabular}

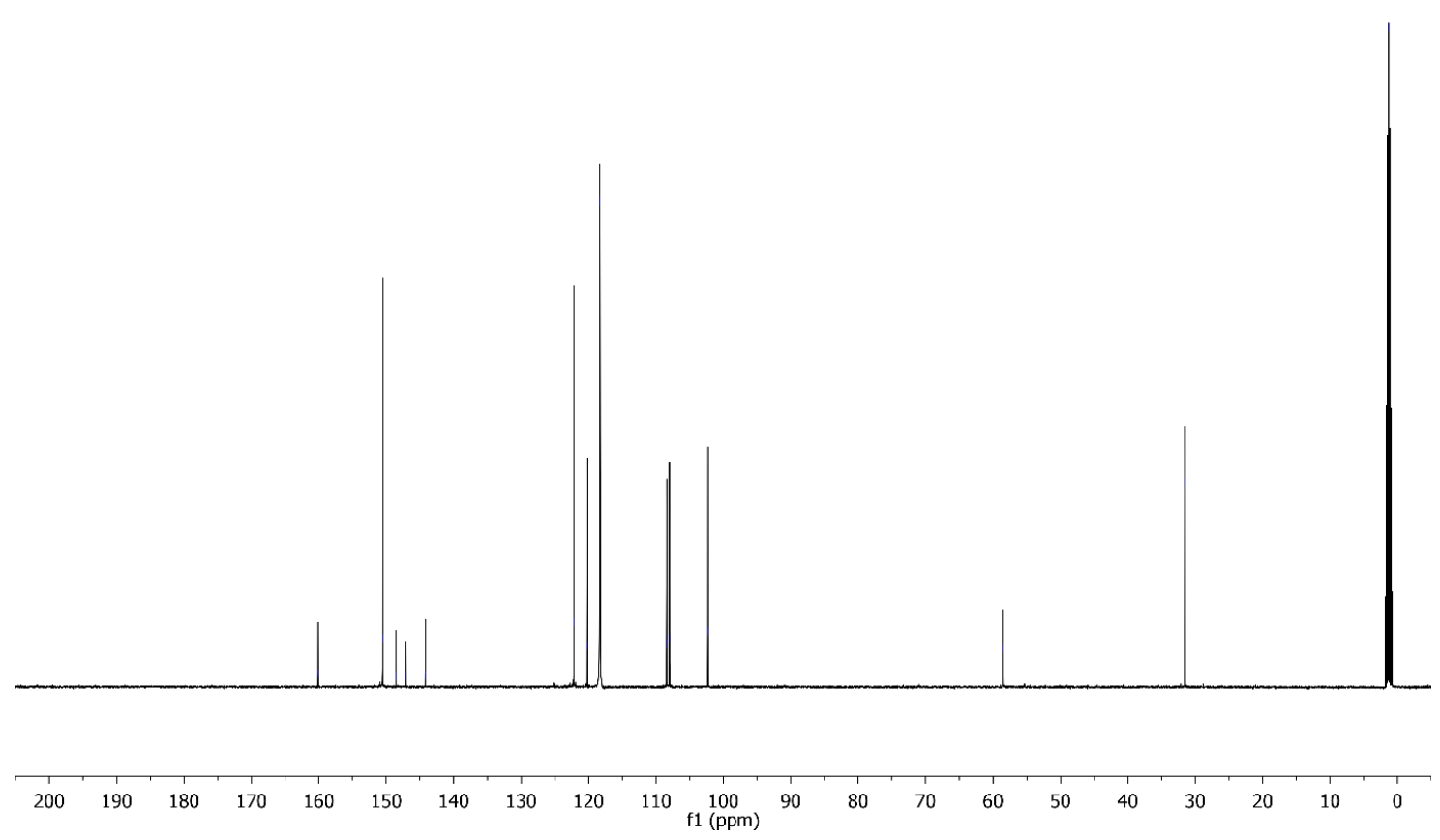

Figure S34. ${ }^{13} \mathrm{C}$ NMR $(126 \mathrm{MHz})$ spectrum of $\mathbf{5 j}$ in $\mathrm{CD}_{3} \mathrm{CN}$. 
<smiles>CC(C)COC(=O)c1ccc(C(C)(N)c2ccncc2)cc1</smiles>

Compound 50 was synthesized following General Procedure A using 4-pyridinecarbonitrile 4a (69 mg, $0.66 \mathrm{mmol})$, PC1 (6 mg, 6E-3 mmol), benzoic acid (37 mg, $0.3 \mathrm{mmol})$, DIPA (153 $\mu \mathrm{L} \mathrm{L}, 1.1 \mathrm{mmol})$ and $O$-benzoyl oxime (3o) $(90 \mathrm{mg}, 0.3 \mathrm{mmol})$ in DMSO $(3 \mathrm{~mL})$. The product was purified according to the general chromatography method for oximes. The product was obtained as a film on a vial $(29 \mathrm{mg}, 32 \%)$. The yield of product $\mathbf{5 0}$ was determined to be $32 \%$ by a calibrated UPLC-MS ${ }^{1} \mathrm{H}$ NMR $\left(500 \mathrm{MHz}, \mathrm{CD}_{3} \mathrm{CN}\right) \delta 8.46(\mathrm{~d}, J=6.0 \mathrm{~Hz}, 2 \mathrm{H}), 7.88(\mathrm{~d}, J=8.5 \mathrm{~Hz}, 2 \mathrm{H})$, 7.47 (d, $J=8.5 \mathrm{~Hz}, 2 \mathrm{H}), 7.33(\mathrm{~d}, J=6.0 \mathrm{~Hz}, 2 \mathrm{H}), 2.11(\mathrm{~s}, 3 \mathrm{H}), 1.79(\mathrm{~s}, 3 \mathrm{H}), 1.55(\mathrm{~s}, 9 \mathrm{H}) .{ }^{13} \mathrm{C}$ NMR $(126 \mathrm{MHz}$, $\mathrm{CD}_{3} \mathrm{CN}$ ) $\delta 166.24,159.42,154.67,150.63,131.35,130.02,127.24,122.27,118.29$ (MeCN residual solvent), 81.69, 58.90, 31.09, 28.33. MS (ESI) m/z calcd. for $\mathrm{C}_{18} \mathrm{H}_{23} \mathrm{~N}_{2} \mathrm{O}_{2}\left([\mathrm{M}+\mathrm{H}]^{+}\right)$299.2, found 299.2. 


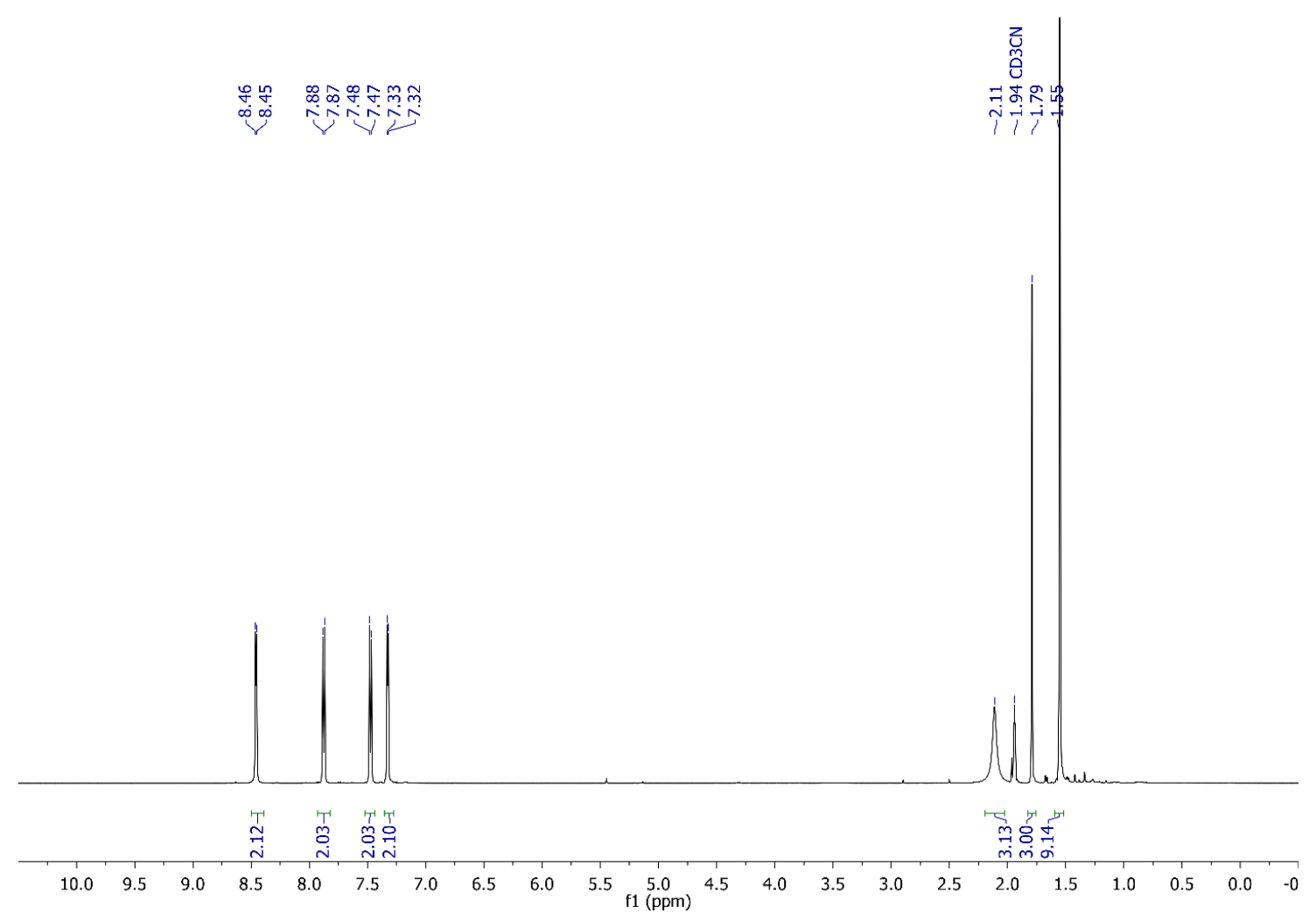

Figure $\mathbf{S 3 5}{ }^{1} \mathrm{H}$ qNMR $(500 \mathrm{MHz})$ spectrum of $\mathbf{5 o}$ in $\mathrm{CD}_{3} \mathrm{CN}$.

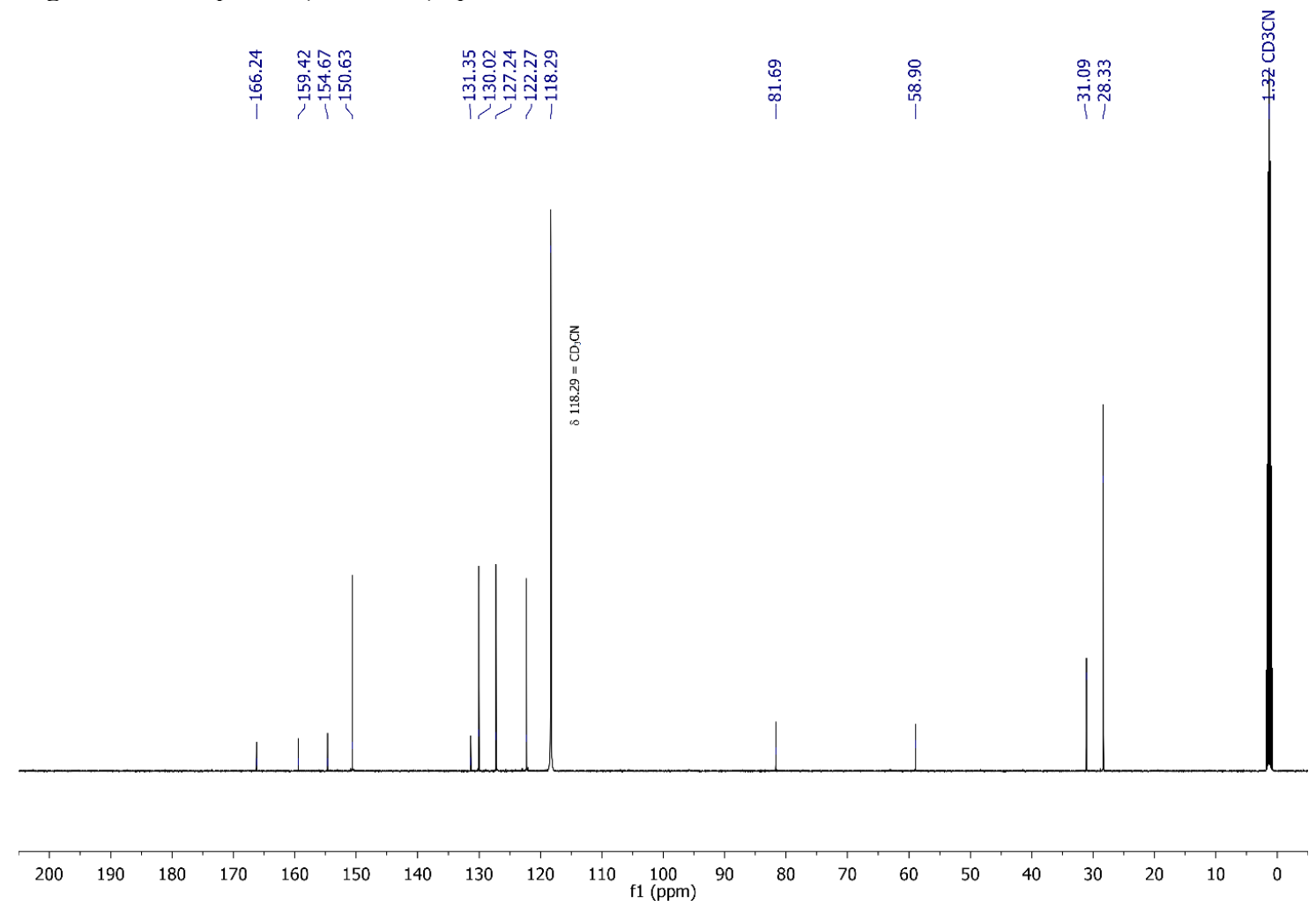

Figure S36. ${ }^{13} \mathrm{C}$ NMR $(126 \mathrm{MHz})$ spectrum of 50 in $\mathrm{CD}_{3} \mathrm{CN}$. 


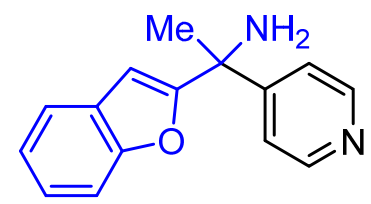

Compound 5k was synthesized following General Procedure A using 4-pyridinecarbonitrile 4a (69 mg, $0.66 \mathrm{mmol})$, PC1 (6 mg, 6E-3 mmol), benzoic acid (37 mg, $0.3 \mathrm{mmol})$, DIPA (153 $\mu \mathrm{L} \mathrm{L,} 1.1 \mathrm{mmol})$ and $O$-benzoyl oxime (3k) $(84 \mathrm{mg}, 0.3 \mathrm{mmol})$ in DMSO $(3 \mathrm{~mL})$. The product was purified according to the general chromatography method for oximes. The product was obtained as a film on a vial $(16 \mathrm{mg}, 31 \%)$. The yield of product $\mathbf{5 k}$ was determined to be $39 \%$ by a calibrated UPLC-MS ${ }^{1} \mathrm{H}$ NMR $\left(500 \mathrm{MHz}, \mathrm{CD}_{3} \mathrm{CN}\right) \delta 8.50(\mathrm{~d}, J=5.9 \mathrm{~Hz}, 2 \mathrm{H}), 7.60(\mathrm{~d}, J=7.1 \mathrm{~Hz}, 1 \mathrm{H})$, $7.43(\mathrm{~d}, J=6.0 \mathrm{~Hz}, 2 \mathrm{H}), 7.38(\mathrm{~d}, J=7.9 \mathrm{~Hz}, 1 \mathrm{H}), 7.29-7.19(\mathrm{~m}, 2 \mathrm{H}), 6.77(\mathrm{~s}, 1 \mathrm{H}), 1.79(\mathrm{~s}, 3 \mathrm{H}) .{ }^{13} \mathrm{C} \mathrm{NMR}(126 \mathrm{MHz}$, $\left.\mathrm{CD}_{3} \mathrm{CN}\right) \delta 150.67,125.17,123.92,122.14,121.94,118.31,111.78,102.91,56.43,29.44$. MS (ESI) M/Z CALCD. FOR $\mathrm{C}_{15} \mathrm{H}_{15} \mathrm{~N}_{2} \mathrm{O}\left([\mathrm{M}+\mathrm{H}]^{+}\right)$239.1, found 239.2. 


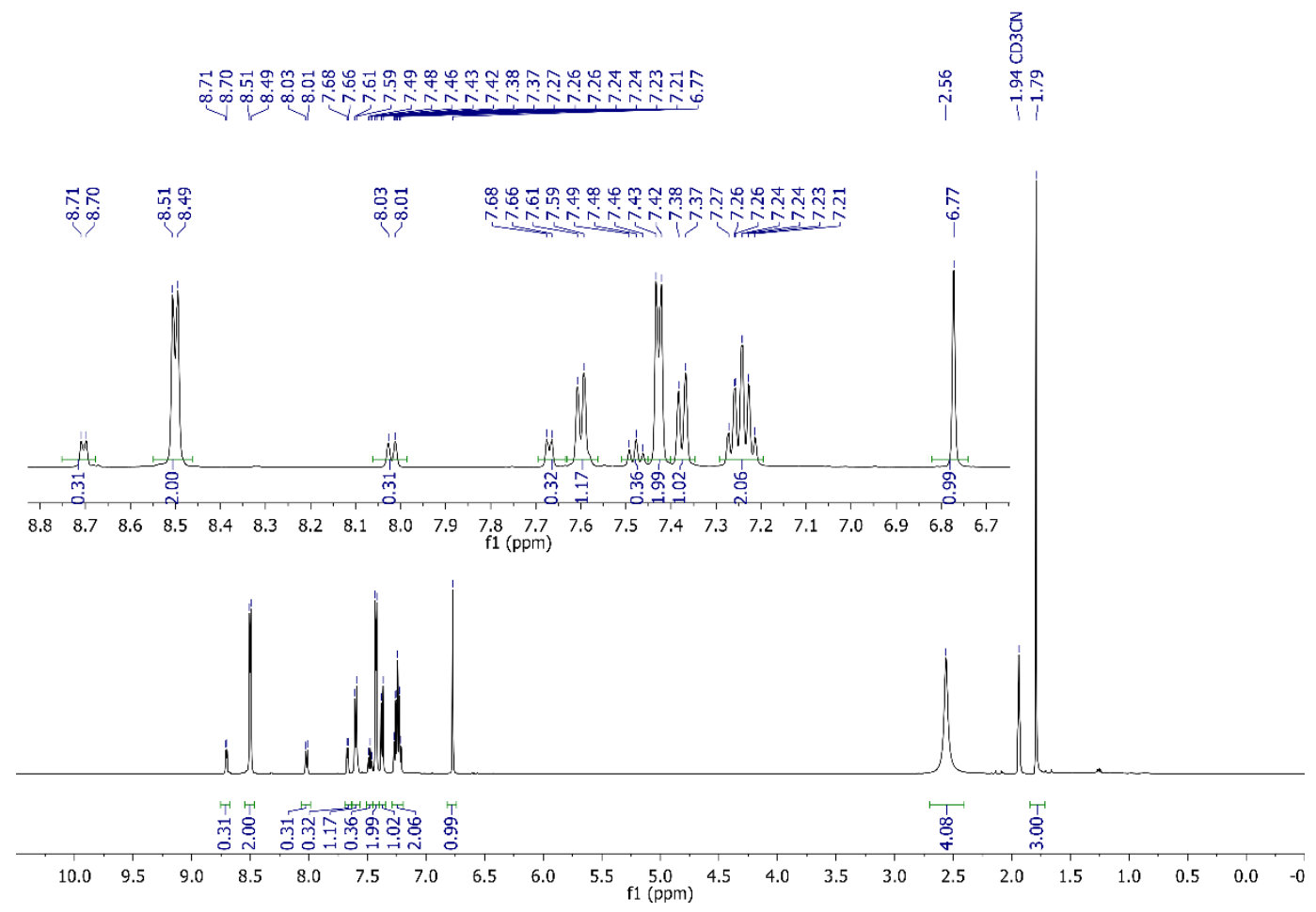

Figure S37. ${ }^{1} \mathrm{H}$ qNMR $(500 \mathrm{MHz})$ spectrum of $\mathbf{5} \mathbf{k}$ in $\mathrm{CD}_{3} \mathrm{CN}$.

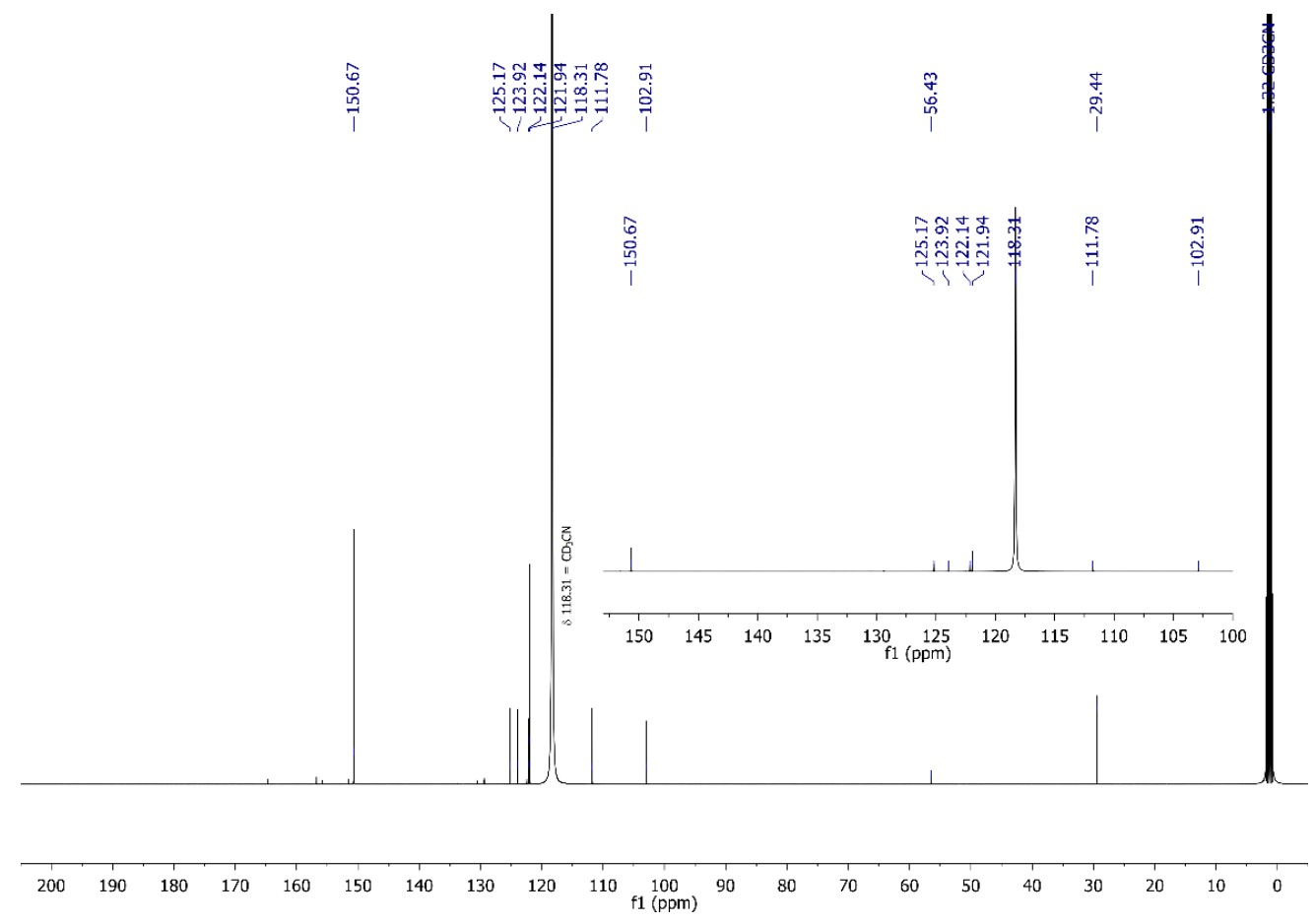

Figure S38. ${ }^{13} \mathrm{C}$ NMR (126 MHz) spectrum of $\mathbf{5 k}$ in $\mathrm{CD}_{3} \mathrm{CN}$. 
<smiles>NC(c1ccccc1)(c1ccncc1)C1CC1</smiles>

Compound 5n was synthesized following General Procedure A using 4-pyridinecarbonitrile 4a (69 mg, $0.66 \mathrm{mmol})$, PC1 $(6 \mathrm{mg}, 6 \mathrm{E}-3 \mathrm{mmol})$, benzoic acid $(37 \mathrm{mg}, 0.3 \mathrm{mmol})$, DIPA $(153 \mu \mathrm{L} \mathrm{L}, 1.1 \mathrm{mmol})$ and $O$-benzoyl oxime (3n) $(80 \mathrm{mg}, 0.3 \mathrm{mmol})$ in DMSO $(3 \mathrm{~mL})$. The product was purified according to the general chromatography method for oximes. The product was obtained as a film on a vial $(38 \mathrm{mg}, 56 \%)$. The yield of product $\mathbf{5 n}$ was determined to be $56 \%$ by a calibrated UPLC-MS ${ }^{1} \mathrm{H}$ NMR $\left(500 \mathrm{MHz}, \mathrm{CD}_{3} \mathrm{CN}\right) \delta 8.46(\mathrm{~d}, J=6.2 \mathrm{~Hz}, 2 \mathrm{H}), 7.45-7.41(\mathrm{~m}, 2 \mathrm{H}), 7.36(\mathrm{~d}$, $J=6.2 \mathrm{~Hz}, 2 \mathrm{H}), 7.31(\mathrm{t}, J=7.6 \mathrm{~Hz}, 2 \mathrm{H}), 7.26-7.20(\mathrm{~m}, 1 \mathrm{H}), 1.88(\mathrm{~s}, 2 \mathrm{H}), 1.63(\mathrm{tt}, J=8.3,5.5 \mathrm{~Hz}, 1 \mathrm{H}), 0.59-0.33$ $(\mathrm{m}, 4 \mathrm{H}) .{ }^{13} \mathrm{C}$ NMR $\left(126 \mathrm{MHz}, \mathrm{CD}_{3} \mathrm{CN}\right) \delta 159.52,150.32,149.40,128.96,127.97,127.59,123.09,60.69,22.42,2.08$, 1.71. MS (ESI) $\mathrm{m} / \mathrm{z}$ calcd. for $\mathrm{C}_{15} \mathrm{H}_{17} \mathrm{~N}_{2}\left([\mathrm{M}+\mathrm{H}]^{+}\right)$225.1, found 225.2 


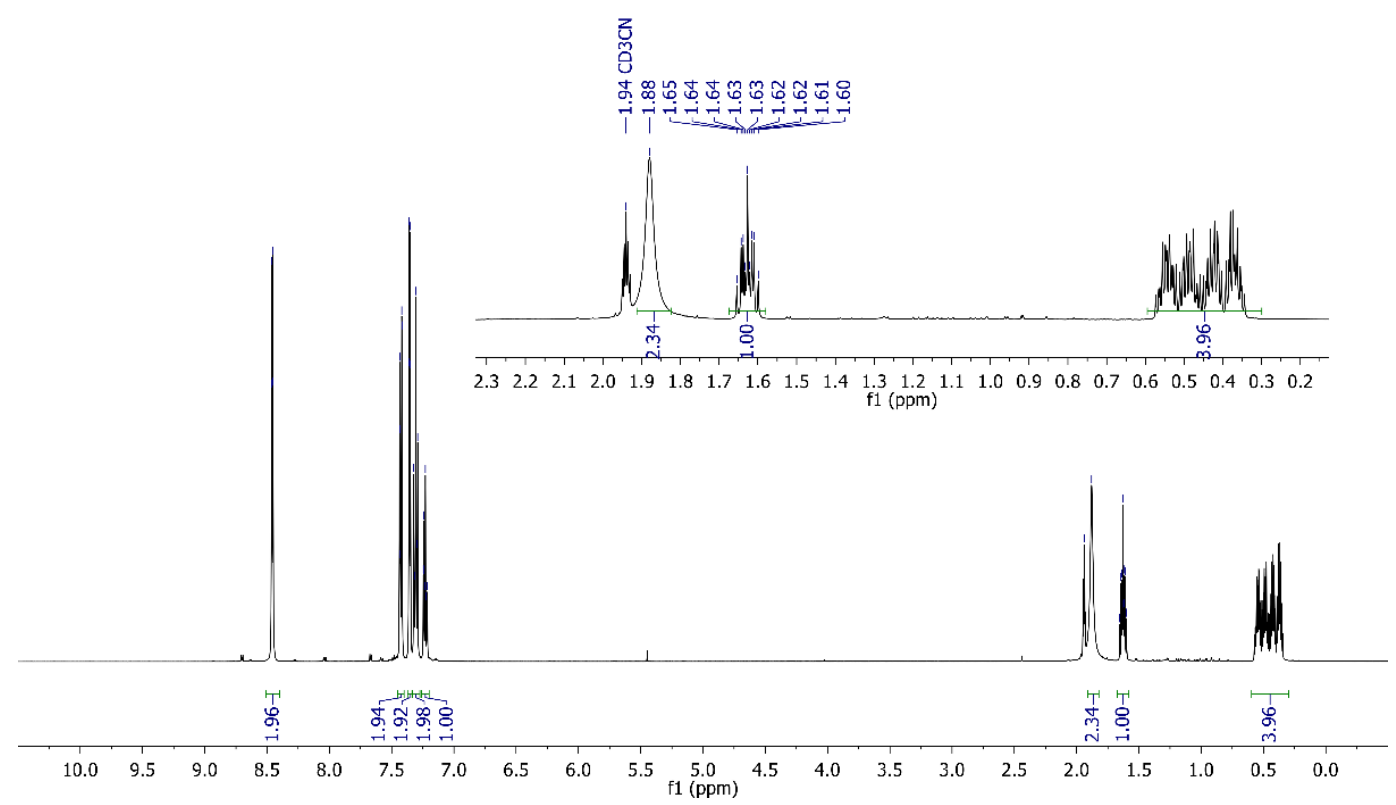

Figure S39. ${ }^{1} \mathrm{H}$ qNMR $(500 \mathrm{MHz})$ spectrum of $\mathbf{5 n}$ in $\mathrm{CD}_{3} \mathrm{CN}$.

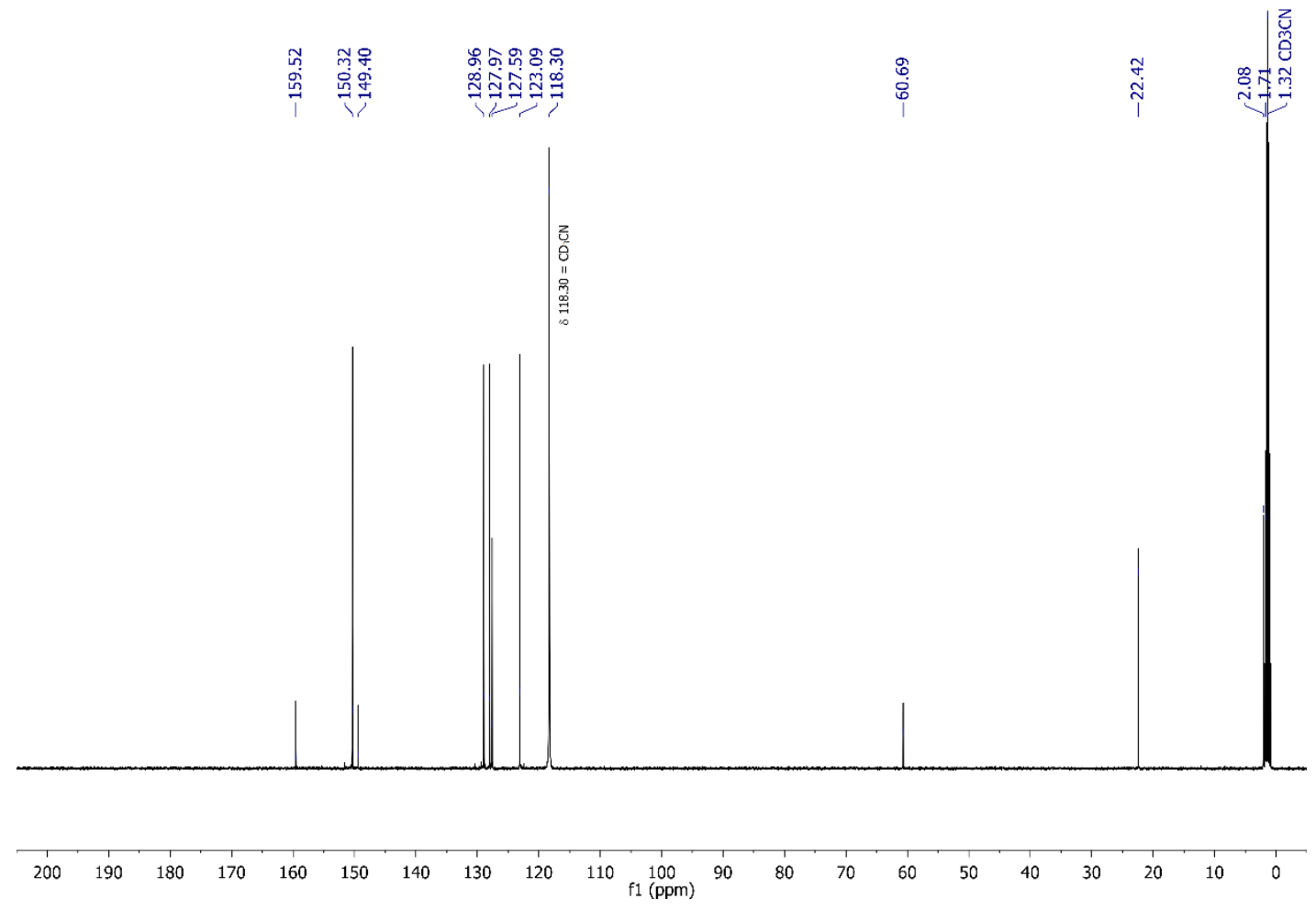

Figure S40. ${ }^{13} \mathrm{C}$ NMR $(126 \mathrm{MHz})$ spectrum of $\mathbf{5 n}$ in $\mathrm{CD}_{3} \mathrm{CN}$. 


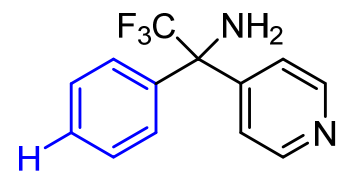

Compound 5p was synthesized following General Procedure A using 4-pyridinecarbonitrile 4a (69 mg, $0.66 \mathrm{mmol})$, PC1 (6 mg, 6E-3 mmol), benzoic acid (37 mg, $0.3 \mathrm{mmol})$, DIPA (153 $\mu \mathrm{L} \mathrm{L,} 1.1 \mathrm{mmol}$ ) and $O$-benzoyl oxime (3p) $(89 \mathrm{mg}, 0.3 \mathrm{mmol})$ in DMSO $(3 \mathrm{~mL})$. The product was purified according to the general chromatography method for oximes. The product was obtained as a film on a vial (44 mg, 58\%). The yield of product $\mathbf{5 p}$ was determined to be $65 \%$ by a calibrated UPLC-MS ${ }^{1} \mathrm{H}$ NMR $\left(500 \mathrm{MHz}\right.$, Acetonitrile- $\left.d_{3}\right) \delta 8.57(\mathrm{~d}, J=6.1 \mathrm{~Hz}, 2 \mathrm{H}), 7.46(\mathrm{~d}, J=7.1 \mathrm{~Hz}$, 2H), $7.43(\mathrm{~d}, J=5.3 \mathrm{~Hz}, 2 \mathrm{H}), 7.41-7.33(\mathrm{~m}, 3 \mathrm{H}), 2.76(\mathrm{~s}, 2 \mathrm{H}) .{ }^{13} \mathrm{C}$ NMR $\left(126 \mathrm{MHz}, \mathrm{CD}_{3} \mathrm{CN}\right) \delta 179.34,168.90$, $162.33,159.04,157.95,157.88,157.11,156.23(\mathrm{q}, J=285.5 \mathrm{~Hz}), 151.92,146.83,94.93(\mathrm{q}, J=26.6 \mathrm{~Hz}) .{ }^{19} \mathrm{~F}$ NMR $\left(471 \mathrm{MHz}, \mathrm{CD}_{3} \mathrm{CN}\right) \delta$-73.24. MS (ESI) m/z calcd. for $\mathrm{C}_{13} \mathrm{H}_{12} \mathrm{~F}_{3} \mathrm{~N}_{2}\left([\mathrm{M}+\mathrm{H}]^{+}\right)$253.1, found 253.2. 


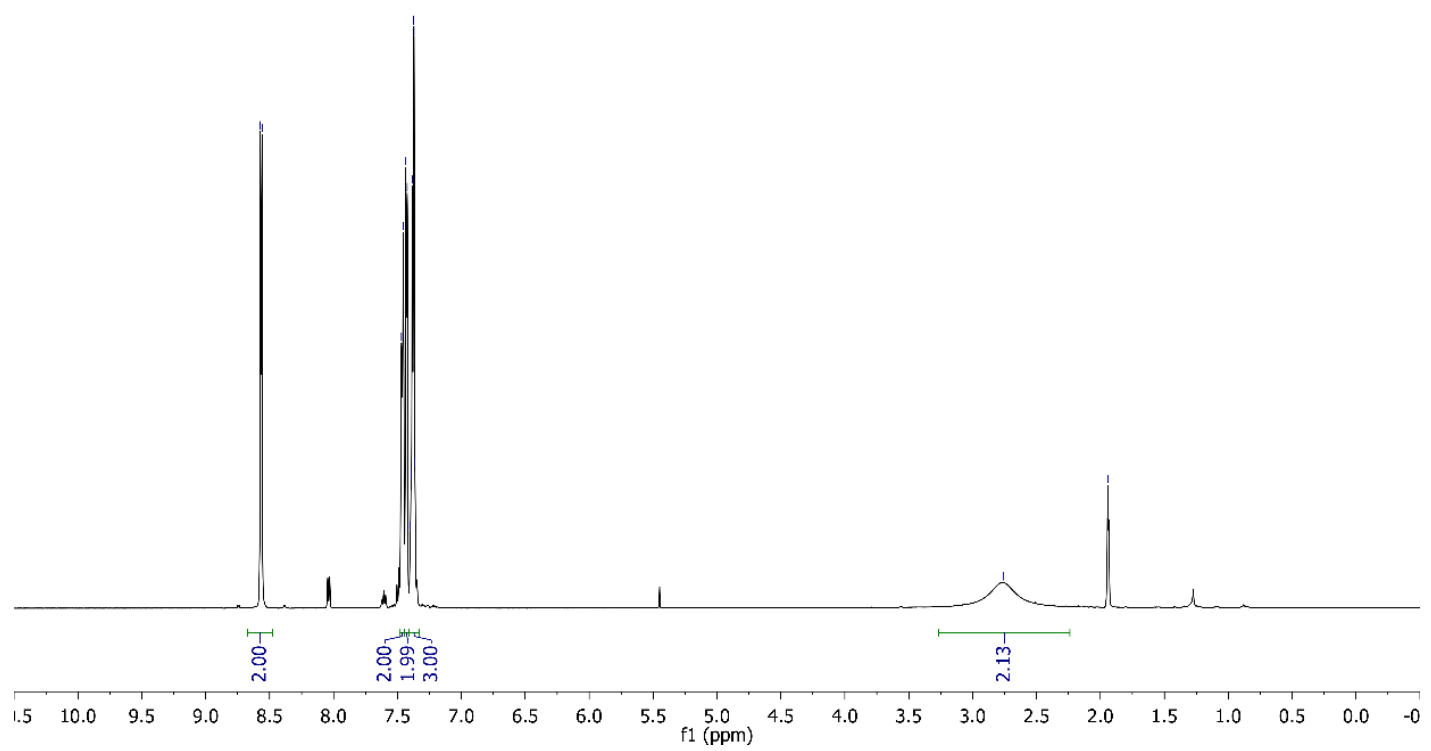

Figure $\mathbf{S 4 1 .}{ }^{1} \mathrm{H}$ qNMR $(500 \mathrm{MHz})$ spectrum of $\mathbf{5 p}$ in $\mathrm{CD}_{3} \mathrm{CN}$.

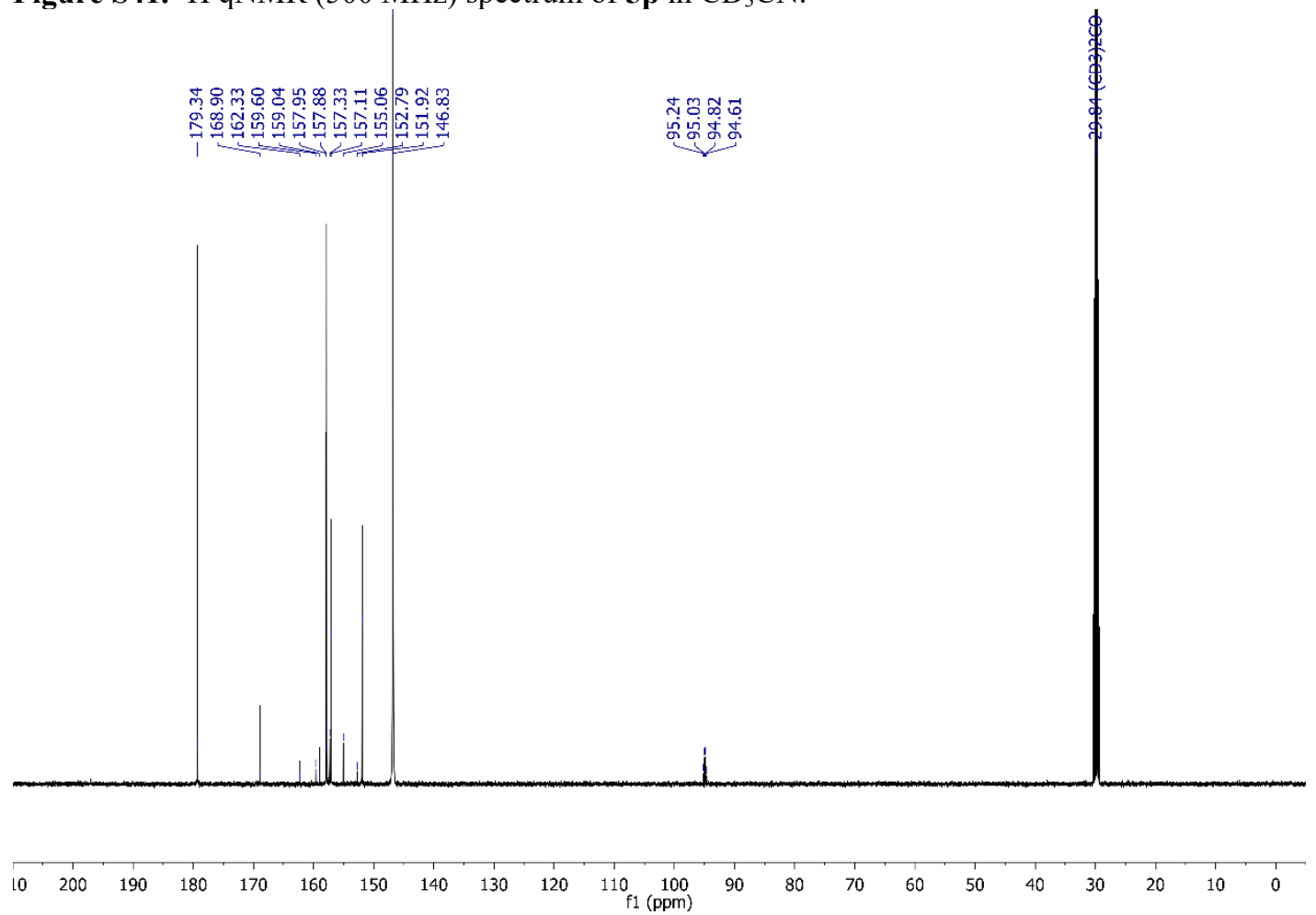

Figure S42. ${ }^{13} \mathrm{C}$ NMR $(126 \mathrm{MHz})$ spectrum of $\mathbf{5 p}$ in $\mathrm{CD}_{3} \mathrm{CN}$. 


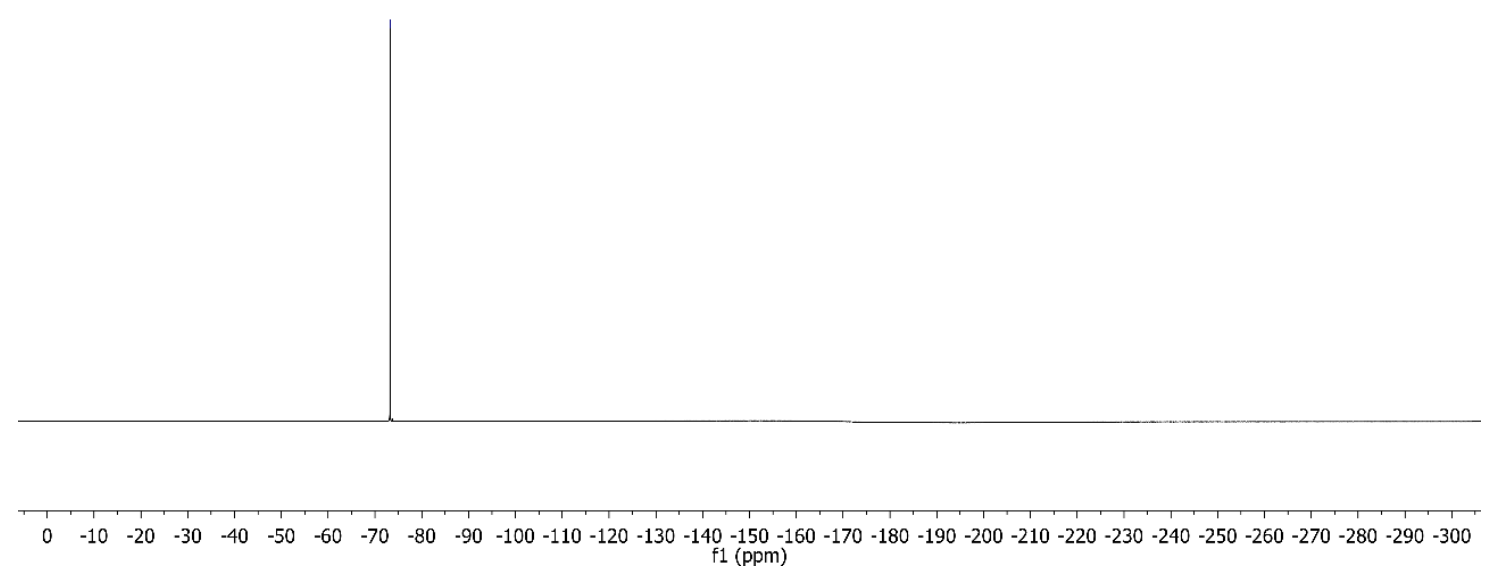

Figure S43. ${ }^{19} \mathrm{~F}$ NMR (471 MHz) spectrum of compound $\mathbf{5 p}$ in $\mathrm{CD}_{3} \mathrm{CN}$. 
<smiles>CC(N)(c1ccncc1)c1ccc(Br)cc1</smiles>

Compound 5q was synthesized following General Procedure A using 4-pyridinecarbonitrile 4a (69 $\mathrm{mg}, 0.66 \mathrm{mmol})$, PC1 (6 mg, 6E-3 mmol), benzoic acid (37 mg, $0.3 \mathrm{mmol})$, DIPA (153 $\mu \mathrm{L} \mathrm{L}, 1.1 \mathrm{mmol})$ and $O$-benzoyl oxime (3q) $(99 \mathrm{mg}, 0.3 \mathrm{mmol})$ in DMSO $(3 \mathrm{~mL})$. The product was purified according to the general chromatography method for oximes. The product was obtained as a film on a vial $(25 \mathrm{mg}, 25 \%)$. The yield of product $\mathbf{5 q}$ was determined to be $29 \%$ by a calibrated UPLC-MS. ${ }^{1} \mathrm{H}$ NMR $\left(500 \mathrm{MHz}\right.$, Acetonitrile- $\left.d_{3}\right) \delta 8.57(\mathrm{~d}, J=6.3 \mathrm{~Hz}, 2 \mathrm{H}), 7.54(\mathrm{~d}, J=8.8 \mathrm{~Hz}$, $2 \mathrm{H}), 7.43-7.32(\mathrm{~m}, 4 \mathrm{H}), 2.60(\mathrm{~s}, 2 \mathrm{H}), 2.16(\mathrm{~s}, 1 \mathrm{H}) .{ }^{13} \mathrm{C} \mathrm{NMR}\left(126 \mathrm{MHz}, \mathrm{CD}_{3} \mathrm{CN}\right) \delta 150.97,150.11,139.80,132.37$, 126.29 (q, $J=285.5 \mathrm{~Hz}), 123.29,123.27,123.18,66.23$ (q, $J=27.0 \mathrm{~Hz}) .{ }^{19} \mathrm{~F}$ NMR $\left(471 \mathrm{MHz}, \mathrm{CD}_{3} \mathrm{CN}\right) \delta-73.40 . \mathrm{MS}$ (ESI) $\mathrm{m} / \mathrm{z}$ calcd. for $\mathrm{C}_{13} \mathrm{H}_{11} \mathrm{BrF}_{3} \mathrm{~N}_{2}\left([\mathrm{M}+\mathrm{H}]^{+}\right)$331.0, found 331.0. 


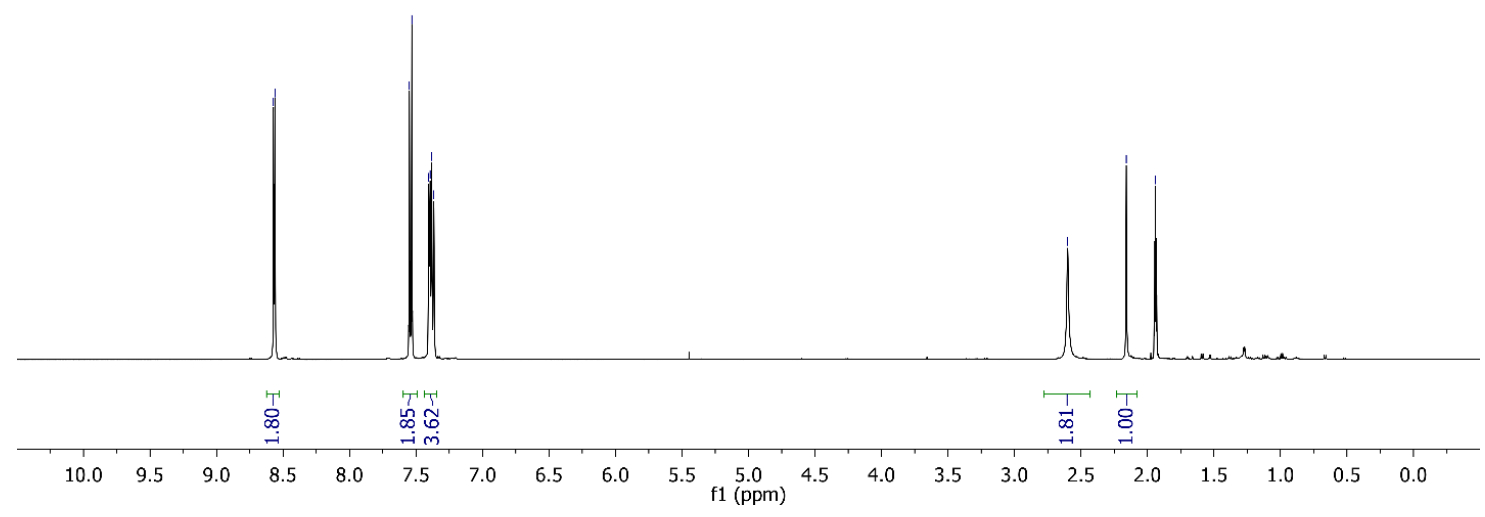

Figure S44. ${ }^{1} \mathrm{H}$ qNMR $(500 \mathrm{MHz})$ spectrum of $\mathbf{5 q}$ in $\mathrm{CD}_{3} \mathrm{CN}$.

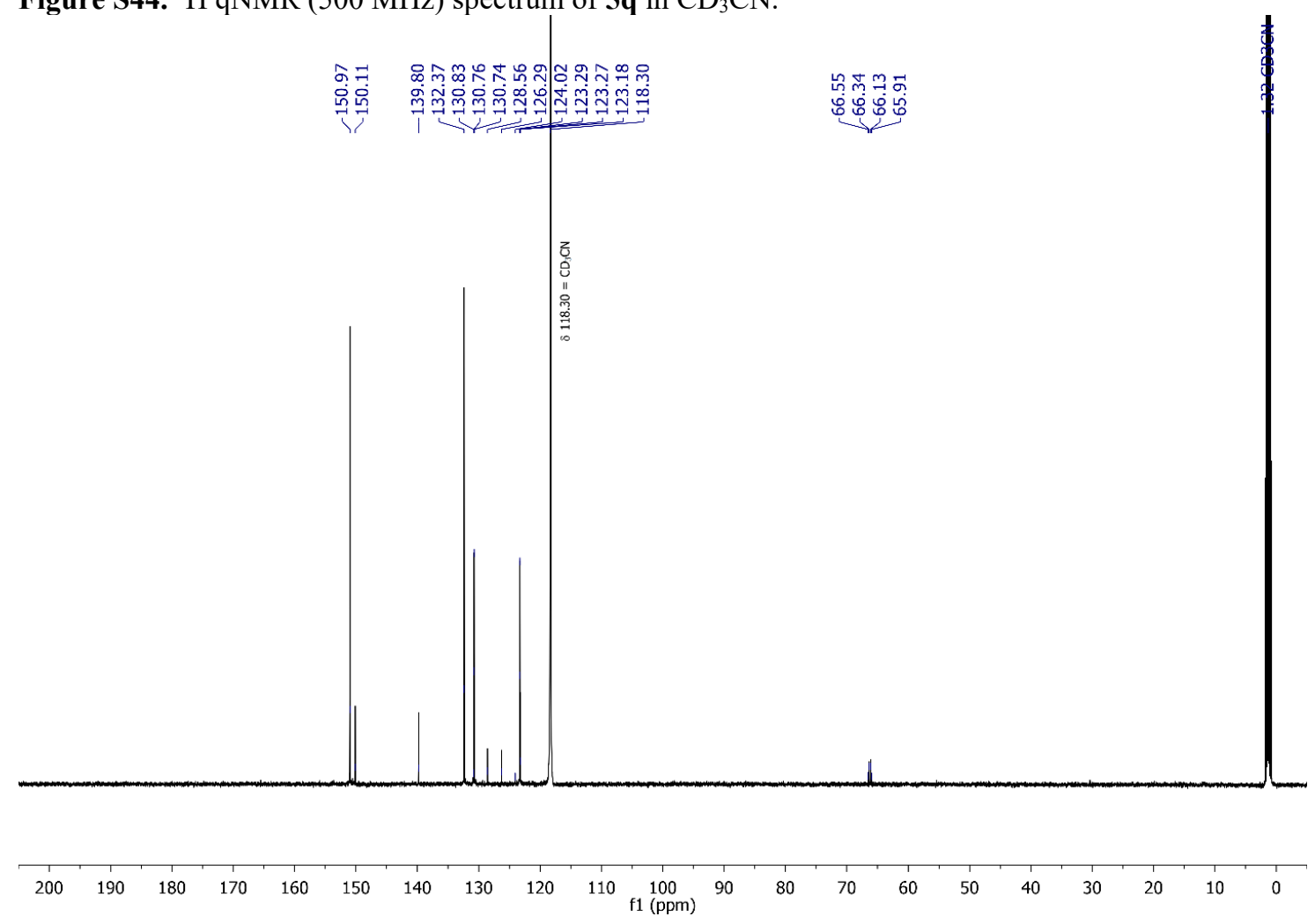

Figure S45. ${ }^{13} \mathrm{C}$ NMR $(126 \mathrm{MHz})$ spectrum of $\mathbf{5 q}$ in $\mathrm{CD}_{3} \mathrm{CN}$. 


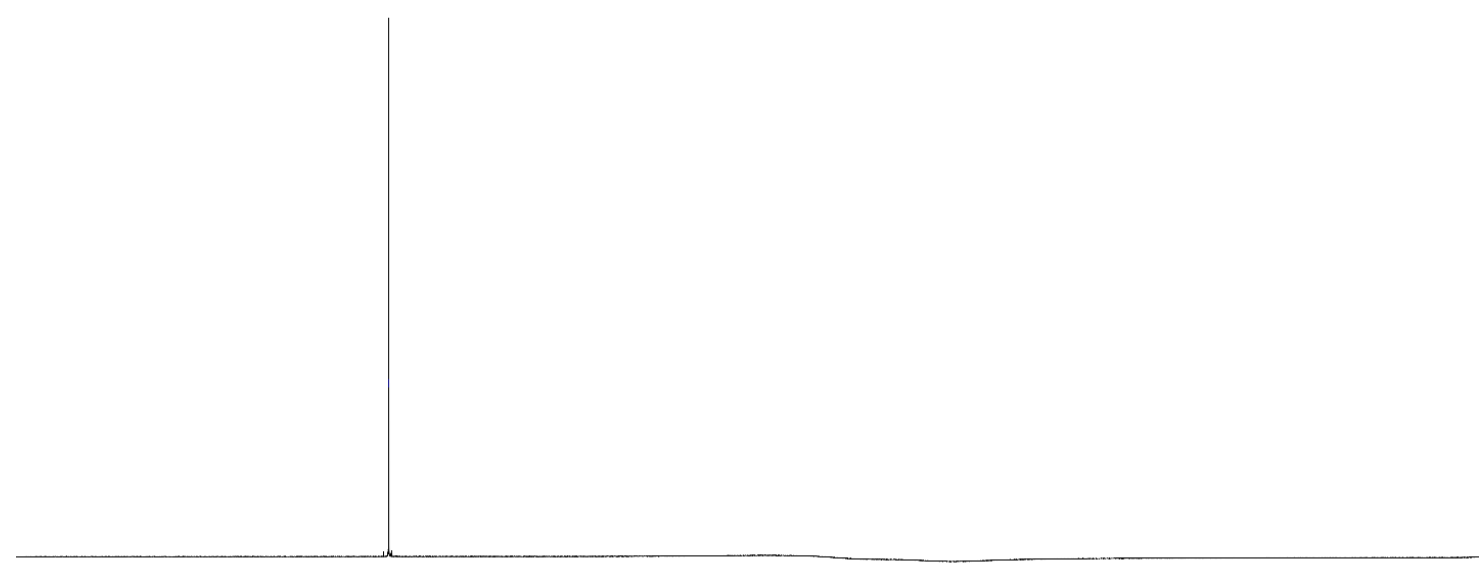

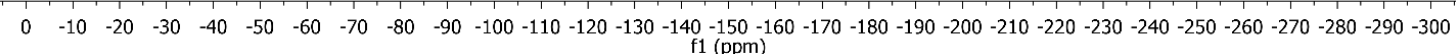

Figure S46. ${ }^{19} \mathrm{~F}$ NMR (471 MHz) spectrum of compound $\mathbf{5 q}$ in $\mathrm{CD}_{3} \mathrm{CN}$. 


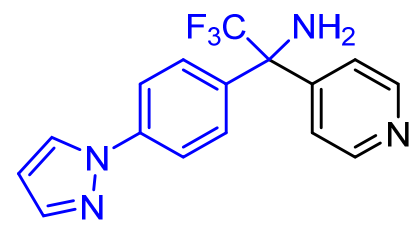

Compound 5r was synthesized following General Procedure A using 4-pyridinecarbonitrile 4a (69 mg, $0.66 \mathrm{mmol})$, PC1 (6 mg, 6E-3 mmol), benzoic acid $(37 \mathrm{mg}, 0.3 \mathrm{mmol})$, DIPA $(153 \mu \mathrm{L} \mathrm{L}, 1.1 \mathrm{mmol})$ and $O$-benzoyl oxime (3r) $(108 \mathrm{mg}, 0.3 \mathrm{mmol})$ in DMSO $(3 \mathrm{~mL})$. The product was purified according to the general chromatography method for oximes. The product was obtained as a film on a vial $(46 \mathrm{mg}, 48 \%)$. The yield of product $\mathbf{5 r}$ was determined to be $57 \%$ by a calibrated UPLC-MS ${ }^{1} \mathrm{H}$ NMR $\left(500 \mathrm{MHz}, \mathrm{CD}_{3} \mathrm{CN}, 1.7: 1\right.$ mixture of rotamers) $\delta 8.58(\mathrm{~d}, J=5.3 \mathrm{~Hz}, 2 \mathrm{H})$, $8.14(\mathrm{~d}, J=1.6 \mathrm{~Hz}, 1 \mathrm{H}), 7.77(\mathrm{~d}, J=8.7 \mathrm{~Hz}, 2 \mathrm{H}), 7.70(\mathrm{~s}, 1 \mathrm{H}), 7.56(\mathrm{~d}, J=8.5 \mathrm{~Hz}, 2 \mathrm{H}), 7.45(\mathrm{~d}, J=4.7 \mathrm{~Hz}, 2 \mathrm{H}), 6.50$ (s, $1 \mathrm{H}), 2.63$ (br s, 1.3H), 2.14 (br s, 0.8H). ${ }^{13} \mathrm{C}$ NMR $\left(126 \mathrm{MHz}, \mathrm{CD}_{3} \mathrm{CN}, 1.7: 1\right.$ mixture of rotamers) $\delta 150.96,150.43$, $150.40,142.25,140.96,138.21,138.18,129.92(\mathrm{q}, J=1.7 \mathrm{~Hz}), 128.32,127.64$ (q, $J=285.8 \mathrm{~Hz}), 123.35$ (q, $J=1.7$ $\mathrm{Hz}), 119.36,108.87,66.21(\mathrm{q}, J=26.8 \mathrm{~Hz}), 66.13(\mathrm{q}, J=26.7 \mathrm{~Hz}) .{ }^{19} \mathrm{~F}\left\{{ }^{1} \mathrm{H}\right\}$ NMR $\left(471 \mathrm{MHz}, \mathrm{CD}_{3} \mathrm{CN}\right) \delta-73.55(\mathrm{~s}$, 3F).MS (ESI) $\mathrm{m} / \mathrm{z}$ calcd. for $\mathrm{C}_{16} \mathrm{H}_{14} \mathrm{~F}_{3} \mathrm{~N}_{4}\left([\mathrm{M}+\mathrm{H}]^{+}\right) 319.1$, found 318.8 


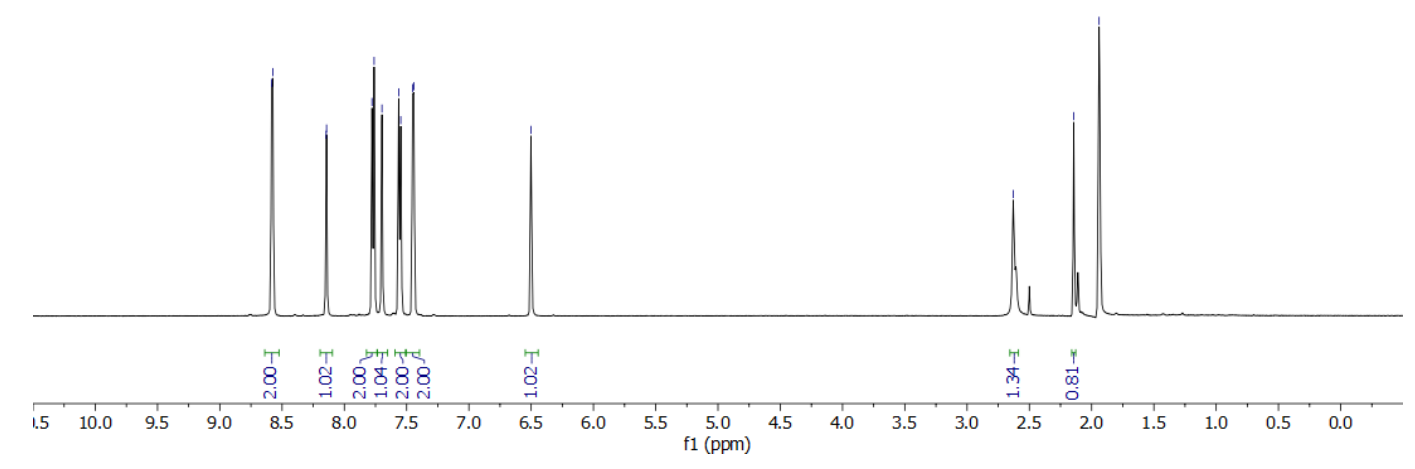

Figure $\mathbf{S 4 7 .}{ }^{1} \mathrm{H}$ qNMR $(500 \mathrm{MHz})$ spectrum of $\mathbf{5 r}$ in $\mathrm{CD}_{3} \mathrm{CN}$.

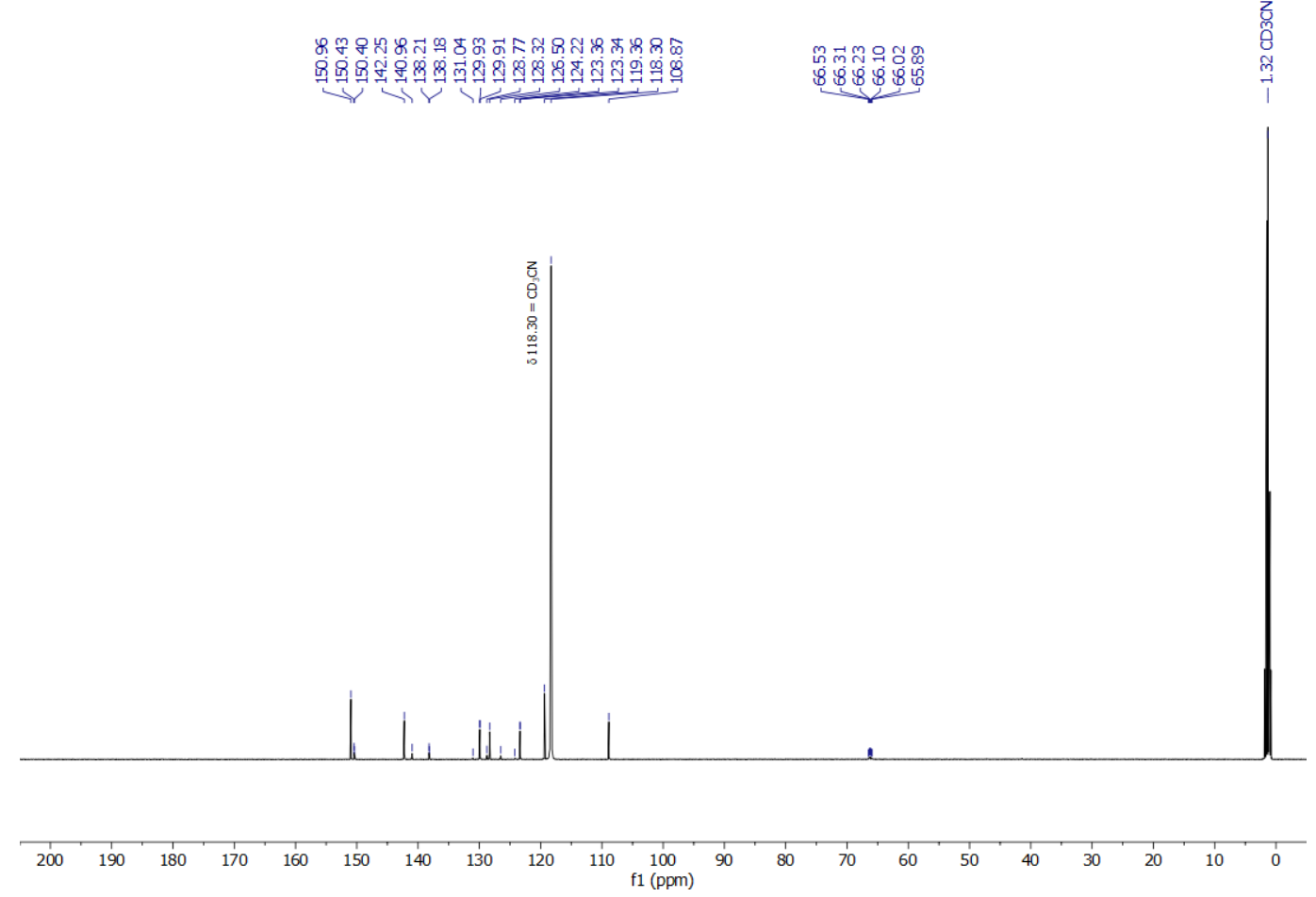

Figure S48. ${ }^{13} \mathrm{C}$ NMR $(126 \mathrm{MHz})$ spectrum of $\mathbf{5 r}$ in $\mathrm{CD}_{3} \mathrm{CN}$. 


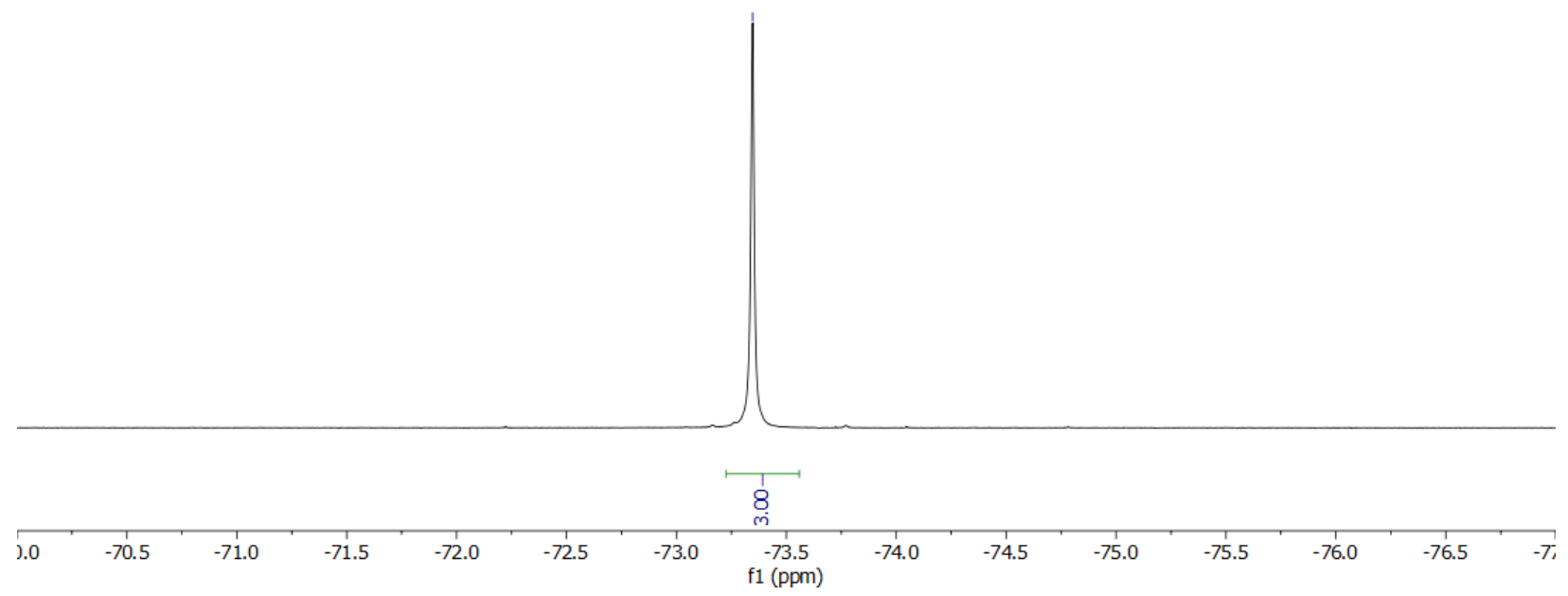

Figure S49. ${ }^{19} \mathrm{~F}$ NMR (471 MHz) spectrum of compound $\mathbf{5 r}$ in $\mathrm{CD}_{3} \mathrm{CN}$ 


\section{Cyanoarene Scope}

Table S5. Table of Purchased Cyanoarenes Starting Materials

4-pyridyl<smiles>N#Cc1ccncc1</smiles>

$4 a$<smiles>N#Cc1ccnc(-c2cccs2)c1</smiles>

4b<smiles>Cn1ccnc1-n1ccnc1</smiles>

4c

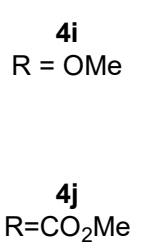<smiles>N#Cc1ccnc(C2CC2)c1</smiles>

40<smiles>N#Cc1ccnc(-n2cccn2)c1</smiles>

$4 d$<smiles>C#Cc1cc(C#N)ccn1</smiles><smiles>CC(C)(C)c1cc(C#N)ccn1</smiles>

$4 q$<smiles>N#Cc1ccnc(-c2ccco2)c1</smiles>

$4 e$<smiles>N#Cc1ccnc(CO)c1</smiles><smiles>N#Cc1ccnc(Cl)c1</smiles><smiles>N#Cc1ccnc(C(F)(F)F)c1</smiles>

$4 n$<smiles>CSc1cc(C#N)ccn1</smiles><smiles>COc1cc(C#N)ccn1</smiles>

$4 \mathrm{~s}$<smiles>COC(=O)c1cc(C#N)ccn1</smiles><smiles>N#Cc1ccnc(-c2ccncc2)c1</smiles>

$4 \mathrm{~g}$<smiles>N#Cc1ccnc(C(N)=O)c1</smiles><smiles>N#Cc1ccncc1-c1ccccc1</smiles><smiles>N#Cc1ccncc1N</smiles>

2-pyridyl

non-heterocyclic arenes<smiles>N#Cc1cc(-c2ccccc2)ccn1</smiles><smiles>CC(=O)c1ccnc(C#N)c1</smiles><smiles>N#Cc1ccnc(C#N)c1</smiles>

$4 z$

4aa<smiles>N#Cc1ccc(C#N)cc1</smiles>

$4 a c$<smiles>N#Cc1ccncn1</smiles>

$4 y$

non-pyridyl heterocycles<smiles>N#Cc1ccnc2[nH]ccc12</smiles>

$4 a e$<smiles>Cc1cc(C#N)nc(C)n1</smiles>

4af<smiles>Cc1nc(C#N)c(C#N)nc1C</smiles>

4ag<smiles>N#Cc1ncnc2[nH]ccc12</smiles>

4ah<smiles>N#Cc1cccc2ccccc12</smiles>

4ad 
Authentic samples of compound 5s-5ad, 5ag, 5ai - 5az were synthesized and characterized as described previously. ${ }^{5}$ NMR characterization of the authentic samples included as line listing for each compound.

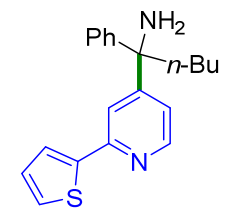

Compound 5s was synthesized following General Procedure A using cyanoarene 4b (123 mg, $0.66 \mathrm{mmol})$, PC1 (6 $\mathrm{mg}, 6 \mathrm{E}-3 \mathrm{mmol})$, benzoic acid (37 mg, $0.3 \mathrm{mmol})$, DIPA (153 $\mu \mathrm{L}, 1.1 \mathrm{mmol})$ and $O$-benzoyl oxime (3I) $(84 \mathrm{mg}, 0.3$ mmol) in DMSO (3 mL). MS (ESI) M/Z CALCD. FOR $\mathrm{C}_{20} \mathrm{H}_{23} \mathrm{~N}_{2} \mathrm{~S}\left([\mathrm{M}+\mathrm{H}]^{+}\right) 323.2$, found 323.2. The product was synthesized in $42 \%$ yield (retention time $\mathrm{t}=2.783, \mathrm{~m} / \mathrm{z}=323.2(\mathrm{M}+\mathrm{H}]^{+}$) as determined by a calibrated UPLC generated from an authentic sample using the UPLC-MS Method for Yield Determination.

Compound $\mathbf{5 s}$ was also synthesized from the corresponding iminium $\mathrm{HCl}$ salt $\mathbf{6} \mathbf{l}$ and cyanoarene $\mathbf{4 c}$. The reaction was conducted by following the General Iminium Salt Reaction Procedure using iminium HCl salt $6 \mathbf{1}(130 \mathrm{mg}, 0.66 \mathrm{mmol}$, 2.2 equiv.); photocatalyst $\operatorname{Ir}\left[\mathrm{dF}(\mathrm{Me}) \mathrm{ppy}_{2} \mathrm{dtbbpyPF}_{6}(6 \mathrm{mg}, 2 \mathrm{E}-3 \mathrm{mmol}, 2 \mathrm{~mol} \%)\right.$; DIPA (153 $\mu \mathrm{L}, 1.1 \mathrm{mmol}, 3.6$ equiv.); and cyanoarene $4 \mathrm{~s}$ (41 mg, $0.3 \mathrm{mmol}, 1.0$ equiv.). The product was synthesized in $54 \%$ yield (retention time $\left.\mathrm{t}=2.783, \mathrm{~m} / \mathrm{z}=323.2(\mathrm{M}+\mathrm{H}]^{+}\right)$as determined by a calibrated UPLC generated from an authentic sample using the UPLC-MS Method for Yield Determination.

${ }^{1} \mathrm{H}$ NMR $\left(500 \mathrm{MHz}, \mathrm{CD}_{3} \mathrm{CN}\right) \delta 8.36(\mathrm{~d}, J=5.2 \mathrm{~Hz}, 1 \mathrm{H}), 7.85(\mathrm{~s}, 1 \mathrm{H}), 7.62(\mathrm{~d}, J=3.4 \mathrm{~Hz}, 1 \mathrm{H}), 7.46-7.41(\mathrm{~m}, 3 \mathrm{H})$, $7.30(\mathrm{t}, J=7.6 \mathrm{~Hz}, 2 \mathrm{H}), 7.22-7.15(\mathrm{~m}, 2 \mathrm{H}), 7.10(\mathrm{t}, J=4.3 \mathrm{~Hz}, 1 \mathrm{H}), 2.27-2.17(\mathrm{~m}, 2 \mathrm{H}), 1.99$ (br s, 2H), 1.38-1.28 $(\mathrm{m}, 2 \mathrm{H}), 1.26-1.09(\mathrm{~m}, 2 \mathrm{H}), 0.85(\mathrm{t}, J=7.3 \mathrm{~Hz}, 3 \mathrm{H}) .{ }^{13} \mathrm{C} \mathrm{NMR}\left(126 \mathrm{MHz}, \mathrm{CD}_{3} \mathrm{CN}\right) \delta 160.38,153.17,150.07,148.94$, $146.28,129.14,128.61,127.43,127.41,125.57,121.52,117.38,61.56,42.14,26.95,23.81,14.38$. ESI LRMS $m / z$ calcd. for $\mathrm{C}_{20} \mathrm{H}_{23} \mathrm{~N}_{2} \mathrm{~S}\left([\mathrm{M}+\mathrm{H}]^{+}\right)$323.2, found 323.2.<smiles>Cc1nccn1-c1cc(C(C)(N)c2ccccc2)ccn1</smiles>

Compound 5t was synthesized following General Procedure A using cyanoarene $4 \mathbf{c}$ (122 mg, $0.66 \mathrm{mmol})$, PC1 (6 $\mathrm{mg}, 6 \mathrm{E}-3 \mathrm{mmol})$, benzoic acid (37 mg, $0.3 \mathrm{mmol})$, DIPA $(153 \mu \mathrm{L}, 1.1 \mathrm{mmol})$ and $O$-benzoyl oxime (3I) $(84 \mathrm{mg}, 0.3$ mmol) in DMSO $(3 \mathrm{~mL})$. MS (ESI) $\mathrm{m} / z$ calcd. for $\mathrm{C}_{20} \mathrm{H}_{25} \mathrm{~N}_{4}\left([\mathrm{M}+\mathrm{H}]^{+}\right) 321.2$, found 321.3. The product was synthesized in $73 \%$ yield (retention time $\mathrm{t}=1.930, \mathrm{~m} / \mathrm{z}=321.3(\mathrm{M}+\mathrm{H}]^{+}$) as determined by a calibrated UPLC generated from an authentic sample using the UPLC-MS Method for Yield Determination

${ }^{1} \mathrm{H}$ NMR (500 MHz, $\left.\mathrm{CD}_{3} \mathrm{CN}\right) \delta 8.37(\mathrm{~d}, J=5.4 \mathrm{~Hz}, 1 \mathrm{H}), 7.48(\mathrm{~s}, 1 \mathrm{H}), 7.45(\mathrm{~d}, J=7.6 \mathrm{~Hz}, 2 \mathrm{H}), 7.35-7.28(\mathrm{~m}, 4 \mathrm{H})$, $7.21(\mathrm{t}, J=7.3 \mathrm{~Hz}, 1 \mathrm{H}), 6.89(\mathrm{~s}, 1 \mathrm{H}), 2.41(\mathrm{~s}, 3 \mathrm{H}), 2.29-2.18(\mathrm{~m}, 2 \mathrm{H}), 2.01(\mathrm{~s}, 2 \mathrm{H}), 1.38-1.29(\mathrm{~m}, 2 \mathrm{H}), 1.27-1.10(\mathrm{~m}$, $2 \mathrm{H}), 0.86(\mathrm{t}, \mathrm{J}=7.3 \mathrm{~Hz}, 3 \mathrm{H}) .{ }^{13} \mathrm{C} \mathrm{NMR}\left(126 \mathrm{MHz}, \mathrm{CD}_{3} \mathrm{CN}\right) \delta 163.04,151.75,149.57,148.60,145.45,129.23,128.33$, $127.60,127.42$, 121.76, 120.26, 116.45, 61.73, 42.12, 26.91, 23.78, 15.40, 14.33 . 


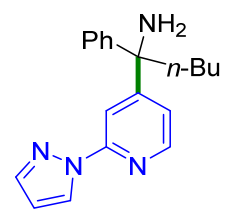

Compound 5u was synthesized following General Procedure A using cyanoarene 4d (123 mg, $0.66 \mathrm{mmol})$, PC1 (6 $\mathrm{mg}, 6 \mathrm{E}-3 \mathrm{mmol})$, benzoic acid (37 mg, $0.3 \mathrm{mmol})$, DIPA $(153 \mu \mathrm{L}, 1.1 \mathrm{mmol})$ and $O$-benzoyl oxime $(\mathrm{X})(84 \mathrm{mg}, 0.3$ mmol) in DMSO (3 mL). MS (ESI) M/Z CALCD. FOR $\mathrm{C}_{19} \mathrm{H}_{23} \mathrm{~N}_{4}\left([\mathrm{M}+\mathrm{H}]^{+}\right) 307.2$, found 307.2. The product 5u was synthesized in $82 \%$ yield (retention time $\mathrm{t}=2.662, \mathrm{~m} / \mathrm{z}=307.2(\mathrm{M}+\mathrm{H}]^{+}$) as determined by a calibrated UPLC generated from an authentic sample using the UPLC-MS Method for Yield Determination

Compound $\mathbf{5 u}$ was also synthesized from the corresponding iminium $\mathrm{HCl}$ salt $\mathbf{6} \mathbf{l}$ and cyanoarene $\mathbf{4 d}$. The reaction was conducted by following the General Iminium Salt Reaction Procedure using iminium HCl salt 31 ( $130 \mathrm{mg}, 0.66 \mathrm{mmol}$, 2.2 equiv.); photocatalyst $\operatorname{Ir}\left[\mathrm{dF}(\mathrm{Me}) \mathrm{ppy}_{2} \mathrm{dtbbpyPF}_{6}(6 \mathrm{mg}, 2 \mathrm{E}-3 \mathrm{mmol}, 2 \mathrm{~mol} \%)\right.$; DIPA (153 $\mu \mathrm{L}, 1.1 \mathrm{mmol}, 3.6$ equiv.); and cyanoarene $4 \mathbf{d}$ (41 $\mathrm{mg}, 0.3 \mathrm{mmol}, 1.0$ equiv.). The product $5 \mathbf{u}$ was synthesized in $69 \%$ yield (retention time $\left.\mathrm{t}=2.662, \mathrm{~m} / \mathrm{z}=307.2(\mathrm{M}+\mathrm{H}]^{+}\right)$as determined by a calibrated UPLC generated from an authentic sample using the UPLC-MS Method for Yield Determination

${ }^{1} \mathrm{H}$ NMR $\left(500 \mathrm{MHz}, \mathrm{CD}_{3} \mathrm{CN}\right) \delta 8.53(\mathrm{~d}, J=1.0 \mathrm{~Hz}, 1 \mathrm{H}), 8.28(\mathrm{~d}, J=5.2 \mathrm{~Hz}, 1 \mathrm{H}), 8.00(\mathrm{~s}, 1 \mathrm{H}), 7.70(\mathrm{~s}, 1 \mathrm{H}), 7.44(\mathrm{~d}$, $J=7.8 \mathrm{~Hz}, 2 \mathrm{H}), 7.31(\mathrm{t}, J=7.7 \mathrm{~Hz}, 2 \mathrm{H}), 7.25(\mathrm{~d}, J=5.2 \mathrm{~Hz}, 1 \mathrm{H}), 7.21(\mathrm{t}, J=7.4 \mathrm{~Hz}, 1 \mathrm{H}), 6.47(\mathrm{~s}, 1 \mathrm{H}), 2.28-2.18$ $(\mathrm{m}, 2 \mathrm{H}), 2.05(\mathrm{br} \mathrm{s}, 2 \mathrm{H}), 1.37-1.28(\mathrm{~m}, 2 \mathrm{H}), 1.24-1.09(\mathrm{~m}, 2 \mathrm{H}), 0.85(\mathrm{t}, J=7.3 \mathrm{~Hz}, 3 \mathrm{H}) .{ }^{13} \mathrm{C} \mathrm{NMR}\left(126 \mathrm{MHz}, \mathrm{CD}_{3} \mathrm{CN}\right)$ $\delta 163.05,152.61,148.86,148.78,142.77,129.20,127.95,127.55,127.45,120.97,110.94,108.63,61.75,42.17,26.90$, $23.78,14.34$.

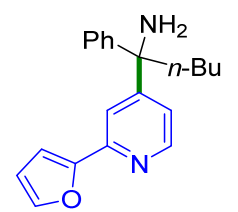

Compound 5v was synthesized following General Procedure A using cyanoarene 4e (112 mg, $0.66 \mathrm{mmol})$, PC1 (6 $\mathrm{mg}, 6 \mathrm{E}-3 \mathrm{mmol})$, benzoic acid (37 mg, $0.3 \mathrm{mmol})$, DIPA (153 $\mu \mathrm{L}, 1.1 \mathrm{mmol})$ and $O$-benzoyl oxime (31) $(84 \mathrm{mg}, 0.3$ $\mathrm{mmol})$ in DMSO $(3 \mathrm{~mL})$. MS (ESI) M/Z CALCD. FOR $\mathrm{C}_{20} \mathrm{H}_{23} \mathrm{~N}_{2} \mathrm{O}\left([\mathrm{M}+\mathrm{H}]^{+}\right) 307.2$, found 307.2. The product was synthesized in $35 \%$ yield (retention time $\mathrm{t}=2.558, \mathrm{~m} / \mathrm{z}=307.2(\mathrm{M}+\mathrm{H}]^{+}$) as determined by a calibrated UPLC generated from an authentic sample using the UPLC-MS Method for Yield Determination

Compound $\mathbf{5 v}$ was also synthesized from the corresponding iminium $\mathrm{HCl}$ salt $\mathbf{6} \mathbf{l}$ and cyanoarene $\mathbf{4 e}$. The reaction was conducted by following the General Iminium Salt Reaction Procedure using iminium $\mathrm{HCl}$ salt $61(130 \mathrm{mg}, 0.66 \mathrm{mmol}$, 2.2 equiv.); photocatalyst $\operatorname{Ir}\left[\mathrm{dF}(\mathrm{Me}) \mathrm{ppy}_{2} \mathrm{dtbbpyPF}_{6}(6 \mathrm{mg}, 2 \mathrm{E}-3 \mathrm{mmol}, 2 \mathrm{~mol} \%)\right.$; DIPA (153 $\mu \mathrm{L}, 1.1 \mathrm{mmol}, 3.6$ equiv.); and cyanoarene $4 \mathrm{e}(37 \mathrm{mg}, 0.3 \mathrm{mmol}, 1.0$ equiv.). The product was synthesized in $69 \%$ yield (retention time $\left.\mathrm{t}=2.558, \mathrm{~m} / \mathrm{z}=307.2(\mathrm{M}+\mathrm{H}]^{+}\right)$as determined by a calibrated UPLC generated from an authentic sample using the UPLC-MS Method for Yield Determination.

${ }^{1} \mathrm{H}$ NMR $\left(500 \mathrm{MHz}, \mathrm{CD}_{3} \mathrm{CN}\right) \delta 8.42(\mathrm{dd}, J=0.4,5.2 \mathrm{~Hz}, 1 \mathrm{H}), 7.75(\mathrm{~d}, J=1.1 \mathrm{~Hz}, 1 \mathrm{H}), 7.59(\mathrm{dd}, J=0.6,1.6 \mathrm{~Hz}, 1 \mathrm{H})$, $7.44(\mathrm{~d}, J=7.3 \mathrm{~Hz}, 2 \mathrm{H}), 7.31(\mathrm{t}, J=8.1 \mathrm{~Hz}, 2 \mathrm{H}), 7.23-7.18(\mathrm{~m}, 2 \mathrm{H}), 7.02(\mathrm{dd}, J=0.4,3.4 \mathrm{~Hz}, 1 \mathrm{H}), 6.56(\mathrm{dd}, J=1.8$, $3.4 \mathrm{~Hz}, 1 \mathrm{H}), 2.28-2.17(\mathrm{~m}, 2 \mathrm{H}), 1.84(\mathrm{br} \mathrm{s}, 2 \mathrm{H}), 1.38-1.28(\mathrm{~m}, 2 \mathrm{H}), 1.24-1.08(\mathrm{~m}, 2 \mathrm{H}), 0.86(\mathrm{t}, J=7.30 \mathrm{~Hz}, 3 \mathrm{H}) .{ }^{13} \mathrm{C}$ NMR $\left(126 \mathrm{MHz}, \mathrm{CD}_{3} \mathrm{CN}\right) \delta 160.37,155.04,150.42,150.00,149.03,144.50,129.17,127.46,127.45,121.35,117.17$, $113.05,109.42,61.56,42.17,26.94,23.81,14.35$. 


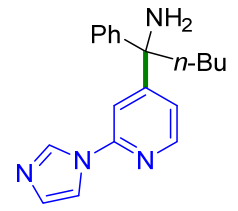

Compound 5w was also synthesized from the corresponding iminium $\mathrm{HCl}$ salt $\mathbf{6 l}$ and cyanoarene $\mathbf{4 f}$. The reaction was conducted by following the General Iminium Salt Reaction Procedure using iminium HCl salt 61 (130 mg, 0.66 mmol, 2.2 equiv.); photocatalyst $\operatorname{Ir}\left[\mathrm{dF}(\mathrm{Me}) \mathrm{ppy}_{2} \mathrm{dtbbpyPF}_{6}(6 \mathrm{mg}, 2 \mathrm{E}-3 \mathrm{mmol}, 2 \mathrm{~mol} \%)\right.$; DIPA (153 $\mu \mathrm{L}, 1.1 \mathrm{mmol}, 3.6$ equiv.); and cyanoarene $6 \mathbf{l}$ (41 mg, $0.3 \mathrm{mmol}, 1.0$ equiv.). $\mathrm{MS}$ (ESI) $\mathrm{m} / z$ calcd. for $\mathrm{C}_{19} \mathrm{H}_{23} \mathrm{~N}_{4}([\mathrm{M}+\mathrm{H}]+) 308.2,308.2$. The product $5 \mathrm{w}$ was synthesized in $25 \%$ yield (retention time $\mathrm{t}=2.245, \mathrm{~m} / \mathrm{z}=321.3(\mathrm{M}+\mathrm{H}]^{+}$) as determined by a calibrated UPLC generated from an authentic sample using the UPLC-MS Method for Yield Determination.

${ }^{1} \mathrm{H}$ NMR $\left(500 \mathrm{MHz}, \mathrm{CD}_{3} \mathrm{CN}\right) \delta 8.33-8.29(\mathrm{~m}, 2 \mathrm{H}), 7.73(\mathrm{~s}, 1 \mathrm{H}), 7.64(\mathrm{~s}, 1 \mathrm{H}), 7.45(\mathrm{~d}, J=8.0 \mathrm{~Hz}, 2 \mathrm{H}), 7.31(\mathrm{t}, J=7.7$ $\mathrm{Hz}, 2 \mathrm{H}), 7.24(\mathrm{~d}, J=5.3 \mathrm{~Hz}, 1 \mathrm{H}), 7.21(\mathrm{t}, J=7.3 \mathrm{~Hz}, 1 \mathrm{H}), 7.09 \mathrm{~s},(1 \mathrm{H}), 2.30-2.20$ (m, 2H), 2.10 (br s, 2H), $1.39-1.29$ $(\mathrm{m}, 2 \mathrm{H}), 1.26-1.09(\mathrm{~m}, 2 \mathrm{H}), 0.87$ (t, $J=7.3 \mathrm{~Hz}, 3 \mathrm{H}) .{ }^{13} \mathrm{C} \mathrm{NMR}\left(126 \mathrm{MHz}, \mathrm{CD}_{3} \mathrm{CN}\right) \delta 163.43,150.41,149.48,148.63$, $136.07,131.04,129.23,127.60,127.41,121.61,117.54,111.50,61.79,42.09,26.89,23.79,14.33$.<smiles>CCCC(N)(c1ccccc1)c1ccnc(-c2ccncc2)c1</smiles>

Compound 5x was synthesized following General Procedure A using cyanoarene 4g (120 mg, 0.66 mmol), PC1 (6 $\mathrm{mg}, 6 \mathrm{E}-3 \mathrm{mmol})$, benzoic acid $(37 \mathrm{mg}, 0.3 \mathrm{mmol})$, DIPA $(153 \mu \mathrm{L}, 1.1 \mathrm{mmol})$ and $O$-benzoyl oxime (3l) $(84 \mathrm{mg}, 0.3$ mmol) in DMSO (3 mL). MS (ESI) $\mathrm{m} / z$ calcd. for $\mathrm{C}_{21} \mathrm{H}_{24} \mathrm{~N}_{3}([\mathrm{M}+\mathrm{H}]+)$ 318.2, found 318.3. The product was synthesized in $32 \%$ yield (retention time $\mathrm{t}=2.342, \mathrm{~m} / \mathrm{z}=318.3(\mathrm{M}+\mathrm{H}]^{+}$) as determined by a calibrated UPLC generated from an authentic sample using the UPLC-MS Method for Yield Determination.

Compound $\mathbf{5 x}$ was also synthesized from the corresponding iminium $\mathrm{HCl}$ salt $\mathbf{6 l}$ and cyanoarene $\mathbf{4 g}$. The reaction was conducted by following the General Iminium Salt Reaction Procedure using iminium HCl salt 61 (130 mg, 0.66 mmol, 2.2 equiv.); photocatalyst $\operatorname{Ir}\left[\mathrm{dF}(\mathrm{Me}){ }{ }_{2}\right]_{2} \mathrm{dtbbpyPF}_{6}(6 \mathrm{mg}, 2 \mathrm{E}-3 \mathrm{mmol}, 2 \mathrm{~mol} \%)$; DIPA (153 $\mu \mathrm{L}, 1.1 \mathrm{mmol}, 3.6$ equiv.); and cyanoarene $\mathbf{4 g}$ (40 mg, $0.3 \mathrm{mmol}, 1.0$ equiv.). The product $\mathbf{5 x w a s}$ synthesized in $18 \%$ yield (retention time $\left.\mathrm{t}=2.342, \mathrm{~m} / \mathrm{z}=318.3(\mathrm{M}+\mathrm{H}]^{+}\right)$as determined by a calibrated UPLC generated from an authentic sample using the UPLC-MS Method for Yield Determination.

${ }^{1} \mathrm{H}$ NMR $\left(500 \mathrm{MHz}, \mathrm{CD}_{3} \mathrm{CN}\right) \delta 8.65(\mathrm{dd}, J=1.7,4.5 \mathrm{~Hz}, 2 \mathrm{H}), 8.57(\mathrm{~d}, J=5.2 \mathrm{~Hz}, 1 \mathrm{H}), 8.00(\mathrm{dd}, J=0.5,1.6 \mathrm{~Hz}, 1 \mathrm{H})$, $7.93(\mathrm{dd}, J=1.7,4.5 \mathrm{~Hz}, 2 \mathrm{H}), 7.48-7.44(\mathrm{~m}, 2 \mathrm{H}), 7.39$ (dd, $J=1.7,5.2, \mathrm{~Hz}, 1 \mathrm{H}), 7.28-7.33(\mathrm{~m}, 2 \mathrm{H}), 7.21(\mathrm{tt}, J=1.1$, $6.9 \mathrm{~Hz}, 1 \mathrm{H}), 2.31-2.22(\mathrm{~m}, 2 \mathrm{H}), 2.00$ (br s, $2 \mathrm{H}), 1.39-1.30(\mathrm{~m}, 2 \mathrm{H}), 1.27-1.10(\mathrm{~m}, 2 \mathrm{H}), 0.87(\mathrm{t}, J=7.3 \mathrm{~Hz}, 3 \mathrm{H}) .{ }^{13} \mathrm{C}$ NMR $\left(126 \mathrm{MHz}, \mathrm{CD}_{3} \mathrm{CN}\right) \delta 160.91,155.12,151.35,150.75,148.91,147.43,129.20,127.51,127.45,123.20,121.90$, 119.84, 61.69, 42.19, 26.96, 23.81, 14.34 . 


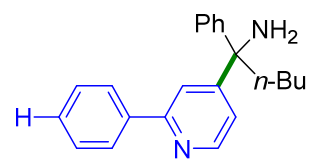

Compound 5y was synthesized following General Procedure A using cyanoarene $4 \mathrm{~h}$ (119 mg, $0.66 \mathrm{mmol})$, PC1 (6 $\mathrm{mg}, 6 \mathrm{E}-3 \mathrm{mmol})$, benzoic acid ( $37 \mathrm{mg}, 0.3 \mathrm{mmol})$, DIPA $(153 \mu \mathrm{L}, 1.1 \mathrm{mmol})$ and $O$-benzoyl oxime (3I) $(84 \mathrm{mg}, 0.3$ mmol) in DMSO $(3 \mathrm{~mL})$. MS (ESI) $m / z$ calcd. for $\mathrm{C}_{22} \mathrm{H}_{25} \mathrm{~N}_{2}\left([\mathrm{M}+\mathrm{H}]^{+}\right) 317.2$, found 317.2. The product $\mathbf{5 y}$ was synthesized in $89 \%$ yield (retention time $\mathrm{t}=2.342, \mathrm{~m} / \mathrm{z}=317.2(\mathrm{M}+\mathrm{H}]^{+}$) as determined by a calibrated UPLC generated from an authentic sample using the UPLC-MS Method for Yield Determination.

Compound $\mathbf{5 y}$ was also synthesized from the corresponding iminium $\mathrm{HCl}$ salt $\mathbf{6 l}$ and cyanoarene $\mathbf{4 h}$. The reaction was conducted by following the General Iminium Salt Reaction Procedure using iminium HCl salt $61(130 \mathrm{mg}, 0.66 \mathrm{mmol}$, 2.2 equiv.); photocatalyst $\operatorname{Ir}\left[\mathrm{dF}(\mathrm{Me}) \mathrm{ppy}_{2} \mathrm{dtbbpyPF}_{6}(6 \mathrm{mg}, 2 \mathrm{E}-3 \mathrm{mmol}, 2 \mathrm{~mol} \%)\right.$; DIPA (153 $\mu \mathrm{L}, 1.1 \mathrm{mmol}, 3.6$ equiv.); and cyanoarene $4 \mathrm{~h}(40 \mathrm{mg}, 0.3 \mathrm{mmol}, 1.0$ equiv.). The product $5 \mathbf{y}$ was synthesized in $66 \%$ yield (retention time $\left.\mathrm{t}=2.342, \mathrm{~m} / \mathrm{z}=317.2(\mathrm{M}+\mathrm{H}]^{+}\right)$as determined by a calibrated UPLC generated from an authentic sample using the UPLC-MS Method for Yield Determination.

${ }^{1} \mathrm{H}$ NMR $\left(500 \mathrm{MHz}, \mathrm{CD}_{3} \mathrm{CN}\right) \delta 8.51(\mathrm{~d}, J=5.2 \mathrm{~Hz}, 1 \mathrm{H}), 8.03-7.99(\mathrm{~m}, 2 \mathrm{H}), 7.89(\mathrm{~d}, 1.0 \mathrm{~Hz}, 1 \mathrm{H}), 7.49-7.43(\mathrm{~m}, 4 \mathrm{H})$, 7.43-7.38 (m, 1H), 7.32-7.25 (m, 3H), 7.22-7.17 (m, 1H), 2.26 (t, $J=8.4 \mathrm{~Hz}, 2 \mathrm{H}), 2.05$ (br s, 2H), 1.34 (sextet, $J=$ $7.3 \mathrm{~Hz}, 2 \mathrm{H}), 1.27-1.11(\mathrm{~m}, 2 \mathrm{H}), 0.86(\mathrm{t}, J=7.3 \mathrm{~Hz}, 3 \mathrm{H}) .{ }^{13} \mathrm{C}$ NMR $\left(126 \mathrm{MHz}, \mathrm{CD}_{3} \mathrm{CN}\right) \delta 160.38,157.73,150.34$, $149.09,140.60,129.66,129.13,127.74,127.44,127.41,121.56,119.19,61.64,42.22,26.99,23.82,14.38$. ESI LRMS $\mathrm{m} / z$ calcd. for $\mathrm{C}_{22} \mathrm{H}_{25} \mathrm{~N}_{2}\left([\mathrm{M}+\mathrm{H}]^{+}\right)$317.2, found 317.2.

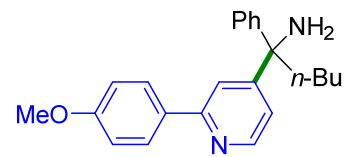

Compound $5 \mathbf{z}$ was synthesized following General Procedure A using cyanoarene $4 \mathbf{i}$ (139 mg, $0.66 \mathrm{mmol}), \mathbf{P C 1}$ (6 mg, $6 \mathrm{E}-3 \mathrm{mmol})$, benzoic acid ( $37 \mathrm{mg}, 0.3 \mathrm{mmol})$, DIPA ( $153 \mu \mathrm{L}, 1.1 \mathrm{mmol})$ and $O$-benzoyl oxime (3I) $(84 \mathrm{mg}, 0.3 \mathrm{mmol})$ in DMSO (3 mL). MS (ESI) $\mathrm{m} / z$ calcd. for $\mathrm{C}_{23} \mathrm{H}_{27} \mathrm{~N}_{2} \mathrm{O}\left([\mathrm{M}+\mathrm{H}]^{+}\right) 347.2$, found 347.3. The product $\mathbf{5 z}$ was synthesized in $97 \%$ yield (retention time $\mathrm{t}=2.790, \mathrm{~m} / \mathrm{z}=347.3(\mathrm{M}+\mathrm{H}]^{+}$) as determined by a calibrated UPLC generated from an authentic sample using the UPLC-MS Method for Yield Determination.

Compound $\mathbf{5 z}$ was also synthesized from the corresponding iminium $\mathrm{HCl}$ salt $\mathbf{6} \mathbf{l}$ and cyanoarene $4 \mathbf{i}$. The reaction was conducted by following the General Iminium Salt Reaction Procedure using iminium $\mathrm{HCl}$ salt $61(130 \mathrm{mg}, 0.66 \mathrm{mmol}$, 2.2 equiv.); photocatalyst $\operatorname{Ir}[\mathrm{dF}(\mathrm{Me}) \mathrm{ppy}]_{2} \mathrm{dtbbpyPF}_{6}(6 \mathrm{mg}, 2 \mathrm{E}-3 \mathrm{mmol}, 2 \mathrm{~mol} \%)$; DIPA (153 $\mu \mathrm{L}, 1.1 \mathrm{mmol}, 3.6$ equiv.); and cyanoarene $4 \mathbf{i}$ (46 mg, $0.3 \mathrm{mmol}, 1.0$ equiv.). The product $5 \mathbf{z}$ was synthesized in $95 \%$ yield (retention time $\left.\mathrm{t}=2.790, \mathrm{~m} / \mathrm{z}=347.3(\mathrm{M}+\mathrm{H}]^{+}\right)$as determined by a calibrated UPLC generated from an authentic sample using the UPLC-MS Method for Yield Determination.

${ }^{1} \mathrm{H}$ NMR $\left(500 \mathrm{MHz}, \mathrm{CD}_{3} \mathrm{CN}\right) \delta 8.46(\mathrm{~d}, J=5.1 \mathrm{~Hz}, 1 \mathrm{H}), 7.96(\mathrm{~d}, J=8.7 \mathrm{~Hz}, 2 \mathrm{H}), 7.82(\mathrm{~s}, 1 \mathrm{H}), 7.45(\mathrm{~d}, J=7.6 \mathrm{~Hz}$, $2 \mathrm{H}), 7.30(\mathrm{t}, J=7.7 \mathrm{~Hz}, 2 \mathrm{H}), 7.20(\mathrm{~d}, J=6.0 \mathrm{~Hz}, 2 \mathrm{H}), 7.00(\mathrm{~d}, J=8.7 \mathrm{~Hz}, 2 \mathrm{H}), 3.82(\mathrm{~s}, 3 \mathrm{H}), 2.25(\mathrm{t}, J=8.2 \mathrm{~Hz}, 2 \mathrm{H})$, $2.04(\mathrm{~s}, 2 \mathrm{H}), 1.39-1.29(\mathrm{~m}, 2 \mathrm{H}), 1.26-1.09(\mathrm{~m}, 2 \mathrm{H}), 0.86(\mathrm{t}, J=7.3 \mathrm{~Hz}, 3 \mathrm{H}) .{ }^{13} \mathrm{C}$ NMR $\left(126 \mathrm{MHz}, \mathrm{CD}_{3} \mathrm{CN}\right) \delta 161.50$, 160.24 , 157.46, 150.19, 149.18, 133.10, 129.12, 129.05, 127.45, 127.38, 120.85, 118.29, 114.98, 61.63, 56.02, 42.21, $27.01,23.83,14.37$. 


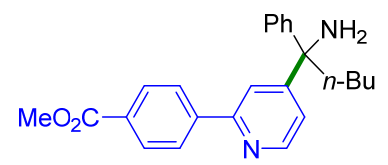

Compound 5aa was synthesized following General Procedure A using cyanoarene 4j (157 mg, 0.66 mmol), PC1 (6 $\mathrm{mg}$, 6E-3 mmol), benzoic acid (37 mg, $0.3 \mathrm{mmol})$, DIPA $(153 \mu \mathrm{L}, 1.1 \mathrm{mmol})$ and $O$-benzoyl oxime (3I) $(84 \mathrm{mg}, 0.3$ $\mathrm{mmol})$ in DMSO $(3 \mathrm{~mL})$. MS (ESI) $\mathrm{m} / z$ calcd. for $\mathrm{C}_{24} \mathrm{H}_{27} \mathrm{~N}_{2} \mathrm{O}_{2}\left([\mathrm{M}+\mathrm{H}]^{+}\right) 375.2$, found 375.3. The product 5aa was synthesized in $25 \%$ yield (retention time $\mathrm{t}=2.905, \mathrm{~m} / \mathrm{z}=375.3(\mathrm{M}+\mathrm{H}]^{+}$) as determined by a calibrated UPLC generated from an authentic sample using the UPLC-MS Method for Yield Determination.

Compound 5aa was also synthesized from the corresponding iminium $\mathrm{HCl}$ salt $\mathbf{6} \mathbf{l}$ and cyanoarene $\mathbf{4 j}$. The reaction was conducted by following the General Iminium Salt Reaction Procedure using iminium HCl salt 61 (130 mg, 0.66 mmol, 2.2 equiv.); photocatalyst $\operatorname{Ir}[\mathrm{dF}(\mathrm{Me}) \text { ppy }]_{2} \mathrm{dtbbpyPF}_{6}(6 \mathrm{mg}, 2 \mathrm{E}-3 \mathrm{mmol}, 2 \mathrm{~mol} \%)$; DIPA (153 $\mu \mathrm{L}, 1.1 \mathrm{mmol}$, 3.6 equiv.); and cyanoarene $4 \mathbf{j}$ ( $52 \mathrm{mg}, 0.3 \mathrm{mmol}, 1.0$ equiv.). The product $5 \mathbf{a a}$ was synthesized in $46 \%$ yield (retention time $\left.\mathrm{t}=2.905, \mathrm{~m} / \mathrm{z}=375.3(\mathrm{M}+\mathrm{H}]^{+}\right)$as determined by a calibrated UPLC generated from an authentic sample using the UPLC-MS Method for Yield Determination.

${ }^{1} \mathrm{H}$ NMR (500 MHz, $\left.\mathrm{CD}_{3} \mathrm{CN}\right) \delta 8.54(\mathrm{~d}, J=5.1 \mathrm{~Hz}, 1 \mathrm{H}), 8.11(\mathrm{~d}, J=8.3 \mathrm{~Hz}, 2 \mathrm{H}), 8.06(\mathrm{~d}, J=8.4 \mathrm{~Hz}, 2 \mathrm{H}), 7.96(\mathrm{~s}$, $1 \mathrm{H}), 7.45(\mathrm{~d}, J=7.8 \mathrm{~Hz}, 2 \mathrm{H}), 7.37-7.27(\mathrm{~m}, 3 \mathrm{H}), 7.20(\mathrm{t}, J=7.3 \mathrm{~Hz}, 1 \mathrm{H}), 3.89(\mathrm{~s}, 3 \mathrm{H}), 2.31-2.21(\mathrm{~m}, 2 \mathrm{H}), 1.99$ (br $\mathrm{s}, 2 \mathrm{H}), 1.39-1.29(\mathrm{~m}, 2 \mathrm{H}), 1.26-1.10(\mathrm{~m}, 2 \mathrm{H}), 0.86(\mathrm{t}, J=7.3 \mathrm{~Hz}, 4 \mathrm{H}) .{ }^{13} \mathrm{C}$ NMR $\left(126 \mathrm{MHz}, \mathrm{CD}_{3} \mathrm{CN}\right) \delta 13 \mathrm{C} \mathrm{NMR}$ $(126 \mathrm{MHz}, \mathrm{CD} 3 \mathrm{CN}) \delta 167.49,160.68,156.46,150.57,149.00,144.76,131.34,130.65,129.17,127.85,127.46$, $127.44,122.34,119.81,61.67,52.77,42.20,26.98,23.82,14.36$.

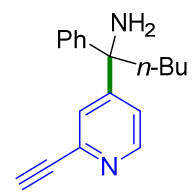

Compound 5ab was synthesized following General Procedure A using cyanoarene 4k ( $85 \mathrm{mg}, 0.66 \mathrm{mmol})$, PC1 (6 $\mathrm{mg}, 6 \mathrm{E}-3 \mathrm{mmol})$, benzoic acid (37 mg, $0.3 \mathrm{mmol})$, DIPA $(153 \mu \mathrm{L}, 1.1 \mathrm{mmol})$ and $O$-benzoyl oxime (3I) $(84 \mathrm{mg}, 0.3$ mmol) in DMSO $(3 \mathrm{~mL})$. MS (ESI) $\mathrm{m} / z$ calcd. for $\mathrm{C}_{18} \mathrm{H}_{22} \mathrm{~N}_{2}\left([\mathrm{M}+\mathrm{H}]^{+}\right) 265.2$, found 265.2. The product 5ab was synthesized in $9 \%$ yield (retention time $\mathrm{t}=2.362, \mathrm{~m} / \mathrm{z}=265.2(\mathrm{M}+\mathrm{H}]^{+}$) as determined by a calibrated UPLC generated from an authentic sample using the UPLC-MS Method for Yield Determination.

Compound 5ab was also synthesized from the corresponding iminium $\mathrm{HCl}$ salt $\mathbf{6} \mathbf{l}$ and cyanoarene $\mathbf{4 k}$. The reaction was conducted by following the General Iminium Salt Reaction Procedure using iminium $\mathrm{HCl}$ salt $6 \mathbf{1}$ (130 mg, 0.66 mmol, 2.2 equiv.); photocatalyst $\operatorname{Ir}[\mathrm{dF}(\mathrm{Me}) \text { ppy }]_{2} \mathrm{dtbbpyPF}_{6}(6 \mathrm{mg}, 2 \mathrm{E}-3 \mathrm{mmol}, 2 \mathrm{~mol} \%)$; DIPA (153 $\mu \mathrm{L}, 1.1 \mathrm{mmol}$, 3.6 equiv.); and cyanoarene $4 \mathbf{k}$ ( $28 \mathrm{mg}, 0.3 \mathrm{mmol}, 1.0$ equiv.). The product $\mathbf{5 a b}$ was synthesized in $45 \%$ yield (retention time $\mathrm{t}=2.362, \mathrm{~m} / \mathrm{z}=265.2(\mathrm{M}+\mathrm{H}]^{+}$) as determined by a calibrated UPLC generated from an authentic sample using the UPLC-MS Method for Yield Determination.

${ }^{1} \mathrm{H}$ NMR $\left(500 \mathrm{MHz}, \mathrm{CD}_{3} \mathrm{CN}\right) \delta 8.44(\mathrm{~d}, J=5.2 \mathrm{~Hz}, 1 \mathrm{H}), 7.58(\mathrm{~s}, 1 \mathrm{H}), 7.43(\mathrm{~d}, J=8.0 \mathrm{~Hz}, 2 \mathrm{H}), 7.40-7.36(\mathrm{~m}, 1 \mathrm{H})$, $7.33(\mathrm{t}, J=7.6 \mathrm{~Hz}, 2 \mathrm{H}), 7.23(\mathrm{t}, J=7.3 \mathrm{~Hz}, 1 \mathrm{H}), 3.42(\mathrm{~s}, 3 \mathrm{H}), 2.27-2.15(\mathrm{~m}, 2 \mathrm{H}), 1.95(\mathrm{br} \mathrm{s}, 2 \mathrm{H}), 1.39-1.30(\mathrm{~m}, 2 \mathrm{H})$, $1.25-1.08(\mathrm{~m}, 2 \mathrm{H}), 0.88(\mathrm{t}, J=7.3 \mathrm{~Hz}, 3 \mathrm{H})(\mathrm{s}, 6 \mathrm{H}) .{ }^{13} \mathrm{C} \mathrm{NMR}\left(126 \mathrm{MHz}, \mathrm{CD}_{3} \mathrm{CN}\right) \delta 160.08,150.80,148.58,142.82$, $129.19,127.53,127.39,126.54,122.78,84.09,77.90,61.33,42.01,26.84,23.74,14.32$. 
<smiles>CCCCC(N)(c1ccccc1)c1ccnc(CO)c1</smiles>

Compound 5ac was synthesized following General Procedure A using cyanoarene 41 (89 mg, 0.66 mmol), PC1 (6 mg, $6 \mathrm{E}-3 \mathrm{mmol})$, benzoic acid $(37 \mathrm{mg}, 0.3 \mathrm{mmol})$, DIPA $(153 \mu \mathrm{L}, 1.1 \mathrm{mmol})$ and $O$-benzoyl oxime $(3 \mathrm{l})(84 \mathrm{mg}, 0.3 \mathrm{mmol})$ in DMSO (3 mL). MS (ESI) $\mathrm{m} / z$ calcd. for $\mathrm{C}_{17} \mathrm{H}_{23} \mathrm{~N}_{2} \mathrm{O}\left([\mathrm{M}+\mathrm{H}]^{+}\right)$271.2, found 271.3. The product 5ac was synthesized in $89 \%$ yield (retention time $\mathrm{t}=1.968, \mathrm{~m} / \mathrm{z}=265.2(\mathrm{M}+\mathrm{H}]^{+}$) as determined by a calibrated UPLC generated from an authentic sample using the UPLC-MS Method for Yield Determination.

Compound 5ac was also synthesized from the corresponding iminium $\mathrm{HCl}$ salt $\mathbf{6} \mathbf{1}$ and cyanoarene $\mathbf{4 l}$. The reaction was conducted by following the General Iminium Salt Reaction Procedure using iminium $\mathrm{HCl}$ salt 61 (130 mg, 0.66 mmol, 2.2 equiv.); photocatalyst $\operatorname{Ir}[\mathrm{dF}(\mathrm{Me}) \text { ppy }]_{2} \mathrm{dtbbpyPF}_{6}(6 \mathrm{mg}, 2 \mathrm{E}-3 \mathrm{mmol}, 2 \mathrm{~mol} \%)$; DIPA (153 $\mu \mathrm{L}, 1.1 \mathrm{mmol}$, 3.6 equiv.); and cyanoarene $\mathbf{4 l}$ (30 mg, $0.3 \mathrm{mmol}, 1.0$ equiv.). The product 5ac was synthesized in $90 \%$ yield (retention time $\left.\mathrm{t}=1.968, \mathrm{~m} / \mathrm{z}=265.2(\mathrm{M}+\mathrm{H}]^{+}\right)$as determined by a calibrated UPLC generated from an authentic sample using the UPLC-MS Method for Yield Determination

${ }^{1} \mathrm{H}$ NMR $\left(500 \mathrm{MHz}, \mathrm{CD}_{3} \mathrm{CN}\right) \delta 8.36(\mathrm{~d}, J=5.2 \mathrm{~Hz}, 1 \mathrm{H}), 7.44(\mathrm{~s}, 1 \mathrm{H}), 7.41(\mathrm{~d}, J=7.6 \mathrm{~Hz}, 2 \mathrm{H}), 7.29(\mathrm{t}, J=7.9 \mathrm{~Hz}$, $2 \mathrm{H}), 7.23(\mathrm{~d}, J=5.1,1 \mathrm{H}), 7.20(\mathrm{t}, J=7.3 \mathrm{~Hz}, 1 \mathrm{H}), 4.59(\mathrm{~s}, 2 \mathrm{H}), 2.30-2.05(\mathrm{~m}, 3 \mathrm{H}), 1.37-1.27(\mathrm{~m}, 2 \mathrm{H}), 1.22-1.07(\mathrm{~m}$, $2 \mathrm{H}), 0.86(\mathrm{t}, J=7.4 \mathrm{~Hz}, 3 \mathrm{H}) .{ }^{13} \mathrm{C} \mathrm{NMR}\left(126 \mathrm{MHz}, \mathrm{CD}_{3} \mathrm{CN}\right) \delta 161.63,160.09,149.37,149.08,129.12,127.40,121.16$, $119.10,65.41,61.52,42.28,26.93,23.81,14.35$.<smiles>CCCC(N)(c1ccccc1)c1ccnc(Cl)c1</smiles>

Compound 5ai was synthesized following General Procedure A using cyanoarene 4m (91 mg, 0.66 mmol), PC1 (6 $\mathrm{mg}, 6 \mathrm{E}-3 \mathrm{mmol})$, benzoic acid $(37 \mathrm{mg}, 0.3 \mathrm{mmol})$, DIPA $(153 \mu \mathrm{L}, 1.1 \mathrm{mmol})$ and $O$-benzoyl oxime (31) $(84 \mathrm{mg}, 0.3$

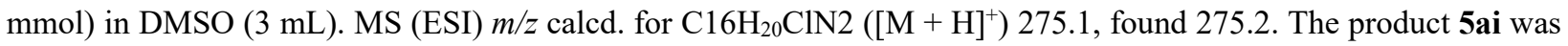
synthesized in $0 \%$ yield (retention time $\mathrm{t}=2.568, \mathrm{~m} / \mathrm{z}=275.2(\mathrm{M}+\mathrm{H}]^{+}$) as determined by a calibrated UPLC generated from an authentic sample using the UPLC-MS Method for Yield Determination.

Compound 5ai was also synthesized from the corresponding iminium $\mathrm{HCl}$ salt $\mathbf{6 l}$ and cyanoarene $\mathbf{4 m}$. The reaction was conducted by following the General Iminium Salt Reaction Procedure using iminium $\mathrm{HCl} \mathrm{salt} 61$ (130 mg, 0.66 mmol, 2.2 equiv.); photocatalyst $\operatorname{Ir}[\mathrm{dF}(\mathrm{Me}) \text { ppy }]_{2} \mathrm{dtbbpyPF}_{6}(6 \mathrm{mg}, 2 \mathrm{E}-3 \mathrm{mmol}, 2 \mathrm{~mol} \%)$; DIPA (153 $\mu \mathrm{L}, 1.1 \mathrm{mmol}$, 3.6 equiv.); and cyanoarene $\mathbf{4 m}$ (30 mg, $0.3 \mathrm{mmol}, 1.0$ equiv.). The product 5 ai was synthesized in $39 \%$ yield (retention time $\mathrm{t}=2.568, \mathrm{~m} / \mathrm{z}=275.2(\mathrm{M}+\mathrm{H}]^{+}$) as determined by a calibrated UPLC generated from an authentic sample using the UPLC-MS Method for Yield Determination.

${ }^{1} \mathrm{H}$ NMR $\left(500 \mathrm{MHz}, \mathrm{CD}_{3} \mathrm{CN}\right) \delta 8.22(\mathrm{~d}, J=5.3 \mathrm{~Hz}, 1 \mathrm{H}), 7.46(\mathrm{~d}, J=1.0 \mathrm{~Hz}, 1 \mathrm{H}), 7.40(\mathrm{~d}, J=7.4 \mathrm{~Hz}, 2 \mathrm{H}) .7 .31(\mathrm{t}, J$ $=7.4 \mathrm{~Hz}, 2 \mathrm{H}), 7.28(\mathrm{dd}, J=1.5,5.3 \mathrm{~Hz}, 1 \mathrm{H}), 7.22(\mathrm{t}, J=7.3 \mathrm{~Hz}, 1 \mathrm{H}) .{ }^{13} \mathrm{C} \mathrm{NMR}\left(126 \mathrm{MHz}, \mathrm{CD}_{3} \mathrm{CN}\right) \delta 163.47,152.16$, $150.36,148.37,129.25,127.65,127.37,123.01,122.12,61.60,42.03,26.82,23.73,14.30$. 
<smiles>CC(N)(c1ccccc1)c1ccnc(C(F)(F)F)c1</smiles>

Compound 5aj was synthesized following General Procedure A using cyanoarene 4n (114 mg, 0.66 mmol), PC1 (6 $\mathrm{mg}, 6 \mathrm{E}-3 \mathrm{mmol})$, benzoic acid (37 mg, $0.3 \mathrm{mmol})$, DIPA $(153 \mu \mathrm{L}, 1.1 \mathrm{mmol})$ and $O$-benzoyl oxime (3I) $(84 \mathrm{mg}, 0.3$ mmol) in DMSO (3 mL). MS (ESI) $\mathrm{m} / z$ calcd. for $\mathrm{C}_{17} \mathrm{H}_{19} \mathrm{~F}_{3} \mathrm{~N}_{2}\left([\mathrm{M}+\mathrm{H}]^{+}\right)$309.2, found 309.3. The product 5aj was synthesized in $0 \%$ yield (retention time $\mathrm{t}=2.91, \mathrm{~m} / \mathrm{z}=309.3(\mathrm{M}+\mathrm{H}]^{+}$) as determined by a calibrated UPLC generated from an authentic sample using the UPLC-MS Method for Yield Determination.

Compound 5aj was also synthesized from the corresponding iminium $\mathrm{HCl}$ salt $\mathbf{3 1}$ and cyanoarene $\mathbf{4 n}$. The reaction was conducted by following the General Iminium Salt Reaction Procedure using iminium HCl salt 61 (130 mg, 0.66 mmol, 2.2 equiv.); photocatalyst $\operatorname{Ir}[\mathrm{dF}(\mathrm{Me}) \text { ppy }]_{2} \mathrm{dtbbpyPF}_{6}$ (6 mg, $\left.2 \mathrm{E}-3 \mathrm{mmol}, 2 \mathrm{~mol} \%\right)$; DIPA (153 $\mu \mathrm{L}, 1.1 \mathrm{mmol}$, 3.6 equiv.); and cyanoarene $4 \mathbf{n}(38 \mathrm{mg}, 0.3 \mathrm{mmol}, 1.0$ equiv.). The product 5 aj was synthesized in $65 \%$ yield (retention time $\left.\mathrm{t}=2.91, \mathrm{~m} / \mathrm{z}=309.3(\mathrm{M}+\mathrm{H}]^{+}\right)$as determined by a calibrated UPLC generated from an authentic sample using the UPLC-MS Method for Yield Determination.

${ }^{1} \mathrm{H}$ NMR $\left(500 \mathrm{MHz}, \mathrm{CD}_{3} \mathrm{CN}\right) \delta 8.59(\mathrm{~d}, J=5.2 \mathrm{~Hz}, 1 \mathrm{H}), 7.84(\mathrm{~d}, J=1.3 \mathrm{~Hz}, 1 \mathrm{H}), 7.60(\mathrm{dd}, J=1.5,5.2 \mathrm{~Hz}, 1 \mathrm{H}), 7.44-$ 7.39 (m, 2H), 7.34-7.30 (m, 2H), 7.24-7.20 (m, 1H), 2.31-2.16 (m, 2H), 2.00 (br s, 1H), 1.38-1.29 (m, 2H), 1.25$1.06(\mathrm{~m}, 2 \mathrm{H}), 0.86(\mathrm{t}, J=7.4 \mathrm{~Hz}, 3 \mathrm{H}) .{ }^{13} \mathrm{C}$ NMR $\left(126 \mathrm{MHz}, \mathrm{CD}_{3} \mathrm{CN}\right) \delta 161.97,150.90,148.32(\mathrm{q}, J=33.6 \mathrm{~Hz})$, 148.28, 129.32, 127.73, 127.42, 125.89, 123.07 (q, $J=273.4 \mathrm{~Hz}), 119.55$ (q, $J=2.9 \mathrm{~Hz}), 61.71,42.08,26.83,23.73$, 14.30. ${ }^{19} \mathrm{~F}\left\{{ }^{1} \mathrm{H}\right\}$ NMR $\left(471 \mathrm{MHz}, \mathrm{CD}_{3} \mathrm{CN}\right) \delta-68.29(\mathrm{~s}, 3 \mathrm{~F}) .{ }^{19} \mathrm{~F}$ NMR (471 MHz, $\left.\mathrm{CD}_{3} \mathrm{CN}\right) \delta-68.29(\mathrm{~s}, 3 \mathrm{~F})$.

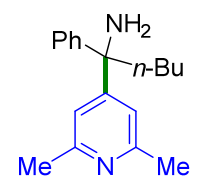

Compound 5ad was synthesized following General Procedure A using cyanoarene 40 ( $87 \mathrm{mg}, 0.66 \mathrm{mmol})$, PC1 (6 $\mathrm{mg}, 6 \mathrm{E}-3 \mathrm{mmol})$, benzoic acid (37 mg, $0.3 \mathrm{mmol})$, DIPA (153 $\mu \mathrm{L}, 1.1 \mathrm{mmol})$ and $O$-benzoyl oxime (3I) $(84 \mathrm{mg}, 0.3$ mmol) in DMSO (3 mL). MS (ESI) $\mathrm{m} / z$ calcd. for $\mathrm{C}_{18} \mathrm{H}_{2} \mathrm{~N}_{2}\left([\mathrm{M}+\mathrm{H}]^{+}\right) 269.2$, found 269.0. The product 5ad was synthesized in $94 \%$ yield (retention time $\mathrm{t}=2.922, \mathrm{~m} / \mathrm{z}=269.0(\mathrm{M}+\mathrm{H}]^{+}$) as determined by a calibrated UPLC generated from an authentic sample using the UPLC-MS Method for Yield Determination.

Compound $\mathbf{5 a d}$ was also synthesized from the corresponding iminium $\mathrm{HCl}$ salt $\mathbf{6} \mathbf{l}$ and cyanoarene 40. The reaction was conducted by following the General Iminium Salt Reaction Procedure using iminium $\mathrm{HCl}$ salt $6 \mathbf{1}$ (130 mg, 0.66 mmol, 2.2 equiv.); photocatalyst $\operatorname{Ir}[\mathrm{dF}(\mathrm{Me}) \text { ppy }]_{2} \mathrm{dtbbpyPF}_{6}(6 \mathrm{mg}, 2 \mathrm{E}-3 \mathrm{mmol}, 2 \mathrm{~mol} \%)$; DIPA (153 $\mu \mathrm{L}, 1.1 \mathrm{mmol}$, 3.6 equiv.); and cyanoarene 40 ( $29 \mathrm{mg}, 0.3 \mathrm{mmol}, 1.0$ equiv.). The product $5 \mathrm{ad}$ was synthesized in $56 \%$ yield (retention time $\left.\mathrm{t}=2.922, \mathrm{~m} / \mathrm{z}=269.0(\mathrm{M}+\mathrm{H}]^{+}\right)$as determined by a calibrated UPLC generated from an authentic sample using the UPLC-MS Method for Yield Determination.

${ }^{1} \mathrm{H}$ NMR $\left(500 \mathrm{MHz}, \mathrm{CD}_{3} \mathrm{CN}\right) \delta 7.40(\mathrm{~d}, J=7.5 \mathrm{~Hz}, 2 \mathrm{H}), 7.28(\mathrm{t}, J=7.5 \mathrm{~Hz}, 2 \mathrm{H}), 7.18(\mathrm{t}, J=7.2 \mathrm{~Hz}, 1 \mathrm{H}), 7.04(\mathrm{~s}$, 2H), 2.38 (s, 6H), 2.22-2.10 (m, 2H), 1.95 (br s, 2H), 1.32 (sextet, $7.4 \mathrm{~Hz}, 2 \mathrm{H}), 1.20-1.08(\mathrm{~m}, 2 \mathrm{H}), 0.86(\mathrm{~s}, 3 \mathrm{H}) .{ }^{13} \mathrm{C}$ NMR $\left(126 \mathrm{MHz}, \mathrm{CD}_{3} \mathrm{CN}\right) \delta 159.87,158.25,149.33,129.02,127.34,127.24,118.92,61.25,42.28,26.93,24.62$, $23.84,14.37$. 


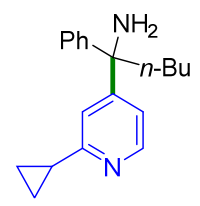

Compound 5ae was synthesized following General Procedure A using cyanoarene 4p (95 mg, 0.66 mmol), PC1 (6 $\mathrm{mg}, 6 \mathrm{E}-3 \mathrm{mmol})$, benzoic acid $(37 \mathrm{mg}, 0.3 \mathrm{mmol})$, DIPA $(153 \mu \mathrm{L}, 1.1 \mathrm{mmol})$ and $O$-benzoyl oxime $(3 \mathrm{l})(84 \mathrm{mg}, 0.3$ $\mathrm{mmol})$ in DMSO $(3 \mathrm{~mL}) .{ }^{1} \mathrm{H}$ NMR $\left(500 \mathrm{MHz}, \mathrm{CD}_{3} \mathrm{CN}\right) \delta 8.27(\mathrm{~d}, J=5.3 \mathrm{~Hz}, 1 \mathrm{H}), 7.44(\mathrm{~d}, J=7.3 \mathrm{~Hz}, 2 \mathrm{H}), 7.34-$ $7.29(\mathrm{~m}, 3 \mathrm{H}), 7.22(\mathrm{t}, J=7.3 \mathrm{~Hz}, 1 \mathrm{H}), 7.09(\mathrm{dd}, J=5.3,1.8 \mathrm{~Hz}, 1 \mathrm{H}), 2.25-2.18(\mathrm{~m}, 2 \mathrm{H}), 2.04-1.93(\mathrm{~m}, 4 \mathrm{H}), 1.36$ $(\mathrm{h}, J=7.3 \mathrm{~Hz}, 2 \mathrm{H}), 1.18$ (dd, $J=16.9,11.6 \mathrm{~Hz}, 2 \mathrm{H}), 0.98-0.91(\mathrm{~m}, 4 \mathrm{H}), 0.89$ (t, $J=7.4 \mathrm{~Hz}, 3 \mathrm{H}) .{ }^{13} \mathrm{C}$ NMR $(126$ $\left.\mathrm{MHz}, \mathrm{CD}_{3} \mathrm{CN}\right) \delta 163.28,159.20,149.89,149.25,129.06,127.36,127.30,120.30,119.60,118.27,61.31,42.20,26.96$, 23.83, 17.68, 14.37, 10.11, 10.09. MS (ESI) $\mathrm{m} / \mathrm{z}$ calcd. for $\mathrm{C}_{19} \mathrm{H}_{25} \mathrm{~N}_{2}\left([\mathrm{M}+\mathrm{H}]^{+}\right)$281.2, found 280.9. The product 5ae was synthesized in $89 \%$ yield (retention time $\mathrm{t}=2.478, \mathrm{~m} / \mathrm{z}=280.9(\mathrm{M}+\mathrm{H}]^{+}$) as determined by a calibrated UPLC generated from an authentic sample using the UPLC-MS Method for Yield Determination. 


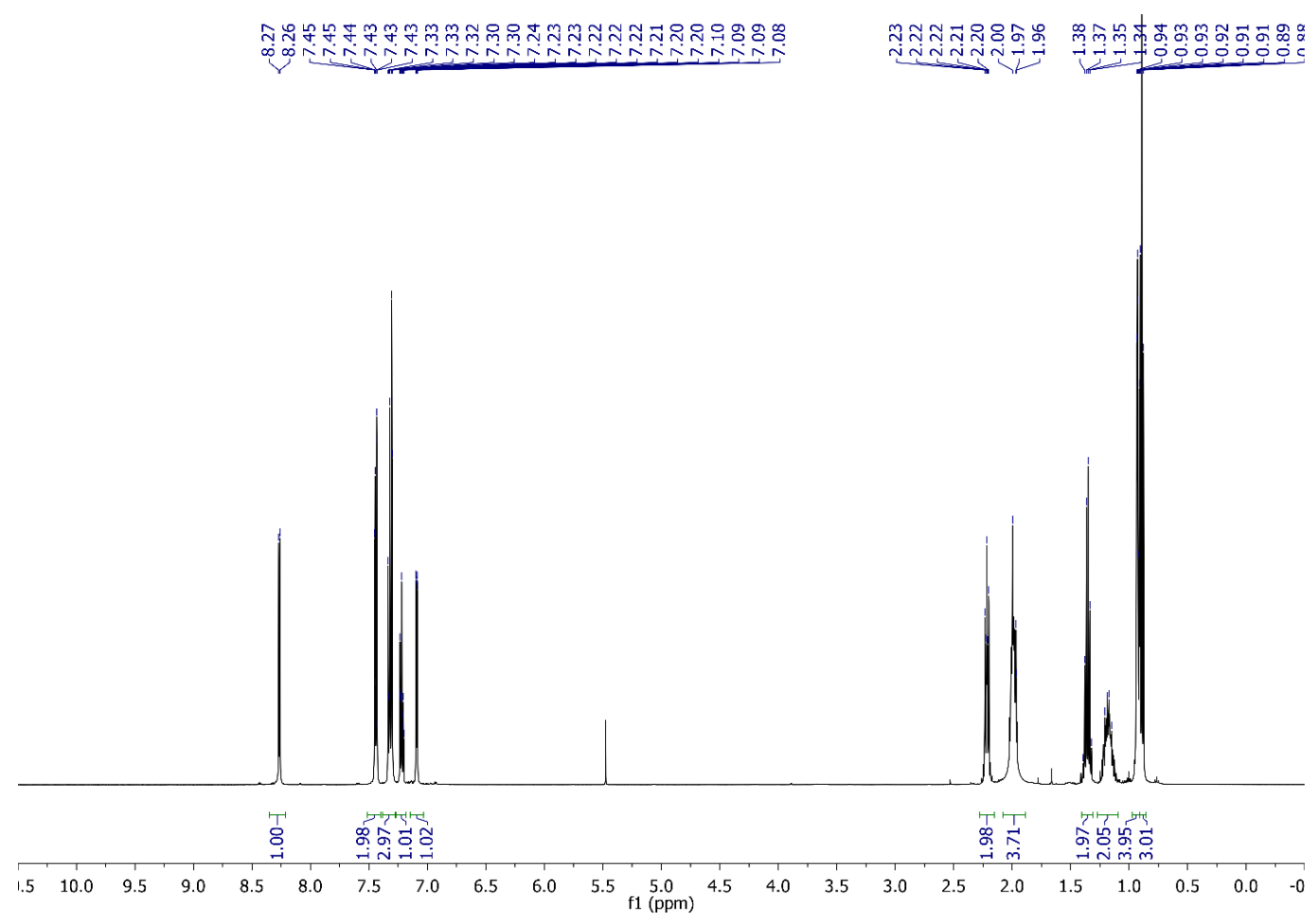

Figure S50. ${ }^{1} \mathrm{H}$ qNMR $(500 \mathrm{MHz})$ spectrum of $5 \mathbf{a e}$ in $\mathrm{CD}_{3} \mathrm{CN}$.

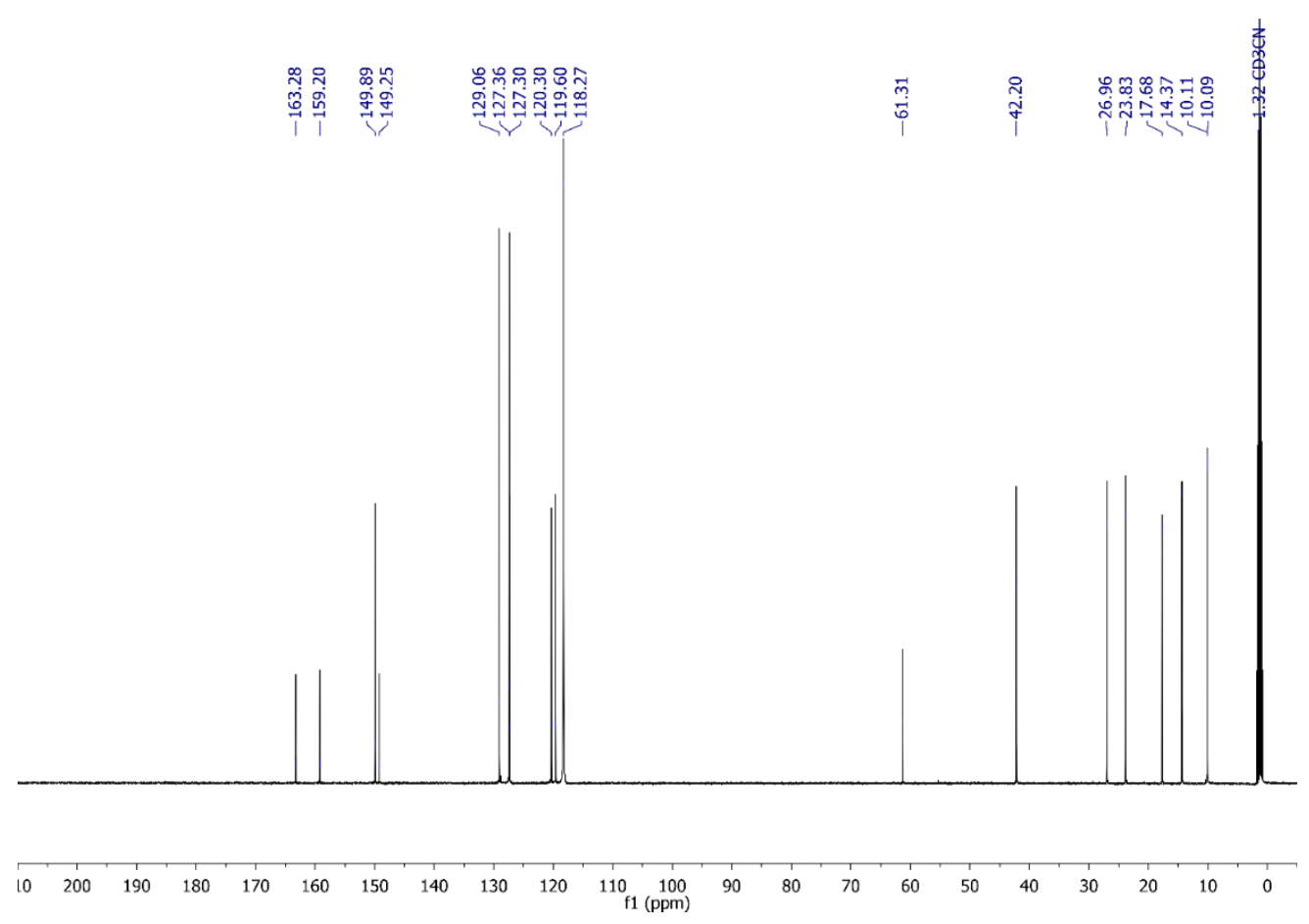

Figure S51. ${ }^{13} \mathrm{C}$ NMR $(126 \mathrm{MHz})$ spectrum of $\mathbf{5 a e}$ in $\mathrm{CD}_{3} \mathrm{CN}$. 


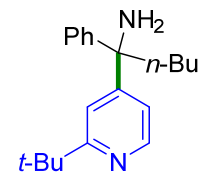

Compound 5af was synthesized following General Procedure A using cyanoarene 4q (106 mg, $0.66 \mathrm{mmol}$ ), PC1 (6 $\mathrm{mg}, 6 \mathrm{E}-3 \mathrm{mmol})$, benzoic acid (37 mg, $0.3 \mathrm{mmol})$, DIPA (153 $\mu \mathrm{L}, 1.1 \mathrm{mmol})$ and $O$-benzoyl oxime (3I) $(84 \mathrm{mg}, 0.3$ mmol) in DMSO (3 mL). ${ }^{1} \mathrm{H}$ NMR $\left(500 \mathrm{MHz}, \mathrm{CD}_{3} \mathrm{CN}\right) \delta 8.35(\mathrm{~d}, J=5.2 \mathrm{~Hz}, 1 \mathrm{H}), 7.48(\mathrm{~s}, 1 \mathrm{H}), 7.42(\mathrm{~d}, J=7.9 \mathrm{~Hz}$, 2H), $7.29(\mathrm{t}, J=7.6 \mathrm{~Hz}, 2 \mathrm{H}), 7.19(\mathrm{t}, J=7.2 \mathrm{~Hz}, 1 \mathrm{H}), 7.11(\mathrm{~d}, J=5.1 \mathrm{~Hz}, 1 \mathrm{H}), 2.20(\mathrm{t}, J=8.0 \mathrm{~Hz}, 2 \mathrm{H}), 1.98(\mathrm{~s}, 2 \mathrm{H})$, $1.30(\mathrm{~s}, 12 \mathrm{H}), 1.16$ (ddq, $J=21.2,14.1,7.5 \mathrm{~Hz}, 2 \mathrm{H}), 0.86(\mathrm{t}, J=7.3 \mathrm{~Hz}, 3 \mathrm{H}) .{ }^{13} \mathrm{C}$ NMR $\left(126 \mathrm{MHz}, \mathrm{CD}_{3} \mathrm{CN}\right) \delta 169.83$, 159.34, 149.26, 149.04, 129.04, 127.36, 127.28, 119.94, 117.71, 61.62, 42.37, 38.19, 30.54, 27.00, 23.80, 14.38. MS (ESI) $\mathrm{m} / \mathrm{z}$ calcd. for $\mathrm{C}_{20} \mathrm{H}_{29} \mathrm{~N}_{2}\left([\mathrm{M}+\mathrm{H}]^{+}\right)$297.2, found 296.9. The product 5af was synthesized in $97 \%$ yield (retention time $\left.\mathrm{t}=2.718, \mathrm{~m} / \mathrm{z}=296.9(\mathrm{M}+\mathrm{H}]^{+}\right)$as determined by a calibrated UPLC generated from an authentic sample using the UPLC-MS Method for Yield Determination. 


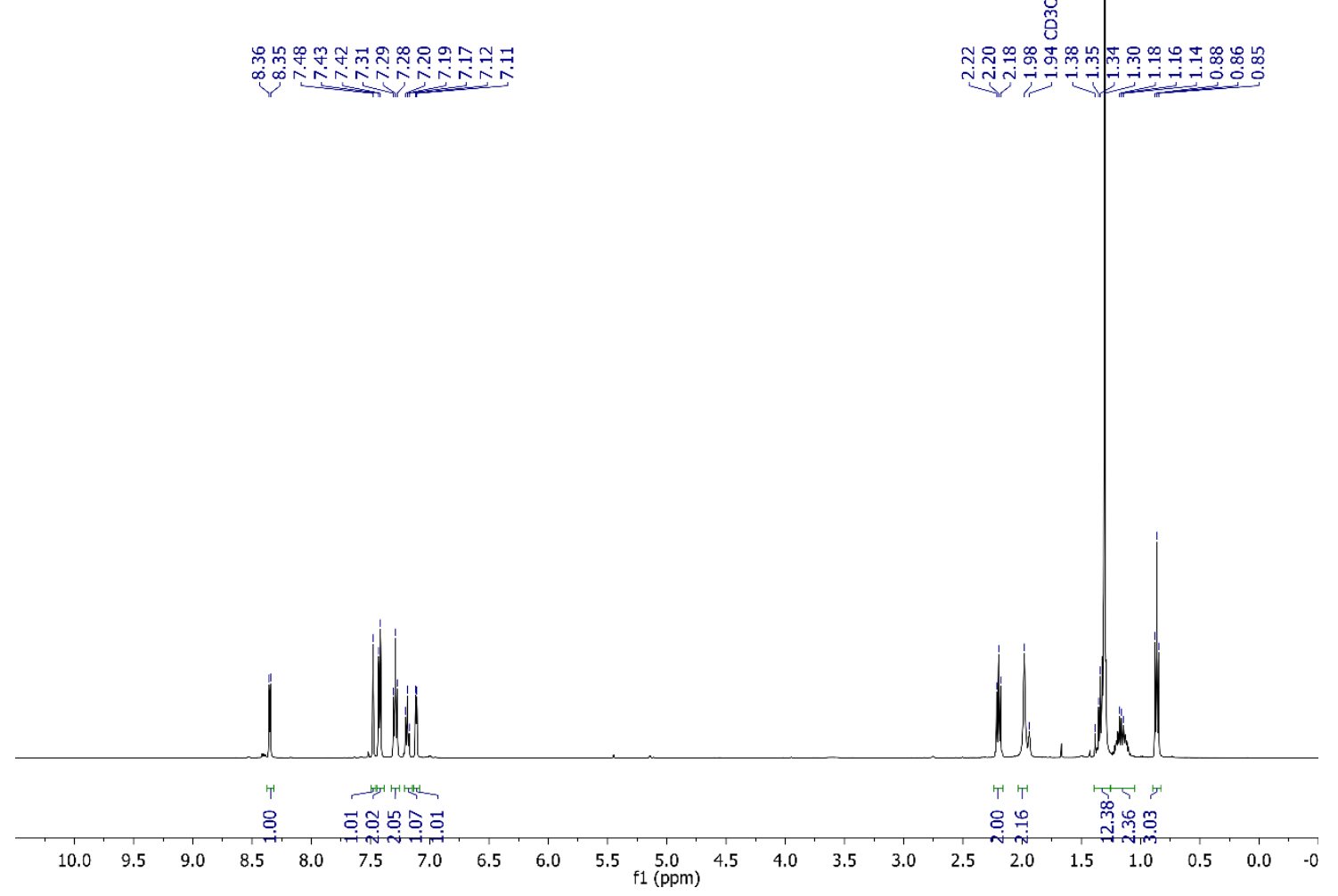

Figure S52. ${ }^{1} \mathrm{H}$ qNMR $(500 \mathrm{MHz})$ spectrum of $5 \mathbf{a f}$ in $\mathrm{CD}_{3} \mathrm{CN}$.

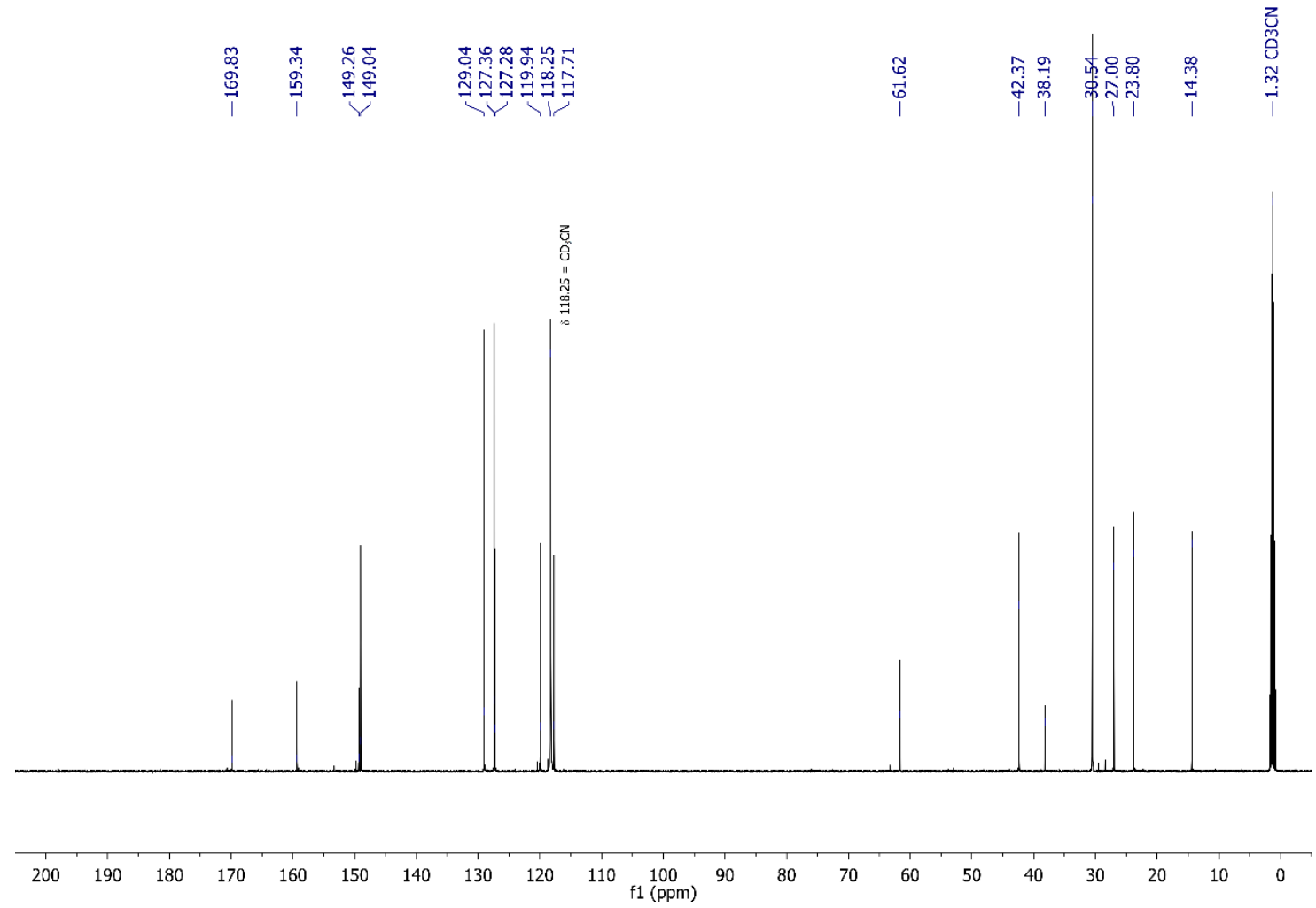

Figure S53. ${ }^{13} \mathrm{C}$ NMR $(126 \mathrm{MHz})$ spectrum of 5 af in $\mathrm{CD}_{3} \mathrm{CN}$. 
<smiles>CS(=O)(=O)c1cc(C(N)(c2ccccc2)c2ccccc2)ccn1</smiles>

Compound 5ag was synthesized following General Procedure A using cyanoarene 4q (99 mg, $0.66 \mathrm{mmol})$, PC1 (6 $\mathrm{mg}, 6 \mathrm{E}-3 \mathrm{mmol})$, benzoic acid (37 mg, $0.3 \mathrm{mmol})$, DIPA $(153 \mu \mathrm{L}, 1.1 \mathrm{mmol})$ and $O$-benzoyl oxime (3l) $(84 \mathrm{mg}, 0.3$ mmol) in DMSO $(3 \mathrm{~mL})$. MS (ESI) $\mathrm{m} / z$ calcd. for $\mathrm{C}_{17} \mathrm{H}_{22} \mathrm{~N}_{2} \mathrm{~S}\left([\mathrm{M}+\mathrm{H}]^{+}\right)$287.2, found 287.2. The product 40 was synthesized in $79 \%$ yield (retention time $\mathrm{t}=2.55, \mathrm{~m} / \mathrm{z}=287.0(\mathrm{M}+\mathrm{H}]^{+}$) as determined by a calibrated UPLC generated from an authentic sample using the UPLC-MS Method for Yield Determination.

Compound 5ag was also synthesized from the corresponding iminium $\mathrm{HCl}$ salt $\mathbf{6} \mathbf{l}$ and cyanoarene 4q. The reaction was conducted by following the General Iminium Salt Reaction Procedure using iminium HCl salt 61 (130 mg, 0.66 mmol, 2.2 equiv.); photocatalyst $\operatorname{Ir}[\mathrm{dF}(\mathrm{Me}) \text { ppy }]_{2} \mathrm{dtbbpyPF}_{6}$ (6 mg, 2E-3 mmol, $\left.2 \mathrm{~mol} \%\right)$; DIPA (153 $\mu \mathrm{L}, 1.1 \mathrm{mmol}$, 3.6 equiv.); and cyanoarene $\mathbf{4 q}(33 \mathrm{mg}, 0.3 \mathrm{mmol}, 1.0$ equiv.). The product $5 \mathrm{ag}$ was synthesized in $60 \%$ yield (retention time $\left.\mathrm{t}=2.55, \mathrm{~m} / \mathrm{z}=287.2(\mathrm{M}+\mathrm{H}]^{+}\right)$as determined by a calibrated UPLC generated from an authentic sample using the UPLC-MS Method for Yield Determination.

${ }^{1} \mathrm{H}$ NMR $\left(500 \mathrm{MHz}, \mathrm{CD}_{3} \mathrm{CN}\right) \delta 8.26(\mathrm{~d}, J=5.4 \mathrm{~Hz}, 1 \mathrm{H}), 7.42-7.38(\mathrm{~m}, 2 \mathrm{H}) 7.32-7.27(\mathrm{~m}, 3 \mathrm{H}), 7.20(\mathrm{t}, J=7.3 \mathrm{~Hz}$, $1 \mathrm{H}), 2.49$ (s, 3H), 2.22-2.12 (m, 2H), 2.07 (br s, 2H), 1.37-1.27 (m, 2H), 1.22-1.06 (m, 2H), $0.86(\mathrm{t}, J=7.4 \mathrm{~Hz}, 3 \mathrm{H})$. ${ }^{13} \mathrm{C}$ NMR $\left(126 \mathrm{MHz}, \mathrm{CD}_{3} \mathrm{CN}\right) \delta 160.61,159.66,149.96,148.84,129.14,127.46,127.37,119.70,118.92,61.45,49.90$, $42.02,26.92,23.79,14.34,13.43$.

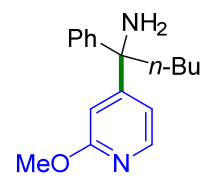

Compound 5ah was synthesized following General Procedure A using cyanoarene 4s (89 mg, $0.66 \mathrm{mmol})$, PC1 (6 $\mathrm{mg}, 6 \mathrm{E}-3 \mathrm{mmol})$, benzoic acid (37 mg, $0.3 \mathrm{mmol})$, DIPA (153 $\mu \mathrm{L}, 1.1 \mathrm{mmol})$ and $O$-benzoyl oxime (3I) $(84 \mathrm{mg}, 0.3$ $\mathrm{mmol})$ in DMSO $(3 \mathrm{~mL})$. The product was isolated as a film on a vial $(54 \mathrm{mg}, 67 \%)$. The product $5 \mathrm{ah}$ was synthesized in $81 \%$ yield (retention time $\mathrm{t}=2.420, \mathrm{~m} / \mathrm{z}=254.2(\mathrm{M}+\mathrm{H}]^{+}$) as determined by a calibrated UPLC generated from an authentic sample using the UPLC-MS Method for Yield Determination.

${ }^{1} \mathrm{H}$ NMR $\left(500 \mathrm{MHz}, \mathrm{CD}_{3} \mathrm{CN}\right) \delta 7.98(\mathrm{~d}, J=6.2 \mathrm{~Hz}, 1 \mathrm{H}), 7.40(\mathrm{~d}, J=7.2 \mathrm{~Hz}, 2 \mathrm{H}), 7.29(\mathrm{t}, J=7.7 \mathrm{~Hz}, 2 \mathrm{H}), 7.19(\mathrm{t}, J$ $=7.3 \mathrm{~Hz}, 1 \mathrm{H}), 6.87-6.83(\mathrm{~m}, 2 \mathrm{H}), 3.85(\mathrm{~s}, 3 \mathrm{H}), 2.20-2.13(\mathrm{~m}, 2 \mathrm{H}), 2.03(\mathrm{~s}, 2 \mathrm{H}), 1.37-1.25(\mathrm{~m}, 3 \mathrm{H}), 1.23-1.07$ $(\mathrm{m}, 2 \mathrm{H}), 0.86(\mathrm{t}, J=7.4 \mathrm{~Hz}, 3 \mathrm{H}) .{ }^{13} \mathrm{C} \mathrm{NMR}\left(126 \mathrm{MHz}, \mathrm{CD}_{3} \mathrm{CN}\right) \delta 165.46,162.57,149.02,147.35,129.07,127.37$, $127.34,118.28,116.63,108.93,61.39,53.81,42.06,26.93,23.81,14.36$. MS (ESI) $m / z$ calcd. for $\mathrm{C}_{17} \mathrm{H}_{23} \mathrm{~N}_{2} \mathrm{O}([\mathrm{M}+$ $\left.\mathrm{H}-\mathrm{NH}_{2}\right]^{+}$) 254.2, found 254.2. 


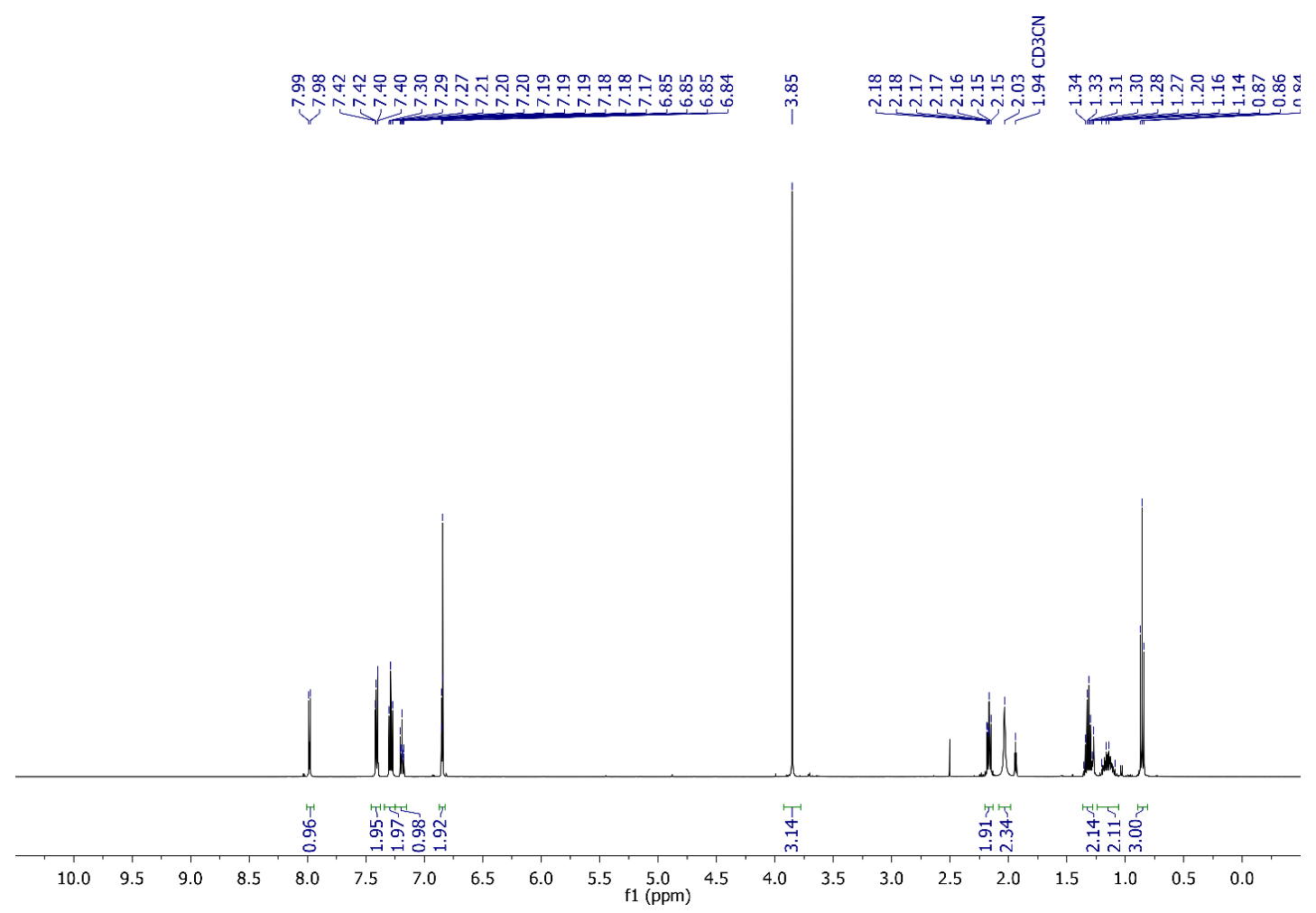

Figure S54. ${ }^{1} \mathrm{H}$ qNMR (500 MHz) spectrum of $\mathbf{5 a h}$ in $\mathrm{CD}_{3} \mathrm{CN}$.
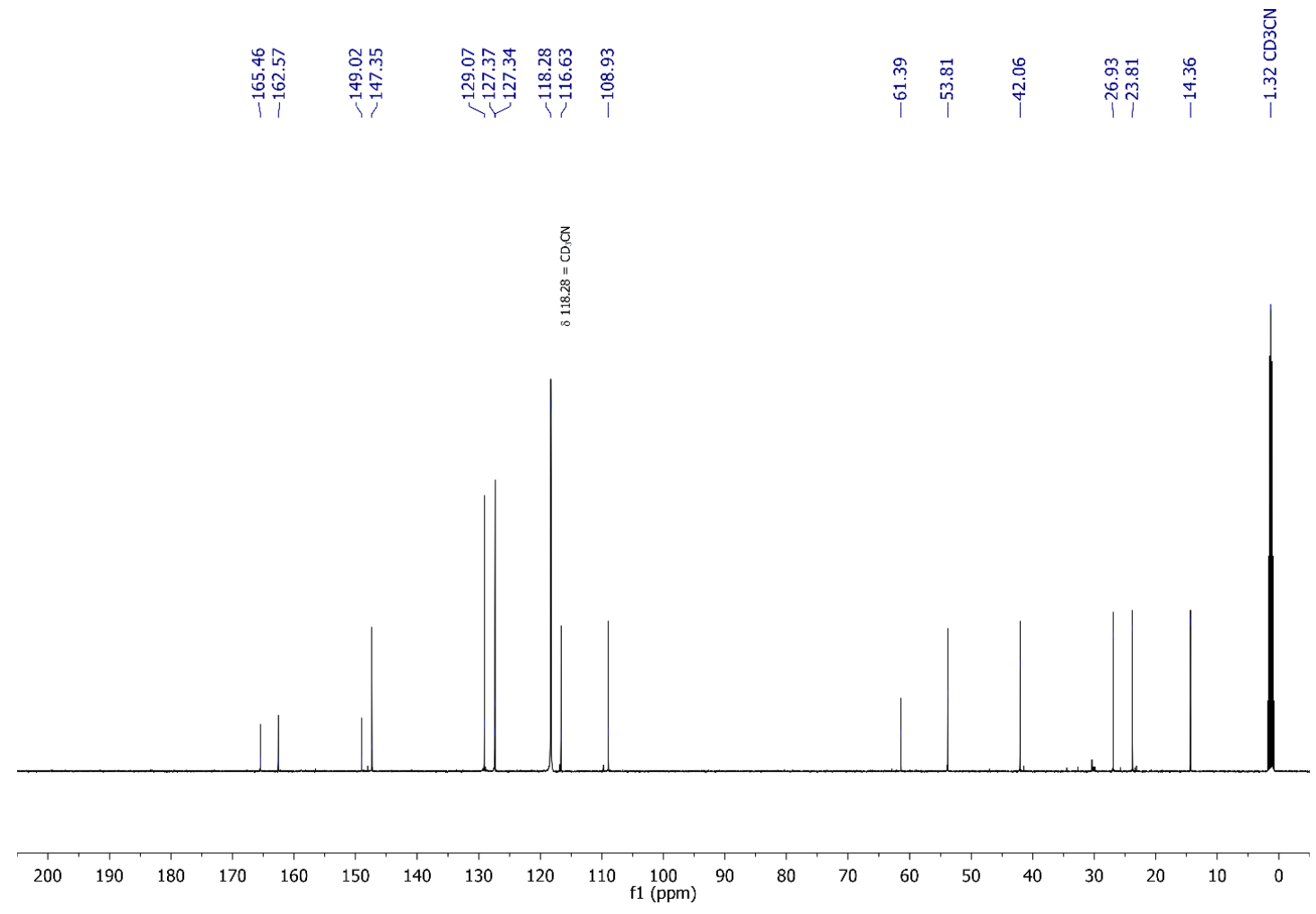

Figure S55. ${ }^{13} \mathrm{C}$ NMR $(126 \mathrm{MHz})$ spectrum of $\mathbf{5 a h}$ in $\mathrm{CD}_{3} \mathrm{CN}$. 
<smiles>CCCCC(N)(c1ccccc1)c1ccnc(C(N)=O)c1</smiles>

Compound 5al was synthesized following General Procedure A using cyanoarene 4u (97 mg, 0.66 mmol), PC1 (6 $\mathrm{mg}, 6 \mathrm{E}-3 \mathrm{mmol})$, benzoic acid $(37 \mathrm{mg}, 0.3 \mathrm{mmol})$, DIPA $(153 \mu \mathrm{L}, 1.1 \mathrm{mmol})$ and $O$-benzoyl oxime (3l) $(84 \mathrm{mg}, 0.3$ mmol) in DMSO $(3 \mathrm{~mL})$. MS (ESI) $\mathrm{m} / z$ calcd. for $\mathrm{C}_{17} \mathrm{H}_{22} \mathrm{~N}_{3} \mathrm{O}\left([\mathrm{M}+\mathrm{H}]^{+}\right)$284.2, found 284.3. The product 5al was synthesized in $22 \%$ yield (retention time $\mathrm{t}=2.110, \mathrm{~m} / \mathrm{z}=284.3(\mathrm{M}+\mathrm{H}]^{+}$) as determined by a calibrated UPLC generated from an authentic sample using the UPLC-MS Method for Yield Determination.

Compound 5al was also synthesized from the corresponding iminium $\mathrm{HCl}$ salt $\mathbf{6 l}$ and cyanoarene $\mathbf{4 u}$. The reaction was conducted by following the General Iminium Salt Reaction Procedure using iminium HCl salt 61 (130 mg, 0.66 mmol, 2.2 equiv.); photocatalyst $\operatorname{Ir}[\mathrm{dF}(\mathrm{Me}) \text { ppy }]_{2} \mathrm{dtbbpyPF}_{6}(6 \mathrm{mg}, 2 \mathrm{E}-3 \mathrm{mmol}, 2 \mathrm{~mol} \%)$; DIPA (153 $\mu \mathrm{L}, 1.1 \mathrm{mmol}$, 3.6 equiv.); and cyanoarene $4 \mathbf{u}$ (32 $\mathrm{mg}, 0.3 \mathrm{mmol}, 1.0$ equiv.). The product 5al was synthesized in $64 \%$ yield (retention time $\left.\mathrm{t}=2.110, \mathrm{~m} / \mathrm{z}=284.3(\mathrm{M}+\mathrm{H}]^{+}\right)$as determined by a calibrated UPLC generated from an authentic sample using the UPLC-MS Method for Yield Determination.

${ }^{1} \mathrm{H}$ NMR $\left(500 \mathrm{MHz}, \mathrm{CD}_{3} \mathrm{CN}\right) \delta 8.45(\mathrm{~d}, J=5.1 \mathrm{~Hz}, 1 \mathrm{H}), 8.12(\mathrm{~s}, 1 \mathrm{H}), 7.82($ br s, $1 \mathrm{H}), 7.55(\mathrm{~d}, J=5.0 \mathrm{~Hz}, 1 \mathrm{H}), 7.40$ $(\mathrm{d}, J=7.9 \mathrm{~Hz}, 2 \mathrm{H}), 7.29(\mathrm{t}, J=7.6 \mathrm{~Hz}, 2 \mathrm{H}), 7.20(\mathrm{t}, J=7.2 \mathrm{~Hz}, 1 \mathrm{H}), 6.47(\mathrm{br} \mathrm{s}, 1 \mathrm{H}), 2.22(\mathrm{~d}$ of quintet, $J=5.2,13.5$ $\mathrm{Hz}, 2 \mathrm{H}), 1.99$ (br s, 2H), 1.35-1.25 (m, 2H), 1.22-1.04 (m, 2H), $0.83(\mathrm{t}, J=7.3 \mathrm{~Hz}, 3 \mathrm{H}) .{ }^{13} \mathrm{C} \mathrm{NMR}\left(126 \mathrm{MHz}, \mathrm{CD}_{3} \mathrm{CN}\right)$ $\delta 167.66,161.27,150.84,149.37,148.64,129.20,127.53,127.44,125.33,120.93,61.61,42.15,26.85,23.74,14.34$.<smiles>CCCCC(N)(c1ccccc1)c1ccnc(C(=O)OC)c1</smiles>

Synthesis of 5ak was synthesized following General Procedure A using cyanoarene 4t (107 mg, 0.66 mmol), PC1 (6 $\mathrm{mg}, 6 \mathrm{E}-3 \mathrm{mmol})$, benzoic acid $(37 \mathrm{mg}, 0.3 \mathrm{mmol})$, DIPA $(153 \mu \mathrm{L}, 1.1 \mathrm{mmol})$ and $O$-benzoyl oxime (3l) $(84 \mathrm{mg}, 0.3$ $\mathrm{mmol})$ in DMSO (3 $\mathrm{mL})$. The reaction was unsuccessful.

Compound 5ak was also synthesized from the corresponding iminium $\mathrm{HCl}$ salt $\mathbf{6 l}$ and cyanoarene $\mathbf{4 t}$. The reaction was conducted by following the General Iminium Salt Reaction Procedure using iminium $\mathrm{HCl}$ salt 61 (130 mg, 0.66 mmol, 2.2 equiv.); photocatalyst $\operatorname{Ir}[\mathrm{dF}(\mathrm{Me}) \text { ppy }]_{2} \mathrm{dtbbpyPF}_{6}(6 \mathrm{mg}, 2 \mathrm{E}-3 \mathrm{mmol}, 2 \mathrm{~mol} \%)$; DIPA (153 $\mu \mathrm{L}, 1.1 \mathrm{mmol}$, 3.6 equiv.); and cyanoarene $4 \mathbf{t}$ (36 $\mathrm{mg}, 0.3 \mathrm{mmol}, 1.0$ equiv.). The reaction was unsuccessful. 
<smiles>CCCCC(N)(c1ccccc1)c1ccncc1-c1ccccc1</smiles>

Compound 5am was synthesized following General Procedure A using cyanoarene 4v (119 mg, $0.66 \mathrm{mmol}$ ), PC1 (6 $\mathrm{mg}, 6 \mathrm{E}-3 \mathrm{mmol})$, benzoic acid (37 mg, $0.3 \mathrm{mmol})$, DIPA (153 $\mu \mathrm{L}, 1.1 \mathrm{mmol})$ and $O$-benzoyl oxime (3I) $(84 \mathrm{mg}, 0.3$ mmol) in DMSO $(3 \mathrm{~mL})$. MS (ESI) $\mathrm{m} / z$ calcd. for $\mathrm{C}_{22} \mathrm{H}_{25} \mathrm{~N}_{2}\left([\mathrm{M}+\mathrm{H}]^{+}\right) 317.2$, found 317.3. The product 5amwas synthesized in $10 \%$ yield (retention time $\mathrm{t}=2.68, \mathrm{~m} / \mathrm{z}=317.3(\mathrm{M}+\mathrm{H}]^{+}$) as determined by a calibrated UPLC generated from an authentic sample using the UPLC-MS Method for Yield Determination.

Compound 5am was also synthesized from the corresponding iminium $\mathrm{HCl}$ salt $\mathbf{6} \mathbf{l}$ and cyanoarene $\mathbf{4 v}$. The reaction was conducted by following the General Iminium Salt Reaction Procedure using iminium HCl salt 61 (130 mg, 0.66 mmol, 2.2 equiv.); photocatalyst $\operatorname{Ir}[\mathrm{dF}(\mathrm{Me}) \text { ppy }]_{2} \mathrm{dtbbpyPF}_{6}(6 \mathrm{mg}, 2 \mathrm{E}-3 \mathrm{mmol}, 2 \mathrm{~mol} \%)$; DIPA (153 $\mu \mathrm{L}, 1.1 \mathrm{mmol}$, 3.6 equiv.); and cyanoarene $4 \mathbf{v}$ ( $40 \mathrm{mg}, 0.3 \mathrm{mmol}, 1.0$ equiv.). The product $\mathbf{5} \mathbf{a m}$ was synthesized in $42 \%$ yield (retention time $\mathrm{t}=2.68, \mathrm{~m} / \mathrm{z}=317.3(\mathrm{M}+\mathrm{H}]^{+}$) as determined by a calibrated UPLC generated from an authentic sample using the UPLC-MS Method for Yield Determination.

${ }^{1} \mathrm{H}$ NMR $\left(500 \mathrm{MHz}, \mathrm{CD}_{3} \mathrm{CN}\right) \delta 8.58(\mathrm{~d}, J=5.3 \mathrm{~Hz}, 1 \mathrm{H}), 8.08(\mathrm{~s}, 1 \mathrm{H}), 7.86(\mathrm{~d}, J=5.3 \mathrm{~Hz}, 1 \mathrm{H}), 7.20(\mathrm{t}, J=7.4 \mathrm{~Hz}$, $1 \mathrm{H}), 7.17-7.11(\mathrm{~m}, 3 \mathrm{H}), 7.06(\mathrm{t}, J=7.5 \mathrm{~Hz}, 2 \mathrm{H}), 6.98-6.94(\mathrm{~m}, 2 \mathrm{H}), 6.51(\mathrm{~d}, J=7.4 \mathrm{~Hz}, 2 \mathrm{H}), 2.02(\mathrm{td}, J=4.9,12.2$ $\mathrm{Hz}, 1 \mathrm{H}), 1.89(\mathrm{td}, J=12.6,4.7 \mathrm{~Hz}, 1 \mathrm{H}), 1.71(\mathrm{br} \mathrm{s}, 2 \mathrm{H}), 1.29-1.17(\mathrm{~m}, 2 \mathrm{H}), 1.14-0.98(\mathrm{~m}, 2 \mathrm{H}), 0.82(\mathrm{t}, J=7.3 \mathrm{~Hz}$, 1H). ${ }^{13} \mathrm{C}$ NMR $\left(126 \mathrm{MHz}, \mathrm{CD}_{3} \mathrm{CN}\right) \delta 155.75,152.92,150.43,149.58,140.30,137.98,130.50,128.86,128.19,127.92$, $127.24,127.12,122.51,61.85,41.60,26.93,23.80,14.34$.<smiles>CC(N)(c1ccccc1)c1ccncc1C#N</smiles>

Compound 5an was synthesized following General Procedure A using cyanoarene 4w (85 mg, $0.66 \mathrm{mmol}$ ), PC1 (6 $\mathrm{mg}$, 6E-3 mmol), benzoic acid (37 mg, $0.3 \mathrm{mmol})$, DIPA $(153 \mu \mathrm{L}, 1.1 \mathrm{mmol})$ and $O$-benzoyl oxime (3I) $(84 \mathrm{mg}, 0.3$ mmol) in DMSO $(3 \mathrm{~mL})$. MS (ESI) $\mathrm{m} / z$ calcd. for $\mathrm{C}_{22} \mathrm{H}_{25} \mathrm{~N}_{2}\left([\mathrm{M}+\mathrm{H}]^{+}\right) 266.2$, found 265.9. The product 5an was synthesized in $0 \%$ yield (retention time $\mathrm{t}=2.24, \mathrm{~m} / \mathrm{z}=265.9(\mathrm{M}+\mathrm{H}]^{+}$) as determined by a calibrated UPLC generated from an authentic sample using the UPLC-MS Method for Yield Determination.

Compound 5an was also synthesized from the corresponding iminium $\mathrm{HCl}$ salt $\mathbf{6} \mathbf{l}$ and cyanoarene $\mathbf{4 w}$. The reaction was conducted by following the General Iminium Salt Reaction Procedure using iminium $\mathrm{HCl}$ salt $\mathbf{6 1}$ (130 mg, 0.66 mmol, 2.2 equiv.); photocatalyst $\operatorname{Ir}\left[\mathrm{dF}(\mathrm{Me}) \mathrm{ppy}_{2} \mathrm{dtbbpyPF}_{6}(6 \mathrm{mg}, 2 \mathrm{E}-3 \mathrm{mmol}, 2 \mathrm{~mol} \%\right.$ ); DIPA (153 $\mu \mathrm{L}, 1.1 \mathrm{mmol}$, 3.6 equiv.); and cyanoarene $4 \mathbf{w}(28 \mathrm{mg}, 0.3 \mathrm{mmol}, 1.0$ equiv.). The product $5 \mathrm{an}$ was synthesized in $0 \%$ yield (retention time $\left.\mathrm{t}=2.24, \mathrm{~m} / \mathrm{z}=265.9(\mathrm{M}+\mathrm{H}]^{+}\right)$as determined by a calibrated UPLC generated from an authentic sample using the UPLC-MS Method for Yield Determination. 
<smiles>CCCCC(N)(c1ccccc1)c1ccncc1Cl</smiles>

Compound 5ao was synthesized following General Procedure A using cyanoarene 4x (91 mg, 0.66 mmol), PC1 (6 $\mathrm{mg}, 6 \mathrm{E}-3 \mathrm{mmol})$, benzoic acid $(37 \mathrm{mg}, 0.3 \mathrm{mmol})$, DIPA $(153 \mu \mathrm{L}, 1.1 \mathrm{mmol})$ and $O$-benzoyl oxime (31) (84 mg, 0.3 mmol) in DMSO (3 mL). MS (ESI) $\mathrm{m} / z$ calcd. for $\mathrm{C}_{16} \mathrm{H}_{20} \mathrm{ClN}_{2}\left([\mathrm{M}+\mathrm{H}]^{+}\right) 275.1$, found 275.2. The product 5ao was synthesized in $17 \%$ yield (retention time $\mathrm{t}=2.587, \mathrm{~m} / \mathrm{z}=275.2(\mathrm{M}+\mathrm{H}]^{+}$) as determined by a calibrated UPLC generated from an authentic sample using the UPLC-MS Method for Yield Determination.

Compound $5 \mathbf{a o}$ was also synthesized from the corresponding iminium $\mathrm{HCl}$ salt $\mathbf{6 l}$ and cyanoarene $\mathbf{4 x}$. The reaction was conducted by following the General Iminium Salt Reaction Procedure using iminium $\mathrm{HCl}$ salt 61 (130 mg, 0.66 mmol, 2.2 equiv.); photocatalyst $\operatorname{Ir}[\mathrm{dF}(\mathrm{Me}) \text { ppy }]_{2} \mathrm{dtbbpyPF}_{6}(6 \mathrm{mg}, 2 \mathrm{E}-3 \mathrm{mmol}, 2 \mathrm{~mol} \%)$; DIPA (153 $\mu \mathrm{L}, 1.1 \mathrm{mmol}$, 3.6 equiv.); and cyanoarene $\mathbf{4 x}$ (30 mg, $0.3 \mathrm{mmol}, 1.0$ equiv.). The product $5 \mathrm{ao}$ was not synthesized by this method. However, des-halogenated product $5 \mathbf{l}$ was synthesized in $23 \%$ yield (retention time $\mathrm{t}=2.587, \mathrm{~m} / \mathrm{z}=275.2(\mathrm{M}+\mathrm{H}]^{+}$) as determined by a calibrated UPLC generated from an authentic sample using the UPLC-MS Method for Yield Determination.

${ }^{1} \mathrm{H}$ NMR $\left(500 \mathrm{MHz}, \mathrm{CD}_{3} \mathrm{CN}\right) \delta 8.51(\mathrm{~d}, J=5.2 \mathrm{~Hz}, 1 \mathrm{H}), 8.38(\mathrm{~s}, 1 \mathrm{H}), 7.86(\mathrm{~d}, J=5.2 \mathrm{~Hz}, 1 \mathrm{H}), 7.28-7.17(\mathrm{~m}, 5 \mathrm{H})$, 2.49 (ddd, $J=4.7,12.3,13.0 \mathrm{~Hz}, 1 \mathrm{H}), 2.15-2.19$ (m, 1H), 2.07 (br s, 2H), 1.29 (sextet, $7.4 \mathrm{~Hz}, 2 \mathrm{H}), 1.18-0.97$ (m, $2 \mathrm{H}), 0.83(\mathrm{~s}, 3 \mathrm{H}) .{ }^{13} \mathrm{C}$ NMR $\left(126 \mathrm{MHz}, \mathrm{CD}_{3} \mathrm{CN}\right) \delta 155.08,151.65,148.97,148.26,131.74,128.94,127.28,127.06$, $124.23,61.98,40.14,26.98,23.78,14.30$.<smiles>CCCCC(N)(c1ccccc1)c1ccncc1F</smiles>

Compound 5ap was synthesized following General Procedure A using cyanoarene 4y (81 mg, 0.66 mmol), PC1 (6 $\mathrm{mg}, 6 \mathrm{E}-3 \mathrm{mmol})$, benzoic acid $(37 \mathrm{mg}, 0.3 \mathrm{mmol})$, DIPA $(153 \mu \mathrm{L}, 1.1 \mathrm{mmol})$ and $O$-benzoyl oxime (3l) $(84 \mathrm{mg}, 0.3$ mmol) in DMSO (3 mL). MS (ESI) $m / z$ calcd. for $\mathrm{C}_{16} \mathrm{H}_{20} \mathrm{FN}_{2}\left([\mathrm{M}+\mathrm{H}]^{+}\right) 259.2$, found 259.3. The product 5ap was synthesized in $44 \%$ yield (retention time $\mathrm{t}=2.310, \mathrm{~m} / \mathrm{z}=259.3(\mathrm{M}+\mathrm{H}]^{+}$) as determined by a calibrated UPLC generated from an authentic sample using the UPLC-MS Method for Yield Determination

Compound 5ap was also synthesized from the corresponding iminium $\mathrm{HCl}$ salt $\mathbf{6 l}$ and cyanoarene $\mathbf{4 y}$. The reaction was conducted by following the General Iminium Salt Reaction Procedure using iminium $\mathrm{HCl} \mathrm{salt} 61$ (130 mg, 0.66 mmol, 2.2 equiv.); photocatalyst $\operatorname{Ir}[\mathrm{dF}(\mathrm{Me}) \text { ppy }]_{2} \mathrm{dtbbpyPF}_{6}(6 \mathrm{mg}, 2 \mathrm{E}-3 \mathrm{mmol}, 2 \mathrm{~mol} \%)$; DIPA (153 $\mu \mathrm{L}, 1.1 \mathrm{mmol}$, 3.6 equiv.); and cyanoarene $4 \mathbf{y}$ ( $28 \mathrm{mg}, 0.3 \mathrm{mmol}, 1.0$ equiv.). The product $5 \mathrm{ap}$ was synthesized in $76 \%$ yield (retention time $\left.\mathrm{t}=2.310, \mathrm{~m} / \mathrm{z}=259.3(\mathrm{M}+\mathrm{H}]^{+}\right)$as determined by a calibrated UPLC generated from an authentic sample using the UPLC-MS Method for Yield Determination

${ }^{1} \mathrm{H}$ NMR $\left(500 \mathrm{MHz}, \mathrm{CD}_{3} \mathrm{CN}\right) \delta 8.42(\mathrm{~d}, J=4.9 \mathrm{~Hz}, 1 \mathrm{H}), 8.25(\mathrm{~d}, J=3.4 \mathrm{~Hz}, 1 \mathrm{H}), 7.71(\mathrm{dd}, J=5.3,7.0 \mathrm{~Hz}, 1 \mathrm{H}), 7.34$ $(\mathrm{d}, J=7.7 \mathrm{~Hz}, 2 \mathrm{H}), 7.29(\mathrm{t}, J=7.6 \mathrm{~Hz}, 2 \mathrm{H}), 7.21(\mathrm{t}, J=7.2 \mathrm{~Hz}, 1 \mathrm{H}), 2.32(\mathrm{dt}, J=4.3,12.6 \mathrm{~Hz}, 1 \mathrm{H}), 2.17(\mathrm{dt}, J=4.4$ $12.8 \mathrm{~Hz}, 1 \mathrm{H}), 2.07$ (br s, $2 \mathrm{H}), 1.37-1.26(\mathrm{~m}, 2 \mathrm{H}), 1.25-1.14(\mathrm{~m}, 1 \mathrm{H}), 1.14-1.04(\mathrm{~m}, 1 \mathrm{H}), 0.85(\mathrm{t}, J=7.4 \mathrm{~Hz}, 3 \mathrm{H}) .{ }^{13} \mathrm{C}$ NMR (126 MHz, CD 3 CN) $\delta 13 C$ NMR (126 MHz, CD3CN) $\delta 159.57,157.55,148.43,146.86,146.82,145.47,145.40$, 139.28, 139.07, 129.05, 127.54, 126.76, 126.75, 123.51, 123.49, 60.33, 60.32, 40.68, 40.66, 26.91, $23.77,14.33$. ${ }^{19} \mathrm{~F}\left\{{ }^{1} \mathrm{H}\right\} \mathrm{NMR}\left(471 \mathrm{MHz}, \mathrm{CD}_{3} \mathrm{CN}\right) \delta-126.87(\mathrm{~s}, 1 \mathrm{~F}) .{ }^{19} \mathrm{~F}$ NMR $\left(471 \mathrm{MHz}, \mathrm{CD}_{3} \mathrm{CN}\right) \delta-126.87(\mathrm{dd}, J=3.5,7.2 \mathrm{~Hz}$, $1 \mathrm{~F})$. 


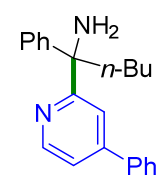

Compound 5aq was synthesized following General Procedure A using cyanoarene 4z (120 mg, 0.66 mmol), PC1 (6 $\mathrm{mg}, 6 \mathrm{E}-3 \mathrm{mmol})$, benzoic acid (37 mg, $0.3 \mathrm{mmol})$, DIPA (153 $\mu \mathrm{L}, 1.1 \mathrm{mmol})$ and $O$-benzoyl oxime (3I) $(84 \mathrm{mg}, 0.3$ mmol) in DMSO $(3 \mathrm{~mL})$. MS (ESI) $\mathrm{m} / z$ calcd. for $\mathrm{C}_{22} \mathrm{H}_{25} \mathrm{~N}_{2}\left([\mathrm{M}+\mathrm{H}]^{+}\right) 317.2$, found 317.2. The product 5aq was synthesized in $95 \%$ yield (retention time $\mathrm{t}=2.832, \mathrm{~m} / \mathrm{z}=317.2(\mathrm{M}+\mathrm{H}]^{+}$) as determined by a calibrated UPLC generated from an authentic sample using the UPLC-MS Method for Yield Determination

Compound 5aq was also synthesized from the corresponding iminium $\mathrm{HCl}$ salt $\mathbf{6} \mathbf{l}$ and cyanoarene $\mathbf{4 z}$. The reaction was conducted by following the General Iminium Salt Reaction Procedure using iminium HCl salt 61 (130 mg, 0.66 mmol, 2.2 equiv.); photocatalyst $\operatorname{Ir}[\mathrm{dF}(\mathrm{Me}) \text { ppy }]_{2} \mathrm{dtbbpyPF}_{6}(6 \mathrm{mg}, 2 \mathrm{E}-3 \mathrm{mmol}, 2 \mathrm{~mol} \%)$; DIPA (153 $\mu \mathrm{L}, 1.1 \mathrm{mmol}$, 3.6 equiv.); and cyanoarene $4 \mathbf{z}$ ( $40 \mathrm{mg}, 0.3 \mathrm{mmol}, 1.0$ equiv.). The product $5 \mathbf{a q}$ was synthesized in $22 \%$ yield (retention time $\left.\mathrm{t}=2.832, \mathrm{~m} / \mathrm{z}=317.2(\mathrm{M}+\mathrm{H}]^{+}\right)$as determined by a calibrated UPLC generated from an authentic sample using the UPLC-MS Method for Yield Determination.

${ }^{1} \mathrm{H}$ NMR $\left(500 \mathrm{MHz}, \mathrm{CD}_{3} \mathrm{CN}\right) \delta 8.52(\mathrm{~d}, J=5.0 \mathrm{~Hz}, 1 \mathrm{H}), 7.79(\mathrm{~s}, 1 \mathrm{H}), 7.68(\mathrm{~d}, J=7.2 \mathrm{~Hz}, 1 \mathrm{H}), 7.53-7.41(\mathrm{~m}, 5 \mathrm{H})$, 7.39 (dd, $J=1.3,5.0 \mathrm{~Hz}, 1 \mathrm{H}), 7.27(\mathrm{t}, J=7.6 \mathrm{~Hz}, 2 \mathrm{H}), 7.16(\mathrm{t}, J=7.3 \mathrm{~Hz}, 1 \mathrm{H}), 2.43-2.28(\mathrm{~m}, 2 \mathrm{H}), 2.20$ (br s, 2H), $1.39-1.29(\mathrm{~m}, 2 \mathrm{H}), 1.29-1.12(\mathrm{~m}, 2 \mathrm{H}), 0.87(\mathrm{t}, J=7.3 \mathrm{~Hz}, 1 \mathrm{H}) .{ }^{13} \mathrm{C} \mathrm{NMR}\left(126 \mathrm{MHz}, \mathrm{CD}_{3} \mathrm{CN}\right) \delta 169.02,149.95$, $149.48,149.27,139.40,130.10,130.00,128.84,127.99$, 127.48, 127.00, 120.09, 119.22, 63.45, 42.25, 27.12, 23.98, 14.46.

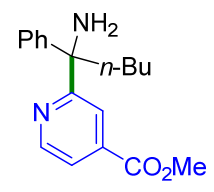

Compound 5ar was synthesized following General Procedure A using cyanoarene 4aa (107 mg, $0.66 \mathrm{mmol}$ ), PC1 (6 $\mathrm{mg}, 6 \mathrm{E}-3 \mathrm{mmol})$, benzoic acid (37 mg, $0.3 \mathrm{mmol})$, DIPA (153 $\mu \mathrm{L}, 1.1 \mathrm{mmol})$ and $O$-benzoyl oxime (3I) $(84 \mathrm{mg}, 0.3$ mmol) in DMSO (3 mL). MS (ESI) $\mathrm{m} / z$ calcd. for $\mathrm{C}_{18} \mathrm{H}_{23} \mathrm{~N}_{2} \mathrm{O}_{2}\left([\mathrm{M}+\mathrm{H}]^{+}\right) 299.2$ found 299.2. The product 5ar was synthesized in $16 \%$ yield (retention time $\mathrm{t}=2.393, \mathrm{~m} / \mathrm{z}=299.2(\mathrm{M}+\mathrm{H}]^{+}$) as determined by a calibrated UPLC generated from an authentic sample using the UPLC-MS Method for Yield Determination

Compound 5ar was also synthesized from the corresponding iminium $\mathrm{HCl}$ salt $\mathbf{6} \mathbf{\mathbf { l }}$ and cyanoarene $\mathbf{4 a a}$. The reaction was conducted by following the General Iminium Salt Reaction Procedure using iminium $\mathrm{HCl}$ salt $6 \mathbf{1}$ (130 mg, 0.66 mmol, 2.2 equiv.); photocatalyst $\operatorname{Ir}\left[\mathrm{dF}(\mathrm{Me}) \mathrm{ppy}_{2} \mathrm{dtbbpyPF}_{6}(6 \mathrm{mg}, 2 \mathrm{E}-3 \mathrm{mmol}, 2 \mathrm{~mol} \%)\right.$; DIPA (153 $\mu \mathrm{L}, 1.1 \mathrm{mmol}$, 3.6 equiv.); and cyanoarene $4 \mathbf{a a}$ ( $36 \mathrm{mg}, 0.3 \mathrm{mmol}, 1.0$ equiv.). The product $5 \mathrm{ar}$ was synthesized in $0 \%$ yield (retention time $\left.\mathrm{t}=2.393, \mathrm{~m} / \mathrm{z}=299.2(\mathrm{M}+\mathrm{H}]^{+}\right)$as determined by a calibrated UPLC generated from an authentic sample using the UPLC-MS Method for Yield Determination.

${ }^{1} \mathrm{H}$ NMR $\left(500 \mathrm{MHz}, \mathrm{CD}_{3} \mathrm{CN}\right) \delta 8.65(\mathrm{~d}, J=4.9 \mathrm{~Hz}, 1 \mathrm{H}), 8.01(\mathrm{~s}, 1 \mathrm{H}), 7.62(\mathrm{dd}, J=1.1,4.9 \mathrm{~Hz}, 1 \mathrm{H}), 7.46(\mathrm{~d}, J=7.5$ $\mathrm{Hz}, 2 \mathrm{H}), 7.27$ (t, $J=7.9 \mathrm{~Hz}, 2 \mathrm{H}), 7.17(\mathrm{t}, J=7.3 \mathrm{~Hz}, 1 \mathrm{H}), 3.88(\mathrm{~s}, 3 \mathrm{H}), 2.38-2.27(\mathrm{~m}, 2 \mathrm{H}), 2.22-2.10$ (m, 2H), 1.36$1.28(\mathrm{~m}, 2 \mathrm{H}), 1.19-1.11(\mathrm{~m}, 2 \mathrm{H}), 0.85(\mathrm{t}, J=7.4 \mathrm{~Hz}, 3 \mathrm{H}) .{ }^{13} \mathrm{C} \mathrm{NMR}\left(126 \mathrm{MHz}, \mathrm{CD}_{3} \mathrm{CN}\right) \delta 169.73,166.75,150.32$, $148.90,138.84,128.96,127.43,127.21,121.17,120.63,63.47,53.28,42.28,26.97,23.91,14.41$. 


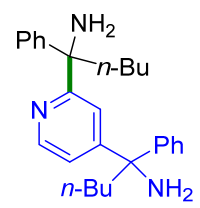

Compound 5as was synthesized following General Procedure A using cyanoarene 4ab (85 mg, 0.66 mmol), PC1 (6 $\mathrm{mg}, 6 \mathrm{E}-3 \mathrm{mmol})$, benzoic acid $(37 \mathrm{mg}, 0.3 \mathrm{mmol})$, DIPA $(153 \mu \mathrm{L}, 1.1 \mathrm{mmol})$ and $O$-benzoyl oxime (3l) $(84 \mathrm{mg}, 0.3$ mmol) in DMSO (3 mL). MS (ESI) $\mathrm{m} / z$ calcd. for $\mathrm{C}_{27} \mathrm{H}_{36} \mathrm{~N}_{3}\left([\mathrm{M}+\mathrm{H}]^{+}\right) 402.3$ found 402.4. The product 5as was synthesized in $0 \%$ yield (retention time $\mathrm{t}=2.435, \mathrm{~m} / \mathrm{z}=402.4(\mathrm{M}+\mathrm{H}]^{+}$) as determined by a calibrated UPLC generated from an authentic sample using the UPLC-MS Method for Yield Determination

Compound 5as was also synthesized from the corresponding iminium $\mathrm{HCl}$ salt $\mathbf{6 l}$ and cyanoarene 4ab. The reaction was conducted by following the General Iminium Salt Reaction Procedure using iminium $\mathrm{HCl}$ salt 61 (130 mg, 0.66 mmol, 2.2 equiv.); photocatalyst $\operatorname{Ir}[\mathrm{dF}(\mathrm{Me}) \text { ppy }]_{2} \mathrm{dtbbpyPF}_{6}(6 \mathrm{mg}, 2 \mathrm{E}-3 \mathrm{mmol}, 2 \mathrm{~mol} \%)$; DIPA (153 $\mu \mathrm{L}, 1.1 \mathrm{mmol}$, 3.6 equiv.); and cyanoarene $\mathbf{4 a b}(28 \mathrm{mg}, 0.3 \mathrm{mmol}, 1.0$ equiv.). The product $\mathbf{5 a s}$ was synthesized in $14 \%$ yield (retention time $\mathrm{t}=2.435, \mathrm{~m} / \mathrm{z}=402.4(\mathrm{M}+\mathrm{H}]^{+}$) as determined by a calibrated UPLC generated from an authentic sample using the UPLC-MS Method for Yield Determination

${ }^{1} \mathrm{H}$ NMR (500 MHz, CD $\left.{ }_{3} \mathrm{OD}\right) \delta 8.66-8.61(\mathrm{~m}, 1 \mathrm{H}), 7.44-7.30(\mathrm{~m}, 8 \mathrm{H}), 7.29-7.23(\mathrm{~m}, 3 \mathrm{H}), 7.17-7.10(\mathrm{~m}, 1 \mathrm{H}), 2.58-$ $2.47(\mathrm{~m}, 1 \mathrm{H}), 2.47-2.39(\mathrm{~m}, 2 \mathrm{H}), 2.32-2.19(\mathrm{~m}, 2 \mathrm{H}) 1.44-1.13(\mathrm{~m}, 6 \mathrm{H}), 1.13-0.94(\mathrm{~m}, 2 \mathrm{H}), 0.90-0.81(\mathrm{~m}, 6 \mathrm{H}) .{ }^{13} \mathrm{C}$ NMR (126 MHz, CD $\mathrm{CD}_{3} \mathrm{OD} \delta 169.93,160.68,157.98,157.87,149.81,149.79,145.81,145.73,141.27,130.16,130.12$, $129.95,129.88,129.69,129.66,128.67,128.62,127.98,127.91,127.63,127.60,122.38,122.35,121.48,66.32,66.31$, $63.29,63.26,27.21,26.83,26.82,23.94,23.90,23.78,23.75,14.29,14.27,14.19,14.18$.<smiles>CCCCCC(N)(c1ccccc1)c1ccc(C#N)cc1</smiles>

Compound 5at was synthesized following General Procedure A using cyanoarene 4ac (85 mg, 0.66 mmol), PC1 (6 $\mathrm{mg}, 6 \mathrm{E}-3 \mathrm{mmol})$, benzoic acid $(37 \mathrm{mg}, 0.3 \mathrm{mmol})$, DIPA $(153 \mu \mathrm{L}, 1.1 \mathrm{mmol})$ and $O$-benzoyl oxime (3I) $(84 \mathrm{mg}, 0.3$ $\mathrm{mmol})$ in DMSO (3 mL). Reaction was unsuccessful.

Compound 5at was also synthesized from the corresponding iminium $\mathrm{HCl}$ salt $\mathbf{6} \mathbf{l}$ and cyanoarene 4ac. The reaction was conducted by following the General Iminium Salt Reaction Procedure using iminium HCl salt 61 (130 mg, 0.66 mmol, 2.2 equiv.); photocatalyst $\operatorname{Ir}[\mathrm{dF}(\mathrm{Me}) \mathrm{ppy}]_{2} \mathrm{dtbbpyPF}_{6}(6 \mathrm{mg}, 2 \mathrm{E}-3 \mathrm{mmol}, 2 \mathrm{~mol} \%)$; DIPA (153 $\mu \mathrm{L}, 1.1 \mathrm{mmol}$, 3.6 equiv.); and cyanoarene $4 \mathbf{a c}$ ( $28 \mathrm{mg}, 0.3 \mathrm{mmol}, 1.0$ equiv.). Reaction was unsuccessful 


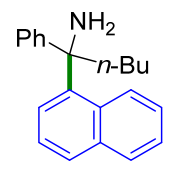

Compound 5au was synthesized following General Procedure A using cyanoarene 4ad (101 mg, 0.66 mmol), PC1 (6 $\mathrm{mg}, 6 \mathrm{E}-3 \mathrm{mmol})$, benzoic acid $(37 \mathrm{mg}, 0.3 \mathrm{mmol})$, DIPA (153 $\mu \mathrm{L}, 1.1 \mathrm{mmol})$ and $O$-benzoyl oxime (3I) $(84 \mathrm{mg}, 0.3$ $\mathrm{mmol})$ in DMSO (3 mL). Reaction was unsuccessful.

Compound 5au was also synthesized from the corresponding iminium $\mathrm{HCl}$ salt $\mathbf{6} \mathbf{1}$ and cyanoarene $\mathbf{4 a d}$. The reaction was conducted by following the General Iminium Salt Reaction Procedure using iminium $\mathrm{HCl}$ salt $\mathbf{6 1}$ (130 mg, 0.66 mmol, 2.2 equiv.); photocatalyst $\operatorname{Ir}\left[\mathrm{dF}(\mathrm{Me}) \mathrm{ppy}_{2} \mathrm{dtbbpyPF}_{6}(6 \mathrm{mg}, 2 \mathrm{E}-3 \mathrm{mmol}, 2 \mathrm{~mol} \%)\right.$; DIPA (153 $\mu \mathrm{L}, 1.1 \mathrm{mmol}$, 3.6 equiv.); and cyanoarene $4 \mathbf{a d}(34 \mathrm{mg}, 0.3 \mathrm{mmol}, 1.0$ equiv.). Reaction was unsuccessful.

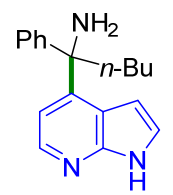

Compound 5av was synthesized following General Procedure A using cyanoarene 4ae (94 mg, $0.66 \mathrm{mmol})$, PC1 (6 $\mathrm{mg}, 6 \mathrm{E}-3 \mathrm{mmol})$, benzoic acid (37 mg, $0.3 \mathrm{mmol})$, DIPA (153 $\mu \mathrm{L}, 1.1 \mathrm{mmol})$ and $O$-benzoyl oxime (3I) $(84 \mathrm{mg}, 0.3$ mmol) in DMSO (3 mL). MS (ESI) $\mathrm{m} / z$ calcd. for $\mathrm{C}_{27} \mathrm{H}_{36} \mathrm{~N}_{3}\left([\mathrm{M}+\mathrm{H}]^{+}\right) 280.2$ found 280.2. The product 5av was synthesized in $39 \%$ yield (retention time $\mathrm{t}=2.18, \mathrm{~m} / \mathrm{z}=280.2(\mathrm{M}+\mathrm{H}]^{+}$) as determined by a calibrated UPLC generated from an authentic sample using the UPLC-MS Method for Yield Determination.

Compound 5av was also synthesized from the corresponding iminium $\mathrm{HCl}$ salt $\mathbf{6} \mathbf{l}$ and cyanoarene 4ae. The reaction was conducted by following the General Iminium Salt Reaction Procedure using iminium $\mathrm{HCl}$ salt 61 (130 mg, 0.66 mmol, 2.2 equiv.); photocatalyst $\operatorname{Ir}\left[\mathrm{dF}(\mathrm{Me}) \mathrm{ppy}_{2} \mathrm{dtbbpyPF}_{6}(6 \mathrm{mg}, 2 \mathrm{E}-3 \mathrm{mmol}, 2 \mathrm{~mol} \%)\right.$; DIPA (153 $\mu \mathrm{L}, 1.1 \mathrm{mmol}$, 3.6 equiv.); and cyanoarene $4 \mathbf{a e}(31 \mathrm{mg}, 0.3 \mathrm{mmol}, 1.0$ equiv.). The product $5 \mathbf{a v}$ was synthesized in $27 \%$ yield (retention time $\mathrm{t}=2.18, \mathrm{~m} / \mathrm{z}=280.2(\mathrm{M}+\mathrm{H}]^{+}$) as determined by a calibrated UPLC generated from an authentic sample using the UPLC-MS Method for Yield Determination.

${ }^{1} \mathrm{H}$ NMR $\left(500 \mathrm{MHz}, \mathrm{CD}_{3} \mathrm{CN}\right) \delta 10.49(\mathrm{~s}, 1 \mathrm{H}), 8.29(\mathrm{~d}, J=5.0 \mathrm{~Hz}, 1 \mathrm{H}), 7.39$ (t, $\left.J=6.5 \mathrm{~Hz}, 3 \mathrm{H}\right), 7.24$ (t, $J=7.6 \mathrm{~Hz}$, $2 \mathrm{H}), 7.19-7.13(\mathrm{~m}, 2 \mathrm{H}), 6.01(\mathrm{~d}, J=3.1 \mathrm{~Hz}, 1 \mathrm{H}), 2.45(\mathrm{td}, J=12.6,4.3 \mathrm{~Hz}, 1 \mathrm{H}), 2.26(\mathrm{td}, J=12.6,3.8 \mathrm{~Hz}, 1 \mathrm{H}), 2.06$ $(\mathrm{s}, 2 \mathrm{H}), 1.35-1.13(\mathrm{~m}, 3 \mathrm{H}), 1.08-0.96(\mathrm{~m}, 1 \mathrm{H}), 0.81(\mathrm{t}, J=7.2 \mathrm{~Hz}, 3 \mathrm{H}) .{ }^{13} \mathrm{C}$ NMR $\left(126 \mathrm{MHz}, \mathrm{CD}_{3} \mathrm{CN}\right) \delta 151.36$, $150.27,149.92$, 143.72, 128.87, 127.44, 127.15, 125.11, 118.57, 113.91, 101.96, 61.92, 41.53, 27.01, 23.88, 14.36. ESI LRMS $m / z$ calcd. for $\mathrm{C}_{18} \mathrm{H}_{22} \mathrm{~N}_{2}\left(\left[\mathrm{M}-\mathrm{NH}_{2}\right]^{+}\right)$280.2, found 280.3 . 


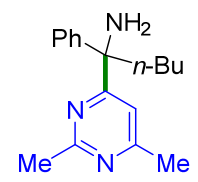

Compound 5aw was synthesized following General Procedure A using cyanoarene 4af ( $88 \mathrm{mg}, 0.66 \mathrm{mmol})$, PC1 (6 $\mathrm{mg}, 6 \mathrm{E}-3 \mathrm{mmol})$, benzoic acid (37 mg, $0.3 \mathrm{mmol})$, DIPA $(153 \mu \mathrm{L}, 1.1 \mathrm{mmol})$ and $O$-benzoyl oxime (3I) $(84 \mathrm{mg}, 0.3$ mmol) in DMSO (3 mL). MS (ESI) $m / z$ calcd. for $\mathrm{C}_{27} \mathrm{H}_{36} \mathrm{~N}_{3}\left([\mathrm{M}+\mathrm{H}]^{+}\right) 270.2$ found 270.2. The product 5 aw was not successfully synthesized.

Compound $\mathbf{5 w}$ was also synthesized from the corresponding iminium $\mathrm{HCl}$ salt $\mathbf{6} \mathbf{l}$ and cyanoarene $\mathbf{4 a f}$. The reaction was conducted by following the General Iminium Salt Reaction Procedure using iminium HCl salt 61 (130 mg, 0.66 mmol, 2.2 equiv.); photocatalyst $\operatorname{Ir}\left[\mathrm{dF}(\mathrm{Me}) \mathrm{ppy}_{2} \mathrm{dtbbpyPF}_{6}(6 \mathrm{mg}, 2 \mathrm{E}-3 \mathrm{mmol}, 2 \mathrm{~mol} \%)\right.$; DIPA (153 $\mu \mathrm{L}, 1.1 \mathrm{mmol}$, 3.6 equiv.); and cyanoarene 4 af $(29 \mathrm{mg}, 0.3 \mathrm{mmol}, 1.0$ equiv.). The product $5 \mathbf{a w}$ was synthesized in $8 \%$ yield (retention time $\left.\mathrm{t}=2.37, \mathrm{~m} / \mathrm{z}=270.2(\mathrm{M}+\mathrm{H}]^{+}\right)$as determined by a calibrated UPLC generated from an authentic sample using the UPLC-MS Method for Yield Determination.

${ }^{1} \mathrm{H}$ NMR $\left(500 \mathrm{MHz}, \mathrm{CD}_{3} \mathrm{CN}\right) \delta 7.46(\mathrm{dd}, J=1.2,8.4,2 \mathrm{H}), 7.27(\mathrm{t}, J=7.7 \mathrm{~Hz}, 2 \mathrm{H}), 7.23(\mathrm{~s}, 1 \mathrm{H}), 7.20-7.15(\mathrm{~m}, 1 \mathrm{H})$, $2.52(\mathrm{~s}, 3 \mathrm{H}), 2.38(\mathrm{~s}, 3 \mathrm{H}), 2.28-2.18(\mathrm{~m}, 2 \mathrm{H}), 2.12(\mathrm{br} \mathrm{s}, 2 \mathrm{H}), 1.38-1.24(\mathrm{~m}, 2 \mathrm{H}), 1.23-1.05(\mathrm{~m}, 2 \mathrm{H}), 0.85(\mathrm{t}, J=7.3$ $\mathrm{Hz}, 3 \mathrm{H}) .{ }^{13} \mathrm{C}$ NMR $\left(126 \mathrm{MHz}, \mathrm{CD}_{3} \mathrm{CN}\right) \delta 176.07,168.20,167.58,148.22,128.93,127.42,127.32,114.97,62.90$, $41.62,26.84,26.18,24.34,23.88,14.36$

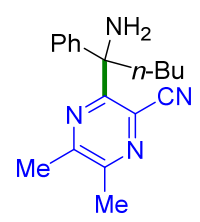

Compound 5ax was synthesized following General Procedure A using cyanoarene 4ag (81 mg, $0.66 \mathrm{mmol})$, PC1 (6 $\mathrm{mg}, 6 \mathrm{E}-3 \mathrm{mmol})$, benzoic acid $(37 \mathrm{mg}, 0.3 \mathrm{mmol})$, DIPA $(153 \mu \mathrm{L}, 1.1 \mathrm{mmol})$ and $O$-benzoyl oxime (3I) $(84 \mathrm{mg}, 0.3$ $\mathrm{mmol}$ ) in DMSO $(3 \mathrm{~mL})$. MS (ESI) $\mathrm{m} / z$ calcd. for $\mathrm{C}_{18} \mathrm{H}_{23} \mathrm{~N}_{4}\left([\mathrm{M}+\mathrm{H}]^{+}\right) 295.2$ found 295.3. The product 5ax was synthesized in $0 \%$ yield (retention time $\mathrm{t}=2.565, \mathrm{~m} / \mathrm{z}=295.3(\mathrm{M}+\mathrm{H}]^{+}$) as determined by a calibrated UPLC generated from an authentic sample using the UPLC-MS Method for Yield Determination.

Compound 5ax was also synthesized from the corresponding iminium $\mathrm{HCl}$ salt $\mathbf{6} \mathbf{l}$ and cyanoarene $\mathbf{4 a g}$. The reaction was conducted by following the General Iminium Salt Reaction Procedure using iminium $\mathrm{HCl}$ salt $6 \mathbf{1}$ (130 mg, 0.66 mmol, 2.2 equiv.); photocatalyst $\operatorname{Ir}\left[\mathrm{dF}(\mathrm{Me}) \mathrm{ppy}_{2} \mathrm{dtbbpyPF}_{6}(6 \mathrm{mg}, 2 \mathrm{E}-3 \mathrm{mmol}, 2 \mathrm{~mol} \%)\right.$; DIPA (153 $\mu \mathrm{L}, 1.1 \mathrm{mmol}$, 3.6 equiv.); and cyanoarene $4 \mathbf{a g}(29 \mathrm{mg}, 0.3 \mathrm{mmol}, 1.0$ equiv.). The product $5 \mathbf{a x}$ was synthesized in $10 \%$ yield (retention time $\mathrm{t}=2.565, \mathrm{~m} / \mathrm{z}=295.3(\mathrm{M}+\mathrm{H}]^{+}$) as determined by a calibrated UPLC generated from an authentic sample using the UPLC-MS Method for Yield Determination.

${ }^{1} \mathrm{H}$ NMR $\left(500 \mathrm{MHz}, \mathrm{CD}_{3} \mathrm{CN}\right) \delta 7.73(\mathrm{~d}, J=7.4 \mathrm{~Hz}, 2 \mathrm{H}), 7.28(\mathrm{t}, J=7.4 \mathrm{~Hz}, 2 \mathrm{H}), 7.19(\mathrm{t}, J=7.3 \mathrm{~Hz}, 1 \mathrm{H}), 5.27$ (very br s, 2H), 2.57 (s, 3H), $2.53(\mathrm{~s}, 3 \mathrm{H}), 2.25(\mathrm{dt}, J=4.2,13.0 \mathrm{~Hz}, 1 \mathrm{H}), 2.06(\mathrm{dt}, J=4.2,13.0 \mathrm{~Hz}, 1 \mathrm{H}), 1.24-1.14(\mathrm{~m}, 2 \mathrm{H})$, $1.14-1.04(\mathrm{~m}, 1 \mathrm{H}), 0.92-0.81(\mathrm{~m}, 1 \mathrm{H}), 0.76(\mathrm{t}, J=7.3 \mathrm{~Hz}, 6 \mathrm{H}) .{ }^{13} \mathrm{C}$ NMR $\left(126 \mathrm{MHz}, \mathrm{CD}_{3} \mathrm{CN}\right) \delta 165.67,162.07$, $152.92,152.60,144.02,143.03,128.84,127.58,118.28,74.75,40.82,27.26,23.58,22.95,22.48,14.27$. 


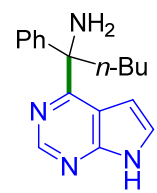

Compound 5ay was synthesized following General Procedure A using cyanoarene 4ah ( $95 \mathrm{mg}, 0.66 \mathrm{mmol})$, PC1 (6 $\mathrm{mg}, 6 \mathrm{E}-3 \mathrm{mmol})$, benzoic acid (37 mg, $0.3 \mathrm{mmol})$, DIPA (153 $\mu \mathrm{L}, 1.1 \mathrm{mmol})$ and $O$-benzoyl oxime (3I) $(84 \mathrm{mg}, 0.3$ mmol) in DMSO (3 mL). MS (ESI) $\mathrm{m} / z$ calcd. for $\mathrm{C}_{18} \mathrm{H}_{23} \mathrm{~N}_{4}\left([\mathrm{M}+\mathrm{H}]^{+}\right) 295.2$ found 295.3. The product 5ay was not successfully synthesized.

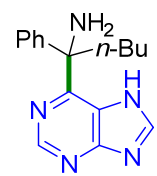

Compound 5az was synthesized following General Procedure A using cyanoarene 4ai (96 mg, $0.66 \mathrm{mmol}$ ), PC1 (6 $\mathrm{mg}, 6 \mathrm{E}-3 \mathrm{mmol})$, benzoic acid (37 mg, $0.3 \mathrm{mmol})$, DIPA (153 $\mu \mathrm{L}, 1.1 \mathrm{mmol})$ and $O$-benzoyl oxime (3I) $(84 \mathrm{mg}, 0.3$ mmol) in DMSO (3 mL). MS (ESI) $\mathrm{m} / z$ calcd. for $\mathrm{C}_{18} \mathrm{H}_{23} \mathrm{~N}_{4}\left([\mathrm{M}+\mathrm{H}]^{+}\right) 295.2$ found 295.3. The product $\mathbf{5 a z}$ was not successfully synthesized. 


\section{Synthesis of $O$-Benzoyl Oximes Starting Materials}

\subsection{Oxime Synthesis:}

Hydroxyl amine was suspended in EtOH (0.5 M), and pyridine (2.5 equivalents) was added. The suspension was heated to $75{ }^{\circ} \mathrm{C}$ to generate a homogeneous solution. The ketone was then added in one portion. The reaction was considered complete when the starting ketone was no longer detected via UPLC-MS. The crude reaction was cooled to room temperature and concentrated under reduced pressure. The concentrate was dissolved in EtOAc and washed with saturated $\mathrm{NH}_{4} \mathrm{Cl}$ ( 1 equal volume). The reaction was washed twice with water ( 2 x 1 equal volume), and once with brine. The organic layer was then collected, dried over sodium sulfate and concentrated to a crude solid. The identity of the crude product was confirmed via UPLC-MS analysis, and the isolated material was utilized in the following benzoylation reaction without further purification.

\subsection{Benzoylation of Oximes:}

General Method A:

Oxime ( 1 equivalent) was added to a round bottom flask containing a stir bar and placed under an atmosphere of nitrogen. The oxime was dissolved in methylene chloride to afford a $1.65 \mathrm{M}$ solution. The solution was then diluted with hexanes until the total concentration of oxime was $0.33 \mathrm{M}$. Pyridine (1.1 equivalent) was then added to the reaction flask via syringe. Benzoyl chloride (1.1 equivalent) was then added to the reaction dropwise via a syringe. If a substantial increase in reaction temperature was observed, addition of benzoyl chloride was halted for two minutes. During addition of benzoyl chloride, a white precipitate was formed. The reaction was stirred at $23{ }^{\circ} \mathrm{C}$, until the oxime is observed to be consumed by LC-MS. Upon completion of the reaction, the reaction was diluted 1:1 with deionized water and contents of the reaction were placed into a separatory funnel. The two immiscible layers were mixed, and then upon separation, the aqueous layer was removed, and the organic layer was washed with water $(3 \times 50 \mathrm{~mL})$. The organic layer was dried over sodium sulfate and concentrated under reduced pressure to afford a mostly pure crude product. The products were then recrystallized to afford pure product.

General Method B:

Note: Used when starting materials were insoluble in high concentrations of hexanes.

Oxime ( 1 equivalent) was added to a round bottom flask containing a stir bar and placed under an atmosphere of nitrogen. The oxime was dissolved in methylene chloride to afford a $0.33 \mathrm{M}$ solution. Pyridine (1.1 equivalent) was then added to the reaction flask via syringe. Benzoyl chloride (1.1 equivalent) was then added to the reaction dropwise via a syringe. If a substantial increase in reaction temperature was observed, addition of benzoyl chloride was halted for two minutes. During addition of benzoyl chloride, a white precipitate was formed. The reaction is stirred at $23{ }^{\circ} \mathrm{C}$, until the oxime is observed to be consumed by LC-MS. Upon completion of the reaction, the reaction was diluted 1:1 with deionized water and contents of the reaction were placed into a separatory funnel. The two immiscible layers were mixed, and then upon separation, the aqueous layer was removed, and the organic layer was washed with water $(3 \times 50 \mathrm{~mL})$. The organic layer was dried over sodium sulfate and concentrated under reduced pressure to afford a mostly pure crude product. The products were then recrystallized to afford pure product.

General Method C:

Note: Used for heterocyclic ketones.

Oxime ( 1 equivalent) was added to a round bottom flask containing a stir bar and placed under an atmosphere of nitrogen. The oxime was dissolved in methylene chloride to afford a $0.33 \mathrm{M}$ solution. Triethylamine (1.1 equivalent) was then added to the reaction flask via syringe. Benzoyl chloride (1.1 equivalent) was then added to the reaction dropwise via a syringe. If a substantial increase in reaction temperature was observed, addition of benzoyl chloride was halted for two minutes. During addition of benzoyl chloride, a white precipitate was formed. The reaction is stirred at $23{ }^{\circ} \mathrm{C}$, until the oxime is observed to be consumed by LC-MS. Upon completion of the reaction, the reaction was diluted 1:1 with deionized water and contents of the reaction were placed into a separatory funnel. The two immiscible layers were mixed, and then upon separation, the aqueous layer was removed, and the organic layer was 
washed with water $(3 \times 50 \mathrm{~mL})$. The organic layer was dried over sodium sulfate and concentrated under reduced pressure to afford a mostly pure crude product. The products were then recrystallized to afford pure product. 
Table S6. Table of $O$-Benzoylated Oximes Synthesized

\section{O-Benzoyl Oximes}

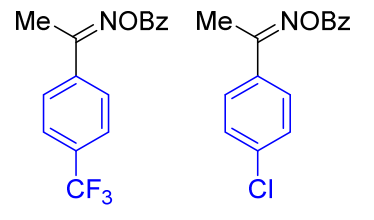

3b

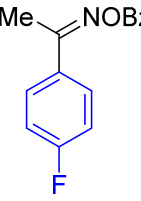

3d

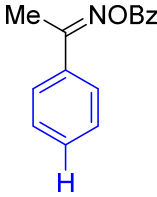

$3 a$

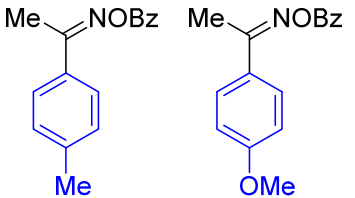

$3 f$

$3 g$

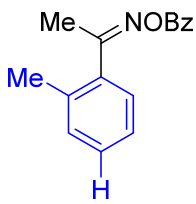

3h

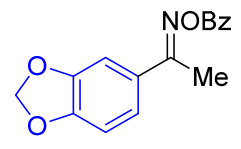

3I

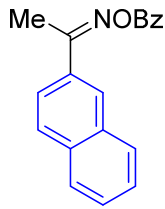

3j<smiles>CCCCC(=NC(=O)OCc1ccccc1)c1ccccc1</smiles>

3k
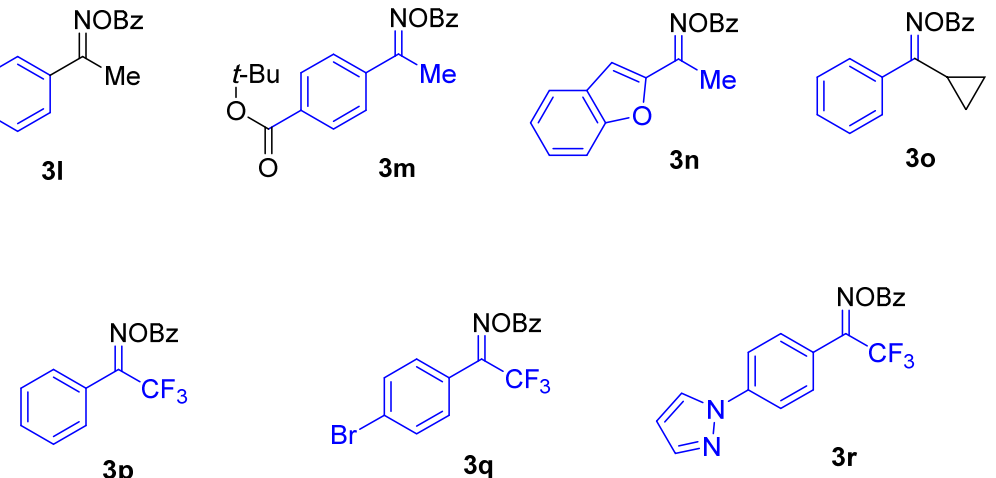

$3 p$<smiles>O=C(c1ccc(Br)cc1)C(F)(F)F</smiles>

$3 q$

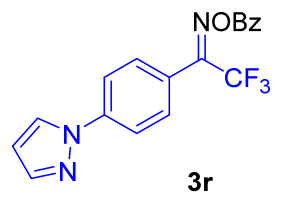




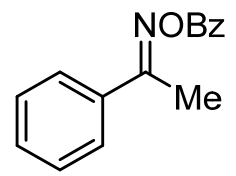

Compound 3a was synthesized according to general method A using acetophenone (9.33 $\mathrm{mL}, 80 \mathrm{mmol})$, pyridine $(16.11 \mathrm{~mL}, 200 \mathrm{mmol})$, hydroxylamine hydrochloride $(8.339 \mathrm{~g}, 120 \mathrm{mmol})$, and ethanol $(160 \mathrm{~mL})$. Then the crude material of the first step was reacted with pyridine $(6450 \mathrm{~mL}, 80 \mathrm{mmol})$, benzoyl chloride $(9.286 \mathrm{~mL}, 80 \mathrm{mmol})$, in methylene chloride $(48 \mathrm{~mL})$ and hexanes $(194 \mathrm{~mL})$ according to the General Method A. The crude product was recrystallized from $\mathrm{CH}_{2} \mathrm{Cl}_{2} / \mathrm{Hexanes}$ to afford the product as a white crystalline solid (15.636 g, $\left.80 \%\right)$. ${ }^{1} \mathrm{H} \mathrm{NMR}(500$ $\left.\mathrm{MHz}, \mathrm{DMSO}-d_{6}\right) \delta 8.09(\mathrm{~d}, J=7.1 \mathrm{~Hz}, 2 \mathrm{H}), 7.83(\mathrm{~d}, J=6.8 \mathrm{~Hz}, 2 \mathrm{H}), 7.71(\mathrm{t}, J=7.5 \mathrm{~Hz}, 1 \mathrm{H}), 7.59(\mathrm{t}, J=7.7 \mathrm{~Hz}, 2 \mathrm{H})$, $7.56-7.48(\mathrm{~m}, 3 \mathrm{H}), 2.52(\mathrm{~s}, 3 \mathrm{H}) .{ }^{13} \mathrm{C}$ NMR $\left(126 \mathrm{MHz}, \mathrm{DMSO}-d_{6}\right) \delta 164.01,162.89,134.48,133.71,130.77,129.24$, 128.97, 128.68, 128.55, 126.94, 14.34. MS (ESI) $m / z$ calcd. for $\mathrm{C}_{15} \mathrm{H}_{14} \mathrm{NO}_{2}\left([\mathrm{M}+\mathrm{H}]^{+}\right) 240.1$, found 240.2 


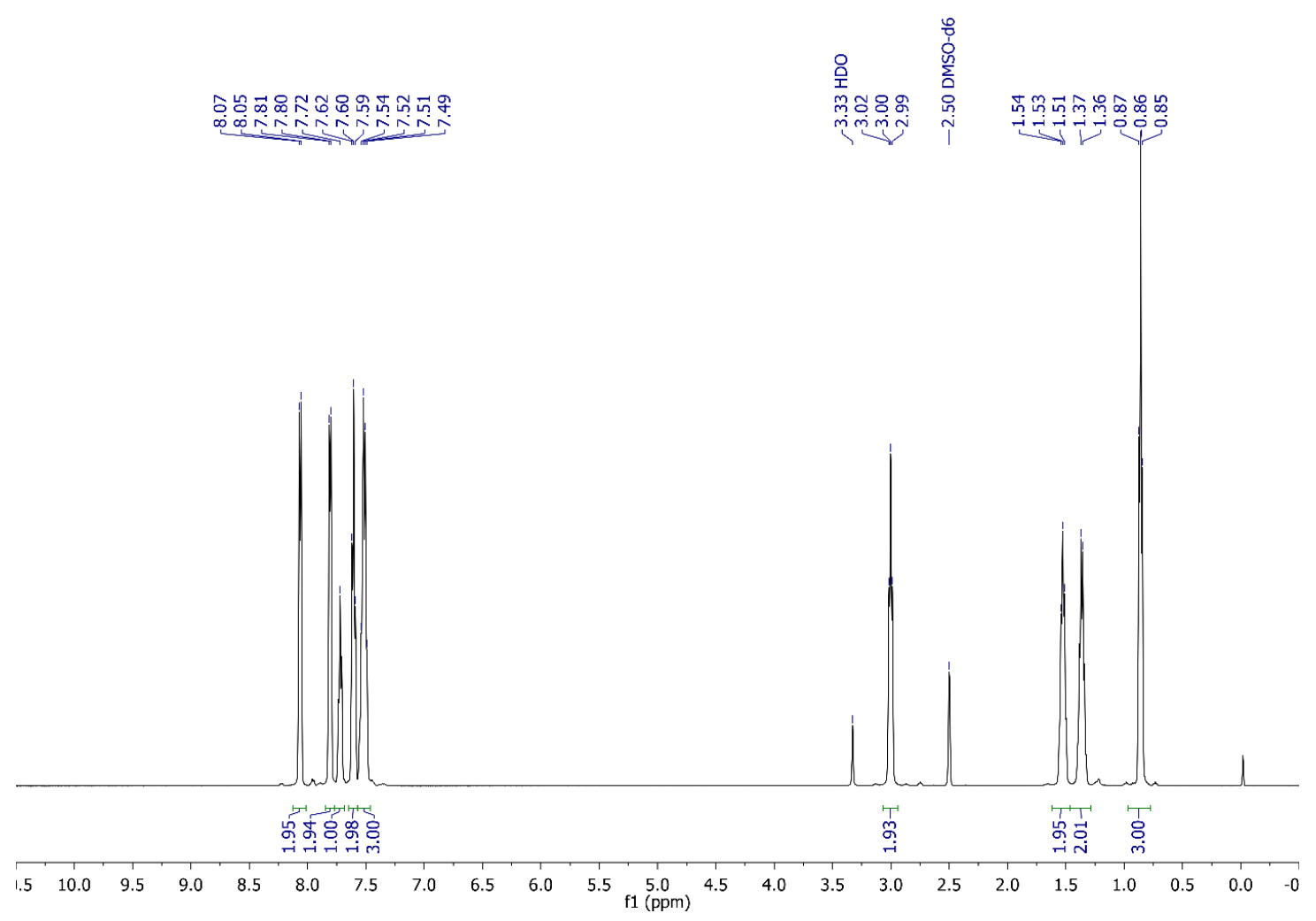

Figure S56. ${ }^{1} \mathrm{H}$ qNMR $(500 \mathrm{MHz})$ spectrum of 3a in DMSO- $d_{6}$.
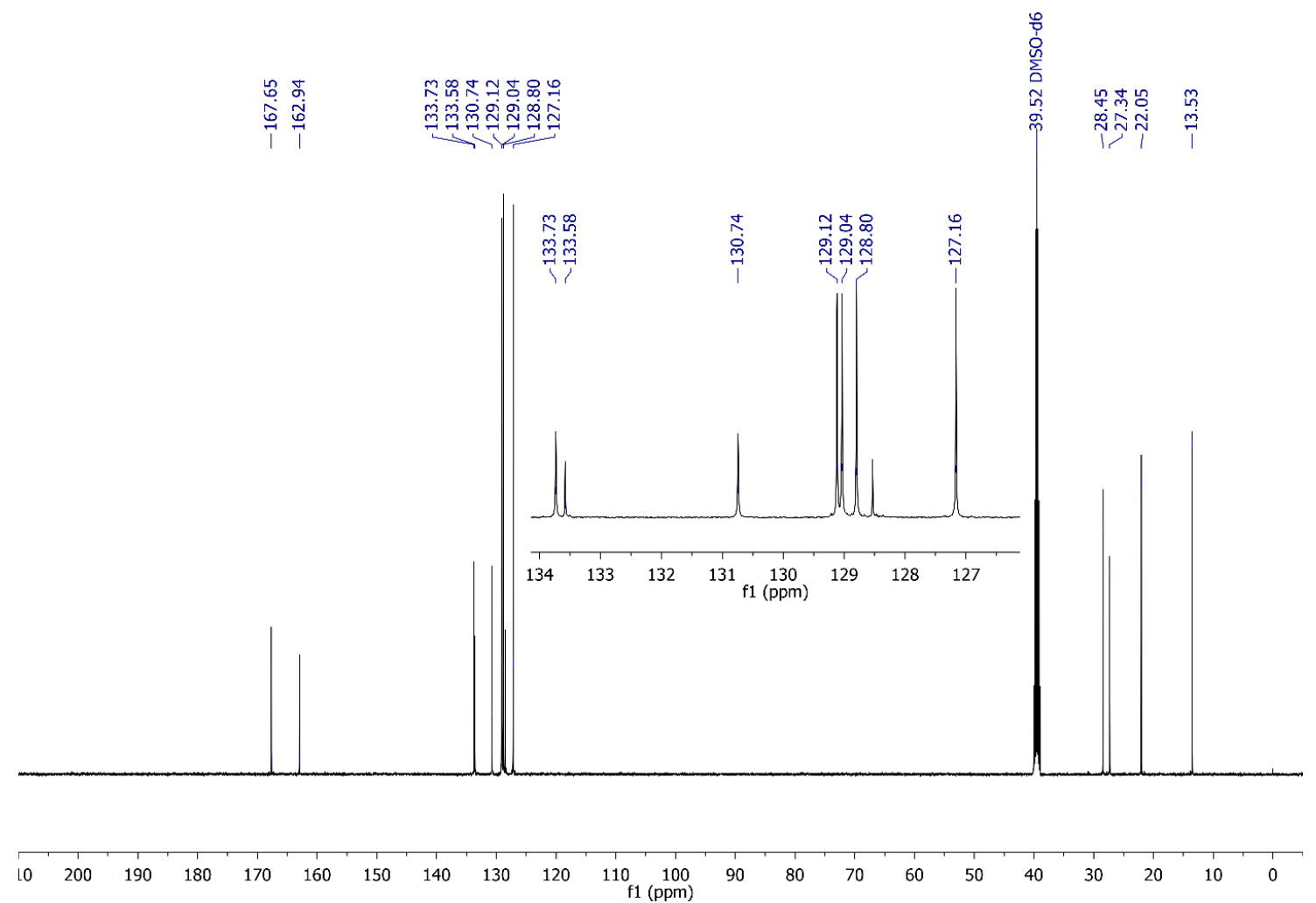

Figure S57. ${ }^{13} \mathrm{C}$ NMR $(126 \mathrm{MHz})$ spectrum of 3a in DMSO- $d_{6}$. 
<smiles>CC(=O)N=C(C)c1ccc(C)cc1</smiles>

Compound $3 \mathbf{f}$ was synthesized according to general method A using 4-methylacetophenone $(3.73 \mathrm{~mL}, 28$ $\mathrm{mmol})$, pyridine $(5.537 \mathrm{~mL}, 70 \mathrm{mmol})$, hydroxylamine hydrochloride $(2.918 \mathrm{~g}, 42 \mathrm{mmol})$, and ethanol $(80 \mathrm{~mL})$. Then the crude material of the first step was reacted with pyridine $(2.258 \mathrm{~mL}, 28 \mathrm{mmol})$, benzoyl chloride $(3.250 \mathrm{~mL}, 28$ $\mathrm{mmol})$, in methylene chloride $(17 \mathrm{~mL})$ and hexanes $(68 \mathrm{~mL})$ according to the general method. The crude product was recrystallized from $\mathrm{CH}_{2} \mathrm{Cl}_{2} / \mathrm{Hexanes}$ to afford the product as a white crystalline solid $(4.449 \mathrm{~g}, 63 \%)$. ${ }^{1} \mathrm{H}$ NMR (500 $\mathrm{MHz}$, DMSO- $\left.d_{6}\right) \delta 8.08(\mathrm{~d}, J=7.3 \mathrm{~Hz}, 2 \mathrm{H}), 7.75-7.69(\mathrm{~m}, 3 \mathrm{H}), 7.59(\mathrm{t}, J=7.7 \mathrm{~Hz}, 2 \mathrm{H}), 7.30(\mathrm{~d}, J=8.0 \mathrm{~Hz}, 2 \mathrm{H})$, $2.49(\mathrm{~s}, 3 \mathrm{H}), 2.36(\mathrm{~s}, 3 \mathrm{H}) .{ }^{13} \mathrm{C}$ NMR (126 MHz, DMSO- $\left.d_{6}\right) \delta 163.78,162.91,140.68,133.67,131.59,129.25,129.22$, 128.97, 128.61, 126.87, 20.89, 14.19. MS (ESI) $\mathrm{m} / z$ calcd. for $\mathrm{C}_{16} \mathrm{H}_{15} \mathrm{NO}_{2}\left([\mathrm{M}+\mathrm{H}]^{+}\right)$254.1, found $\left([\mathrm{M}+\mathrm{H}]^{+}\right)$254.2. 


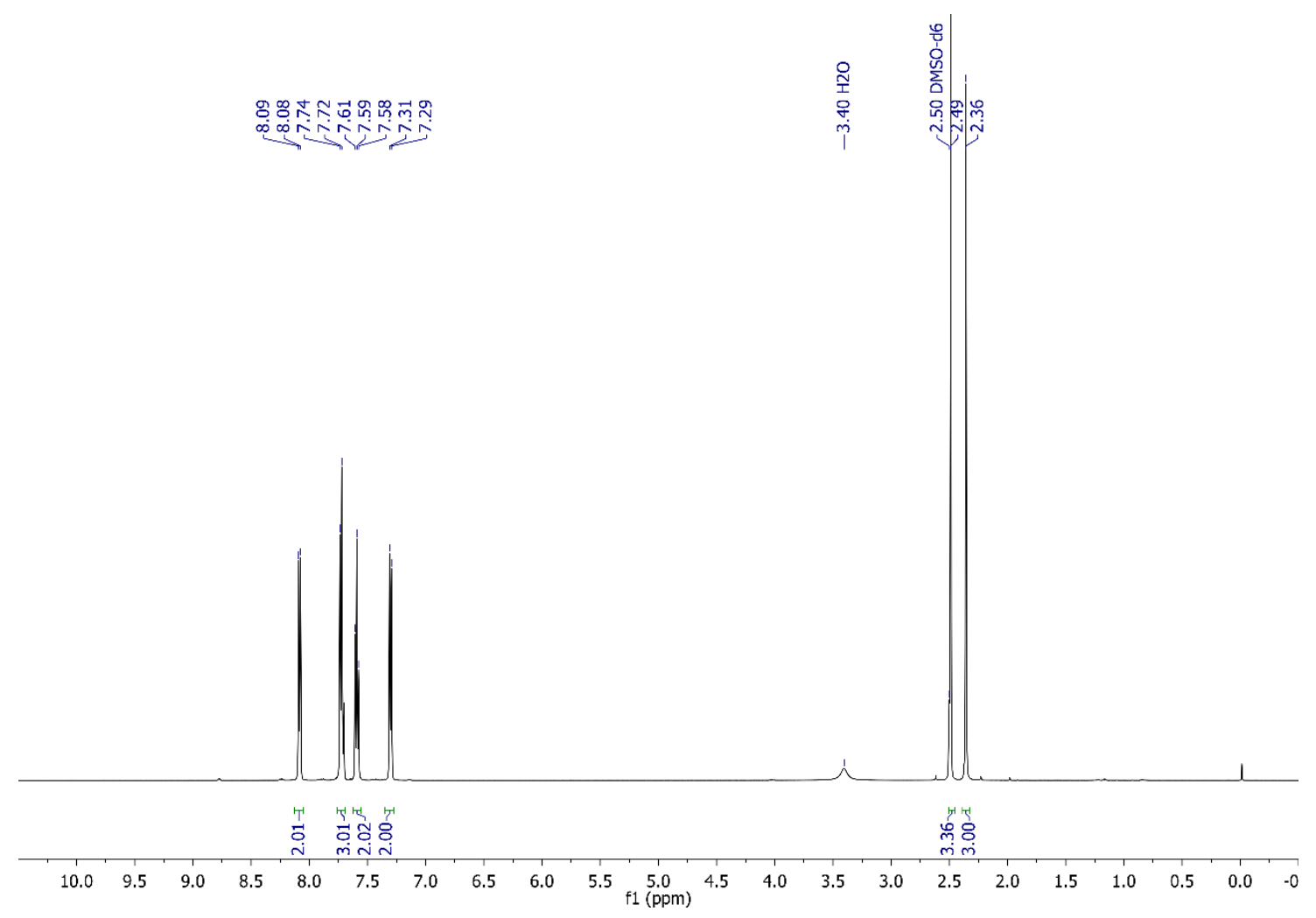

Figure S58. ${ }^{1} \mathrm{H}$ qNMR $(500 \mathrm{MHz})$ spectrum of $\mathbf{3 f}$ in DMSO- $d_{6}$.

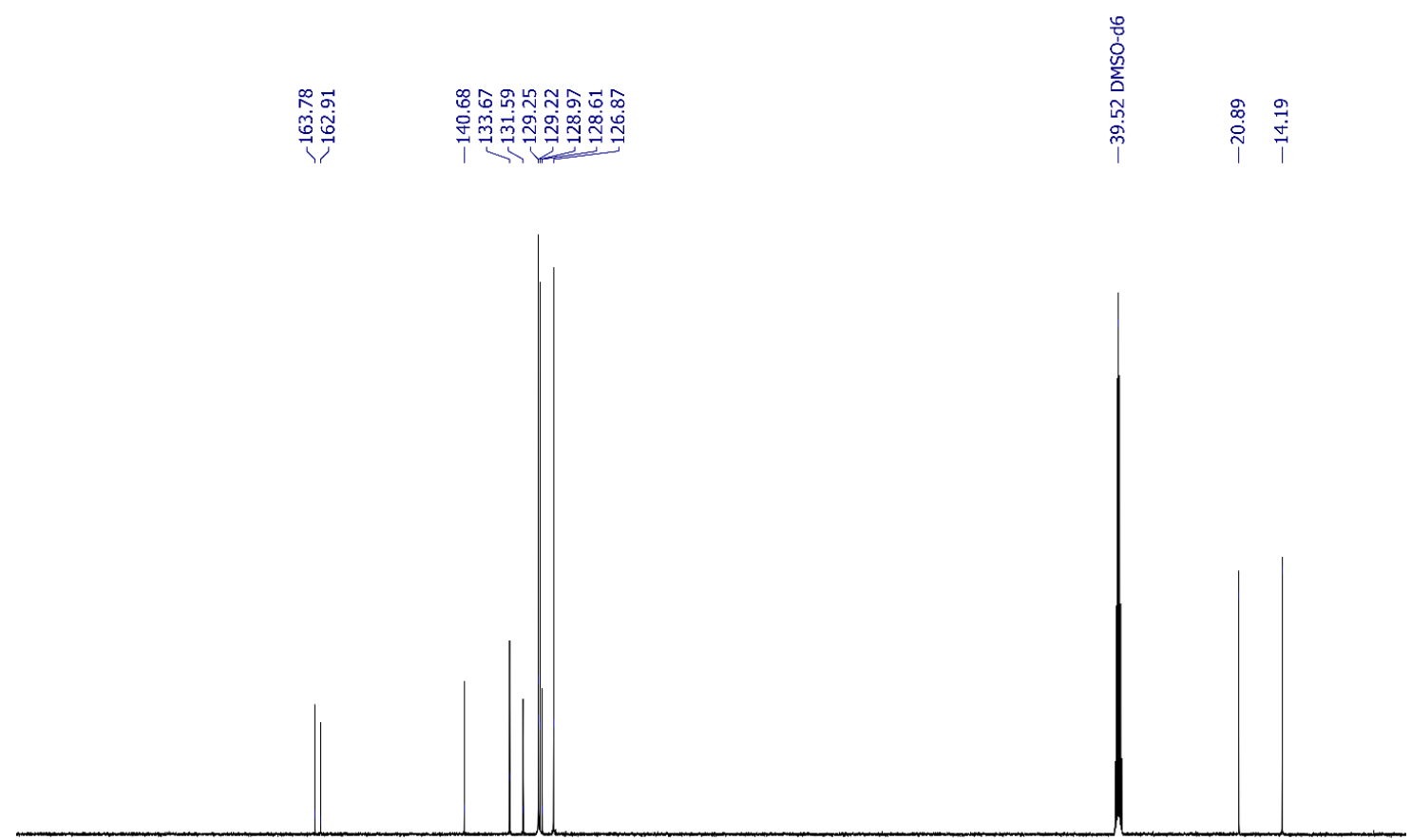

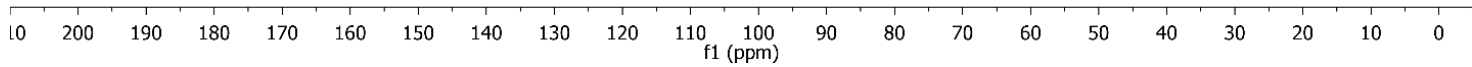

Figure S59. ${ }^{13} \mathrm{C}$ NMR $(126 \mathrm{MHz})$ spectrum of $\mathbf{3 f}$ in DMSO- $d_{6}$. 
<smiles>CC(=O)N=C(C)c1ccc(C(F)(F)F)cc1</smiles>

Compound $\mathbf{3 b}$ was synthesized according to general method A using 4-trifluoromethyl acetophenone (2.822 g, $15 \mathrm{mmol})$, pyridine (3.0 $\mathrm{mL}, 37.5 \mathrm{mmol})$, hydroxylamine hydrochloride $(1.564 \mathrm{~g}, 22.5 \mathrm{mmol})$, and ethanol $(30 \mathrm{~mL})$. Then the crude material of the first step was reacted with pyridine $(1.33 \mathrm{~mL}, 16.5 \mathrm{mmol})$, benzoyl chloride $(1.741 \mathrm{~mL}$, $15 \mathrm{mmol})$, in methylene chloride $(9 \mathrm{~mL})$ and hexanes $(24 \mathrm{~mL})$ according to the general method. The crude product was recrystallized from $\mathrm{CH}_{2} \mathrm{Cl}_{2} / \mathrm{Hexanes}$ to afford the product as a white crystalline solid $(1.41 \mathrm{~g}, 31 \%) .{ }^{1} \mathrm{H}$ NMR $\left(500 \mathrm{MHz}, \mathrm{DMSO}-d_{6}\right) \delta 8.10(\mathrm{~d}, J=7.3 \mathrm{~Hz}, 2 \mathrm{H}), 8.05(\mathrm{~d}, J=8.1 \mathrm{~Hz}, 2 \mathrm{H}), 7.87(\mathrm{~d}, J=8.2 \mathrm{~Hz}, 2 \mathrm{H}), 7.73(\mathrm{t}, J=7.4$ $\mathrm{Hz}, 1 \mathrm{H}), 7.60(\mathrm{t}, J=7.7 \mathrm{~Hz}, 2 \mathrm{H}), 2.57(\mathrm{~s}, 3 \mathrm{H}) .{ }^{13} \mathrm{C}$ NMR $\left(126 \mathrm{MHz}, \mathrm{DMSO}-d_{6}\right) \delta 163.10,162.75,138.47,133.88$, 130.80 (q, $J=31.9 \mathrm{~Hz}), 129.33,129.03,128.29,127.87,125.60$ (q, $J=3.8 \mathrm{~Hz}), 125.26$ (q, $J=272.7,271.6 \mathrm{~Hz})$, 14.41. ${ }^{19} \mathrm{~F}$ NMR (471 MHz, DMSO- $\left.d_{6}\right) \delta-61.41$. MS (ESI) $m / z$ calcd. for $\mathrm{C}_{16} \mathrm{H}_{12} \mathrm{~F}_{3} \mathrm{NO}_{2}\left([\mathrm{M}+\mathrm{H}]^{+}\right) 308.1$, found 308.1 


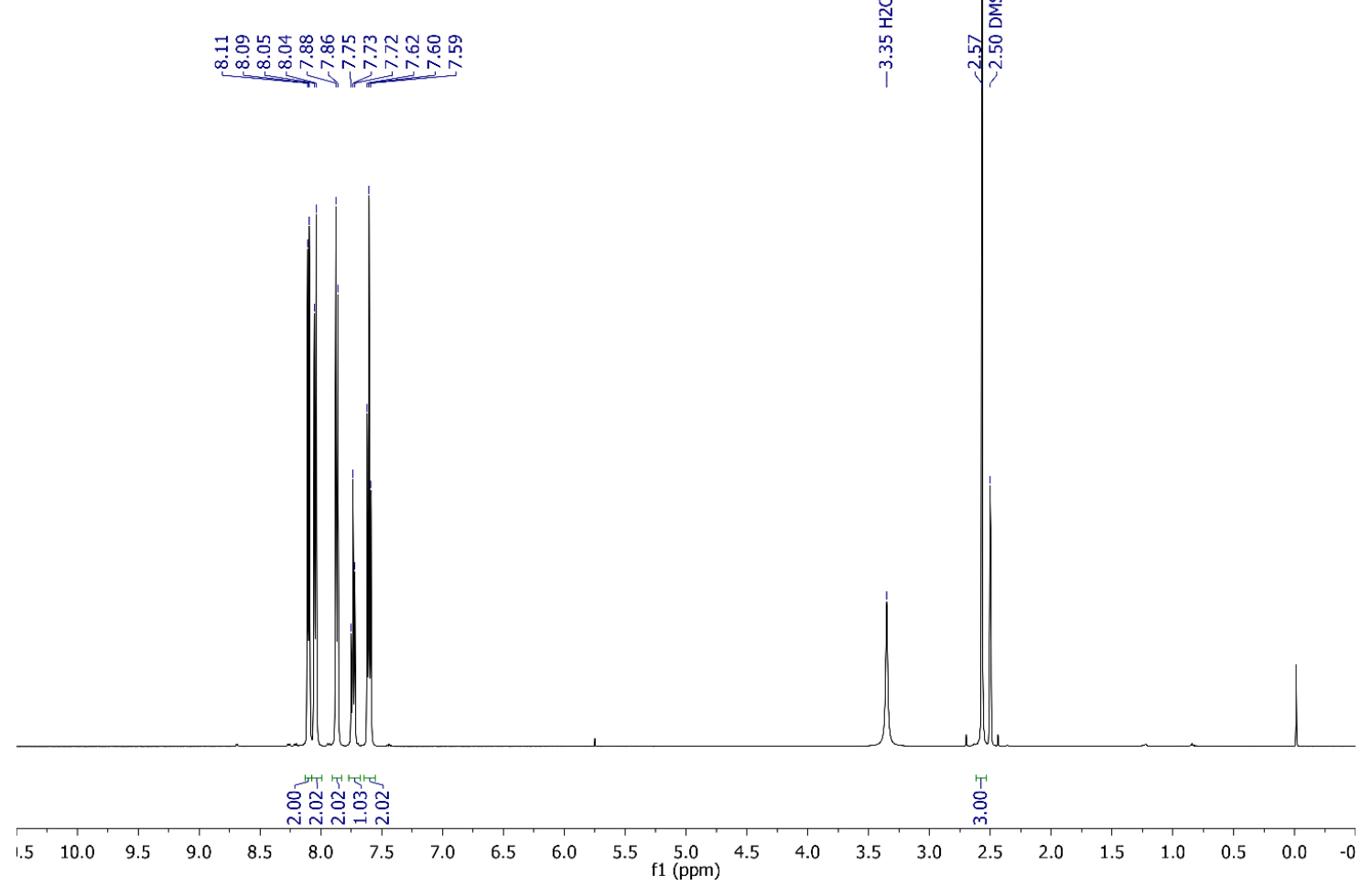

Figure S60. ${ }^{1} \mathrm{H}$ qNMR $(500 \mathrm{MHz})$ spectrum of compound $\mathbf{3 b}$ in DMSO- $d_{6}$
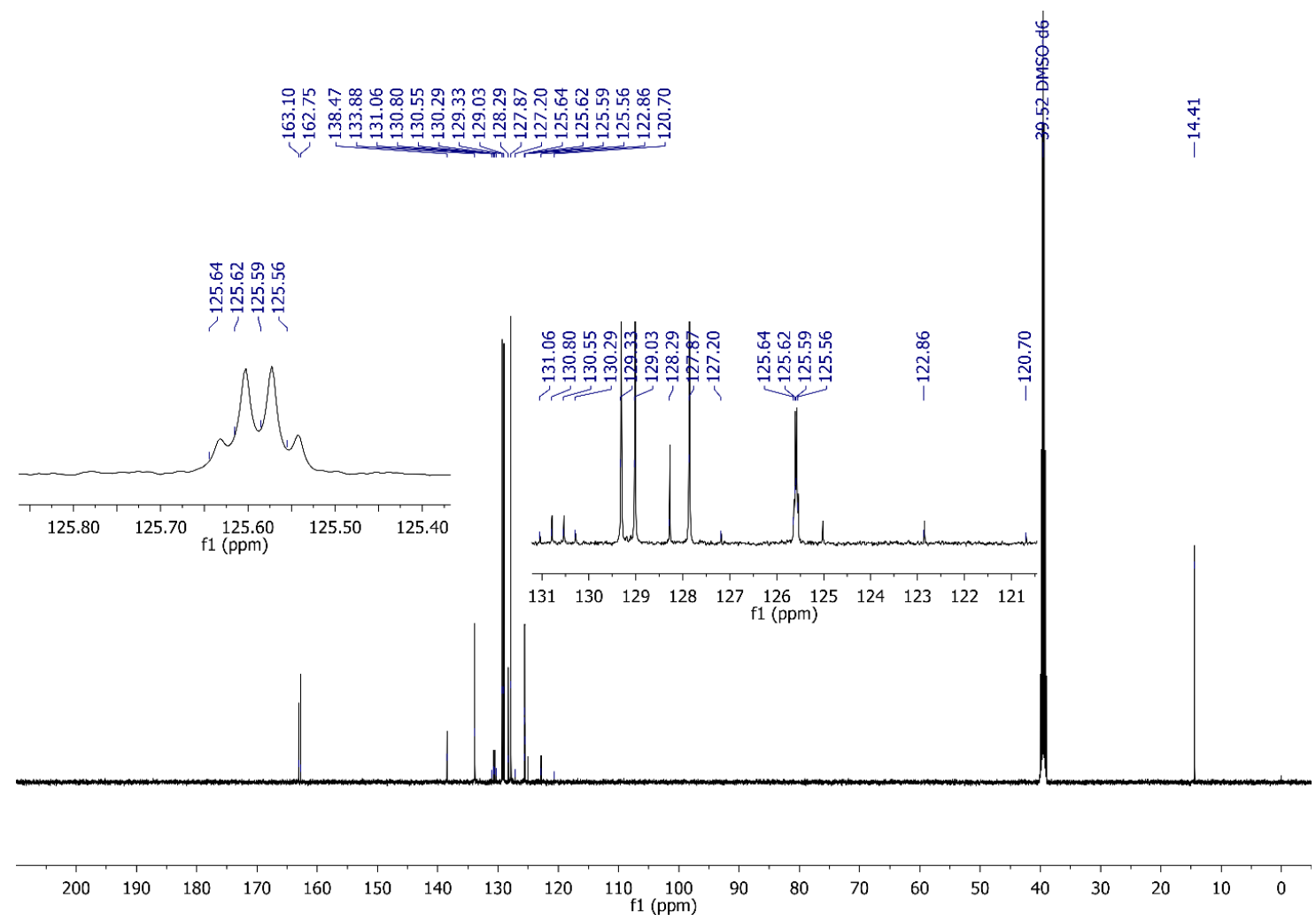

Figure S61. ${ }^{13} \mathrm{C}$ NMR $(126 \mathrm{MHz})$ spectrum of $\mathbf{3 b}$ in DMSO- $d_{6}$. 


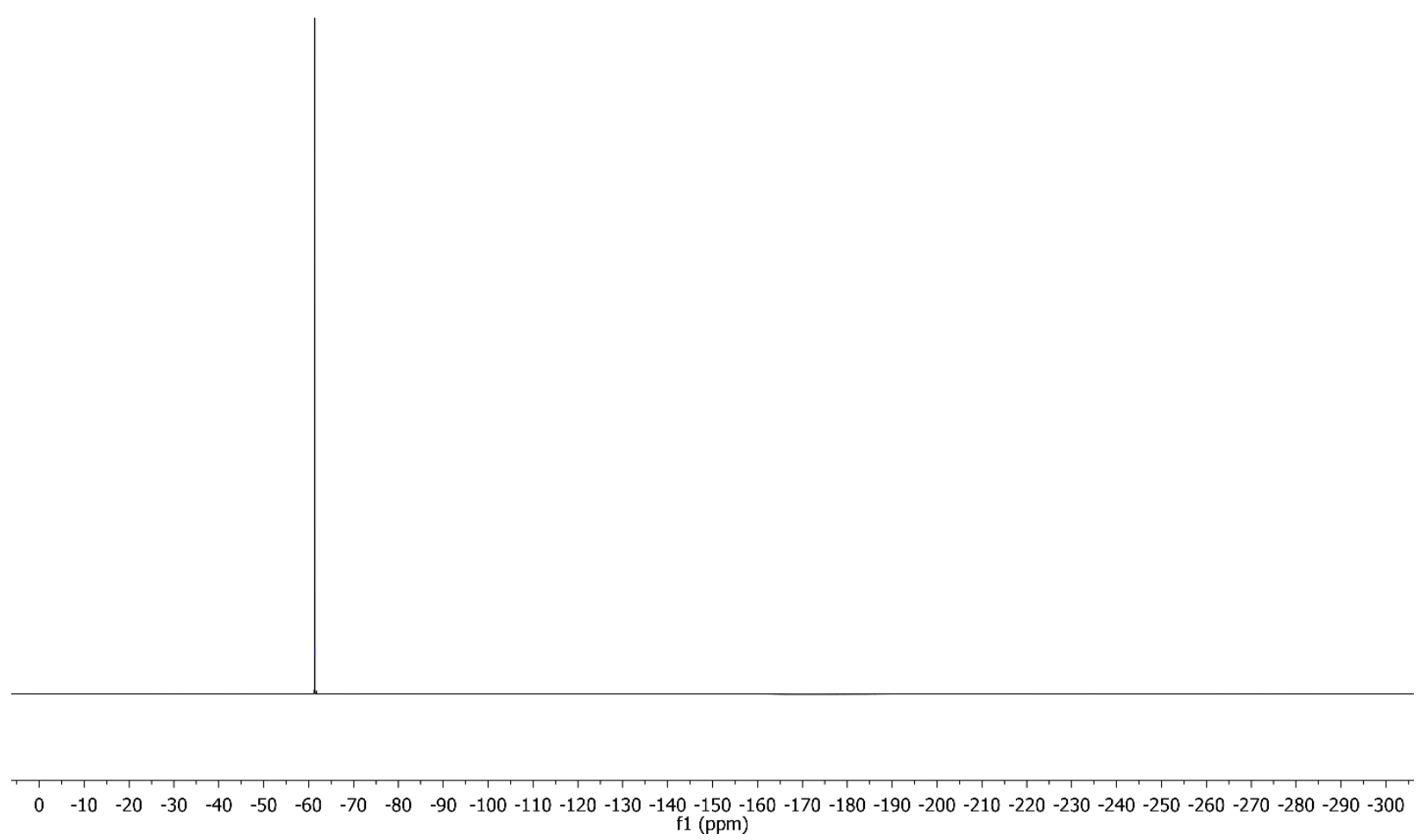

Figure S62. ${ }^{19} \mathrm{~F}$ NMR $(471 \mathrm{MHz})$ spectrum of compound $\mathbf{3 b}$ in DMSO- $d_{6}$ 
<smiles>CC(=O)N=C(C)c1ccc(F)cc1</smiles>

Compound 3d was synthesized according to general method A using 4-fluoroacetophenone $(1.381 \mathrm{~g}, 10$ $\mathrm{mmol})$, pyridine $(2.013 \mathrm{~mL}, 25 \mathrm{mmol})$, hydroxylamine hydrochloride $(1.042 \mathrm{~g}, 15 \mathrm{mmol})$, and ethanol $(20 \mathrm{~mL})$. Then the crude material of the first step was reacted with pyridine $(0.806 \mathrm{~mL}, 10 \mathrm{mmol})$, benzoyl chloride $(1.16 \mathrm{~mL}, 10$ $\mathrm{mmol})$, in methylene chloride $(6 \mathrm{~mL})$ hexanes $(24 \mathrm{~mL})$ according to the general method. The crude product was recrystallized from $\mathrm{CH}_{2} \mathrm{Cl}_{2} / \mathrm{Hexanes}$ to afford the product as a white crystalline solid $(1.311 \mathrm{~g}, 51 \%)$.

${ }^{1} \mathrm{H}$ NMR $\left(500 \mathrm{MHz}, \mathrm{DMSO}-d_{6}\right) \delta 8.09(\mathrm{~d}, J=7.6 \mathrm{~Hz}, 2 \mathrm{H}), 7.94-7.87(\mathrm{~m}, 2 \mathrm{H}), 7.73(\mathrm{t}, J=6.8 \mathrm{~Hz}, 1 \mathrm{H}), 7.60(\mathrm{t}, J=$ $7.6 \mathrm{~Hz}, 2 \mathrm{H}), 7.34(\mathrm{t}, J=8.8 \mathrm{~Hz}, 2 \mathrm{H}), 2.52(\mathrm{~s}, 3 \mathrm{H}) .{ }^{13} \mathrm{C}$ NMR $\left(126 \mathrm{MHz}, \mathrm{DMSO}-d_{6}\right) \delta 164.61,163.07,162.84,162.63$, 133.73, 130.93, 130.90, 129.44, 129.37, 129.24, 128.97, 128.46, 115.77, 115.60, 14.33. MS (ESI) m/z calcd. for $\mathrm{C}_{15} \mathrm{H}_{12} \mathrm{FNO}_{2}\left([\mathrm{M}+\mathrm{H}]^{+}\right)$258.1, found 258.3 


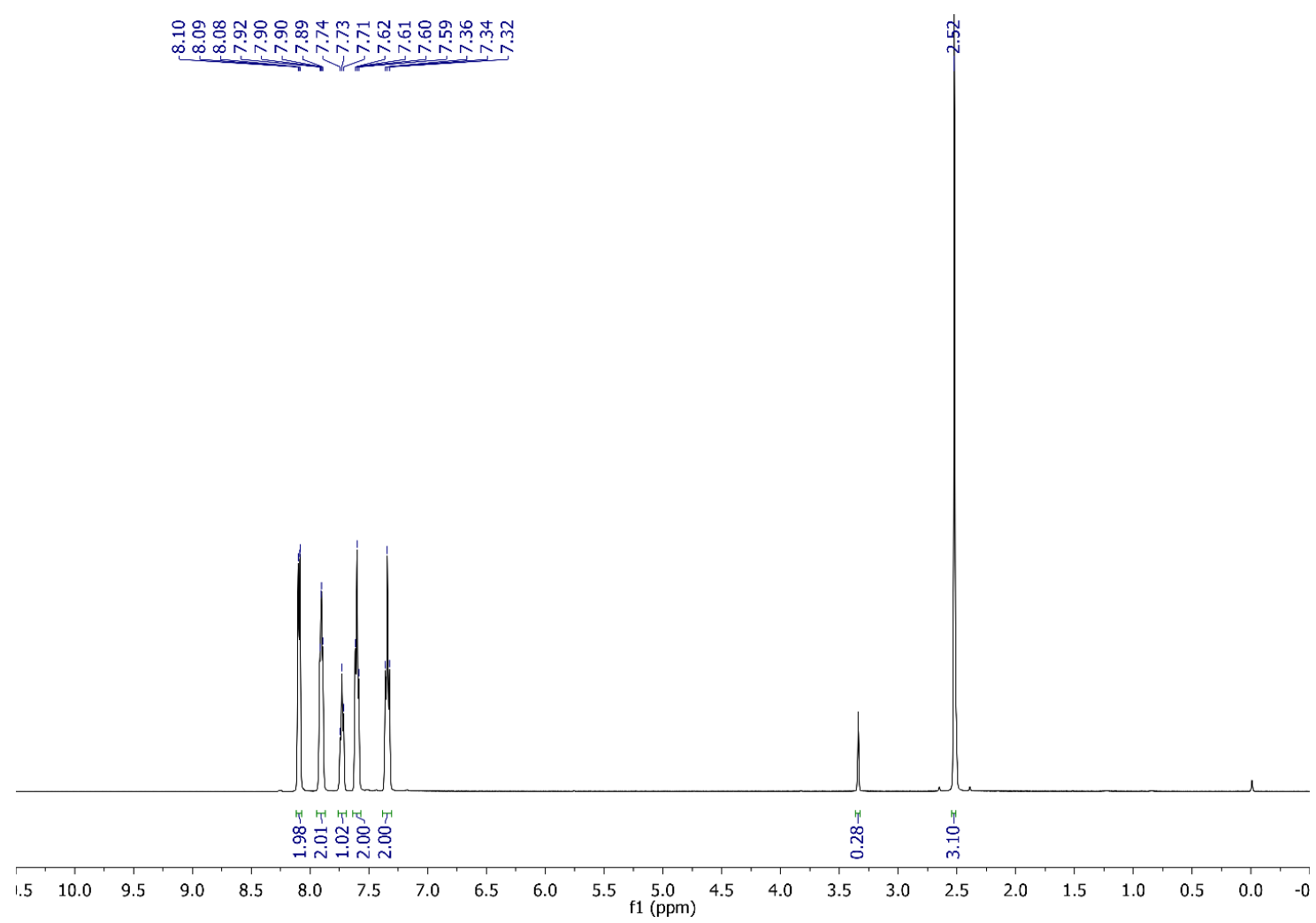

Figure S63. ${ }^{1} \mathrm{H}$ qNMR $(500 \mathrm{MHz})$ spectrum of compound 3d in DMSO- $d_{6}$
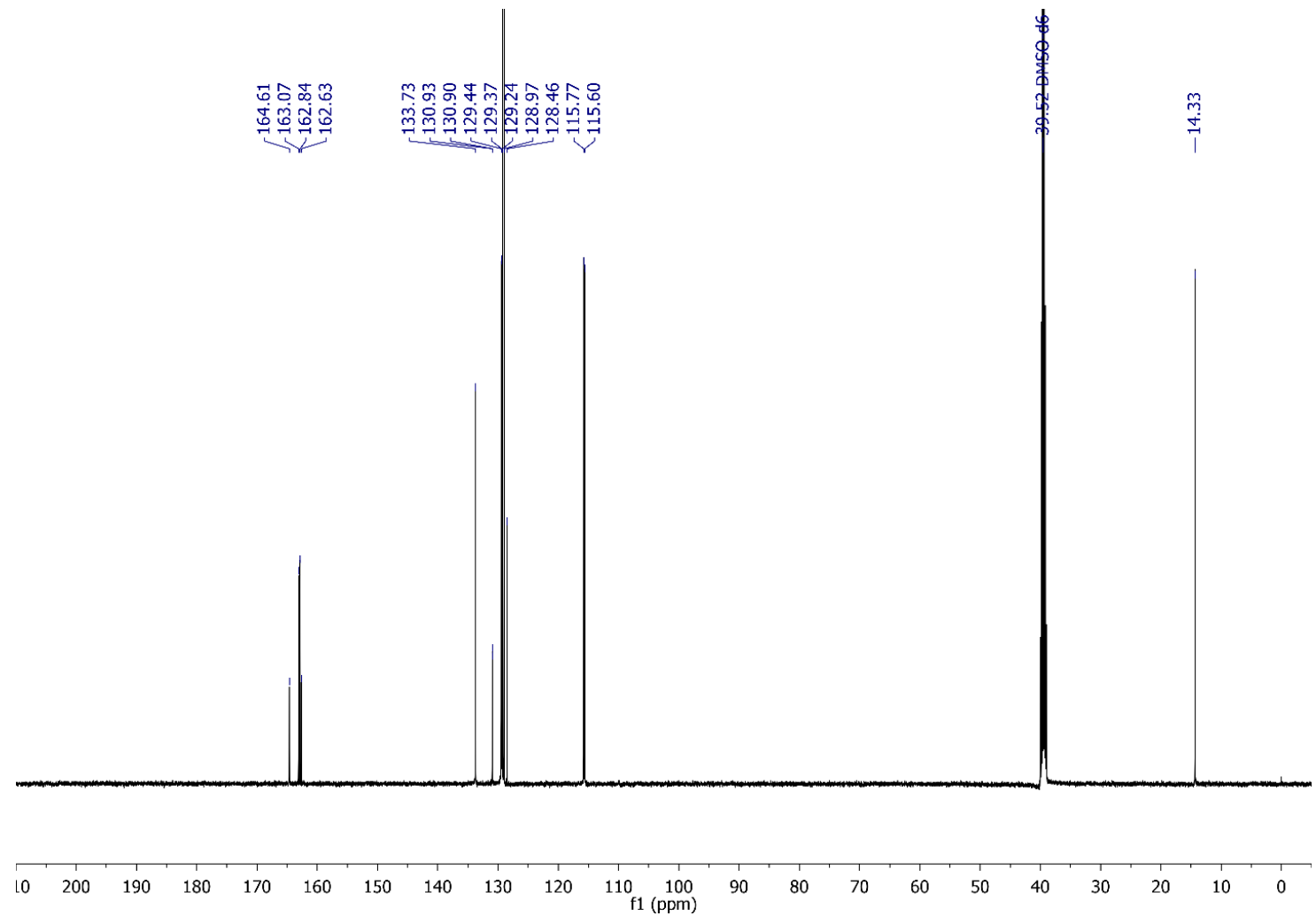

Figure S64. ${ }^{13} \mathrm{C}$ NMR $(126 \mathrm{MHz})$ spectrum of $\mathbf{3 d}$ in DMSO- $d_{6}$. 
<smiles>CC(=O)N=C(C)c1ccc(Cl)cc1</smiles>

Compound 3c was synthesized according to general method A using 4-chloroacetophenone $(3.242 \mathrm{~mL}, 25$ $\mathrm{mmol})$, pyridine $(5.03 \mathrm{~mL}, 62.5 \mathrm{mmol})$, hydroxylamine hydrochloride $(2.61 \mathrm{~g}, 37.5 \mathrm{mmol})$, and ethanol $(50 \mathrm{~mL})$. Then the crude material of the first step was reacted with pyridine $(1.582 \mathrm{~mL}, 20 \mathrm{mmol})$, benzoyl chloride $(2.321 \mathrm{~mL}, 20$ $\mathrm{mmol})$, in methylene chloride $(12 \mathrm{~mL})$ and hexanes $(48 \mathrm{~mL})$ according to the general method. The crude product was recrystallized from $\mathrm{CH}_{2} \mathrm{Cl}_{2} / \mathrm{Hexanes}$ to afford the product as a white crystalline solid (2.556 g, 47\%). ${ }^{1} \mathrm{H}$ NMR (500 MHz, DMSO- $\left.d_{6}\right) \delta 8.09$ (d, $\left.J=9.9 \mathrm{~Hz}, 2 \mathrm{H}\right), 7.86(\mathrm{~d}, J=8.5 \mathrm{~Hz}, 2 \mathrm{H}), 7.73(\mathrm{t}, J=6.8 \mathrm{~Hz}, 1 \mathrm{H}), 7.64-7.53(\mathrm{~m}, 4 \mathrm{H})$, $2.52(\mathrm{~s}, 3 \mathrm{H}) .{ }^{13} \mathrm{C}$ NMR $\left(126 \mathrm{MHz}\right.$, DMSO- $\left.d_{6}\right) \delta 163.06,162.78,135.61,133.78,133.27,129.26,128.99,128.77(2 \mathrm{C})$, 128.39, 14.23. MS (ESI) $m / z$ calcd for $\mathrm{C}_{15} \mathrm{H}_{14} \mathrm{NO}_{2}\left([\mathrm{M}+\mathrm{H}]^{+}\right)$274.1, found 274.1. 


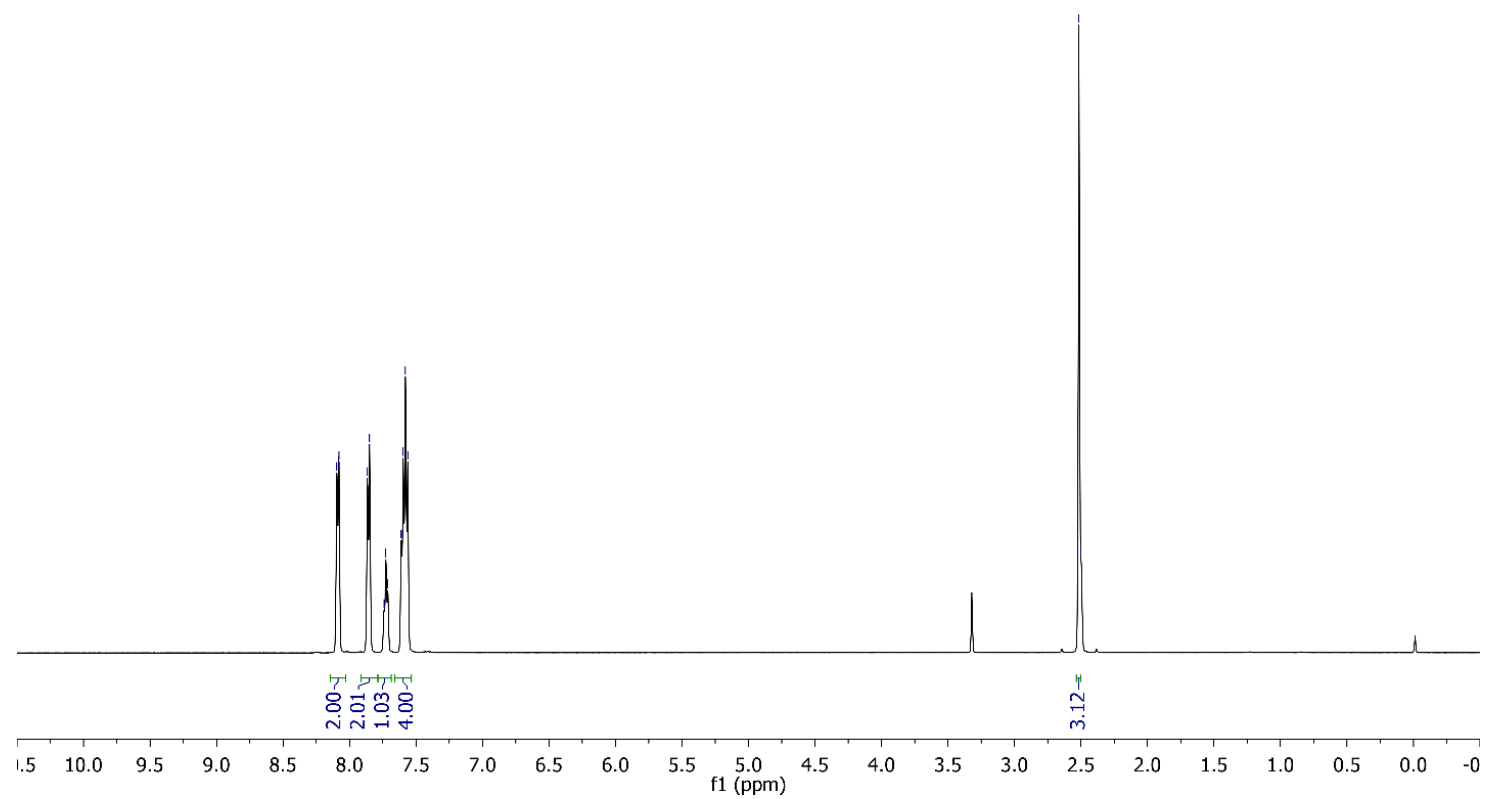

Figure S65. ${ }^{1} \mathrm{H}$ qNMR (500 MHz) spectrum of compound $\mathbf{3 c}$ in DMSO- $d_{6}$

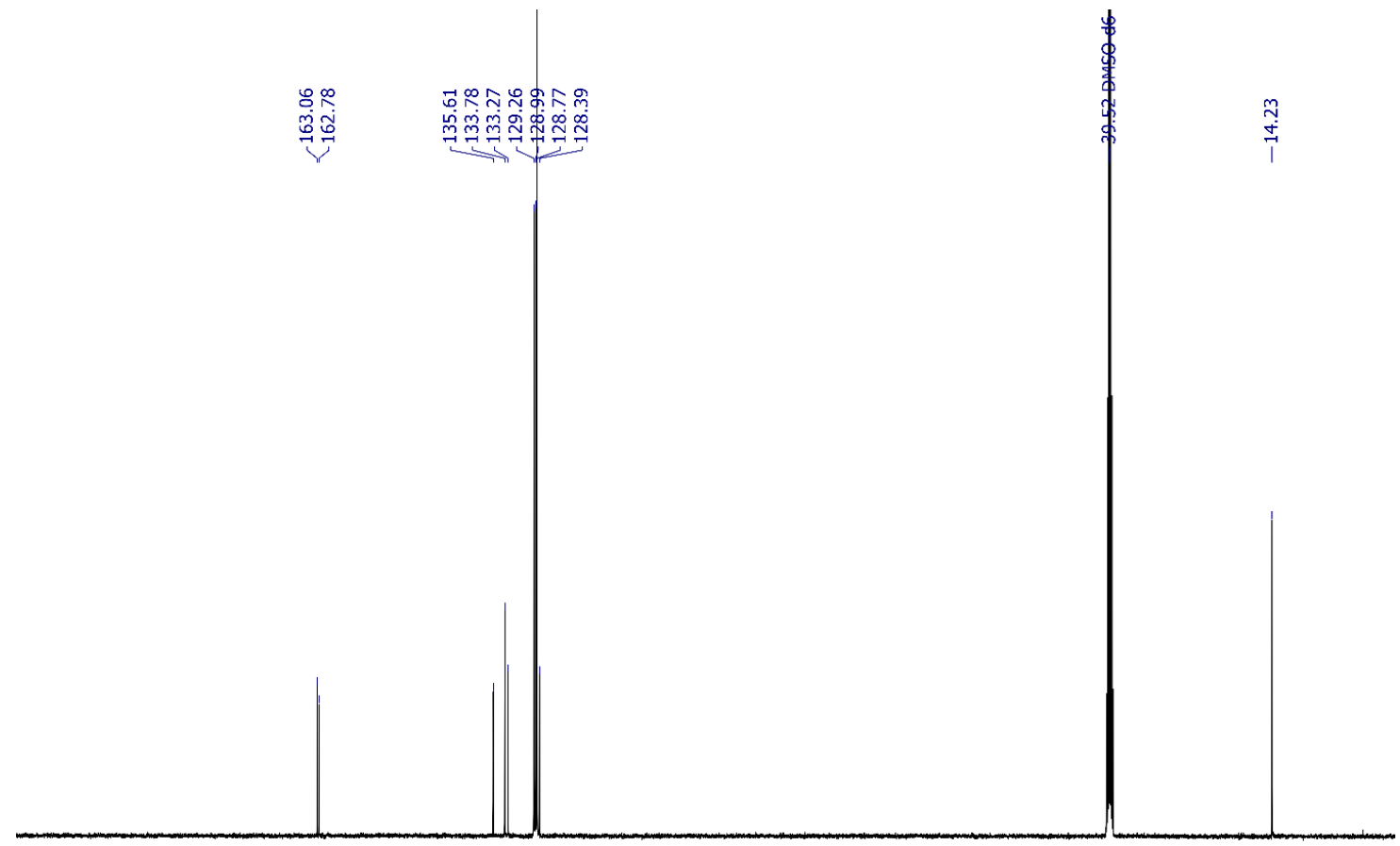

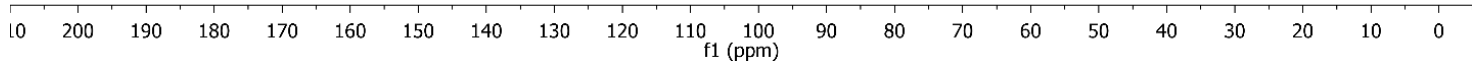

Figure S66. ${ }^{13} \mathrm{C}$ NMR $(126 \mathrm{MHz})$ spectrum of $3 \mathbf{c}$ in DMSO- $d_{6}$. 
Me

Compound 3g was synthesized according to general method A using 4-methoxyacetophenone(4.505 g, 18 $\mathrm{mmol})$, pyridine $(6 \mathrm{~mL}, 27 \mathrm{mmol})$, hydroxylamine hydrochloride $(3.12 \mathrm{~g}, 45 \mathrm{mmol})$, and ethanol $(60 \mathrm{~mL})$. Then the crude material of the first step $(3.3 \mathrm{~g})$ was reacted with pyridine $(1.61 \mathrm{~mL}, 20 \mathrm{mmol})$, benzoyl chloride $(2.32 \mathrm{~mL}, 20$ $\mathrm{mmol})$, in methylene chloride $(12 \mathrm{~mL})$ and hexanes $(49 \mathrm{~mL})$ according to the general method. The crude product was recrystallized from $\mathrm{CH}_{2} \mathrm{Cl}_{2} / \mathrm{Hexanes}$ to afford the product as a white crystalline solid $(3.35 \mathrm{~g}, 62 \%)$. ${ }^{1} \mathrm{H}$ NMR $(500$ MHz, DMSO- $\left.d_{6}\right) \delta 8.08(\mathrm{~d}, J=7.9 \mathrm{~Hz}, 2 \mathrm{H}), 7.94-7.77(\mathrm{~m}, 2 \mathrm{H}), 7.71(\mathrm{t}, J=6.8 \mathrm{~Hz}, 1 \mathrm{H}), 7.59(\mathrm{t}, J=7.1 \mathrm{~Hz}, 2 \mathrm{H})$, $7.04(\mathrm{~d}, J=8.6 \mathrm{~Hz}, 2 \mathrm{H}), 3.82(\mathrm{~s}, 3 \mathrm{H}), 2.48(\mathrm{~s}, 3 \mathrm{H}) .{ }^{13} \mathrm{C}$ NMR $\left(126 \mathrm{MHz}, \mathrm{DMSO}-d_{6}\right) \delta 163.31,162.94,161.32,133.62$, $129.18,128.95,128.67,128.54,126.51,114.06,55.31,14.04$. MS (ESI) $m / z$ calcd for $\mathrm{C}_{16} \mathrm{H}_{16} \mathrm{NO}_{3}\left([\mathrm{M}+\mathrm{H}]^{+}\right) 270.1$, found 270.2 


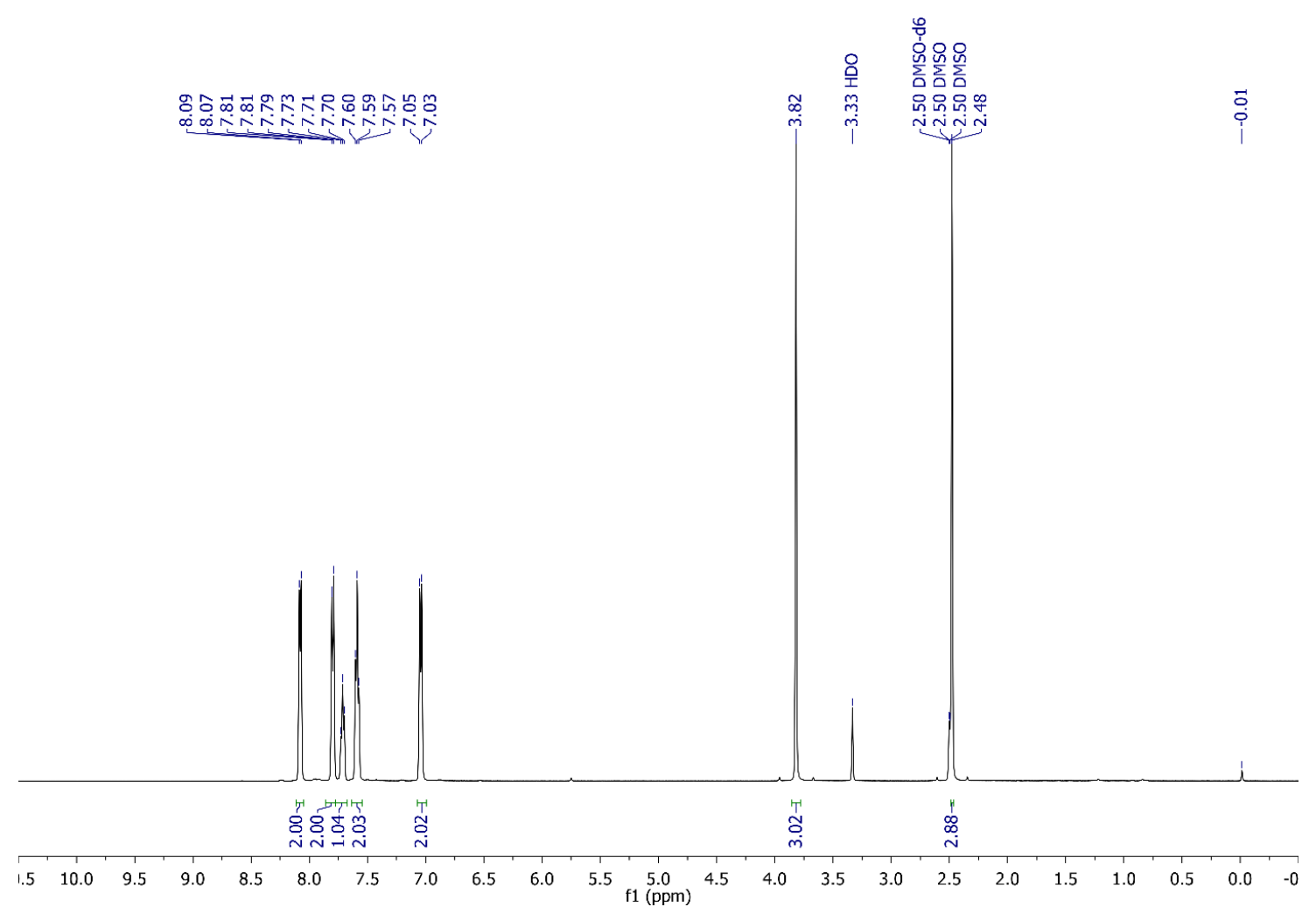

Figure S67. ${ }^{1} \mathrm{H}$ qNMR $(500 \mathrm{MHz})$ spectrum of compound $\mathbf{3 g}$ in DMSO- $d_{6}$

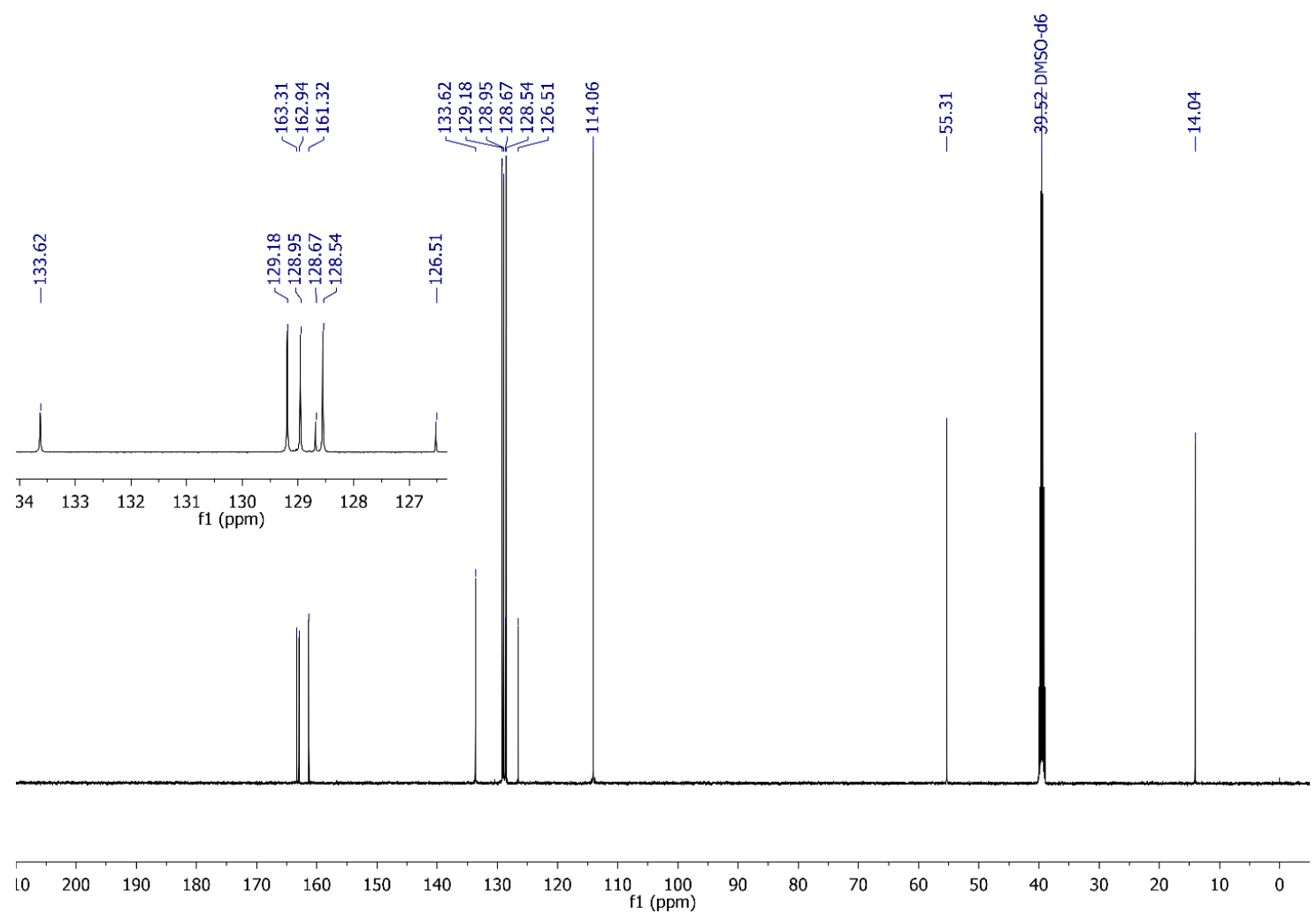

Figure S68. ${ }^{13} \mathrm{C}$ NMR $(126 \mathrm{MHz})$ spectrum of $\mathbf{3 g}$ in DMSO- $d_{6}$. 
<smiles>CCCCCCCC(=O)OC(C)(C)C</smiles>

Compound $3 \mathrm{I}$ was synthesized according to general method A using valerophenone (4.597 g, $28 \mathrm{mmol})$, pyridine $(5.64 \mathrm{~mL}, 70 \mathrm{mmol})$, hydroxylamine hydrochloride $(2.92 \mathrm{~g}, 42 \mathrm{mmol})$, and ethanol $(56 \mathrm{~mL})$. Then the crude material of the first step was reacted with pyridine $(2.258 \mathrm{~mL}, 28 \mathrm{mmol})$, benzoyl chloride $(3.25 \mathrm{~mL}, 28 \mathrm{mmol})$, in methylene chloride $(17 \mathrm{~mL})$ and hexanes $(68 \mathrm{~mL})$ according to the General Method A. The crude product was recrystallized from $\mathrm{CH}_{2} \mathrm{Cl}_{2} / \mathrm{Hexanes}$ to afford the product as a white crystalline solid $(6.09 \mathrm{~g}, 77 \%)$. ${ }^{1} \mathrm{H}$ NMR $(500$ MHz, DMSO- $\left.d_{6}\right) \delta 8.06(\mathrm{~d}, J=7.9 \mathrm{~Hz}, 2 \mathrm{H}), 7.81(\mathrm{~d}, J=7.7 \mathrm{~Hz}, 2 \mathrm{H}), 7.72(\mathrm{t}, J=6.8 \mathrm{~Hz}, 1 \mathrm{H}), 7.60(\mathrm{t}, J=7.4 \mathrm{~Hz}, 2 \mathrm{H})$, $7.51(\mathrm{q}, J=8.1,6.1 \mathrm{~Hz}, 3 \mathrm{H}), 3.00(\mathrm{t}, J=7.4 \mathrm{~Hz}, 2 \mathrm{H}), 1.60-1.48(\mathrm{~m}, 2 \mathrm{H}), 1.36(\mathrm{q}, J=7.1 \mathrm{~Hz}, 2 \mathrm{H}), 0.86(\mathrm{t}, J=7.2$ $\mathrm{Hz}, 3 \mathrm{H}) .{ }^{13} \mathrm{C}$ NMR $\left(126 \mathrm{MHz}\right.$, DMSO- $\left.d_{6}\right) \delta 167.65,162.94,133.73,133.58,130.74,129.12,129.04,128.80,127.16$, 28.45, 27.34, 22.05, 13.53. MS (ESI) $m / z$ calcd. for $\mathrm{C}_{18} \mathrm{H}_{20} \mathrm{NO}_{2}\left([\mathrm{M}+\mathrm{H}]^{+}\right) 280.1$, found 280.2 


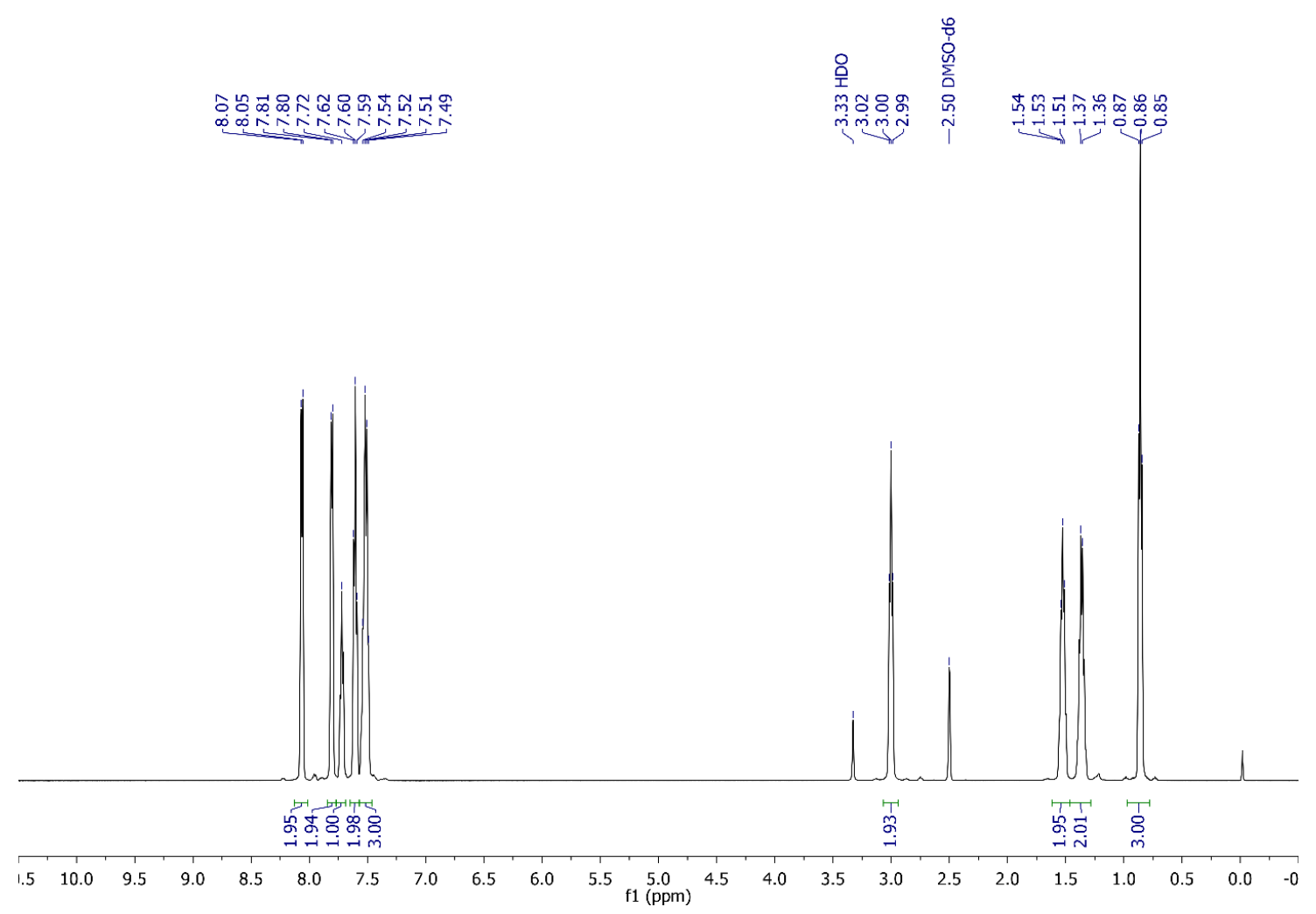

Figure S69. ${ }^{1} \mathrm{H}$ qNMR $(500 \mathrm{MHz})$ spectrum of compound 31 in DMSO- $d_{6}$

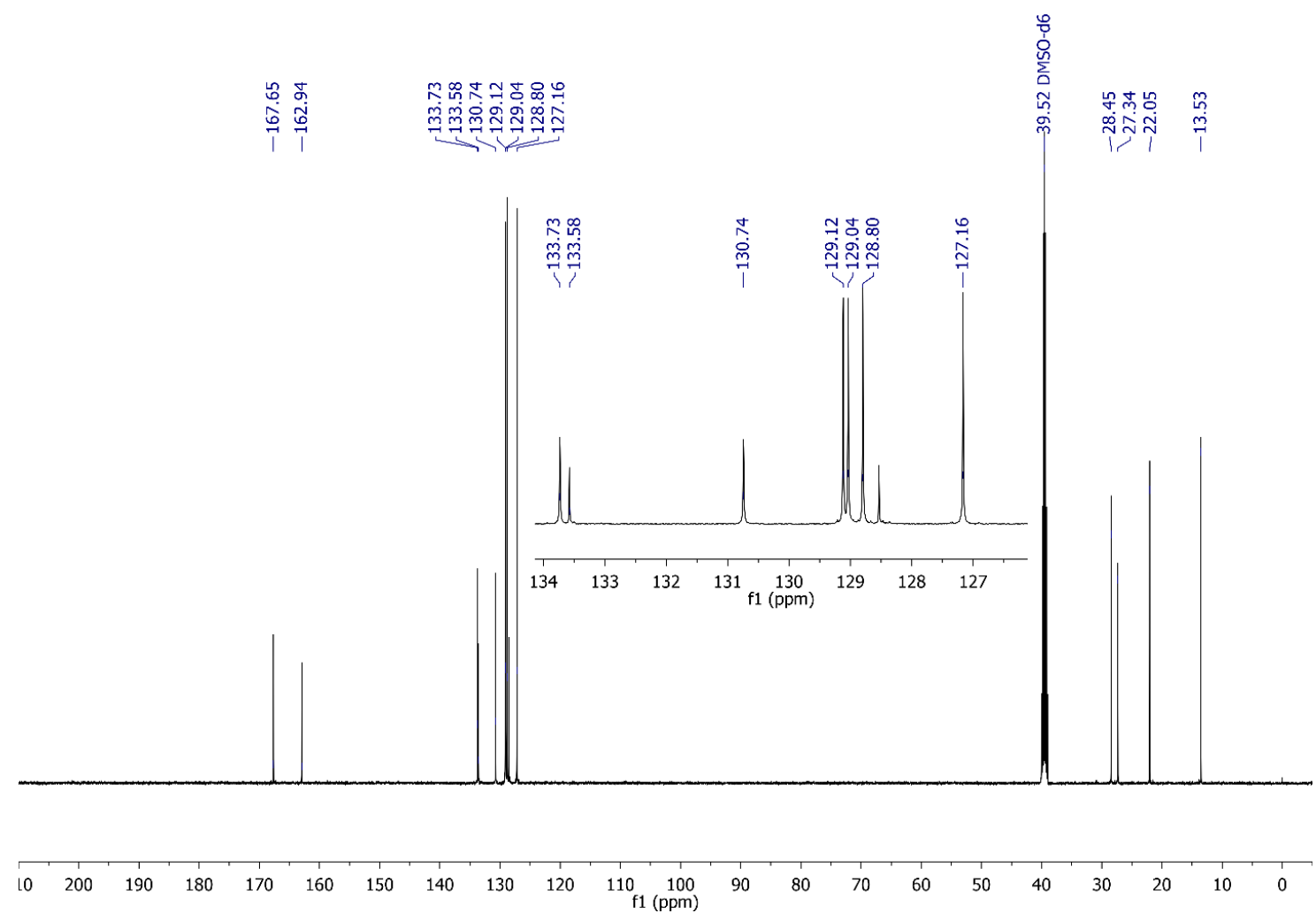

Figure S70. ${ }^{13} \mathrm{C}$ NMR $(126 \mathrm{MHz})$ spectrum of 31 in DMSO- $d_{6}$. 


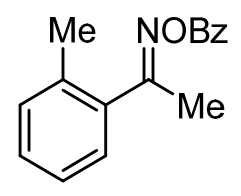

Compound $3 \mathbf{h}$ was synthesized according to general method A using 2-methylacetophenone $(3.9 \mathrm{~mL}, 30$ $\mathrm{mmol})$, pyridine $(6.0 \mathrm{~mL}, 75 \mathrm{mmol})$, hydroxylamine hydrochloride $(3.13 \mathrm{~g}, 45 \mathrm{mmol})$, and ethanol $(60 \mathrm{~mL})$. Then the crude material of the first step was reacted with pyridine $(2.39 \mathrm{~mL}, 29.6 \mathrm{mmol})$, benzoyl chloride $(3.44 \mathrm{~mL}, 29.6$ $\mathrm{mmol})$, in methylene chloride $(18 \mathrm{~mL})$ and hexanes $(72 \mathrm{~mL})$ according to the General Method A. The crude product was recrystallized from $\mathrm{CH}_{2} \mathrm{Cl}_{2} / \mathrm{Hexanes}$, filtered cold $\left(-20^{\circ} \mathrm{C}\right)$ to afford the product as a beige solid $(5.74 \mathrm{~g}, 76 \%)$. ${ }^{1} \mathrm{H}$ NMR $\left(500 \mathrm{MHz}, \mathrm{DMSO}-d_{6}\right) \delta 8.09(\mathrm{~d}, J=8.5 \mathrm{~Hz}, 2 \mathrm{H}), 7.72(\mathrm{t}, J=6.8 \mathrm{~Hz}, 1 \mathrm{H}), 7.60(\mathrm{t}, J=7.5 \mathrm{~Hz}, 2 \mathrm{H}), 7.38(\mathrm{dt}$, $J=7.8,1.6,1.1 \mathrm{~Hz}, 2 \mathrm{H}), 7.34-7.27(\mathrm{~m}, 2 \mathrm{H}), 2.45(\mathrm{~s}, 3 \mathrm{H}), 2.39(\mathrm{~s}, 3 \mathrm{H}) .{ }^{13} \mathrm{C}$ NMR $\left(126 \mathrm{MHz}, \mathrm{DMSO}-d_{6}\right) \delta 166.53$, $162.90,135.42,135.39,133.67,130.67,129.37,129.22,128.95,128.58,128.15,125.87,19.68,17.84$. MS (ESI) $\mathrm{m} / \mathrm{z}$ calcd. for $\mathrm{C}_{16} \mathrm{H}_{16} \mathrm{NO}_{2}\left([\mathrm{M}+\mathrm{H}]^{+}\right) 254.1$, found $\left([\mathrm{M}+\mathrm{H}]^{+}\right) 254.2$ 


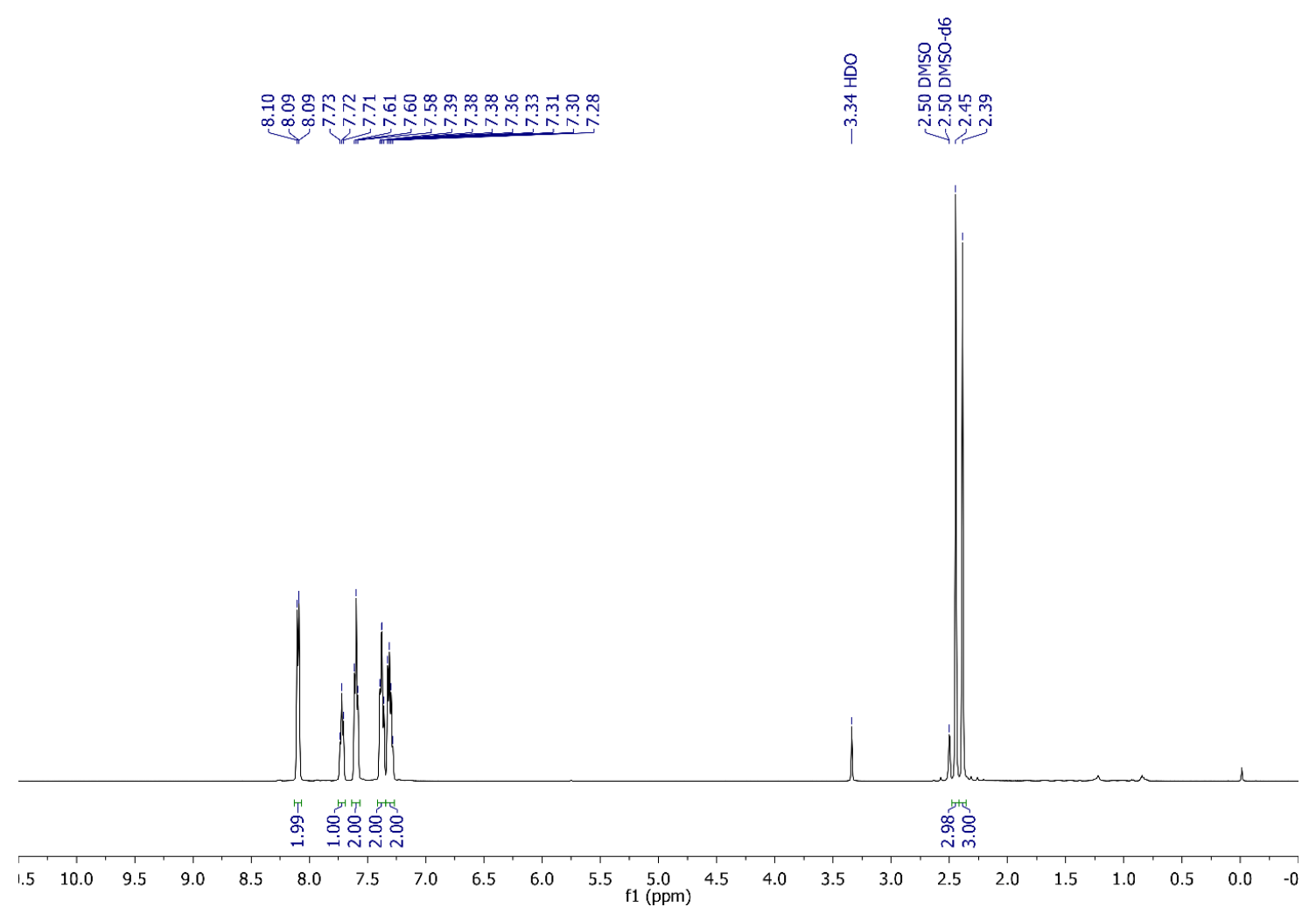

Figure S71. ${ }^{1} \mathrm{H}$ qNMR $(500 \mathrm{MHz})$ spectrum of compound $\mathbf{3 h}$ in DMSO- $d_{6}$

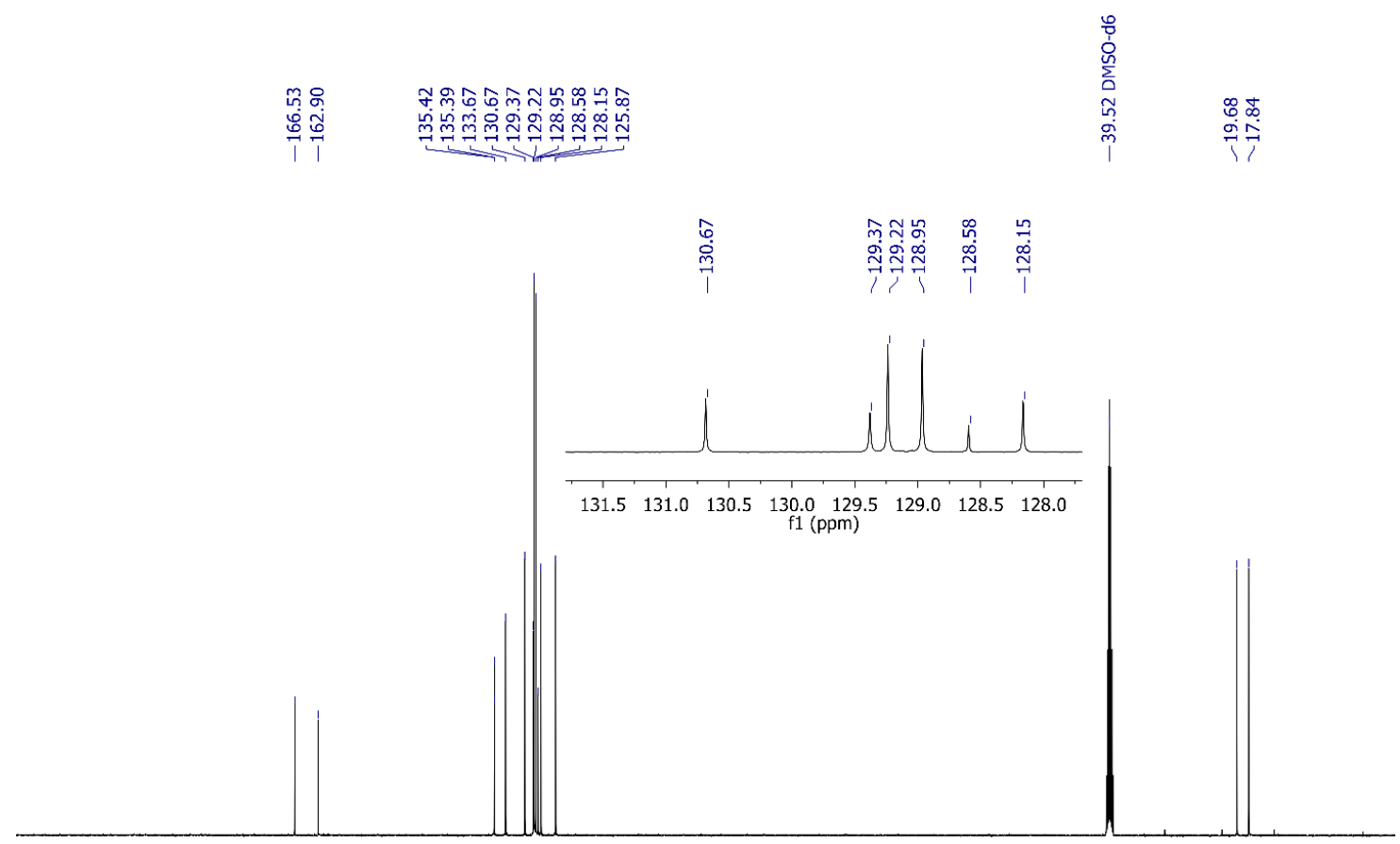

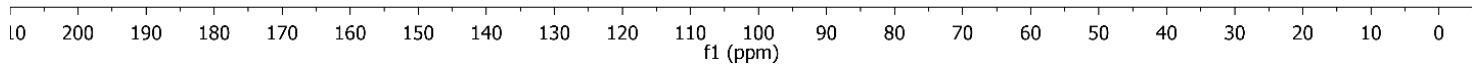

Figure S72. ${ }^{13} \mathrm{C}$ NMR $(126 \mathrm{MHz})$ spectrum of $\mathbf{3 h}$ in DMSO- $d_{6}$. 


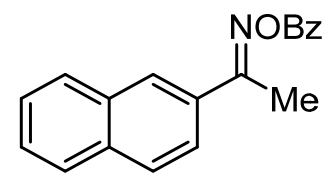

Compound 3j was synthesized according to general method A using acetonapthophenone (4.26 g, $25 \mathrm{mmol})$, pyridine $(5.03 \mathrm{~mL}, \mathrm{mmol})$, hydroxylamine hydrochloride $(2.61 \mathrm{~g}, 37.5 \mathrm{mmol})$, and ethanol $(50 \mathrm{~mL})$. Then the crude material of the first step (ca. $20 \mathrm{mmol}$ recovered) was reacted with pyridine $(1.61 \mathrm{~mL}, 20 \mathrm{mmol})$, benzoyl chloride $(2.32 \mathrm{~mL}, 20 \mathrm{mmol})$, in methylene chloride $(12 \mathrm{~mL})$ and hexanes $(48 \mathrm{~mL})$ according to the General Method A. The crude product was recrystallized from $\mathrm{CH}_{2} \mathrm{Cl}_{2} /$ Hexanes to afford the product as a white crystalline solid (5.04 g, 68\%). ${ }^{1} \mathrm{H}$ NMR $\left(500 \mathrm{MHz}, \mathrm{DMSO}-d_{6}\right) \delta 8.41(\mathrm{~s}, 1 \mathrm{H}), 8.12(\mathrm{~d}, J=7.6 \mathrm{~Hz}, 2 \mathrm{H}), 8.07(\mathrm{~d}, J=7.1 \mathrm{~Hz}, 1 \mathrm{H}), 8.02(\mathrm{~s}, 2 \mathrm{H}), 7.98$ $(\mathrm{d}, J=6.9 \mathrm{~Hz}, 1 \mathrm{H}), 7.74(\mathrm{t}, J=6.8 \mathrm{~Hz}, 1 \mathrm{H}), 7.60(\mathrm{~d}, J=6.5 \mathrm{~Hz}, 4 \mathrm{H}), 2.65(\mathrm{~s}, 3 \mathrm{H}) .{ }^{13} \mathrm{C}$ NMR $\left(126 \mathrm{MHz}, \mathrm{DMSO}-d_{6}\right)$ $\delta 163.74,162.93,133.87,133.78,132.56,131.74,129.30,129.03,128.76,128.54,128.20,127.81,127.59,127.57$, 126.78, 123.39, 14.14. MS (ESI) $m / z$ calcd. for $\mathrm{C}_{19} \mathrm{H}_{16} \mathrm{NO}_{2}\left([\mathrm{M}+\mathrm{H}]^{+}\right) 290.1$, found 290.1 . 


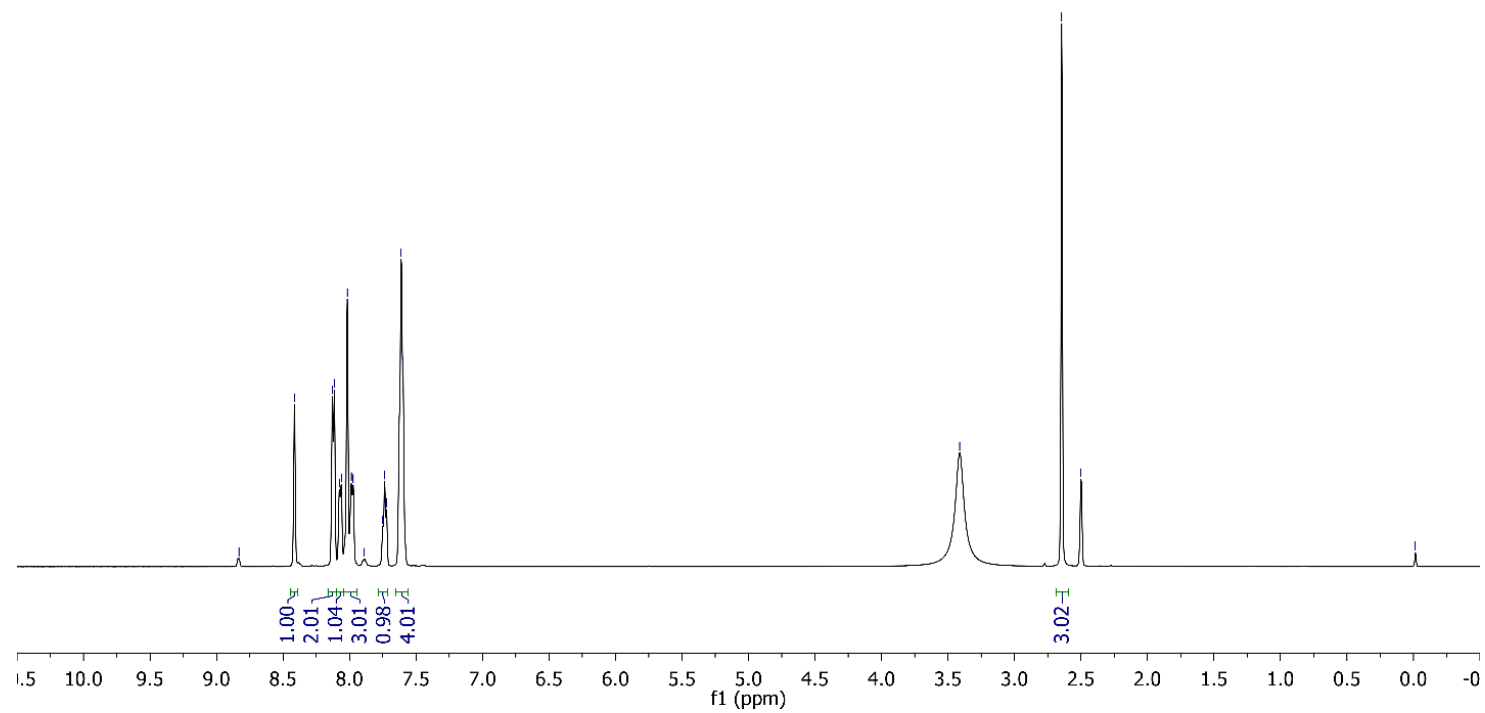

Figure S73. ${ }^{1} \mathrm{H}$ qNMR $(500 \mathrm{MHz})$ spectrum of compound $\mathbf{3 j}$ in DMSO- $d_{6}$

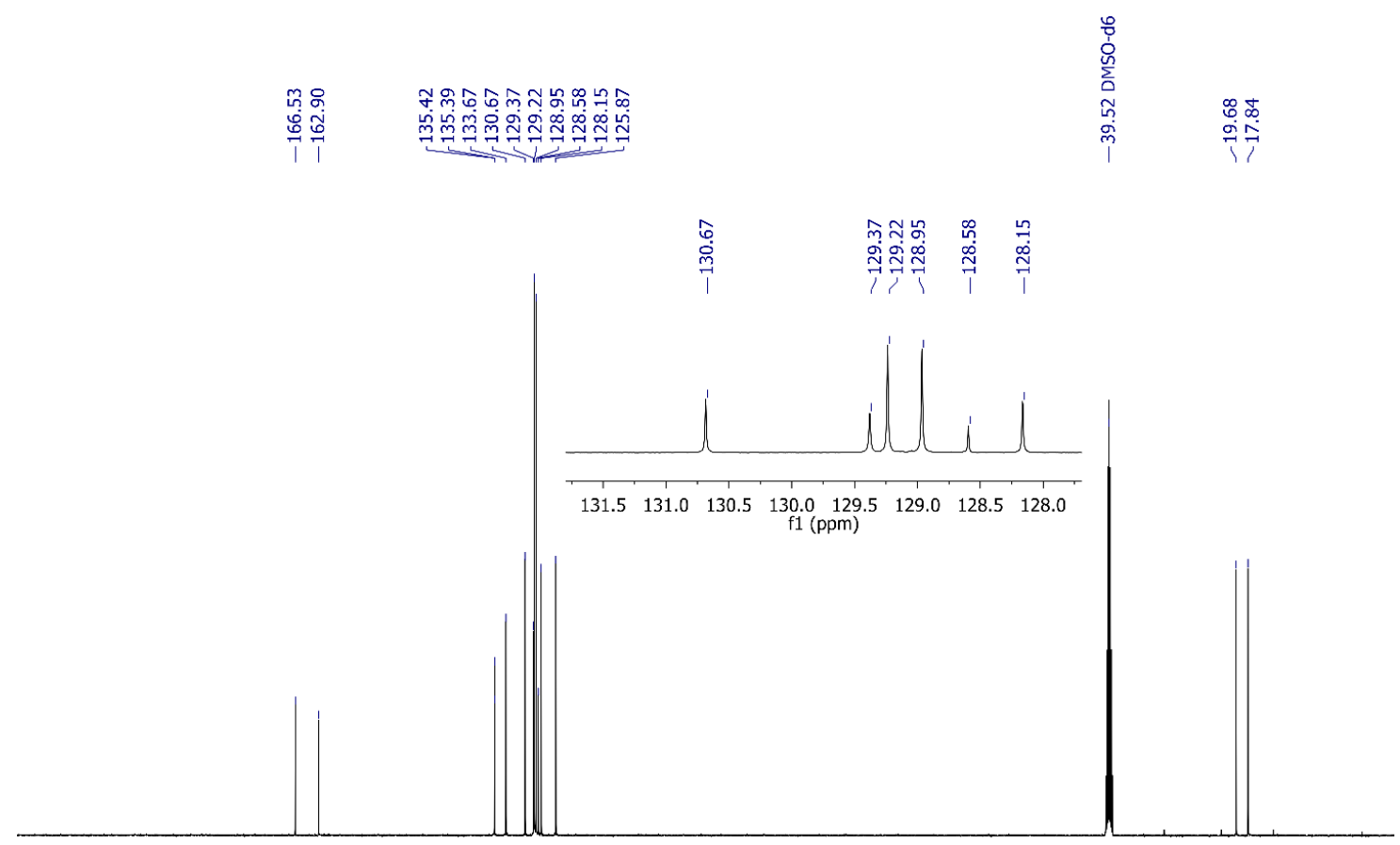

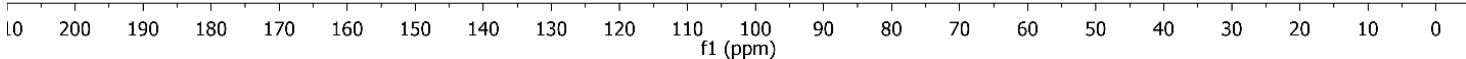

Figure S74. ${ }^{13} \mathrm{C}$ NMR $(126 \mathrm{MHz})$ spectrum of $\mathbf{3 j}$ in DMSO- $d_{6}$. 


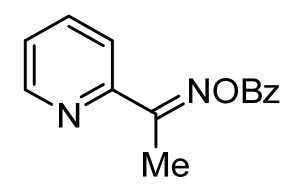

Compound 3s was synthesized according to general method $\mathrm{C}$ using 2-acetylpyridine (3.028 g, $25 \mathrm{mmol})$, triethylamine $(5.03 \mathrm{~mL}, 62.5 \mathrm{mmol})$, hydroxylamine hydrochloride $(2.61 \mathrm{~g}, 37.5 \mathrm{mmol})$, and ethanol $(50 \mathrm{~mL})$. Then the crude material of the first step was reacted with pyridine $(1.451 \mathrm{~mL}, 18 \mathrm{mmol})$, benzoyl chloride $(2.09 \mathrm{~mL}, 18$ $\mathrm{mmol})$, in methylene chloride $(55 \mathrm{~mL})$ according to the general method. The crude product was recrystallized from $\mathrm{CH}_{2} \mathrm{Cl}_{2}$ to afford the product as a white crystalline solid $(3.792 \mathrm{~g}, 63 \%) .{ }^{1} \mathrm{H}$ NMR $\left(500 \mathrm{MHz}\right.$, DMSO- $\left.d_{6}\right) \delta 8.74-8.68$ $(\mathrm{m}, 1 \mathrm{H}), 8.11(\mathrm{~d}, J=8.0 \mathrm{~Hz}, 2 \mathrm{H}), 8.04(\mathrm{~d}, J=7.5 \mathrm{~Hz}, 1 \mathrm{H}), 7.95(\mathrm{dt}, J=15.4,7.6 \mathrm{~Hz}, 1 \mathrm{H}), 7.73(\mathrm{~d}, J=7.3 \mathrm{~Hz}, 1 \mathrm{H})$, $7.61(\mathrm{t}, J=7.2 \mathrm{~Hz}, 2 \mathrm{H}), 7.58-7.52(\mathrm{~m}, 1 \mathrm{H}), 2.58(\mathrm{~s}, 3 \mathrm{H}) .{ }^{13} \mathrm{C}$ NMR $\left(126 \mathrm{MHz}, \mathrm{DMSO}-d_{6}\right) \delta 164.41,162.70,151.85$, $149.34,137.16,133.87,129.32,129.02,128.28,125.56,121.39,12.83$. MS (ESI) m/z calcd. for $\mathrm{C}_{14} \mathrm{H}_{13} \mathrm{~N}_{2} \mathrm{O}_{2}([\mathrm{M}+$ $\mathrm{H}]^{+}$) 241.1, found 241.2 


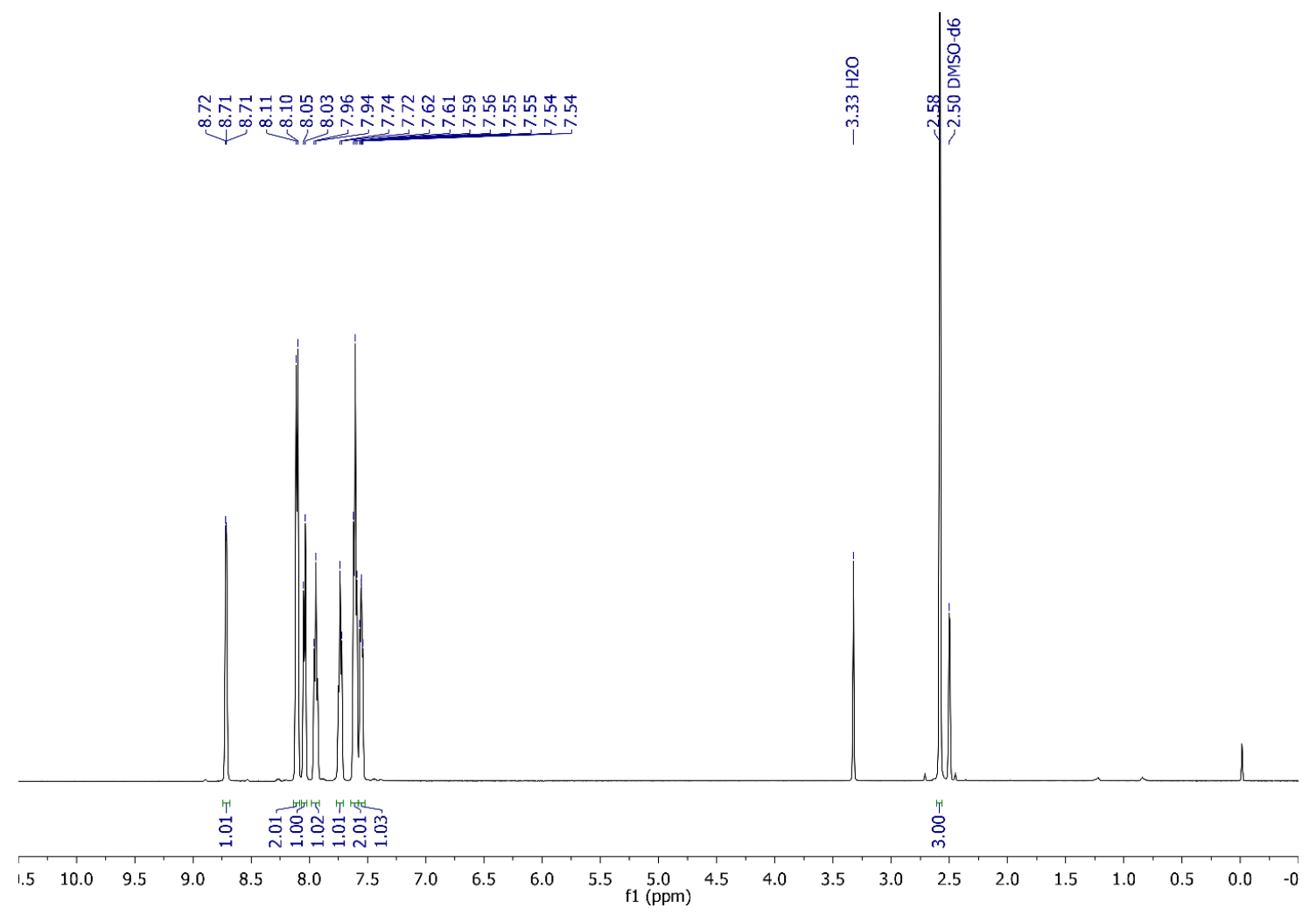

Figure S75. ${ }^{1} \mathrm{H}$ qNMR (500 MHz) spectrum of compound 3s in DMSO- $d_{6}$

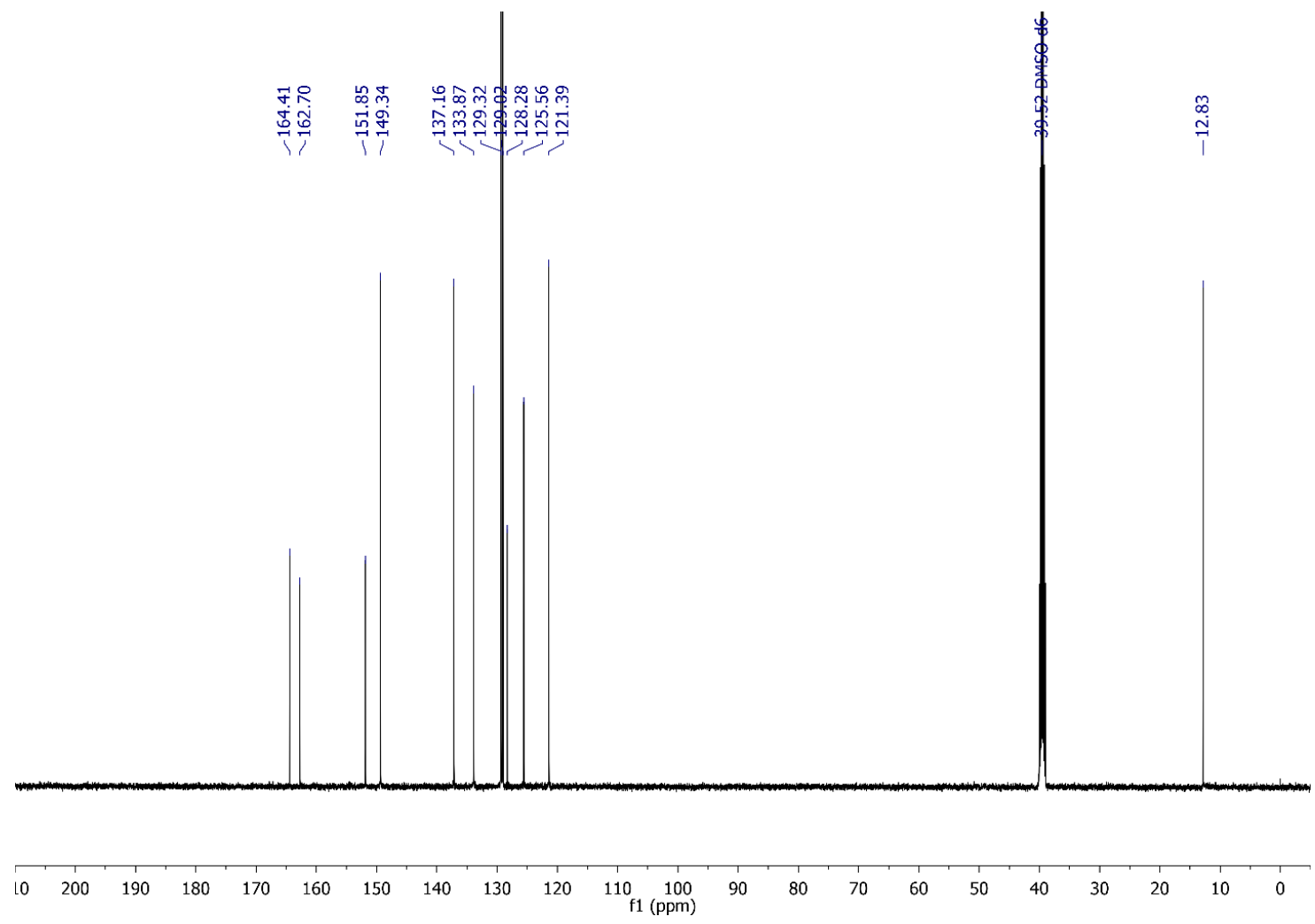

Figure S76. ${ }^{13} \mathrm{C}$ NMR $(126 \mathrm{MHz})$ spectrum of $3 \mathrm{~s}$ in DMSO- $d_{6}$. 


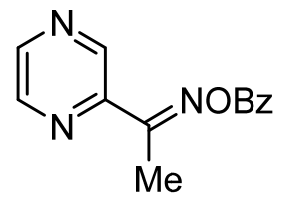

Compound 3t was synthesized according to general method $\mathrm{C}$ using 2-acetylpyrazine (2.422 $\mathrm{g}, 20 \mathrm{mmol})$, $\mathrm{Et}_{3} \mathrm{~N}$ (6.97 mL, $\left.50 \mathrm{mmol}\right)$, hydroxylamine hydrochloride $(2.08 \mathrm{~g}, 30 \mathrm{mmol})$, and ethanol (40 mL). Then the crude material of the first step was reacted with pyridine $(1.45 \mathrm{~mL}, 18 \mathrm{mmol})$, benzoyl chloride $(2.1 \mathrm{~mL}, 18 \mathrm{mmol})$, in methylene chloride $(55 \mathrm{~mL})$ and according to the general method. The crude product was recrystallized from $\mathrm{CH}_{2} \mathrm{Cl}_{2} / \mathrm{Hexanes}$ to afford the product as a white crystalline solid $(2.35 \mathrm{~g}, 54 \%)$. ${ }^{1} \mathrm{H} \mathrm{NMR}\left(500 \mathrm{MHz}, \mathrm{DMSO}-d_{6}\right) \delta$ $9.20(\mathrm{~s}, 1 \mathrm{H}), 8.80(\mathrm{~s}, 2 \mathrm{H}), 8.12(\mathrm{~d}, J=7.9 \mathrm{~Hz}, 2 \mathrm{H}), 7.75(\mathrm{t}, J=6.8 \mathrm{~Hz}, 1 \mathrm{H}), 7.62(\mathrm{t}, J=7.2 \mathrm{~Hz}, 2 \mathrm{H}), 2.59(\mathrm{~s}, 3 \mathrm{H}) .{ }^{13} \mathrm{C}$ NMR (126 MHz, DMSO) $\delta$ 163.08, 162.56, 147.69, 146.04, 144.19, 142.61, 134.04, 129.41, 129.08, $128.03,12.75$. MS (ESI) $\mathrm{m} / \mathrm{z}$ calcd. for $\mathrm{C}_{13} \mathrm{H}_{12} \mathrm{~N}_{3} \mathrm{O}_{2}\left([\mathrm{M}+\mathrm{H}]^{+}\right) 242.1$, found 242.2 


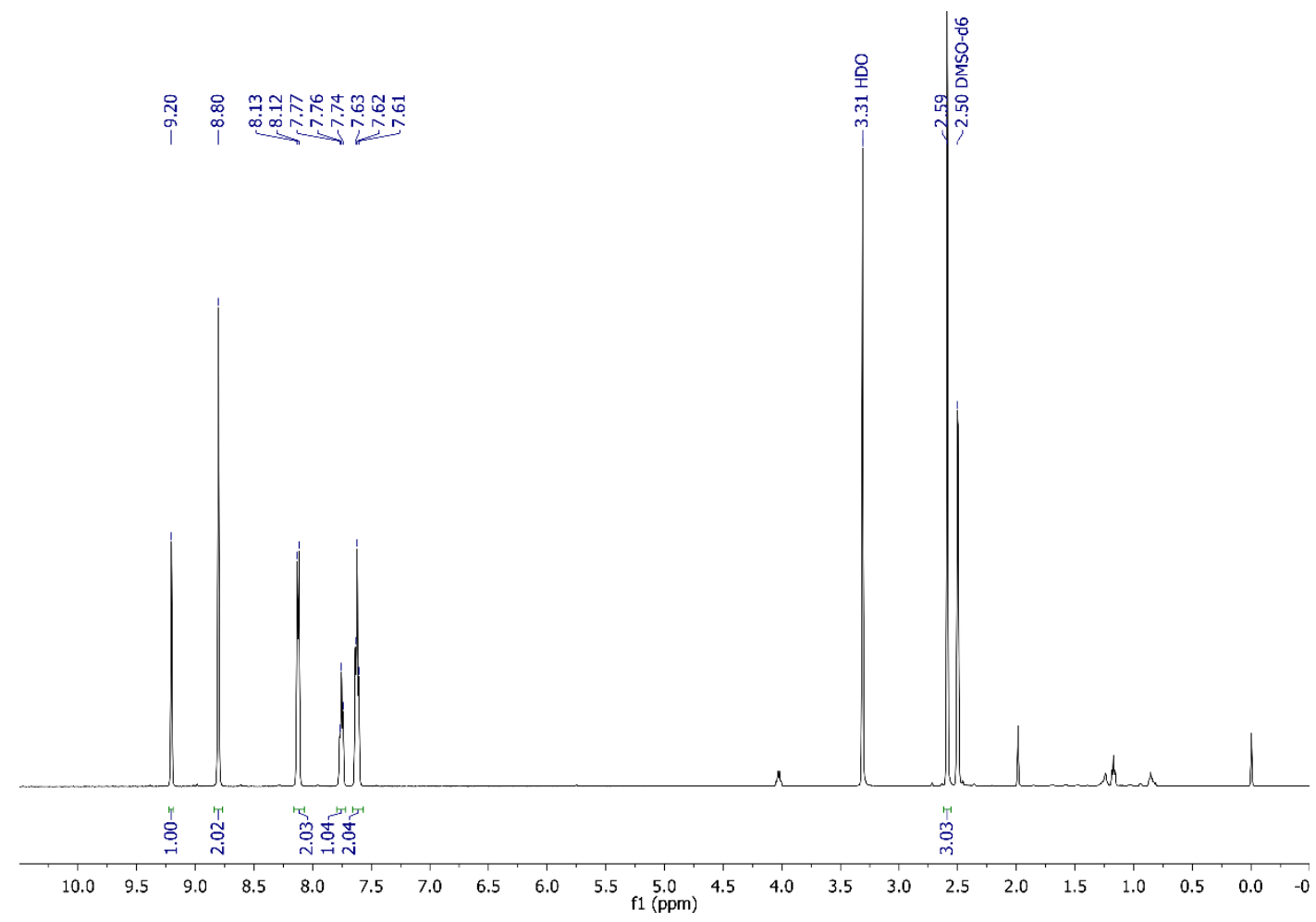

Figure S77. ${ }^{1} \mathrm{H}$ qNMR (500 MHz) spectrum of compound $3 \mathrm{t}$ in DMSO- $d_{6}$
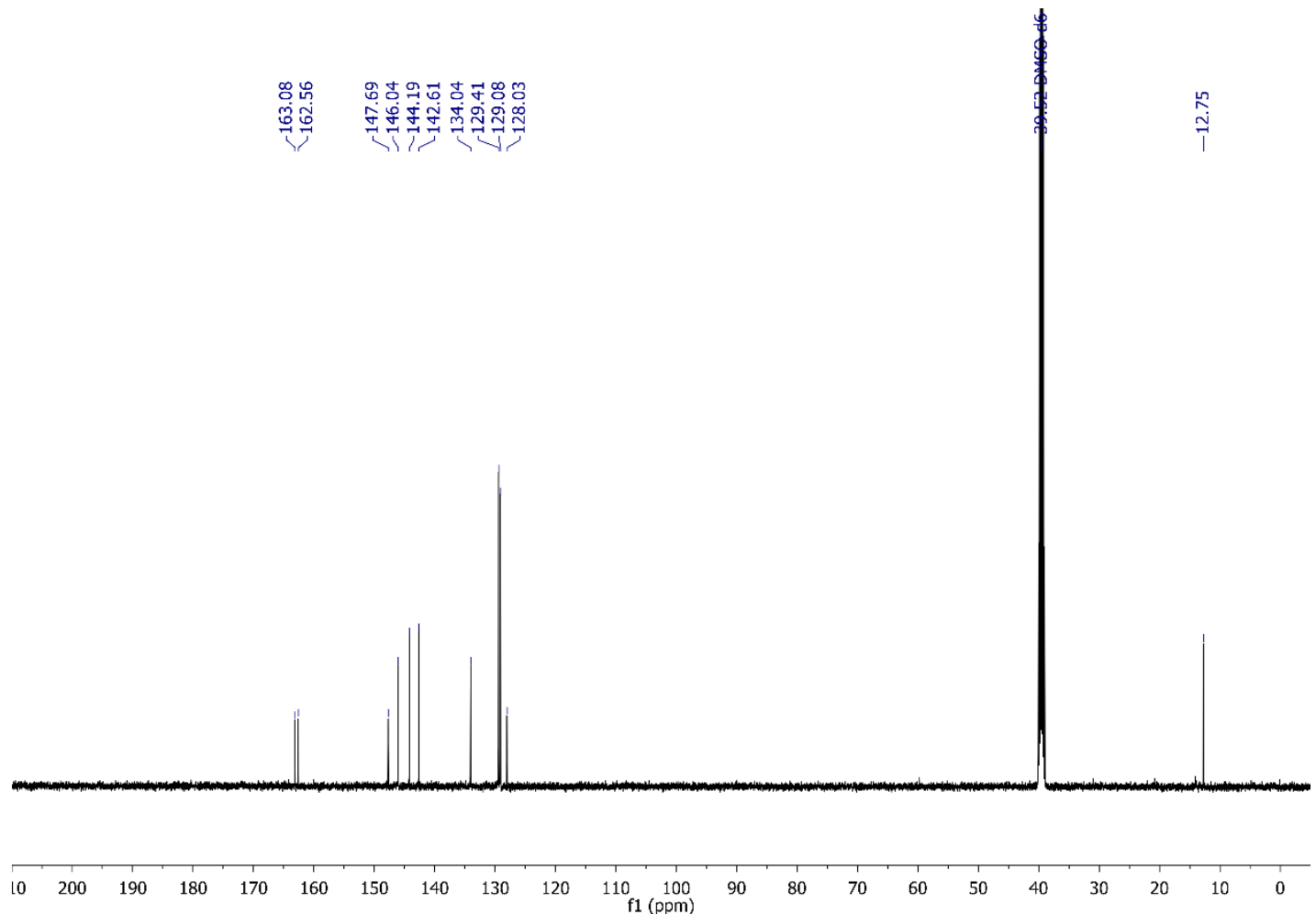

Figure S78. ${ }^{13} \mathrm{C}$ NMR $(126 \mathrm{MHz})$ spectrum of $\mathbf{3 t}$ in DMSO- $d_{6}$. 


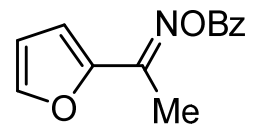

Compound 3u a was synthesized according to general method A using 1-(furan-2-yl)ethan-1-one (5.0 mL, $50 \mathrm{mmol})$, pyridine $(10 \mathrm{~mL}, 125 \mathrm{mmol})$, hydroxylamine hydrochloride $(5.212 \mathrm{~g}, 75 \mathrm{mmol})$, and ethanol $(100 \mathrm{~mL})$. Then the crude material $(5.8 \mathrm{~g})$ of the first step was reacted with pyridine $(3.73 \mathrm{~mL}, 46.4 \mathrm{mmol})$, benzoyl chloride $(5.38 \mathrm{~mL}, 46.4 \mathrm{mmol})$, in methylene chloride $(28 \mathrm{~mL})$ and hexanes $(112 \mathrm{~mL})$ according to the general method. The crude product was recrystallized from $\mathrm{CH}_{2} \mathrm{Cl}_{2} /$ Hexanes to afford the product as a dark brown solid. The product required further purification and was dissolved in hot $\mathrm{CH}_{2} \mathrm{Cl}_{2} / \mathrm{Hexanes,} \mathrm{filtered,} \mathrm{and} \mathrm{recrystallized} \mathrm{to} \mathrm{afford} \mathrm{the} \mathrm{product}$ as a brown solid (4.212 g, 39\%). ${ }^{1} \mathrm{H}$ NMR (500 MHz, DMSO- $\left.d_{6}\right) \delta 8.07(\mathrm{~d}, J=7.3 \mathrm{~Hz}, 2 \mathrm{H}), 7.93(\mathrm{~s}, 1 \mathrm{H}), 7.71(\mathrm{t}, J=$ $7.4 \mathrm{~Hz}, 1 \mathrm{H}), 7.58(\mathrm{t}, J=7.7 \mathrm{~Hz}, 2 \mathrm{H}), 7.22(\mathrm{~d}, J=3.4 \mathrm{~Hz}, 1 \mathrm{H}), 6.68(\mathrm{dd}, J=3.3,1.6 \mathrm{~Hz}, 1 \mathrm{H}), 2.43(\mathrm{~s}, 3 \mathrm{H}) .{ }^{13} \mathrm{C} \mathrm{NMR}$ (126 MHz, DMSO) $\delta 162.68,154.93,147.61,146.12,133.72,129.25,128.94,128.42,115.10,112.13,13.16$. MS (ESI) M/Z CALCD. FOR C13H11NO3 $\left([\mathrm{M}+\mathrm{H}]^{+}\right) 230.1$, found 230.1 


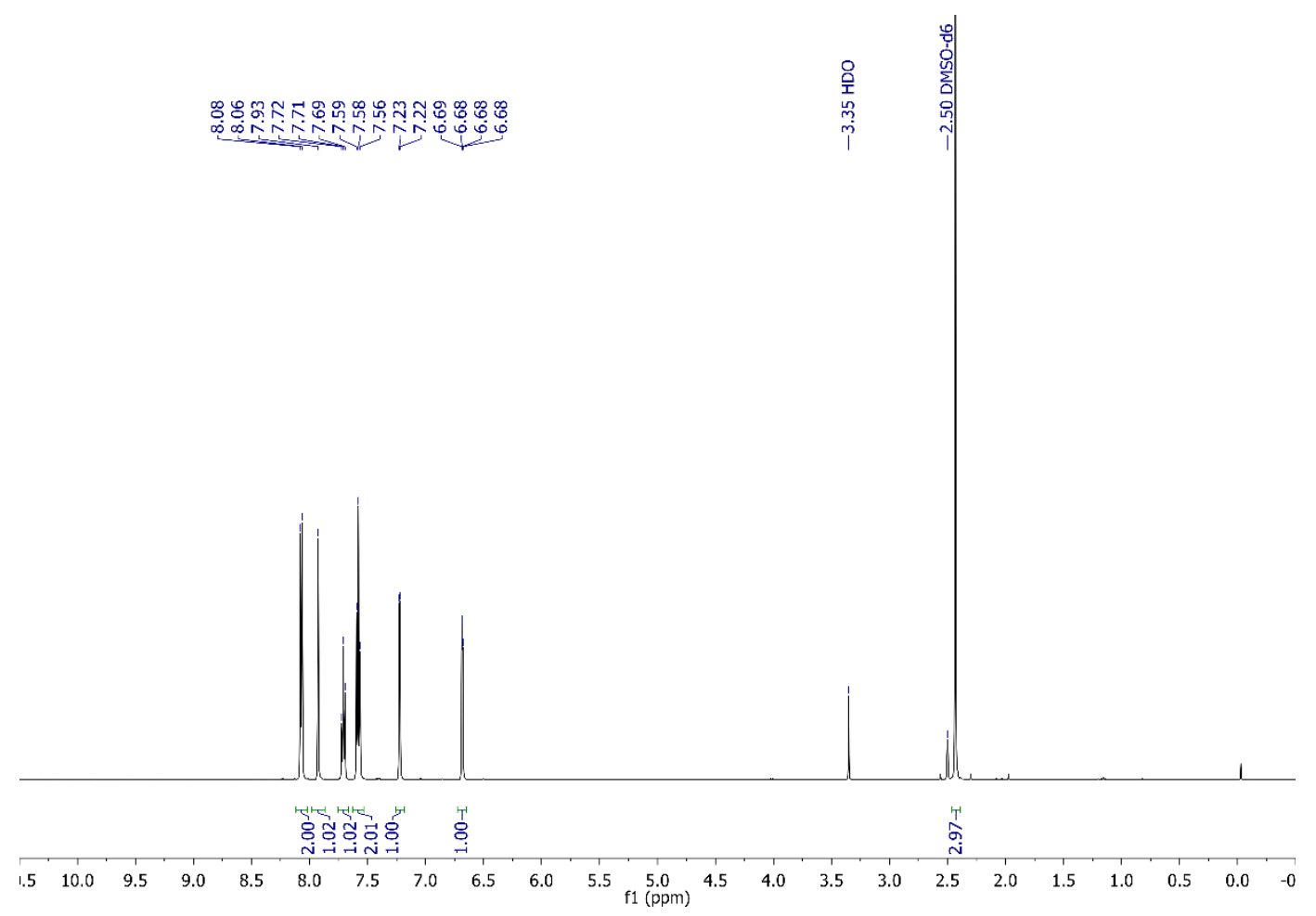

Figure S79. ${ }^{1} \mathrm{H}$ qNMR $(500 \mathrm{MHz})$ spectrum of compound $3 \mathbf{u}$ in DMSO- $d_{6}$
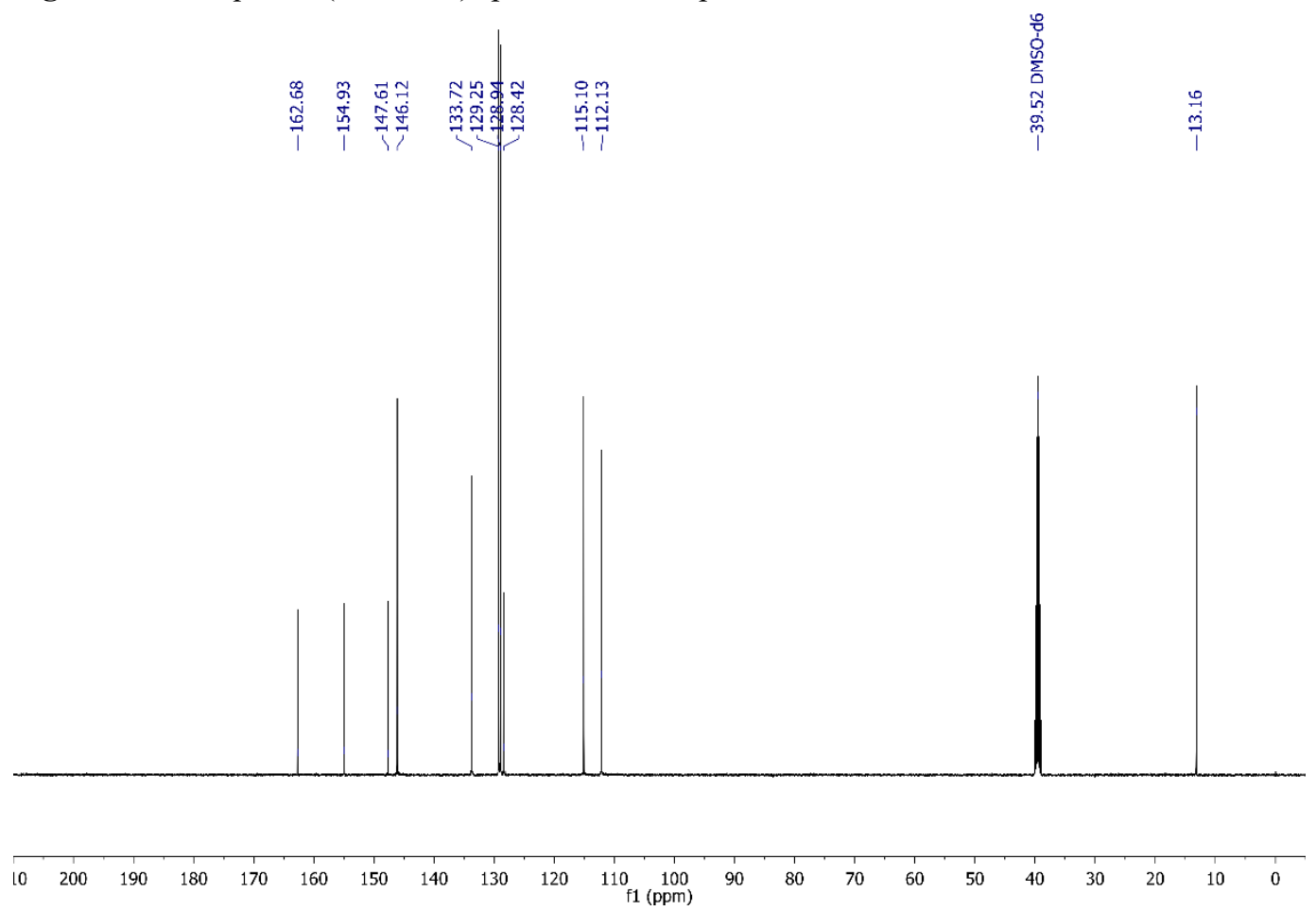

Figure S80. ${ }^{13} \mathrm{C}$ NMR $(126 \mathrm{MHz})$ spectrum of $\mathbf{3 u}$ in DMSO- $d_{6}$. 


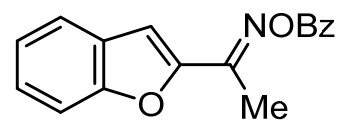

Compound 3n was synthesized according to general method B using 1-(benzofuran-2-yl)ethan-1-one (4.00 g, $25 \mathrm{mmol})$, pyridine (5.03 $\mathrm{mL}, 62.5 \mathrm{mmol})$, hydroxylamine hydrochloride $(2.61 \mathrm{~g}, 37.5 \mathrm{mmol})$, and ethanol (50 $\mathrm{mL})$. Then the crude material of the first step was reacted with pyridine $(1.61 \mathrm{~mL}, 20 \mathrm{mmol})$, benzoyl chloride $(2.32 \mathrm{~mL}$, $20 \mathrm{mmol})$, in methylene chloride $(60 \mathrm{~mL})$ according to the general method. The crude product was recrystallized from $\mathrm{CH}_{2} \mathrm{Cl}_{2} / \mathrm{Hexanes}$ to afford the product as a white crystalline solid (2.645 $\mathrm{g}, 47 \%$ ).

${ }^{1} \mathrm{H}$ NMR $\left(500 \mathrm{MHz}, \mathrm{DMSO}-d_{6}\right) \delta 8.10(\mathrm{~d}, J=7.1 \mathrm{~Hz}, 2 \mathrm{H}), 7.78-7.67(\mathrm{~m}, 4 \mathrm{H}), 7.59(\mathrm{t}, J=6.9 \mathrm{~Hz}, 2 \mathrm{H}), 7.45(\mathrm{t}, J=$ $7.3 \mathrm{~Hz}, 1 \mathrm{H}), 7.32(\mathrm{t}, J=7.1 \mathrm{~Hz}, 1 \mathrm{H}), 2.54(\mathrm{~s}, 3 \mathrm{H}) .{ }^{13} \mathrm{C}$ NMR $\left(126 \mathrm{MHz}, \mathrm{DMSO}-d_{6}\right) \delta 162.59,155.35,154.94,149.57$, $133.89,129.37,129.02,128.23,127.26,126.97,123.63,122.22,111.65,111.62,13.35$. MS (ESI) m/z calcd. for $\mathrm{C}_{17} \mathrm{H}_{15} \mathrm{NO}_{3}\left([\mathrm{M}+\mathrm{H}]^{+}\right) 280.1$, found 280.1 


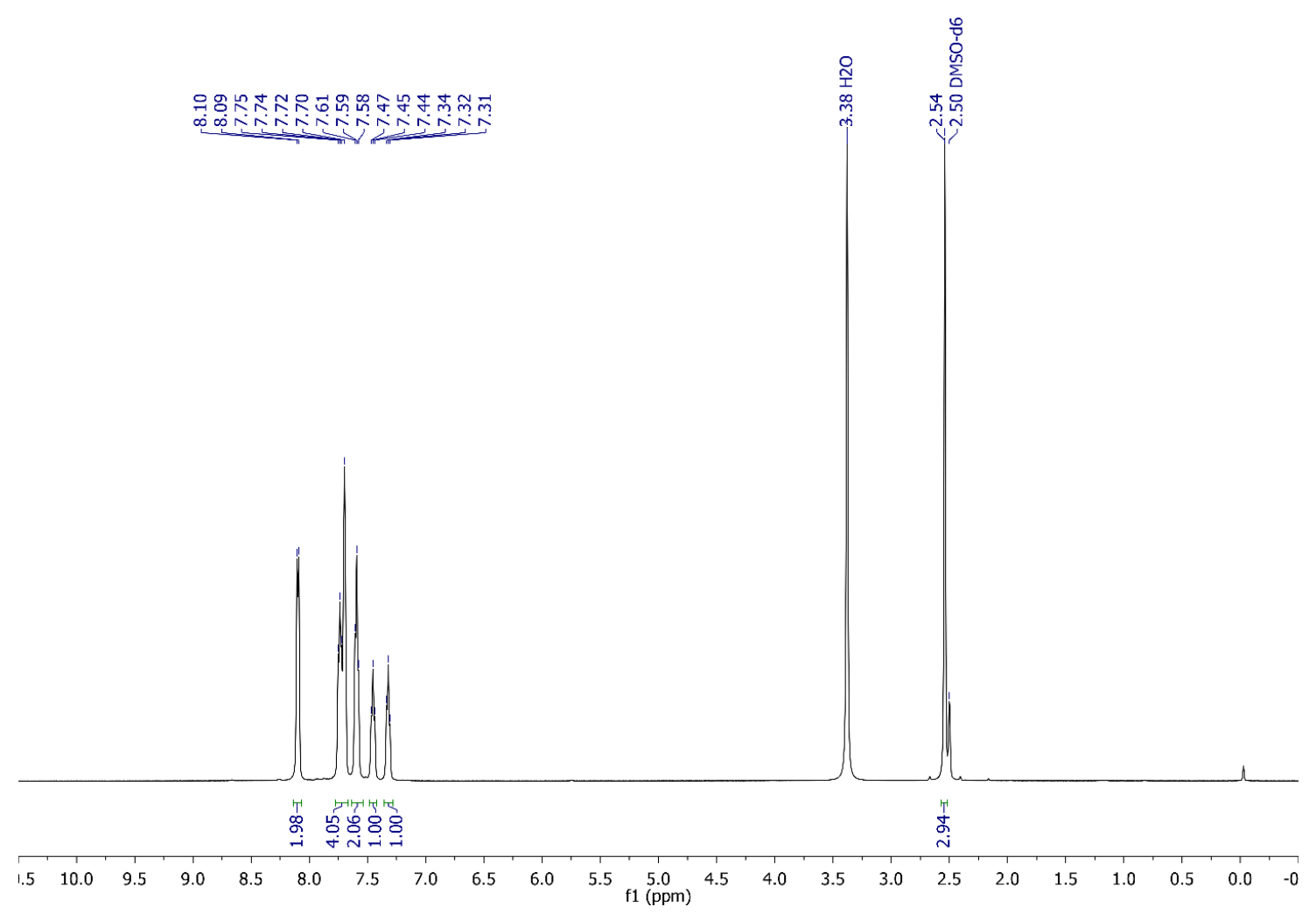

Figure S81. ${ }^{1} \mathrm{H}$ qNMR $(500 \mathrm{MHz})$ spectrum of compound $3 \mathbf{n}$ in DMSO- $d_{6}$

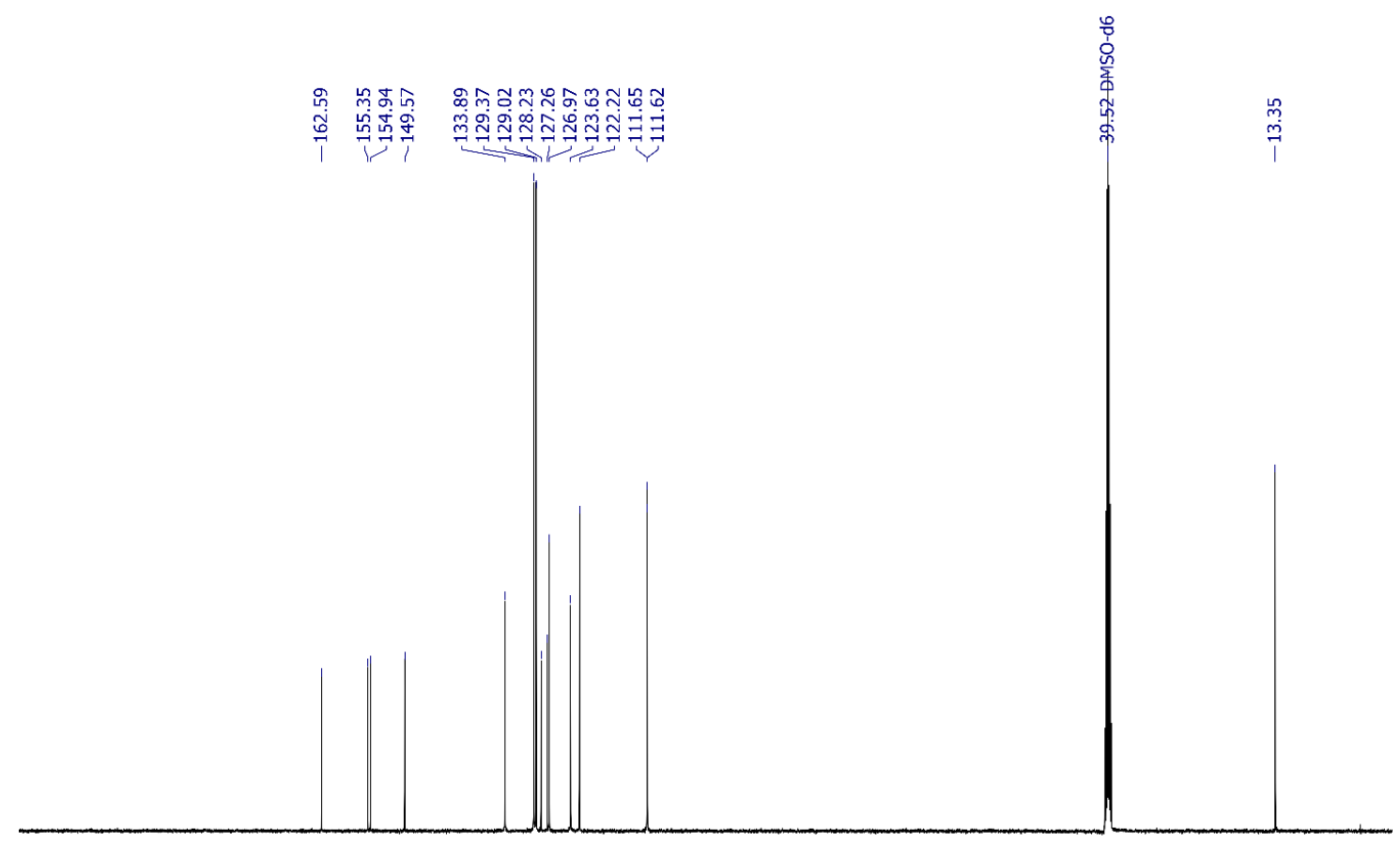

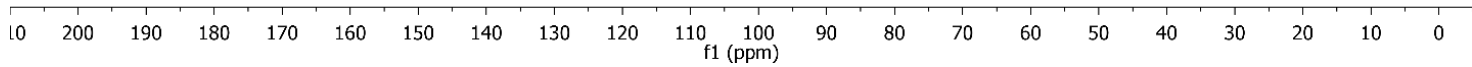

Figure S82. ${ }^{13} \mathrm{C}$ NMR $(126 \mathrm{MHz})$ spectrum of $\mathbf{3 n}$ in DMSO- $d_{6}$. 
<smiles>CC(C)(C)OC(=O)c1ccc2c(c1)OCO2</smiles>

Compound 3k was synthesized according to general method A using 3',4'-(Methylenedioxy)acetophenone $(1.970 \mathrm{~g}, 12.0 \mathrm{mmol})$, pyridine $(2.42 \mathrm{~mL}, 30 \mathrm{mmol})$, hydroxylamine hydrochloride $(1.250 \mathrm{~g}, 18.0 \mathrm{mmol})$, and ethanol $(24 \mathrm{~mL})$. Then the crude material of the first step was reacted with pyridine $(0.90 \mathrm{~mL}, 11.16 \mathrm{mmol})$, benzoyl chloride $(1.30 \mathrm{~mL}, 11.16 \mathrm{mmol})$, in methylene chloride $(7 \mathrm{~mL})$ and hexanes $(27 \mathrm{~mL})$ according to the general method. The crude product was recrystallized from $\mathrm{CH}_{2} \mathrm{Cl}_{2} / \mathrm{Hexanes}$ to afford the product as a white crystalline solid $(2.134 \mathrm{~g}, 63 \%)$.

${ }^{1} \mathrm{H}$ NMR (500 MHz, DMSO- $\left.d_{6}\right) \delta 8.11-8.05(\mathrm{~m}, 2 \mathrm{H}), 7.72(\mathrm{t}, J=6.8 \mathrm{~Hz}, 1 \mathrm{H}), 7.59(\mathrm{t}, J=7.5 \mathrm{~Hz}, 2 \mathrm{H}), 7.40-7.35$ $(\mathrm{m}, 2 \mathrm{H}), 7.06-7.01(\mathrm{~m}, 1 \mathrm{H}), 6.12(\mathrm{~s}, 2 \mathrm{H}), 2.47(\mathrm{~s}, 3 \mathrm{H}) .{ }^{13} \mathrm{C}$ NMR $\left(126 \mathrm{MHz}, \mathrm{DMSO}-d_{6}\right) \delta 163.37,162.89,149.53$, 147.70, 133.68, 129.20, 128.97, 128.58, 128.23, 121.99, 108.28, 106.54, 101.69, 14.28. MS (ESI) m/z calcd. for $\mathrm{C}_{16} \mathrm{H}_{14} \mathrm{NO}_{4}([\mathrm{M}+\mathrm{H}]+)$ 284.1, found 284.1 


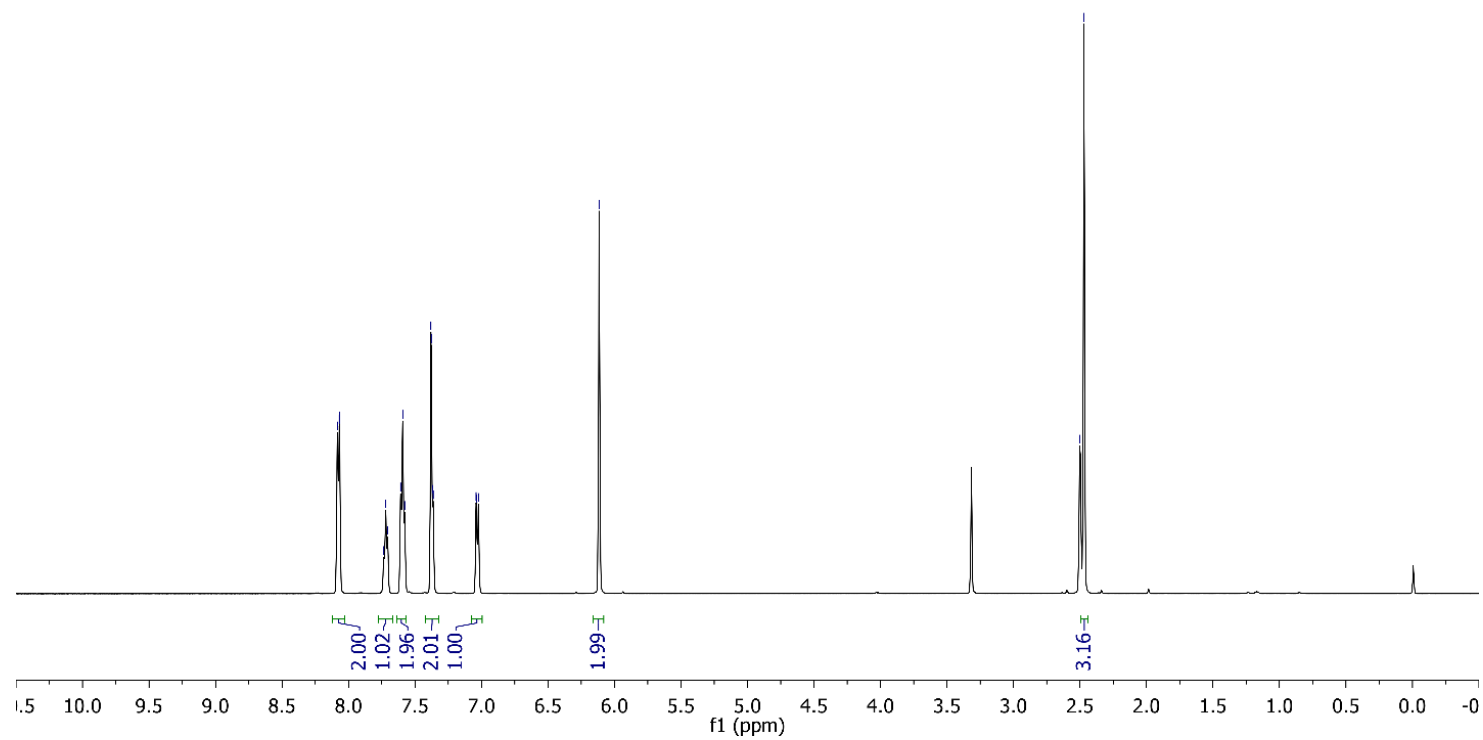

Figure S83. ${ }^{1} \mathrm{H}$ qNMR $(500 \mathrm{MHz})$ spectrum of compound 3k in DMSO- $d_{6}$

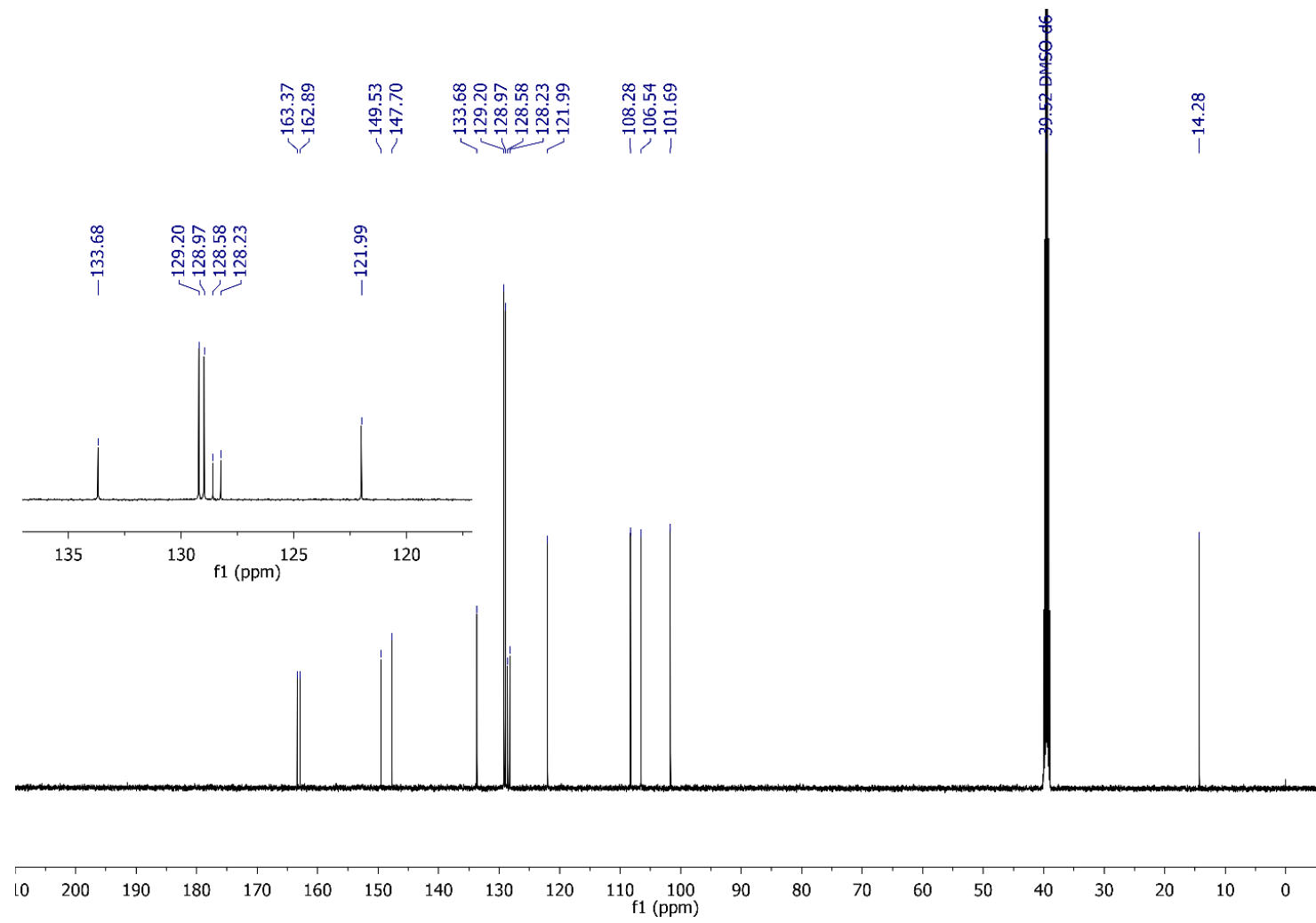

Figure S84. ${ }^{13} \mathrm{C}$ NMR $(126 \mathrm{MHz})$ spectrum of $3 \mathbf{k}$ in DMSO- $d_{6}$. 
(BuO

Compound 3m was synthesized according to general method A using tert-butyl 4-acetylbenzoate (1.652 $\mathrm{g}$, $7.5 \mathrm{mmol})$, pyridine $(1.51 \mathrm{~mL}, 18.75 \mathrm{mmol})$, hydroxylamine hydrochloride $(782 \mathrm{mg}, 11.25 \mathrm{mmol})$, and ethanol $(15.0$ $\mathrm{mL})$. Then the crude material of the first step was reacted with pyridine $(0.610 \mathrm{~mL}, 7.5 \mathrm{mmol})$, benzoyl chloride $(0$. $870 \mathrm{~mL}, 7.5 \mathrm{mmol})$, in methylene chloride $(23 \mathrm{~mL})$ according to the general method. The crude product was recrystallized from $\mathrm{CH}_{2} \mathrm{Cl}_{2}$ to afford the product as a white crystalline solid (1.02 $\mathrm{g}, 40 \%$ ).

${ }^{1} \mathrm{H}$ NMR $\left(500 \mathrm{MHz}\right.$, DMSO- $\left.d_{6}\right) \delta 8.10(\mathrm{~d}, J=8.0 \mathrm{~Hz}, 2 \mathrm{H}), 8.00(\mathrm{~d}, J=8.3 \mathrm{~Hz}, 2 \mathrm{H}), 7.94(\mathrm{~d}, J=8.0 \mathrm{~Hz}, 2 \mathrm{H}), 7.73(\mathrm{t}$, $J=7.4 \mathrm{~Hz}, 1 \mathrm{H}), 7.60(\mathrm{t}, J=7.6 \mathrm{~Hz}, 2 \mathrm{H}), 2.55(\mathrm{~s}, 3 \mathrm{H}), 1.56(\mathrm{~s}, 9 \mathrm{H}) .{ }^{13} \mathrm{C}$ NMR $\left(126 \mathrm{MHz}\right.$, DMSO-$\left.d_{6}\right) \delta 164.37,163.38$, $162.78,138.41,133.85,133.07,129.31,129.27,129.02,128.35,127.19,81.17,27.73,14.41$. MS (ESI) $\mathrm{m} / \mathrm{z}$ calcd. for $\mathrm{C}_{20} \mathrm{H}_{22} \mathrm{NO}_{4}\left([\mathrm{M}+\mathrm{H}]^{+}\right) 340.1$, found 340.2 


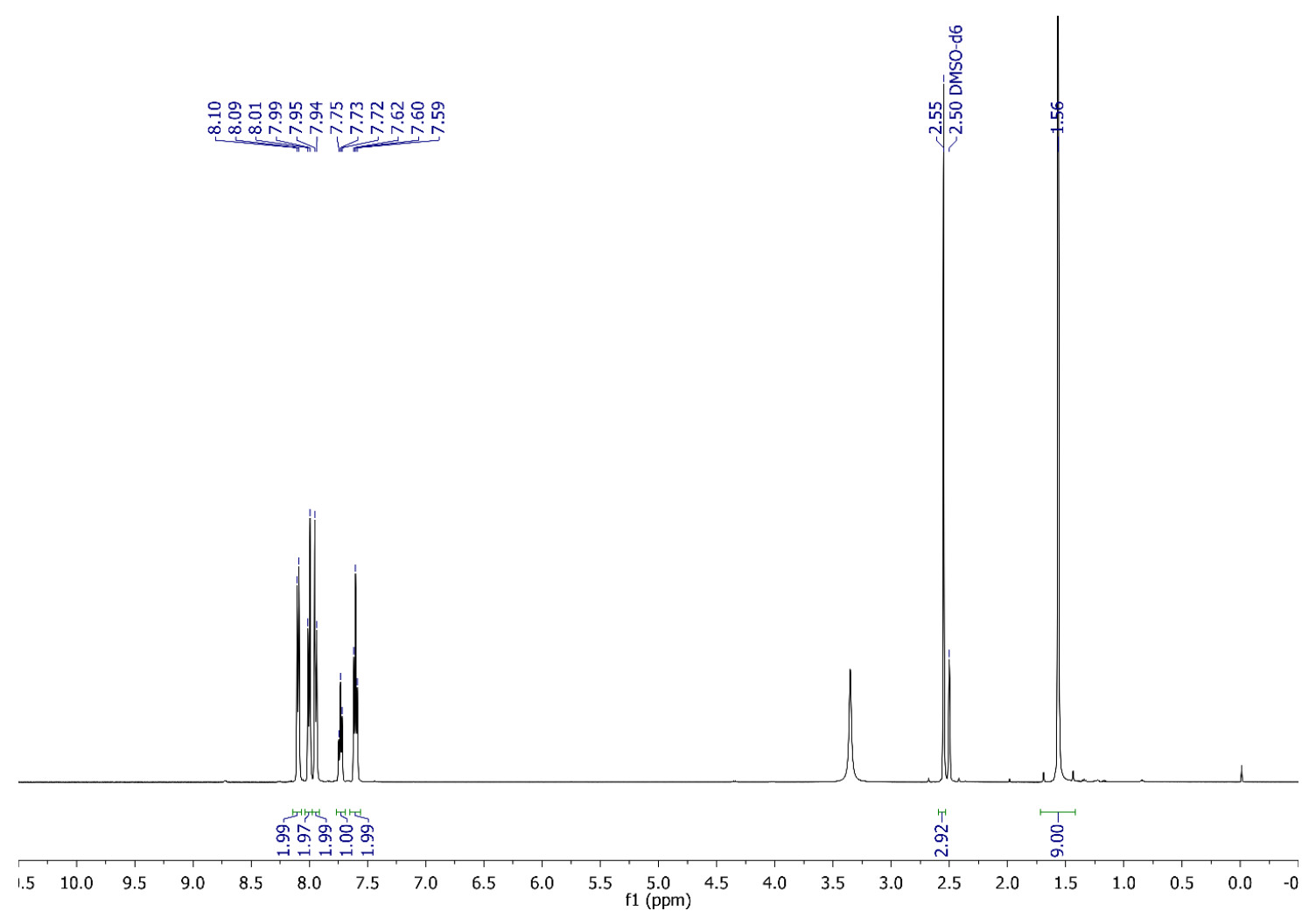

Figure S85. ${ }^{1} \mathrm{H}$ qNMR (500 MHz) spectrum of compound $\mathbf{3 m}$ in DMSO- $d_{6}$

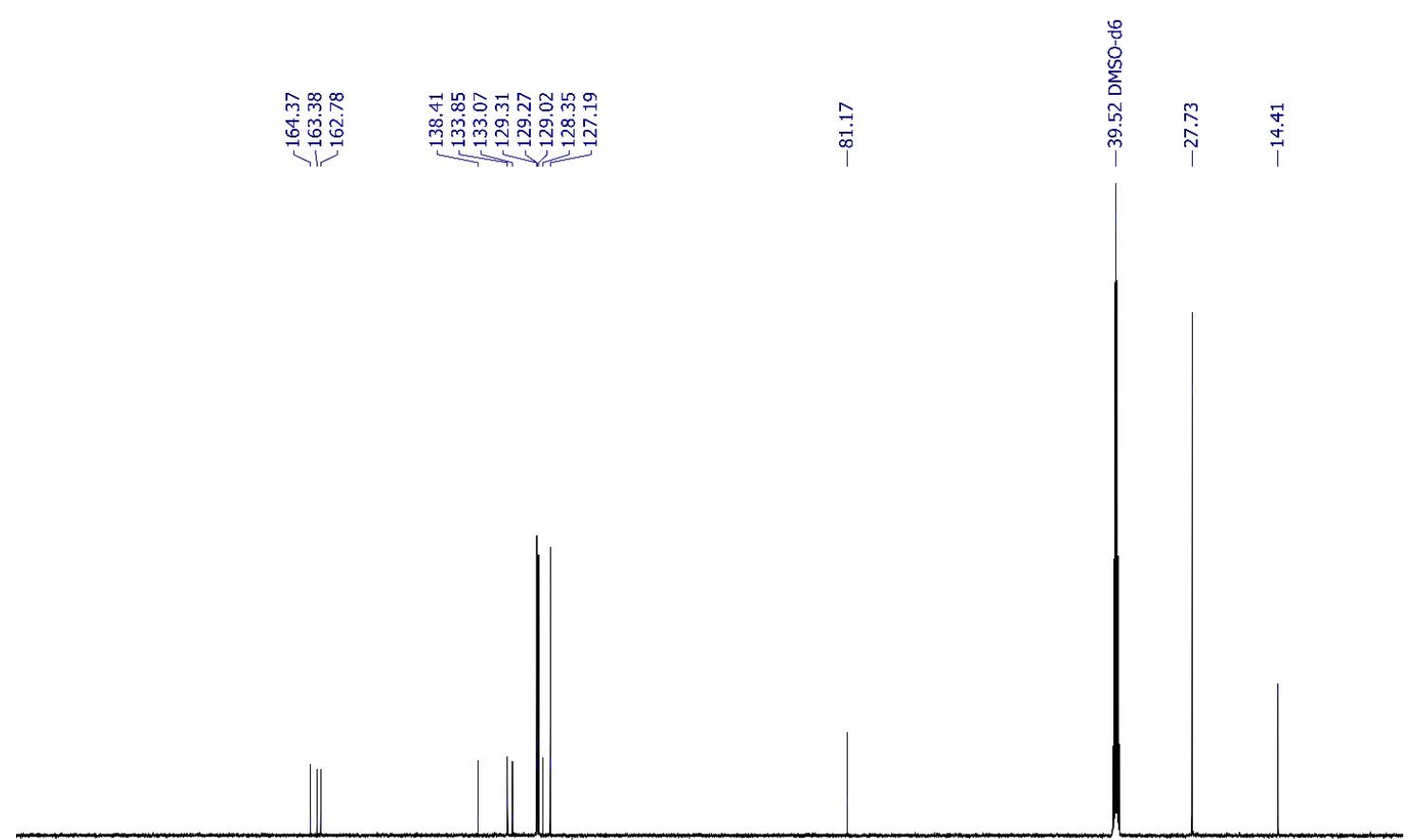

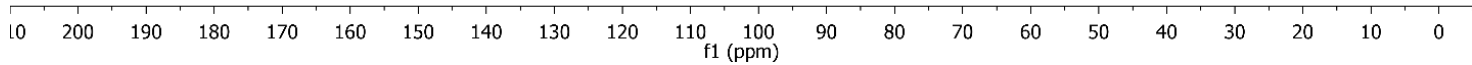

Figure S86. ${ }^{13} \mathrm{C}$ NMR $(126 \mathrm{MHz})$ spectrum of $\mathbf{3 m}$ in DMSO- $d_{6}$. 
<smiles>CC(C)(C)ON=C(c1ccccc1)C(F)(F)F</smiles>

Compound 3p was synthesized according to general method A using 2,2,2-trifluoro-1-phenylethan-1-one $(1.80 \mathrm{~g}, 10.0 \mathrm{mmol})$, pyridine $(2.01 \mathrm{~mL}, 25.0 \mathrm{mmol})$, hydroxylamine hydrochloride $(1.042 \mathrm{~g}, 15.0 \mathrm{mmol})$, and ethanol $(20 \mathrm{~mL})$. Then the crude material of the first step was reacted with pyridine $(0.81 \mathrm{~mL}, 10.0 \mathrm{mmol})$, benzoyl chloride $(1.15 \mathrm{~mL}, 10.0 \mathrm{mmol})$, in methylene chloride $(6 \mathrm{~mL})$ and hexanes $(24 \mathrm{ml})$ according to the general method. The crude product was recrystallized from $\mathrm{CH}_{2} \mathrm{Cl}_{2} / \mathrm{Hexanes}$ to afford the product as a white crystalline solid $(1.299 \mathrm{~g}, 44 \%)$. The remaining mother liquor contained product which could not be purified by further recrystallization. ${ }^{1} \mathrm{H}$ NMR (500 MHz, DMSO- $\left.d_{6}\right) \delta 7.74(\mathrm{~d}, J=7.8 \mathrm{~Hz}, 1 \mathrm{H}), 7.72-7.60(\mathrm{~m}, 3 \mathrm{H}), 7.50(\mathrm{t}, J=7.1 \mathrm{~Hz}, 1 \mathrm{H}) .{ }^{13} \mathrm{C}$ NMR $(126 \mathrm{MHz}$, DMSO- $\left.d_{6}\right) \delta 161.57,153.93(\mathrm{q}, J=32.7 \mathrm{~Hz}), 134.44,131.63,129.35,129.05,128.92,128.23,125.30,120.01(\mathrm{q}, J=$ $276.7 \mathrm{~Hz}) .{ }^{19} \mathrm{~F}$ NMR $(471 \mathrm{MHz}, \mathrm{DMSO}) \delta$-65.93. MS (ESI) $\mathrm{m} / \mathrm{z}$ calcd. for $\mathrm{C}_{15} \mathrm{H}_{10} \mathrm{~F}_{3} \mathrm{NO}_{2}\left([\mathrm{M}+\mathrm{H}]^{+}\right)$294.1, found 294.2 . 


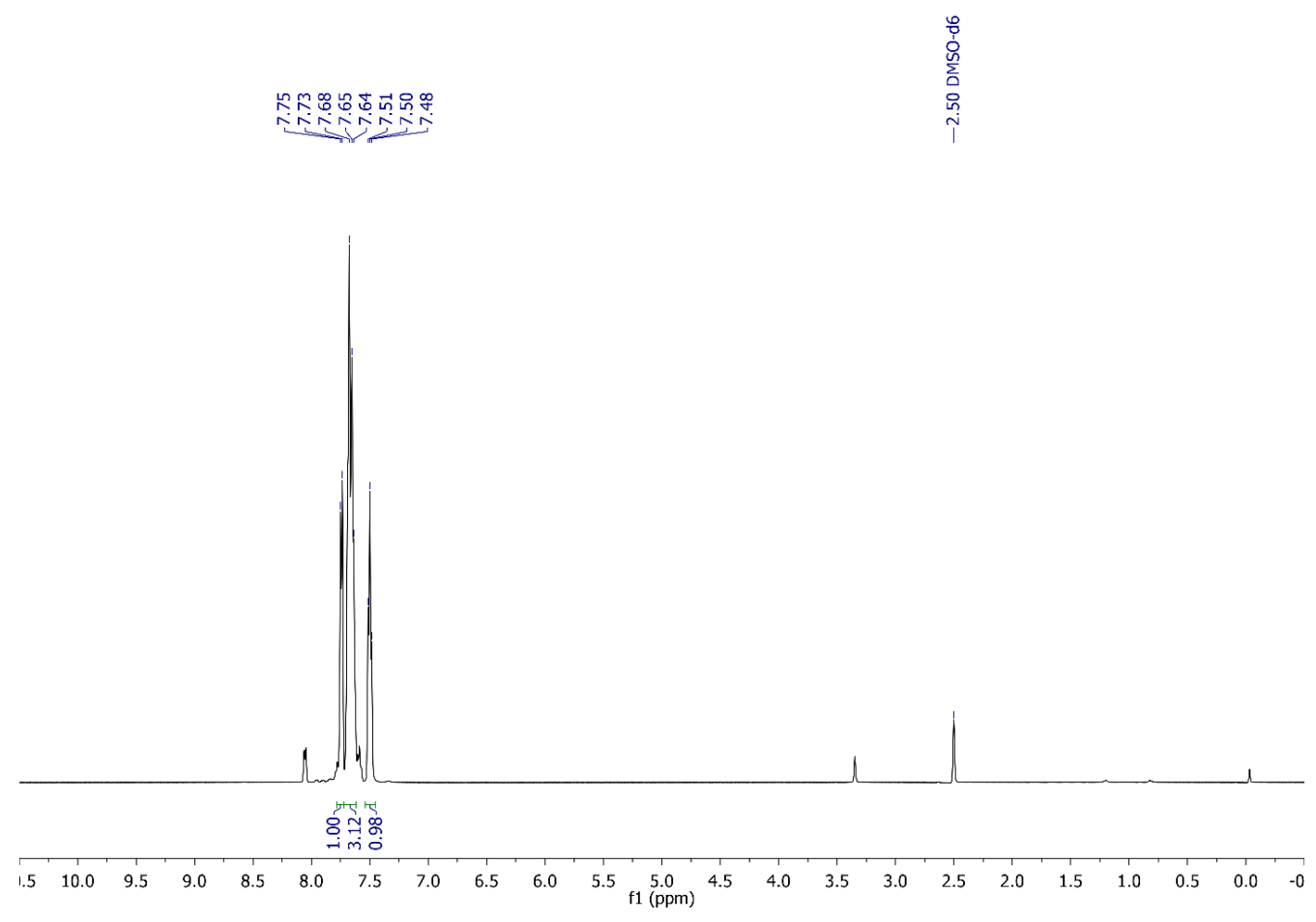

Figure S87. ${ }^{1} \mathrm{H}$ qNMR $(500 \mathrm{MHz})$ spectrum of compound 3p in DMSO- $d_{6}$

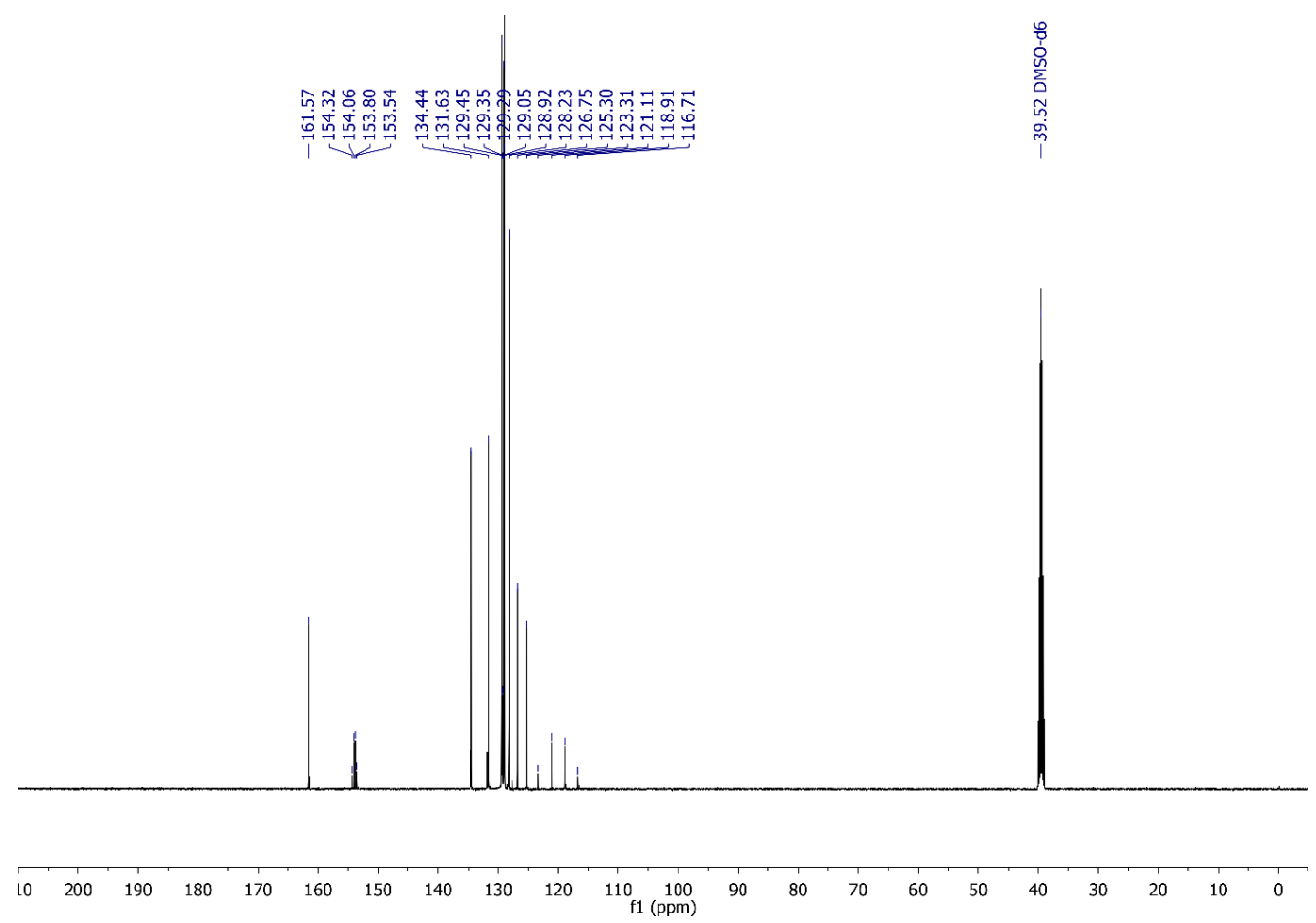

Figure S88. ${ }^{13} \mathrm{C}$ NMR $(126 \mathrm{MHz})$ spectrum of $\mathbf{3 p}$ in DMSO- $d_{6}$. 


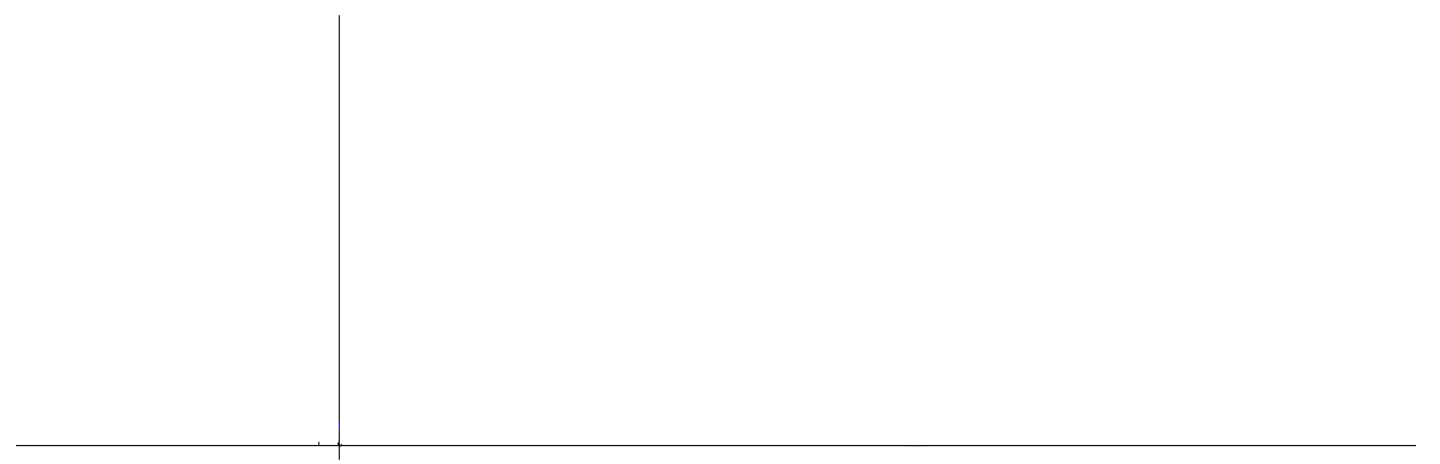

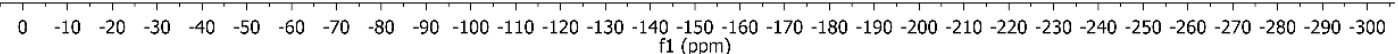

Figure S89. ${ }^{19} \mathrm{~F}$ NMR (471 MHz) spectrum of compound 3p in DMSO- $d_{6}$. 
<smiles>CC(C)(C)ON=C(c1ccc(Br)cc1)C(F)(F)F</smiles>

Compound 3q was synthesized according to general method A using 1-(4-bromophenyl)-2,2,2trifluoroethan-1-one (2.532 g, $10 \mathrm{mmol})$, pyridine $(2.01 \mathrm{~mL}, 25.0 \mathrm{mmol})$, hydroxylamine hydrochloride $(1.042 \mathrm{~g}, 15$ $\mathrm{mmol})$, and ethanol $(20 \mathrm{~mL})$. Then the crude material of the first step was reacted with pyridine $(0.887 \mathrm{~mL}, 11 \mathrm{mmol})$, benzoyl chloride $(1.16 \mathrm{~mL}, 10 \mathrm{mmol})$, in methylene chloride $(6 \mathrm{~mL})$ and hexanes $(24 \mathrm{~mL})$ according to the general method. The crude product was recrystallized from $\mathrm{CH}_{2} \mathrm{Cl}_{2} / \mathrm{Hexanes}\left(-20^{\circ} \mathrm{C}\right.$ and rapid filtration required) to afford the product as a white crystalline solid $(1.1 \mathrm{~g}, 30 \%) .{ }^{1} \mathrm{H}$ NMR $\left(500 \mathrm{MHz}, \mathrm{DMSO}-d_{6}\right) \delta 7.88(\mathrm{~d}, J=8.6 \mathrm{~Hz}, 2 \mathrm{H}), 7.78$ $(\mathrm{d}, J=7.3 \mathrm{~Hz}, 2 \mathrm{H}), 7.74-7.66(\mathrm{~m}, 3 \mathrm{H}), 7.55$ (t, $J=7.8 \mathrm{~Hz}, 2 \mathrm{H}) .{ }^{13} \mathrm{C}$ NMR $(126 \mathrm{MHz}, \mathrm{DMSO}) \delta 161.55,153.24$, $152.97,152.71,152.45,134.57,130.53,129.44,129.19,126.67,125.54,124.35,123.14,120.94,118.74,116.54 .{ }^{19} \mathrm{~F}$ NMR (471 MHz, DMSO) $\delta$-65.84.MS (ESI) m/z calcd. For $\mathrm{C}_{14} \mathrm{H}_{10} \mathrm{~F}_{3} \mathrm{BrNO}_{2}([\mathrm{M}+\mathrm{H}]+)$ ) 372.0, found 372.1 


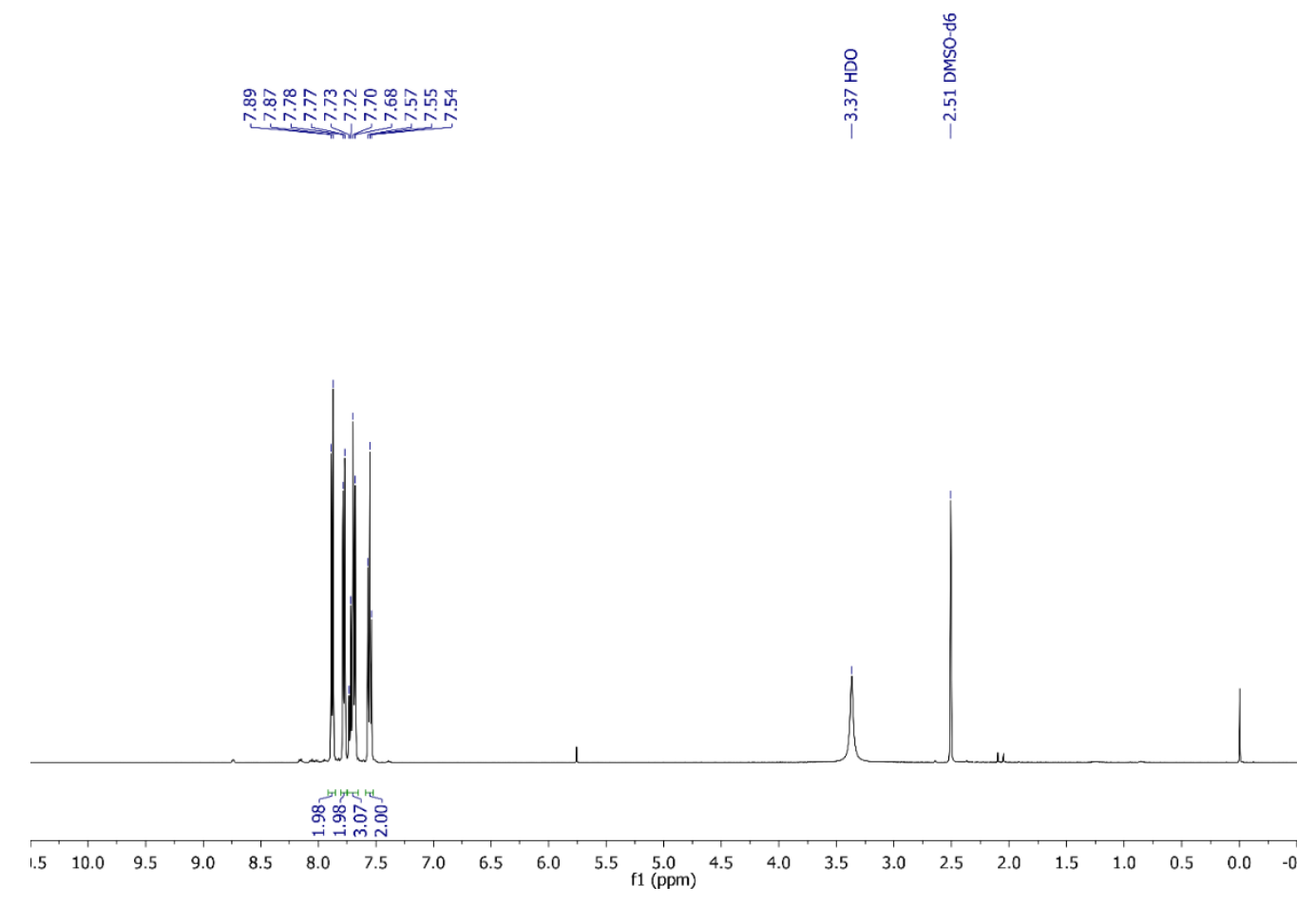

Figure S90. ${ }^{1} \mathrm{H}$ qNMR $(500 \mathrm{MHz})$ spectrum of compound 3q in DMSO- $d_{6}$

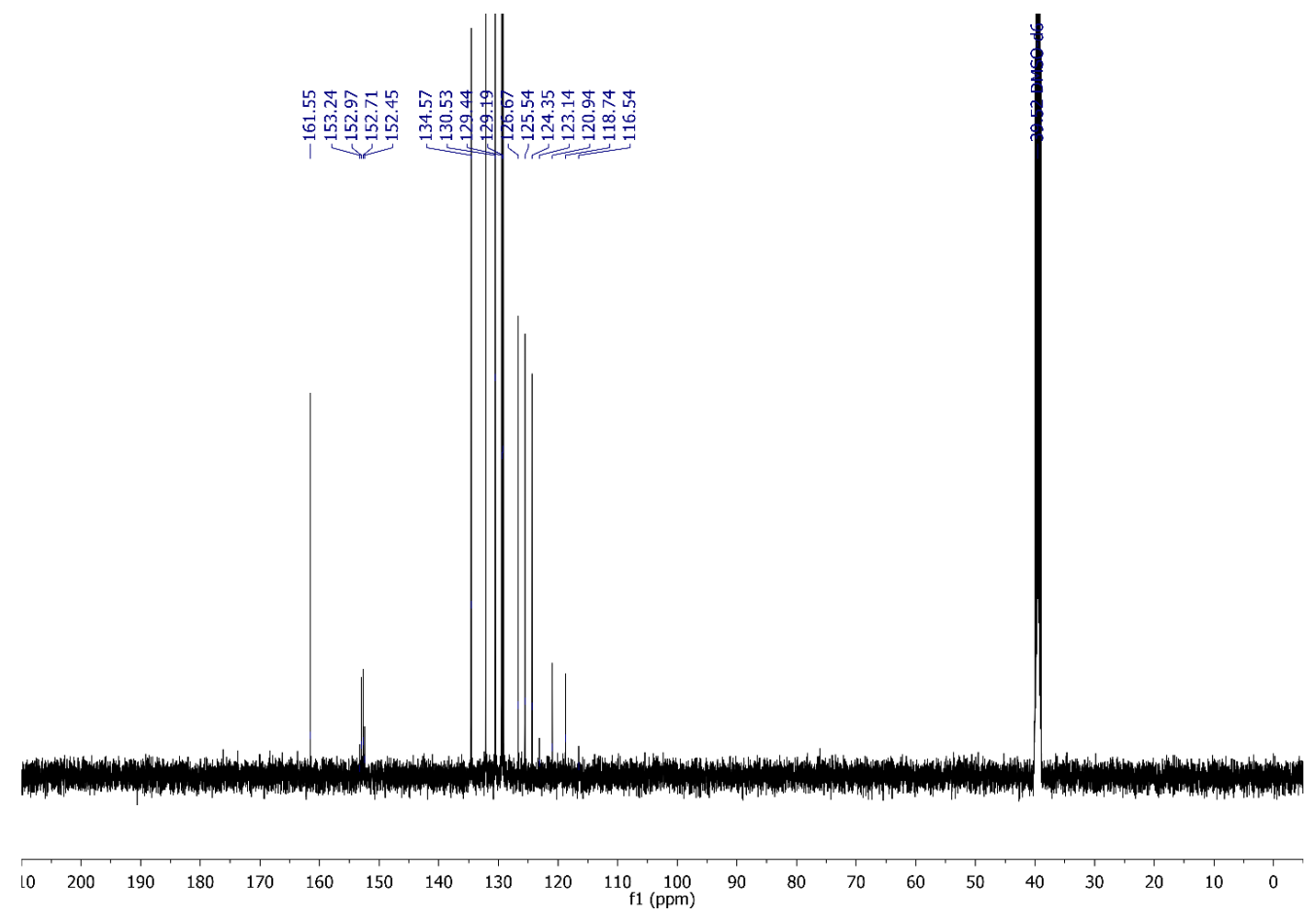

Figure S91. ${ }^{13} \mathrm{C}$ NMR $(126 \mathrm{MHz})$ spectrum of $\mathbf{3 q}$ in DMSO- $d_{6}$. 


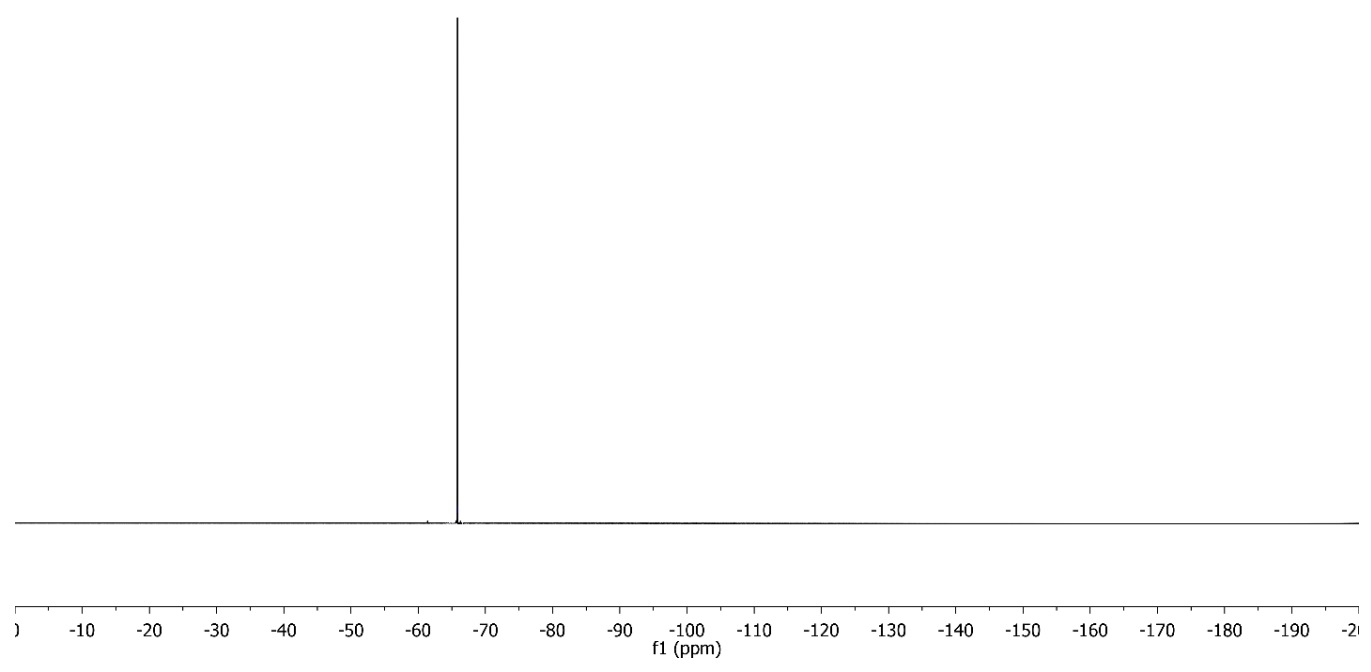

Figure S92. ${ }^{19} \mathrm{~F}$ NMR $(471 \mathrm{MHz})$ spectrum of compound $\mathbf{3 q}$ in DMSO- $d_{6}$. 


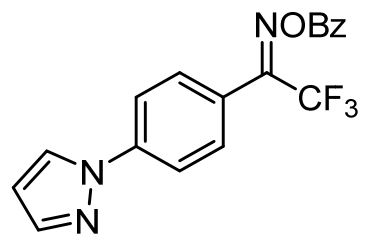

Compound 3r was synthesized according to general method A using 1-(4-(1H-pyrazol-1-yl)phenyl)-2,2,2trifluoroethan-1-one ${ }^{6}(2.40 \mathrm{~g}, 10.0 \mathrm{mmol})$, pyridine $(2.0 \mathrm{~mL}, 25.0 \mathrm{mmol})$, hydroxylamine hydrochloride $(1.042 \mathrm{~g}, 15.0$ $\mathrm{mmol})$, and ethanol $(20 \mathrm{~mL})$. Then the crude material of the first step was reacted with triethylamine $(1.35 \mathrm{~mL}, 9.68$ mmol), benzoyl chloride $(1.06 \mathrm{~mL}, 9.68 \mathrm{mmol})$, in methylene chloride $(6 \mathrm{~mL})$ and hexanes $(24 \mathrm{~mL})$ according to the general method. The crude product was recrystallized from $\mathrm{CH}_{2} \mathrm{Cl}_{2} / \mathrm{Hexanes}$ to afford the product as a white crystalline solid (3.233 g, 90\%). The crystalline solid is observed to be a mixture of isomers the oxime. ${ }^{1} \mathrm{H} \mathrm{NMR}(500 \mathrm{MHz}$, DMSO- $\left.d_{6}\right) \delta 8.66(\mathrm{dd}, J=9.3,2.5 \mathrm{~Hz}, 1 \mathrm{H}), 8.16-8.12(\mathrm{~m}, 1 \mathrm{H}), 8.08(\mathrm{ddd}, J=11.5,7.7,1.6 \mathrm{~Hz}, 2 \mathrm{H}), 7.89-7.76(\mathrm{~m}$, $5 \mathrm{H}), 7.71-7.62(\mathrm{~m}, 1 \mathrm{H}), 7.56-7.50(\mathrm{~m}, 1 \mathrm{H}), 6.62(\mathrm{dt}, J=2.8,1.5 \mathrm{~Hz}, 1 \mathrm{H}) .{ }^{13} \mathrm{C}$ NMR $(126 \mathrm{MHz}, \mathrm{DMSO}) \delta 161.65$, $161.42,153.39,153.35,153.14,153.10,152.88,152.86,152.62,142.04,141.95,141.66,134.68,134.45,130.61$, $130.17,129.47,129.44,129.32,129.13,128.28,128.24,126.78,126.68,124.92,123.37,122.37,121.17,121.03$, 118.97, 118.77, 118.28, 118.26, 116.77, 116.51, 114.26, 108.68, 108.66. MS (ESI) m/z calcd. for $\mathrm{C}_{18} \mathrm{H}_{12} \mathrm{~F}_{3} \mathrm{~N}_{3} \mathrm{O}_{2}([\mathrm{M}$ $+\mathrm{H}]^{+}$) 360.1 , found 360.1 


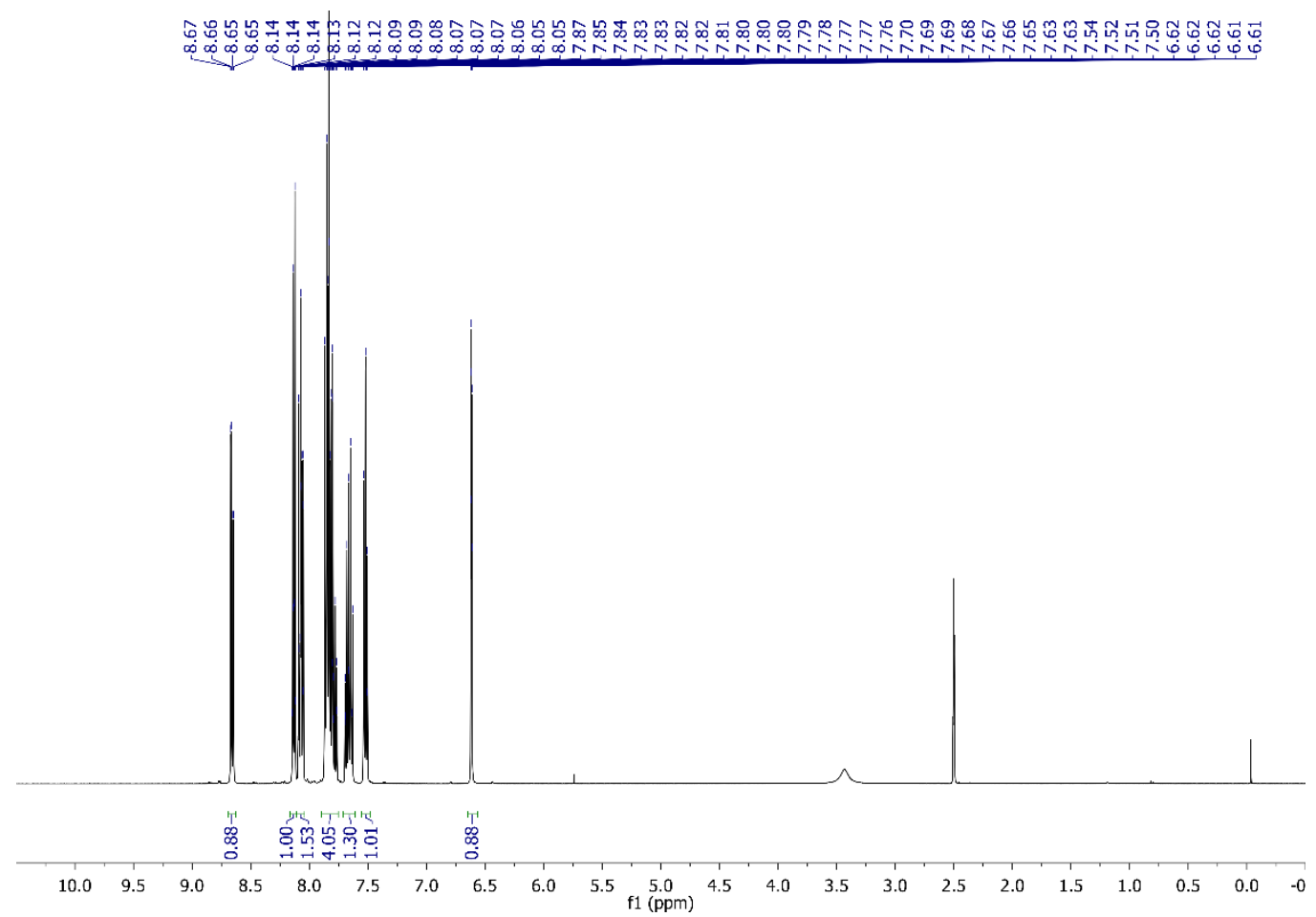

Figure S93. ${ }^{1} \mathrm{H}$ qNMR $(500 \mathrm{MHz})$ spectrum of $3 \mathbf{r}$ in $\mathrm{CD}_{3} \mathrm{CN}$.
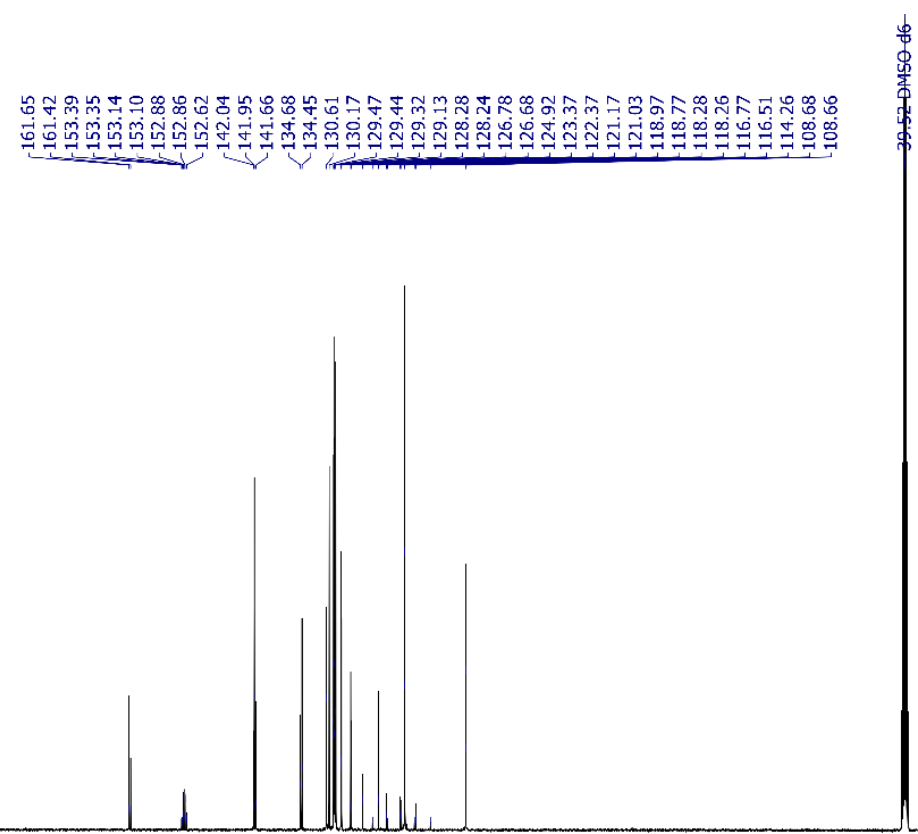

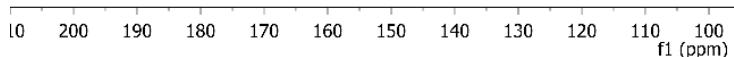

Figure S94. ${ }^{13} \mathrm{C}$ NMR $(126 \mathrm{MHz})$ spectrum of $3 \mathbf{r}$ in DMSO- $d_{6}$. 


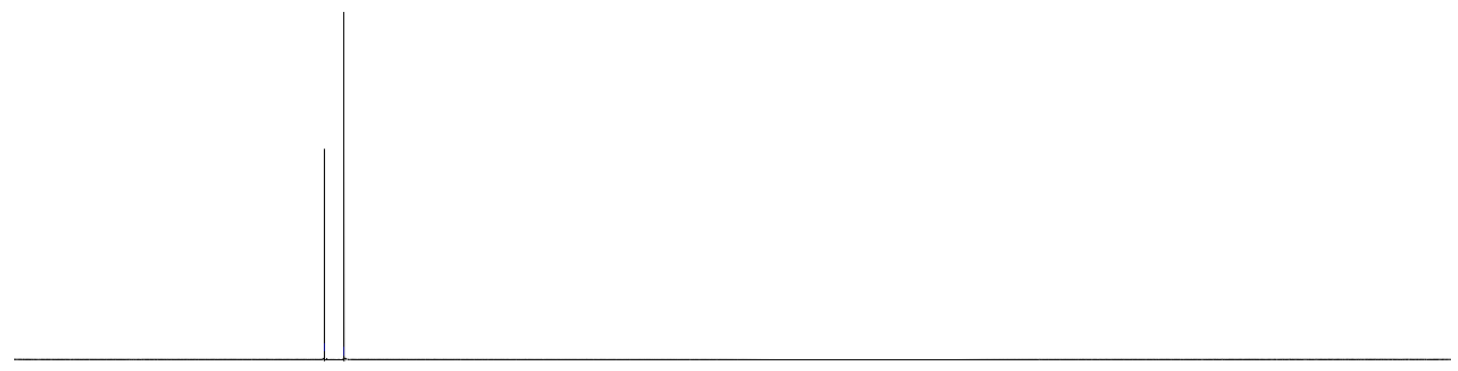

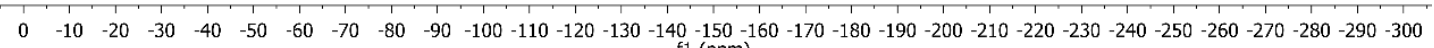
Figure S95. ${ }^{19} \mathrm{~F}$ NMR (471 MHz) spectrum of compound $3 \mathbf{r}$ in DMSO- $d_{6}$. 


\section{Synthesis of Iminium Hydrochlorides}

\section{Synthesis of Substrates}

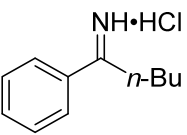

Synthesis of $\mathbf{6 l} \cdot \mathbf{H C l}$ was prepared following a literature procedure.<smiles>CC(=NCl)c1ccc(C(F)(F)F)cc1</smiles>

Synthesis of $6 \mathbf{b} \cdot \mathbf{H C l}$ was prepared following a literature procedure. ${ }^{7}$<smiles>CN=C(Cl)c1ccc(Cl)cc1</smiles>

Synthesis of $\mathbf{6 c} \cdot \mathbf{H C l}$ was prepared following a literature procedure. ${ }^{7}$<smiles></smiles>

Synthesis of $\mathbf{6 d} \bullet \mathbf{H C l}$ was prepared following a literature procedure. ${ }^{7}$<smiles>C[C@H](N=CCl)c1ccccc1</smiles>

Synthesis of $6 \mathbf{6 a} \cdot \mathbf{H C l}$ was prepared following a literature procedure. ${ }^{7}$<smiles>C/C(=N\Cl)c1ccc(C)cc1</smiles>

Synthesis of $6 \mathbf{6 e} \cdot \mathbf{H C l}$ was prepared following a literature procedure. ${ }^{7}$<smiles>COc1ccc(C(C)=NCl)cc1</smiles>

Synthesis of $\mathbf{6 f} \bullet \mathbf{H C l}$ was prepared following a literature procedure. ${ }^{7}$

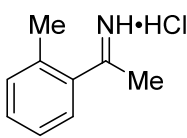

Synthesis of $\mathbf{6 g} \cdot \mathbf{H C l}$ was prepared following a literature procedure. ${ }^{7}$<smiles>Cc1ccc2ccccc2c1</smiles>

Synthesis of $6 \mathbf{h} \cdot \mathbf{H C l}$ was prepared following a literature procedure. ${ }^{7}$ 


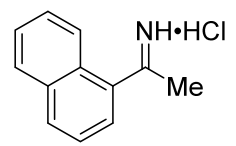

Synthesis of $6 \mathbf{6} \cdot \mathbf{H C l}$ was prepared following a literature procedure. ${ }^{7}$

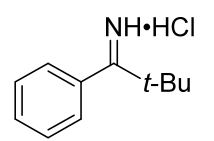

Synthesis of $6 \mathbf{m} \cdot \mathbf{H C l}$ was prepared following a literature procedure. ${ }^{7}$ 


\section{UV-Vis Absorption/Emission Spectra and Stern-Volmer Plot}

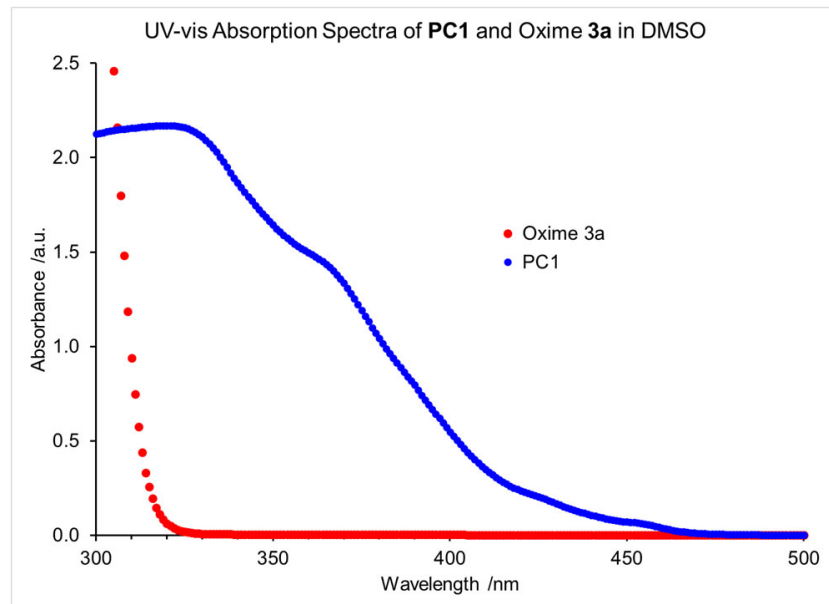

Figure S96. (A) UV-vis absorption spectra of PC1 and oxime 3a in DMSO illustrating the absorbance edge for PC1 (ca. $470 \mathrm{~nm})$ and $\mathbf{3 a}($ ca. $330 \mathrm{~nm})$.
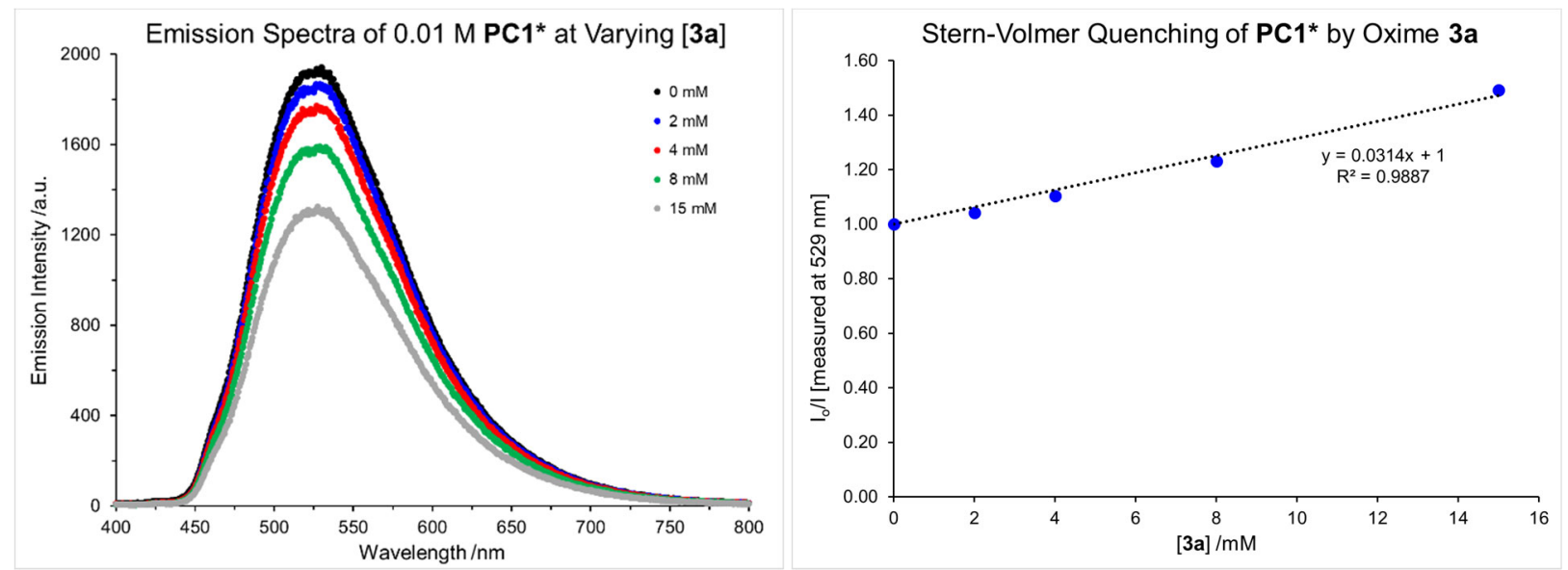

Figure S97. (Left) Emission spectra of PC1* $([\mathrm{PC1}]=0.01 \mathrm{M}$ in DMSO) as a function of concentration of oxime 3a $(0,2,4,8,15 \mathrm{mM})$ in DMSO using $\lambda_{\mathrm{exc}}=380 \mathrm{~nm}$. (Right) Stern-Volmer plot for the quenching of PC1* $([\mathbf{P C 1}]=$ $0.01 \mathrm{M}$ in DMSO) by oxime $\mathbf{3 a}\left(\lambda_{\mathrm{ex}}=380 \mathrm{~nm}, \lambda_{\mathrm{em}}=529 \mathrm{~nm}\right)$.

If one wants to estimate the rate constant for the quenching event $\left(k_{\mathrm{q}}\right)$, the data from the Stern-Volmer plot in Figure S93B can be fit to the following Stern-Volmer equation: ${ }^{8}$

$$
I_{\mathrm{o}} / I=1+K_{\mathrm{Sv}}[\mathbf{3 a}]
$$

where $I_{\mathrm{o}}$ is the emission intensity of PC1* $([\mathbf{P C 1}]=0.01 \mathrm{M})$ at $529 \mathrm{~nm}$ with no oxime present $([3 \mathbf{3 a}]=0 \mathrm{mM}), I$ is the emission intensity of PC1* $([\mathbf{P C 1}]=0.01 \mathrm{M})$ at $529 \mathrm{~nm}$ with oxime present $([\mathbf{3 a}]=2,4,8,15 \mathrm{mM})$ and the $K_{\mathrm{SV}}$ is the Stern-Volmer constant. Since $K_{\mathrm{SV}}=k_{\mathrm{q}} \bullet \tau_{\mathrm{o}}$ where $\tau_{\mathrm{o}}$ is the lifetime of the excited state $(\mathbf{P C} \mathbf{1} *)$, if one estimates this lifetime to be between 0.5 and $2.5 \mu \mathrm{s},{ }^{9}$ typical values for Ir-based photocatalysts containing two phenyl-pyridyl ligands and one bipyridyl ligand (e.g. $\left.\operatorname{Ir}(\mathrm{ppy})_{2} \mathrm{dtbbpy}_{\mathrm{PF}}, \tau_{\mathrm{o}}=0.54 \mu \mathrm{s}\right)$, one arrives at an estimated $k_{\mathrm{q}}$ value between $1.3 \times 10^{7}$ $\mathrm{M}^{-1} \cdot \mathrm{s}^{-1}$ and $6.3 \times 10^{7} \mathrm{M}^{-1} \cdot \mathrm{s}^{-1}$. 


\section{DFT Computational Study}

\subsection{Computational Method}

All of the quantum chemical calculations were performed using the Gaussian 16 program..$^{10}$ Geometry optimizations and frequency calculations were performed at the M06-2X/6-31+G(d,p) level of theory ${ }^{11,12}$ with $\mathrm{SMD}^{13}$ solvation in DMSO. A "tight" geometry convergence (opt=tight), an "ultrafine" integration grid, and a 2-electron integral accuracy of $10^{-14}$ (int $=($ Acc $2 E=14$,ultrafine $)$ ) were used. The stationary points were characterized by the presence of only positive eigenvalues of the Hessian for minima. All Gibbs energies were calculated for a temperature of $298.15 \mathrm{~K}$ and a pressure of $1 \mathrm{~atm}$ using the quasiharmonic approximation proposed by Cramer and Truhlar, in which all of the real vibrational frequencies lower than $100 \mathrm{~cm}^{-1}$ were set to $100 \mathrm{~cm}^{-1} .{ }^{14}$ All molecular structures were rendered in CYLView ${ }^{15}$ in combination with POV-Ray 3.6. ${ }^{16}$ All CYLView generated images have the following color-coding for labeling elements: $\mathrm{C}=$ silver, $\mathrm{H}=$ white, $\mathrm{O}=$ light red, $\mathrm{N}=$ blue.

The DFT-calculated reduction potentials against SCE were obtained using the following equation:

$$
\begin{aligned}
E^{\circ}, \text { calc } & =-\left[(\text { energy of reduced species }- \text { energy of oxidized species }) /\left(n_{\mathrm{e}} \bullet \mathrm{F}\right)\right]-3.96 \mathrm{~V}-0.244 \mathrm{~V} \\
& =\left[E A /\left(n_{\mathrm{e}} \bullet \mathrm{F}\right)\right]-3.96 \mathrm{~V}-0.244 \mathrm{~V}
\end{aligned}
$$

where $n_{\mathrm{e}}=1$, and $n_{\mathrm{e}} \cdot \mathrm{F}=23.061 \mathrm{kcal} \cdot \mathrm{mol}^{-1} \bullet \mathrm{V}^{-1}$. The adoption of the SHE reference potential is explained below. In the second equality, $E A$ refers to the electron affinity and follows the conventional definition, namely, energy of the oxidized species minus energy of the reduced species. More positive values of $E A$ correspond to more favorable singleelectron reductions. To convert $E A$ and $E^{\circ}$,calc to reduction potential values that are comparable to experiment, appropriate values of SHE and conversion factors between different electrodes needed to be identified. The value of SHE in DMSO of $3.96 \mathrm{~V}$ was adopted from the review of Marenich et al., ${ }^{17}$ while the conversion factor from SHE to SCE $(-0.244 \mathrm{~V})$ was taken from Pavlishchuk et al. ${ }^{18}$ 


\subsection{DFT Calculations Regarding the PCET Reduction of 4-Cyanopyridine}

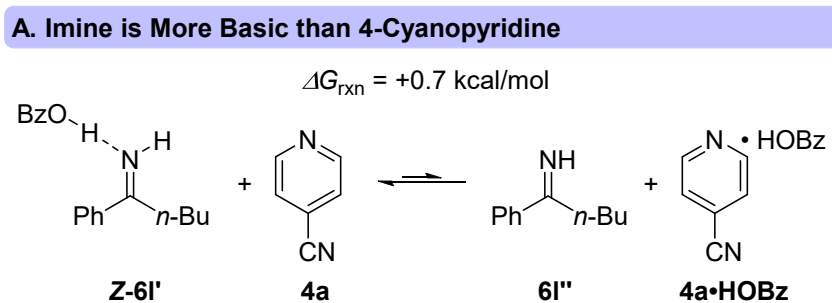

B. Benzoic Acid Protonates DIPA Favorably Over 4-Cyanopyridine

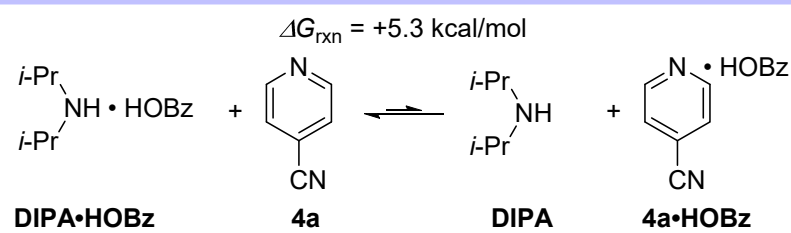

C. Summary of DFT Calculated Redox Potentials

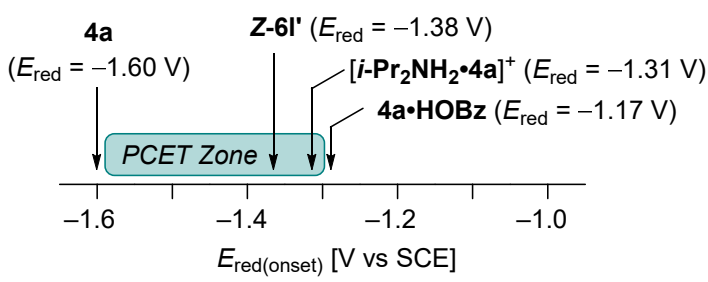

Figure S98. DFT calculated energies and redox potentials using M06-2X/6-31+G(d,p) SMD=DMSO.
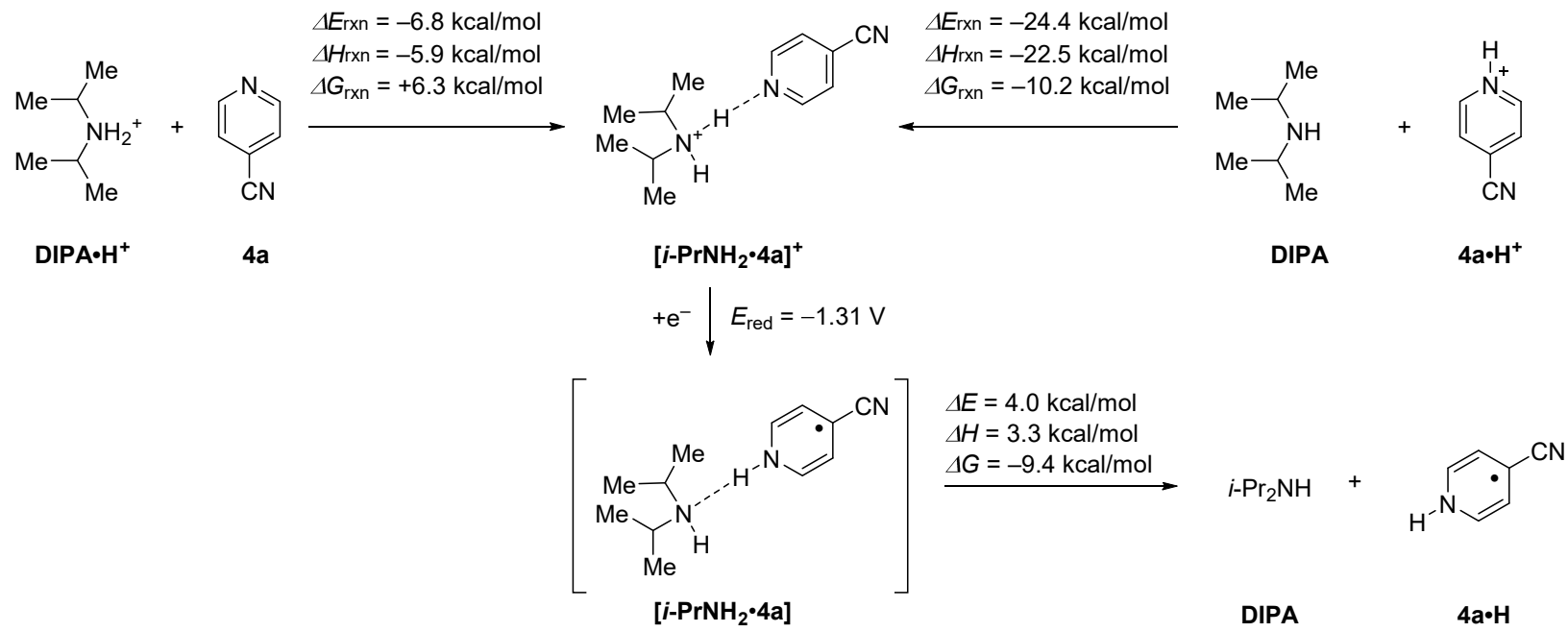

Scheme S99. DFT analysis (using M06-2X/6-31+G(d,p) SMD=DMSO) of hydrogen bonded complex $\left[\boldsymbol{i}-\mathbf{P r N H} \mathbf{N}^{\bullet 4} \mathbf{a}\right]^{+}$ and its reduction potential (vs SCE).

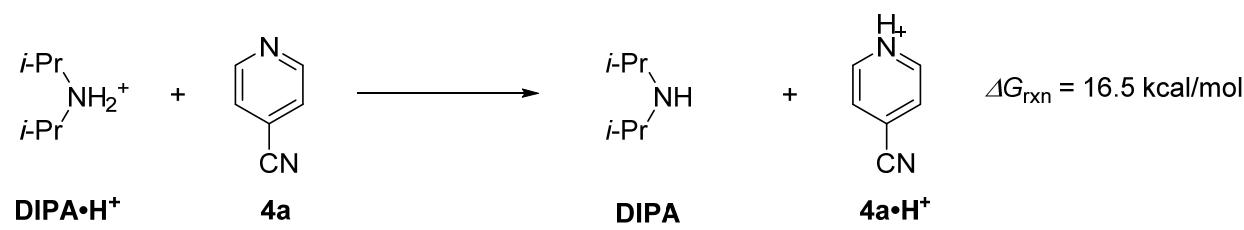

Scheme S100. DFT calculated energies (M06-2X/6-31+G(d,p) SMD=DMSO) for the proton exchange equilibrium between diisopropylammonium DIPA $\cdot \mathbf{H}^{+}$and 4-cyanopyridine (4a). 
To characterize the hydrogen bonded complex $\left[\boldsymbol{i}-\mathbf{P r N H}_{2} \bullet \mathbf{4 a}\right]^{+}$, the DFT data in Scheme S99 (top left) were obtained and the hydrogen-bonding interaction contributes $-5.9 \mathrm{kcal} / \mathrm{mol}$ by enthalpy but is disfavored by entropy, resulting in a net $+6.3 \mathrm{kcal} / \mathrm{mol}$ in Gibbs energy relative to infinitely separated DIPA $\cdot \mathrm{H}^{+}+\mathbf{4 a}$. Thus, the hydrogen-bonding is enthalpically favorable but not strong enough to overcome the entropy loss due to complex formation. The Gibbs energy change suggests that complex formation between DIPA $\cdot \mathrm{H}^{+}$ and $4 \mathbf{a}$ is slightly endergonic but not prohibitive.

We also calculated the energy of $\left[\boldsymbol{i}-\mathbf{P r N H}_{2} \cdot \mathbf{4 a}\right]^{+}$relative to the pyridinium ion $\mathbf{4 a} \cdot \mathrm{H}^{+}$and neutral DIPA. $[\boldsymbol{i}$ $\left.\mathbf{P r N H}_{2} \cdot \mathbf{4 a}\right]^{+}$is lower in energy than infinitely separated DIPA and $\mathbf{4 a} \cdot \mathrm{H}^{+}$by $22.5 \mathrm{kcal} / \mathrm{mol}$ by enthalpy and $10.2 \mathrm{kcal} / \mathrm{mol}$ by Gibbs energy (Scheme S99, top right). The more favorable energies in this equation compared with the energy changes discussed in the preceding paragraph are a consequence of the lower stability of pyridinium $\mathbf{4 a} \cdot \mathrm{H}^{+}$relative to DIPA $\cdot \mathbf{H}^{+}$. This is further supported by the computed equilibrium in Scheme S100.

To characterize the association of the diisoproylammonium $\left(\right.$ DIPA $\left.\cdot \mathrm{H}^{+}\right)$and 4-cyanopyridine $(\mathbf{4 a})$, we used DFT calculations to evaluate the energies of two tautomerically related hydrogen-bonded complexes: the diisopropylammonium $\bullet$ pyridine complex DIPA $\cdot \mathrm{H}^{+} \ldots \mathbf{4 a}$ and the diisopropylamine $\bullet$ pyridinium complex DIPA...4a $\mathbf{a}^{+} \mathrm{H}$ (Scheme $\left.\mathrm{S} 101\right)$. The DIPA $\cdot \mathrm{H}^{+} \ldots \mathbf{4 a}$ complex could be located as a minimum. However, the "pyridinium complex" (DIPA...4a $\left.\mathbf{a} \cdot \mathrm{H}^{+}\right)$could not be located as a stationary point. All attempts of full optimization of the "pyridinium complex" (DIPA ...4a $\left.\cdot \mathrm{H}^{+}\right)$led back to DIPA $\cdot \mathrm{H}^{+} \ldots \mathbf{4 a}$ in which the proton in the $\mathrm{N}-\mathrm{H}^{+} \ldots \mathrm{N}$ hydrogen bond resides on the diisopropylammonium. Constraining the $\mathrm{N}^{+}-\mathrm{H}$ bond of the pyridinium moiety of the complex DIPA...4a $\cdot \mathrm{H}^{+}$at $1.02 \AA$ (that is, the bond length found in 4cyanopyridinium ion) led to a structure that is $12.5 \mathrm{kcal} / \mathrm{mol}$ less stable than DIPA $\cdot \mathrm{H}^{+} \ldots$.4a. These calculations suggest that the hydrogen-bonded complex associating the proton donor and acceptor is best described by the diisopropylammonium-pyridine form DIPA $\cdot \mathrm{H}^{+} \ldots$. 4a. Thus, the proton in the PCET step to generated $\mathbf{4 a} \cdot \mathbf{H}$ is best understood as coming from diisopropylammonium at early stages of the reaction. At later stages of the reaction, the iminium benzoate (61') may be the source of the proton for the PCET to generated $\mathbf{4 a} \cdot \mathbf{H}$.
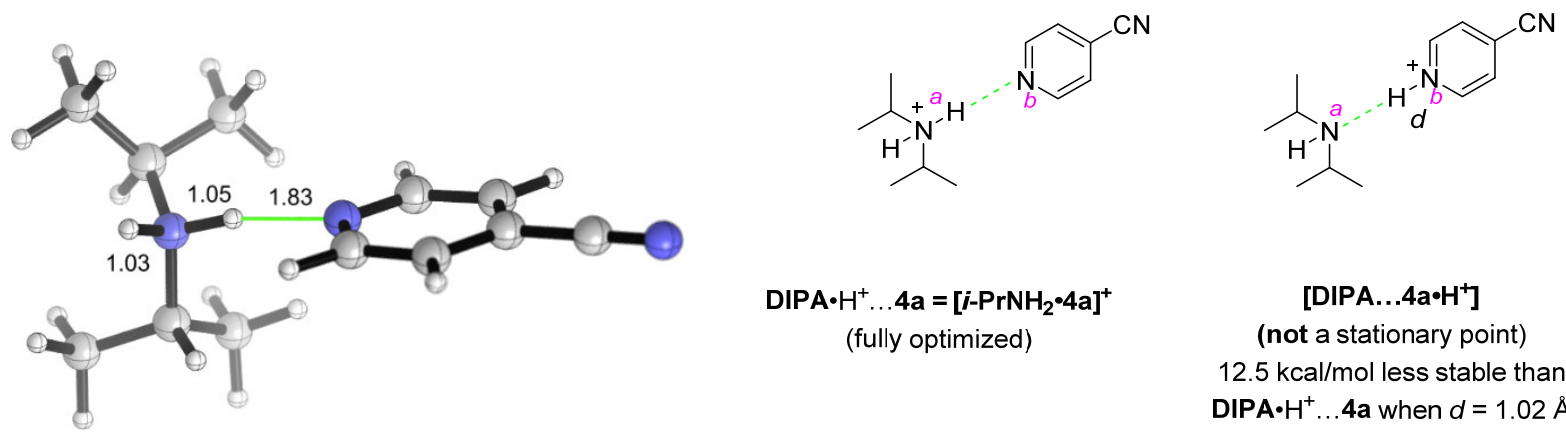

$\mathrm{DIPA} \cdot \mathrm{H}^{+} \ldots \mathbf{4 a}=\left[i-\mathrm{PrNH}_{2} \cdot 4 \mathrm{a}\right]^{+}$

(fully optimized)
[DIPA...4a*H']

(not a stationary point)

$12.5 \mathrm{kcal} / \mathrm{mol}$ less stable than

DIPA $\cdot \mathrm{H}^{+} \ldots$ a when $d=1.02 \AA$

Figure S101. DFT computed structure of DIPA $\bullet H^{+} \ldots \mathbf{4 a}=\left[i-\operatorname{PrNH}_{2} \bullet 4 a\right]^{+}$. 


\subsection{DFT Illustrations of Alpha-Amino Radical Without and With ortho-Methyl Substituent}<smiles>CC(N)c1ccccc1</smiles>

XII

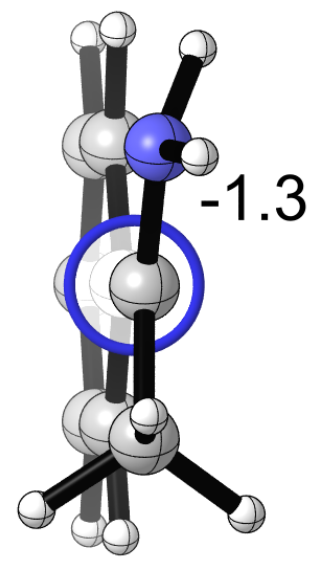<smiles>CC(=[14C])c1ccccc1C</smiles>

conformer 1

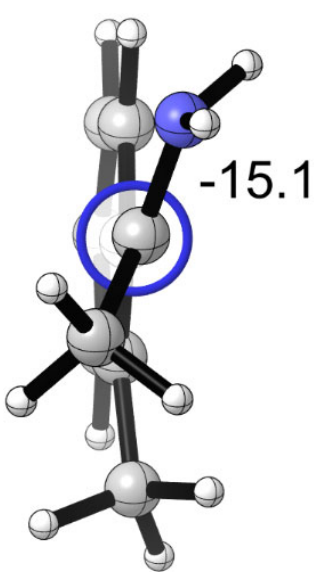<smiles>Cc1ccccc1C(C)N</smiles>

conformer 2

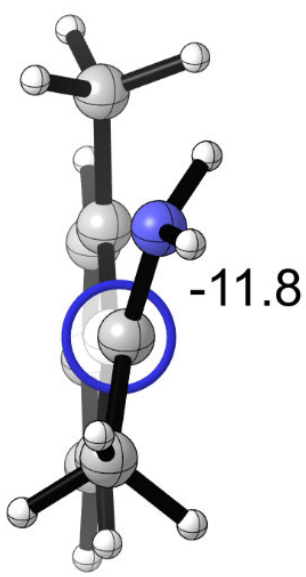

Figure S103. Newman projection (blue circle) illustrating the dihedral angle between the arene and the imine derived $\pi$-systems for alpha-amino radicals derived from iminium chloride $\mathbf{6 a}($ left) and $\mathbf{6 g}$ (middle $=$ conformer $\mathbf{1}$; right $=$ conformer 2), conformer 2 is $+0.25 \mathrm{kcal} / \mathrm{mol}$ relative to conformer 1 . SOMO energies of alpha-amino radicals (left) $-4.74 \mathrm{eV}$ vs vacuum, (middle) $-4.74 \mathrm{eV}$ vs vacuum, and (right) $-4.64 \mathrm{eV}$ vs vacuum. 


\subsection{Illustration of Data Against Cyclopropane Ring Opening}<smiles>NC(=C1CC1)c1ccccc1</smiles>

S1-closed<smiles>NC(=C1CC1)c1ccccc1</smiles>

S1-closed
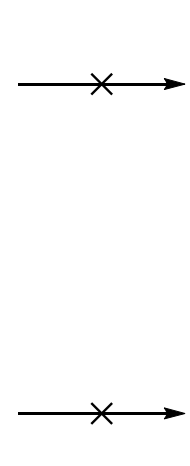<smiles>N/C(=C\CI)c1ccccc1</smiles>

Z-S2-open<smiles>CC/C=C(/N)c1ccccc1</smiles>

\section{E-S2-open}

$\Delta G_{\mathrm{rxn}}=+11.8 \mathrm{kcal} / \mathrm{mol}$

Figure S104. DFT calculated Gibbs free energy for ring opening of methylcyclopropyl radical illustrating the process is unfavored. S1-closed is the preferred form of the radical due to the radical being benzylic and $\alpha$ - to a heteroatom. 


\subsection{Cartesian Coordinates and Energies of DFT Optimized Structures}

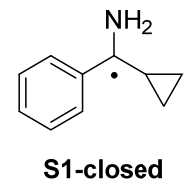

\section{Structure: S1-closed}

Charge $=0$, Multiplicity $=2$

Number of imaginary frequencies: 0

SCF Energy: $\quad-442.819949$ hartree

SCF Energy + ZPVE: -442.623246 hartree

Enthalpy: $\quad-442.612292$ hartree

Free Energy: $\quad-442.659624$ hartree

Free Energy with quasiharmonic correction: -442.658537 hartree
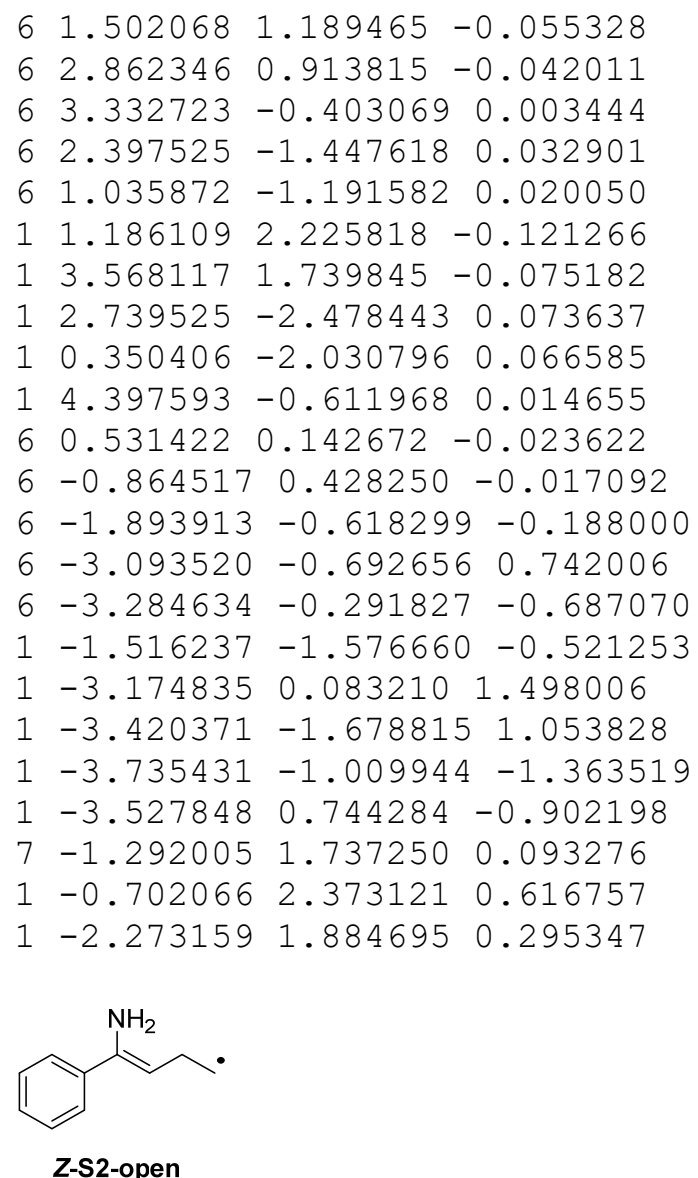

Structure: $\boldsymbol{Z}$-S2-open

Charge $=0$, Multiplicity $=2$

Number of imaginary frequencies: 0

SCF Energy: $\quad-442.797087$ hartree

SCF Energy + ZPVE: -442.603118 hartree

Enthalpy: $\quad-442.591141$ hartree

Free Energy: $\quad-442.640989$ hartree

Free Energy with quasiharmonic correction: -442.639757 hartree 

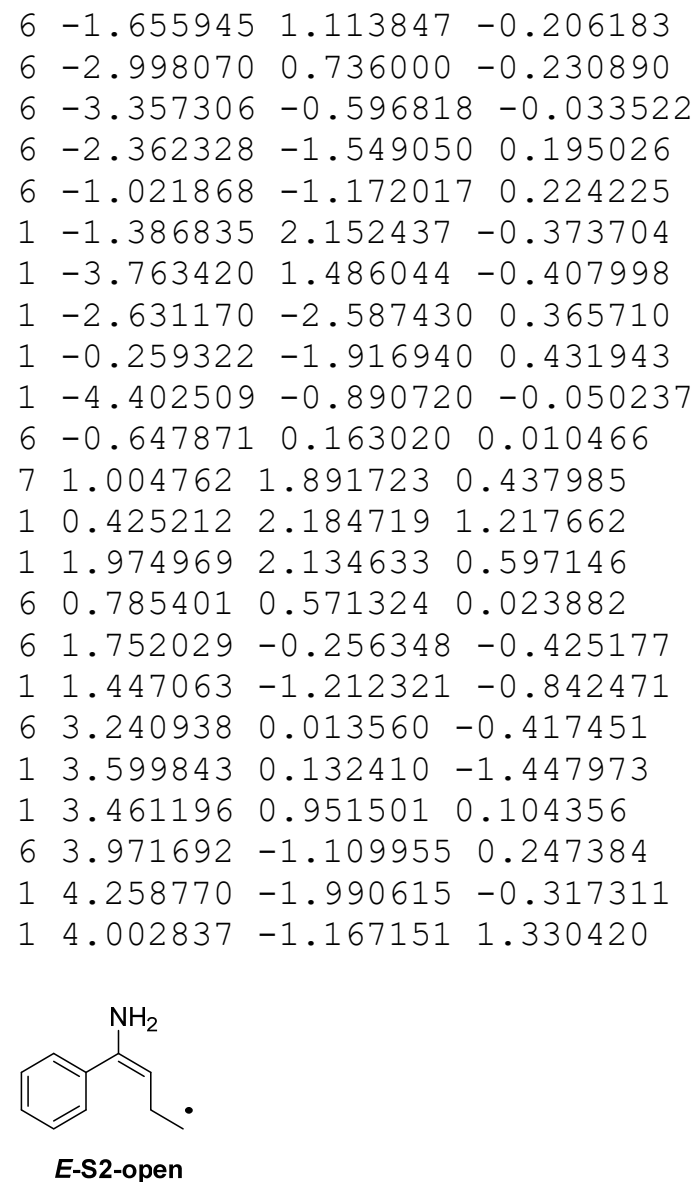

\section{Structure: $\boldsymbol{E}$-S2-open}

Charge $=0$, Multiplicity $=2$

Number of imaginary frequencies: 0

SCF Energy: $\quad-442.794540$ hartree

SCF Energy + ZPVE: -442.600941 hartree

Enthalpy: $\quad-442.588890$ hartree

Free Energy: $\quad-442.638817$ hartree

Free Energy with quasiharmonic correction: -442.637674 hartree
$\begin{array}{llll}6 & 1.367039 & 0.894038 & 0.771167\end{array}$
$\begin{array}{lllll}6 & 2.625698 & 0.318292 & 0.934805\end{array}$
$\begin{array}{lllll}6 & 2.989525 & -0.798916 & 0.181642\end{array}$
$\begin{array}{lllll}6 & 2.088004 & -1.334251 & -0.738863\end{array}$
$\begin{array}{lllll}6 & 0.827521 & -0.760614 & -0.900281\end{array}$
11.0862551 .7632391 .358968
$\begin{array}{lllll}1 & 3.323009 & 0.742418 & 1.651395\end{array}$
$\begin{array}{lllll}1 & 2.368301 & -2.194742 & -1.339259\end{array}$
$\begin{array}{lllll}1 & 0.136491 & -1.167878 & -1.633373\end{array}$
$\begin{array}{lllll}1 & 3.971598 & -1.245308 & 0.306525\end{array}$
$\begin{array}{lllll}6 & 0.447718 & 0.352407 & -0.138544\end{array}$
$\begin{array}{lllll}7 & -0.842948 & 2.390877 & -0.352861\end{array}$
$\begin{array}{lllll}1 & -0.085667 & 2.761910 & -0.918161\end{array}$
$\begin{array}{lllll}1 & -1.727074 & 2.817398 & -0.610500\end{array}$
$\begin{array}{lllll}6 & -0.888753 & 0.985026 & -0.315440\end{array}$ 


$$
\begin{array}{llll}
6 & -2.052547 & 0.305611 & -0.365554 \\
1 & -2.958271 & 0.886568 & -0.545443 \\
6 & -2.273727 & -1.165825 & -0.080272 \\
1 & -2.619820 & -1.675630 & -0.988542 \\
1 & -1.329506 & -1.634416 & 0.214667 \\
6 & -3.284678 & -1.334975 & 1.009743 \\
1 & -4.343960 & -1.385979 & 0.780936 \\
1 & -2.995524 & -1.168473 & 2.042405
\end{array}
$$

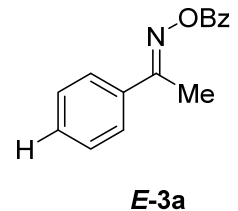

\section{Structure: $\boldsymbol{E}-\mathbf{3 a}$}

Charge $=0$, Multiplicity $=1$

Number of imaginary frequencies: 0

SCF Energy: -784.309711 hartree

SCF Energy + ZPVE: -784.063000 hartree

Enthalpy: $\quad-784.046606$ hartree

Free Energy: $\quad-784.108172$ hartree

Free Energy with quasiharmonic correction: -784.104475 hartree

$$
\begin{array}{llll}
6 & 1.687461 & 2.522671 & 0.829926 \\
6 & 1.336856 & 1.204200 & 0.206520 \\
6 & 2.378408 & 0.160440 & 0.009200 \\
6 & 3.719934 & 0.530430 & -0.141905 \\
6 & 4.697335 & -0.442003 & -0.349896 \\
6 & 4.345757 & -1.790370 & -0.395577 \\
6 & 3.010019 & -2.165281 & -0.235531 \\
6 & 2.030400 & -1.197203 & -0.035916 \\
7 & 0.167459 & 0.881938 & -0.209455 \\
8 & -0.746949 & 1.935786 & -0.015996 \\
6 & -2.050976 & 1.606203 & -0.228217 \\
6 & -2.486523 & 0.191958 & -0.047652 \\
6 & -1.981840 & -0.611878 & 0.980797 \\
6 & -2.494343 & -1.892892 & 1.166667 \\
6 & -3.490909 & -2.379460 & 0.318302 \\
6 & -3.993043 & -1.576485 & -0.706100 \\
6 & -3.502188 & -0.284535 & -0.880678 \\
8 & -2.808172 & 2.506219 & -0.503039 \\
1 & 2.570011 & 2.426848 & 1.462611 \\
1 & 1.898740 & 3.259221 & 0.046513 \\
1 & 0.853998 & 2.897650 & 1.425090 \\
1 & 4.003712 & 1.578350 & -0.112107 \\
1 & 5.733790 & -0.143685 & -0.474558 \\
1 & 5.108932 & -2.547302 & -0.549576 \\
1 & 2.732196 & -3.214636 & -0.260049 \\
1 & 0.992809 & -1.488837 & 0.098293 \\
1 & -1.206440 & -0.236765 & 1.641279 \\
1 & -2.113370 & -2.512888 & 1.972180 \\
1 & -3.878589 & -3.383955 & 0.459163
\end{array}
$$


$1-4.769670-1.952808-1.364479$

$\begin{array}{llll}1 & -3.895451 & 0.354423 & -1.665526\end{array}$

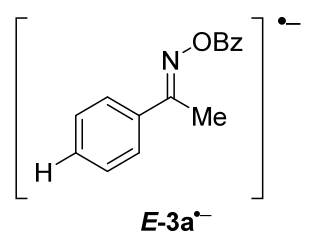

Structure: $\boldsymbol{E}-\mathbf{3 a}{ }^{\cdot-}$

Charge $=-1$, Multiplicity $=2$

Number of imaginary frequencies: 0

SCF Energy: $\quad-784.385248$ hartree

SCF Energy + ZPVE: -784.141864 hartree

Enthalpy: $\quad-784.125164$ hartree

Free Energy: $\quad-784.187921$ hartree

Free Energy with quasiharmonic correction: -784.184134 hartree

$\begin{array}{llll}6 & 1.086306 & 2.272483 & 1.171533 \\ 6 & 1.119836 & 1.204291 & 0.110997 \\ 6 & 2.234482 & 0.326179 & -0.055276 \\ 6 & 3.462517 & 0.460337 & 0.673036 \\ 6 & 4.518419 & -0.416748 & 0.489994 \\ 6 & 4.442889 & -1.479783 & -0.430218 \\ 6 & 3.258803 & -1.620492 & -1.172320 \\ 6 & 2.190679 & -0.753477 & -1.007264 \\ 7 & 0.085863 & 1.027403 & -0.736860 \\ 8 & -0.946609 & 1.993432 & -0.436337 \\ 6 & -2.187912 & 1.504598 & -0.402721 \\ 6 & -2.395553 & 0.065686 & -0.042808 \\ 6 & -1.682900 & -0.534879 & 1.002256 \\ 6 & -1.981381 & -1.839968 & 1.382722 \\ 6 & -2.975348 & -2.557801 & 0.711384 \\ 6 & -3.686480 & -1.958839 & -0.327534 \\ 6 & -3.408355 & -0.641889 & -0.693554 \\ 8 & -3.124051 & 2.254168 & -0.617386 \\ 1 & 1.881663 & 2.141415 & 1.905056 \\ 1 & 1.184111 & 3.278431 & 0.742257 \\ 1 & 0.131620 & 2.253497 & 1.707680 \\ 1 & 3.587269 & 1.270601 & 1.384374 \\ 1 & 5.427069 & -0.270024 & 1.070110 \\ 1 & 5.275841 & -2.161614 & -0.568081 \\ 1 & 3.171931 & -2.427737 & -1.897169 \\ 1 & 1.291231 & -0.898091 & -1.596781 \\ 1 & -0.902084 & 0.018354 & 1.516344 \\ 1 & -1.434591 & -2.301203 & 2.199523 \\ 1 & -3.195924 & -3.580553 & 1.002406 \\ 1 & -4.461826 & -2.512290 & -0.848576 \\ 1 & -3.968080 & -0.161604 & -1.490701\end{array}$




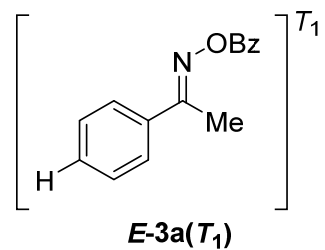

Structure: $\boldsymbol{E}-\mathbf{3 a}\left(\boldsymbol{T}_{\mathbf{1}}\right)$

Charge $=0$, Multiplicity $=3$

Number of imaginary frequencies: 0

SCF Energy: $\quad-784.218448$ hartree

SCF Energy + ZPVE: -783.975083 hartree

Enthalpy: $\quad-783.958257$ hartree

Free Energy: $\quad-784.021835$ hartree

Free Energy with quasiharmonic correction: -784.018044 hartree
$\begin{array}{llll}6 & 1.254270 & 2.461851-0.650418\end{array}$
$61.3194361 .050463-0.157735$
$\begin{array}{lllll}6 & 2.530580 & 0.349773 & 0.134881\end{array}$
$\begin{array}{llll}6 & 3.786766 & 0.986099 & -0.023920\end{array}$

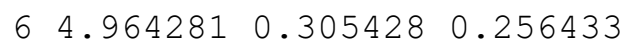
$\begin{array}{llll}6 & 4.931745 & -1.019564 & 0.702199\end{array}$
$\begin{array}{llll}6 & 3.698927 & -1.661243 & 0.865622\end{array}$
$\begin{array}{llll}6 & 2.514478 & -0.994551 & 0.588067\end{array}$
$\begin{array}{lllll}7 & 0.091063 & 0.399234 & 0.045567\end{array}$
$8-0.251123-0.234752-1.141878$
$6-1.465006-0.904153-1.115385$
$6-2.561999-0.358115-0.276528$
$\begin{array}{llll}6 & -2.739365 & 1.017176 & -0.085379\end{array}$
$\begin{array}{llll}6 & -3.840788 & 1.472436 & 0.633590\end{array}$
$\begin{array}{llll}6 & -4.749400 & 0.561760 & 1.175914\end{array}$
$\begin{array}{llll}6 & -4.570116 & -0.808817 & 0.984740\end{array}$
$\begin{array}{llll}6 & -3.484121 & -1.270320 & 0.246265\end{array}$
$8-1.559213-1.869049-1.826871$
$11.813023 \quad 3.139476 \quad 0.004817$
$\begin{array}{lllll}1 & 1.685648 & 2.545215 & -1.656569\end{array}$
$\begin{array}{lllll}1 & 0.216959 & 2.801450 & -0.694881\end{array}$
$\begin{array}{lllll}1 & 3.830681 & 2.013404 & -0.372178\end{array}$
$\begin{array}{lllll}1 & 5.917029 & 0.810236 & 0.125717\end{array}$
$\begin{array}{lllll}1 & 5.855524 & -1.546291 & 0.920160\end{array}$
$\begin{array}{lllll}1 & 3.664677 & -2.689811 & 1.212556\end{array}$
$\begin{array}{llll}1 & 1.565406 & -1.505398 & 0.723013\end{array}$
$\begin{array}{llll}1 & -2.035792 & 1.727727 & -0.507077\end{array}$
$\begin{array}{lllll}1 & -3.988593 & 2.538611 & 0.772726\end{array}$
$\begin{array}{lllll}1 & -5.600598 & 0.921989 & 1.745817\end{array}$
$\begin{array}{llll}1 & -5.277062 & -1.516517 & 1.405803\end{array}$
$\begin{array}{llll}1 & -3.339782 & -2.333642 & 0.081063\end{array}$ 
$\overbrace{E-3 b}^{M e}$

Structure: $\boldsymbol{E}-\mathbf{3 b}$

Charge $=0$, Multiplicity $=1$

Number of imaginary frequencies: 0

SCF Energy: $\quad-1121.270489$ hartree

SCF Energy + ZPVE: -1121.018920 hartree

Enthalpy: $\quad-1120.998874$ hartree

Free Energy: $\quad-1121.070562$ hartree

Free Energy with quasiharmonic correction: -1121.064238 hartree

$\begin{array}{llll}6 & 3.244790 & -1.006756 & 0.524240 \\ 6 & 4.113940 & -0.051294 & 0.003231 \\ 6 & 3.638234 & 1.177677 & -0.454598 \\ 6 & 2.277492 & 1.452486 & -0.387705 \\ 6 & 1.389205 & 0.496719 & 0.122072 \\ 6 & 1.882406 & -0.730161 & 0.580675 \\ 6 & -0.070553 & 0.783341 & 0.155302 \\ 7 & -0.821243 & -0.245164 & -0.000454 \\ 8 & -2.168101 & 0.135290 & 0.045078 \\ 6 & -3.039800 & -0.898007 & -0.100376 \\ 6 & -4.447121 & -0.408627 & -0.064798 \\ 8 & -2.710435 & -2.049185 & -0.237447 \\ 6 & -4.763045 & 0.948528 & 0.067466 \\ 6 & -6.098041 & 1.344961 & 0.089571 \\ 6 & -7.113643 & 0.393954 & -0.018814 \\ 6 & -6.796770 & -0.959355 & -0.149744 \\ 6 & -5.464905 & -1.361558 & -0.173191 \\ 6 & -0.579079 & 2.182666 & 0.341128 \\ 6 & 5.589481 & -0.311134 & -0.066122 \\ 9 & 6.291860 & 0.543471 & 0.709246 \\ 9 & 5.922760 & -1.551185 & 0.327332 \\ 9 & 6.071154 & -0.156483 & -1.317524 \\ 1 & 3.622570 & -1.956136 & 0.888193 \\ 1 & 4.324198 & 1.915322 & -0.861016 \\ 1 & 1.908368 & 2.406227 & -0.751180 \\ 1 & 1.198414 & -1.463995 & 0.993432 \\ 1 & -3.974284 & 1.688906 & 0.151520 \\ 1 & -6.345382 & 2.396979 & 0.190939 \\ 1 & -8.153039 & 0.707700 & -0.001121 \\ 1 & -7.586709 & -1.699066 & -0.233900 \\ 1 & -5.203108 & -2.409979 & -0.275026 \\ 1 & -1.353588 & 2.194860 & 1.111991 \\ 1 & 0.223588 & 2.857765 & 0.634462 \\ 1 & -1.031549 & 2.545856 & -0.587681\end{array}$




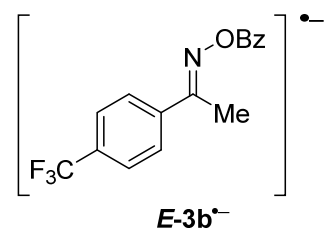

Structure: $\boldsymbol{E}-\mathbf{3} \mathbf{b}^{--}$

Charge $=-1$, Multiplicity $=2$

Number of imaginary frequencies: 0

SCF Energy: $\quad-1121.356768$ hartree

SCF Energy + ZPVE: -1121.108423 hartree

Enthalpy: $\quad-1121.088097$ hartree

Free Energy: $\quad-1121.161452$ hartree

Free Energy with quasiharmonic correction: -1121.154437 hartree

$\begin{array}{llll}6 & -3.303220 & -1.150084 & -0.236288 \\ 6 & -4.120184 & -0.011257 & -0.051611 \\ 6 & -3.510432 & 1.208610 & 0.324316 \\ 6 & -2.145420 & 1.292037 & 0.490831 \\ 6 & -1.288870 & 0.154598 & 0.304312 \\ 6 & -1.938811 & -1.075020 & -0.073653 \\ 6 & 0.125330 & 0.235525 & 0.471687 \\ 7 & 0.826008 & -0.877726 & 0.219874 \\ 8 & 2.228362 & -0.689601 & 0.455612 \\ 6 & 2.930237 & -0.156979 & -0.549875 \\ 6 & 4.396984 & -0.137506 & -0.247246 \\ 8 & 2.450768 & 0.271200 & -1.578757 \\ 6 & 4.918047 & -0.663911 & 0.939756 \\ 6 & 6.290795 & -0.616343 & 1.174072 \\ 6 & 7.143720 & -0.044213 & 0.229169 \\ 6 & 6.623695 & 0.481712 & -0.954762 \\ 6 & 5.252540 & 0.435957 & -1.192564 \\ 6 & 0.745524 & 1.542689 & 0.896605 \\ 6 & -5.584735 & -0.118657 & -0.149061 \\ 9 & -6.205496 & -0.463134 & 1.026862 \\ 9 & -6.184313 & 1.040482 & -0.516814 \\ 9 & -5.995975 & -1.061233 & -1.032833 \\ 1 & -3.757237 & -2.097614 & -0.516447 \\ 1 & -4.121872 & 2.094560 & 0.475160 \\ 1 & -1.717897 & 2.252536 & 0.760193 \\ 1 & -1.336838 & -1.963249 & -0.231896 \\ 1 & 4.253887 & -1.108197 & 1.673201 \\ 1 & 6.694679 & -1.025851 & 2.094810 \\ 1 & 8.213014 & -0.008159 & 0.415419 \\ 1 & 7.286000 & 0.927427 & -1.690524 \\ 1 & 4.833337 & 0.841624 & -2.107882 \\ 1 & 0.207840 & 1.966810 & 1.750045 \\ 1 & 1.788519 & 1.423954 & 1.189763 \\ 1 & 0.702336 & 2.279464 & 0.085134\end{array}$




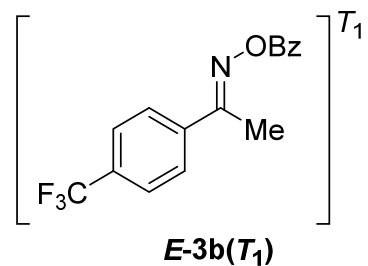

Structure: $E-3 b\left(T_{1}\right)$

Charge $=0$, Multiplicity $=3$

Number of imaginary frequencies: 0

SCF Energy: $\quad-1121.180623$ hartree

SCF Energy + ZPVE: -1120.932202 hartree

Enthalpy: $\quad-1120.911795$ hartree

Free Energy: $\quad-1120.985396$ hartree

Free Energy with quasiharmonic correction: -1120.978951 hartree
$\begin{array}{llll}6 & 2.451490 & -0.525248 & 0.942240\end{array}$
$\begin{array}{lll}6 & 3.512995-0.305913 \quad 0.061089\end{array}$
$\begin{array}{llll}6 & 3.434384 & 0.697378 & -0.912851\end{array}$
$62.2991321 .481678-1.006464$
$\begin{array}{llll}6 & 1.205688 & 1.278392 & -0.124702\end{array}$
$\begin{array}{llll}6 & 1.310463 & 0.255738 & 0.852112\end{array}$
$60.0342172 .084285-0.230433$
$\begin{array}{llll}7 & -1.017497 \quad 1.876987 & 0.680370\end{array}$
$\begin{array}{lllll}8 & -1.886696 & 0.977171 & 0.104240\end{array}$
$\begin{array}{lllll}6 & -3.004850 & 0.705526 & 0.876024\end{array}$
$\begin{array}{llll}6 & -3.884582 & -0.282088 & 0.204093\end{array}$
$\begin{array}{llll}8 & -3.199035 & 1.222988 & 1.941456\end{array}$
$6-3.561008-0.854246-1.032100$
$6-4.429819-1.777480-1.608130$
$\begin{array}{lllll}6 & -5.613118 & -2.127902 & -0.956967\end{array}$
$\begin{array}{lllll}6 & -5.933697 & -1.556067 & 0.275968\end{array}$
$\begin{array}{lllll}6 & -5.070911 & -0.633773 & 0.858071\end{array}$
$\begin{array}{lllll}6 & -0.155258 & 3.183031 & -1.226473\end{array}$
$\begin{array}{lllll}6 & 4.754586 & -1.136514 & 0.127405\end{array}$
$\begin{array}{lllll}9 & 5.865613 & -0.384475 & 0.295134\end{array}$
$94.737753-2.027711 \quad 1.133490$
$94.960783-1.841791-1.008486$
$12.516265-1.3019851 .696970$
$\begin{array}{lllll}1 & 4.263858 & 0.859625 & -1.595906\end{array}$
$\begin{array}{lllll}1 & 2.246324 & 2.254578 & -1.766074\end{array}$
$\begin{array}{lllll}1 & 0.492344 & 0.083272 & 1.544527\end{array}$
$\begin{array}{lllll}1 & -2.641587 & -0.584618 & -1.540052\end{array}$
$1-4.182038-2.223754-2.565891$
$1-6.287146-2.848115-1.410948$
$\begin{array}{lllll}1 & -6.854292 & -1.829218 & 0.781790\end{array}$
$1-5.306723-0.1812161 .815945$
$\begin{array}{lllll}1 & -1.084193 & 3.720280 & -1.024044\end{array}$
$\begin{array}{lllll}1 & 0.675215 & 3.896822 & -1.193069\end{array}$
$\begin{array}{llll}1 & -0.205299 & 2.779167 & -2.245936\end{array}$ 


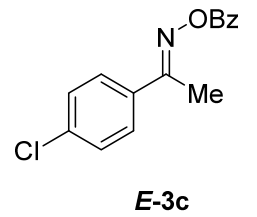

Structure: $\boldsymbol{E}-\mathbf{3 c}$

Charge $=0$, Multiplicity $=1$

Number of imaginary frequencies: 0

SCF Energy: $\quad-1243.885638$ hartree

SCF Energy + ZPVE: -1243.648406 hartree

Enthalpy: $\quad-1243.630784$ hartree

Free Energy: $\quad-1243.696147$ hartree

Free Energy with quasiharmonic correction: -1243.691383 hartree
$63.930814-1.108366 \quad 0.502753$
$6 \quad 4.807598-0.162883-0.022603$
$\begin{array}{llll}6 & 4.359434 & 1.068381 & -0.488424\end{array}$
$\begin{array}{llll}6 & 2.998902 & 1.358099 & -0.421534\end{array}$

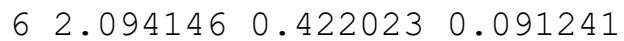
$\begin{array}{llll}6 & 2.573765 & -0.810121 & 0.554658\end{array}$
$\begin{array}{lllll}6 & 0.639977 & 0.727791 & 0.122746\end{array}$
$\begin{array}{lllll}7 & -0.128181 & -0.291231 & -0.014658\end{array}$
$8-1.469500 \quad 0.112407 \quad 0.038698$
$\begin{array}{lllll}17 & 6.516276 & -0.531007 & -0.091254\end{array}$
$\begin{array}{llll}6 & -2.359548 & -0.902020 & -0.116594\end{array}$
$6-3.758103-0.388522-0.060440$
$8-2.052815-2.056697-0.277524$
$\begin{array}{llll}6 & -4.048427 & 0.970729 & 0.104710\end{array}$
$6-5.375726 \quad 1.3908390 .146122$
$\begin{array}{lllll}6 & -6.409391 & 0.461219 & 0.024058\end{array}$
$6-6.118209-0.894171-0.140220$
$\begin{array}{llll}6 & -4.794038 & -1.319896 & -0.182905\end{array}$
$\begin{array}{llll}6 & 0.149105 & 2.136136 & 0.291037\end{array}$
$14.301506-2.058852 \quad 0.871836$
$\begin{array}{lllll}1 & 5.057462 & 1.791417 & -0.896973\end{array}$
$\begin{array}{lllll}1 & 2.648205 & 2.316487 & -0.791829\end{array}$
$\begin{array}{lllll}1 & 1.881932 & -1.534807 & 0.971333\end{array}$
$\begin{array}{lllll}1 & -3.245815 & 1.694696 & 0.199431\end{array}$
$\begin{array}{lllll}1 & -5.602920 & 2.444594 & 0.273389\end{array}$
$\begin{array}{lllll}1 & -7.442713 & 0.793310 & 0.057060\end{array}$
$1-6.922080-1.617391-0.235087$
$1-4.552331-2.370304-0.310565$
$\begin{array}{lllll}1 & 0.962815 & 2.808776 & 0.557890\end{array}$
$\begin{array}{lllll}1 & -0.317911 & 2.486549 & -0.635367\end{array}$
$\begin{array}{llll}1 & -0.610835 & 2.170147 & 1.075794\end{array}$ 


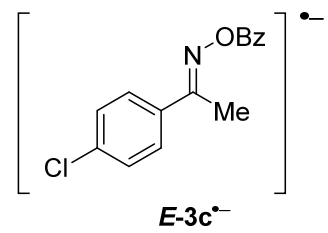

Structure: $\boldsymbol{E}-\mathbf{3 c}^{\mathbf{*}}$

Charge $=-1$, Multiplicity $=2$

Number of imaginary frequencies: 0

SCF Energy: $\quad-1243.958380$ hartree

SCF Energy + ZPVE: -1243.724631 hartree

Enthalpy: $\quad-1243.706621$ hartree

Free Energy: $\quad-1243.773059$ hartree

Free Energy with quasiharmonic correction: -1243.768575 hartree
$62.868148-0.864633-0.948771$
$\begin{array}{llll}6 & 3.952674-0.446719-0.170566\end{array}$
$\begin{array}{lllll}6 & 3.812445 & 0.643081 & 0.702774\end{array}$
$\begin{array}{llll}6 & 2.598841 & 1.298331 & 0.803813\end{array}$
$\begin{array}{llll}6 & 1.451306 & 0.900157 & 0.042009\end{array}$
$61.651759-0.211453-0.852855$
$\begin{array}{lll}6 & 0.184969 & 1.553702 \quad 0.122750\end{array}$
$\begin{array}{lllll}7 & -0.764307 & 1.125312 & -0.730137\end{array}$
$\begin{array}{llll}8 & -1.963002 & 1.905112 & -0.534675\end{array}$
$\begin{array}{lllll}17 & 5.499847 & -1.275514 & -0.296408\end{array}$
$\begin{array}{llll}6 & -3.101312 & 1.207398 & -0.518615\end{array}$
$6-3.075006-0.226728-0.088498$
$8-4.1412391 .772065-0.806417$
$6-2.305849-0.649406 \quad 1.002520$
$\begin{array}{lllll}6 & -2.398794 & -1.965371 & 1.446224\end{array}$
$\begin{array}{llll}6 & -3.240329 & -2.869539 & 0.792308\end{array}$
$6-4.006448-2.448591-0.293920$
$\begin{array}{llll}6 & -3.936402 & -1.123590 & -0.723941\end{array}$
$\begin{array}{llll}6 & -0.092506 & 2.659870 & 1.105397\end{array}$
$12.980026-1.704789-1.629252$
$\begin{array}{lllll}1 & 4.658444 & 0.976712 & 1.297217\end{array}$
$\begin{array}{lllll}1 & 2.541841 & 2.144084 & 1.480762\end{array}$
$\begin{array}{lllll}1 & 0.823451 & -0.556519 & -1.462067\end{array}$
$1-1.6442850 .050068 \quad 1.505173$
$\begin{array}{llll}1 & -1.810457 & -2.288472 & 2.299654\end{array}$
$1-3.300456-3.898720 \quad 1.133635$
$1-4.664064-3.147585-0.801714$
$1-4.542658-0.781898-1.557698$
$\begin{array}{lllll}1 & 0.699603 & 2.755337 & 1.847273\end{array}$
$\begin{array}{lllll}1 & -0.205380 & 3.628819 & 0.602698\end{array}$
$\begin{array}{llll}1 & -1.030375 & 2.473037 & 1.639170\end{array}$ 


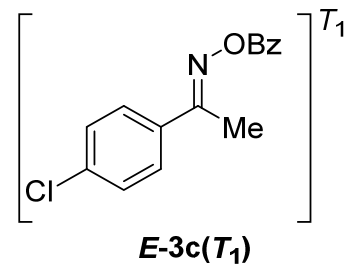

Structure: $\boldsymbol{E}-\mathbf{3 c}\left(\boldsymbol{T}_{1}\right)$

Charge $=0$, Multiplicity $=3$

Number of imaginary frequencies: 0

SCF Energy: $\quad-1243.794686$ hartree

SCF Energy + ZPVE: -1243.560851 hartree

Enthalpy: $\quad-1243.542786$ hartree

Free Energy: $\quad-1243.610674$ hartree

Free Energy with quasiharmonic correction: -1243.605375 hartree

$\begin{array}{llll}6 & 2.939841 & -0.935680 & 0.983988 \\ 6 & 4.019807 & -0.825482 & 0.106714 \\ 6 & 4.058247 & 0.163399 & -0.876031 \\ 6 & 3.001136 & 1.054282 & -0.984812 \\ 6 & 1.884954 & 0.972227 & -0.115408 \\ 6 & 1.883560 & -0.046292 & 0.872444 \\ 6 & 0.796456 & 1.885553 & -0.240582 \\ 7 & -0.277056 & 1.787934 & 0.663466 \\ 8 & -1.213686 & 0.947753 & 0.095814 \\ 17 & 5.350623 & -1.951771 & 0.240636 \\ 6 & -2.346752 & 0.763293 & 0.868473 \\ 6 & -3.298177 & -0.159603 & 0.200645 \\ 8 & -2.502764 & 1.295402 & 1.933467 \\ 6 & -3.020488 & -0.753165 & -1.036641 \\ 6 & -3.954513 & -1.612113 & -1.610038 \\ 6 & -5.157521 & -1.877663 & -0.955099 \\ 6 & -5.432558 & -1.284578 & 0.278857 \\ 6 & -4.504332 & -0.426208 & 0.858328 \\ 6 & 0.714018 & 2.975592 & -1.261374 \\ 1 & 2.928020 & -1.709678 & 1.744643 \\ 1 & 4.906102 & 0.233130 & -1.549747 \\ 1 & 3.035623 & 1.819230 & -1.753922 \\ 1 & 1.047014 & -0.133495 & 1.559339 \\ 1 & -2.086049 & -0.549110 & -1.547629 \\ 1 & -3.742070 & -2.074726 & -2.568561 \\ 1 & -5.882487 & -2.548264 & -1.406595 \\ 1 & -6.368576 & -1.491849 & 0.787648 \\ 1 & -4.704411 & 0.041849 & 1.816927 \\ 1 & 1.603040 & 3.615588 & -1.233966 \\ 1 & 0.637630 & 2.557923 & -2.273780 \\ 1 & -0.165511 & 3.597368 & -1.080459\end{array}$ 


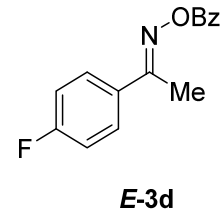

Structure: $\boldsymbol{E}-\mathbf{3 d}$

Charge $=0$, Multiplicity $=1$

Number of imaginary frequencies: 0

SCF Energy: $\quad-883.527583$ hartree

SCF Energy + ZPVE: -883.289015 hartree

Enthalpy: $\quad-883.271775$ hartree

Free Energy: $\quad-883.335964$ hartree

Free Energy with quasiharmonic correction: -883.331397 hartree

$\begin{array}{llll}6 & 4.329488 & -1.236996 & 0.470638 \\ 6 & 5.204289 & -0.293705 & -0.046817 \\ 6 & 4.793543 & 0.948603 & -0.500910 \\ 6 & 3.437647 & 1.258441 & -0.428151 \\ 6 & 2.518860 & 0.330641 & 0.077752 \\ 6 & 2.977710 & -0.915448 & 0.527991 \\ 6 & 1.070673 & 0.660678 & 0.115089 \\ 7 & 0.281955 & -0.341581 & -0.031954 \\ 8 & -1.051542 & 0.089392 & 0.031142 \\ 9 & 6.521923 & -0.598877 & -0.103439 \\ 6 & -1.963610 & -0.904649 & -0.120660 \\ 6 & -3.350560 & -0.360190 & -0.058789 \\ 8 & -1.683740 & -2.066231 & -0.282303 \\ 6 & -3.609480 & 1.005538 & 0.105226 \\ 6 & -4.926690 & 1.455658 & 0.153046 \\ 6 & -5.981729 & 0.549443 & 0.038487 \\ 6 & -5.722002 & -0.812417 & -0.124816 \\ 6 & -4.407946 & -1.268045 & -0.173849 \\ 6 & 0.605457 & 2.075955 & 0.300870 \\ 1 & 4.704669 & -2.191255 & 0.824880 \\ 1 & 5.518144 & 1.650709 & -0.899213 \\ 1 & 3.099627 & 2.225486 & -0.787364 \\ 1 & 2.272682 & -1.630859 & 0.938363 \\ 1 & -2.790504 & 1.711640 & 0.194076 \\ 1 & -5.129311 & 2.514525 & 0.279486 \\ 1 & -7.007132 & 0.904759 & 0.076552 \\ 1 & -6.542419 & -1.517581 & -0.213719 \\ 1 & -4.190843 & -2.323909 & -0.300739 \\ 1 & -0.154446 & 2.114221 & 1.085445 \\ 1 & 1.431189 & 2.730041 & 0.576692 \\ 1 & 0.145705 & 2.446853 & -0.621186\end{array}$




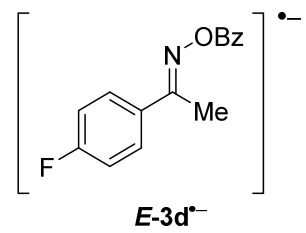

Structure: $\boldsymbol{E}-\mathbf{3 d}^{-}$

Charge $=-1$, Multiplicity $=2$

Number of imaginary frequencies: 0

SCF Energy: $\quad-883.604701$ hartree

SCF Energy + ZPVE: -883.369685 hartree

Enthalpy: $\quad-883.352195$ hartree

Free Energy: $\quad-883.416815$ hartree

Free Energy with quasiharmonic correction: -883.412918 hartree

$\begin{array}{llll}6 & 4.325546 & -1.266492 & 0.373489 \\ 6 & 5.210152 & -0.300452 & -0.081664 \\ 6 & 4.807623 & 0.970410 & -0.454708 \\ 6 & 3.452680 & 1.284518 & -0.364607 \\ 6 & 2.520805 & 0.338147 & 0.082997 \\ 6 & 2.976624 & -0.936871 & 0.453706 \\ 6 & 1.076187 & 0.675510 & 0.146445 \\ 7 & 0.264212 & -0.311942 & -0.003995 \\ 8 & -1.040851 & 0.105909 & 0.069977 \\ 9 & 6.527784 & -0.613093 & -0.158079 \\ 6 & -2.001691 & -0.924004 & -0.064423 \\ 6 & -3.317836 & -0.387562 & -0.052918 \\ 8 & -1.659520 & -2.111938 & -0.163622 \\ 6 & -3.615397 & 1.016033 & 0.056709 \\ 6 & -4.923252 & 1.464183 & 0.063658 \\ 6 & -6.012632 & 0.571977 & -0.035113 \\ 6 & -5.734979 & -0.808299 & -0.142899 \\ 6 & -4.437745 & -1.283193 & -0.152204 \\ 6 & 0.619282 & 2.090270 & 0.362056 \\ 1 & 4.691517 & -2.245272 & 0.665755 \\ 1 & 5.536968 & 1.691696 & -0.808059 \\ 1 & 3.124983 & 2.274711 & -0.665721 \\ 1 & 2.266675 & -1.671862 & 0.818444 \\ 1 & -2.800668 & 1.729717 & 0.133507 \\ 1 & -5.112262 & 2.532655 & 0.147151 \\ 1 & -7.034578 & 0.936844 & -0.028384 \\ 1 & -6.557752 & -1.516432 & -0.220040 \\ 1 & -4.241987 & -2.348441 & -0.235351 \\ 1 & -0.153033 & 2.111391 & 1.136173 \\ 1 & 1.442910 & 2.736391 & 0.665228 \\ 1 & 0.168466 & 2.493204 & -0.552016\end{array}$




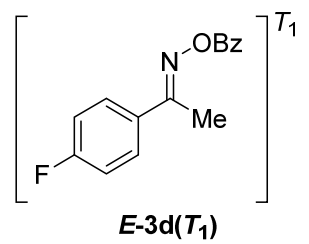

Structure: $\boldsymbol{E}-\mathbf{3 d}\left(\boldsymbol{T}_{\mathbf{1}}\right)$

Charge $=0$, Multiplicity $=3$

Number of imaginary frequencies: 0

SCF Energy: $\quad-883.435774$ hartree

SCF Energy + ZPVE: -883.200541 hartree

Enthalpy: $\quad-883.182924$ hartree

Free Energy: $\quad-883.248728$ hartree

Free Energy with quasiharmonic correction: -883.244417 hartree

$\begin{array}{llll}6 & 3.174802 & -1.290526 & 1.111466 \\ 6 & 4.261882 & -1.300473 & 0.246190 \\ 6 & 4.406961 & -0.388115 & -0.789904 \\ 6 & 3.423073 & 0.574297 & -0.967081 \\ 6 & 2.292616 & 0.628831 & -0.113704 \\ 6 & 2.193529 & -0.328211 & 0.929463 \\ 6 & 1.286122 & 1.622865 & -0.309658 \\ 7 & 0.196916 & 1.674249 & 0.578404 \\ 8 & -0.804369 & 0.883564 & 0.045978 \\ 9 & 5.221034 & -2.242249 & 0.421065 \\ 6 & -1.968783 & 0.874742 & 0.791696 \\ 6 & -2.982730 & -0.022502 & 0.183236 \\ 8 & -2.106563 & 1.522461 & 1.793572 \\ 6 & -2.718503 & -0.782577 & -0.962360 \\ 6 & -3.711686 & -1.610031 & -1.480174 \\ 6 & -4.960452 & -1.678343 & -0.861431 \\ 6 & -5.222163 & -0.918590 & 0.280587 \\ 6 & -4.234779 & -0.091300 & 0.804674 \\ 6 & 1.313364 & 2.653404 & -1.393863 \\ 1 & 3.108228 & -2.025374 & 1.907165 \\ 1 & 5.274617 & -0.438398 & -1.439632 \\ 1 & 3.525450 & 1.290459 & -1.775796 \\ 1 & 1.340974 & -0.309095 & 1.601774 \\ 1 & -1.748531 & -0.731741 & -1.444605 \\ 1 & -3.509558 & -2.201875 & -2.367166 \\ 1 & -5.731533 & -2.324913 & -1.269509 \\ 1 & -6.193858 & -0.972092 & 0.760993 \\ 1 & -4.423653 & 0.504525 & 1.692030 \\ 1 & 0.486909 & 3.356520 & -1.268295 \\ 1 & 2.252549 & 3.218163 & -1.384167 \\ 1 & 1.218610 & 2.185286 & -2.382431\end{array}$




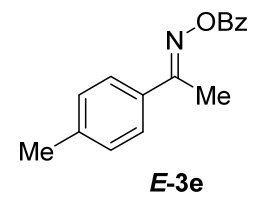

Structure: $\boldsymbol{E}-\mathbf{3 e}$

Charge $=0$, Multiplicity $=1$

Number of imaginary frequencies: 0

SCF Energy: $\quad-823.615733$ hartree

SCF Energy + ZPVE: -823.341478 hartree

Enthalpy: $\quad-823.323202$ hartree

Free Energy: $\quad-823.390032$ hartree

Free Energy with quasiharmonic correction: -823.384736 hartree
$6 \quad 4.314425-1.2030120 .457287$
$65.243983-0.279522-0.042645$
$\begin{array}{lllll}6 & 4.771275 & 0.960170 & -0.481415\end{array}$
$6 \quad 3.414660 \quad 1.277210-0.418491$

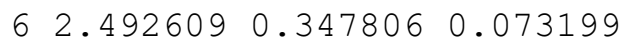
$\begin{array}{llll}6 & 2.960204 & -0.899593 & 0.514310\end{array}$
$\begin{array}{llll}6 & 1.043246 & 0.670320 & 0.104509\end{array}$
$\begin{array}{lllll}7 & 0.257503 & -0.336087 & -0.035635\end{array}$
$8-1.078566 \quad 0.091436 \quad 0.024092$
$6-1.986909-0.905603-0.121660$
$6-3.376106-0.366293-0.057485$
$8-1.704446-2.067237-0.280484$
$\begin{array}{lllll}6 & -3.639980 & 0.998346 & 0.107428\end{array}$
$\begin{array}{llll}6 & -4.958803 & 1.443505 & 0.157918\end{array}$
$\begin{array}{llll}6 & -6.010670 & 0.533353 & 0.045246\end{array}$
$6-5.746042-0.827457-0.118855$
$6-4.430325-1.278034-0.170608$
$\begin{array}{lllll}6 & 0.568655 & 2.084266 & 0.279099\end{array}$
$66.709656-0.619721-0.083954$
$\begin{array}{lllll}1 & 4.662245 & -2.170237 & 0.812503\end{array}$
$\begin{array}{lllll}1 & 5.472416 & 1.690119 & -0.877655\end{array}$
$\begin{array}{lllll}1 & 3.078470 & 2.246737 & -0.774526\end{array}$
$\begin{array}{lllll}1 & 2.256738 & -1.623224 & 0.914398\end{array}$
$\begin{array}{lllll}1 & -2.823333 & 1.707325 & 0.194996\end{array}$
$\begin{array}{lllll}1 & -5.165185 & 2.501562 & 0.285102\end{array}$
$\begin{array}{lllll}1 & -7.037339 & 0.884758 & 0.085477\end{array}$
$1-6.563944-1.535738-0.206283$
$1-4.209390-2.333027-0.298109$
$\begin{array}{lllll}1 & 0.095844 & 2.441426 & -0.641715\end{array}$
$\begin{array}{lllll}1 & -0.183405 & 2.125470 & 1.071223\end{array}$
$\begin{array}{llll}1 & 1.391835 & 2.747787 & 0.539081\end{array}$
$17.280314 \quad 0.154205-0.602712$
$\begin{array}{lllll}1 & 7.112237 & -0.718884 & 0.929835\end{array}$
$16.874822-1.573703-0.594328$ 


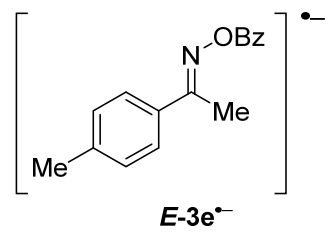

Structure: $\boldsymbol{E}-\mathbf{3} \mathrm{e}^{--}$

Charge $=-1$, Multiplicity $=2$

Number of imaginary frequencies: 0

SCF Energy: $\quad-823.692363$ hartree

SCF Energy + ZPVE: -823.421632 hartree

Enthalpy: $\quad-823.403121$ hartree

Free Energy: $\quad-823.470164$ hartree

Free Energy with quasiharmonic correction: -823.465776 hartree

$\begin{array}{llll}6 & -5.250416 & -0.286697 & 0.076402 \\ 6 & -4.784180 & 0.978888 & 0.440202 \\ 6 & -3.428742 & 1.300536 & 0.358942 \\ 6 & -2.494834 & 0.355331 & -0.080907 \\ 6 & -2.960546 & -0.918001 & -0.448557 \\ 6 & -4.312225 & -1.229223 & -0.369412 \\ 6 & -1.048448 & 0.685276 & -0.137185 \\ 6 & -0.582521 & 2.099443 & -0.339386 \\ 7 & -0.239870 & -0.306346 & 0.006706 \\ 8 & 1.067921 & 0.108464 & -0.061216 \\ 6 & 2.024727 & -0.924496 & 0.069098 \\ 8 & 1.679464 & -2.111532 & 0.170059 \\ 6 & 3.343259 & -0.393685 & 0.052924 \\ 6 & 4.459887 & -1.293938 & 0.149313 \\ 6 & 5.759026 & -0.824595 & 0.135493 \\ 6 & 6.042286 & 0.554569 & 0.025940 \\ 6 & 4.956171 & 1.451284 & -0.070115 \\ 6 & 3.646509 & 1.008670 & -0.058774 \\ 1 & -3.101690 & 2.291238 & 0.661447 \\ 6 & -6.714512 & -0.633432 & 0.138131 \\ 1 & -5.489317 & 1.726521 & 0.795114 \\ 1 & -2.253090 & -1.659397 & -0.807069 \\ 1 & -4.651539 & -2.219211 & -0.666266 \\ 1 & -0.121964 & 2.488108 & 0.575962 \\ 1 & -1.403553 & 2.754944 & -0.628774 \\ 1 & 0.184643 & 2.124578 & -1.118619 \\ 1 & 4.259881 & -2.358302 & 0.233744 \\ 1 & 6.579034 & -1.536192 & 0.210440 \\ 1 & 7.065735 & 0.915107 & 0.015684 \\ 1 & 5.149437 & 2.518900 & -0.155016 \\ 1 & 2.834384 & 1.725557 & -0.133665 \\ 1 & -6.872572 & -1.576009 & 0.671441 \\ 1 & -7.284364 & 0.149737 & 0.644292 \\ 1 & -7.127673 & -0.756177 & -0.869053\end{array}$




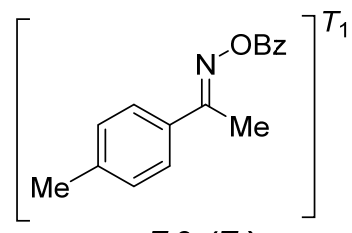

$E-3 \mathrm{e}\left(T_{1}\right)$

Structure: $E-3 e\left(T_{1}\right)$

Charge $=0$, Multiplicity $=3$

Number of imaginary frequencies: 0

SCF Energy: $\quad-823.523970$ hartree

SCF Energy + ZPVE: -823.253063 hartree

Enthalpy: $\quad-823.234397$ hartree

Free Energy: $\quad-823.302932$ hartree

Free Energy with quasiharmonic correction: -823.297904 hartree
$6 \quad 3.175950-1.2435821 .065355$
$\begin{array}{llll}6 & 4.299932 & -1.274144 & 0.221189\end{array}$
$\begin{array}{lllll}6 & 4.386379-0.308897-0.790470\end{array}$
$\begin{array}{lllll}6 & 3.398131 & 0.652300 & -0.962903\end{array}$
$\begin{array}{llll}6 & 2.264736 & 0.684869 & -0.115433\end{array}$
$\begin{array}{llll}6 & 2.179830 & -0.295007 & 0.908988\end{array}$
$\begin{array}{lllll}6 & 1.246579 & 1.668368 & -0.295056\end{array}$

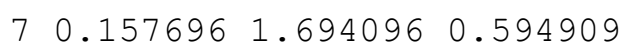
$\begin{array}{lllll}8 & -0.834784 & 0.895736 & 0.053963\end{array}$
$\begin{array}{lllll}6 & -1.996148 & 0.859686 & 0.801428\end{array}$
$\begin{array}{lllll}6 & -2.997071 & -0.047773 & 0.185532\end{array}$
$8-2.143766 \quad 1.493786 \quad 1.810954$
$\begin{array}{lllll}6 & -2.722326 & -0.792887 & -0.967369\end{array}$
$6-3.703065-1.630709-1.492241$
$6-4.949990-1.724342-0.873117$
$\begin{array}{lllll}6 & -5.222202 & -0.979694 & 0.276356\end{array}$
$\begin{array}{llll}6 & -4.247232 & -0.142070 & 0.807343\end{array}$
$\begin{array}{llll}6 & 1.258883 & 2.717532 & -1.362280\end{array}$
$\begin{array}{llll}6 & 5.368582 & -2.316339 & 0.408857\end{array}$
$\begin{array}{llll}1 & 3.088226 & -1.982683 & 1.858731\end{array}$
$\begin{array}{lllll}1 & 5.246107 & -0.313050 & -1.456224\end{array}$
$13.4973451 .383083-1.759883$
$\begin{array}{llll}1 & 1.325667 & -0.298179 & 1.580713\end{array}$
$1-1.753702-0.722396-1.449849$
$1-3.492669-2.210903-2.385002$
$1-5.711333-2.378916-1.286685$
$\begin{array}{lllll}1 & -6.192383 & -1.053049 & 0.757238\end{array}$
$\begin{array}{lllll}1 & -4.444394 & 0.442351 & 1.700471\end{array}$
$\begin{array}{llll}1 & 1.168152 & 2.265642 & -2.358770\end{array}$
$10.424437 \quad 3.408928-1.224082$
$\begin{array}{lllll}1 & 2.191141 & 3.293511 & -1.345005\end{array}$
$\begin{array}{lllll}1 & 6.175230 & -2.189431 & -0.317328\end{array}$
$\begin{array}{lllll}1 & 5.799636 & -2.258892 & 1.414126\end{array}$
$\begin{array}{lllll}1 & 4.957260 & -3.324722 & 0.290772\end{array}$ 


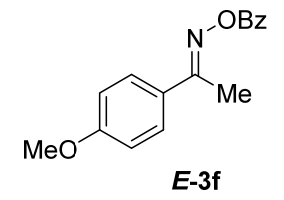

Structure: $\boldsymbol{E}-\mathbf{3 f}$

Charge $=0$, Multiplicity $=1$

Number of imaginary frequencies: 0

SCF Energy: $\quad-898.801821$ hartree

SCF Energy + ZPVE: -898.521756 hartree

Enthalpy: $\quad-898.502892$ hartree

Free Energy: $\quad-898.570307$ hartree

Free Energy with quasiharmonic correction: -898.565703 hartree
$63.977506-0.9459610 .398136$
$\begin{array}{lllll}6 & 4.844295 & 0.038214 & -0.091849\end{array}$
$\begin{array}{llll}6 & 4.329767 & 1.273985 & -0.508548\end{array}$
$\begin{array}{lllll}6 & 2.968344 & 1.522754 & -0.433883\end{array}$

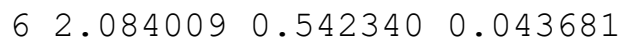
$\begin{array}{llll}6 & 2.610528 & -0.685371 & 0.458046\end{array}$
$\begin{array}{llll}6 & 0.624335 & 0.797843 & 0.083403\end{array}$
$\begin{array}{lllll}7 & -0.118955 & -0.239689 & -0.068297\end{array}$
$\begin{array}{lllll}8 & -1.472515 & 0.129304 & 0.014809\end{array}$
$\begin{array}{lllll}6 & -2.338484 & -0.900818 & -0.148376\end{array}$
$6-3.749141-0.424574-0.055780$
$8-2.009366-2.045074-0.341813$
$\begin{array}{llll}6 & -4.070517 & 0.920990 & 0.156389\end{array}$
$\begin{array}{llll}6 & -5.406997 & 1.306153 & 0.231734\end{array}$
$\begin{array}{llll}6 & -6.419363 & 0.355052 & 0.096979\end{array}$
$6-6.097303-0.986756-0.114232$
$\begin{array}{lllll}6 & -4.763734 & -1.377349 & -0.190748\end{array}$
$\begin{array}{lllll}6 & 0.087732 & 2.186440 & 0.283049\end{array}$
$\begin{array}{lllll}8 & 6.185006 & -0.111584 & -0.192184\end{array}$
$\begin{array}{lllll}6 & 6.745511 & -1.355331 & 0.213100\end{array}$
$\begin{array}{lllll}1 & 4.350595 & -1.904024 & 0.740185\end{array}$
$\begin{array}{lllll}1 & 5.014096 & 2.026136 & -0.888333\end{array}$
$\begin{array}{lllll}1 & 2.591005 & 2.483437 & -0.771395\end{array}$
$\begin{array}{lllll}1 & 1.943687 & -1.447850 & 0.848546\end{array}$
$\begin{array}{lllll}1 & -3.284166 & 1.661297 & 0.261100\end{array}$
$\begin{array}{lllll}1 & -5.657942 & 2.349401 & 0.395698\end{array}$
$\begin{array}{llll}1 & -7.459960 & 0.659709 & 0.156589\end{array}$
$1-6.884456-1.726827-0.219131$
$1-4.498079-2.416862-0.354768$
$\begin{array}{llll}1 & -0.429212 & 2.527028 & -0.619968\end{array}$
$\begin{array}{lllll}1 & -0.641667 & 2.184982 & 1.097395\end{array}$
$\begin{array}{lllll}1 & 0.884254 & 2.887813 & 0.526173\end{array}$
$16.344903-2.180168-0.385646$
$\begin{array}{lllll}1 & 7.818317 & -1.269372 & 0.043093\end{array}$
$16.557375-1.543704 \quad 1.275436$ 


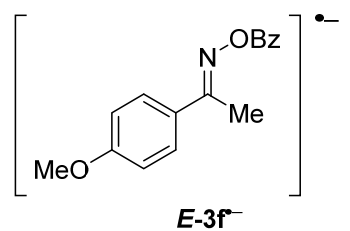

Structure: $\boldsymbol{E}-\mathbf{3 f}$

Charge $=-1$, Multiplicity $=2$

Number of imaginary frequencies: 0

SCF Energy: $\quad-898.877977$ hartree

SCF Energy + ZPVE: -898.601541 hartree

Enthalpy: $\quad-898.582437$ hartree

Free Energy: $\quad-898.650079$ hartree

Free Energy with quasiharmonic correction: -898.646341 hartree
$63.983930-0.956342 \quad 0.346870$
$6 \quad 4.850564 \quad 0.040072-0.116065$
$\begin{array}{lllll}6 & 4.335146 & 1.290570 & -0.480885\end{array}$
$\begin{array}{llll}6 & 2.973886 & 1.540094 & -0.384068\end{array}$
$\begin{array}{llll}6 & 2.086362 & 0.550297 & 0.069318\end{array}$
$\begin{array}{lll}6 & 2.618101-0.691585 & 0.434220\end{array}$
$\begin{array}{llll}6 & 0.627540 & 0.810206 & 0.136683\end{array}$
$\begin{array}{lllll}7 & -0.136584 & -0.214873 & -0.015161\end{array}$
$\begin{array}{lllll}8 & -1.462415 & 0.141564 & 0.075835\end{array}$
$6-2.375141-0.925982-0.080153$
$6-3.714984-0.452102-0.051823$
$8-1.982130-2.095322-0.210602$
$\begin{array}{lllll}6 & -4.078377 & 0.933279 & 0.090837\end{array}$
$\begin{array}{lllll}6 & -5.405871 & 1.318866 & 0.109921\end{array}$
$\begin{array}{llll}6 & -6.452693 & 0.378749 & -0.007673\end{array}$
$6-6.110242-0.984588-0.146498$
$6-4.792296-1.397051-0.168952$
$\begin{array}{lllll}6 & 0.098184 & 2.198746 & 0.361749\end{array}$
$\begin{array}{lllll}8 & 6.192640 & -0.111741 & -0.237660\end{array}$
$\begin{array}{llll}6 & 6.748839 & -1.370912 & 0.118458\end{array}$
$\begin{array}{lllll}1 & 4.355872 & -1.928407 & 0.649038\end{array}$
$\begin{array}{lllll}1 & 5.017294 & 2.054538 & -0.841162\end{array}$
$\begin{array}{lllll}1 & 2.598141 & 2.513626 & -0.685168\end{array}$
$\begin{array}{lllll}1 & 1.953419 & -1.465784 & 0.804989\end{array}$
$\begin{array}{lllll}1 & -3.297631 & 1.682415 & 0.182419\end{array}$
$\begin{array}{lllll}1 & -5.644845 & 2.375106 & 0.217938\end{array}$
$\begin{array}{lllll}1 & -7.490678 & 0.694726 & 0.008890\end{array}$
$1-6.898887-1.728988-0.238116$
$1-4.546637-2.449631-0.276910$
$\begin{array}{llll}1 & -0.411988 & 2.566407 & -0.535612\end{array}$
$\begin{array}{lllll}1 & -0.643220 & 2.181990 & 1.166001\end{array}$
$\begin{array}{lllll}1 & 0.893732 & 2.894076 & 0.628551\end{array}$
$16.336980-2.173592-0.502800$
$17.820594-1.286821-0.059999$
$\begin{array}{lllll}1 & 6.571494 & -1.595457 & 1.175851\end{array}$ 


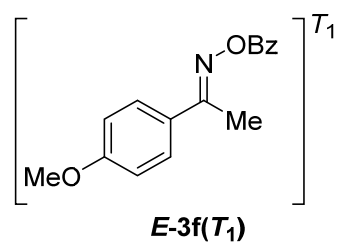

Structure: $E-\mathbf{3 f}\left(T_{1}\right)$

Charge $=0$, Multiplicity $=3$

Number of imaginary frequencies: 0

SCF Energy: $\quad-898.709600$ hartree

SCF Energy + ZPVE: -898.433106 hartree

Enthalpy: $\quad-898.413720$ hartree

Free Energy: $\quad-898.483978$ hartree

Free Energy with quasiharmonic correction: -898.478632 hartree

$\begin{array}{llll}6 & 3.018587 & -0.962467 & 0.731800\end{array}$

$64.091310-0.728063-0.139709$

$64.0657010 .394693-0.985110$

$\begin{array}{llll}6 & 2.994322 & 1.265323-0.963708\end{array}$

$\begin{array}{llll}6 & 1.892911 & 1.051970 & -0.091362\end{array}$

$61.941326-0.085706 \quad 0.750486$

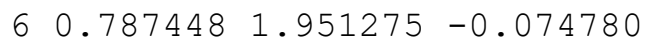

$\begin{array}{llll}7 & -0.260995 & 1.724262 & 0.835297\end{array}$

$\begin{array}{llll}8 & -1.202510 & 0.936993 & 0.190433\end{array}$

$\begin{array}{lllll}6 & -2.307884 & 0.639401 & 0.961500\end{array}$

$\begin{array}{lllll}6 & -3.274384 & -0.198704 & 0.206846\end{array}$

$\begin{array}{llll}8 & -2.439453 & 1.022240 & 2.092854\end{array}$

$\begin{array}{llll}6 & -3.043497 & -0.602677 & -1.113449\end{array}$

$6-3.990488-1.387964-1.766042$

$6-5.159902-1.768924-1.106970$

$\begin{array}{lllll}6 & -5.387974 & -1.365498 & 0.210253\end{array}$

$\begin{array}{lllll}6 & -4.446849 & -0.581006 & 0.868287\end{array}$

$\begin{array}{lllll}6 & 0.656603 & 3.156562 & -0.953327\end{array}$

$8 \quad 5.185274-1.520632-0.236482$

$\begin{array}{llll}6 & 5.245682 & -2.670375 & 0.598700\end{array}$

$13.013332-1.8198891 .394843$

$\begin{array}{lllll}1 & 4.903553 & 0.561727 & -1.655426\end{array}$

$\begin{array}{lllll}1 & 2.998218 & 2.123249 & -1.629166\end{array}$

$\begin{array}{llll}1 & 1.120907 & -0.283032 & 1.434891\end{array}$

$1-2.135150-0.308138-1.627496$

$1-3.814839-1.702511-2.789822$

$\begin{array}{lllll}1 & -5.895154 & -2.381260 & -1.620378\end{array}$

$\begin{array}{lllll}1 & -6.297697 & -1.662263 & 0.722496\end{array}$

$\begin{array}{lllll}1 & -4.610228 & -0.259749 & 1.892054\end{array}$

$\begin{array}{lllll}1 & 0.544561 & 2.867741 & -2.006882\end{array}$

$\begin{array}{lllll}1 & -0.221231 & 3.740077 & -0.665937\end{array}$

$\begin{array}{lllll}1 & 1.538781 & 3.802723 & -0.880984\end{array}$

$\begin{array}{lllll}1 & 4.415891 & -3.353715 & 0.387824\end{array}$

$\begin{array}{lllll}1 & 6.189866 & -3.161697 & 0.364871\end{array}$

$\begin{array}{llll}1 & 5.232209 & -2.388953 & 1.657122\end{array}$ 


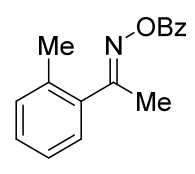

\section{$E-3 g$}

Structure: $\boldsymbol{E}-\mathbf{3 g}$

Charge $=0$, Multiplicity $=1$

Number of imaginary frequencies: 0

SCF Energy: $\quad-823.613519$ hartree

SCF Energy + ZPVE: -823.338914 hartree

Enthalpy: $\quad-823.321006$ hartree

Free Energy: $\quad-823.385867$ hartree

Free Energy with quasiharmonic correction: -823.381690 hartree

$$
\begin{array}{llll}
6 & -4.468487 & -1.615178 & 0.809986 \\
6 & -5.409241 & -0.837868 & 0.136933 \\
6 & -5.012677 & 0.336434 & -0.501867 \\
6 & -3.683771 & 0.771603 & -0.475836 \\
6 & -2.742534 & -0.017854 & 0.212430 \\
6 & -3.137986 & -1.204512 & 0.840816 \\
6 & -1.310405 & 0.388490 & 0.287310 \\
7 & -0.478820 & -0.511538 & -0.090196 \\
8 & 0.835862 & -0.038421 & 0.072330 \\
6 & 1.792873 & -0.912675 & -0.329539 \\
6 & 3.153388 & -0.337643 & -0.122795 \\
8 & 1.568064 & -2.003966 & -0.790555 \\
6 & 3.349157 & 0.943287 & 0.406159 \\
6 & 4.644007 & 1.427431 & 0.576560 \\
6 & 5.739384 & 0.638978 & 0.221817 \\
6 & 5.542629 & -0.638511 & -0.305691 \\
6 & 4.251194 & -1.127405 & -0.478579 \\
6 & -0.915679 & 1.736129 & 0.820599 \\
6 & -3.296541 & 2.036397 & -1.200624 \\
1 & -4.765099 & -2.533580 & 1.307098 \\
1 & -6.449844 & -1.146581 & 0.101572 \\
1 & -5.745872 & 0.928520 & -1.043949 \\
1 & -2.393697 & -1.796609 & 1.365357 \\
1 & 2.498020 & 1.556962 & 0.681915 \\
1 & 4.797587 & 2.420783 & 0.986316 \\
1 & 6.747262 & 1.020145 & 0.356402 \\
1 & 6.394618 & -1.251910 & -0.581514 \\
1 & 4.083192 & -2.118664 & -0.887581 \\
1 & -0.399411 & 2.320023 & 0.051880 \\
1 & -0.222692 & 1.605998 & 1.657331 \\
1 & -1.789571 & 2.286905 & 1.166853 \\
1 & -3.249310 & 2.890297 & -0.516210 \\
1 & -2.319867 & 1.944907 & -1.684854 \\
1 & -4.036841 & 2.270032 & -1.969516
\end{array}
$$




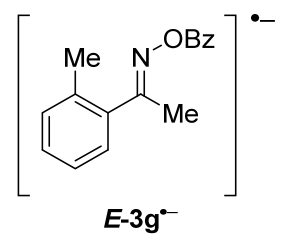

Structure: $\boldsymbol{E}-\mathbf{3 g}^{\mathbf{*}}$

Charge $=-1$, Multiplicity $=2$

Number of imaginary frequencies: 0

SCF Energy: $\quad-823.689560$ hartree

SCF Energy + ZPVE: -823.418403 hartree

Enthalpy: $\quad-823.400243$ hartree

Free Energy: $\quad-823.465797$ hartree

Free Energy with quasiharmonic correction: -823.461975 hartree
$6 \quad 4.950752-1.207134-0.736419$
$65.5283550 .018576-0.408939$
$\begin{array}{lllll}6 & 4.737348 & 1.027683 & 0.135901\end{array}$
$\begin{array}{lllll}6 & 3.364102 & 0.859466 & 0.355654\end{array}$
$\begin{array}{llll}6 & 2.782741-0.381522 & 0.013897\end{array}$
$63.590847-1.400215-0.514938$
$\begin{array}{llll}6 & 1.334129 & -0.676514 & 0.186298\end{array}$
$\begin{array}{lllll}7 & 0.512503 & 0.257891 & -0.138079\end{array}$
$\begin{array}{lllll}8 & -0.789185 & -0.152710 & 0.030712\end{array}$
$\begin{array}{lllll}6 & -1.760693 & 0.836911 & -0.245358\end{array}$
$\begin{array}{lllll}6 & -3.072156 & 0.299914 & -0.136996\end{array}$
$8-1.4312521 .999786-0.524416$
$\begin{array}{llll}6 & -3.356470 & -1.073112 & 0.187498\end{array}$
$\begin{array}{lllll}6 & -4.660310 & -1.523866 & 0.278160\end{array}$
$\begin{array}{lllll}6 & -5.758615 & -0.664134 & 0.058957\end{array}$
$\begin{array}{lllll}6 & -5.493931 & 0.686063 & -0.259657\end{array}$
$\begin{array}{llll}6 & -4.201043 & 1.162779 & -0.356476\end{array}$
$\begin{array}{lllll}6 & 0.887400 & -2.026443 & 0.678358\end{array}$
$\begin{array}{lllll}6 & 2.587271 & 1.997670 & 0.969263\end{array}$
$\begin{array}{lllll}1 & 5.551886 & -2.007108 & -1.157833\end{array}$
$\begin{array}{lllll}1 & 6.589748 & 0.186577 & -0.566476\end{array}$
$\begin{array}{lllll}1 & 5.193998 & 1.975821 & 0.409505\end{array}$
$\begin{array}{lllll}1 & 3.139030 & -2.355042 & -0.770823\end{array}$
$\begin{array}{lllll}1 & -2.534414 & -1.760783 & 0.362009\end{array}$
$\begin{array}{lllll}1 & -4.839424 & -2.568662 & 0.524548\end{array}$
$\begin{array}{lllll}1 & -6.777250 & -1.030863 & 0.132872\end{array}$
$1-6.3236001 .368722-0.433039$
$1-4.0150592 .204496-0.601969$
$\begin{array}{lllll}1 & 1.729624 & -2.615105 & 1.042461\end{array}$
$\begin{array}{lllll}1 & 0.161568 & -1.900327 & 1.487278\end{array}$
$\begin{array}{lllll}1 & 0.381998 & -2.581142 & -0.120361\end{array}$
$\begin{array}{lllll}1 & 2.021088 & 2.548965 & 0.212691\end{array}$
$\begin{array}{lllll}1 & 1.864753 & 1.640573 & 1.707789\end{array}$
$\begin{array}{lllll}1 & 3.273667 & 2.695308 & 1.456320\end{array}$ 


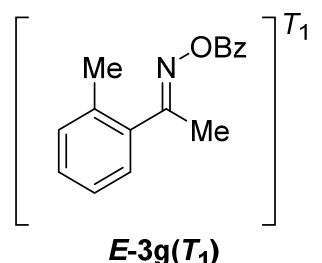

Structure: $\boldsymbol{E}-\mathbf{3 g}\left(\boldsymbol{T}_{\mathbf{1}}\right)$

Charge $=0$, Multiplicity $=3$

Number of imaginary frequencies: 0

SCF Energy: $\quad-823.517432$ hartree

SCF Energy + ZPVE: -823.245648 hartree

Enthalpy: $\quad-823.227426$ hartree

Free Energy: $\quad-823.295414$ hartree

Free Energy with quasiharmonic correction: -823.289394 hartree

$\begin{array}{llll}6 & -2.817413 & -2.180901 & 1.012903 \\ 6 & -3.978358 & -2.270585 & 0.242432 \\ 6 & -4.354260 & -1.182690 & -0.546917 \\ 6 & -3.617629 & 0.001200 & -0.601769 \\ 6 & -2.423714 & 0.100173 & 0.182659 \\ 6 & -2.059481 & -1.022570 & 0.980827 \\ 6 & -1.565312 & 1.248235 & 0.219761 \\ 7 & -0.442597 & 1.186228 & 1.076123 \\ 8 & 0.611723 & 0.690832 & 0.328933 \\ 6 & 1.794134 & 0.576621 & 1.033090 \\ 6 & 2.880391 & 0.051759 & 0.167127 \\ 8 & 1.894602 & 0.868459 & 2.194183 \\ 6 & 2.677054 & -0.257268 & -1.183039 \\ 6 & 3.737597 & -0.745028 & -1.942428 \\ 6 & 4.992634 & -0.924779 & -1.359846 \\ 6 & 5.193199 & -0.616698 & -0.012720 \\ 6 & 4.138847 & -0.128859 & 0.751963 \\ 6 & -1.657850 & 2.548967 & -0.522958 \\ 6 & -4.133627 & 1.107228 & -1.488164 \\ 1 & -2.503075 & -3.012123 & 1.636906 \\ 1 & -4.583699 & -3.171596 & 0.253203 \\ 1 & -5.256811 & -1.251763 & -1.148797 \\ 1 & -1.162365 & -0.968855 & 1.590045 \\ 1 & 1.702380 & -0.118741 & -1.637734 \\ 1 & 3.583361 & -0.985019 & -2.989579 \\ 1 & 5.816252 & -1.305616 & -1.956336 \\ 1 & 6.169622 & -0.757194 & 0.439886 \\ 1 & 4.280140 & 0.114731 & 1.800148 \\ 1 & -1.465712 & 2.414625 & -1.594407 \\ 1 & -0.900146 & 3.234323 & -0.133675 \\ 1 & -2.633090 & 3.027481 & -0.411278 \\ 1 & -4.436887 & 1.983956 & -0.907638 \\ 1 & -3.389521 & 1.434080 & -2.219019 \\ 1 & -5.010143 & 0.754959 & -2.037033\end{array}$


<smiles>Cc1ccc(/C(=N/O)c2ccccc2)cc1</smiles>

Z-3a

Structure: $\boldsymbol{Z}-\mathbf{3 a}$

Charge $=0$, Multiplicity $=1$

Number of imaginary frequencies: 0

SCF Energy: $\quad-784.313665$ hartree

SCF Energy + ZPVE: -784.066908 hartree

Enthalpy: $\quad-784.050533$ hartree

Free Energy: $\quad-784.112326$ hartree

Free Energy with quasiharmonic correction: -784.108060 hartree

$$
\begin{array}{llll}
6 & -3.154865 & 2.530655 & 0.050003 \\
6 & -2.031313 & 1.537659 & 0.038426 \\
6 & -2.367219 & 0.085919 & 0.048924 \\
6 & -1.803594 & -0.775114 & 0.999971 \\
6 & -2.165082 & -2.119472 & 1.026830 \\
6 & -3.079304 & -2.619309 & 0.097635 \\
6 & -3.642114 & -1.766012 & -0.850441 \\
6 & -3.298413 & -0.414494 & -0.867878 \\
7 & -0.849156 & 2.034784 & 0.031574 \\
8 & 0.122583 & 1.019571 & -0.029565 \\
6 & 1.399766 & 1.469070 & 0.021588 \\
6 & 2.357655 & 0.327890 & -0.053807 \\
6 & 1.926829 & -1.001695 & -0.132162 \\
6 & 2.867460 & -2.027154 & -0.195077 \\
6 & 4.230858 & -1.729738 & -0.180955 \\
6 & 4.658738 & -0.403179 & -0.102727 \\
6 & 3.723737 & 0.625624 & -0.038068 \\
8 & 1.713605 & 2.629700 & 0.119430 \\
1 & -2.765957 & 3.549588 & 0.086477 \\
1 & -3.798781 & 2.349791 & 0.915911 \\
1 & -3.768412 & 2.414608 & -0.848728 \\
1 & -1.091452 & -0.389742 & 1.723233 \\
1 & -1.730148 & -2.777842 & 1.772760 \\
1 & -3.354147 & -3.669626 & 0.116046 \\
1 & -4.354192 & -2.148766 & -1.575143 \\
1 & -3.744076 & 0.245037 & -1.606894 \\
1 & 0.866339 & -1.233119 & -0.143168 \\
1 & 2.535425 & -3.058850 & -0.255421 \\
1 & 4.960529 & -2.532478 & -0.231180 \\
1 & 5.719199 & -0.171778 & -0.091671 \\
1 & 4.041430 & 1.661618 & 0.024256
\end{array}
$$




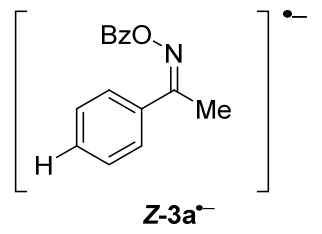

Structure: $\boldsymbol{Z}-\mathbf{3 a} \mathbf{a}^{-}$

Charge $=-1$, Multiplicity $=2$

Number of imaginary frequencies: 0

SCF Energy: $\quad-784.389331$ hartree

SCF Energy + ZPVE: - 784.145927 hartree

Enthalpy: $\quad-784.129353$ hartree

Free Energy: $\quad-784.191302$ hartree

Free Energy with quasiharmonic correction: -784.187945 hartree

$\begin{array}{llll}6 & -3.087075 & 2.567880 & 0.208567 \\ 6 & -2.003463 & 1.527814 & 0.135997 \\ 6 & -2.408282 & 0.095476 & 0.059503 \\ 6 & -1.794268 & -0.883046 & 0.854943 \\ 6 & -2.224245 & -2.206722 & 0.802700 \\ 6 & -3.264168 & -2.576222 & -0.052466 \\ 6 & -3.882118 & -1.609561 & -0.844639 \\ 6 & -3.465230 & -0.280194 & -0.780255 \\ 7 & -0.797713 & 1.973711 & 0.147666 \\ 8 & 0.144915 & 0.977673 & 0.037866 \\ 6 & 1.481248 & 1.440621 & -0.031240 \\ 6 & 2.390453 & 0.348991 & -0.052845 \\ 6 & 1.993771 & -1.033247 & 0.018902 \\ 6 & 2.936574 & -2.043514 & -0.009789 \\ 6 & 4.317906 & -1.766247 & -0.109598 \\ 6 & 4.720974 & -0.414456 & -0.185088 \\ 6 & 3.800195 & 0.614733 & -0.159166 \\ 8 & 1.740580 & 2.652591 & -0.076622 \\ 1 & -2.648046 & 3.557786 & 0.347408 \\ 1 & -3.768696 & 2.354227 & 1.038048 \\ 1 & -3.680771 & 2.577138 & -0.711333 \\ 1 & -0.983955 & -0.602895 & 1.519474 \\ 1 & -1.743835 & -2.951850 & 1.430194 \\ 1 & -3.592424 & -3.610375 & -0.097457 \\ 1 & -4.693321 & -1.886759 & -1.511227 \\ 1 & -3.958223 & 0.464607 & -1.398158 \\ 1 & 0.939276 & -1.281968 & 0.093886 \\ 1 & 2.600637 & -3.077213 & 0.046344 \\ 1 & 5.048085 & -2.568743 & -0.129352 \\ 1 & 5.779526 & -0.174491 & -0.265428 \\ 1 & 4.128146 & 1.648619 & -0.219177\end{array}$




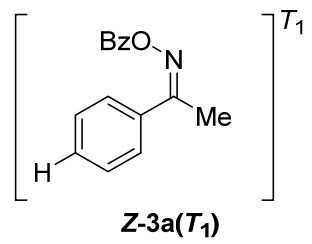

Structure: $Z-3 \mathbf{a}\left(T_{1}\right)$

Charge $=0$, Multiplicity $=3$

Number of imaginary frequencies: 0

SCF Energy: $\quad-784.224593$ hartree

SCF Energy + ZPVE: -783.981171 hartree

Enthalpy: $\quad-783.964320$ hartree

Free Energy: $\quad-784.028637$ hartree

Free Energy with quasiharmonic correction: -784.024079 hartree

$\begin{array}{llll}6 & -1.880166 & 2.179925 & -1.599787 \\ 6 & -1.752600 & 1.289135 & -0.404792 \\ 6 & -2.659645 & 0.235423 & -0.075982 \\ 6 & -2.462746 & -0.576589 & 1.070913 \\ 6 & -3.350449 & -1.598639 & 1.374158 \\ 6 & -4.455676 & -1.848474 & 0.552729 \\ 6 & -4.663310 & -1.056898 & -0.581337 \\ 6 & -3.784597 & -0.028945 & -0.896577 \\ 7 & -0.661502 & 1.538649 & 0.447117 \\ 8 & 0.401278 & 0.787175 & -0.018408 \\ 6 & 1.564053 & 0.945189 & 0.712397 \\ 6 & 2.654028 & 0.098518 & 0.165654 \\ 6 & 2.463220 & -0.749829 & -0.931526 \\ 6 & 3.525901 & -1.521575 & -1.394505 \\ 6 & 4.770744 & -1.447885 & -0.768567 \\ 6 & 4.959020 & -0.600814 & 0.325497 \\ 6 & 3.902202 & 0.172289 & 0.794196 \\ 8 & 1.644815 & 1.678967 & 1.659698 \\ 1 & -1.140612 & 2.982538 & -1.553909 \\ 1 & -2.877573 & 2.629552 & -1.656668 \\ 1 & -1.717633 & 1.616497 & -2.528203 \\ 1 & -1.611615 & -0.392569 & 1.720582 \\ 1 & -3.182483 & -2.207692 & 2.257561 \\ 1 & -5.146174 & -2.650448 & 0.794024 \\ 1 & -5.517815 & -1.245385 & -1.224728 \\ 1 & -3.959100 & 0.574029 & -1.782444 \\ 1 & 1.496385 & -0.808939 & -1.418985 \\ 1 & 3.381215 & -2.181183 & -2.244099 \\ 1 & 5.596100 & -2.051825 & -1.133569 \\ 1 & 5.927654 & -0.544059 & 0.811707 \\ 1 & 4.033544 & 0.834840 & 1.643763\end{array}$




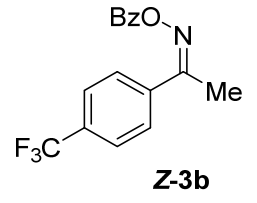

Structure: $\boldsymbol{Z}-\mathbf{3 b}$

Charge $=0$, Multiplicity $=1$

Number of imaginary frequencies: 0

SCF Energy: $\quad-1121.267979$ hartree

SCF Energy + ZPVE: -1121.016619 hartree

Enthalpy: $\quad-1120.996597$ hartree

Free Energy: $\quad-1121.068824$ hartree

Free Energy with quasiharmonic correction: -1121.061604 hartree

$\begin{array}{llll}6 & 1.884413 & -0.293337 & 1.009880 \\ 6 & 2.889624 & -0.256850 & 0.042322 \\ 6 & 2.922508 & 0.749656 & -0.918972 \\ 6 & 1.942052 & 1.738802 & -0.905485 \\ 6 & 0.920198 & 1.704584 & 0.047371 \\ 6 & 0.897818 & 0.684998 & 1.007436 \\ 6 & -0.104945 & 2.787670 & 0.062941 \\ 7 & -1.373647 & 2.608687 & 0.062259 \\ 8 & -1.688193 & 1.241071 & -0.007914 \\ 6 & -3.019467 & 0.977864 & 0.015163 \\ 6 & -3.262452 & -0.491755 & -0.052743 \\ 8 & -3.879376 & 1.820162 & 0.084130 \\ 6 & -2.214341 & -1.418766 & -0.095205 \\ 6 & -2.502101 & -2.780408 & -0.152118 \\ 6 & -3.827461 & -3.216823 & -0.167479 \\ 6 & -4.871482 & -2.290963 & -0.124960 \\ 6 & -4.590500 & -0.929228 & -0.066827 \\ 6 & 0.363351 & 4.210784 & 0.091139 \\ 6 & 3.919775 & -1.347656 & 0.018988 \\ 9 & 5.055449 & -0.974493 & -0.596944 \\ 9 & 4.261210 & -1.756801 & 1.255696 \\ 9 & 3.478052 & -2.447616 & -0.631520 \\ 1 & 1.869636 & -1.078512 & 1.759656 \\ 1 & 3.706901 & 0.772722 & -1.668367 \\ 1 & 1.970731 & 2.529427 & -1.648954 \\ 1 & 0.115448 & 0.662373 & 1.759127 \\ 1 & -1.183060 & -1.080424 & -0.082885 \\ 1 & -1.690574 & -3.500639 & -0.184779 \\ 1 & -4.047209 & -4.279333 & -0.212595 \\ 1 & -5.902401 & -2.630319 & -0.136837 \\ 1 & -5.392370 & -0.198446 & -0.032330 \\ 1 & 1.014078 & 4.368642 & 0.956480 \\ 1 & 0.947887 & 4.430216 & -0.807816 \\ 1 & -0.487323 & 4.892368 & 0.139966\end{array}$




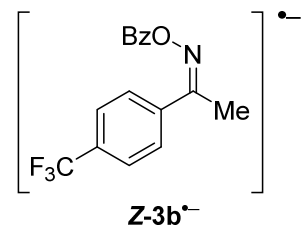

Structure: $\boldsymbol{Z}-\mathbf{3} \mathbf{b}^{--}$

Charge $=-1$, Multiplicity $=2$

Number of imaginary frequencies: 0

SCF Energy: $\quad-1121.355564$ hartree

SCF Energy + ZPVE: -1121.106654 hartree

Enthalpy: $\quad-1121.086605$ hartree

Free Energy: $\quad-1121.158741$ hartree

Free Energy with quasiharmonic correction: -1121.152297 hartree
$\begin{array}{lllll}6 & 1.785467 & -0.685094 & 0.459615\end{array}$
$\begin{array}{llll}6 & 3.110680 & -0.332911 & 0.109858\end{array}$
$\begin{array}{llll}6 & 3.363001 & 0.992275 & -0.307959\end{array}$
$\begin{array}{llll}6 & 2.345953 & 1.918323-0.363619\end{array}$
$\begin{array}{llll}6 & 0.979107 & 1.594203 & -0.029050\end{array}$
$\begin{array}{lllll}6 & 0.760444 & 0.230380 & 0.394750\end{array}$
$6-0.0110762 .622125-0.086049$
$\begin{array}{lllll}7 & -1.343921 & 2.568225 & -0.019723\end{array}$
$\begin{array}{lllll}8 & -1.792185 & 1.217472 & -0.064344\end{array}$
$\begin{array}{lllll}6 & -3.096954 & 1.049261 & 0.164298\end{array}$
$\begin{array}{llll}6 & -3.493220 & -0.383977 & -0.009104\end{array}$
$\begin{array}{lllll}8 & -3.873474 & 1.926371 & 0.481504\end{array}$
$6-2.626806-1.337831-0.555977$
$6-3.050499-2.657441-0.699405$
$\begin{array}{llll}6 & -4.333727 & -3.030264 & -0.296476\end{array}$
$\begin{array}{llll}6 & -5.198584 & -2.079052 & 0.247390\end{array}$
$\begin{array}{lllll}6 & -4.780608 & -0.758322 & 0.388326\end{array}$
$60.437003 \quad 4.059590-0.244066$
$\begin{array}{llll}6 & 4.168435 & -1.355275 & 0.109540\end{array}$
$95.417056-0.8349250 .109430$
$94.104590-2.197409 \quad 1.175532$
$94.138650-2.195741-0.975516$
$\begin{array}{lllll}1 & 1.569498 & -1.695823 & 0.798877\end{array}$
$\begin{array}{lllll}1 & 4.370134 & 1.290542 & -0.586603\end{array}$
$\begin{array}{lllll}1 & 2.593745 & 2.922930 & -0.688611\end{array}$
$\begin{array}{lllll}1 & -0.224565 & -0.087027 & 0.705729\end{array}$
$1-1.630244-1.048464-0.873830$
$1-2.378324-3.394691-1.127660$
$\begin{array}{lllll}1 & -4.659865 & -4.060104 & -0.407892\end{array}$
$1-6.197432-2.366546 \quad 0.561149$
$\begin{array}{lllll}1 & -5.443514 & -0.008040 & 0.807752\end{array}$
$\begin{array}{lllll}1 & 1.209687 & 4.326191 & 0.484045\end{array}$
$10.847023 \quad 4.244322-1.244054$
$1-0.417771 \quad 4.725105-0.105560$ 


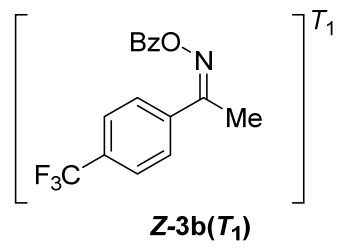

Structure: $Z-3 b\left(T_{1}\right)$

Charge $=0$, Multiplicity $=3$

Number of imaginary frequencies: 0

SCF Energy: $\quad-1121.180600$ hartree

SCF Energy + ZPVE: -1120.932215 hartree

Enthalpy: $\quad-1120.911792$ hartree

Free Energy: $\quad-1120.985562$ hartree

Free Energy with quasiharmonic correction: -1120.978943 hartree

$\begin{array}{llll}6 & 2.457037-0.5287120 .889338\end{array}$

$\begin{array}{lllll}6 & 3.520538 & -0.286097 & 0.009631\end{array}$

$\begin{array}{llll}6 & 3.439675 & 0.737580 & -0.936114\end{array}$

$\begin{array}{llll}6 & 2.298327 & 1.522270 & -1.006422\end{array}$

$\begin{array}{llll}6 & 1.208600 & 1.296599 & -0.129948\end{array}$

$\begin{array}{llll}6 & 1.316496 & 0.248685 & 0.823297\end{array}$

$\begin{array}{lllll}6 & 0.035474 & 2.102955 & -0.211559\end{array}$

$\begin{array}{lllll}7 & -1.010699 & 1.874686 & 0.700479\end{array}$

$\begin{array}{llll}8 & -1.881475 & 0.984395 & 0.111912\end{array}$

$\begin{array}{lllll}6 & -2.993227 & 0.694063 & 0.886242\end{array}$

$\begin{array}{llll}6 & -3.872173-0.287302 & 0.204283\end{array}$

$\begin{array}{llll}8 & -3.183017 & 1.192712 & 1.961367\end{array}$

$\begin{array}{llll}6 & -3.555597 & -0.835694 & -1.044424\end{array}$

$6-4.423295-1.754715-1.628879$

$\begin{array}{llll}6 & -5.598493 & -2.124447 & -0.973702\end{array}$

$\begin{array}{lllll}6 & -5.912181 & -1.576109 & 0.271621\end{array}$

$\begin{array}{lllll}6 & -5.050477 & -0.658208 & 0.862183\end{array}$

$\begin{array}{lllll}6 & -0.159553 & 3.223471 & -1.181784\end{array}$

$\begin{array}{lllll}6 & 4.739108 & -1.146391 & 0.114549\end{array}$

$95.684757-0.830263-0.786816$

$95.322826-1.0621761 .331741$

$94.457633-2.457102-0.063535$

$\begin{array}{lllll}1 & 2.527981 & -1.326368 & 1.623684\end{array}$

$14.264466 \quad 0.922275-1.616321$

$\begin{array}{lllll}1 & 2.243281 & 2.313562 & -1.746687\end{array}$

$\begin{array}{lllll}1 & 0.499682 & 0.057580 & 1.512384\end{array}$

$1-2.642492-0.551011-1.555570$

$1-4.180936-2.182578-2.596379$

$1-6.271584-2.841363-1.434246$

$\begin{array}{llll}1 & -6.826481 & -1.864216 & 0.780548\end{array}$

$\begin{array}{lllll}1 & -5.280897 & -0.223940 & 1.829795\end{array}$

$\begin{array}{lllll}1 & 0.669167 & 3.938522 & -1.133927\end{array}$

$\begin{array}{lllll}1 & -0.210415 & 2.842558 & -2.209968\end{array}$

$\begin{array}{lllll}1 & -1.089446 & 3.753493 & -0.965283\end{array}$ 


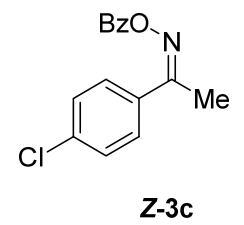

Structure: $Z$-3c

Charge $=0$, Multiplicity $=1$

Number of imaginary frequencies: 0

SCF Energy: $\quad-1243.883202$ hartree

SCF Energy + ZPVE: -1243.645809 hartree

Enthalpy: $\quad-1243.628343$ hartree

Free Energy: $\quad-1243.692615$ hartree

Free Energy with quasiharmonic correction: -1243.688536 hartree

$\begin{array}{llll}6 & 3.235980 & -1.101729 & -0.034727 \\ 6 & 3.491390 & -0.103005 & 0.897861 \\ 6 & 2.710517 & 1.050483 & 0.877615 \\ 6 & 1.670750 & 1.191141 & -0.046801 \\ 6 & 1.435369 & 0.170186 & -0.975855 \\ 6 & 2.220885 & -0.977934 & -0.979500 \\ 6 & 0.869679 & 2.447229 & -0.065123 \\ 6 & 1.598910 & 3.756970 & -0.087589 \\ 7 & -0.410301 & 2.519913 & -0.072307 \\ 8 & -0.989298 & 1.240934 & -0.003703 \\ 6 & -2.345810 & 1.247417 & -0.017320 \\ 8 & -3.022769 & 2.243332 & -0.080631 \\ 6 & -2.876360 & -0.144550 & 0.052677 \\ 6 & -4.264882 & -0.308860 & 0.068923 \\ 6 & -4.811268 & -1.587379 & 0.128803 \\ 6 & -3.972394 & -2.702574 & 0.170777 \\ 6 & -2.586662 & -2.538722 & 0.153557 \\ 6 & -2.033891 & -1.261589 & 0.095164 \\ 17 & 4.219879 & -2.547841 & -0.026344 \\ 1 & 4.288270 & -0.217667 & 1.625020 \\ 1 & 2.910608 & 1.834477 & 1.601516 \\ 1 & 0.643102 & 0.273321 & -1.710354 \\ 1 & 2.044219 & -1.764619 & -1.705487 \\ 1 & 2.207063 & 3.864213 & 0.815932 \\ 1 & 2.274686 & 3.789174 & -0.947508 \\ 1 & 0.892844 & 4.587068 & -0.142327 \\ 1 & -4.904367 & 0.567514 & 0.034612 \\ 1 & -5.889124 & -1.714926 & 0.142209 \\ 1 & -4.398965 & -3.700135 & 0.216851 \\ 1 & -1.934876 & -3.406176 & 0.185484 \\ 1 & -0.956034 & -1.134968 & 0.081937\end{array}$




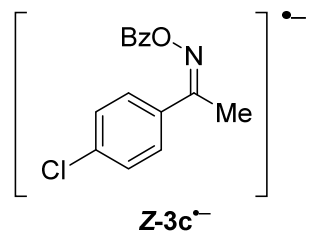

Structure: $\boldsymbol{Z}-\mathbf{3} \mathbf{c}^{-}$

Charge $=-1$, Multiplicity $=2$

Number of imaginary frequencies: 0

SCF Energy: $\quad-1243.959502$ hartree

SCF Energy + ZPVE: -1243.725598 hartree

Enthalpy: $\quad-1243.707868$ hartree

Free Energy: $\quad-1243.772724$ hartree

Free Energy with quasiharmonic correction: -1243.769202 hartree

$\begin{array}{llll}6 & -3.655500 & -0.003742 & -0.788937 \\ 6 & -3.332379 & -1.091711 & 0.013245 \\ 6 & -2.217568 & -1.075886 & 0.846754 \\ 6 & -1.403138 & 0.052146 & 0.861500 \\ 6 & -1.699764 & 1.165233 & 0.062023 \\ 6 & -2.840045 & 1.125421 & -0.749996 \\ 6 & -0.871177 & 2.402669 & 0.105870 \\ 7 & 0.413672 & 2.451175 & 0.107518 \\ 8 & 0.998223 & 1.210589 & 0.016413 \\ 17 & -4.355019 & -2.512369 & -0.020109 \\ 6 & 2.414614 & 1.229853 & -0.021295 \\ 6 & 2.933833 & -0.092080 & -0.057399 \\ 8 & 3.042460 & 2.299029 & -0.025367 \\ 6 & 2.120595 & -1.279875 & -0.042773 \\ 6 & 2.699013 & -2.534766 & -0.075769 \\ 6 & 4.100063 & -2.704577 & -0.125793 \\ 6 & 4.909966 & -1.547534 & -0.146002 \\ 6 & 4.358322 & -0.281607 & -0.115447 \\ 6 & -1.579276 & 3.728104 & 0.155154 \\ 1 & -4.529710 & -0.031831 & -1.430903 \\ 1 & -1.986116 & -1.930608 & 1.474151 \\ 1 & -0.533376 & 0.067307 & 1.508751 \\ 1 & -3.097288 & 1.975412 & -1.374434 \\ 1 & 1.039271 & -1.185873 & -0.006729 \\ 1 & 2.053633 & -3.411033 & -0.063482 \\ 1 & 4.541395 & -3.695644 & -0.149311 \\ 1 & 5.992541 & -1.651469 & -0.187574 \\ 1 & 4.996245 & 0.597469 & -0.133211 \\ 1 & -2.142954 & 3.904760 & -0.766681 \\ 1 & -2.290923 & 3.751935 & 0.986556 \\ 1 & -0.853900 & 4.534789 & 0.276908\end{array}$




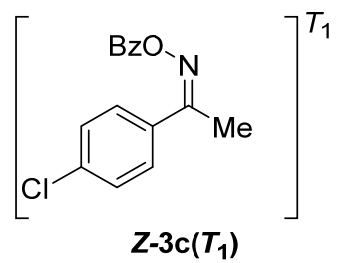

Structure: $Z-3 \mathbf{c}\left(T_{1}\right)$

Charge $=0$, Multiplicity $=3$

Number of imaginary frequencies: 0

SCF Energy: $\quad-1243.794686$ hartree

SCF Energy + ZPVE: -1243.560851 hartree

Enthalpy: $\quad-1243.542786$ hartree

Free Energy: $\quad-1243.610674$ hartree

Free Energy with quasiharmonic correction: -1243.605375 hartree

$\begin{array}{llll}6 & -4.019776 & -0.825490 & 0.106728 \\ 6 & -4.058229 & 0.163384 & -0.876024 \\ 6 & -3.001129 & 1.054276 & -0.984814 \\ 6 & -1.884944 & 0.972238 & -0.115412 \\ 6 & -1.883536 & -0.046274 & 0.872445 \\ 6 & -2.939806 & -0.935671 & 0.984000 \\ 6 & -0.796459 & 1.885578 & -0.240592 \\ 6 & -0.714031 & 2.975598 & -1.261405 \\ 7 & 0.277060 & 1.787975 & 0.663452 \\ 8 & 1.213683 & 0.947782 & 0.095805 \\ 6 & 2.346755 & 0.763330 & 0.868455 \\ 8 & 2.502788 & 1.295467 & 1.933431 \\ 6 & 3.298158 & -0.159599 & 0.200640 \\ 6 & 4.504325 & -0.426192 & 0.858308 \\ 6 & 5.432530 & -1.284592 & 0.278848 \\ 6 & 5.157461 & -1.877720 & -0.955080 \\ 6 & 3.954441 & -1.612182 & -1.610003 \\ 6 & 3.020437 & -0.753203 & -1.036618 \\ 17 & -5.350580 & -1.951794 & 0.240661 \\ 1 & -4.906087 & 0.233102 & -1.549737 \\ 1 & -3.035625 & 1.819220 & -1.753927 \\ 1 & -1.046986 & -0.133465 & 1.559338 \\ 1 & -2.927975 & -1.709664 & 1.744661 \\ 1 & -0.637678 & 2.557908 & -2.273806 \\ 1 & -1.603041 & 3.615608 & -1.233985 \\ 1 & 0.165513 & 3.597363 & -1.080525 \\ 1 & 4.704428 & 0.041898 & 1.816886 \\ 1 & 6.368556 & -1.491854 & 0.787628 \\ 1 & 5.882410 & -2.548345 & -1.406567 \\ 1 & 3.741973 & -2.074829 & -2.568504 \\ 1 & 2.085989 & -0.549159 & -1.547594\end{array}$




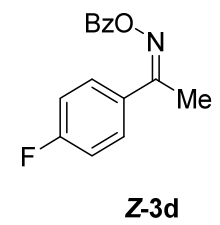

Structure: $\boldsymbol{Z}-\mathbf{3 d}$

Charge $=0$, Multiplicity $=1$

Number of imaginary frequencies: 0

SCF Energy: $\quad-883.517194$ hartree

SCF Energy + ZPVE: -883.278816 hartree

Enthalpy: $\quad-883.261609$ hartree

Free Energy: $\quad-883.325461$ hartree

Free Energy with quasiharmonic correction: -883.320947 hartree

$\begin{array}{llll}6 & -3.685544 & -1.172264 & 0.398763 \\ 6 & -4.430616 & -0.409829 & -0.487639 \\ 6 & -4.013103 & 0.822662 & -0.960749 \\ 6 & -2.788396 & 1.314759 & -0.514771 \\ 6 & -1.996660 & 0.567292 & 0.364537 \\ 6 & -2.455213 & -0.676528 & 0.818140 \\ 6 & -0.708725 & 1.135082 & 0.850412 \\ 7 & 0.424907 & 0.538191 & 0.851404 \\ 8 & 0.334158 & -0.763973 & 0.312956 \\ 9 & -5.624427 & -0.891918 & -0.906235 \\ 6 & 1.528331 & -1.382778 & 0.105879 \\ 6 & 2.751386 & -0.563360 & -0.127962 \\ 8 & 1.521226 & -2.590183 & 0.064526 \\ 6 & 2.722172 & 0.603304 & -0.899610 \\ 6 & 3.910689 & 1.272278 & -1.179181 \\ 6 & 5.120794 & 0.793064 & -0.673515 \\ 6 & 5.147496 & -0.369479 & 0.097242 \\ 6 & 3.964820 & -1.057664 & 0.357845 \\ 6 & -0.706168 & 2.527791 & 1.407983 \\ 1 & -4.062520 & -2.128420 & 0.745765 \\ 1 & -4.633689 & 1.380546 & -1.653788 \\ 1 & -2.447086 & 2.281254 & -0.872316 \\ 1 & -1.854529 & -1.257576 & 1.509387 \\ 1 & 1.782012 & 0.977992 & -1.291498 \\ 1 & 3.892315 & 2.169625 & -1.789639 \\ 1 & 6.043794 & 1.324763 & -0.884685 \\ 1 & 6.088053 & -0.743880 & 0.488856 \\ 1 & 3.975061 & -1.972230 & 0.942939 \\ 1 & -1.407986 & 2.590962 & 2.245124 \\ 1 & 0.294130 & 2.803239 & 1.746118 \\ 1 & -1.034718 & 3.240913 & 0.645930\end{array}$




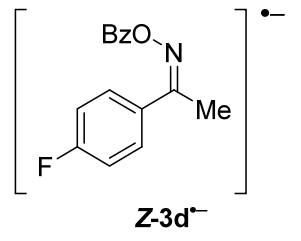

Structure: $\boldsymbol{Z}-\mathbf{3 d} \mathbf{d}^{-}$

Charge $=-1$, Multiplicity $=2$

Number of imaginary frequencies: 0

SCF Energy: $\quad-883.601181$ hartree

SCF Energy + ZPVE: -883.366041 hartree

Enthalpy: $\quad-883.348651$ hartree

Free Energy: $\quad-883.412579$ hartree

Free Energy with quasiharmonic correction: -883.409054 hartree

$\begin{array}{llll}6 & -2.256579 & 1.653986 & -0.814823 \\ 6 & -3.358971 & 1.817795 & 0.009421 \\ 6 & -3.873762 & 0.796360 & 0.789139 \\ 6 & -3.248835 & -0.448210 & 0.735263 \\ 6 & -2.117619 & -0.654930 & -0.065794 \\ 6 & -1.634954 & 0.409163 & -0.842642 \\ 6 & -1.495385 & -2.007361 & -0.122638 \\ 7 & -0.235167 & -2.262253 & -0.122580 \\ 8 & 0.543644 & -1.133456 & -0.017845 \\ 9 & -3.962603 & 3.030947 & 0.049148 \\ 6 & 1.936409 & -1.387552 & 0.034001 \\ 6 & 2.669791 & -0.171065 & 0.058674 \\ 8 & 2.376795 & -2.546394 & 0.060280 \\ 6 & 2.068713 & 1.136546 & 0.017673 \\ 6 & 2.850182 & 2.276501 & 0.042276 \\ 6 & 4.259135 & 2.209061 & 0.110395 \\ 6 & 4.862088 & 0.932422 & 0.156487 \\ 6 & 4.105340 & -0.222736 & 0.134460 \\ 6 & -2.403519 & -3.204232 & -0.186828 \\ 1 & -1.897323 & 2.482885 & -1.415980 \\ 1 & -4.741370 & 0.972295 & 1.416256 \\ 1 & -3.646138 & -1.258692 & 1.338392 \\ 1 & -0.771134 & 0.262460 & -1.481012 \\ 1 & 0.987568 & 1.226136 & -0.031293 \\ 1 & 2.361794 & 3.248602 & 0.009779 \\ 1 & 4.860833 & 3.111931 & 0.127862 \\ 1 & 5.946118 & 0.853014 & 0.211963 \\ 1 & 4.585923 & -1.196235 & 0.171485 \\ 1 & -3.112236 & -3.104814 & -1.015056 \\ 1 & -1.814632 & -4.113492 & -0.321867 \\ 1 & -2.985535 & -3.302534 & 0.735336\end{array}$




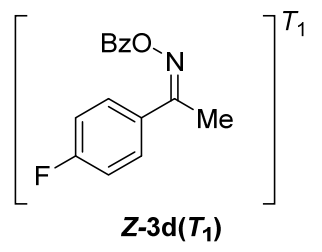

Structure: $\boldsymbol{Z}-\mathbf{3 d}\left(\boldsymbol{T}_{\mathbf{1}}\right)$

Charge $=0$, Multiplicity $=3$

Number of imaginary frequencies: 0

SCF Energy: $\quad-883.428977$ hartree

SCF Energy + ZPVE: -883.194126 hartree

Enthalpy: $\quad-883.176410$ hartree

Free Energy: $\quad-883.242397$ hartree

Free Energy with quasiharmonic correction: -883.238052 hartree

$\begin{array}{llll}6 & -3.041283 & 1.550857 & -0.550134 \\ 6 & -4.306844 & 1.055922 & -0.262156 \\ 6 & -4.513061 & -0.183670 & 0.327752 \\ 6 & -3.406638 & -0.959076 & 0.643754 \\ 6 & -2.092226 & -0.502062 & 0.372477 \\ 6 & -1.939426 & 0.771382 & -0.233511 \\ 6 & -0.963782 & -1.314505 & 0.699902 \\ 7 & 0.335384 & -0.821118 & 0.486398 \\ 8 & 0.725951 & -1.214888 & -0.786376 \\ 9 & -5.385955 & 1.814741 & -0.572365 \\ 6 & 2.053997 & -0.995161 & -1.109127 \\ 6 & 2.848502 & 0.005032 & -0.347065 \\ 8 & 2.482744 & -1.643619 & -2.027238 \\ 6 & 2.332530 & 1.244136 & 0.048630 \\ 6 & 3.167921 & 2.166944 & 0.671851 \\ 6 & 4.504768 & 1.850267 & 0.919054 \\ 6 & 5.017214 & 0.614268 & 0.524425 \\ 6 & 4.194277 & -0.303435 & -0.122628 \\ 6 & -1.055967 & -2.685899 & 1.292123 \\ 1 & -2.932300 & 2.525558 & -1.014275 \\ 1 & -5.522500 & -0.526268 & 0.530555 \\ 1 & -3.558488 & -1.929663 & 1.104458 \\ 1 & -0.947068 & 1.148623 & -0.458496 \\ 1 & 1.295568 & 1.498929 & -0.140765 \\ 1 & 2.773014 & 3.133739 & 0.967293 \\ 1 & 5.148177 & 2.570005 & 1.416118 \\ 1 & 6.056928 & 0.367258 & 0.713939 \\ 1 & 4.585440 & -1.262977 & -0.446567 \\ 1 & -1.545615 & -3.380010 & 0.596910 \\ 1 & -0.057876 & -3.070029 & 1.513757 \\ 1 & -1.638811 & -2.681962 & 2.220386\end{array}$ 

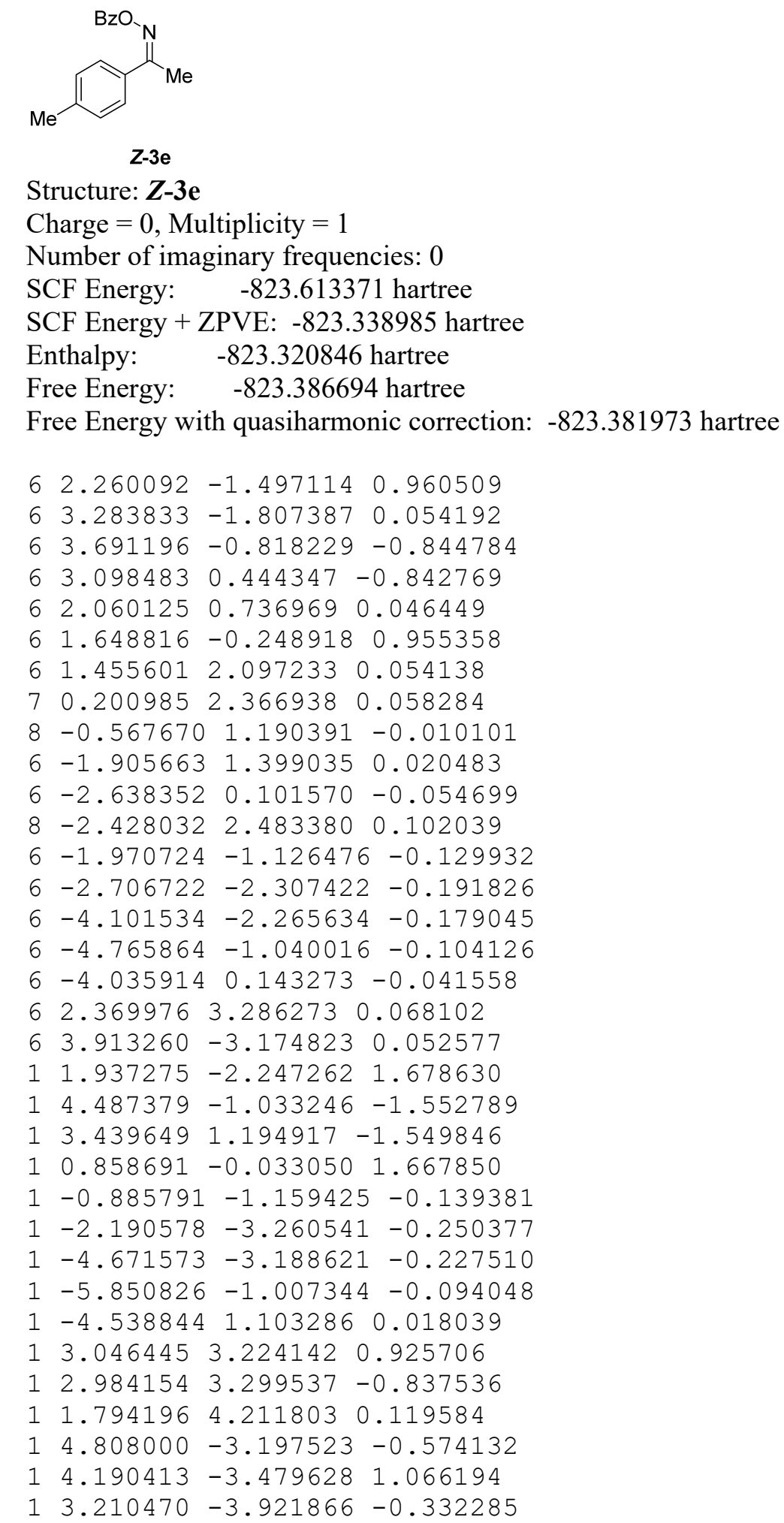


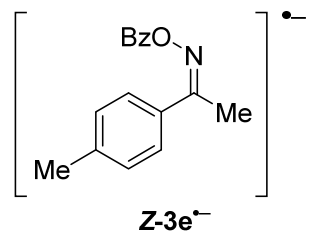

Structure: $\boldsymbol{Z}-\mathbf{3} \mathrm{e}^{-}$

Charge $=-1$, Multiplicity $=2$

Number of imaginary frequencies: 0

SCF Energy: $\quad-823.688813$ hartree

SCF Energy + ZPVE: -823.418020 hartree

Enthalpy: $\quad-823.399567$ hartree

Free Energy: $\quad-823.466507$ hartree

Free Energy with quasiharmonic correction: -823.461941 hartree

$\begin{array}{lllll}6 & 2.302524 & -1.577921 & 0.794770\end{array}$

$63.431296-1.765313-0.015044$

$63.884030-0.680209-0.769852$

$\begin{array}{lllll}6 & 3.232612 & 0.552362 & -0.721489\end{array}$

$\begin{array}{llll}6 & 2.090317 & 0.728424 & 0.068379\end{array}$

$\begin{array}{llll}6 & 1.637122 & -0.357753 & 0.833668\end{array}$

$\begin{array}{llll}6 & 1.432466 & 2.062945 & 0.130189\end{array}$

$\begin{array}{llllll}7 & 0.166257 & 2.288689 & 0.130635\end{array}$

$\begin{array}{llll}8 & -0.585917 & 1.141189 & 0.020740\end{array}$

$\begin{array}{llll}6 & -1.982124 & 1.363544 & -0.044318\end{array}$

$\begin{array}{lllll}6 & -2.688765 & 0.130901 & -0.059883\end{array}$

$\begin{array}{lllll}8 & -2.448608 & 2.511739 & -0.089508\end{array}$

$6-2.058282-1.162045 \quad 0.003381$

$6-2.813028-2.319861-0.014069$

$6-4.222567-2.285830-0.096718$

$6-4.854109-1.023871-0.164801$

$6-4.1243230 .148611-0.150106$

$\begin{array}{llll}6 & 2.309065 & 3.283453 & 0.199526\end{array}$

$64.121569-3.102289-0.070650$

$11.939849-2.4033661 .403500$

$\begin{array}{lllll}1 & 4.761635 & -0.796211 & -1.400977\end{array}$

$\begin{array}{lllll}1 & 3.615585 & 1.376668 & -1.316267\end{array}$

$\begin{array}{lllll}1 & 0.765883 & -0.240670 & 1.469021\end{array}$

$\begin{array}{lllll}1 & -0.975855 & -1.225141 & 0.064144\end{array}$

$\begin{array}{lllll}1 & -2.302825 & -3.279964 & 0.035830\end{array}$

$1-4.803341-3.202392-0.108336$

$1-5.939094-0.970271-0.231704$

$1-4.6266421 .110295-0.204139$

$13.016725 \quad 3.201638 \quad 1.030626$

$\begin{array}{llll}1 & 2.892705 & 3.396495 & -0.719919\end{array}$

$\begin{array}{llll}1 & 1.697106 & 4.177725 & 0.332466\end{array}$

$15.084366-3.028880-0.582448$

$\begin{array}{lllll}1 & 4.292063 & -3.498459 & 0.934999\end{array}$

$13.507432-3.832609-0.608989$ 


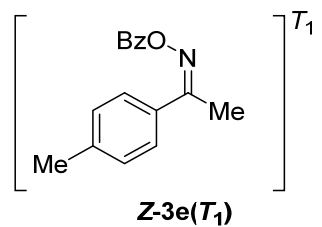

Structure: $Z-3 \mathrm{e}\left(T_{1}\right)$

Charge $=0$, Multiplicity $=3$

Number of imaginary frequencies: 0

SCF Energy: $\quad-823.516414$ hartree

SCF Energy + ZPVE: -823.245601 hartree

Enthalpy: $\quad-823.227129$ hartree

Free Energy: $\quad-823.294729$ hartree

Free Energy with quasiharmonic correction: -823.289914 hartree
$\begin{array}{lllll}6 & 3.661487 & 0.646269 & 0.880098\end{array}$
$\begin{array}{lllll}6 & 3.316913 & 1.777852 & 0.128082\end{array}$
$\begin{array}{llll}6 & 2.272442 & 1.654523 & -0.803115\end{array}$
$\begin{array}{lllll}6 & 1.593932 & 0.458966 & -0.976123\end{array}$
$\begin{array}{llll}6 & 1.940293 & -0.688963 & -0.214925\end{array}$
$\begin{array}{lllll}6 & 2.996586 & -0.562247 & 0.719593\end{array}$
$\begin{array}{llll}6 & 1.235330 & -1.923588 & -0.354510\end{array}$
$\begin{array}{llll}7 & 0.292439 & -2.039760 & -1.390029\end{array}$
$\begin{array}{lllll}8 & -0.944592 & -1.447544 & -1.165829\end{array}$
$\begin{array}{lllll}6 & -1.395115 & -1.177037 & 0.110133\end{array}$
$\begin{array}{llll}6 & -2.438770 & -0.123205 & 0.101498\end{array}$
$8-0.994176-1.768888 \quad 1.078505$
$\begin{array}{llll}6 & -2.722084 & 0.631943 & -1.042592\end{array}$
$\begin{array}{llll}6 & -3.699621 & 1.622019 & -0.985390\end{array}$
$\begin{array}{lllll}6 & -4.392292 & 1.854290 & 0.203449\end{array}$
$\begin{array}{llll}6 & -4.108876 & 1.098447 & 1.343148\end{array}$
$\begin{array}{llll}6 & -3.131665 & 0.110314 & 1.294938\end{array}$
$\begin{array}{lllll}6 & 1.542119 & -3.161324 & 0.426540\end{array}$
$\begin{array}{lllll}6 & 4.032619 & 3.089097 & 0.306698\end{array}$
14.4685120 .7168091 .605451
$\begin{array}{lllll}1 & 1.989528 & 2.518327 & -1.400702\end{array}$
$\begin{array}{llllll}1 & 0.793320 & 0.402744 & -1.708594\end{array}$
$\begin{array}{lllll}1 & 3.291279 & -1.418084 & 1.319307\end{array}$
$\begin{array}{lllll}1 & -2.181657 & 0.451978 & -1.965677\end{array}$
$\begin{array}{lllll}1 & -3.920073 & 2.212235 & -1.869073\end{array}$
$\begin{array}{lllll}1 & -5.154776 & 2.626501 & 0.242861\end{array}$
$1-4.649018 \quad 1.2805292 .266745$
$\begin{array}{lllll}1 & -2.899494 & -0.484285 & 2.172790\end{array}$
$\begin{array}{lllll}1 & 2.558253 & -3.518295 & 0.217907\end{array}$
$\begin{array}{lllll}1 & 1.465084 & -2.970107 & 1.502490\end{array}$
$\begin{array}{lllll}1 & 0.838115 & -3.955386 & 0.169243\end{array}$
$\begin{array}{lllll}1 & 3.348332 & 3.860917 & 0.675957\end{array}$
14.8555102 .9950561 .019599
$14.4403713 .446707-0.644645$ 


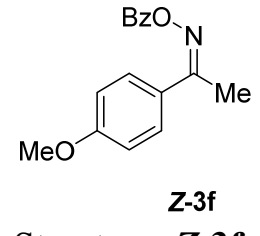

Structure: $\boldsymbol{Z}-\mathbf{3 f}$

Charge $=0$, Multiplicity $=1$

Number of imaginary frequencies: 0

SCF Energy: $\quad-898.799554$ hartree

SCF Energy + ZPVE: -898.519495 hartree

Enthalpy: $\quad-898.500735$ hartree

Free Energy: $\quad-898.567379$ hartree

Free Energy with quasiharmonic correction: -898.563224 hartree

$\begin{array}{llll}6 & 3.552120 & -0.094413 & -0.667714 \\ 6 & 3.234931 & -1.120508 & 0.227018 \\ 6 & 2.117612 & -0.992170 & 1.066672 \\ 6 & 1.319061 & 0.136423 & 0.997004 \\ 6 & 1.620867 & 1.175556 & 0.099322 \\ 6 & 2.750883 & 1.047327 & -0.711203 \\ 6 & 0.808742 & 2.418298 & 0.040843 \\ 7 & -0.473097 & 2.491511 & 0.011606 \\ 8 & -1.053466 & 1.209996 & -0.028978 \\ 6 & -2.407781 & 1.215889 & -0.027854 \\ 6 & -2.937788 & -0.177990 & -0.079146 \\ 8 & -3.088262 & 2.211297 & 0.014434 \\ 6 & -2.094542 & -1.294783 & -0.111570 \\ 6 & -2.646011 & -2.573253 & -0.152122 \\ 6 & -4.031584 & -2.738985 & -0.161243 \\ 6 & -4.871388 & -1.624146 & -0.129577 \\ 6 & -4.326102 & -0.344309 & -0.087889 \\ 6 & 1.521202 & 3.739316 & 0.022932 \\ 8 & 3.945436 & -2.264065 & 0.357506 \\ 6 & 5.090426 & -2.431521 & -0.470904 \\ 1 & 4.410689 & -0.164552 & -1.324942 \\ 1 & 1.892559 & -1.790992 & 1.766522 \\ 1 & 0.461506 & 0.220069 & 1.656664 \\ 1 & 3.012947 & 1.837144 & -1.408976 \\ 1 & -1.016841 & -1.166729 & -0.104931 \\ 1 & -1.993226 & -3.440241 & -0.176451 \\ 1 & -4.457224 & -3.737522 & -0.193009 \\ 1 & -5.949167 & -1.753013 & -0.136702 \\ 1 & -4.966374 & 0.531786 & -0.061663 \\ 1 & 2.175170 & 3.820351 & 0.896224 \\ 1 & 2.149652 & 3.817239 & -0.869433 \\ 1 & 0.803706 & 4.561456 & 0.022609 \\ 1 & 5.508396 & -3.403510 & -0.210653 \\ 1 & 4.813392 & -2.424102 & -1.530436 \\ 1 & 5.832937 & -1.650173 & -0.277181\end{array}$




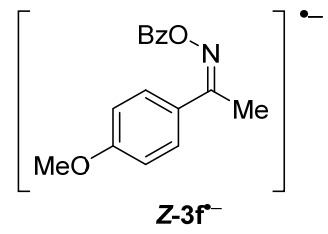

Structure: $\boldsymbol{Z}-3 \mathbf{f}^{-}$

Charge $=-1$, Multiplicity $=2$

Number of imaginary frequencies: 0

SCF Energy: $\quad-898.874815$ hartree

SCF Energy + ZPVE: -898.598222 hartree

Enthalpy: $\quad-898.579176$ hartree

Free Energy: $\quad-898.646594$ hartree

Free Energy with quasiharmonic correction: -898.642856 hartree

$\begin{array}{llll}6 & 3.682913 & -0.022653 & -0.590403 \\ 6 & 3.324307 & -1.128902 & 0.184215 \\ 6 & 2.136804 & -1.096357 & 0.930154 \\ 6 & 1.312360 & 0.015816 & 0.885333 \\ 6 & 1.650136 & 1.137803 & 0.105752 \\ 6 & 2.850712 & 1.098423 & -0.609685 \\ 6 & 0.814302 & 2.367603 & 0.076497 \\ 7 & -0.471568 & 2.421828 & 0.058863 \\ 8 & -1.062218 & 1.178651 & 0.015212 \\ 6 & -2.475714 & 1.200786 & -0.038228 \\ 6 & -2.998658 & -0.120173 & -0.073524 \\ 8 & -3.103962 & 2.270054 & -0.050144 \\ 6 & -2.188200 & -1.310342 & -0.060122 \\ 6 & -2.769473 & -2.563843 & -0.091805 \\ 6 & -4.171265 & -2.730822 & -0.139163 \\ 6 & -4.978521 & -1.571466 & -0.158615 \\ 6 & -4.423926 & -0.306922 & -0.129642 \\ 6 & 1.511141 & 3.701644 & 0.059233 \\ 8 & 4.057221 & -2.264316 & 0.281194 \\ 6 & 5.271638 & -2.331435 & -0.455900 \\ 1 & 4.596132 & -0.014990 & -1.173694 \\ 1 & 1.876582 & -1.956695 & 1.539514 \\ 1 & 0.399760 & 0.020853 & 1.470280 \\ 1 & 3.151989 & 1.949861 & -1.212598 \\ 1 & -1.106600 & -1.218759 & -0.027254 \\ 1 & -2.125897 & -3.441514 & -0.081021 \\ 1 & -4.614912 & -3.720887 & -0.161600 \\ 1 & -6.061454 & -1.672856 & -0.198298 \\ 1 & -5.059967 & 0.573560 & -0.146676 \\ 1 & 2.235731 & 3.769967 & 0.876626 \\ 1 & 2.056919 & 3.845181 & -0.879117 \\ 1 & 0.779560 & 4.506129 & 0.156249 \\ 1 & 5.699068 & -3.310672 & -0.241441 \\ 1 & 5.084666 & -2.241819 & -1.531467 \\ 1 & 5.969749 & -1.550239 & -0.136224\end{array}$




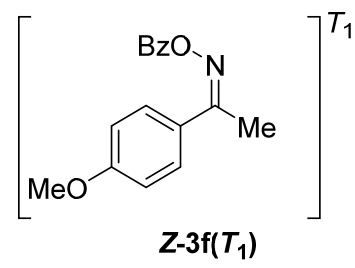

Structure: $\boldsymbol{Z}-\mathbf{3 f}\left(\boldsymbol{T}_{\mathbf{1}}\right)$

Charge $=0$, Multiplicity $=3$

Number of imaginary frequencies: 0

SCF Energy: $\quad-898.702943$ hartree

SCF Energy + ZPVE: -898.426423 hartree

Enthalpy: $\quad-898.407123$ hartree

Free Energy: $\quad-898.475758$ hartree

Free Energy with quasiharmonic correction: -898.472077 hartree

$\begin{array}{llll}6 & -4.107039 & -0.483990 & 0.015188 \\ 6 & -2.879981 & -1.112595 & 0.268157 \\ 6 & -1.694480 & -0.420397 & 0.056137 \\ 6 & -1.684956 & 0.914643 & -0.413950 \\ 6 & -2.943696 & 1.527160 & -0.660087 \\ 6 & -4.123763 & 0.842671 & -0.449400 \\ 6 & -0.473122 & 1.632440 & -0.632682 \\ 6 & -0.407864 & 3.061454 & -1.077272 \\ 7 & 0.763848 & 0.991190 & -0.443686 \\ 8 & 1.144526 & 1.200240 & 0.879854 \\ 6 & 2.429614 & 0.814225 & 1.209933 \\ 8 & 2.890487 & 1.315121 & 2.202804 \\ 6 & 3.147338 & -0.179705 & 0.367352 \\ 6 & 4.527390 & -0.002602 & 0.224053 \\ 6 & 5.274725 & -0.932443 & -0.493656 \\ 6 & 4.649921 & -2.053320 & -1.041016 \\ 6 & 3.276698 & -2.241937 & -0.875867 \\ 6 & 2.519057 & -1.302880 & -0.181557 \\ 8 & -5.315893 & -1.068467 & 0.188890 \\ 6 & -5.341250 & -2.411313 & 0.657470 \\ 1 & -2.838127 & -2.133578 & 0.629674 \\ 1 & -0.755799 & -0.925696 & 0.262390 \\ 1 & -2.984597 & 2.551325 & -1.017726 \\ 1 & -5.082237 & 1.316918 & -0.638282 \\ 1 & 0.629847 & 3.358040 & -1.246277 \\ 1 & -0.965939 & 3.217519 & -2.007743 \\ 1 & -0.836147 & 3.730433 & -0.319441 \\ 1 & 5.005055 & 0.866117 & 0.666573 \\ 1 & 6.342645 & -0.785127 & -0.619701 \\ 1 & 5.233426 & -2.783444 & -1.593780 \\ 1 & 2.793222 & -3.120525 & -1.290947 \\ 1 & 1.452798 & -1.454978 & -0.056379 \\ 1 & -4.879943 & -2.491243 & 1.647640 \\ 1 & -4.831937 & -3.081856 & -0.042991 \\ 1 & -6.393705 & -2.685860 & 0.723581\end{array}$




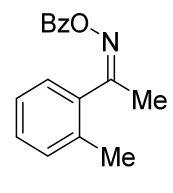

Z-3g

Structure: $\boldsymbol{Z}-\mathbf{3 g}$

Charge $=0$, Multiplicity $=1$

Number of imaginary frequencies: 0

SCF Energy: $\quad-823.614467$ hartree

SCF Energy + ZPVE: -823.339645 hartree

Enthalpy: $\quad-823.321903$ hartree

Free Energy: $\quad-823.385823$ hartree

Free Energy with quasiharmonic correction: -823.381965 hartree

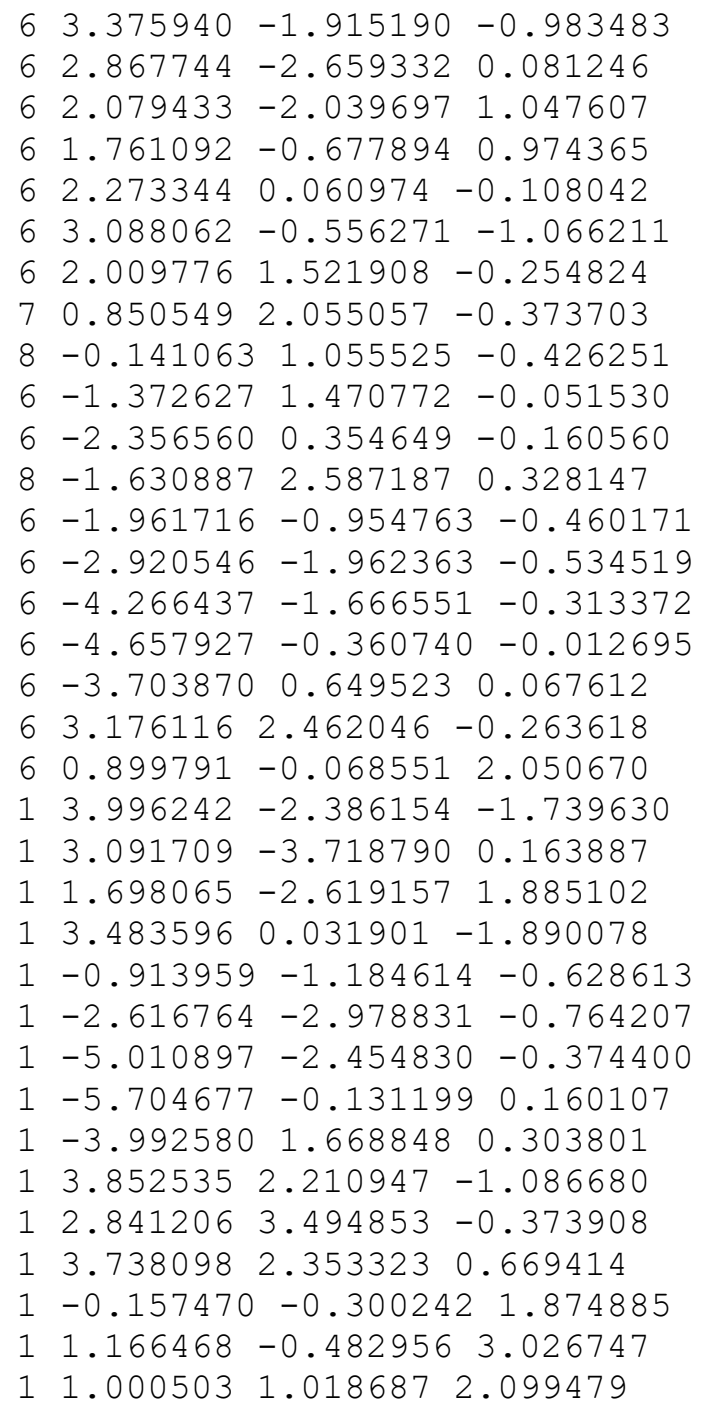




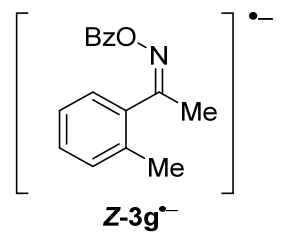

Structure: $\boldsymbol{Z}-\mathbf{3 g}^{\mathbf{*}}$

Charge $=-1$, Multiplicity $=2$

Number of imaginary frequencies: 0

SCF Energy: $\quad-823.689881$ hartree

SCF Energy + ZPVE: -823.418668 hartree

Enthalpy: $\quad-823.400588$ hartree

Free Energy: $\quad-823.465541$ hartree

Free Energy with quasiharmonic correction: -823.462018 hartree
$63.494501-1.857945-0.971678$
$63.035339-2.6095290 .109073$
$\begin{array}{llll}6 & 2.226526 & -2.011723 & 1.073563\end{array}$
$\begin{array}{llll}6 & 1.841782 & -0.668620 & 0.982419\end{array}$
$\begin{array}{llll}6 & 2.305503 & 0.082513-0.115725\end{array}$
$63.137758-0.515319-1.071190$
$61.9802431 .531483-0.270640$
$\begin{array}{lllll}7 & 0.793410 & 2.013245 & -0.350070\end{array}$
$\begin{array}{llll}8 & -0.158895 & 1.014706 & -0.358817\end{array}$
$\begin{array}{llll}6 & -1.464836 & 1.441669 & -0.036988\end{array}$
$\begin{array}{lllll}6 & -2.387910 & 0.367100 & -0.149260\end{array}$
$\begin{array}{lllll}8 & -1.691016 & 2.611730 & 0.310020\end{array}$
$\begin{array}{lllll}6 & -2.022204 & -0.969267 & -0.543973\end{array}$
$6-2.974718-1.966957-0.632362$
$6-4.334555-1.722889-0.337754$
$6-4.705655-0.4182190 .058469$
$\begin{array}{llll}6 & -3.774953 & 0.597507 & 0.155133\end{array}$
$\begin{array}{llll}6 & 3.116473 & 2.510329 & -0.336607\end{array}$
$\begin{array}{lllll}6 & 0.956526 & -0.085459 & 2.052663\end{array}$
$\begin{array}{lllll}1 & 4.129585 & -2.309339 & -1.727832\end{array}$
$\begin{array}{lllll}1 & 3.311534 & -3.655467 & 0.206263\end{array}$
$\begin{array}{llll}1 & 1.881227 & -2.595957 & 1.923490\end{array}$
$13.497214 \quad 0.076443-1.909109$
$1-0.984531-1.191242-0.775982$
$\begin{array}{llll}1 & -2.663591 & -2.964898 & -0.935435\end{array}$
$1-5.072226-2.515344-0.411194$
$\begin{array}{lllll}1 & -5.746719 & -0.205290 & 0.293901\end{array}$
$\begin{array}{llll}1 & -4.078120 & 1.594833 & 0.461036\end{array}$
$\begin{array}{lllll}1 & 3.761322 & 2.294984 & -1.195138\end{array}$
$\begin{array}{lllll}1 & 2.737391 & 3.530458 & -0.426420\end{array}$
$\begin{array}{lllll}1 & 3.734085 & 2.430083 & 0.564252\end{array}$
$\begin{array}{llll}1 & -0.097351 & -0.279591 & 1.822463\end{array}$
$\begin{array}{llll}1 & 1.181231 & -0.539953 & 3.021500\end{array}$
$\begin{array}{llll}1 & 1.075449 & 0.998022 & 2.138211\end{array}$ 


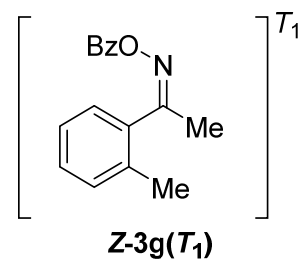

Structure: $Z-3 g\left(T_{1}\right)$

Charge $=0$, Multiplicity $=3$

Number of imaginary frequencies: 0

SCF Energy: $\quad-823.487101$ hartree

SCF Energy + ZPVE: -823.217451 hartree

Enthalpy: $\quad-823.199139$ hartree

Free Energy: $\quad-823.264414$ hartree

Free Energy with quasiharmonic correction: -823.261251 hartree

$\begin{array}{llll}6 & -0.968646 & 2.686947 & -0.267545 \\ 6 & -0.368388 & 1.881730 & 0.704306 \\ 6 & -0.999765 & 0.726049 & 1.137335 \\ 6 & -2.134229 & 0.225031 & 0.302557 \\ 6 & -2.964368 & 1.263850 & -0.313701 \\ 6 & -2.340602 & 2.413022 & -0.670380 \\ 6 & -2.344310 & -1.133198 & -0.018126 \\ 6 & -3.686091 & -1.593978 & -0.528797 \\ 7 & -1.456299 & -2.123105 & 0.085970 \\ 8 & -0.137633 & -1.655486 & 0.267604 \\ 6 & 0.443994 & -1.011979 & -0.776511 \\ 8 & -0.095604 & -0.830155 & -1.841952 \\ 6 & 1.824896 & -0.569715 & -0.430565 \\ 6 & 2.387412 & -0.804716 & 0.829012 \\ 6 & 3.670313 & -0.339173 & 1.107282 \\ 6 & 4.388839 & 0.358958 & 0.135106 \\ 6 & 3.825422 & 0.592868 & -1.120499 \\ 6 & 2.543403 & 0.130394 & -1.404164 \\ 1 & -0.493421 & 3.604411 & -0.595473 \\ 6 & -0.742336 & 0.114316 & 2.476621 \\ 1 & 0.530117 & 2.222607 & 1.215332 \\ 1 & -3.985253 & 1.055417 & -0.616279 \\ 1 & -2.869852 & 3.160325 & -1.255071 \\ 1 & -3.747385 & -2.681888 & -0.458003 \\ 1 & -4.509249 & -1.151224 & 0.036070 \\ 1 & -3.800877 & -1.315223 & -1.582070 \\ 1 & 1.825968 & -1.343478 & 1.585151 \\ 1 & 4.108855 & -0.520209 & 2.083657 \\ 1 & 5.388363 & 0.721170 & 0.356522 \\ 1 & 4.383460 & 1.136850 & -1.876183 \\ 1 & 2.089545 & 0.310164 & -2.373718 \\ 1 & -1.534108 & 0.424666 & 3.173171 \\ 1 & -0.752970 & -0.978860 & 2.447866 \\ 1 & 0.213526 & 0.459692 & 2.880436\end{array}$




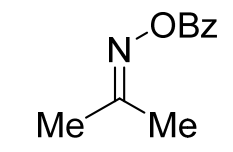

Structure: acetone $\boldsymbol{O}$-benzoyl oxime

Charge $=0$, Multiplicity $=1$

Number of imaginary frequencies: 0

SCF Energy: $\quad-592.643006$ hartree

SCF Energy + ZPVE: -592.449797 hartree

Enthalpy: $\quad-592.436514$ hartree

Free Energy: $\quad-592.489651$ hartree

Free Energy with quasiharmonic correction: -592.487633 hartree

$\begin{array}{llll}6 & 3.134898 & 0.266235 & -0.000000 \\ 7 & 2.255580 & -0.665154 & -0.000003 \\ 8 & 0.963613 & -0.091663 & -0.000002 \\ 6 & -0.043761 & -0.994455 & 0.000001 \\ 6 & -1.370290 & -0.309614 & 0.000000 \\ 8 & 0.109104 & -2.191667 & 0.000002 \\ 6 & -1.490915 & 1.084723 & -0.000000 \\ 6 & -2.756498 & 1.666774 & -0.000001 \\ 6 & -3.897716 & 0.863475 & -0.000001 \\ 6 & -3.776011 & -0.527171 & -0.000000 \\ 6 & -2.514035 & -1.114041 & 0.000000 \\ 6 & 2.822925 & 1.733468 & 0.000003 \\ 6 & 4.564597 & -0.173423 & -0.000001 \\ 1 & -0.604195 & 1.710070 & -0.000000 \\ 1 & -2.851728 & 2.748082 & -0.000001 \\ 1 & -4.882412 & 1.321332 & -0.000001 \\ 1 & -4.663279 & -1.152528 & -0.000000 \\ 1 & -2.404044 & -2.193852 & 0.000001 \\ 1 & 2.231350 & 1.996016 & -0.882313 \\ 1 & 2.231345 & 1.996011 & 0.882317 \\ 1 & 3.744517 & 2.316605 & 0.000008 \\ 1 & 5.070137 & 0.233319 & 0.882070 \\ 1 & 4.648214 & -1.261482 & -0.000004 \\ 1 & 5.070136 & 0.233322 & -0.882072\end{array}$




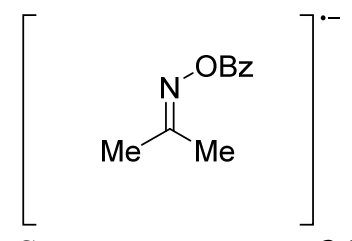

Structure: acetone $\boldsymbol{O}$-benzoyl oxime ${ }^{-}$

Charge $=-1$, Multiplicity $=2$

Number of imaginary frequencies: 0

SCF Energy: $\quad-592.717432$ hartree

SCF Energy + ZPVE: -592.527922 hartree

Enthalpy: $\quad-592.514296$ hartree

Free Energy: $\quad-592.568669$ hartree

Free Energy with quasiharmonic correction: -592.566650 hartree

$\begin{array}{llll}6 & 3.145231 & 0.268784 & 0.000000 \\ 7 & 2.244415 & -0.643854 & 0.000001 \\ 8 & 0.979008 & -0.077789 & -0.000001 \\ 6 & -0.083488 & -1.005517 & -0.000000 \\ 6 & -1.339174 & -0.339532 & -0.000000 \\ 8 & 0.126579 & -2.228467 & -0.000001 \\ 6 & -1.492836 & 1.092785 & -0.000000 \\ 6 & -2.748364 & 1.670726 & 0.000000 \\ 6 & -3.925377 & 0.889248 & 0.000001 \\ 6 & -3.789196 & -0.517730 & 0.000000 \\ 6 & -2.547385 & -1.121191 & 0.000000 \\ 6 & 2.844572 & 1.739838 & -0.000001 \\ 6 & 4.574267 & -0.181431 & 0.000001 \\ 1 & -0.608311 & 1.722804 & -0.000000 \\ 1 & -2.827316 & 2.756315 & 0.000000 \\ 1 & -4.905184 & 1.355689 & 0.000001 \\ 1 & -4.680429 & -1.142566 & 0.000001 \\ 1 & -2.461772 & -2.204204 & 0.000000 \\ 1 & 2.250851 & 2.008103 & -0.880133 \\ 1 & 2.250852 & 2.008104 & 0.880131 \\ 1 & 3.768030 & 2.321714 & -0.000002 \\ 1 & 5.091513 & 0.213173 & 0.881627 \\ 1 & 4.645143 & -1.271155 & 0.000002 \\ 1 & 5.091513 & 0.213172 & -0.881625\end{array}$


$\left[\mathrm{Me}_{\mathrm{Me}}^{\mathrm{N}^{-\mathrm{OBz}}}\right]^{T_{1}}$

Structure: acetone $\boldsymbol{O}$-benzoyl oxime $\left(T_{1}\right)$

Charge $=0$, Multiplicity $=3$

Number of imaginary frequencies: 0

SCF Energy: $\quad-592.510127$ hartree

SCF Energy + ZPVE: -592.321912 hartree

Enthalpy: $\quad-592.307628$ hartree

Free Energy: $\quad-592.364485$ hartree

Free Energy with quasiharmonic correction: -592.361554 hartree

$\begin{array}{llll}6 & 3.138673 & 0.254642 & 0.000005 \\ 7 & 2.243507 & -0.662453 & -0.000001 \\ 8 & 0.966528 & -0.078637 & -0.000014 \\ 6 & -0.063703 & -0.984716 & -0.000024 \\ 6 & -1.338694 & -0.319672 & -0.000011 \\ 8 & 0.089969 & -2.199813 & -0.000003 \\ 6 & -1.481161 & 1.152759 & -0.000014 \\ 6 & -2.710199 & 1.705988 & -0.000002 \\ 6 & -3.911169 & 0.881923 & 0.000014 \\ 6 & -3.776949 & -0.565704 & 0.000017 \\ 6 & -2.563116 & -1.150656 & 0.000005 \\ 6 & 2.843538 & 1.725397 & 0.000000 \\ 6 & 4.563233 & -0.202681 & 0.000018 \\ 1 & -0.586745 & 1.764853 & -0.000025 \\ 1 & -2.822158 & 2.785965 & -0.000004 \\ 1 & -4.890248 & 1.342617 & 0.000023 \\ 1 & -4.677109 & -1.172757 & 0.000029 \\ 1 & -2.444988 & -2.228107 & 0.000007 \\ 1 & 2.253641 & 1.994636 & -0.881783 \\ 1 & 2.253627 & 1.994640 & 0.881774 \\ 1 & 3.770809 & 2.299785 & 0.000006 \\ 1 & 5.075932 & 0.195706 & 0.881960 \\ 1 & 4.632038 & -1.291951 & 0.000020 \\ 1 & 5.075947 & 0.195703 & -0.881917\end{array}$ 
<smiles>CN=C(C)c1ccccc1</smiles>

VII

Structure: VII

Charge $=0$, Multiplicity $=1$ Number of imaginary frequencies: 0

SCF Energy: $\quad-364.226956$ hartree

SCF Energy + ZPVE: -364.088768 hartree

Free Energy: $\quad-364.122983$ hartree

Free Energy with quasiharmonic correction: -364.121473 hartree

$\begin{array}{lllll}6 & 0.585379 & 1.241645 & 0.000002\end{array}$

$\begin{array}{lllll}6 & 1.969686 & 1.111680 & 0.000004\end{array}$

$\begin{array}{llll}6 & 2.555109 & -0.157227 & 0.000002\end{array}$

$\begin{array}{lllll}6 & 1.748237 & -1.293036 & -0.000003\end{array}$

$60.358510-1.166823-0.000004$

$12.5941161 .999902 \quad 0.000008$

$12.196808-2.281714-0.000006$

$1-0.256865-2.060752-0.000007$

$13.636332-0.2573970 .000004$

$6-0.2310010 .101150-0.000002$

$\begin{array}{lllll}7 & -2.267590 & 1.382556 & -0.000007\end{array}$

$6-1.722202 \quad 0.247425-0.000002$

$6-2.597779-0.9887440 .000007$

$1-2.387106-1.590696 \quad 0.888384$

$1-2.387112-1.590704-0.888365$

$1-3.649932-0.7013770 .000009$

$\begin{array}{lllll}1 & 0.131261 & 2.228431 & 0.000004\end{array}$<smiles>CC(C)NC(C)C</smiles>

Structure: DIPA

Charge $=0$, Multiplicity $=1$

Number of imaginary frequencies: 0

SCF Energy: $\quad-292.293965$ hartree

SCF Energy + ZPVE: -292.087399 hartree

Free Energy: $\quad-292.120483$ hartree

Free Energy with quasiharmonic correction: -292.120121 hartree

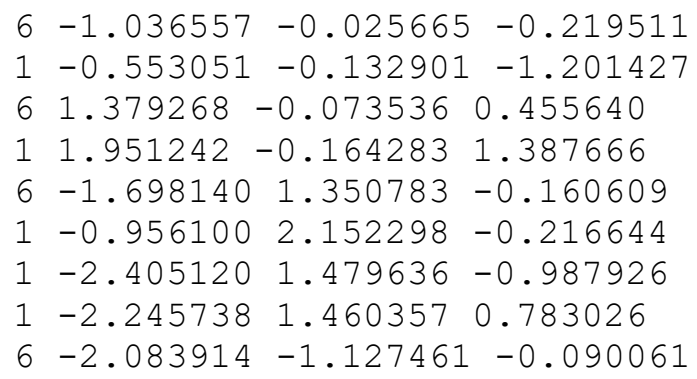


$\begin{array}{llll}1 & -2.871739 & -1.009398 & -0.840241\end{array}$

$1-1.631179-2.116844-0.218599$

$\begin{array}{lllll}1 & -2.551447 & -1.088837 & 0.901724\end{array}$

$\begin{array}{llll}6 & 1.694252 & 1.289639 & -0.152509\end{array}$

$\begin{array}{lllll}1 & 2.768107 & 1.372668 & -0.347165\end{array}$

$\begin{array}{lllll}1 & 1.172340 & 1.430598 & -1.105924\end{array}$

$\begin{array}{lllll}1 & 1.402819 & 2.099364 & 0.523942\end{array}$

$\begin{array}{lllll}6 & 1.827623 & -1.206959 & -0.474231\end{array}$

$\begin{array}{llll}1 & 1.611350 & -2.182865 & -0.025583\end{array}$

$\begin{array}{lllll}1 & 1.321780 & -1.155472 & -1.444795\end{array}$

$12.906014-1.149441-0.658040$

$7-0.035934-0.166283 \quad 0.856326$

$\begin{array}{lllll}1 & -0.162934 & -1.071705 & 1.303389\end{array}$

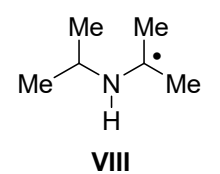

\section{Structure: VII}

Charge $=0$, Multiplicity $=2$

Number of imaginary frequencies: 0

SCF Energy: $\quad-291.647225$ hartree

SCF Energy + ZPVE: -291.454572 hartree

Free Energy: $\quad-291.488651$ hartree

Free Energy with quasiharmonic correction: -291.488589 hartree

$\begin{array}{llll}6 & 1.207181 & 0.136252 & 0.234945 \\ 1 & 1.071087 & 0.804982 & 1.092836 \\ 6 & -1.252814 & 0.015976 & -0.132048 \\ 6 & 1.524579 & 0.976935 & -1.003147 \\ 1 & 0.709526 & 1.672485 & -1.227507 \\ 1 & 2.441424 & 1.556557 & -0.853387 \\ 1 & 1.667225 & 0.324348 & -1.873124 \\ 6 & 2.343644 & -0.829197 & 0.549299 \\ 1 & 3.280691 & -0.284526 & 0.696386 \\ 1 & 2.129945 & -1.405229 & 1.454684 \\ 1 & 2.487039 & -1.531469 & -0.281162 \\ 6 & -1.543214 & 1.207628 & 0.728100 \\ 1 & -2.506558 & 1.644066 & 0.449304 \\ 1 & -1.604028 & 0.929618 & 1.796483 \\ 1 & -0.783605 & 1.992170 & 0.642956 \\ 6 & -2.393218 & -0.904086 & -0.442648 \\ 1 & -2.109834 & -1.661367 & -1.182728 \\ 1 & -2.737317 & -1.442248 & 0.459160 \\ 1 & -3.249898 & -0.348078 & -0.834904 \\ 7 & -0.030693 & -0.635262 & 0.085255 \\ 1 & 0.102201 & -1.425520 & -0.542790\end{array}$


<smiles>N=C([Mg][Mg])c1ccccc1</smiles>

Structure: $\boldsymbol{E}$-IX

Charge $=0$, Multiplicity $=1$

Number of imaginary frequencies: 0

SCF Energy: $\quad-364.881258$ hartree

SCF Energy + ZPVE: -364.729494 hartree

Free Energy: $\quad-364.762756$ hartree

Free Energy with quasiharmonic correction: -364.761480 hartree

$6-0.586895-1.231667-0.000001$

$6-1.975183-1.148384-0.000001$

$6-2.603806 \quad 0.099703-0.000000$

$6-1.832784 \quad 1.2599590 .000001$

$6-0.439651 \quad 1.176160 \quad 0.000001$

$1-2.570210-2.056843-0.000002$

$1-2.312163 \quad 2.2342350 .000002$

$\begin{array}{llll}1 & 0.142200 & 2.092051 & 0.000004\end{array}$

$\begin{array}{llll}1 & -3.687868 & 0.164563 & 0.000000\end{array}$

$60.199338-0.069850-0.000000$

$\begin{array}{lllll}7 & 2.224080 & -1.338784 & 0.000002\end{array}$

$\begin{array}{lllll}6 & 1.693286 & -0.175127 & 0.000001\end{array}$

$\begin{array}{lllll}6 & 2.488134 & 1.105254 & -0.000002\end{array}$

$\begin{array}{lllll}1 & 2.250247 & 1.707141 & 0.882803\end{array}$

$12.2502451 .707130-0.882815$

$\begin{array}{lllll}1 & 3.557850 & 0.886558 & 0.000000\end{array}$

$13.243091-1.2600330 .000002$

$1-0.096589-2.199612-0.000001$<smiles>[R1]c1ccccc1C(N)=[W]</smiles>

Structure: $\boldsymbol{Z}$-IX

Charge $=0$, Multiplicity $=1$

Number of imaginary frequencies: 0

SCF Energy: $\quad-364.879818$ hartree

SCF Energy + ZPVE: -364.727918 hartree

Free Energy: $\quad-364.760470$ hartree

Free Energy with quasiharmonic correction: -364.760068 hartree
$\begin{array}{lllll}6 & 0.573945 & 1.212247 & -0.178404\end{array}$
$\begin{array}{lllll}6 & 1.963360 & 1.123662 & -0.190972\end{array}$
$\begin{array}{llll}6 & 2.588743-0.110481 & -0.006269\end{array}$
$\begin{array}{lllll}6 & 1.815641 & -1.255780 & 0.179066\end{array}$
$\begin{array}{lllll}6 & 0.424030 & -1.169427 & 0.179257\end{array}$
$\begin{array}{lllll}1 & 2.557958 & 2.017659 & -0.352753\end{array}$
$\begin{array}{lllll}1 & 2.294424 & -2.220009 & 0.320590\end{array}$
$\begin{array}{lllll}1 & -0.163335 & -2.071059 & 0.321431\end{array}$ 

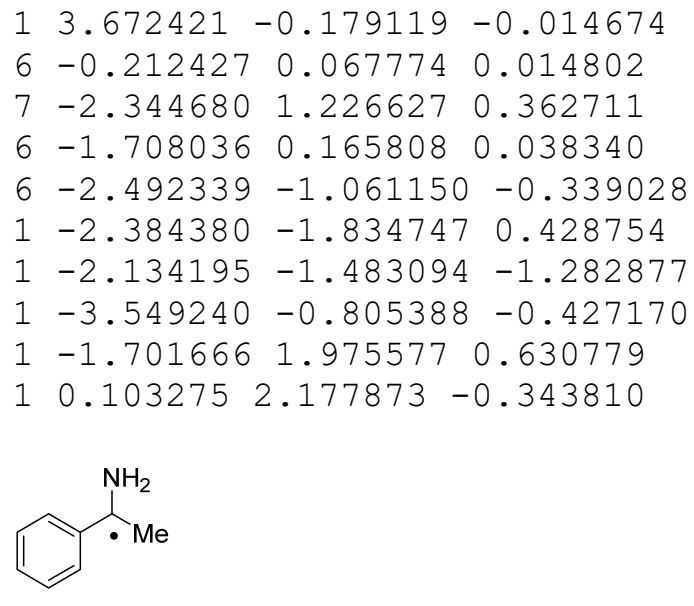

XII

Structure: XII

Charge $=0$, Multiplicity $=2$

Number of imaginary frequencies: 0

SCF Energy: $\quad-365.457969$ hartree

SCF Energy + ZPVE: -365.296556 hartree

Free Energy: $\quad-365.330292$ hartree

Free Energy with quasiharmonic correction: -365.330194 hartree
$\begin{array}{llll}6 & 0.592860 & 1.226386 & -0.042749\end{array}$
$\begin{array}{llll}6 & 1.979068 & 1.170347 & -0.034586\end{array}$
$\begin{array}{llll}6 & 2.654689 & -0.055898 & 0.008158\end{array}$
$\begin{array}{llll}6 & 1.901219 & -1.238414 & 0.037306\end{array}$
$\begin{array}{llll}6 & 0.515905 & -1.203183 & 0.029252\end{array}$
$\begin{array}{llll}1 & 2.545407 & 2.097574 & -0.067611\end{array}$
$12.406246-2.200404 \quad 0.068334$
$1-0.036763-2.137152 \quad 0.058472$
$13.739390-0.0901160 .014503$
$\begin{array}{llll}6 & -0.194053 & 0.035152 & -0.004688\end{array}$
$\begin{array}{llll}7 & -2.306689 & 1.244078 & 0.000155\end{array}$
$\begin{array}{llll}6 & -1.612495 & 0.055952 & 0.003235\end{array}$
$6-2.427696-1.199136-0.055130$
$\begin{array}{lllll}1 & -2.296004 & -1.807040 & 0.849047\end{array}$
$1-2.145809-1.826146-0.908792$
$1-3.490667-0.963000-0.147207$
$\begin{array}{llll}1 & -3.292195 & 1.194597 & 0.224531\end{array}$
$\begin{array}{lllll}1 & 0.115243 & 2.200234 & -0.096504\end{array}$
$\begin{array}{llll}1 & -1.855012 & 2.075677 & 0.359357\end{array}$ 


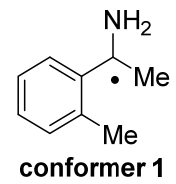

Structure: conformer 1

Charge $=0$, Multiplicity $=2$

Number of imaginary frequencies: 0

SCF Energy: $\quad-404.749598$ hartree

SCF Energy + ZPVE: -404.559996 hartree

Enthalpy: $\quad-404.549084$ hartree

Free Energy: $\quad-404.595247$ hartree

Free Energy with quasiharmonic correction: -404.594976 hartree

$\begin{array}{llll}6 & 0.599998 & -1.464713 & 0.070297\end{array}$

$61.986123-1.4631760 .113364$

$\begin{array}{lllll}6 & 2.688654 & -0.256373 & 0.086236\end{array}$

$\begin{array}{lllll}6 & 1.963613 & 0.937123 & -0.007080\end{array}$

$\begin{array}{lllll}6 & 0.571946 & 0.975448 & -0.054261\end{array}$

$12.519332-2.407892 \quad 0.179148$

$12.503093 \quad 1.879759-0.073079$

$\begin{array}{lllll}1 & 3.773549 & -0.239706 & 0.117792\end{array}$

$\begin{array}{lllll}6 & -0.161900 & -0.258280 & 0.010955\end{array}$

$\begin{array}{llll}7 & -2.179068 & -1.565891 & -0.289054\end{array}$

$\begin{array}{lllll}6 & -1.589503 & -0.345345 & -0.009282\end{array}$

$\begin{array}{llll}6 & -2.554404 & 0.711502 & 0.439751\end{array}$

$\begin{array}{llll}1 & -3.004000 & 1.257369 & -0.400317\end{array}$

$1-2.094793 \quad 1.4351151 .111366$

$\begin{array}{lllll}1 & -3.375788 & 0.231930 & 0.985636\end{array}$

$\begin{array}{lllll}1 & -3.181844 & -1.546617 & -0.428560\end{array}$

$\begin{array}{lllll}1 & 0.081589 & -2.416517 & 0.133356\end{array}$

$1-1.705378-2.181132-0.940279$

$\begin{array}{llll}6 & -0.088419 & 2.317797 & -0.264426\end{array}$

$\begin{array}{llll}1 & -0.912245 & 2.253305 & -0.981197\end{array}$

$\begin{array}{lllll}1 & -0.490417 & 2.742227 & 0.661398\end{array}$

$\begin{array}{lllll}1 & 0.643724 & 3.029501 & -0.655219\end{array}$

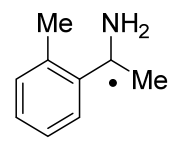

conformer 2

Structure: conformer 2

Charge $=0$, Multiplicity $=2$

Number of imaginary frequencies: 0

SCF Energy: $\quad-404.749874$ hartree

SCF Energy + ZPVE: -404.559818 hartree

Enthalpy: $\quad-404.549060$ hartree

Free Energy: $\quad-404.594753$ hartree

Free Energy with quasiharmonic correction: -404.594584 hartree

$6-0.484738-1.476702-0.032480$

$6-1.864874-1.580689-0.064827$

$6-2.659112-0.429655-0.059150$ 
$\begin{array}{llll}6 & -2.023505 & 0.815559 & -0.000257\end{array}$

$\begin{array}{lllll}6 & -0.638833 & 0.963585 & 0.033836\end{array}$

$\begin{array}{lllll}1 & -2.324038 & -2.565141 & -0.099595\end{array}$

$\begin{array}{llll}1 & -2.631838 & 1.716872 & 0.038434\end{array}$

$\begin{array}{lllll}1 & -3.742192 & -0.494957 & -0.083938\end{array}$

$\begin{array}{llll}6 & 0.191654 & -0.215183 & -0.001176\end{array}$

$\begin{array}{lllll}7 & 2.389995 & 0.894911 & -0.220562\end{array}$

$\begin{array}{lllll}6 & 1.615541 & -0.227548 & -0.009835\end{array}$

$\begin{array}{lllll}6 & 2.391518 & -1.495193 & 0.202497\end{array}$

$\begin{array}{lllll}1 & 2.376331 & -2.136807 & -0.689456\end{array}$

$\begin{array}{llll}1 & 1.995230 & -2.084375 & 1.034845\end{array}$

$\begin{array}{llll}1 & 3.437655 & -1.264464 & 0.422667\end{array}$

$\begin{array}{lllll}1 & 3.362475 & 0.724070 & -0.442564\end{array}$

$\begin{array}{llll}1 & 0.101501 & -2.388922 & -0.057092\end{array}$

$\begin{array}{lllll}1 & 1.997534 & 1.672709 & -0.731176\end{array}$

$\begin{array}{llll}6 & -0.095225 & 2.366936 & 0.179862\end{array}$

$\begin{array}{lllll}1 & 0.673429 & 2.429638 & 0.955643\end{array}$

$\begin{array}{llllll}1 & 0.336144 & 2.755554 & -0.750863\end{array}$

$\begin{array}{llll}1 & -0.906758 & 3.044783 & 0.456215\end{array}$

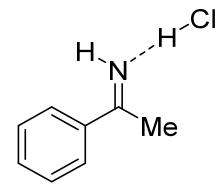

\section{$\mathrm{E}-\mathrm{IX} \cdot \mathrm{HCl}$}

\section{Structure: $\boldsymbol{E}-\mathbf{I X} \cdot \mathbf{H C I}$}

Charge $=0$, Multiplicity $=1$

Number of imaginary frequencies: 0

SCF Energy: $\quad-825.693063$ hartree

SCF Energy + ZPVE: -825.527686 hartree

Free Energy: $\quad-825.565009$ hartree

Free Energy with quasiharmonic correction: -825.563459 hartree

$$
\begin{array}{lllll}
6 & -1.517376 & -1.221950 & -0.186491 \\
6 & -2.895375 & -1.402116 & -0.162564 \\
6 & -3.740801 & -0.309828 & 0.040758 \\
6 & -3.206978 & 0.967933 & 0.206546 \\
6 & -1.829040 & 1.157562 & 0.170147 \\
1 & -3.309992 & -2.393819 & -0.311236 \\
1 & -3.862875 & 1.818487 & 0.360759 \\
1 & -1.422592 & 2.155231 & 0.299816 \\
1 & -4.816593 & -0.454977 & 0.060908 \\
6 & -0.974376 & 0.060579 & -0.011237 \\
7 & 1.296443 & -0.689309 & 0.233737 \\
6 & 0.482320 & 0.276258 & -0.030552 \\
6 & 1.052295 & 1.615673 & -0.347111 \\
1 & 0.827984 & 2.298444 & 0.479486 \\
1 & 0.586253 & 2.018143 & -1.249362 \\
1 & 2.134446 & 1.555392 & -0.470664 \\
1 & 2.332062 & -0.553308 & 0.183363 \\
1 & -0.877388 & -2.078176 & -0.378917 \\
1 & 0.959948 & -1.609076 & 0.510018 \\
17 & 4.361743 & -0.282109 & 0.047217
\end{array}
$$




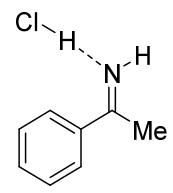

$Z-I X \cdot H C l$

Structure: $\boldsymbol{Z}$-IX・HCI

Charge $=0$, Multiplicity $=1$

Number of imaginary frequencies: 0

SCF Energy: $\quad-825.692971$ hartree

SCF Energy + ZPVE: -825.527489 hartree

Free Energy: $\quad-825.564852$ hartree

Free Energy with quasiharmonic correction: -825.563305 hartree

$\begin{array}{lllll}6 & 0.272585 & -0.902898 & 0.381308\end{array}$

$61.136256-1.9912150 .345015$

$\begin{array}{llll}6 & 2.473494-1.813825-0.015336\end{array}$

$62.950849-0.542282-0.332494$

$\begin{array}{lllll}6 & 2.094712 & 0.553412 & -0.289671\end{array}$

$10.766773-2.9781320 .604148$

$13.990385-0.401964-0.610241$

$\begin{array}{lllll}1 & 2.476201 & 1.538028 & -0.538164\end{array}$

$\begin{array}{llll}1 & 3.144621 & -2.667001 & -0.042496\end{array}$

$\begin{array}{lllll}6 & 0.746267 & 0.378628 & 0.056434\end{array}$

$\begin{array}{llll}7 & -1.429306 & 1.395725 & 0.078792\end{array}$

$\begin{array}{lllll}6 & -0.146642 & 1.547382 & 0.088027\end{array}$

$\begin{array}{llll}6 & 0.409315 & 2.929263 & 0.127317\end{array}$

$\begin{array}{lllll}1 & 0.912797 & 3.138981 & -0.822129\end{array}$

$\begin{array}{llll}1 & 1.151833 & 3.011312 & 0.924690\end{array}$

$\begin{array}{llllll}1 & -0.382664 & 3.663387 & 0.276788\end{array}$

$\begin{array}{lllll}1 & -1.928492 & 0.485385 & -0.005692\end{array}$

$\begin{array}{lllll}1 & -0.761731 & -1.057978 & 0.674862\end{array}$

$\begin{array}{lllll}1 & -2.025631 & 2.219617 & 0.126552\end{array}$

$17-3.350586-1.039559-0.194321$

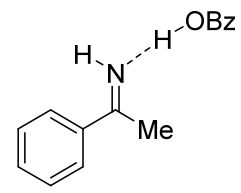

$E-I X \cdot H O B z$

Structure: $\boldsymbol{E}-\mathbf{I X} \cdot \mathbf{H O B z}$

Charge $=0$, Multiplicity $=1$

Number of imaginary frequencies: 0

SCF Energy: $\quad-785.583353$ hartree

SCF Energy + ZPVE: -785.314716 hartree

Free Energy: $\quad-785.363686$ hartree

Free Energy with quasiharmonic correction: -785.357477 hartree

$$
\begin{array}{llll}
6 & -3.827251 & -1.163774 & -0.323316 \\
6 & -5.125855 & -1.663809 & -0.305289 \\
6 & -6.195069 & -0.824421 & 0.012107 \\
6 & -5.959804 & 0.519467 & 0.299283 \\
6 & -4.661064 & 1.024564 & 0.270501
\end{array}
$$


$\begin{array}{llll}1 & -5.303989 & -2.707281 & -0.546128 \\ 1 & -6.787436 & 1.178223 & 0.543485 \\ 1 & -4.493506 & 2.073340 & 0.493729 \\ 1 & -7.207873 & -1.215598 & 0.027564 \\ 6 & -3.580484 & 0.184095 & -0.027071 \\ 7 & -1.155884 & -0.017620 & 0.140645 \\ 6 & -2.187161 & 0.720596 & -0.036070 \\ 6 & -1.992376 & 2.192205 & -0.257727 \\ 1 & -2.360164 & 2.753016 & 0.607903 \\ 1 & -2.553641 & 2.530484 & -1.133177 \\ 1 & -0.932969 & 2.415540 & -0.390894 \\ 1 & 0.360045 & 0.356279 & 0.089077 \\ 1 & -3.008507 & -1.824661 & -0.594064 \\ 6 & 4.467019 & -1.297511 & 0.081352 \\ 6 & 3.587875 & -0.211387 & 0.041051 \\ 6 & 4.089692 & 1.089949 & -0.057948 \\ 6 & 5.466197 & 1.300924 & -0.116044 \\ 6 & 6.342236 & 0.215287 & -0.076216 \\ 6 & 5.841829 & -1.084542 & 0.022523 \\ 1 & 4.062726 & -2.301966 & 0.158262 \\ 1 & 3.403158 & 1.929400 & -0.088434 \\ 1 & 5.855027 & 2.311746 & -0.192586 \\ 1 & 7.414462 & 0.381855 & -0.122045 \\ 1 & 6.522874 & -1.929587 & 0.053168 \\ 1 & -1.375375 & -0.998553 & 0.321022 \\ 6 & 2.114190 & -0.476859 & 0.105085 \\ 8 & 1.657674 & -1.606431 & 0.202218 \\ 8 & 1.369391 & 0.609230 & 0.047192\end{array}$

$\mathrm{BzO}_{\mathrm{H}}$<smiles>C=NCc1ccccc1</smiles>

Z-IX·HOBz

Charge $=0$, Multiplicity $=1$

Number of imaginary frequencies: 0

SCF Energy: $\quad-785.584112$ hartree

SCF Energy + ZPVE: -785.315453 hartree

Free Energy: $\quad-785.364560$ hartree

Free Energy with quasiharmonic correction: -785.358157 hartree
$\begin{array}{llll}6 & -2.434677 & 0.898157 & 0.742710\end{array}$
$\begin{array}{llll}6 & -2.865453 & 2.217927 & 0.646141\end{array}$
$\begin{array}{llll}6 & -3.973500 & 2.536807 & -0.141428\end{array}$
$6-4.654358 \quad 1.528170-0.822121$
$6-4.229955 \quad 0.204238-0.718961$
$1-2.341114 \quad 2.997342 \quad 1.190638$
$1-5.518934 \quad 1.769720-1.432723$
$1-4.769674-0.570916-1.254083$
$\begin{array}{lllll}1 & -4.308937 & 3.566821 & -0.217198\end{array}$
$\begin{array}{llll}6 & -3.107798 & -0.120540 & 0.053690\end{array}$
$\begin{array}{lllll}7 & -1.409207 & -1.746550 & 0.463188\end{array}$ 
$\begin{array}{llll}6 & -2.633454 & -1.529657 & 0.154314\end{array}$

$6-3.613592-2.634537-0.118472$

$1-3.886258-2.636878-1.178907$

$\begin{array}{lllll}1 & -4.531412 & -2.488479 & 0.457905\end{array}$

$\begin{array}{lllll}1 & -3.174681 & -3.602838 & 0.128527\end{array}$

$\begin{array}{lllll}1 & -0.136185 & -0.816552 & 0.426054\end{array}$

$\begin{array}{lllll}1 & -1.584821 & 0.650537 & 1.371107\end{array}$

$\begin{array}{lllll}6 & 4.232145 & -0.870844 & -0.272412\end{array}$

$\begin{array}{lllll}6 & 3.044567 & -0.181758 & -0.009716\end{array}$

$\begin{array}{llll}6 & 3.060741 & 1.209096 & 0.133323\end{array}$

$\begin{array}{lllll}6 & 4.262084 & 1.905492 & 0.013468\end{array}$

$\begin{array}{lllll}6 & 5.446611 & 1.216015 & -0.249069\end{array}$

$65.431203-0.172941-0.391828$

$14.204036-1.950549-0.380903$

$\begin{array}{lllll}1 & 2.136449 & 1.739068 & 0.337296\end{array}$

$14.273936 \quad 2.985415 \quad 0.124828$

$\begin{array}{llllll}1 & 6.381724 & 1.760381 & -0.342475\end{array}$

$\begin{array}{lllll}1 & 6.352541 & -0.709747 & -0.596272\end{array}$

$\begin{array}{lllll}1 & -1.195363 & -2.743485 & 0.521860\end{array}$

$\begin{array}{lllll}6 & 1.771775 & -0.962607 & 0.111940\end{array}$

$8 \quad 1.732958-2.177041-0.014272$

$\begin{array}{lllll}8 & 0.710181 & -0.220721 & 0.365842\end{array}$

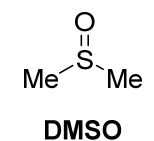

Structure: DMSO

Charge $=0$, Multiplicity $=1$

Number of imaginary frequencies: 0

SCF Energy: $\quad-553.097263$ hartree

SCF Energy + ZPVE: -553.017022 hartree

Free Energy: $\quad-553.045180$ hartree

Free Energy with quasiharmonic correction: -553.045180 hartree

$\begin{array}{llll}6 & -1.352098 & -0.795160 & 0.189660\end{array}$

$1-1.326178-1.776815-0.290295$

$1-2.285956-0.286855-0.058080$

$1-1.244702-0.887067 \quad 1.273701$

$16-0.000000 \quad 0.215521-0.447472$

$8-0.0000091 .499366 \quad 0.379121$

$\begin{array}{llllll}6 & 1.352106 & -0.795146 & 0.189661\end{array}$

$\begin{array}{lllll}1 & 1.244722 & -0.887047 & 1.273704\end{array}$

$\begin{array}{lllll}1 & 1.326176 & -1.776807 & -0.290283\end{array}$

$\begin{array}{lllll}1 & 2.285962 & -0.286848 & -0.058098\end{array}$ 


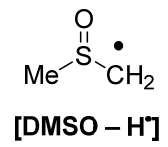

Structure: [DMSO $-\mathbf{H}^{*}$ ]

Charge $=0$, Multiplicity $=2$

Number of imaginary frequencies: 0

SCF Energy: $\quad$-552.422394 hartree

SCF Energy + ZPVE: -552.356643 hartree

Free Energy: $\quad-552.385654$ hartree

Free Energy with quasiharmonic correction: -552.385654 hartree

$\begin{array}{llll}6 & -1.549713-0.202832 & 0.199899\end{array}$

$\begin{array}{lllll}1 & -1.897861 & -1.144824 & -0.230830\end{array}$

$\begin{array}{lllll}1 & -2.187164 & 0.618901 & -0.131246\end{array}$

$\begin{array}{llll}1 & -1.517652 & -0.255313 & 1.290302\end{array}$

$\begin{array}{lllll}16 & 0.119548 & 0.139090 & -0.414815\end{array}$

$\begin{array}{lllll}8 & 0.640146 & 1.358191 & 0.333464\end{array}$

$\begin{array}{lllll}6 & 0.906741 & -1.277999 & 0.224670\end{array}$

$\begin{array}{lllll}1 & 0.472501 & -2.248279 & 0.006401\end{array}$

$\begin{array}{lllll}1 & 1.954073 & -1.176470 & 0.487288\end{array}$

$\mathrm{H}^{\bullet}$

Structure: $\mathbf{H}^{\bullet}$

Charge $=0$, Multiplicity $=2$

Number of imaginary frequencies: 0

SCF Energy: $\quad-0.495776$ hartree

SCF Energy + ZPVE: -0.495776 hartree

Free Energy: $\quad-0.506430$ hartree

Free Energy with quasiharmonic correction: -0.506430 hartree

10.0000000 .0000000 .000000 


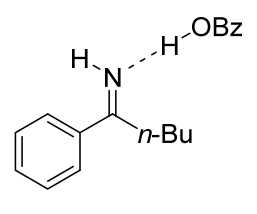

E-6I'

Structure: $\boldsymbol{E}-6 \mathbf{1}$ '

Charge $=0$, Multiplicity $=1$

Number of imaginary frequencies: 0

SCF Energy: $\quad-903.470003$ hartree

SCF Energy + ZPVE: -903.115298 hartree

Enthalpy: $\quad-903.093692$ hartree

Free Energy: $\quad-903.170461$ hartree

Free Energy with quasiharmonic correction: -903.162211 hartree
$6 \quad 3.571313-2.130876 \quad 0.170310$
$64.860593-2.627858 \quad 0.003574$
$65.933870-1.750561-0.159245$
$6 \quad 5.712734-0.374013-0.140343$

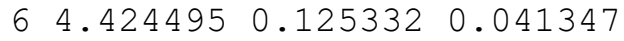
$15.028385-3.7004130 .007979$
$16.543225 \quad 0.314353-0.262588$
$14.2695891 .199686 \quad 0.058517$
$\begin{array}{lllll}1 & 6.939220 & -2.138949 & -0.291373\end{array}$
$\begin{array}{lllll}6 & 3.338014 & -0.748343 & 0.183243\end{array}$
$\begin{array}{lllll}7 & 0.907068 & -0.879729 & 0.025610\end{array}$
$\begin{array}{lllll}6 & 1.953931 & -0.214126 & 0.345585\end{array}$
$\begin{array}{llll}6 & 1.757339 & 1.188230 & 0.866288\end{array}$
$12.602437 \quad 1.489574 \quad 1.491826$
$10.860415 \quad 1.192894 \quad 1.494555$
$\begin{array}{llll}1 & -0.585015 & -0.436522 & 0.166047\end{array}$
$\begin{array}{lllll}1 & 2.750579 & -2.827721 & 0.316928\end{array}$
$6-4.824629-1.565176-0.455296$
$\begin{array}{lllll}6 & -3.851640 & -0.652362 & -0.037830\end{array}$
$\begin{array}{lllll}6 & -4.234665 & 0.592721 & 0.470289\end{array}$
$\begin{array}{lllll}6 & -5.586429 & 0.920550 & 0.559401\end{array}$
$\begin{array}{lllll}6 & -6.556235 & 0.007946 & 0.142019\end{array}$
$6-6.174591-1.235631-0.365443$
$1-4.511931-2.527776-0.847643$
$\begin{array}{llll}1 & -3.475822 & 1.297596 & 0.793059\end{array}$
$\begin{array}{lllll}1 & -5.883135 & 1.887584 & 0.953909\end{array}$
$\begin{array}{lllll}1 & -7.609033 & 0.265308 & 0.212138\end{array}$
$1-6.928630-1.945959-0.690472$
$\begin{array}{lllll}1 & 1.099031 & -1.795977 & -0.381922\end{array}$
$6-2.407653-1.039223-0.149135$
$\begin{array}{lllll}8 & -2.054150 & -2.121916 & -0.592417\end{array}$
$8-1.569870-0.1123090 .270389$
$\begin{array}{lllll}6 & 1.566311 & 2.191608 & -0.282833\end{array}$
$12.424042 \quad 2.149130-0.966878$
$\begin{array}{lllll}1 & 0.683446 & 1.896978 & -0.864559\end{array}$
$\begin{array}{llll}6 & 1.392866 & 3.619633 & 0.228066\end{array}$
$\begin{array}{llllll}1 & 0.543615 & 3.651406 & 0.922714\end{array}$
$\begin{array}{llllll}1 & 2.281807 & 3.906064 & 0.804442\end{array}$
$\begin{array}{lllll}6 & 1.169873 & 4.615947 & -0.906199\end{array}$ 
$11.0483615 .634512-0.525003$

$12.018400 \quad 4.615700-1.599329$

$10.2707314 .361653-1.478170$<smiles>N#Cc1ccncc1</smiles>

$4 a$

Structure: $\mathbf{4 a}$

Charge, Spin Multiplicity: 0, 1

Number of imaginary frequencies: 0

SCF Energy: $\quad-340.410271$ hartree

SCF Energy + ZPVE: -340.322040 hartree

Free Energy: $\quad-340.352278$ hartree

Free Energy with quasiharmonic correction: -340.352278 hartree
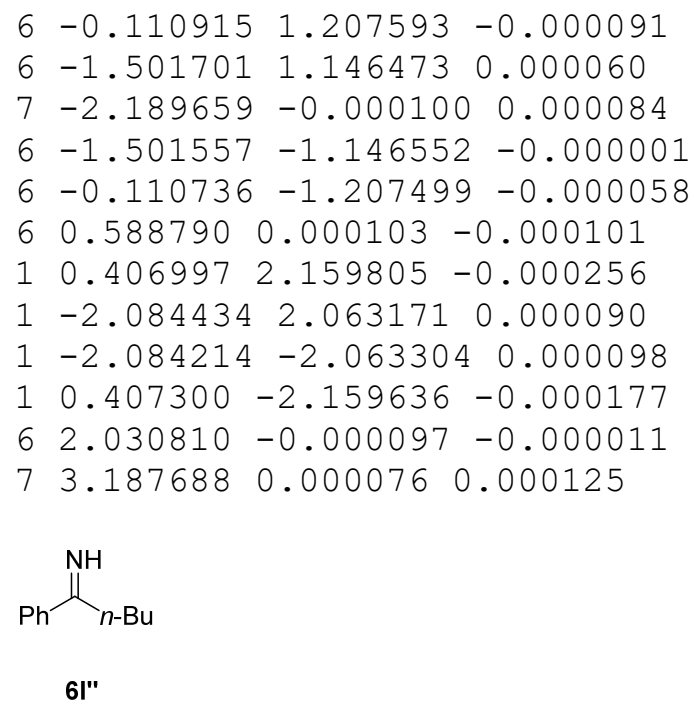

Structure: 6l',

Charge $=0$, Multiplicity $=1$

Number of imaginary frequencies: 0

SCF Energy: $\quad-482.767340$ hartree

SCF Energy + ZPVE: -482.529939 hartree

Enthalpy: $\quad-482.517093$ hartree

Free Energy: $\quad-482.568993$ hartree

Free Energy with quasiharmonic correction: -482.567357 hartree
$61.644928-1.116826-0.376437$
$\begin{array}{lll}6 & 1.296765 & 0.211409-0.097759\end{array}$
$\begin{array}{llll}6 & 2.292978 & 1.092741 & 0.344846\end{array}$
$\begin{array}{lllll}6 & 3.608638 & 0.660737 & 0.492496\end{array}$
$\begin{array}{lllll}6 & 3.949547 & -0.659468 & 0.195001\end{array}$
$\begin{array}{llll}6 & 2.964781-1.545518 & -0.240403\end{array}$
$10.892263-1.820849-0.716753$
$\begin{array}{llll}1 & 2.037238 & 2.118524 & 0.597896\end{array}$
$14.366051 \quad 1.353246 \quad 0.847157$
$\begin{array}{lllll}1 & 4.975582 & -0.996511 & 0.308074\end{array}$ 


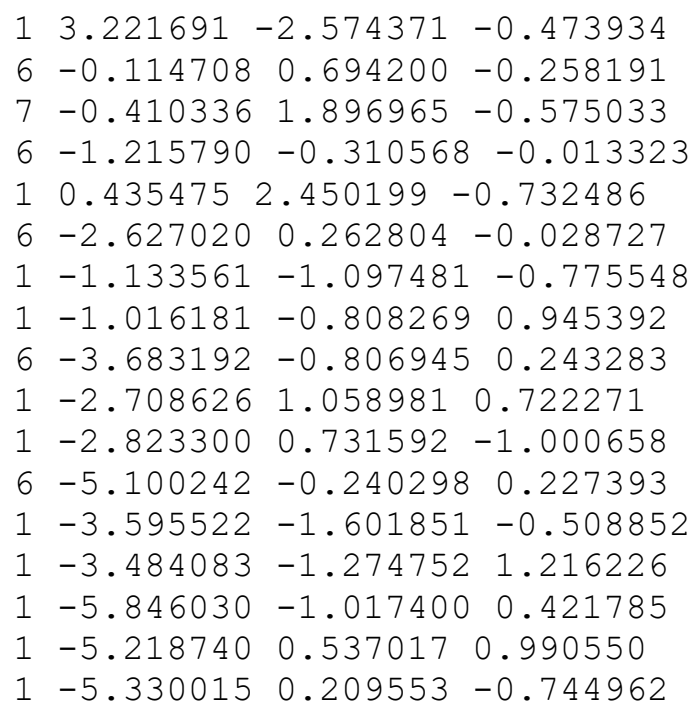<smiles>N#Cc1ccncc1</smiles>

\section{4a•HOBz}

\section{Structure: $\mathbf{4 a} \cdot \mathbf{H O B z}$}

Charge $=0$, Multiplicity $=1$

Number of imaginary frequencies: 0

SCF Energy: $\quad-761.111988$ hartree

SCF Energy + ZPVE: -760.906352 hartree

Enthalpy: $\quad-760.890660$ hartree

Free Energy: $\quad-760.951720$ hartree

Free Energy with quasiharmonic correction: -760.947475 hartree
$63.539637-1.099109-0.000001$
$62.181519-0.801044-0.000012$
$\begin{array}{lll}7 & 1.721236 & 0.454599-0.000016\end{array}$
$\begin{array}{llll}6 & 2.589152 & 1.472327-0.000010\end{array}$
63.9659851 .2826120 .000000
$64.438220-0.0311320 .000006$
$13.883134-2.126853 \quad 0.000002$
$\begin{array}{llll}1 & 1.429533 & -1.584590 & -0.000017\end{array}$
$12.1678392 .473076-0.000016$
$14.644412 \quad 2.127773 \quad 0.000004$
$\begin{array}{llll}6 & 5.858199 & -0.284796 & 0.000013\end{array}$
$\begin{array}{lllll}7 & 6.996753 & -0.488068 & 0.000020\end{array}$
$\begin{array}{lllll}1 & 0.110882 & 0.708034 & -0.000008\end{array}$
$8-0.8956220 .892638-0.000008$
$\begin{array}{llll}6 & -1.554614 & -0.253054 & -0.000007\end{array}$
$8-1.001403-1.342199-0.000013$
$6-3.042231-0.100735-0.000001$
$6-3.6436651 .1617290 .000004$
$\begin{array}{llll}6 & -3.833560 & -1.253321 & 0.000001\end{array}$
$6-5.0331501 .2675690 .000010$ 


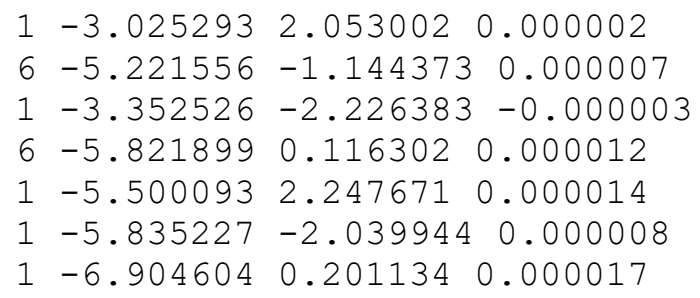<smiles>CC(C)NC(C)C</smiles>

DIPA•HOBz

Structure: DIPA $\cdot \mathbf{H O B z}$

Charge $=0$, Multiplicity $=1$

Number of imaginary frequencies: 0

SCF Energy: $\quad-713.003123$ hartree

SCF Energy + ZPVE: -712.680401 hartree

Enthalpy: $\quad-712.661543$ hartree

Free Energy: $\quad-712.729307$ hartree

Free Energy with quasiharmonic correction: -712.723736 hartree

$\begin{array}{llll}6 & -3.216242 & 0.994656 & -0.301426 \\ 1 & -4.122775 & 0.637401 & 0.201505 \\ 6 & -2.461883 & -1.258458 & 0.574630 \\ 1 & -1.579734 & -1.898150 & 0.449077 \\ 6 & -3.576313 & 1.379594 & -1.731060 \\ 1 & -4.032651 & 0.536771 & -2.261259 \\ 1 & -4.283112 & 2.214334 & -1.736943 \\ 1 & -2.677957 & 1.689247 & -2.278549 \\ 6 & -2.666387 & 2.193111 & 0.467493 \\ 1 & -3.433311 & 2.969634 & 0.551523 \\ 1 & -2.349998 & 1.914383 & 1.476623 \\ 1 & -1.803530 & 2.618299 & -0.057357 \\ 6 & -3.698439 & -2.056583 & 0.168334 \\ 1 & -3.789430 & -2.950538 & 0.793268 \\ 1 & -4.614725 & -1.468843 & 0.289909 \\ 1 & -3.627952 & -2.376877 & -0.876653 \\ 6 & -2.527361 & -0.810752 & 2.029854 \\ 1 & -1.630067 & -0.253326 & 2.316080 \\ 1 & -3.404350 & -0.181643 & 2.217283 \\ 1 & -2.603703 & -1.689854 & 2.676430 \\ 7 & -2.213436 & -0.105218 & -0.330131 \\ 1 & -0.854285 & 0.304264 & -0.078393 \\ 1 & -2.173125 & -0.484276 & -1.277435 \\ 8 & 0.196561 & 0.550516 & 0.136525 \\ 6 & 0.990690 & -0.341707 & -0.399796 \\ 8 & 0.598826 & -1.306096 & -1.049518 \\ 6 & 2.454174 & -0.098495 & -0.160113 \\ 6 & 3.384958 & -0.995782 & -0.691696 \\ 6 & 2.893892 & 1.002130 & 0.581528\end{array}$


$\begin{array}{lllll}6 & 4.747439 & -0.794629 & -0.483964\end{array}$

$\begin{array}{lllll}1 & 3.029390 & -1.845841 & -1.265557\end{array}$

$\begin{array}{lllll}6 & 4.257770 & 1.202519 & 0.788765\end{array}$

$\begin{array}{lllll}1 & 2.166828 & 1.694842 & 0.992152\end{array}$

$\begin{array}{lllll}6 & 5.184897 & 0.305133 & 0.256947\end{array}$

$\begin{array}{lllll}1 & 5.467902 & -1.493401 & -0.898304\end{array}$

$\begin{array}{lllll}1 & 4.597143 & 2.057978 & 1.365006\end{array}$

16.2472130 .4623290 .419477<smiles>CC(C)[NH2+]C(C)C</smiles>

\section{DIPA $\cdot \mathrm{H}^{+}$}

\section{Structure: DIPA $\cdot \mathbf{H}^{+}$}

Charge $=1$, Multiplicity $=1$

Number of imaginary frequencies: 0

SCF Energy: $\quad-292.767070$ hartree

SCF Energy + ZPVE: -292.545052 hartree

Enthalpy: $\quad-292.534812$ hartree

Free Energy: $\quad-292.578317$ hartree

Free Energy with quasiharmonic correction: -292.577862 hartree

$\begin{array}{llll}6 & -1.068164 & -0.027757 & -0.265583 \\ 1 & -0.537098 & -0.144499 & -1.212104 \\ 6 & 1.433178 & -0.063246 & 0.421973 \\ 1 & 1.970494 & -0.134380 & 1.371230 \\ 6 & -1.696172 & 1.353825 & -0.180787 \\ 1 & -0.951364 & 2.148460 & -0.271049 \\ 1 & -2.417718 & 1.466353 & -0.993858 \\ 1 & -2.228277 & 1.472331 & 0.769535 \\ 6 & -2.088663 & -1.142841 & -0.108653 \\ 1 & -2.837391 & -1.061133 & -0.900355 \\ 1 & -1.612815 & -2.125650 & -0.183908 \\ 1 & -2.598538 & -1.066115 & 0.857654 \\ 6 & 1.706127 & 1.285664 & -0.218838 \\ 1 & 2.780776 & 1.367535 & -0.401119 \\ 1 & 1.193077 & 1.388039 & -1.179993 \\ 1 & 1.407132 & 2.108355 & 0.437259 \\ 6 & 1.808549 & -1.241955 & -0.460814 \\ 1 & 1.552170 & -2.192476 & 0.017229 \\ 1 & 1.321763 & -1.191213 & -1.439032 \\ 1 & 2.889176 & -1.222790 & -0.623230 \\ 7 & -0.022022 & -0.174993 & 0.826567 \\ 1 & -0.138619 & -1.088220 & 1.280322 \\ 1 & -0.207747 & 0.518211 & 1.561650\end{array}$


$\overbrace{\mathrm{Me}}^{\mathrm{Me}}$

Structure: $\left[i-\mathrm{PrNH}_{2} \bullet \mathbf{4 a}\right]^{+}$

Charge $=1$, Multiplicity $=1$

Number of imaginary frequencies: 0

SCF Energy: $\quad-633.188236$ hartree

SCF Energy + ZPVE: -632.877512 hartree

Enthalpy: $\quad-632.859391$ hartree

Free Energy: $\quad-632.924526$ hartree

Free Energy with quasiharmonic correction: -632.920133 hartree
$\begin{array}{lllll}6 & 2.917961 & 1.044649 & -0.010644\end{array}$
$\begin{array}{lllll}1 & 3.724450 & 0.621115 & 0.591757\end{array}$
$\begin{array}{llll}6 & 2.326530 & -1.467821 & 0.120892\end{array}$
$\begin{array}{lllll}1 & 1.576245 & -2.109968 & -0.351075\end{array}$
$\begin{array}{llll}6 & 2.037254 & 1.936261 & 0.852524\end{array}$
$\begin{array}{llll}1 & 1.593600 & 1.383211 & 1.685312\end{array}$
$\begin{array}{lllll}1 & 2.642681 & 2.747844 & 1.264401\end{array}$
$\begin{array}{lllll}1 & 1.232849 & 2.376594 & 0.252698\end{array}$
$\begin{array}{lllll}6 & 3.510074 & 1.786130 & -1.198804\end{array}$
$\begin{array}{lllll}1 & 4.113414 & 2.624218 & -0.841083\end{array}$
$\begin{array}{lllll}1 & 4.151570 & 1.127422 & -1.792845\end{array}$
$\begin{array}{lllll}1 & 2.715603 & 2.182099 & -1.840845\end{array}$
$\begin{array}{lllll}6 & 2.058410 & -1.399419 & 1.615396\end{array}$
$\begin{array}{lllll}1 & 2.125916 & -2.410415 & 2.025524\end{array}$
$\begin{array}{lllll}1 & 2.796444 & -0.777201 & 2.131120\end{array}$
$11.056081-1.012242 \quad 1.824058$
$\begin{array}{llll}6 & 3.716531 & -1.980072 & -0.219467\end{array}$
$13.879971-1.990012-1.301474$
$\begin{array}{lllll}1 & 4.497200 & -1.375300 & 0.251728\end{array}$
$\begin{array}{lllll}1 & 3.816061 & -3.003686 & 0.150702\end{array}$
$\begin{array}{lllll}7 & 2.097954 & -0.121541 & -0.521057\end{array}$
$12.262983-0.234782-1.527026$
$\begin{array}{lllll}1 & 1.074044 & 0.096027 & -0.417917\end{array}$
$\begin{array}{lllll}7 & -0.738643 & 0.129301 & -0.199972\end{array}$
$\begin{array}{lllll}6 & -1.398226 & 0.665250 & 0.832091\end{array}$
$6-1.434062-0.475533-1.168815$
$\begin{array}{llll}6 & -2.783821 & 0.623630 & 0.945236\end{array}$
$\begin{array}{llll}1 & -0.797175 & 1.147285 & 1.598261\end{array}$
$\begin{array}{llll}6 & -2.821247 & -0.573795 & -1.155744\end{array}$
$1-0.860066-0.898891-1.988588$
$6-3.498193-0.009343-0.073507$
$1-3.2872091 .068768 \quad 1.795590$
$1-3.353218-1.070810-1.958567$
$6-4.937364-0.080430-0.007926$
$\begin{array}{lllll}7 & -6.091387 & -0.137519 & 0.044012\end{array}$ 


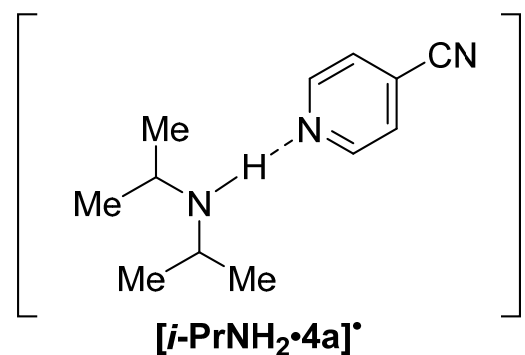

Structure: $\left[i-\operatorname{PrNH}_{2} \bullet \mathbf{A a}^{\circ}\right.$

Charge $=0$, Multiplicity $=2$

Number of imaginary frequencies: 0

SCF Energy: $\quad-633.289051$ hartree

SCF Energy + ZPVE: -632.983122 hartree

Enthalpy: $\quad-632.964847$ hartree

Free Energy: $\quad-633.031051$ hartree

Free Energy with quasiharmonic correction: -633.026422 hartree

$62.849962 \quad 1.025988 \quad 0.004607$

$\begin{array}{llll}1 & 3.691292 & 0.584051 & 0.544900\end{array}$

$62.243108-1.466904 \quad 0.084041$

$\begin{array}{lllll}1 & 1.466133 & -2.091944 & -0.369455\end{array}$

$62.0358501 .896416 \quad 0.953569$

11.6267401 .3154281 .784905

$\begin{array}{llll}1 & 2.678760 & 2.678632 & 1.366623\end{array}$

$\begin{array}{llll}1 & 1.207301 & 2.375789 & 0.420977\end{array}$

$\begin{array}{lllll}6 & 3.378914 & 1.816679 & -1.183150\end{array}$

$\begin{array}{lllll}1 & 3.996977 & 2.646292 & -0.830057\end{array}$

$\begin{array}{llll}1 & 3.989790 & 1.184023 & -1.835428\end{array}$

$\begin{array}{llll}1 & 2.549702 & 2.230185 & -1.768243\end{array}$

$\begin{array}{llll}6 & 2.038413 & -1.445615 & 1.591324\end{array}$

$\begin{array}{lllll}1 & 2.110104 & -2.468804 & 1.969884\end{array}$

$\begin{array}{lllll}1 & 2.804966 & -0.847272 & 2.094188\end{array}$

$\begin{array}{lllll}1 & 1.051180 & -1.052101 & 1.853558\end{array}$

$\begin{array}{llll}6 & 3.610327 & -1.996445 & -0.322481\end{array}$

$13.729888-1.983141-1.410511$

$\begin{array}{lllll}1 & 4.419897 & -1.413264 & 0.127462\end{array}$

$\begin{array}{lllll}1 & 3.712006 & -3.029958 & 0.019794\end{array}$

$\begin{array}{llll}7 & 1.998885 & -0.107179 & -0.503036\end{array}$

$\begin{array}{lllll}1 & 2.128164 & -0.191232 & -1.515519\end{array}$

$\begin{array}{lllll}1 & 0.908134 & 0.106982 & -0.349294\end{array}$

$\begin{array}{lllll}7 & -0.621815 & 0.176173 & -0.197237\end{array}$

$6-1.323588 \quad 0.5858790 .904146$

$6-1.360239-0.331174-1.232109$

$\begin{array}{llll}6 & -2.686422 & 0.521316 & 1.016682\end{array}$

$\begin{array}{lllll}1 & -0.728151 & 0.984053 & 1.723329\end{array}$

$6-2.724607-0.442320-1.227269$

$\begin{array}{lllll}1 & -0.789660 & -0.657937 & -2.099870\end{array}$

$6-3.464612-0.008138-0.071875$

$\begin{array}{lllll}1 & -3.172599 & 0.867153 & 1.923349\end{array}$

$\begin{array}{lllll}1 & -3.239398 & -0.855792 & -2.088601\end{array}$

$6-4.858461-0.097501-0.009814$

$\begin{array}{lllll}7 & -6.030368 & -0.173311 & 0.041985\end{array}$ 


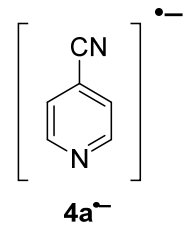

Structure: $\mathbf{4 a}^{\cdot-}$

Charge $=-1$, Multiplicity $=2$

Number of imaginary frequencies: 0

SCF Energy: $\quad-340.502002$ hartree

SCF Energy + ZPVE: -340.416655 hartree

Free Energy: $\quad-340.447861$ hartree

Free Energy with quasiharmonic correction: -340.447861 hartree
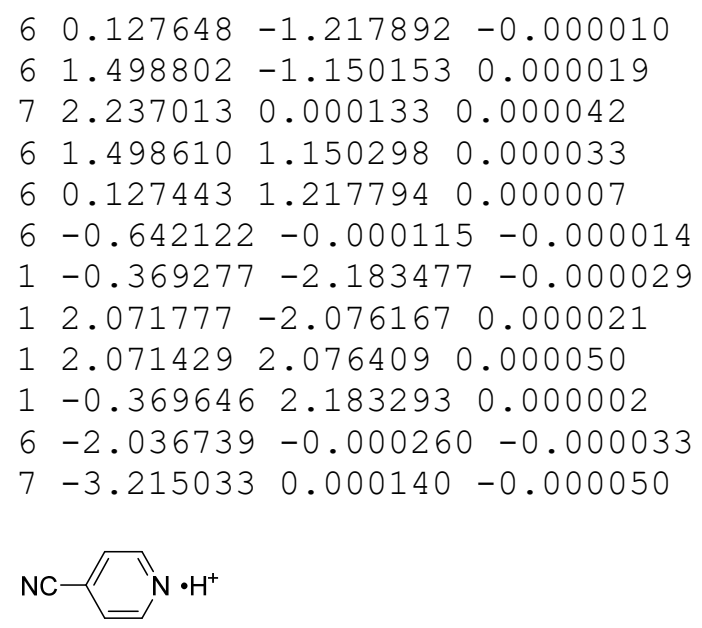

$4 \mathrm{a} \cdot \mathrm{H}^{+}$

Structure: $\mathbf{4 a} \cdot \mathbf{H}^{+}$

Charge $=1$, Multiplicity $=1$

Number of imaginary frequencies: 0

SCF Energy: $\quad-340.855358$ hartree

SCF Energy + ZPVE: -340.753396 hartree

Free Energy: $\quad-340.783778$ hartree

Free Energy with quasiharmonic correction: -340.783778 hartree
60.0589751 .2164340 .000010
$61.4405901 .184402-0.000027$
$72.070626-0.000003-0.000054$
$61.440586-1.184405-0.000044$
$60.058970-1.216432-0.000006$
$6-0.6280460 .0000030 .000018$
$1-0.464048 \quad 2.1645340 .000030$
$\begin{array}{llll}1 & 2.062800 & 2.070229 & -0.000037\end{array}$
$12.062793-2.070235-0.000065$
$1-0.464056-2.164530 \quad 0.000001$
$6-2.0709910 .000006 \quad 0.000051$
$\begin{array}{llll}7 & -3.226357 & -0.000004 & 0.000074\end{array}$
$13.092125-0.000005-0.000084$ 


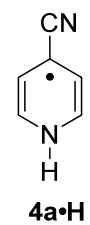

Structure: $\mathbf{4 a} \cdot \mathbf{H}$

Charge $=0$, Multiplicity $=2$

Number of imaginary frequencies: 0

SCF Energy: -340.988685 hartree

SCF Energy + ZPVE: -340.889906 hartree

Free Energy: -340.921302 hartree

Free Energy with quasiharmonic correction: -340.921302 hartree

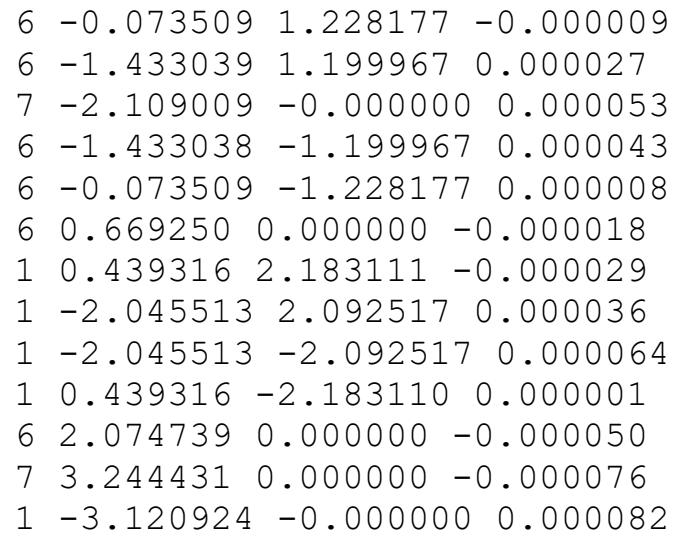

$[\underbrace{-\cdot}_{\mathrm{CN}}$

\section{$[4 \mathrm{a} \cdot \mathrm{HOBz}]^{\cdot-}$}

Structure: [4a-HOBz] ${ }^{-}$

Charge $=-1$, Multiplicity $=2$

Number of imaginary frequencies: 0

SCF Energy: $\quad-761.219615$ hartree

SCF Energy + ZPVE: -761.016945 hartree

Enthalpy: $\quad-761.000778$ hartree

Free Energy: $\quad-761.064441$ hartree

Free Energy with quasiharmonic correction: -761.058957 hartree

$\begin{array}{llll}6 & 3.505073-1.128019 & -0.000008\end{array}$

$\begin{array}{lllll}6 & 2.171414 & -0.848969 & 0.000001\end{array}$

$\begin{array}{lllll}7 & 1.712359 & 0.444286 & 0.000012\end{array}$

$\begin{array}{lllll}6 & 2.598606 & 1.492491 & 0.000005\end{array}$

$\begin{array}{llll}6 & 3.945101 & 1.286522 & -0.000003\end{array}$

$64.461295-0.054378-0.000006$

$13.836846-2.160309-0.000017$ 


$\begin{array}{llll}1 & 1.395022 & -1.605538 & 0.000000 \\ 1 & 2.161021 & 2.484378 & 0.000006 \\ 1 & 4.618004 & 2.136771 & -0.000009 \\ 6 & 5.841557 & -0.304915 & -0.000005 \\ 7 & 6.994363 & -0.514831 & -0.000005 \\ 1 & 0.671426 & 0.634362 & 0.000015 \\ 8 & -0.914328 & 0.996751 & 0.000022 \\ 6 & -1.517093 & -0.119849 & 0.000009 \\ 8 & -0.969892 & -1.243250 & -0.000001 \\ 6 & -3.039629 & -0.067027 & 0.000003 \\ 6 & -3.710462 & 1.159602 & -0.000008 \\ 6 & -3.782458 & -1.251263 & 0.000007 \\ 6 & -5.104669 & 1.202897 & -0.000015 \\ 1 & -3.125450 & 2.073923 & -0.000011 \\ 6 & -5.176426 & -1.211984 & 0.000001 \\ 1 & -3.252007 & -2.198494 & 0.000015 \\ 6 & -5.840312 & 0.016372 & -0.000010 \\ 1 & -5.618067 & 2.160268 & -0.000025 \\ 1 & -5.745927 & -2.137068 & 0.000006 \\ 1 & -6.926145 & 0.048634 & -0.000015\end{array}$<smiles>CC(C)N(C(C)C)[C@H](Cl)[n+]1ccc(C#N)cc1</smiles>

\section{[DIPA...4a・H']}

(optimized with constraint $d=1.02 \AA$ )

The complex [DIPA...4a $\mathbf{a}^{\bullet} \mathbf{H}^{+}$] was obtained by a constrained optimization with the $\mathrm{N}^{b}-\mathrm{H}$ distance fixed at $1.02 \AA$, the bond length found in the optimized structure of 4-cyanopyridinium ion $\left(\mathbf{4} \mathbf{a} \cdot \mathbf{H}^{+}\right)$. Submitting the resultant geometry of [DIPA...4a $\mathbf{a}^{\cdot} \mathbf{H}^{+}$] to another optimization without any distance constraint led back to $\left[i-\operatorname{PrNH}_{2} \bullet 4\right]^{+}$.

Structure: [DIPA...4a・ $\mathbf{H}^{+}$]

Charge $=1$, Multiplicity $=1$

SCF Energy: $\quad-633.168238$ hartree
$62.8442141 .029460-0.044055$
13.7290950 .6359860 .472549
$62.281226-1.4260830 .100561$
$\begin{array}{lllll}1 & 1.517071 & -2.096079 & -0.315391\end{array}$
$\begin{array}{llll}6 & 2.072641 & 1.922347 & 0.925032\end{array}$
$\begin{array}{llll}1 & 1.696622 & 1.352598 & 1.780447\end{array}$
$\begin{array}{lllll}1 & 2.715380 & 2.723153 & 1.305166\end{array}$
$\begin{array}{lllll}1 & 1.217989 & 2.383528 & 0.414162\end{array}$
$\begin{array}{lllll}6 & 3.313520 & 1.824072 & -1.257313\end{array}$
$\begin{array}{lllll}1 & 3.906135 & 2.689450 & -0.946623\end{array}$
$\begin{array}{lllll}1 & 3.928686 & 1.204027 & -1.918337\end{array}$
$\begin{array}{lllll}1 & 2.451091 & 2.189514 & -1.828124\end{array}$
$\begin{array}{llll}6 & 2.108638 & -1.399827 & 1.615517\end{array}$ 


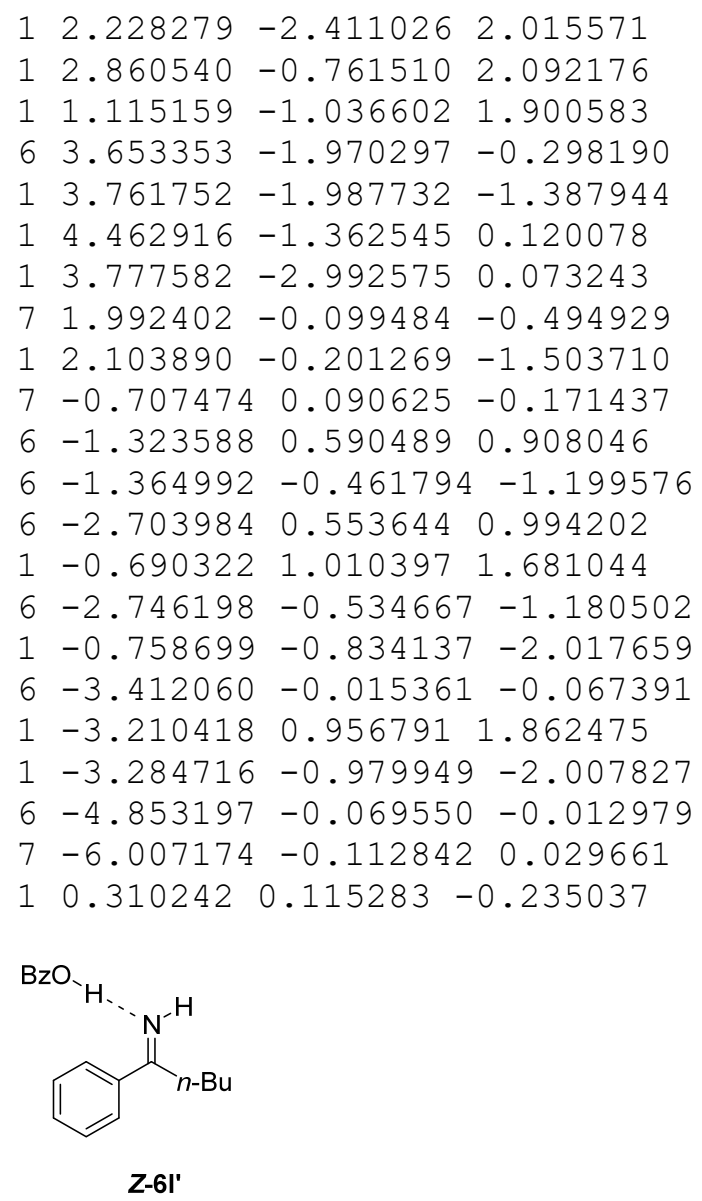

Structure: $\boldsymbol{Z}-6 \mathbf{l}$ '

Charge $=0$, Multiplicity $=1$

SCF Energy: $\quad-903.471306$ hartree

SCF Energy + ZPVE: -903.116823 hartree

Enthalpy: $\quad-903.095176$ hartree

Free Energy: $\quad-903.171887$ hartree

Free Energy with quasiharmonic correction: -903.163717 hartree
61.1902431 .6417960 .674165
$\begin{array}{llll}6 & 1.284471 & 3.025288 & 0.797981\end{array}$
$\begin{array}{lllll}6 & 2.207373 & 3.735069 & 0.028086\end{array}$
$63.0428593 .053550-0.856718$
$\begin{array}{llll}6 & 2.957728 & 1.667397 & -0.973521\end{array}$
$\begin{array}{lllll}1 & 0.642027 & 3.548567 & 1.499663\end{array}$
$13.7653193 .599976-1.455183$
$13.6165921 .150443-1.664778$
$\begin{array}{lllll}1 & 2.280385 & 4.814290 & 0.123641\end{array}$
$\begin{array}{lllll}6 & 2.019956 & 0.950511 & -0.219045\end{array}$
$\begin{array}{lllll}7 & 0.756911 & -1.068969 & -0.122197\end{array}$
$\begin{array}{lllll}6 & 1.895987 & -0.529298 & -0.353357\end{array}$
$63.118616-1.329620-0.721539$
$\begin{array}{llll}1 & 3.500611 & -0.979785 & -1.688306\end{array}$
$12.819689-2.375156-0.849633$
$\begin{array}{lllll}1 & -0.689778 & -0.461492 & 0.026145\end{array}$
$\begin{array}{lllll}1 & 0.483847 & 1.088960 & 1.285859\end{array}$ 

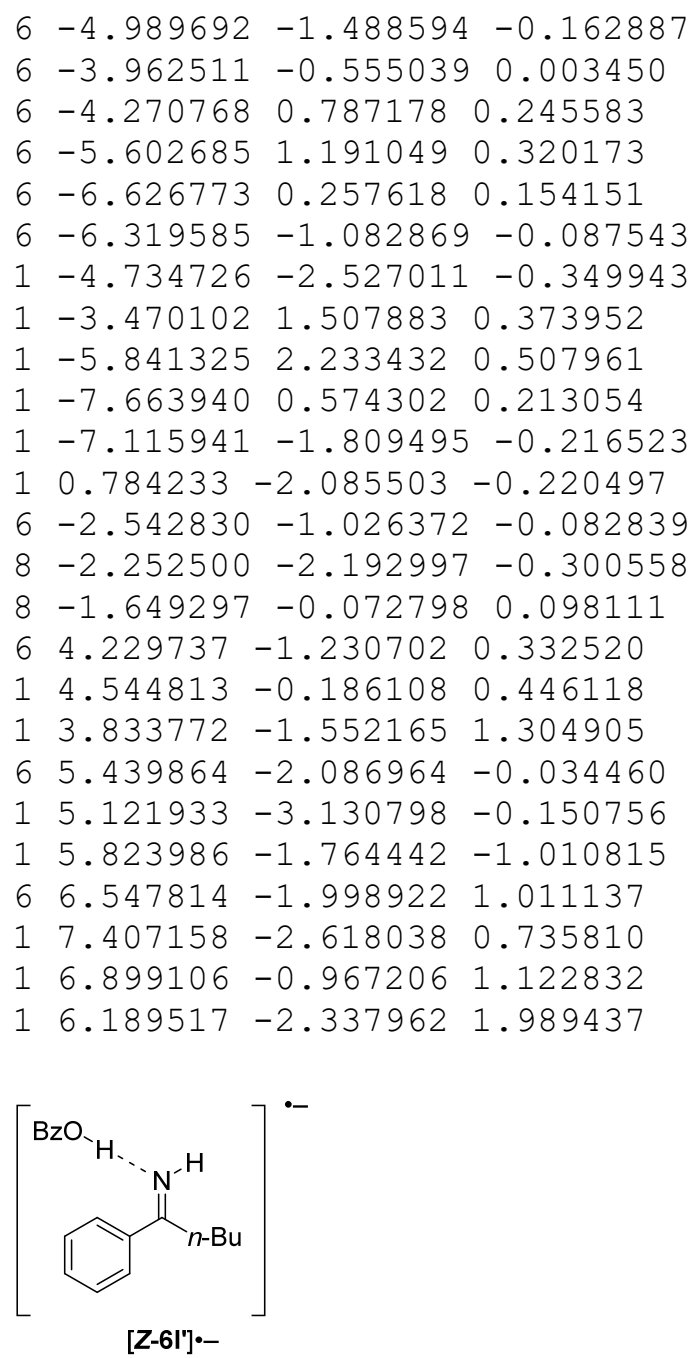

Structure: $\left[\boldsymbol{Z}-6 \mathbf{l}^{\prime}\right]^{-}$

Charge $=-1$, Multiplicity $=2$

SCF Energy: -903.570946 hartree

SCF Energy + ZPVE: -903.219410 hartree

Enthalpy: -903.197009 hartree

Free Energy: -903.276338 hartree

Free Energy with quasiharmonic correction: -903.267578 hartree

$\begin{array}{llll}6 & 1.172807 & 2.055873 & 0.002198 \\ 6 & 1.401574 & 3.396694 & 0.274135 \\ 6 & 2.694949 & 3.937387 & 0.249093 \\ 6 & 3.769008 & 3.086214 & -0.057169 \\ 6 & 3.564643 & 1.743869 & -0.331354 \\ 1 & 0.555204 & 4.037360 & 0.511081 \\ 1 & 4.781953 & 3.480839 & -0.077947 \\ 1 & 4.423866 & 1.118825 & -0.555441 \\ 1 & 2.862845 & 4.987783 & 0.464398 \\ 6 & 2.253268 & 1.172238 & -0.318813 \\ 7 & 0.794903 & -0.751729 & -0.531043 \\ 6 & 2.036738 & -0.201069 & -0.606901 \\ 6 & 3.160265 & -1.149320 & -0.918634\end{array}$


$13.905966-0.664286-1.559214$

$12.758203-1.994406-1.492066$

$\begin{array}{lllll}1 & -0.071126 & -0.243468 & -0.316675\end{array}$

$\begin{array}{lllll}1 & 0.153170 & 1.680549 & 0.032060\end{array}$

$\begin{array}{llll}6 & -4.850898 & -1.509990 & -0.219871\end{array}$

$\begin{array}{lllll}6 & -3.984426 & -0.436551 & 0.007279\end{array}$

$\begin{array}{lllll}6 & -4.516579 & 0.794106 & 0.403584\end{array}$

$\begin{array}{lllll}6 & -5.892606 & 0.950789 & 0.571403\end{array}$

$6-6.751454-0.125846 \quad 0.341881$

$6-6.227621-1.357908-0.054750$

$\begin{array}{lllll}1 & -4.428287 & -2.461747 & -0.527055\end{array}$

$\begin{array}{llll}1 & -3.836369 & 1.622065 & 0.577571\end{array}$

$1-6.296094 \quad 1.9109890 .880648$

$\begin{array}{lllll}1 & -7.823272 & -0.005361 & 0.471682\end{array}$

$\begin{array}{lllll}1 & -6.892532 & -2.198098 & -0.234734\end{array}$

$\begin{array}{lllll}1 & 0.661451 & -1.714679 & -0.804675\end{array}$

$6-2.476485-0.609499-0.176630$

$8-2.064491-1.742341-0.513605$

$8-1.7655490 .416000 \quad 0.028768$

$\begin{array}{lllll}6 & 3.854212 & -1.692353 & 0.341097\end{array}$

$\begin{array}{lllll}1 & 4.252160 & -0.853789 & 0.928280\end{array}$

$\begin{array}{lllll}1 & 3.107137 & -2.193628 & 0.971304\end{array}$

$\begin{array}{llllll}6 & 4.984802 & -2.665301 & 0.015293\end{array}$

$14.584846-3.496213-0.580466$

$\begin{array}{lllll}1 & 5.725636 & -2.156593 & -0.615538\end{array}$

$\begin{array}{lllll}6 & 5.664292 & -3.211371 & 1.268224\end{array}$

$16.472869-3.9050261 .017058$

$16.092793-2.399027 \quad 1.865796$

$\begin{array}{lllll}1 & 4.946637 & -3.747047 & 1.899535\end{array}$ 


\section{References}

(1) (a) Gottlieb, H. E.; Kotlyar, V.; Nudelman, A. NMR Chemical Shifts of Common Laboratory Solvents as Trace Impurities. J. Org. Chem. 1997, 62, 7512-7515. (b) Fulmer, G. R.; Miller, A. J. M. Sherden, N. H.; Gottlieb, H. E.; Nudelman, A.; Stoltz, B. M. Bercaw, J. E.; Goldberg, K. I. NMR Chemical Shifts of Trace Impurities: Common Laboratory Solvents, Organics, and Gases in Deuterated Solvents Relevant to the Organometallic Chemist. Organometallics 2010, 29, 2176-2179.

(2) DiRocco, D. A.; Dykstra, K.; Krska, S.; Vachal, P.; Conway, D. V.; Tudge, M. Late-Stage Functionalization of Biologically Active Heterocycles Through Photoredox Catalysis. Angew. Chem. int. Ed. 2014, 53, 4802-4806.

(3) The Photoreactor TAK120. http://wppr.photoreactor.de/tak120 (accessed August 28, 2019).

(4) Le, C.; Wismer, M. K.; Shi, Z.-C.; Zhang, R.; Conway, D. V.; Li, G.; Vachal, P.; Davies, I. W.; MacMillan, D. W. C. A General Small Scale Reactor To Enable Standardization and Acceleration of Photocatalytic Reactions. $A C S$ Cent. Sci. 2017, 3, 647-653.

(5) Lehnherr, D.; Nicastri, M. C.; Lam, Y.-h.; Liu, J.; Newman, J. A.; Regalado, E. L.; DiRocco, D. A.; Rovis, T. Electrochemical Synthesis of Hindered Primary- and Secondary-Amines via Proton-Coupled Electron Transfer. J. Am. Chem. Soc., accepted.

(6) Sebhat, I. K.; Franklin, C.; Lo, M. M. C.; Chen, D.; Jewell, J. P.; Miller, R.; Pang, J.; Palyha, O.; Kan, Y.; Kelly, T. M.; Guan, X.M; Marsh, D.J.; Kosinski, J.A.; Metzger, J.M.; Lyons, K.; Dragovic, J.; Guzzo, P.R.; Henderson, A.J.; Reitman, M.L; Nargund, R.P.; Wyvratt, M.J.; Lin, L.S. Discovery of MK-5046, a Potent, Selective Bombesin Receptor Subtype-3 Agonist for the Treatment of Obesity. ACS Med. Chem. Lett. 2011, $2,43-47$.

(7) G. Hou, F. Gosselin, W. Li, J. C. McWilliams, Y. Sun, M. Weisel, P. D. O’Shea, C.-y. Chen, I. W. Davies, X. Zhang, Enantioselective Hydrogenation of N-H Imines. J. Am. Chem. Soc. 2009, 131, 9882-9883.

(8) Arias-Rotondo D. M.; McCusker, J. K. The photophysics of photoredox catalysis: a roadmap for catalyst design. Chem. Soc. Rev. 2016, 45, 5803-5820.

(9) Ochola, J. R.; Wolf, M. O. The effect of photocatalyst excited state lifetime on the rate of photoredox catalysis. Org. Biomol. Chem. 2016, 14, 9088-9092.

(10) Gaussian 16, Revision A.03, Frisch, M. J.; Trucks, G. W.; Schlegel, H. B.; Scuseria, G. E.; Robb, M. A.; Cheeseman, J. R.; Scalmani, G.; Barone, V.; Petersson, G. A.; Nakatsuji, H.; Li, X.; Caricato, M.; Marenich, A. V.; Bloino, J.; Janesko, B. G.; Gomperts, R.; Mennucci, B.; Hratchian, H. P.; Ortiz, J. V.; Izmaylov, A. F.; Sonnenberg, J. L.; Williams-Young, D.; Ding, F.; Lipparini, F.; Egidi, F.; Goings, J.; Peng, B.; Petrone, A.; Henderson, T.; Ranasinghe, D.; Zakrzewski, V. G.; Gao, J.; Rega, N.; Zheng, G.; Liang, W.; Hada, M.; Ehara, M.; Toyota, K.; Fukuda, R.; Hasegawa, J.; Ishida, M.; Nakajima, T.; Honda, Y.; Kitao, O.; Nakai, H.; Vreven, T.; Throssell, K.; Montgomery, J. A., Jr.; Peralta, J. E.; Ogliaro, F.; Bearpark, M. J.; Heyd, J. J.; Brothers, E. N.; Kudin, K. N.; Staroverov, V. N.; Keith, T. A.; Kobayashi, R.; Normand, J.; Raghavachari, K.; Rendell, A. P.; Burant, J. C.; Iyengar, S. S.; Tomasi, J.; Cossi, M.; Millam, J. M.; Klene, M.; Adamo, C.; Cammi, R.; Ochterski, J. W.; Martin, R. L.; Morokuma, K.; Farkas, O.; Foresman, J. B.; Fox, D. J. Gaussian, Inc., Wallingford CT, 2016.

(11) Zhao, Y.; Truhlar, D. G. The M06 suite of density functionals for main group thermochemistry, thermochemical kinetics, noncovalent interactions, excited states, and transition elements: two new functionals and systematic testing of four M06-class functionals and 12 other functionals. Theor. Chem. Acc. 2008, 120, $215-241$.

(12) Rassolov, V. A.; Ratner, M. A.; Pople, J. A.; Redfern, P. C.; Curtiss, L.A. J. Comp. Chem. 2001, 22, 976-984.

(13) Marenich, A. V.; Cramer, C. J.; Truhlar, D. G. Universal Solvation Model Based on Solute Electron Density and on a Continuum Model of the Solvent Defined by the Bulk Dielectric Constant and Atomic Surface Tensions $J$. Phys. Chem. B, 2009, 113, 6378-6396.

(14) Ribeiro, R. F.; Marenich, A. V.; Cramer, C. J.; Truhlar, D. G. Use of Solution-Phase Vibrational Frequencies in Continuum Models. J. Phys. Chem. B 2011, 115, 14556-14562.

(15) CYLview, 1.0.561 BETA; Legault, C. Y., Université de Sherbrooke, 2009 (http://www.cylview.org).

(16) Persistence of Vision Pty. Ltd. (2004), Persistence of Vision Raytracer (Version 3.6), [Computer software]. Retrieved from http://www.povray.org/download/

(17) Marenich, A. V.; Ho, J.; Coote, M. L.; Cramer, C. J.; Truhlar, D. G. Computational electrochemistry: prediction of liquid-phase reduction potentials. Phys. Chem. Chem. Phys. 2014, 16, 15068-15106.

(18) Pavlishchuk, V. V.; Addison, A. W. Conversion constants for redox potentials measured versus different reference electrodes in acetonitrile solutions at $25^{\circ} \mathrm{C}$. Inorg. Chim. Acta 2000, 298, 97-101. 